\title{
Revised composite depth scales and integration of IODP Sites U1331-U1334 and ODP Sites 1218-1220'
}

Thomas Westerhold, ${ }^{2}$ Ursula Röhl, ${ }^{2}$ Roy Wilkens, ${ }^{3}$ Heiko Pälike, ${ }^{4}$ Mitch Lyle, ${ }^{5}$ Tom Dunkley Jones, ${ }^{6}$ Paul Bown, ${ }^{7}$ Ted Moore, ${ }^{8}$ Shin-ichi Kamikuri, ${ }^{9}$ Gary Acton, ${ }^{10}$ Christian Ohneiser, ${ }^{11}$ Yuhji Yamamoto, ${ }^{12}$ Carl Richter, ${ }^{13}$ Peter Fitch, ${ }^{6}$ Howie Scher, ${ }^{14}$ Diederik Liebrand, ${ }^{4}$ and the Expedition 320/321 Scientists ${ }^{15}$

\section{Chapter contents}

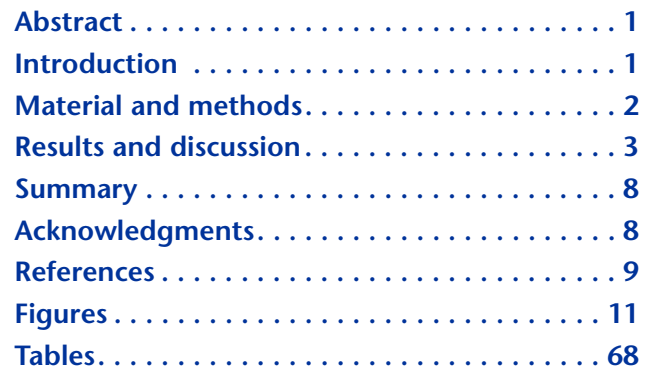

${ }^{1}$ Westerhold, T., Röhl, U., Wilkens, R., Pälike, H., Lyle, M., Jones, T.D., Bown, P., Moore, T., Kamikuri, S., Acton, G., Ohneiser, C., Yamamoto, Y., Richter, C., Fitch, P., Scher, H., Liebrand, D., and the Expedition 320/321 Scientists, 2012. Revised composite depth scales and integration of IODP Sites U1331-U1334 and ODP Sites 1218-1220. In Pälike, H., Lyle, M., Nishi, H., Raffi, I., Gamage, K., Klaus, A., and the Expedition 320/321 Scientists, Proc. IODP, 320/321: Tokyo (Integrated Ocean Drilling Program Management International, Inc.).

doi:10.2204/iodp.proc.320321.201.2012

${ }^{2}$ Center for Marine Environmental Sciences, University of Bremen, 28359 Germany. Correspondence author: twesterhold@marum.de

${ }^{3}$ Institute of Geophysics and Planetology, Univ. Hawaii, Honolulu HI 96822, USA.

${ }^{4}$ School of Ocean \& Earth Sci., Univ. Southampton S014 3ZH, UK.

${ }^{5}$ Dept. of Oceanography, Texas A\&M Univ., College Station TX 77845, USA.

${ }^{6}$ Dept. of Earth Sci. and Engineering, Imperial College, London SW7 2AZ, UK.

${ }^{7}$ Dept. of Earth Sci., Univ. College, London WC1E 6BT, UK.

${ }^{8}$ Dept. of Geological Sci., Univ. Michigan, Ann Arbor MI 48109, USA.

${ }^{9}$ Univ. Tsukuba, Ibaraki 305-8572, Japan.

${ }^{10}$ Dept. of Geology, Univ. California, Davis CA 95616, USA.

${ }^{11}$ Geology Dept., Univ. Otago, Dunedin, NZ.

${ }^{12}$ Marine and Core Research Center, Kochi Univ., Kochi 783-8502, Japan.

${ }^{13}$ Dept. of Geology and Energy Institute, Univ. Louisiana, Lafayette LA 70504, USA.

${ }^{14}$ Dept. of Earth and Ocean Sci., Univ. South Carolina, Columbia SC 29208, USA.

${ }^{15}$ Expedition 320/321 Scientists' addresses.

\section{Abstract}

To reconstruct the climate history of the equatorial Pacific, one major objective of the Pacific Equatorial Age Transect (PEAT) program is to compile a Cenozoic megasplice that integrates all available bio-, chemo-, and magnetostratigraphic data including key records from Ocean Drilling Program (ODP) Leg 199. In order to do so, extended postcruise refinements of the shipboard composite depth scales and composite records are required. Here, we present a revised depth scale of Integrated Ocean Drilling Program (IODP) Expedition 320 Sites U1331, U1332, U1333, and U1334 as well as Leg 199 Sites 1218, 1219, and 1220. The revised composite records were used to perform site-to-site correlation and integration of Leg 199 and Expedition 320 sites. Based on this decimeterscale correlation, a high-resolution integrated paleomagnetic, calcareous nannofossil, and radiolarian stratigraphy for the equatorial Pacific is established that covers the time from 20 to $40 \mathrm{Ma}$. This sedimentary compendium from the equatorial Pacific will be the backbone for paleoceanographic reconstructions for the late Paleogene.

\section{Introduction}

Integrated Ocean Drilling Program (IODP) Expedition 320/321 (Pacific Equatorial Age Transect [PEAT]) cored eight sites (U1331U1338) during March-July in 2009 (Fig. F1), recovering an age transect at the Pacific paleoequatorial region from the time of maximum Cenozoic warmth in the Eocene, through initial major glaciations in the Oligocene, to the present (see the "Expedition 320/321 summary" chapter [Expedition 320/321 Scientists, 2010a]; Lyle et al., 2009). The overall aim was to obtain continuous and well-preserved calcareous sediment sections for specific time slices. Of major importance for the objectives of the PEAT expeditions is the assembly of an integrated bio-, chemo-, and magnetostratigraphy at the Equator, hereafter referred to as the Cenozoic megasplice.

In addition to those from the PEAT drill sites, key records for the breakthrough in reconstructing the equatorial climate system of the late Eocene and Oligocene epochs were recovered during Ocean Drilling Program (ODP) Leg 199 (Lyle, Wilson, Janecek, et al., 2002), Site 1218 in particular (Fig. F1). Data from Site 1218 allowed astronomical calibration of the entire Oligocene (Wade and 
Pälike, 2004; Coxall et al., 2005; Pälike et al., 2006), but the lack of carbonate in the uppermost Eocene at this site made detailed time control much less robust. Although the paleomagnetic record for these time intervals is of high quality (e.g., Lanci et al., 2004, 2005), global stratigraphic correlation is hindered by the lower mass accumulation rate, the absence of a detailed isotope stratigraphy, and low-resolution biostratigraphic control. In order to facilitate the development of an integrated magneto- and biostratigraphic framework with a stable isotope stratigraphy (necessary to enable global correlation), recovery of Eocene carbonate sediment with a highquality magnetostratigraphy was targeted and successfully retrieved during Expedition 320.

During Expedition 320/321, at least three holes at each site were cored and used to construct composite sections (see the "Methods" chapter [Expedition $320 / 321$ Scientists, 2010b]) in order to assure the recovery of a complete stratigraphic section needed for the assembly of the Cenozoic megasplice. As shown at ODP Leg 199 Sites 1218 and 1219 (Pälike et al., 2005), extensive postcruise work is required to reevaluate the shipboard composite depth stratigraphy and to provide a high-resolution revised meters composite depth (rmcd) scale. In addition, squeezing and stretching of cored intervals is necessary to compensate for depth distortion within individual cores (Hagelberg et al., 1992). To locate hiatuses and condensed intervals, it is essential to do a site-to-site correlation using physical property data (Shackleton et al., 1995, 1999; Shackleton and Crowhurst, 1997; Pälike et al., 2005; Westerhold and Röhl, 2006; Westerhold et al., 2007, 2008). Subsequently, the correlation allows integration of any kind of data from one site to another. A prerequisite for correlation is that decimeter-scale features in the sedimentary record can be correlated between holes and, if possible, between sites.

Both Leg 199 and Expedition 320/321 magnetic susceptibility and gamma ray attenuation (GRA) bulk density data can be correlated over large distances $(>1000 \mathrm{~km})$ across the Pacific seafloor (Pälike et al., 2005,2009 ). Physical property data, a proxy for calcium carbonate oscillations, at Sites U1331 and U1332 show a remarkable match with those from ODP Site 1220, a site with an excellent magnetostratigraphy. Similarly, Sites U1333 and U1334 can be correlated to Site 1218. Together, these sites provide a coherent and integrated record for the equatorial Pacific and enable study of sedimentation patterns and mass accumulation rates at orbital resolution (Pälike et al., 2009). Here, we present revised composite depth scales and revised spliced composite records for Expedition 320 Sites U1331,
U1332, U1333, and U1334 and Leg 199 Sites 1218, 1219, and 1220 (Fig. F1). We also correlate and integrate Leg 199 and Expedition 320 physical property and stratigraphic data (including shipboard and revised biostratigraphic data) to define a complete high-resolution time series for the middle-late Eocene, the entire Oligocene, and the early Miocene.

\section{Material and methods}

Magnetic susceptibility and GRA bulk density data from the Whole-Round Multisensor Logger and virtual geomagnetic pole (VGP) data calculated from natural remanent magnetization measurements in a superconducting rock magnetometer collected at Expedition 320 sites (see the "Expedition 320/321 summary" chapter [Expedition 320/321 Scientists, 2010a]) were used to refine depth offsets and to revise the shipboard composite section for Sites U1331, U1332, U1333, and U1334. Similarly, magnetic susceptibility, GRA, and VGP data from Leg 199 sites (Shipboard Scientific Party, 2002a, 2002b, 2002c; Pälike et al., 2005) were used to refine offsets and splices at Sites 1218, 1219, and 1220. Refinements are also based on detailed high-resolution Xray fluorescence (XRF) core scanning data (T. Westerhold et al., unpubl. data; D. Liebrand et al., unpubl. data).

In 2006-2007 new classification and nomenclature for depth scale types were defined for IODP (see IODP Depth Scales Terminology at www.iodp.org/ program-policies/). The new methods and nomenclature for calculating sample depth in a hole has changed to be method specific to ensure that data acquisition, mapping of scales, and construction of composite scales and splices are unequivocal. Because this study integrates data with different depth scale nomenclatures, we will describe in detail the classifications and definitions of depth scales as used here for Leg 199 and Expedition 320. A much more detailed definition of IODP depth scales used during Expedition 320/321 is given in the "Methods" chapter (Expedition 320/321 Scientists, 2010b).

For this study, the most important depth is the core depth below seafloor. This depth for each drilled core is based on the actual length of the recovered core and the drillers depth. It is defined as core depth below seafloor (CSF) for Expedition 320 and meters below seafloor (mbsf) for Leg 199. For consistency, we suggest the use of "mbsf (m CSF-A)" for Expedition 320 sites and "mbsf" for Leg 199 sites. Each point in the core can now be located by adding the offset between sample and core top to the drilling depth below seafloor (DSF) of the top of the core. To construct an initial continuous stratigraphic reference 
during the expedition, individual cores were depth shifted to maximize correlation between multiple adjacent holes and spliced together into a composite record. The new shipboard depth scale of the spliced section is defined as the composite core depth below seafloor (CCSF-A) for Expedition 320 and meters composite depth (mcd) for Leg 199. For consistency we suggest the use of "mcd (m CCSF-A)" for Expedition 320 and "mcd" for Leg 199. The addendum "-A" to Expedition 320 cores to the CCSF denotes that individual cores were shifted vertically without permitting expansion or contraction of the relative depth scale within any core.

Postcruise, we evaluated and revised the shipboard spliced composite section, establishing new core offsets and refined the shipboard splice if necessary. Intervals having significant disturbance or distortion were not used for composite section construction. For construction of the revised records, we tried to maintain those tie points given in the shipboard composite, where possible. Changes in the position of tie points in the revised spliced record have been highlighted as bold letters in the splice tables of each site. The new refined depth scale is defined as the revised composite core depth below seafloor (revised CCSF-A) for Expedition 320 and revised meters composite depth (rmcd) for Leg 199. For consistency we suggest the use of "rmcd (m revised CCSF-A)" for Expedition 320 and "rmcd" for Leg 199. Correction to the rmcd depth scales of Leg 199 (Pälike et al., 2005) are indicated by the corrected revised meters composite depth (corrected rmcd).

After assembling the new composite records, we adjusted sedimentary sections outside the revised composite splice by squeezing and stretching to conform to the overall rmcd and rmcd ( $\mathrm{m}$ revised CCSF-A) depth scales. To indicate this adjustment we added the prefix "adjusted" to the rmcd (m revised CCSF-A) and the corrected rmcd if necessary. This mapping procedure allows data and samples located outside the spliced composite record to be placed in the new revised composite depth scale at each site.

Finally, to integrate all available data we correlated magnetic susceptibility, GRA, and VGP data between sites using the time series analysis program AnalySeries (Paillard et al., 1996). All tables and cleaned composite records of magnetic susceptibility and GRA data from Sites 1218, 1219, 1220, U1331, U1332, U1333, and U1334 and VGP data from Sites 1218,1219 , and 1220 are available online in the WDC-MARE PANGAEA database (doi.pangaea.de/ 10.1594/PANGAEA.757215).

Composite core images were created as an additional aid in site-to-site correlation using an approach modified from that described in Wilkens et al. (2009). In- dividual section images collected by the shipboard Section Half Imaging Logger (SHIL) during Expedition 320 were initially assigned a core depth below seafloor (CSF-A) depth range based on site coring data. Section images were then mapped onto a single image of an entire core. Core image depth ranges were then shifted by constant offsets to revised CCSF-A depths based on the revised composite splice for each site (e.g., Table T1). The final step in creating a single image of the entire revised composite section involved another mapping of depth intervals from individual core images defined in site splice tables (e.g., Table T2) onto a composite image. As the resolution of the original SHIL images is on the order of $50 \mu \mathrm{m}$, each mapping step included image interpolation to a coarser scale-250 $\mu \mathrm{m}$ for the individual core images and $1 \mathrm{~mm}$ for the composite image. Leg 199 was the first use of an earlier version of the shipboard digital imaging system (DIS), and unfamiliarity with its operation led to inconsistent image exposures. Rather than attempt to correct the DIS images, we elected to use the digitized core photos available online at the IODP data website to construct composite site images. Images of each section of each core were digitally cut from the core images and then combined as described above. As the core photo images were much lower resolution (nominally $2.5 \mathrm{~mm}$ ), there was no need to downsize while mapping. A slight unevenness in lighting of the core photos (darker around the perimeter) produced an artifact when cutting and combining section images from digitized core photos into total core images. Apparent $1.5 \mathrm{~m}$ wavelength banding is particularly evident in lighter colored sediments. An example can be seen in the composite image of Site 1218 between 150 and 200 corrected rmcd.

\section{Results and discussion}

\section{Revised composite depth scales} Revised composite depth scales for Expedition
320 Sites U1331-U1334

\section{Site U1331}

The shipboard splice for Site U1331 (see the "Site U1331" chapter [Expedition 320/321 Scientists, 2010c]) was extensively revised (Figs. F2, F3, F4; Tables T1, T2, T3). The weak magnetic susceptibility and GRA signals in the siliceous ooze dominated the Eocene section of Site U1331, and frequent turbidites hampered straightforward correlation. The exact position and extent of turbidites is given in Table T4. The most problematic section is located around 35$40 \mathrm{rmcd}$ ( $\mathrm{m}$ revised CCSF-A), where a turbidite occurs that shows different thicknesses in each cored hole. This could be due to coring disturbance at the 
top of the Hole U1331C or strong thickness variations of the turbidite itself. We improved the splice to $175 \mathrm{rmcd}$ ( $\mathrm{m}$ revised CCSF-A), resulting in a growth factor of 1.12 (Fig. F4). Detailed correlation showed that the shipboard declination record of Cores $320-\mathrm{U} 1331 \mathrm{~B}-4 \mathrm{H}$ and $9 \mathrm{H}$ had to be flipped by $180^{\circ}$.

\section{Site $U 1332$}

The shipboard splice for Site U1332 (see the "Site U1332" chapter [Expedition 320/321 Scientists, 2010d]) had to be moderately revised (Figs. F5, F6, F7; Tables T5, T6, T7). Good VGP, magnetic susceptibility, and GRA data enable improvement of the shipboard splice to $141 \mathrm{~m}$ revised CCSF-A, resulting in a growth factor of 1.09 (Fig. F7). At around 83 rmcd ( $m$ revised CCSF-A), a small gap in the data (Fig. F5) marks an uncertain tie point in the splice. However, detailed correlation to Sites U1331 and 1220 suggests no major break at the base of Chron C15n in the composite record of Site U1332 (see Fig. F23, 50-100 corrected rmcd).

\section{Site $U 1333$}

The splice at Site U1333 (see the "Site U1333" chapter [Expedition 320/321 Scientists, 2010e]) needed no change in the upper $48 \mathrm{rmcd}$ ( $\mathrm{m}$ revised CCSF-A) (Figs. F8, F9, F10; Tables T8, T9, T10). Pronounced cycles in magnetic susceptibility and GRA data in this interval allowed the construction of a robust shipboard splice. Correlation to Sites U1334 and 1218 revealed an incorrect splice interval in the shipboard splice at Site U1333 around $48 \mathrm{rmcd}$ (m revised CCSF-A). Readjustments of the splice reveal a $2 \mathrm{~m}$ gap in the shipboard splice, which has been eliminated by the new revised splice. Although the magnetic susceptibility signal is low between 82 and 132 rmcd (m revised CCSF-A), small distinct peaks can be correlated and then verified by VGP data (Fig. F8, 50-100 m). A minor change in the shipboard splice is required at $126 \mathrm{rmcd}$ ( $\mathrm{m}$ revised CCSF-A). We then follow the tie points of the shipboard splice to $151 \mathrm{rmcd}$ ( $\mathrm{m}$ revised CCSF-A) and maintain the complete uninterrupted splice to $156.44 \mathrm{rmcd}$ (m revised CCSF-A). Below this depth, the splice can only be appended because there is no clear overlap between cores from adjacent holes. Between 180 and 200 rmcd (m revised CCSF-A), a composite record could be established mainly based on the VGP and magnetic susceptibility data. The new splice has a growth factor of 1.14 (Fig. F10).

\section{Site U1334}

At Site U1334 (see the "Site U1334" chapter [Expedition 320/321 Scientists, 2010f]), the shipboard splice was verified to $210 \mathrm{rmcd}$ (m revised CCSF-A) (Figs. F11, F12, F13; Tables T11, T12, T13). Because of geochemical alteration of the magnetic susceptibility record, splicing was uncertain between 150 and 270 rmcd ( $m$ revised CCSF-A). Through extensive usage of augmented magnetic susceptibility, GRA, VGP, core images, and especially postcruise XRF core scanning data (T. Westerhold et al., unpubl. data; D. Liebrand et al., unpubl. data), we secured a complete composite record across the geochemically altered interval to $271 \mathrm{rmcd}$ (m revised CCSF-A). Below this depth, we follow the shipboard splice with a major change in the interval from 297 to $306 \mathrm{rmcd}$ (m revised CCSF-A). This change is important because it covers the interval before the Eocene/Oligocene boundary, which is characterized by strong fluctuations in calcium carbonate content (see the "Expedition 320/321 summary" chapter [Expedition 320/321 Scientists, 2010a]). Splicing this interval was a challenge because extended core barrel drilling produced strong biscuiting of the sediment. A complete splice was assembled for Site U1334 to $341 \mathrm{rmcd}$ (m revised CCSF-A) with a growth factor of 1.16 (Fig. F13).

\section{Revised composite depth scales for Leg 199 Sites 1218-1220}

Before we accomplished a site-to-site correlation of Expedition 320 and Leg 199 sites, it was necessary to recheck the revised splices of Sites 1218 and 1219 (Pälike et al., 2005) and the shipboard splice of Site 1220 (Shipboard Scientific Party, 2002c).

\section{Site 1218}

At Site 1218 (Figs. F14, F15, F16; Tables T14, T15, T16), the revised splice had to be corrected below 210 corrected rmcd. Most of these adjustments benefited from detailed comparison to Site U1334. Prior to Expedition 320, Site 1218 was the only stratigraphically expanded and complete site from the equatorial Pacific covering the late Eocene and early Oligocene. The revisions are mainly in intervals with very high calcium carbonate content and low magnetic susceptibility. A complete splice can be constructed to 287 corrected rmcd, adding a growth factor of 1.11 (Fig. F16).

\section{Site 1219}

Changes to the splice of Site 1219 (Figs. F17, F18; Tables T17, T18) are very small, and thus we suggest continuing to use the table by Pälike et al. (2005) to construct a composite record.

\section{Site 1220}

In contrast, the shipboard splice of Site 1220 had to be corrected below 71 rmcd (Figs. F19, F20, F21; Ta- 
bles T19, T20, T21). Compared to the shipboard splice the changes are minor, a few decimeters at most. The new revised composite record reached 136 rmcd and provided a growth factor of 1.10 (Fig. F21). Please note that Site 1220 was not part of the Pälike et al. (2005) splice revision. Therefore, this study is the first revision of the shipboard splice of Site 1220 and is indicated by the depth scale nomenclature revised meters composite depth (rmcd).

\section{Cleaned magnetic susceptibility, GRA, and VGP data sets}

For reference, we provide cleaned magnetic susceptibility and GRA density data sets for every spliced composite section of Sites U1331 (Table T22), U1332 (Table T23), U1333 (Table T24), and U1334 (Table T25). Cleaned magnetic susceptibility, GRA density, and VGP latitude data are compiled for Sites 1218 (Table T26), 1219 (Table T27), and 1220 (Table T28) (data sets are also available in "Supplementary material"). To obtain cleaned data we removed outliers and data collected close to end caps and cut out disturbed intervals (e.g., core tops). These data sets have been used for the subsequent site-to-site correlation and squeezing and stretching of core sections outside the spliced records. The mapping pairs from the squeezing and stretching can be used to position samples taken outside the splice to be placed into the new revised composite depth scales.

\section{Site-to-site correlation}

More than 800 dated paleomagnetic reversals are available for all PEAT sites (Pälike et al., 2009) and thus provide the perfect framework for the detailed intercalibration of all major fossil groups and refinement of magnetic polarity chrons, particularly in the Eocene. However, the shipboard preliminary paleomagnetic data from Expedition 320 used here have to be considered incomplete. To improve the quality of the magnetostratigraphy, stepwise demagnetization of U-channel samples accompanied by rock magnetic studies are being done as part of the postcruise science. High-quality and high-resolution paleomagnetic records covering the late Eocene, Oligocene, and early Miocene are available from Leg 199 (Pälike et al., 2005; Lanci et al., 2004, 2005). The sites from both expeditions presented here are ideal for the establishment of a fully integrated calibrated bio-, chemo-, and magnetostratigraphy for the early Eocene-early Miocene time interval for the equatorial Pacific. A prerequisite for successful integration of the stratigraphic data and subsequent assembly of the proposed equatorial Pacific Cenozoic megasplice is the correlation of decimeter-scale features in the sedimentary record from the drilled sites from both
Leg 199 and Expedition 320/321 (Pälike et al., 2005, 2009). We follow the successful approach of previous deep-sea drilling expeditions (Shackleton et al., 1995, 1999; Shackleton and Crowhurst, 1997; Pälike et al., 2005; Westerhold and Röhl, 2006; Westerhold et al., 2007, 2008) by using physical property data (magnetic susceptibility and GRA) and XRF core scanning data to correlate site to site. In doing so we can transfer, for example, the high-resolution biostratigraphic data from one site to intervals of another site where, due to poor preservation, datums are not well constrained. Furthermore, we can locate hiatuses and condensed intervals that otherwise would not have been identified. For correlation, we first identified a reference site that has the most complete record and high sedimentation rates compared to the other sites. Then we correlated the other sites to the reference site by selecting tie points. We applied a linear interpolation of depth between tie points. Tie points are listed in Tables T29 and T30.

\section{Correlation between Sites 1218, 1219, U1333, and U1334}

Physical property data at Sites 1218, 1219, U1333, and U1334 show a remarkable match (Fig. F22) even though the sites are between 375 and $1100 \mathrm{~km}$ apart. All sites have an excellent magnetostratigraphy, and thus comparison of the VGP data indicate the high quality of correlation. We have chosen Site 1218 to be the reference site because Site 1218 is the most complete down to the Eocene/Oligocene boundary and has no geochemically altered interval, as found in the mid-Oligocene of Site U1334. The integrated record spans the interval from Chron C1 (Pleistocene) back to Chron C20 (middle Eocene) covering $>40$ m.y. of equatorial Pacific history. We correlated Sites 1219, U1333, and U1334 to Site 1218 (Table T29), providing a coherent and integrated record for the equatorial Pacific.

The correlation shows full coverage of magnetostratigraphy back to the base of early Oligocene Chron C11n.2n using Sites 1218 and U1334 alone. All four sites cover the interval from the base of Chron C6n to the base of C10n.2n ( 20 to 28 Ma) with a complete magnetostratigraphy. In the time span older than Chron C12n ( 30.8 Ma), the magnetostratigraphic boundary positions can be transferred from Site U1333 to Sites 1218, 1219, and U1334 when necessary. The complete magnetostratigraphic record reaches back to the top of middle Eocene Chron C19n ( 41 Ma).

Sedimentation rates in the section from 0 to $20 \mathrm{Ma}$ at all sites are highest at Site 1218 (a low $0.35 \mathrm{~cm} / \mathrm{k} . \mathrm{y}$.). Sites 1219 and U1333 have even lower sedimentation rates in that interval and a hiatus between the 
Pliocene-Pleistocene and the lower Miocene (Pälike et al., 2009). All these sediments consist of clays deposited near or below the calcium carbonate compensation depth. In the upper $40 \mathrm{~m}$ of the integrated stratigraphy (Fig. F22), correlations are based on the VGP data because magnetic susceptibility and GRA data do not provide patterns that can be matched with certainty. Below that interval, matching of different records was straightforward. From $20 \mathrm{Ma}$ to the Eocene/Oligocene boundary, Site U1334 has the highest sedimentation rate $(1.6 \mathrm{~cm} / \mathrm{k} . \mathrm{y}$.) of all the sites (Site $1218=1.3 \mathrm{~cm} / \mathrm{k} . y$. , Site $1219=1.2 \mathrm{~cm} / \mathrm{k} . \mathrm{y}$., and Site U1333 = $1.1 \mathrm{~cm} / \mathrm{k} . \mathrm{y}$.). In the upper Eocene section, sedimentation rates are slightly lower because of the decreased carbonate content (see the "Expedition 320/321 summary" chapter [Expedition 320/321 Scientists, 2010a]). Two short condensed intervals were discovered: one at Site 1219 between 112 and 114 corrected rmcd and one at Site U1333 between 137 and 140 rmcd (m revised CCSF-A) (Fig. F22).

\section{Correlation between Sites 1220, U1331, and U1332}

Physical property data from Sites 1220, U1331, and U1332 show a remarkable match (Fig. F23), being only 120 to $270 \mathrm{~km}$ apart. Sites 1220 and U1332 have an excellent magnetostratigraphy from Chrons C6n to C20n (Table T31). Site U1331 sediment covers Chrons C11-C20n. We chose Site 1220 to be the reference site because it is the most complete for this interval. The correlation with Sites U1331 and U1332 (Table T30) provides a coherent and integrated record.

All three sites show rather low sedimentation rates $(\sim 0.5 \mathrm{~cm} / \mathrm{k} . \mathrm{y}$.) compared to the shallower sites (1218, 1219, U1333, and U1334) (Fig. F24). The upper Eocene sediments are dominated by siliceous ooze and almost entirely lack carbonate sediment. The dominance of siliceous ooze leads to low variability in the GRA density; hence, correlation could only be achieved using magnetic susceptibility data. The comparison of the VGP data suggests a very good match of the three sites in the Eocene. The increased sedimentation rate at Site U1331 in the Eocene is an artifact of the frequent turbidites in the record. The correlation of the Oligocene and Miocene section is straightforward to $28 \mathrm{rmcd}$ (Fig. F23). Above this, Site 1220 can only be matched to Site U1332 using VGP data.

\section{Radiolarians in the tropical Pacific}

Cenozoic radiolarian stratigraphy of the tropics was largely developed in sediments from the Pacific
Ocean; however, it did not begin to reach its full potential until Leg 199 studies were completed by Nigrini et al. (2006). This work, combined with that of earlier studies (e.g., Moore, 1995), sought to tie radiolarian datums to a paleomagnetic timescale that could be tuned to orbital frequencies. These studies also greatly expanded the number of first and last occurrences of species that were recorded and calibrated. This effort took advantage of the many important taxonomic and stratigraphic papers that have appeared over the last 50 years, in particular those written by such authors as William Riedel, Annika Sanfilippo, Catherine Nigrini, David Johnson, and Jean Westberg, who focused much of their efforts on material collected in the tropical Pacific.

Expedition 320 was very successful in recovering Pacific Cenozoic sections deposited on or very close to the paleoequator. Two of these drilled sites (U1333 and U1334) recovered what appear to be complete sections across the Eocene/Oligocene boundary. Only one other section, from Site 1218, has been recovered in the tropical Pacific that clearly shows the "two-step" shift in lithology and geochemistry at this boundary that we believe marks a truly complete stratigraphic section (Coxall et al., 2005). Using the stratigraphic datums defined primarily in Nigrini et al. (2006), we were able to provide very detailed stratigraphic control on the sections recovered during Expedition 320 (Tables T32, T33, T34, T35, T36, T37, T38). While producing this detailed integrated stratigraphy of the equatorial Pacific, we have had to deal with some complicated stratigraphic problems that still need to be fully addressed.

\section{Reworking and mixing of older specimens into younger sections}

Finding reworked older radiolarian specimens in younger sediments plagued the development of a reliable radiolarian stratigraphy in its early days. Such reworking was commonly found in piston cores and gravity cores from the tropical Pacific (e.g., Riedel and Funnell, 1964), and it was not until the Deep Sea Drilling Project (DSDP) and ODP started to collect thick pelagic sections that we were able to begin to develop a reliable sequence of first and last appearances of species. In studying these sections, several important observations have been made: (1) the reworked older forms were never older than the age of the crust on which the sediment lay, (2) reworking of older forms is most common in the upper parts of recovered sections, and (3) reworking of older forms from the Eocene is commonly found around the Eocene/Oligocene boundary and is often associated with a hiatus at this boundary (Moore et al., 1978; 
Moore, 1995). Because many of the biostratigraphic datums near the uppermost part of the Eocene are last appearances, the dependability of such datums are highly suspect and their calibration to a timescale is still open to question.

\section{Taxonomic definition}

Nigrini et al. (2006) described 12 new species, several of which are important in defining the Eocene/Oligocene boundary and in refining the stratigraphy of the Oligocene. These new species require the test of time and usage to make sure their definitions adequately encompass the characteristics and variability of their form. Similarly, other species may need modification of their descriptions in order to more consistently define biostratigraphic datums. Only a small percentage of the total number of radiolarian species present at any given time has been identified as being stratigraphically useful (Riedel and Sanfilippo, 1978). Further work in this area will continue to expand the resolution possible using radiolarian stratigraphy.

\section{Preservation}

Radiolarians are generally well preserved in the tropical Pacific; however, they are subject to dissolution, particularly just above basement and at levels of chert formation. Aside from these two problems, Eocene radiolarians are particularly robust (Moore, 1969; Lazarus et al., 2009), and, with their very diverse fauna, usually provide good stratigraphic control. Preservation in the Oligocene of the sites studied, however, is often only moderate and sometimes quite poor. It has yet to be determined if this variation in Oligocene preservation is site specific or time specific.

The radiolarian stratigraphic data presented herein represent a work in progress. Additional samples are being studied and the detailed site-to-site correlation that has been developed by the work presented here will be used to further refine the positions of individual biostratigraphic datums. Some of this more detailed work is shown in the radiolarian data tables (denoted by "Revised" in the column labeled "Source"). There remain many apparent small discrepancies in the levels of individual datums at different sites. It is yet to be determined whether these discrepancies are a result of reworking of radiolarians above or below the true level of the datum, a failure to recognize the presence of a rare species near its first or last appearance, a true diachrony of the datum, or a minor miscorrelation of the lithologic records themselves. Until these discrepancies can be studied further, we use the age assigned each of the datums as published by Nigrini et al. (2006).

\section{Calcareous nannofossils}

Shipboard calcareous nannofossil biostratigraphy provided critical age control during Leg 199 and Expedition 320, allowing for the identification of paleomagnetic reversals and the development of composite sections, especially within the successions of carbonate-rich Oligocene-Miocene nannofossil oozes. The new correlations presented here enable more refined assessments of the timing and controls on the expression of calcareous nannofossil datums in the equatorial Pacific. The presented tables of nannofossil datums (Tables T39, T40, T41, T42, T43, $\mathrm{T} 44, \mathrm{~T} 45)$ are a compilation of data from both shipboard and postcruise biostratigraphy from Expedition 320 and Leg 199 (Shipboard Scientific Party, 2002a, 2002b, 2002c; Pälike et al., 2006; see also "Biostratigraphy" in each site chapter [Expedition 320/321 Scientists, 2010c, 2010d, 2010e, 2010f]). Calibration ages for calcareous nannofossil datums from the Leg 199 timescale were made consistent with those of Expedition 320 (bottom [B] Sphenolithus ciperoensis at $27.1 \mathrm{Ma}$ rather than $28.1 \mathrm{Ma}$; B Sphenolithus distentus at 30.0 Ma rather than $30.4 \mathrm{Ma}$; top [T] Reticulofenestra umbilicus at $32.0 \mathrm{Ma}$ rather than $31.7 \mathrm{Ma}$ ), as were taxonomic concepts (use of Coccolithus formosus rather than Ericsonia formosa). These changes do not imply that the datum ages used during Expedition 320 are better calibrated than those used during Leg 199; revisions were undertaken prior to Expedition 320 partly based on postcruise work from Leg 199 material (e.g., Blaj et al., 2009). For example, during Expedition 320 it became clear that the Leg 199 biostratigraphic datum age of 28.1 Ma for B S. ciperoensis produced a better fit within the integrated stratigraphy than the revised age provided by Blaj et al. (2009) used during Expedition 320/321 of 27.1 Ma. These discrepancies are likely due to differences in taxonomic concept and boundaries within the intergrading Oligocene sphenolith lineage Sphenolithus predistentus-distentusciperoensis. Ongoing postcruise taxonomic and biostratigraphic work will address these issues.

Placing the existing calcareous nannofossil biostratigraphy of Leg 199 and Expedition 320 within the framework of these new stratigraphic correlations clearly shows that the accurate placement of calcareous nannofossil events is compromised by the occurrence of intervals with low or no carbonate deposition during the middle to late Eocene. A clear example of this is the placement of the latest Eocene event T Discoaster saipanensis (see Tables T42, T43, T44). This event is well constrained at Site 1218 at $244.52 \pm 0.06$ corrected rmcd and at Site U1334 at $301.33 \pm 0.53 \mathrm{rmcd}$ (m revised CCSF-A) (equal to $243.29 \pm 0.45$ corrected rmcd [Site 1218]). But this 
event is poorly constrained at Site 1219 within the interval $190.06 \pm 13.83 \mathrm{rmcd}$ (equal to $254.66 \pm 12.03$ corrected rmcd [Site 1218]), although the identified range is fully consistent with the stratigraphy of Sites 1218 and U1334. Where there is continuous carbonate sedimentation and reasonable nannofossil preservation, most of the nannofossil datums correlate among these equatorial Pacific sites within the accuracy of the current sampling resolution (e.g., top of R. umbilicus placed at 221.42, 222.97, 224.44, and 220.99 corrected rmcd [Site 1218] at Sites 1218, 1219 , U1333, and U1334, respectively). Notable exceptions to this are the placement of the base and top of $S$. ciperoensis and the top of $S$. distentus. The base of $S$. ciperoensis is relatively consistent between Sites 1218, 1219, and U1333 at 144 corrected rmcd (Site 1218), but $S$. ciperoensis is first noted at low abundance $\sim 20 \mathrm{~m}$ lower at Site U1334 at $\sim 164$ corrected rmcd (Site 1218). This suggests the initial evolutionary appearance of $S$. ciperoensis is followed by a period of low abundance in the equatorial Pacific and then a marked abundance increase that is picked as the "B S. ciperoensis" in the majority of these study sites. The top of $S$. ciperoensis is also depressed by $\sim 20 \mathrm{~m}$ at Site U1333 ( 130 corrected rmcd [Site 1218]) with respect to the other sites $(\sim 111$ corrected rmcd [Site 1218] at Sites 1218, 1219, and U1334); again, this may be due to low abundances at the top of this species' range. The top of $S$. distentus is placed $\sim 10 \mathrm{~m}$ higher in the biostratigraphy of Leg 199 than that of Expedition $320(\sim 130$ corrected rmcd [Site 1218] at Sites 1218 and 1219 versus $\sim 140$ corrected rmcd [Site 1218] at Sites U1333 and U1334). This most likely reflects slightly different taxonomic concepts applied by different workers or simply the difficulty in applying consistent taxonomy in a complex species plexus undergoing gradual change, as we observe within this lineage of Oligocene sphenoliths. Improving the taxonomic definition of these sphenolith lineages and determining the abundance patterns and timing of their origin and extinction will be the focus of ongoing detailed biostratigraphic studies.

Reworked calcareous nannofossils were identified in limited intervals of the Oligocene at the top of Site U1331, associated with suspected gravity flow deposits. These intervals were easily identified during shipboard biostratigraphy, and reworking of older nannofossils into younger strata is not thought to have affected the placement of nannofossil datums. The stratigraphic framework presented here is an excellent basis for ongoing detailed assessments of late Eocene-Oligocene nannofossil bioevents, with a particular focus on the improved age resolution and the identification of genuine diachrony across the eastern equatorial Pacific. The integration of both radiolarian and calcareous nannofossil biostratigraphy proved essential for shipboard operations during both Leg 199 and Expedition 320, which both spanned the major lithologic transition from Eocene radiolarian oozes to Oligocene-Miocene calcareous nannofossil oozes. Continued biostratigraphic work on material recovered during these two expeditions and Expedition 321 should produce a greatly improved integrated tropical Pacific radiolarian-nannofossil biostratigraphy of the last $\sim 50 \mathrm{~m} . \mathrm{y}$.

\section{Summary}

We revised the shipboard composite sections of Sites U1331, U1332, U1333, and U1334 from Expedition 320 and Sites 1218, 1219, and 1220 from Leg 199 using shipboard magnetic susceptibility data, GRA bulk density data, natural remanent magnetization data, and core images. Drilling distortions in cores are compensated by differential squeezing and stretching of parallel cores outside the splice at all investigated sites. This is of major importance because we want to integrate all available data from all drilled holes. A detailed site-to-site correlation was performed. We linked the revised composite sections of Sites U1333, U1334, and 1219 to the corrected rmcd of Site 1218. Sites U1331 and U1332 were correlated to the revised composite record of Site 1220 . We chose Sites 1218 and 1220 as reference sites because of their stratigraphic completeness. The decimeterscale correlation was used to integrate and transfer paleomagnetic and biostratigraphic information. Our integrated stratigraphic framework presented here can be used as the backbone for the late Eocene, Oligocene, and early Miocene intervals of the equatorial Pacific Cenozoic megasplice. Because of the presence of clear paleomagnetic records and decimeter-scale cyclic features, the investigated sediments are exceptionally suitable for further working on cyclostratigraphy and orbital tuning. The integration of Expedition 320 and Leg 199 data has the potential to substantially improve the existing geological timescale (Gradstein et al., 2004) and even extend the astronomically calibrated timescale (Lourens et al., 2004; Pälike et al., 2006) far back into the Eocene.

\section{Acknowledgments}

This manuscript benefitted from reviews by David J. Mallinson and Lucas J. Lourens. This research used samples and/or data provided by the Integrated Ocean Drilling Program (IODP). Funding for this research was provided by the Deutsche Forschungsgemeinschaft, the DFG-Leibniz Center for Surface Pro- 
cess and Climate Studies at the University of Potsdam, and by the National Science Foundation (NSF) through the US Science Support Program. We are indebted to the IODP Bremen Core Repository (BCR) and Gulf Coast Repository (GCR) staff for core handling.

\section{References}

Blaj, T., Backman, J., and Raffi, I., 2009. Late Eocene to Oligocene preservation history and biochronology of calcareous nannofossils from paleo-equatorial Pacific Ocean sediments. Riv. Ital. Paleontol. Stratigr., 115(1):6784.

Cande, S.C., and Kent, D.V., 1995. Revised calibration of the geomagnetic polarity timescale for the Late Cretaceous and Cenozoic. J. Geophys. Res., [Solid Earth], 100(B4):6093-6095. doi:10.1029/94JB03098

Coxall, H.K., Wilson, P.A., Pälike, H., Lear, C.H., and Backman, J., 2005. Rapid stepwise onset of Antarctic glaciation and deeper calcite compensation in the Pacific Ocean. Nature (London, U. K.), 433(7021):53-57. doi:10.1038/nature03135

Expedition 320/321 Scientists, 2010a. Expedition 320/321 summary. In Pälike, H., Lyle, M., Nishi, H., Raffi, I., Gamage, K., Klaus, A., and the Expedition 320/321 Scientists, Proc. IODP, 320/321: Tokyo (Integrated Ocean Drilling Program Management International, Inc.). doi:10.2204/iodp.proc.320321.101.2010

Expedition 320/321 Scientists, 2010b. Methods. In Pälike, H., Lyle, M., Nishi, H., Raffi, I., Gamage, K., Klaus, A., and the Expedition 320/321 Scientists, Proc. IODP, 320/ 321: Tokyo (Integrated Ocean Drilling Program Management International, Inc.). doi:10.2204/ iodp.proc.320321.102.2010

Expedition 320/321 Scientists, 2010c. Site U1331. In Pälike, H., Lyle, M., Nishi, H., Raffi, I., Gamage, K., Klaus, A., and the Expedition 320/321 Scientists, Proc. IODP, 320/321: Tokyo (Integrated Ocean Drilling Program Management International, Inc.). doi:10.2204/ iodp.proc.320321.103.2010

Expedition 320/321 Scientists, 2010d. Site U1332. In Pälike, H., Lyle, M., Nishi, H., Raffi, I., Gamage, K., Klaus, A., and the Expedition 320/321 Scientists, Proc. IODP, 320/321: Tokyo (Integrated Ocean Drilling Program Management International, Inc.). doi:10.2204/ iodp.proc.320321.104.2010

Expedition 320/321 Scientists, 2010e. Site U1333. In Pälike, H., Lyle, M., Nishi, H., Raffi, I., Gamage, K., Klaus, A., and the Expedition 320/321 Scientists, Proc. IODP, 320/321: Tokyo (Integrated Ocean Drilling Program Management International, Inc.). doi:10.2204/ iodp.proc.320321.105.2010

Expedition 320/321 Scientists, 2010f. Site U1334. In Pälike, H., Lyle, M., Nishi, H., Raffi, I., Gamage, K., Klaus, A., and the Expedition 320/321 Scientists, Proc. IODP, 320/ 321: Tokyo (Integrated Ocean Drilling Program Management International, Inc.). doi:10.2204/ iodp.proc.320321.106.2010
Gradstein, F.M., Ogg, J.G., and Smith, A.' $\square($ Eds.), 2004. A Geologic Time Scale 2004: Cambridge (Cambridge Univ. Press). doi:10.2277/0521786738

Hagelberg, T., Shackleton, N., Pisias, N., and Shipboard Scientific Party, 1992. Development of composite depth sections for Sites 844 through 854. In Mayer, L., Pisias, N., Janecek, T., et al., Proc. ODP, Init. Repts., 138: College Station, TX (Ocean Drilling Program), 67-77. doi:10.2973/odp.proc.ir.138.105.1992

Lanci, L., Parés, J.M., Channell, J.E.T., and Kent, D.V., 2004. Miocene magnetostratigraphy from equatorial Pacific sediments (ODP Site 1218, Leg 199). Earth Planet. Sci. Lett., 226(1-2):207-224. doi:10.1016/ j.epsl.2004.07.025

Lanci, L., Parés, J.M., Channell, J.E.T., and Kent, D.V., 2005. Oligocene magnetostratigraphy from equatorial Pacific sediments (ODP Sites 1218 and 1219, Leg 199). Earth Planet. Sci. Lett., 237(3-4):617-634. doi:10.1016/ j.epsl.2005.07.004

Lazarus, D.B, Kotrc, B., Wulf, G., and Schmidt, D.N., 2009. Radiolarians decreased silicification as an evolutionary response to reduced Cenozoic ocean silica availability. Proc. Natl. Acad. Sci. U. S. A., 106(23):9333-9338. doi:10.1073/pnas.0812979106

Lourens, L., Hilgen, F., Shackleton, N.J., Laskar, J., and Wilson, D., 2004. The Neogene period. In Gradstein, F.M., Ogg, J.G., and Smith, A.' $\mathrm{\square}$ (Eds.), A Geologic Time Scale 2004: Cambridge (Cambridge Univ. Press), 409-440. doi:10.2277/0521786738

Lyle, M., Raffi, I., Pälike, H., Nishi, H., Gamage, K., Klaus, A., and the Expedition 320/321 Scientists, 2009. Pacific Equatorial Transect. IODP Prel. Rept., 321. doi:10.2204/ iodp.pr.321.2009

Lyle, M., Wilson, P.A., Janecek, T.R., et al., 2002. Proc. ODP, Init. Repts., 199: College Station, TX (Ocean Drilling Program). doi:10.2973/odp.proc.ir.199.2002

Moore, T.C., Jr., 1969. Radiolaria: change in skeletal weight and resistance to solution. Geol. Soc. Am. Bull., 80(10):2103-2108. doi:10.1130/ 0016-7606(1969)80[2103:RCISWA]2.0.CO;2

Moore, T.C., Jr., 1995. Radiolarian stratigraphy, Leg 138. In Pisias, N.G., Mayer, L.A., Janecek, T.R., Palmer-Julson, A., and van Andel, T.H. (Eds.), Proc. ODP, Sci. Results, 138: College Station, TX (Ocean Drilling Program), 191232. doi:10.2973/odp.proc.sr.138.111.1995

Moore, T.C., Jr., van Andel, T.H., Sancetta, C., and Pisias, N., 1978. Cenozoic hiatuses in the pelagic sediments. Micropaleontology, 24(2):113-138. doi:10.2307/1485246

Nigrini, C., Sanfilippo, A., and Moore, T.C., Jr., 2006. Cenozoic radiolarian biostratigraphy: a magnetobiostratigraphic chronology of Cenozoic sequences from ODP Sites 1218, 1219, and 1220, equatorial Pacific. In Wilson, P.A., Lyle, M., and Firth, J.V. (Eds.), Proc. ODP, Sci. Results, 199: College Station, TX (Ocean Drilling Program), 1-76. doi:10.2973/odp.proc.sr.199.225.2006

Paillard, D., Labeyrie, L., and Yiou, P., 1996. Macintosh program performs time-series analysis. Eos, Trans. Am. Geophys. Union, 77(39):379. doi:10.1029/96EO00259

Pälike, H., Moore, T., Backman, J., Raffi, I., Lanci, L., Parés, J.M., and Janecek, T., 2005. Integrated stratigraphic cor- 
relation and improved composite depth scales for ODP Sites 1218 and 1219. In Wilson, P.A., Lyle, M., and Firth, J.V. (Eds.), Proc. ODP, Sci. Results, 199: College Station, TX (Ocean Drilling Program), 1-41. doi:10.2973/ odp.proc.sr.199.213.2005

Pälike, H., Nishi, H., Lyle, M., Raffi, I., Klaus, A., Gamage, K., and the Expedition 320/321 Scientists, 2009. Pacific Equatorial Age Transect. IODP Prel. Rept., 320. doi:10.2204/iodp.pr.320.2009

Pälike, H., Norris, R.D., Herrle, J.O., Wilson, P.A., Coxall, H.K., Lear, C.H., Shackleton, N.J., Tripati, A.K., and Wade, B.S., 2006. The heartbeat of the Oligocene climate system. Science, 314(5807):1894-1898. doi:10.1126/science.1133822

Riedel, W.R., and Funnell, B.M., 1964. Tertiary sediment cores and microfossils from the Pacific Ocean floor. Q. J. Geol. Soc. London, 120:305-368.

Riedel, W.R., and Sanfilippo, A., 1978. Stratigraphy and evolution of tropical Cenozoic radiolarians. Micropaleontology, 24(1):61-96. doi:10.2307/1485420

Shackleton, N.J., and Crowhurst, S., 1997. Sediment fluxes based on an orbitally tuned time scale $5 \mathrm{Ma}$ to $14 \mathrm{Ma}$, Site 926. In Shackleton, N.J., Curry, W.B., Richter, C., and Bralower, T.J. (Eds.), Proc. ODP, Sci. Results, 154: College Station, TX (Ocean Drilling Program), 69-82. doi:10.2973/odp.proc.sr.154.102.1997

Shackleton, N.J., Crowhurst, S., Hagelberg, T., Pisias, N.G., and Schneider, D.A., 1995. A new late Neogene time scale: application to Leg 138 sites. In Pisias, N.G., Mayer, L.A., Janecek, T.R., Palmer-Julson, A., and van Andel, T.H. (Eds.), Proc. ODP, Sci. Results, 138: College Station, TX (Ocean Drilling Program), 73-101. doi:10.2973/ odp.proc.sr.138.106.1995

Shackleton, N.J., Crowhurst, S.J., Weedon, G.P., and Laskar, J., 1999. Astronomical calibration of Oligocene-Miocene time. In Shackleton N.J., McCave, I.N., and Graham, P.W. (Eds.), Astronomical (Milankovitch) Calibration of the Geological Time-Scale. Philos. Trans. R. Soc., Ser. A., 357(1757):1907-1929. doi:10.1098/rsta.1999.0407

Shipboard Scientific Party, 2002a. Site 1218. In Lyle, M., Wilson, P.A., Janecek, T.R., et al., Proc. ODP, Init. Repts., 199: College Station, TX (Ocean Drilling Program), 1125. doi:10.2973/odp.proc.ir.199.111.2002
Shipboard Scientific Party, 2002b. Site 1219. In Lyle, M., Wilson, P.A., Janecek, T.R., et al., Proc. ODP, Init. Repts., 199: College Station, TX (Ocean Drilling Program), 1128. doi:10.2973/odp.proc.ir.199.112.2002

Shipboard Scientific Party, 2002c. Site 1220. In Lyle, M., Wilson, P.A., Janecek, T.R., et al., Proc. ODP, Init. Repts., 199: College Station, TX (Ocean Drilling Program), 192. doi:10.2973/odp.proc.ir.199.113.2002

Wade, B.S., and Pälike, H., 2004. Oligocene climate dynamics. Paleoceanography, 19(4)PA4019. doi:10.1029/ 2004PA001042

Westerhold, T., and Röhl, U., 2006. Data report: revised composite depth records for Shatsky Rise Sites 1209, 1210, and 1211. In Bralower, T.J., Premoli Silva, I., and Malone, M.J. (Eds.), Proc. ODP, Sci. Results, 198: College Station, TX (Ocean Drilling Program), 1-26. doi:10.2973/odp.proc.sr.198.122.2006

Westerhold, T., Röhl, U., Laskar, J., Raffi, I., Bowles, J., Lourens, L.J., and Zachos, J.C., 2007. On the duration of magnetochrons C24r and C25n and the timing of early Eocene global warming events: implications from the Ocean Drilling Program Leg 208 Walvis Ridge depth transect. Paleoceanography, 22:PA2201-PA2219. doi:10.1029/2006PA001322

Westerhold, T., Röhl, U., Raffi, I., Fornaciari, E., Monechi, S., Reale, V., Bowles, J., and Evans, H.F., 2008. Astronomical calibration of the Paleocene time. Palaeogeogr., Palaeoclimatol., Palaeoecol., 257(4):377-403. doi:10.1016/j.palaeo.2007.09.016

Wilkens, R.H., Niklis, N., and Frazer, M., 2009. Data report: digital core images as data: an example from IODP Expedition 303. In Channell, J.E.T., Kanamatsu, T., Sato, T., Stein, R., Alvarez Zarikian, C.A., Malone, M.J., and the Expedition 303/306 Scientists, Proc. IODP, 303/306: College Station, TX (Integrated Ocean Drilling Program Management International, Inc.). doi:10.2204/ iodp.proc.303306.201.2009

Initial receipt: 3 February 2011

Acceptance: 29 June 2011

Publication: 2 January 2012

MS 320321-201 
Figure F1. Location map of sites used in this study (red stars) and additional IODP/ODP/DSDP sites. F.Z. = fracture zone (modified from Pälike et al., 2009).

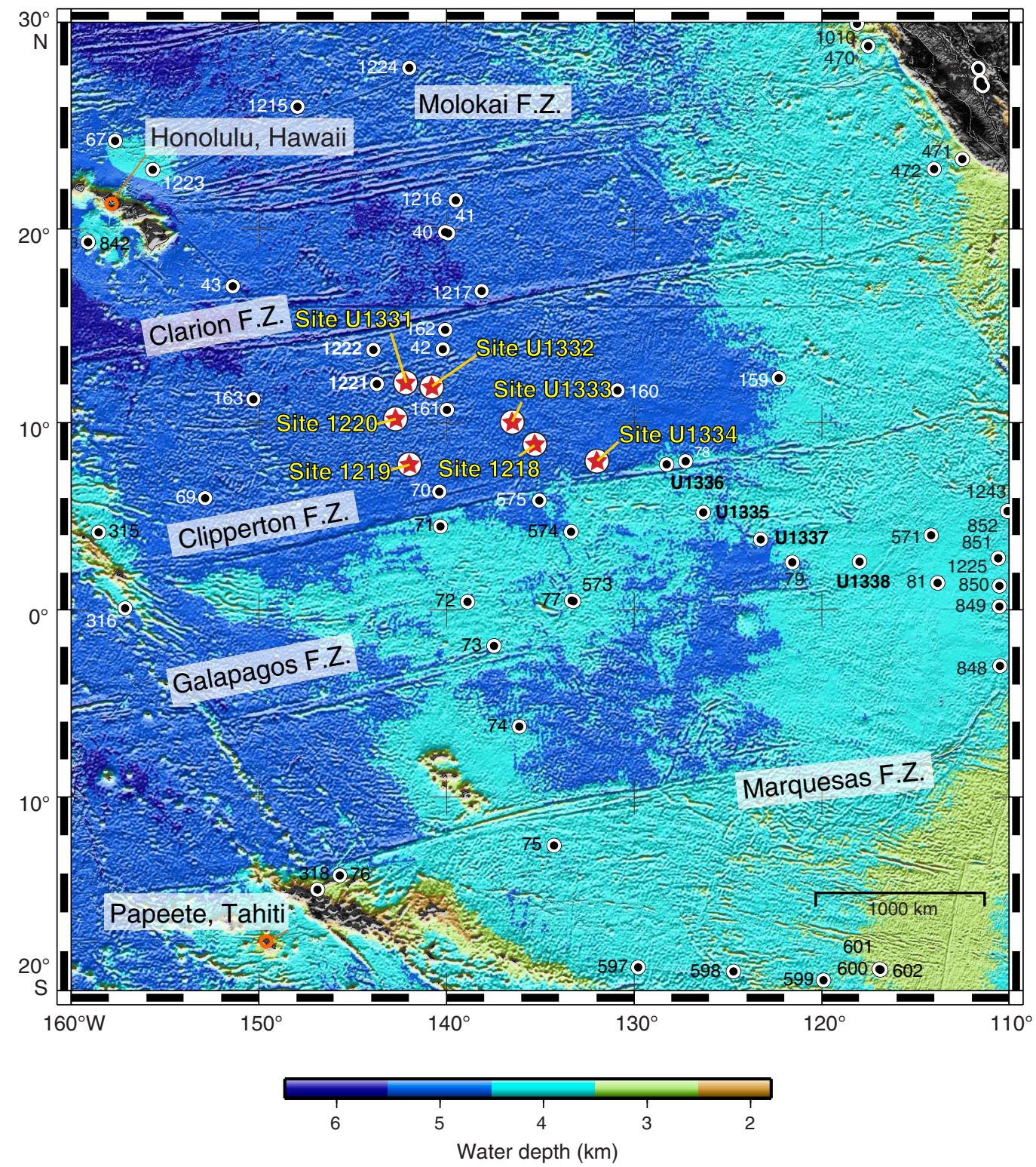


Figure F2. Site U1331 paleomagnetic and physical property data on rmcd (m revised CCSF-A) scale. Splice map and spliced core image on the left side, turbidite location on the right side. VGP $=$ virtual geomagnetic pole, GRA = gamma ray attenuation . Red $=$ Hole U1331A, blue $=$ Hole U1331B, green $=$ Hole U1331C , black $=$ composite record. Composite record line is discontinuous because of distortion and data gaps. DI = drilled interval. (Continued on next three pages.)

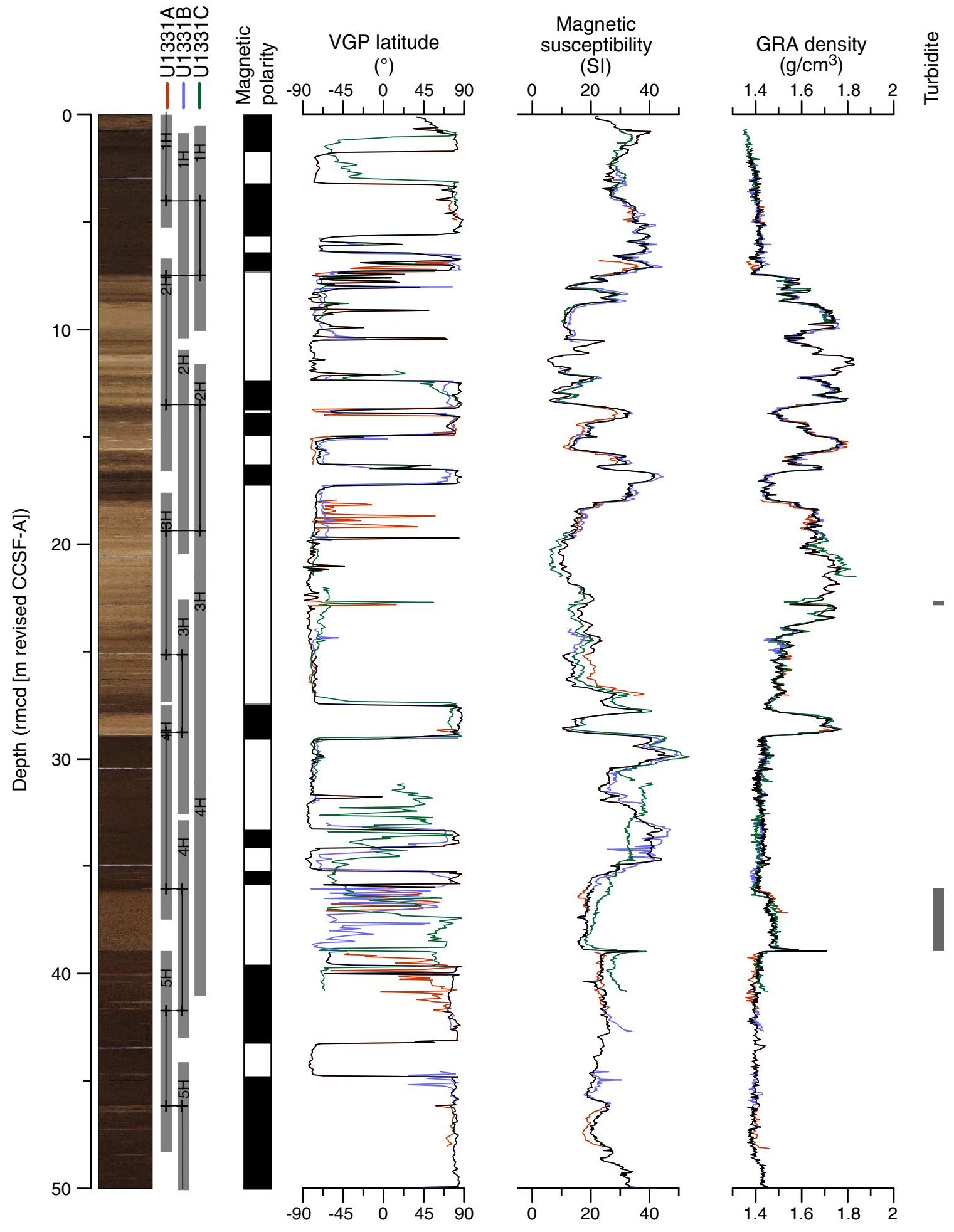


Figure F2 (continued). (Continued on next page.)

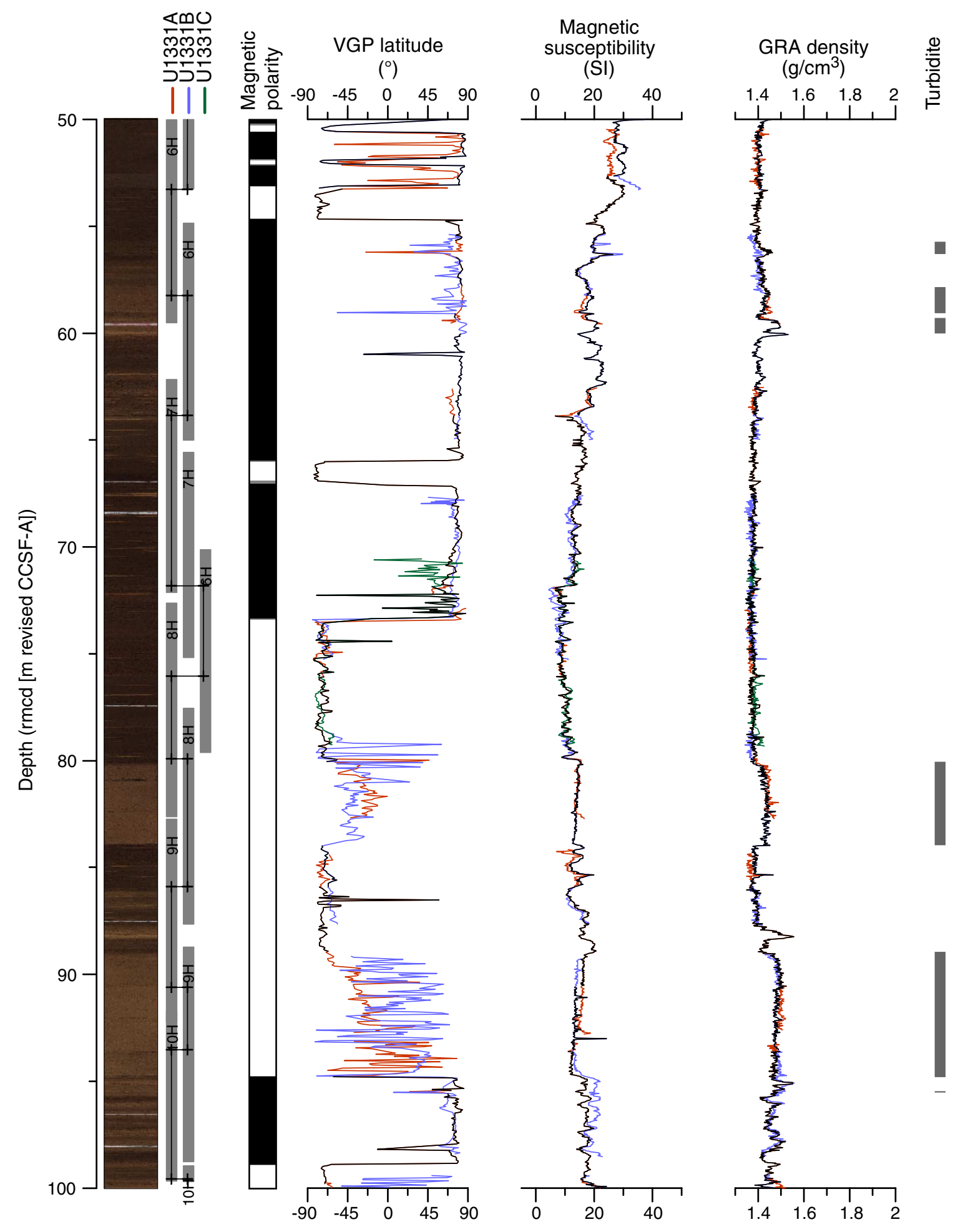


Figure F2 (continued). (Continued on next page.)

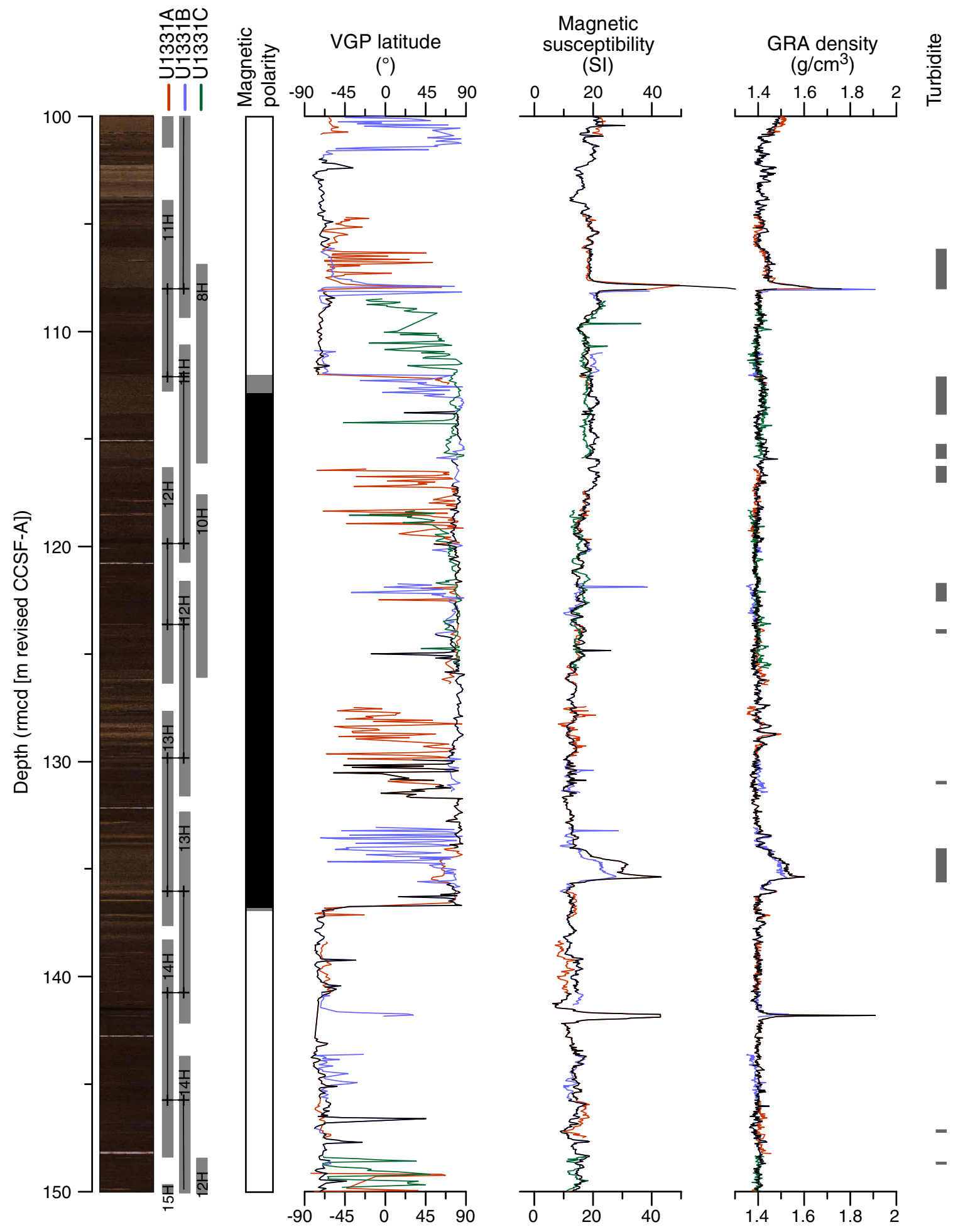


Figure F2 (continued).

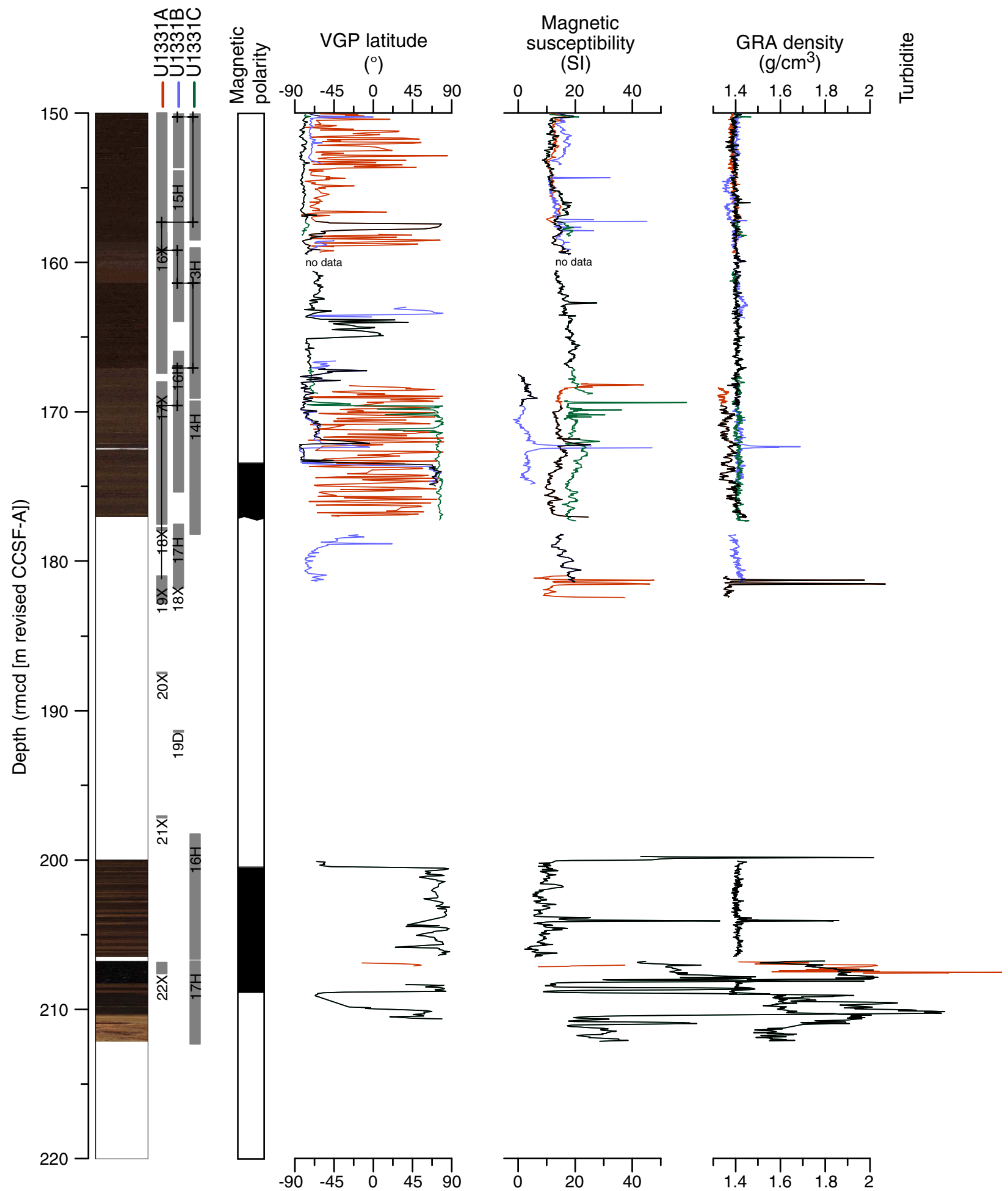


Figure F3. Digital line scan images, Site U1331. Red lines are the tops of splice sections and yellow lines are the bases of those sections (tie to next section). Images were depth registered by R. Wilkens using IGOR-Pro software.

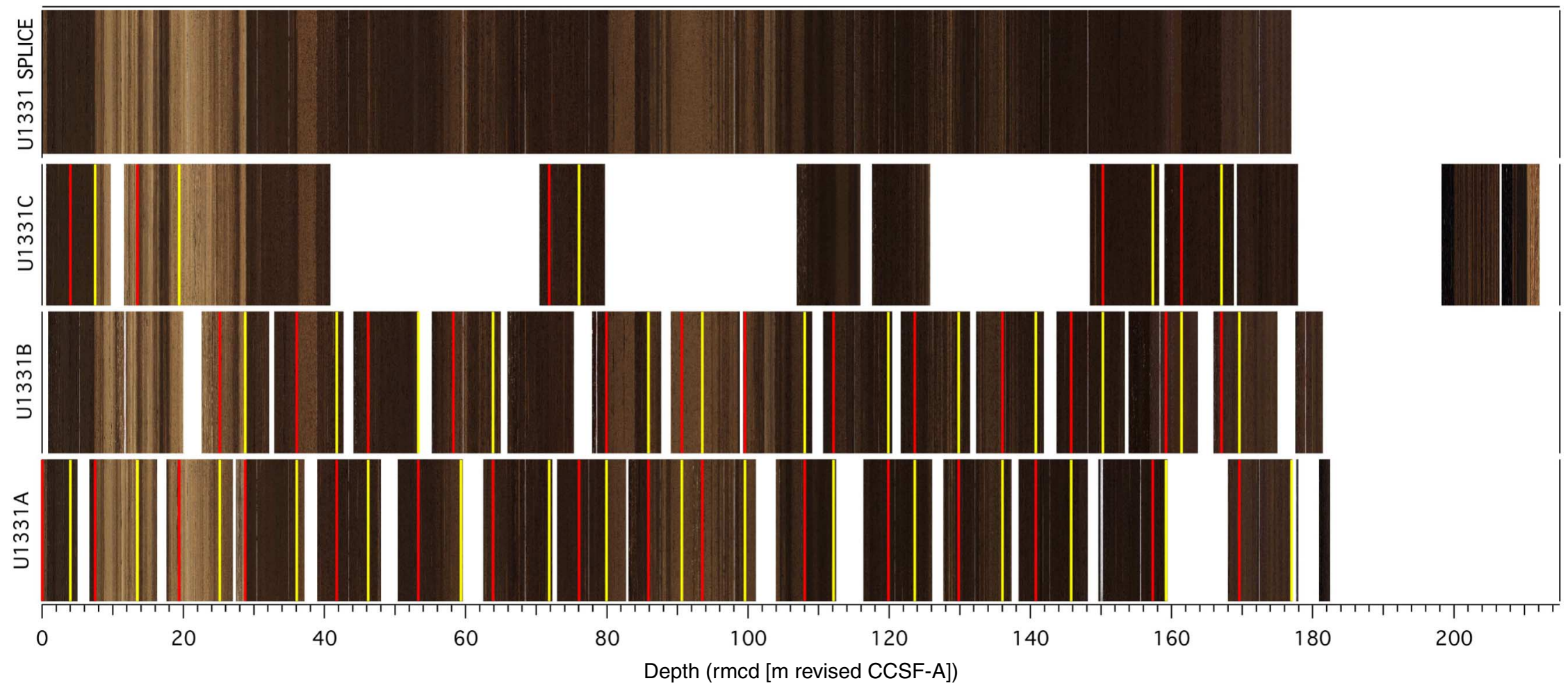


Figure F4. Growth factor calculated by plotting revised composite depth (rmcd [m revised CCSF-A]) against drilled core depth (CSF-A), Site U1331.

\section{Site U1331}

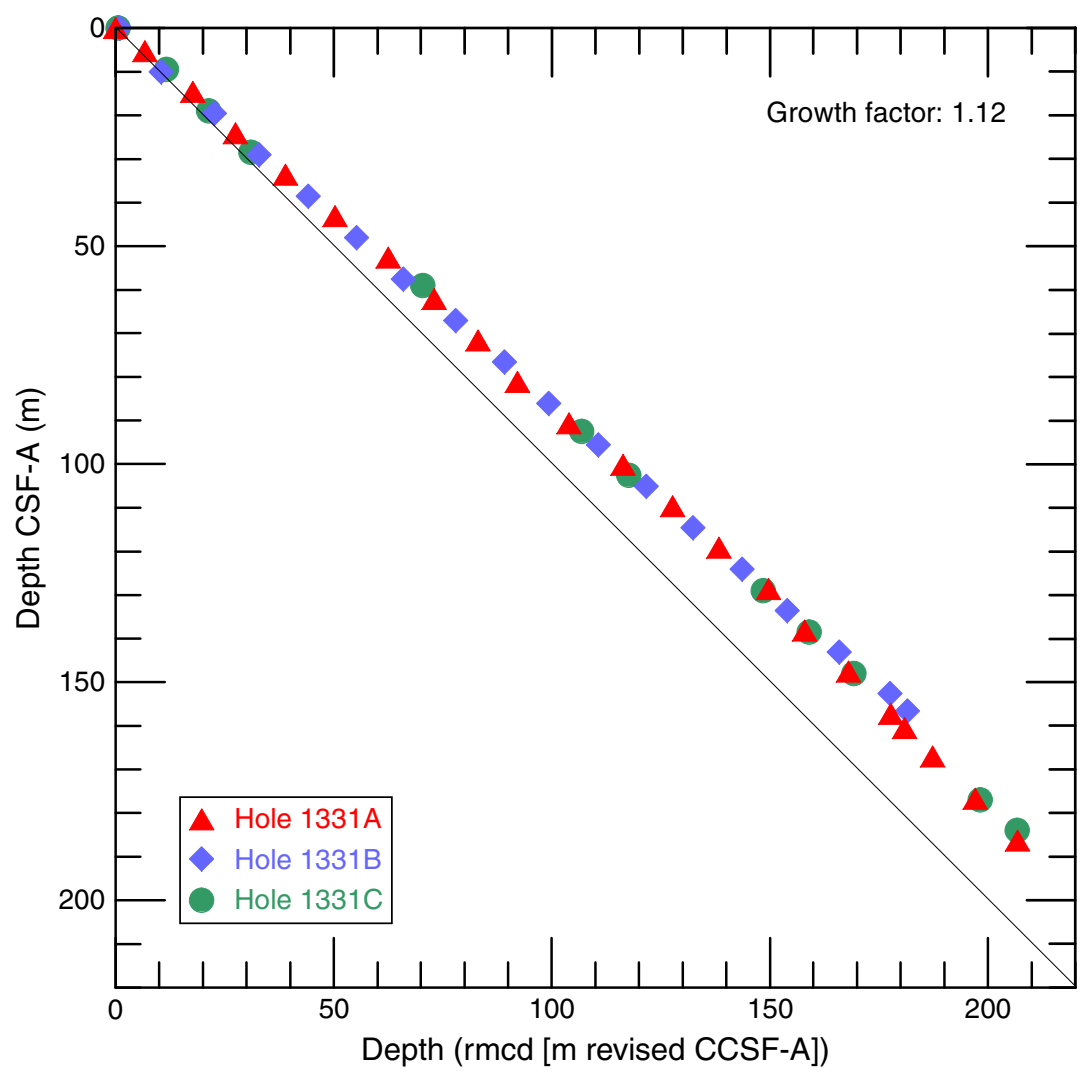


Figure F5. Site U1332 paleomagnetic and physical property data on rmcd (m revised CCSF-A) scale. Splice map and spliced core image on the left side. VGP = virtual geomagnetic pole, GRA = gamma ray attenuation. Red = Hole U1332A, blue $=$ Hole U1332B, green $=$ Hole U1332C, black = composite record . Composite record line is discontinuous because of distortion and data gaps. (Continued on next two pages.)

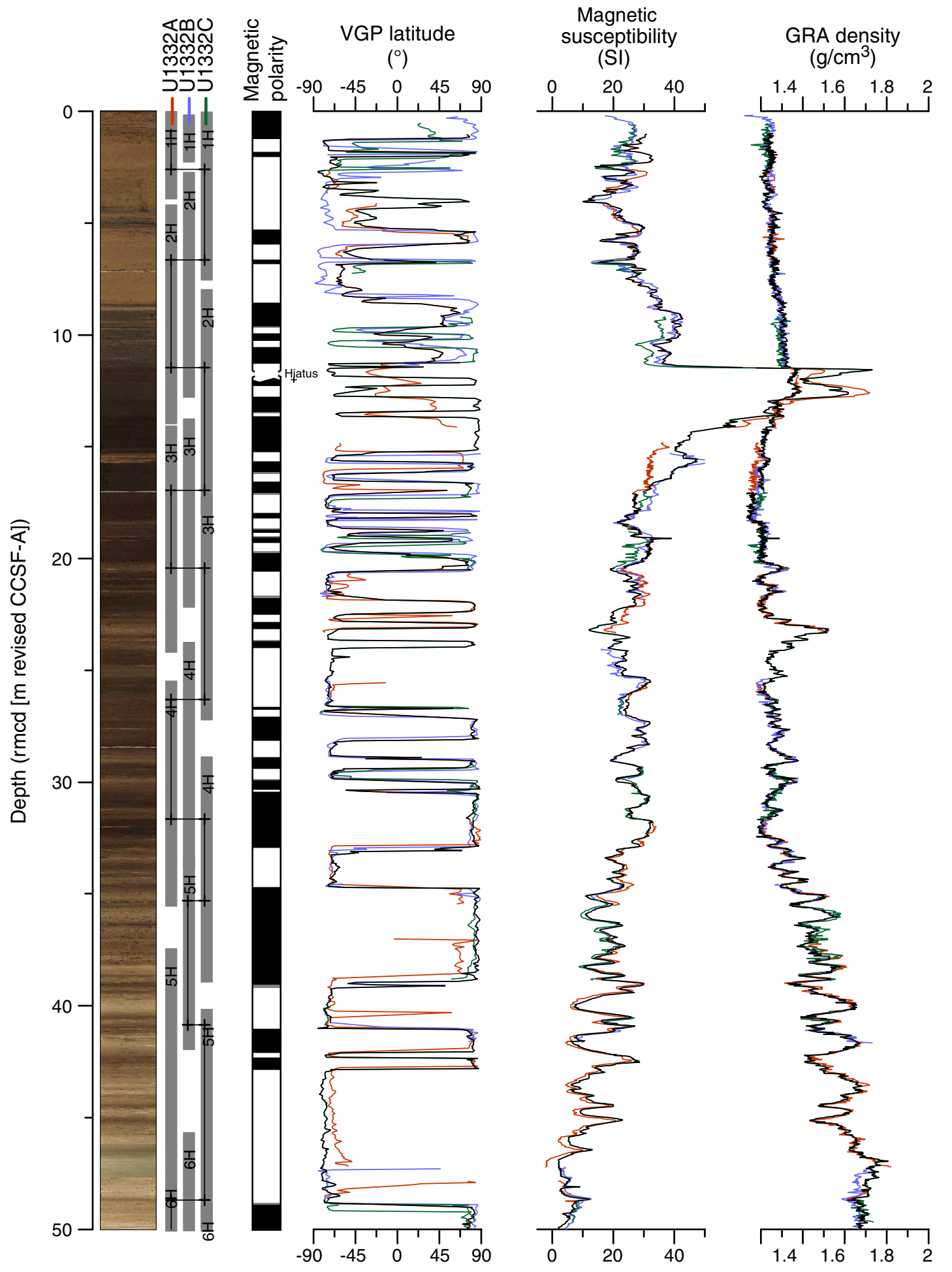


Figure F5 (continued). (Continued on next page.)

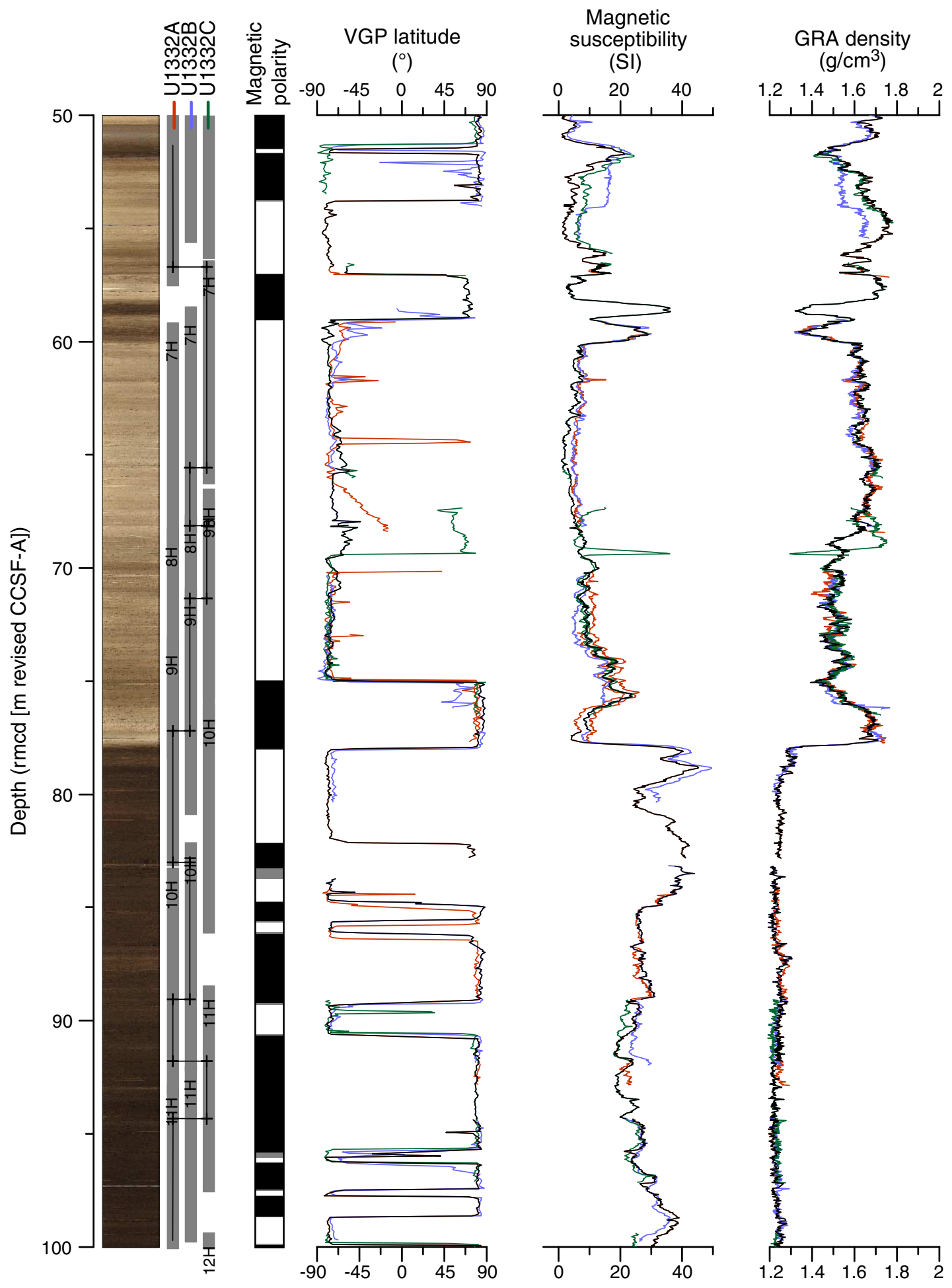


Figure F5 (continued).
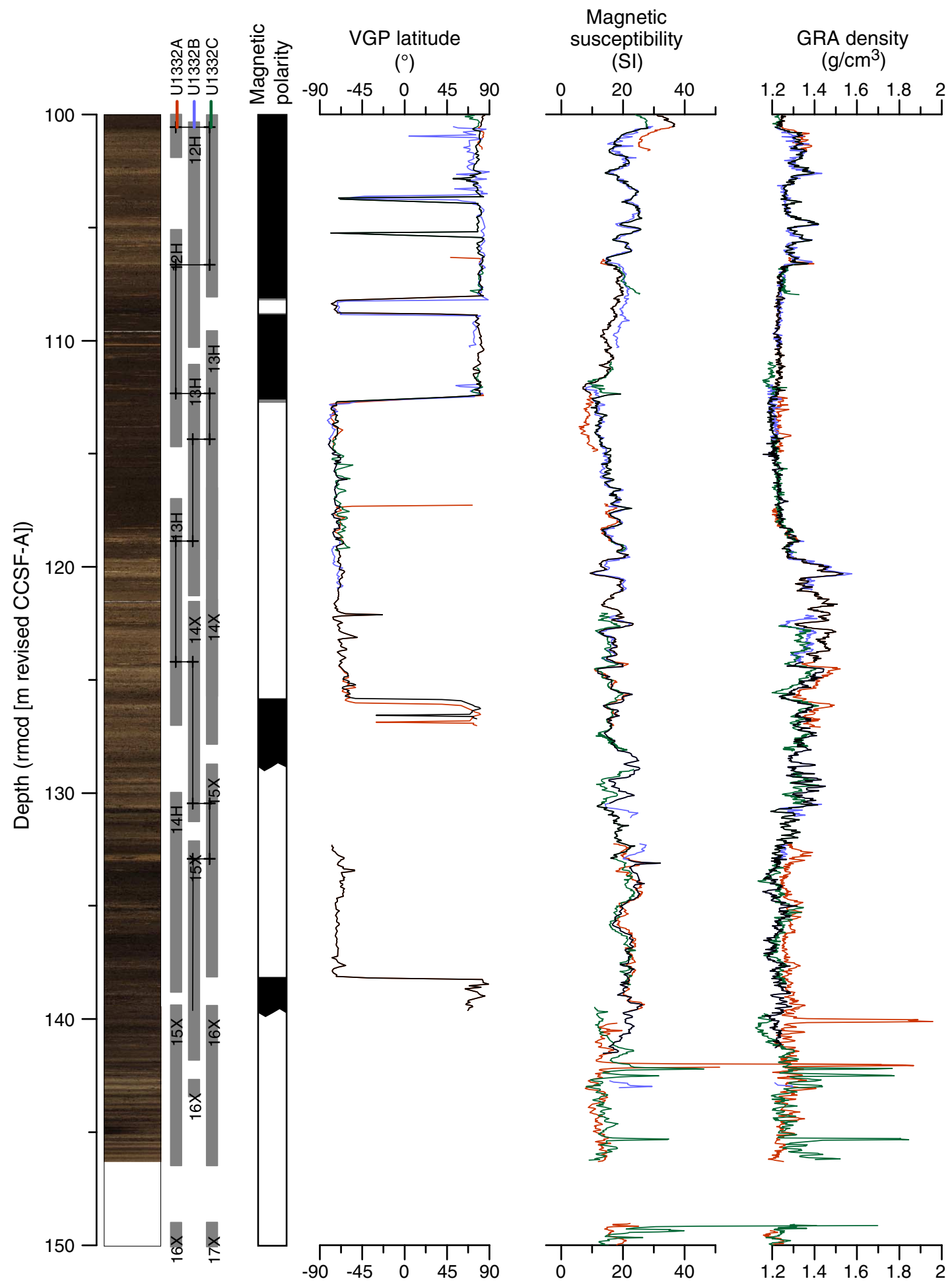
Figure F6. Digital line scan images, Site U1332. Red lines are the tops of splice sections and yellow lines are the bases of those sections (tie to next section). Images were depth registered by R. Wilkens using IGOR-Pro software.

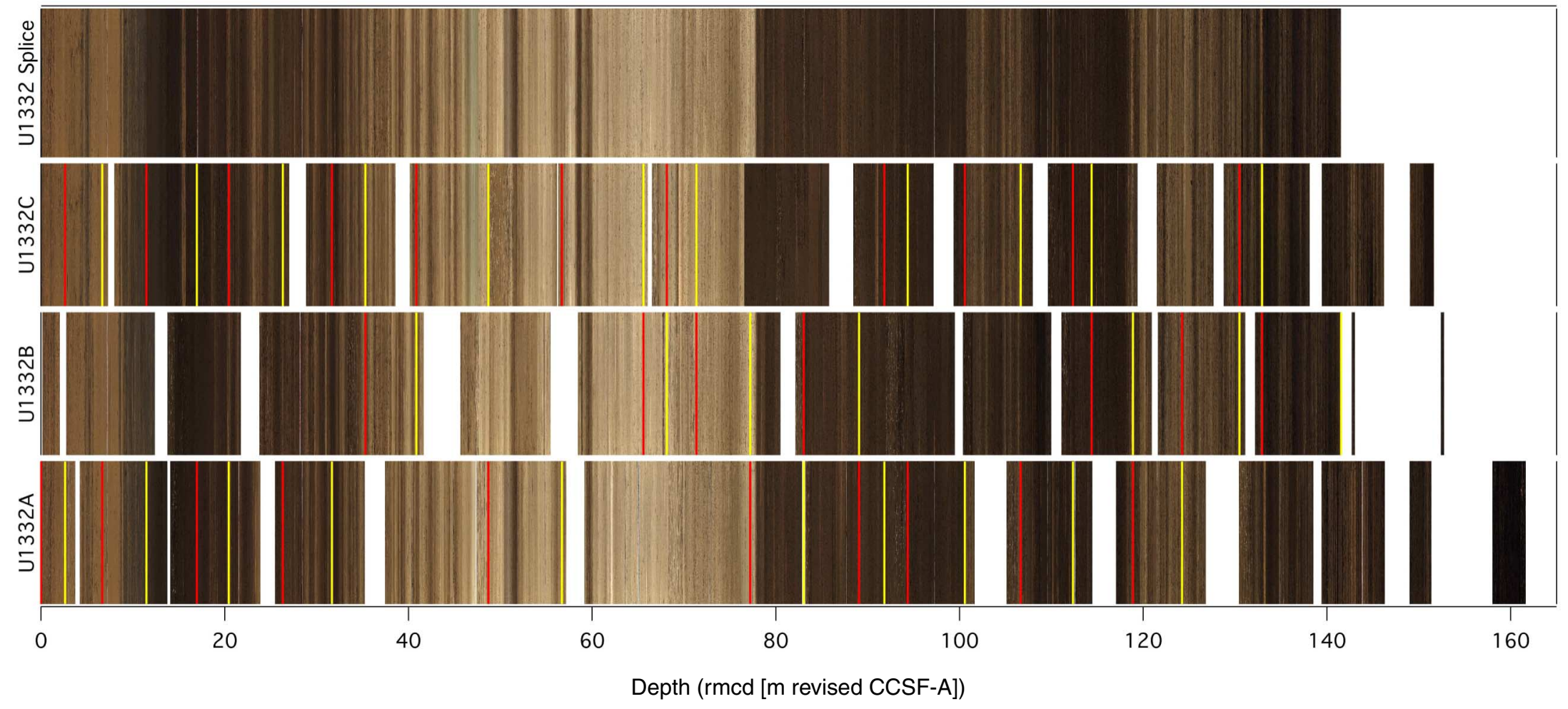


Figure F7. Growth factor calculated by plotting revised composite depth (rmcd [m revised CCSF-A]) against drilled core depth (CSF-A), Site U1332.

\section{Site U1332}

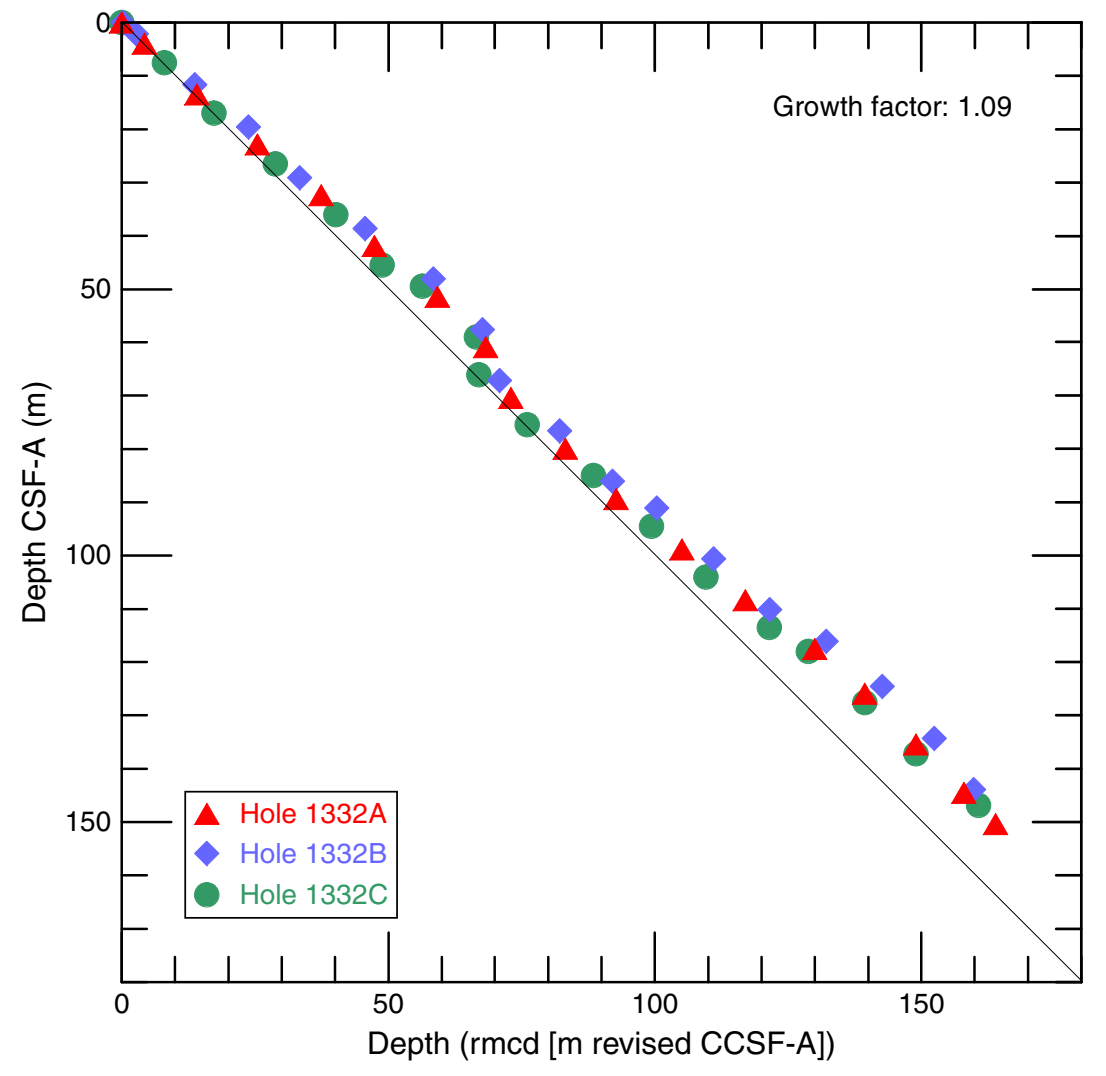


Figure F8. Site U1333 paleomagnetic and physical property data on rmcd (m revised CCSF-A) scale. Splice map and spliced core image on the left side. VGP = virtual geomagnetic pole, GRA = gamma ray attenuation. Red = Hole U1333A, blue $=$ Hole U1333B, green $=$ Hole U1333C, black = composite record . Composite record line is discontinuous because of distortion and data gaps. (Continued on next three pages.)

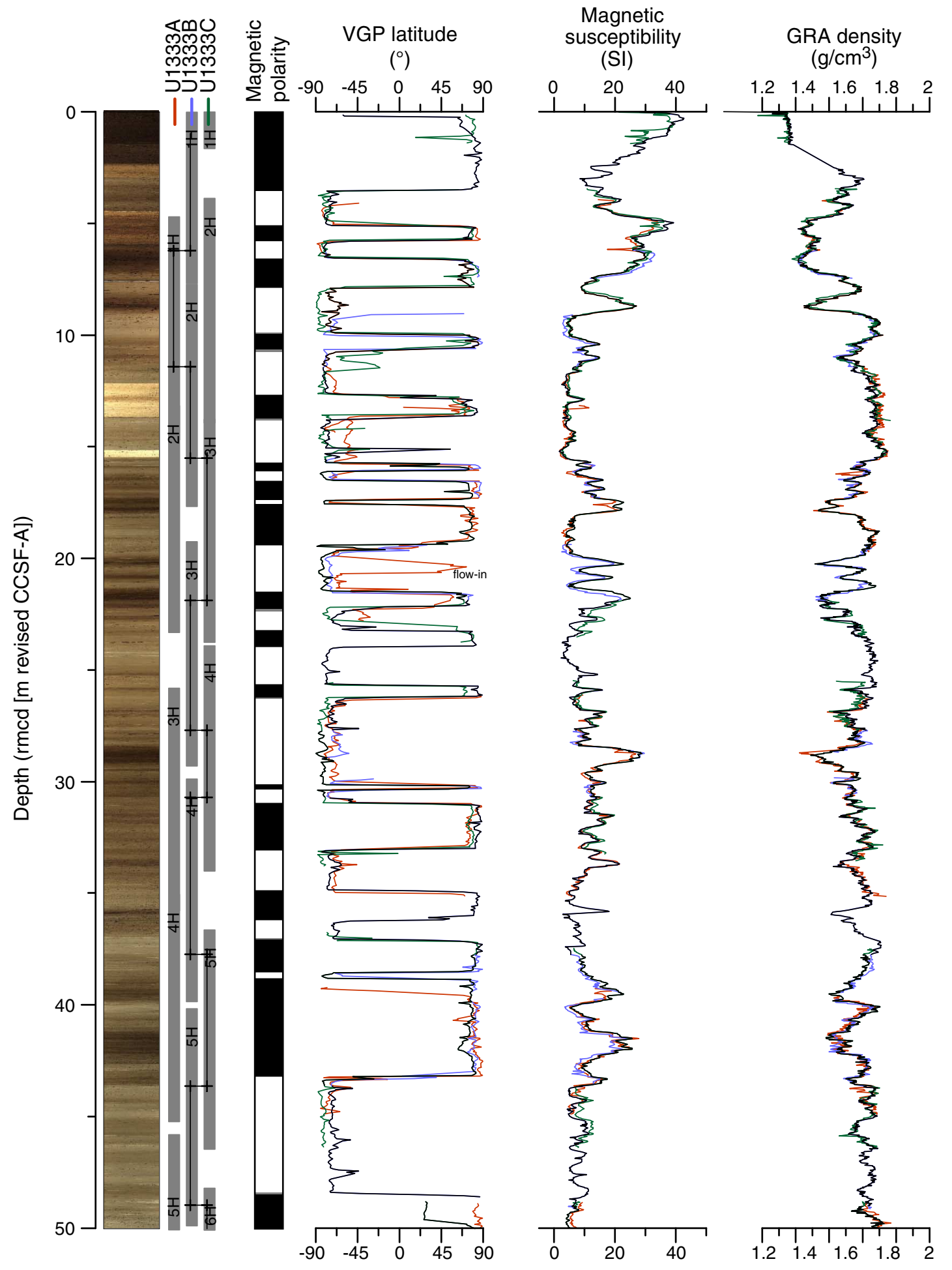


Figure F8 (continued). (Continued on next page.)

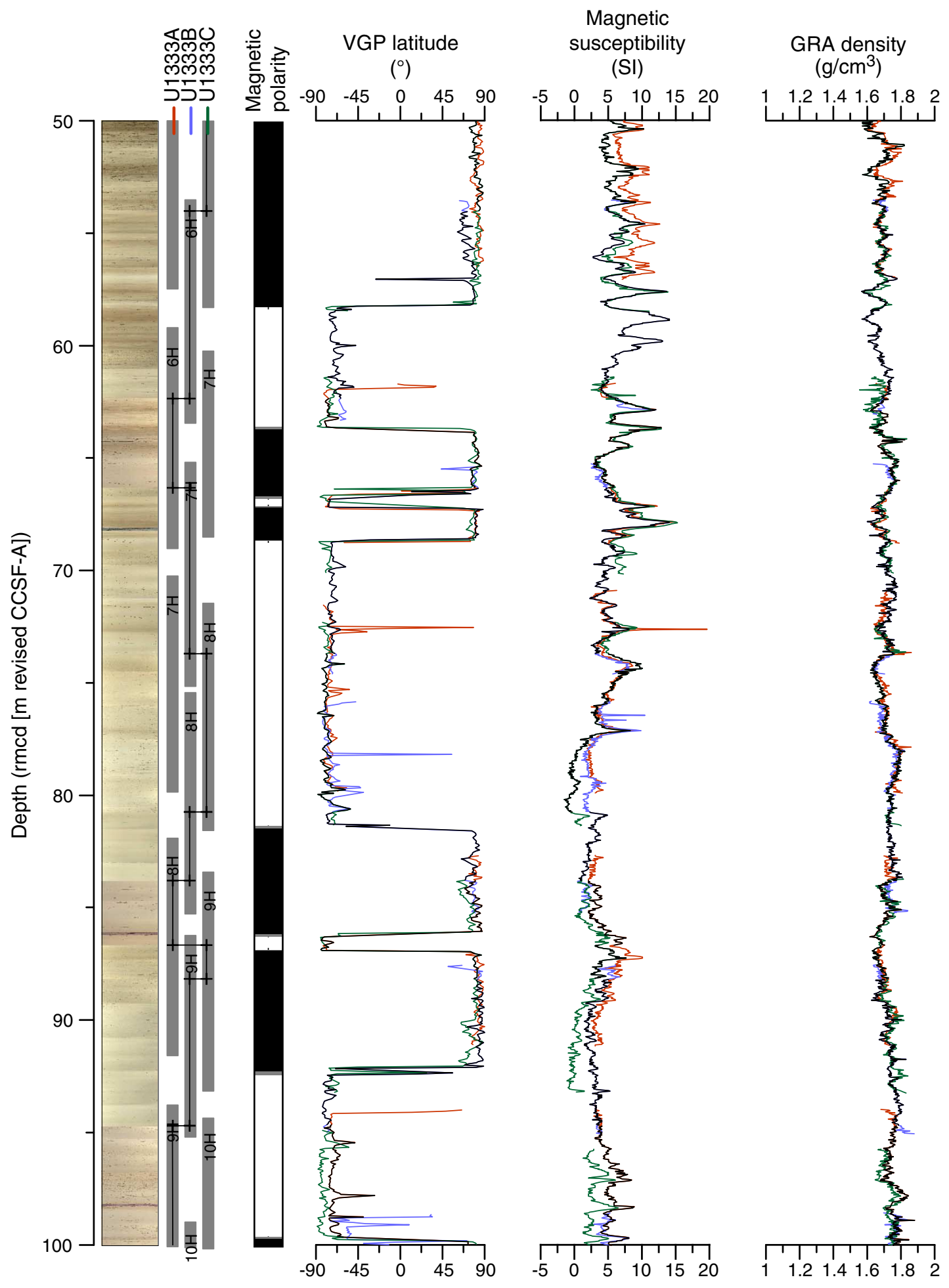


Figure F8 (continued). (Continued on next page.)

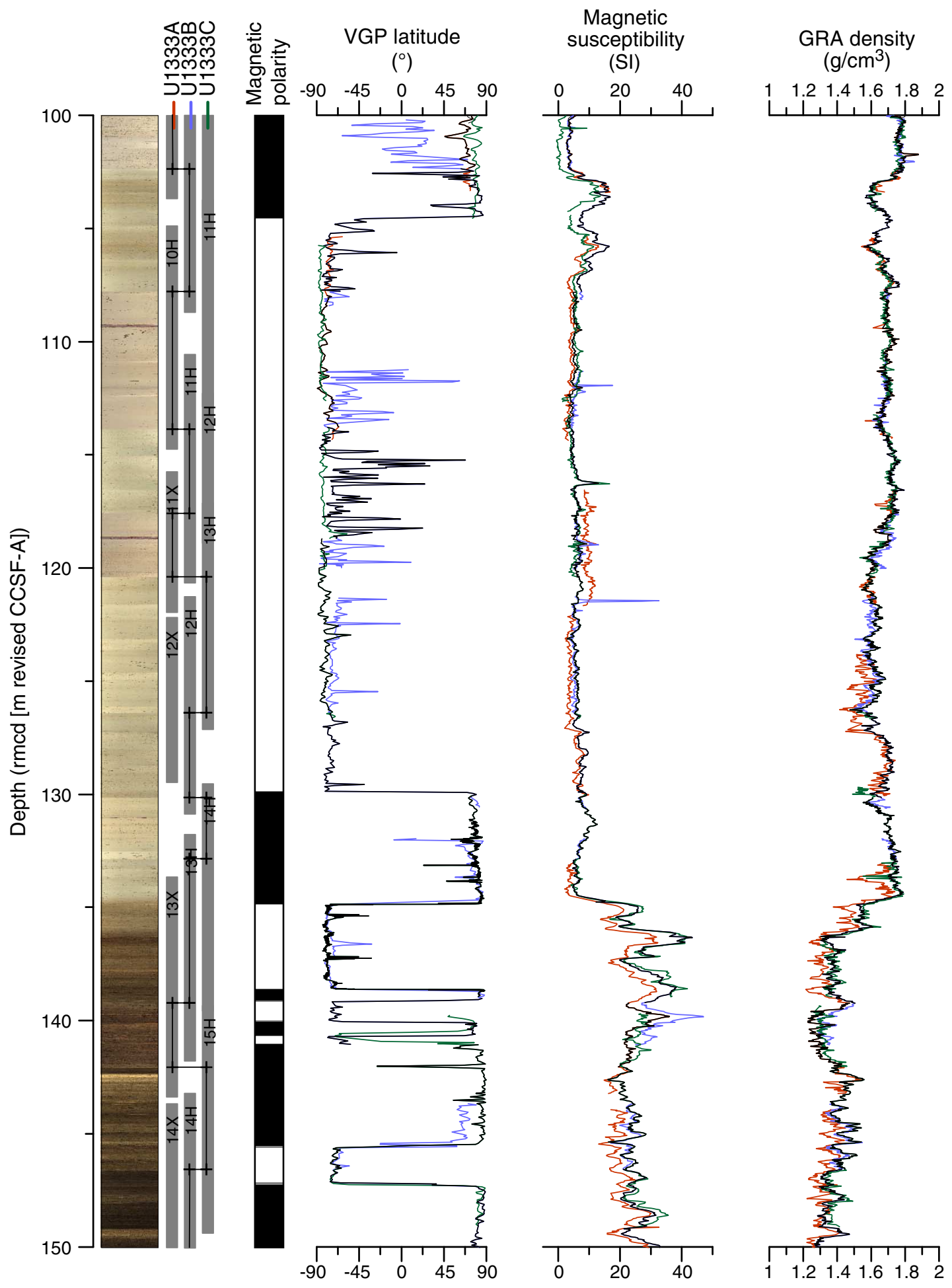


Figure F8 (continued).

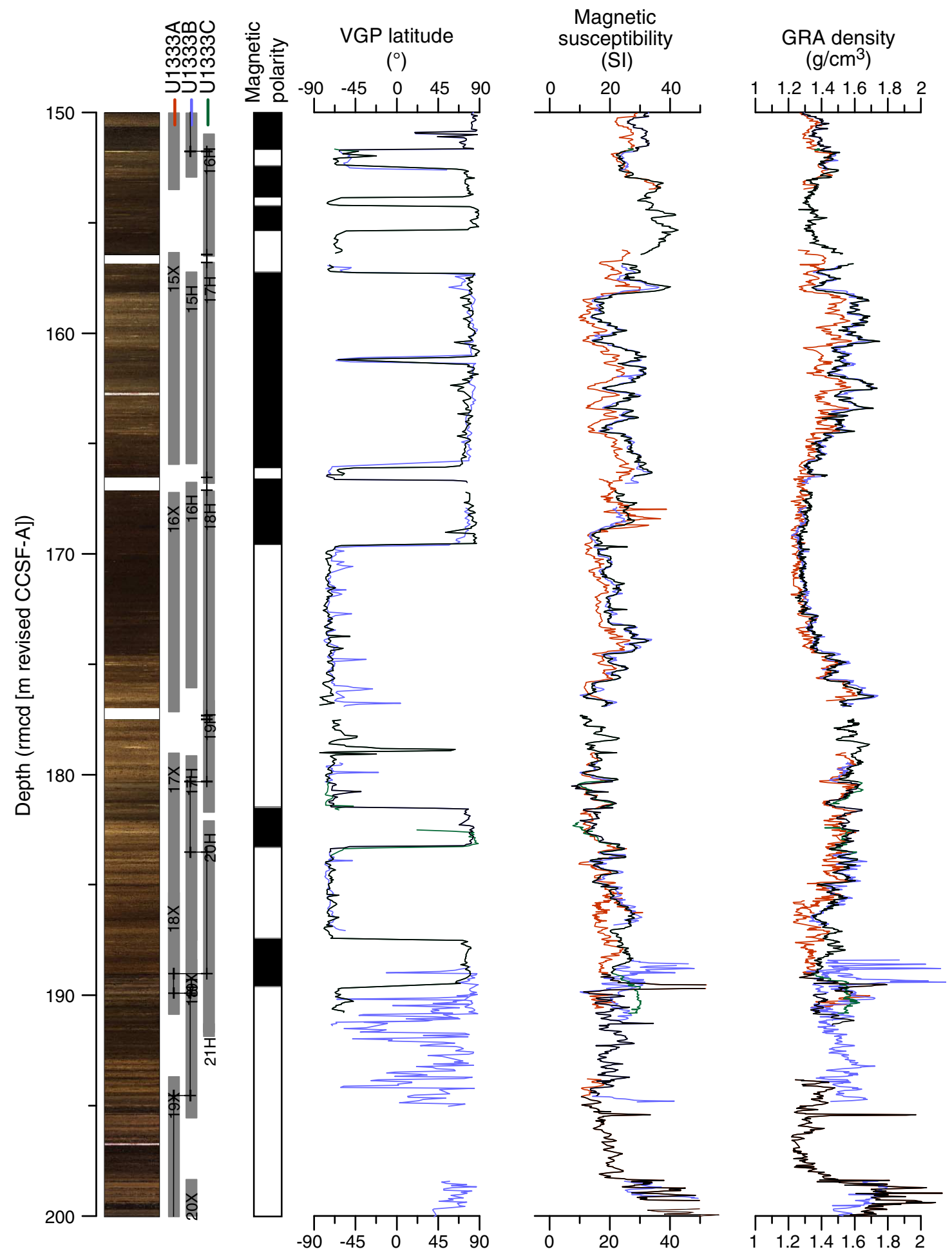


Figure F9. Digital line scan images, Site U1333. Red lines are the tops of splice sections and yellow lines are the bases of those sections (tie to next section). Images were depth registered by R. Wilkens using IGOR-Pro software.
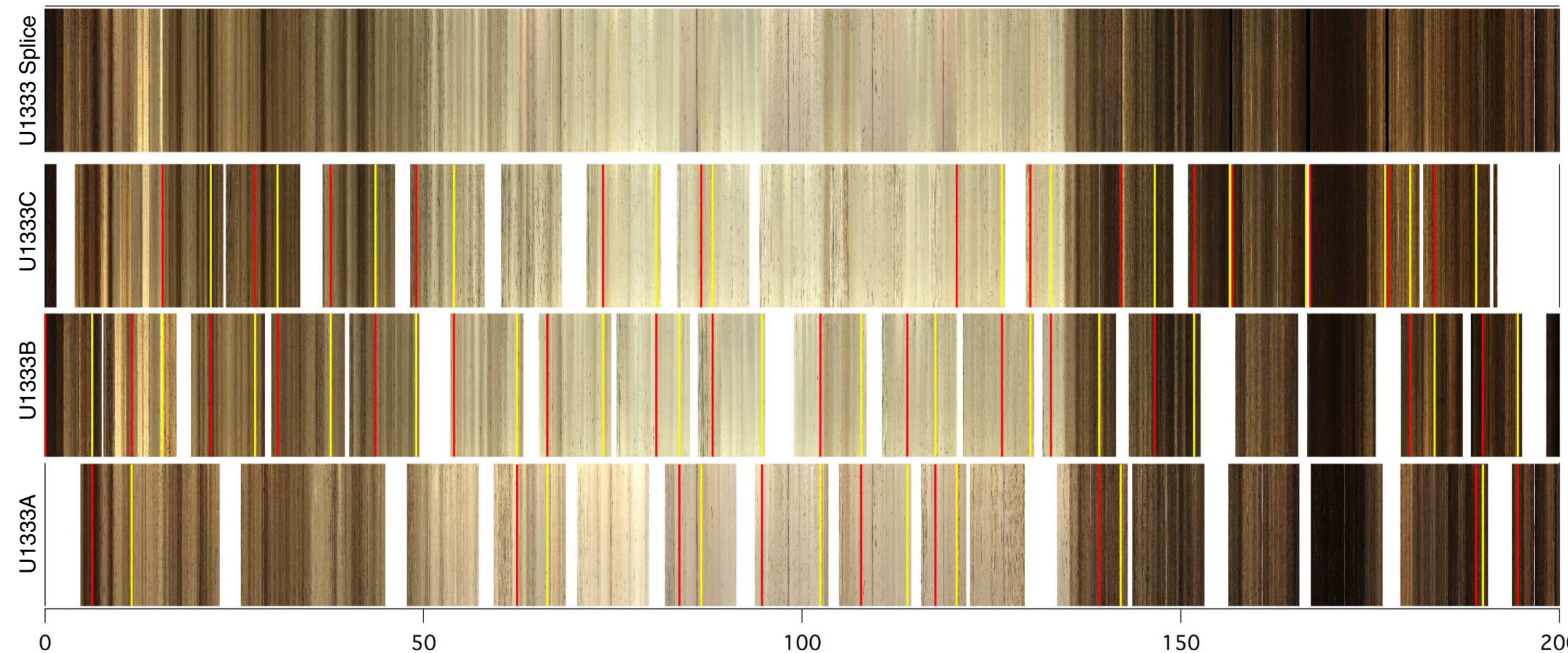

50
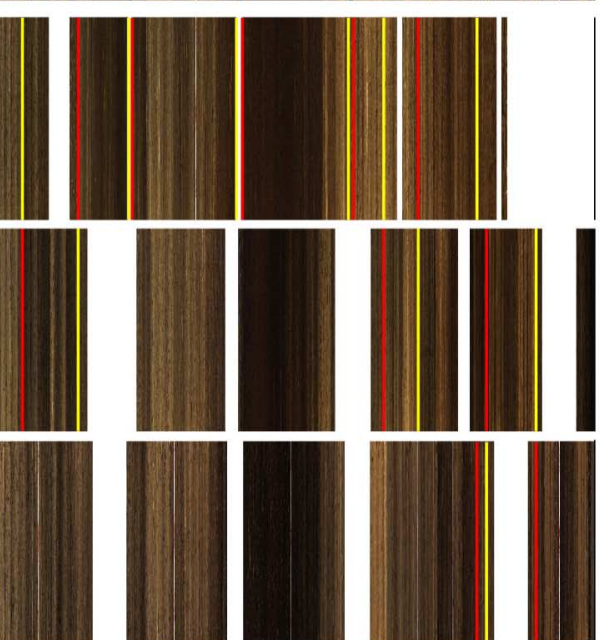

$$
0
$$
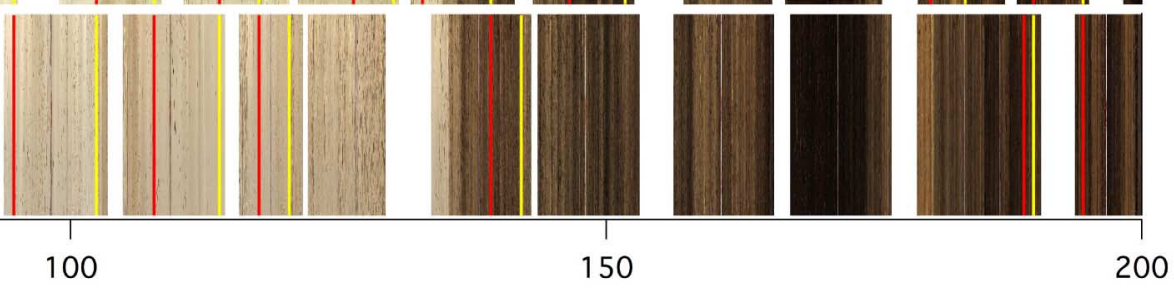

Depth (rmcd [m revised CCSF-A]) 
Figure F10. Growth factor calculated by plotting revised composite depth (rmcd [m revised CCSF-A]) against drilled core depth (CSF-A), Site U1333.

\section{Site U1333}

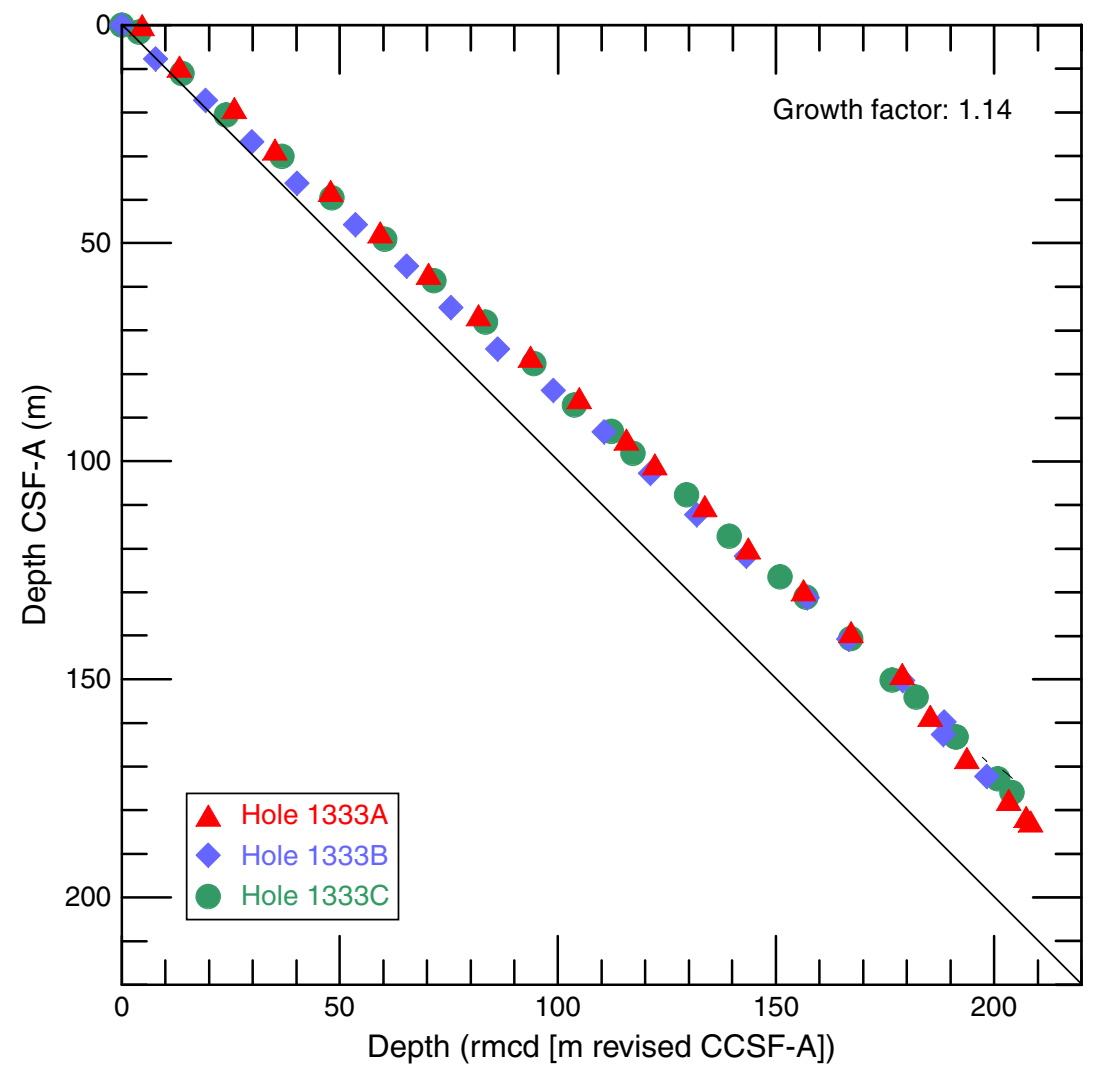


Figure F11. Site U1334 paleomagnetic and physical property data on rmcd (m revised CCSF-A) scale. Splice map and spliced core image on the left side. VGP = virtual geomagnetic pole, GRA = gamma ray attenuation. Red $=$ Hole U1334A, blue $=$ Hole U1334B, green $=$ Hole U1334C, black = composite record . Composite record line is discontinuous because of distortion and data gaps. (Continued on next six pages.)

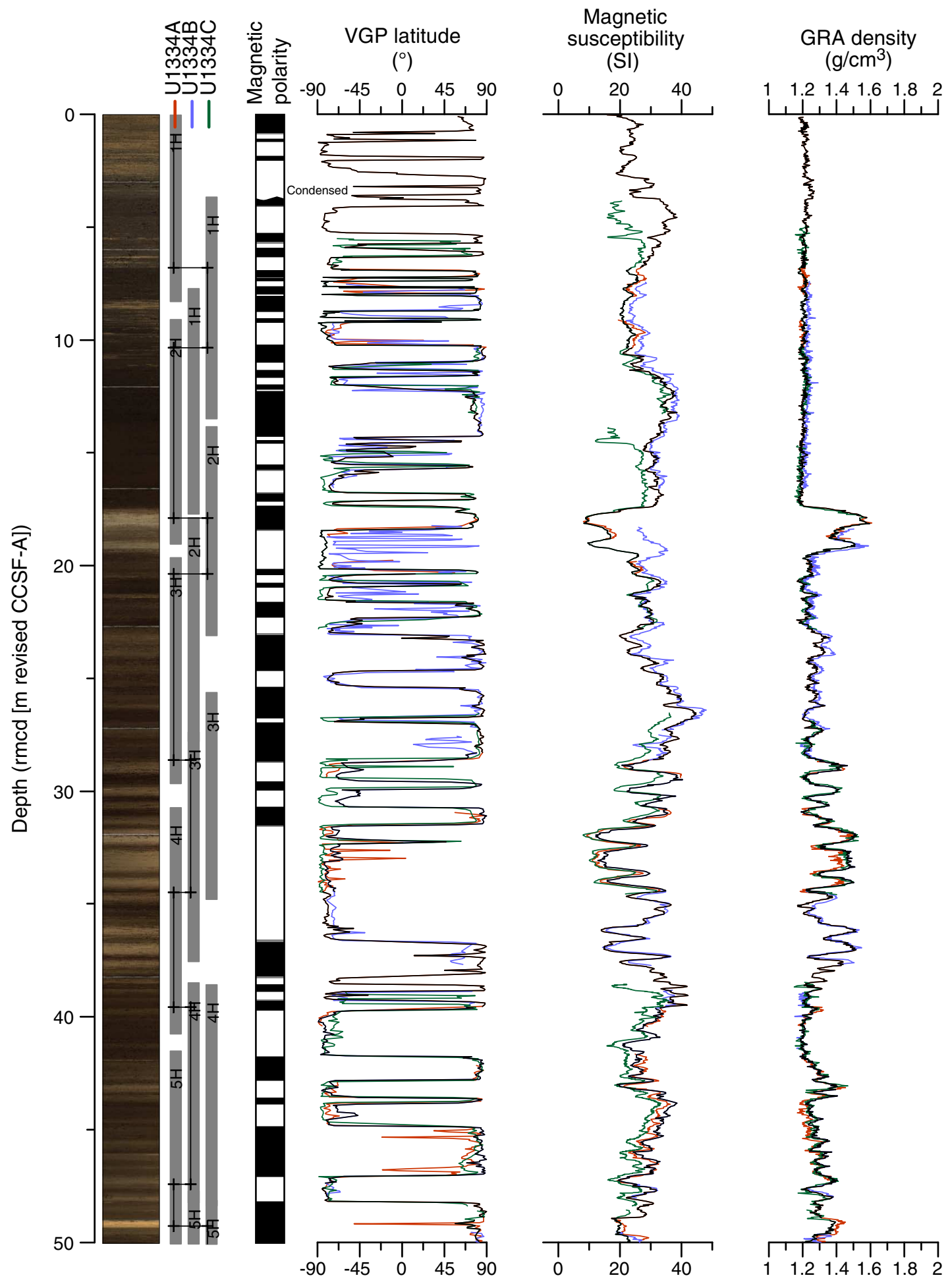


Figure F11 (continued). (Continued on next page.)

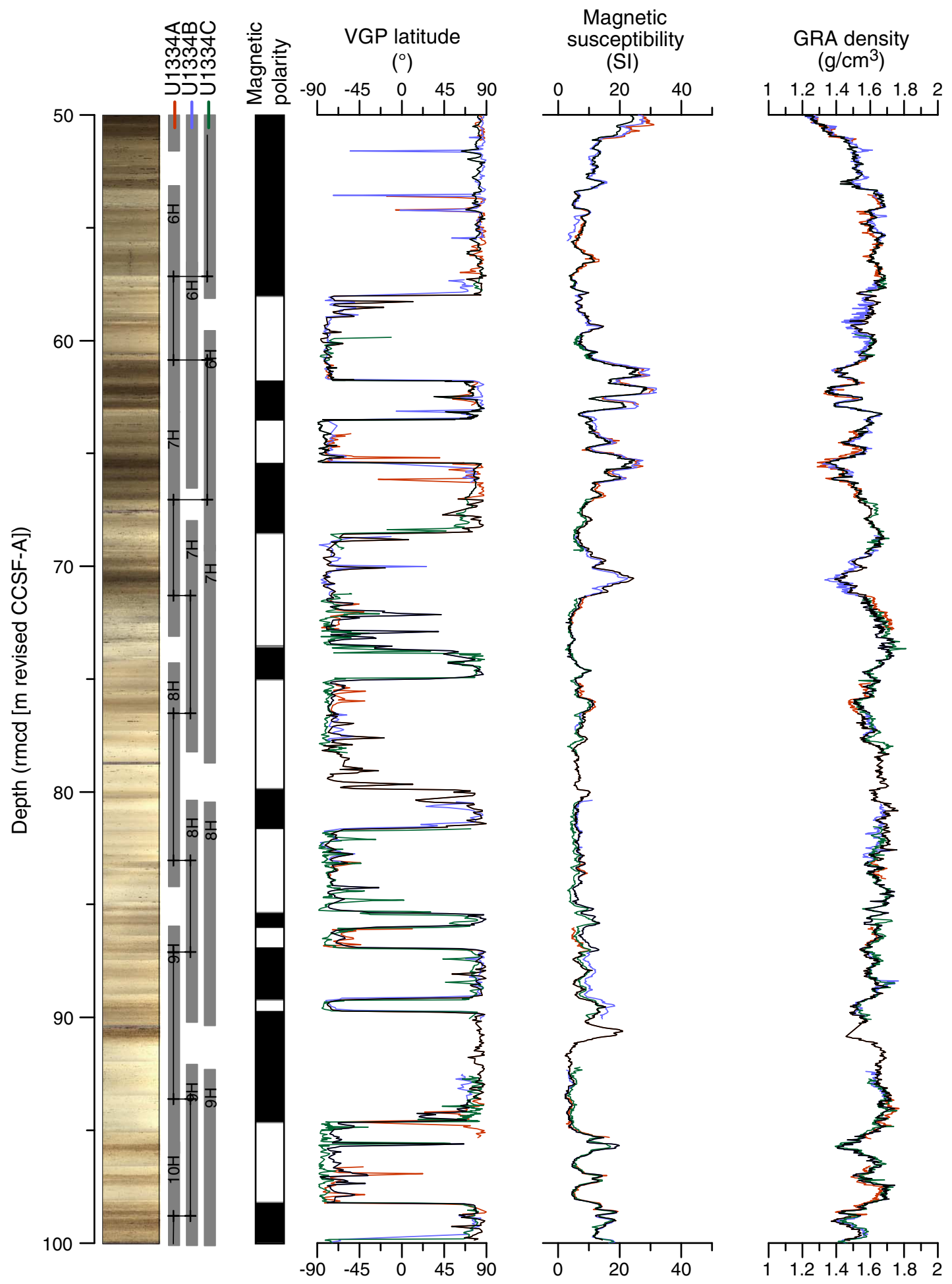


Figure F11 (continued). (Continued on next page.)
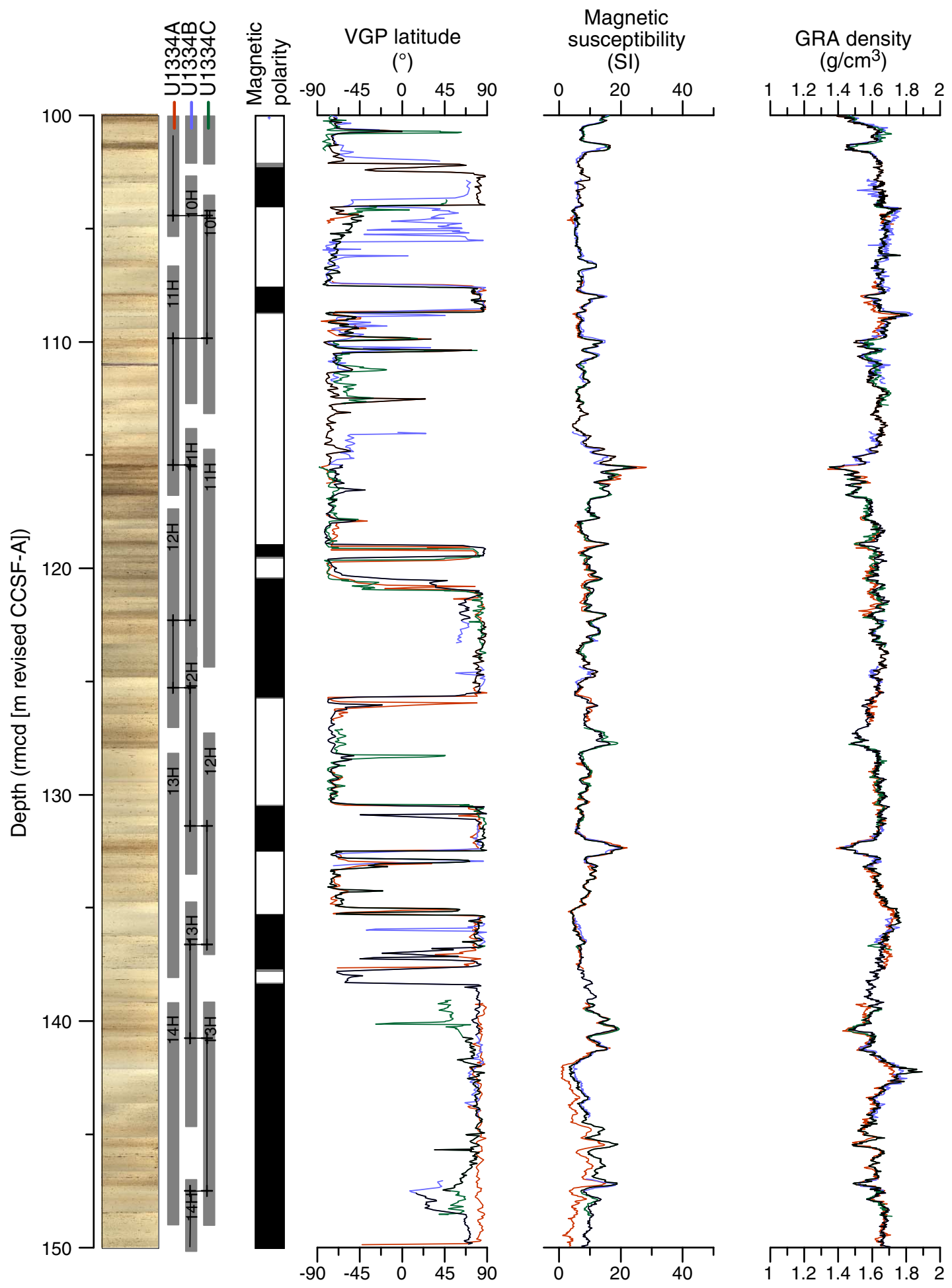
Figure F11 (continued). (Continued on next page.)

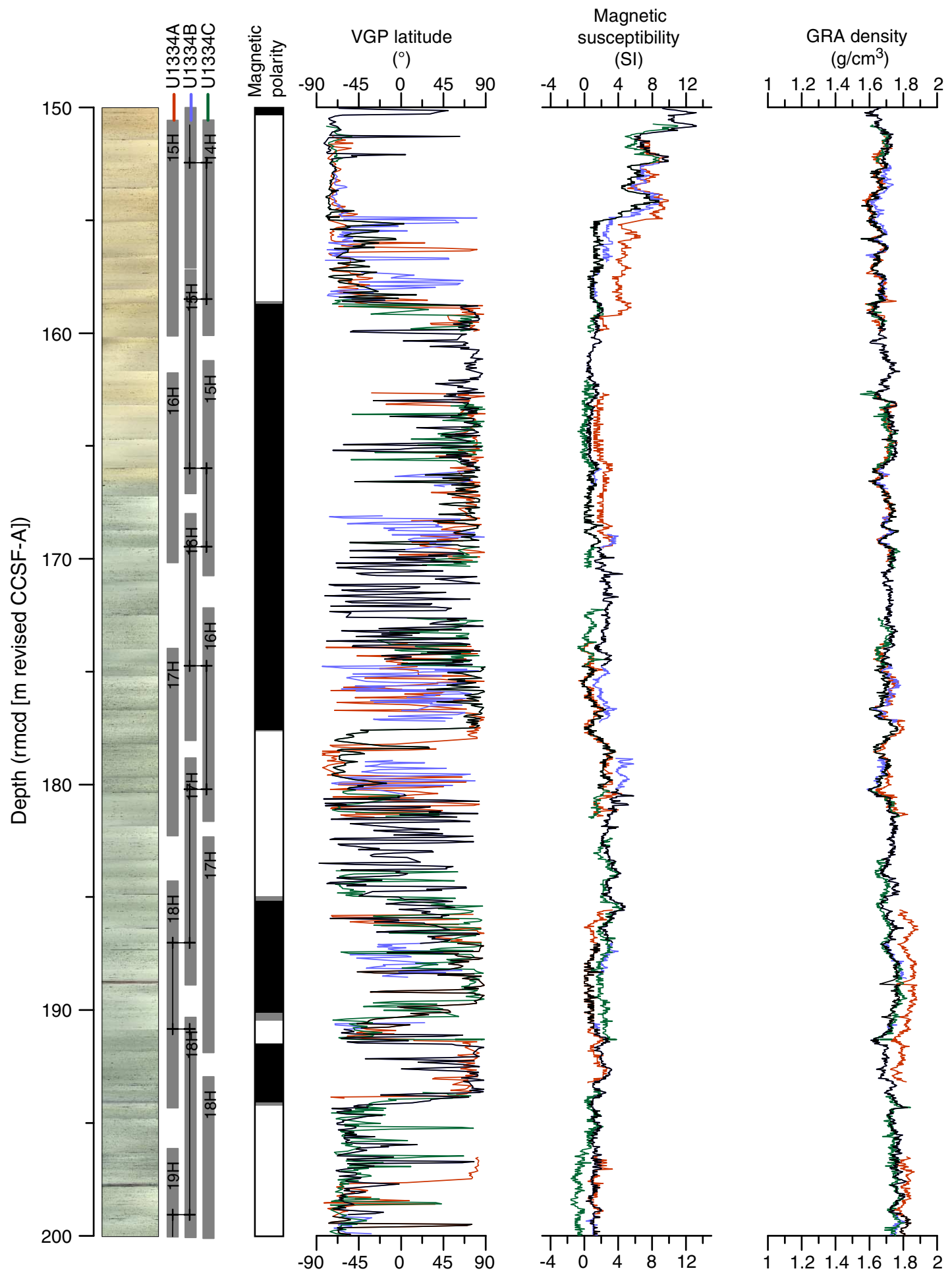


Figure F11 (continued). (Continued on next page.)

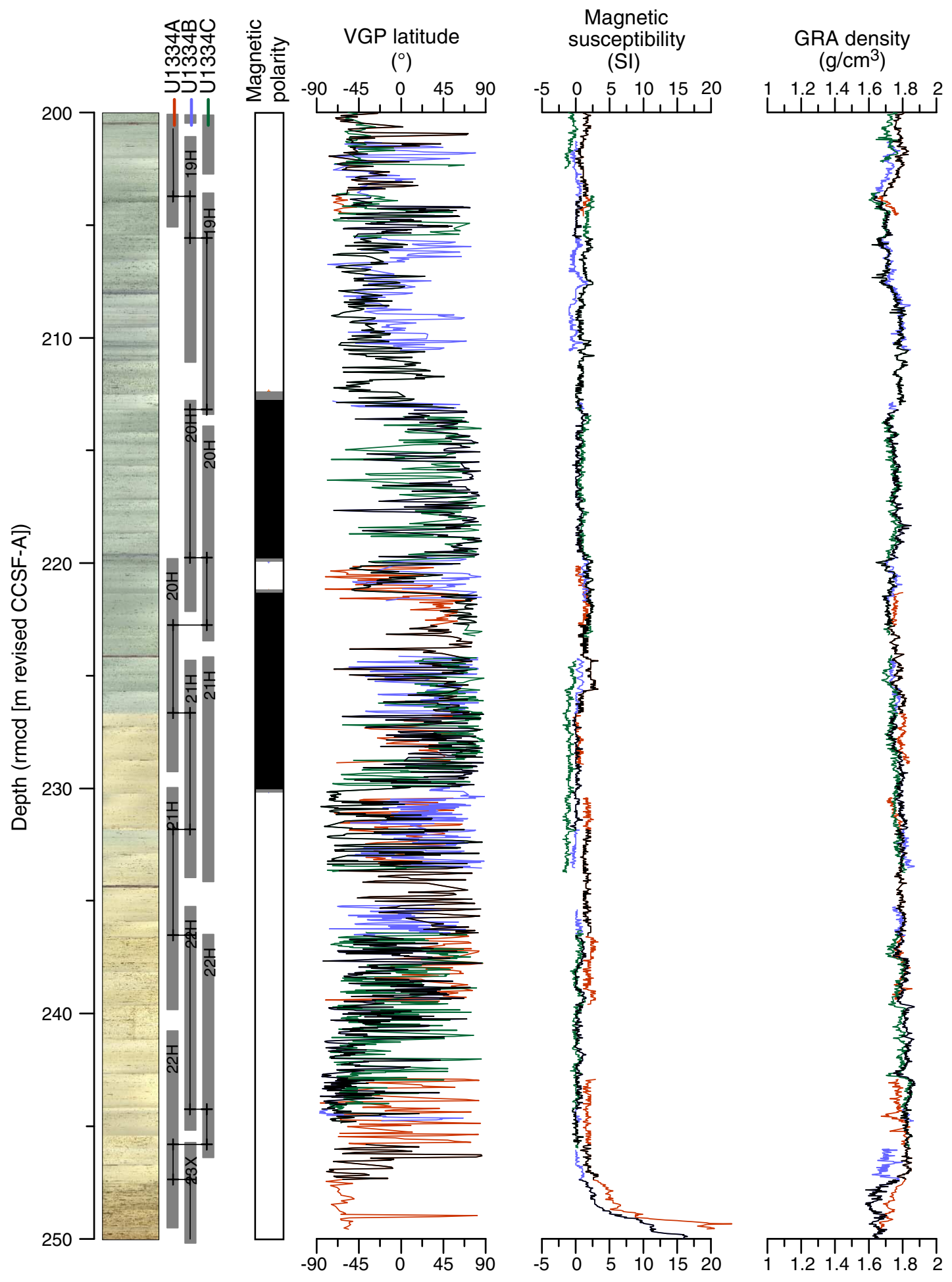


Figure F11 (continued). (Continued on next page.)
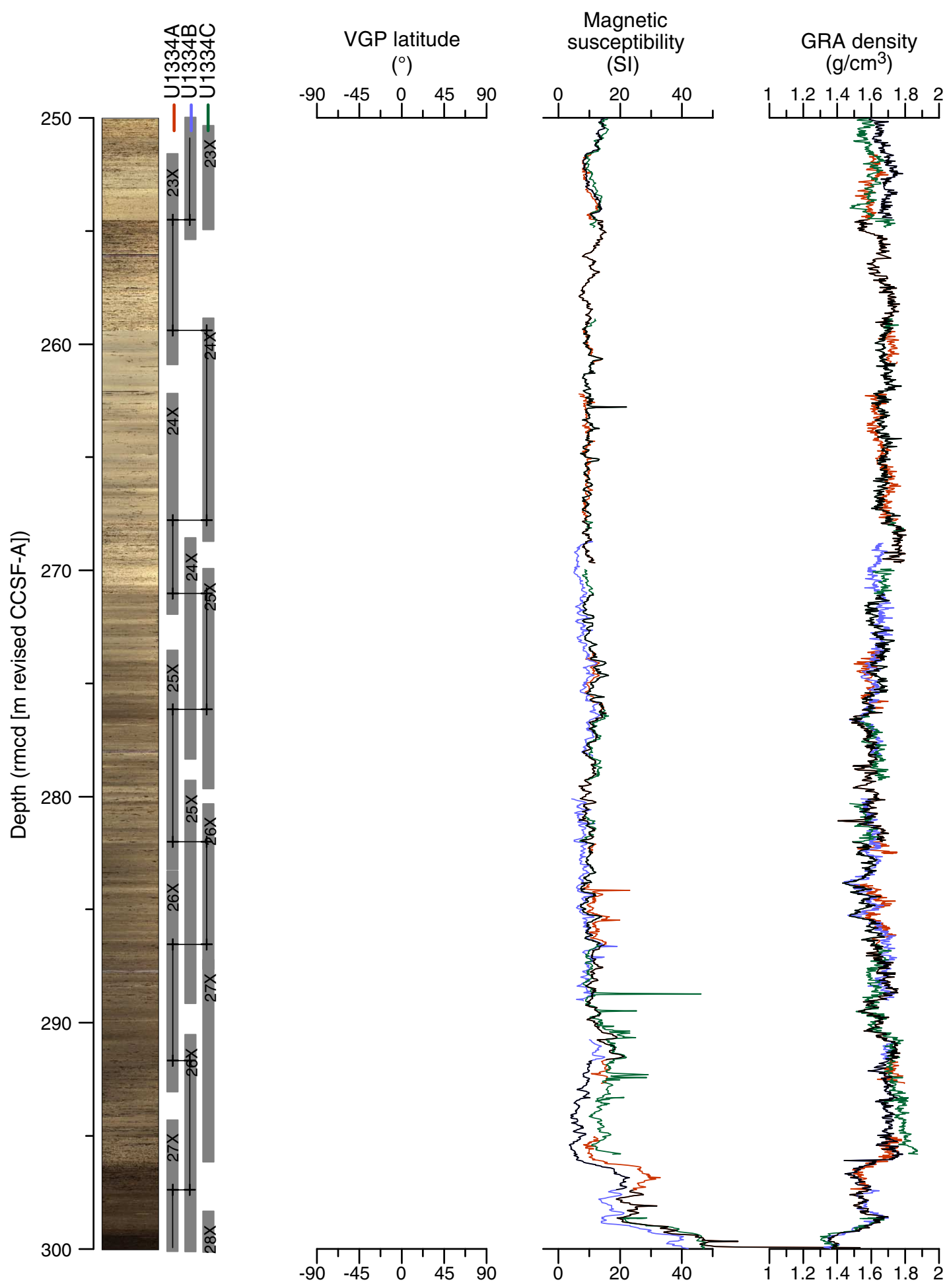
Figure F11 (continued).

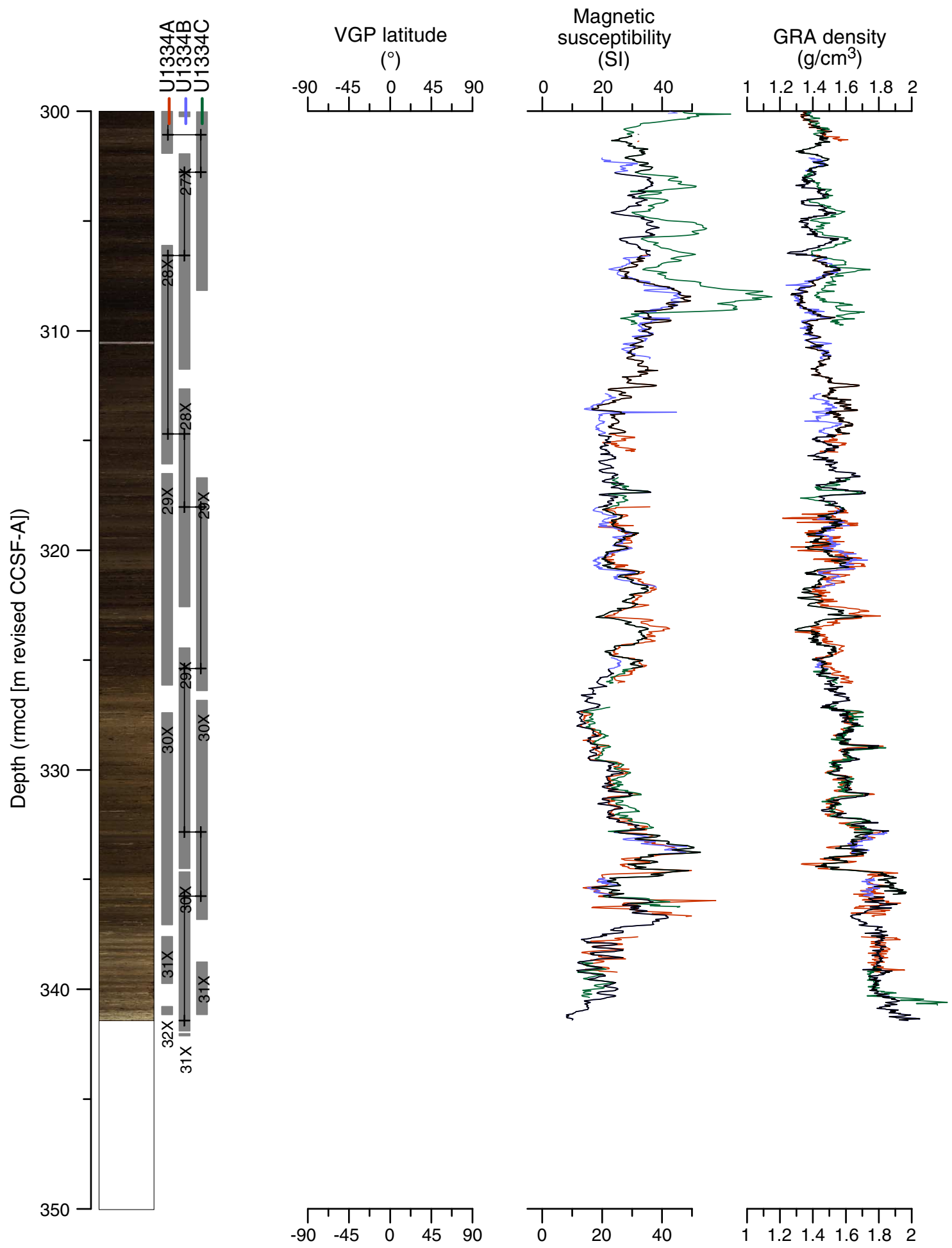


Figure F12. Digital line scan images, Site U1334. Red lines are the tops of splice sections and yellow lines are the bases of those sections (tie to next section). Images were depth registered by R. Wilkens using IGOR-Pro software.

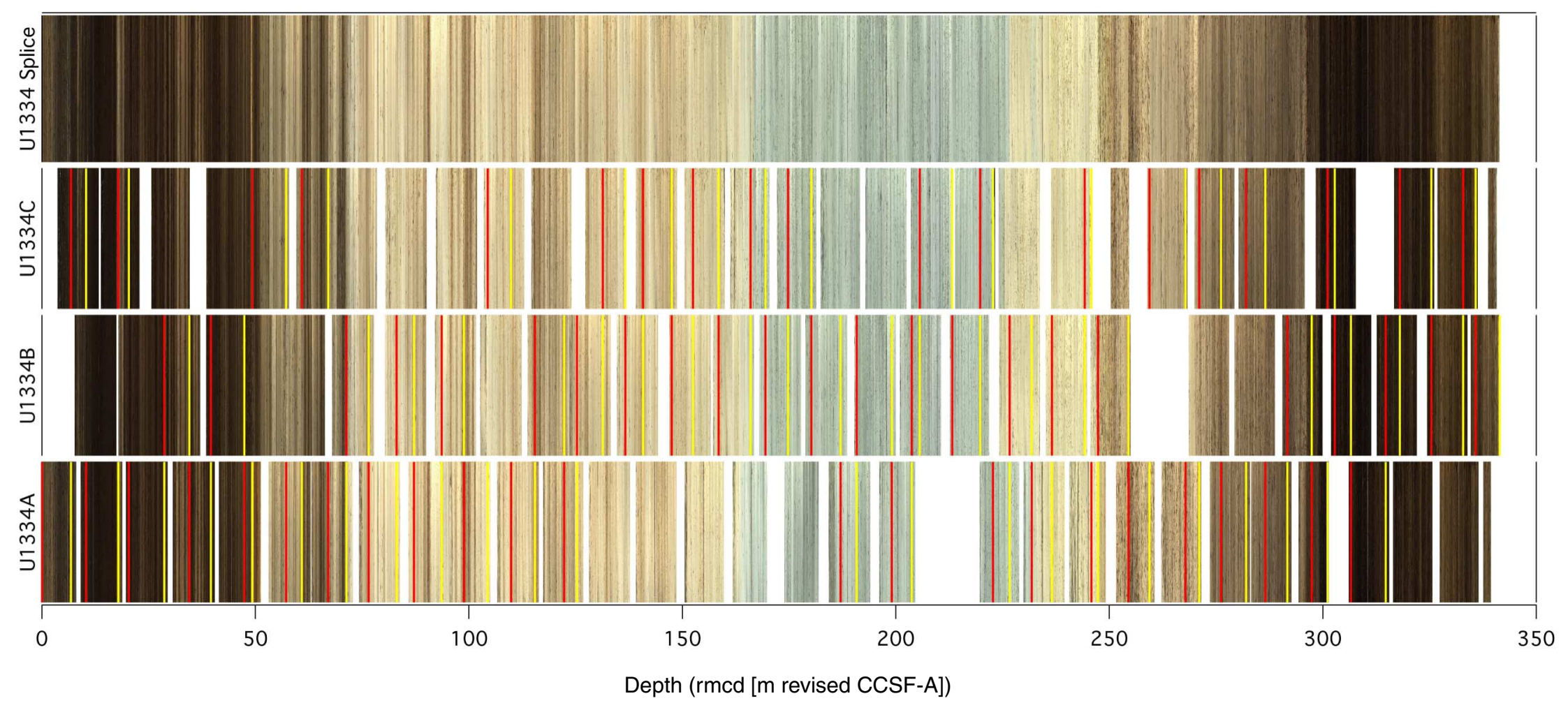

Depth (rmcd [m revised CCSF-A]) 
Figure F13. Growth factor calculated by plotting revised composite depth (rmcd [m revised CCSF-A]) against drilled core depth (CSF-A), Site U1334.

Site U1334

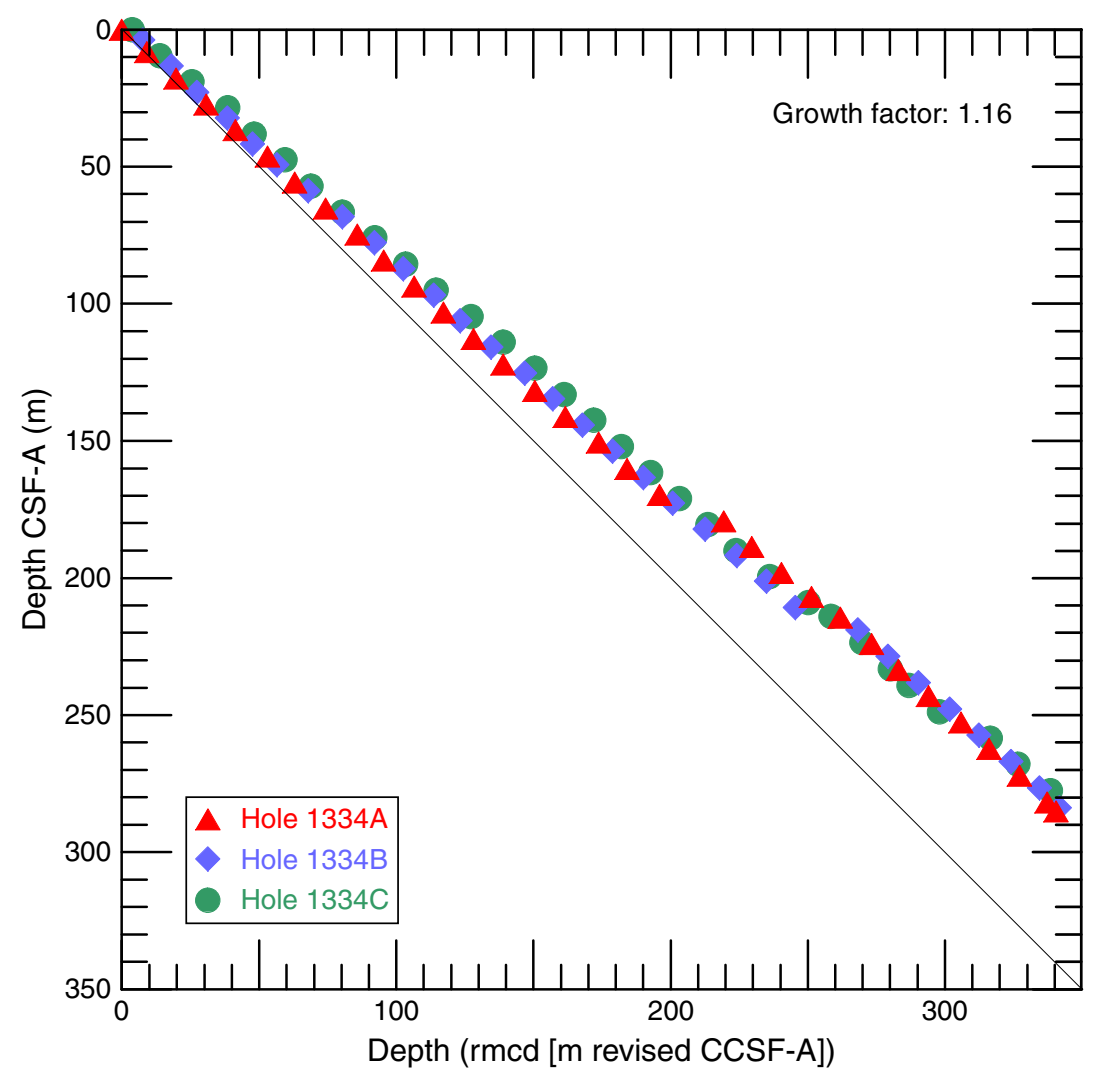


Figure F14. Site 1218 paleomagnetic and physical property data on corrected rmcd scale. Splice map and spliced core image on the left side. VGP = virtual geomagnetic pole, GRA = gamma ray attenuation. Red = Hole $1218 \mathrm{~A}$, blue $=$ Hole $1218 \mathrm{~B}$, green $=$ Hole $1218 \mathrm{C}$, black $=$ composite record . Composite record line is discontinuous because of distortion and data gaps. (Continued on next five pages.)

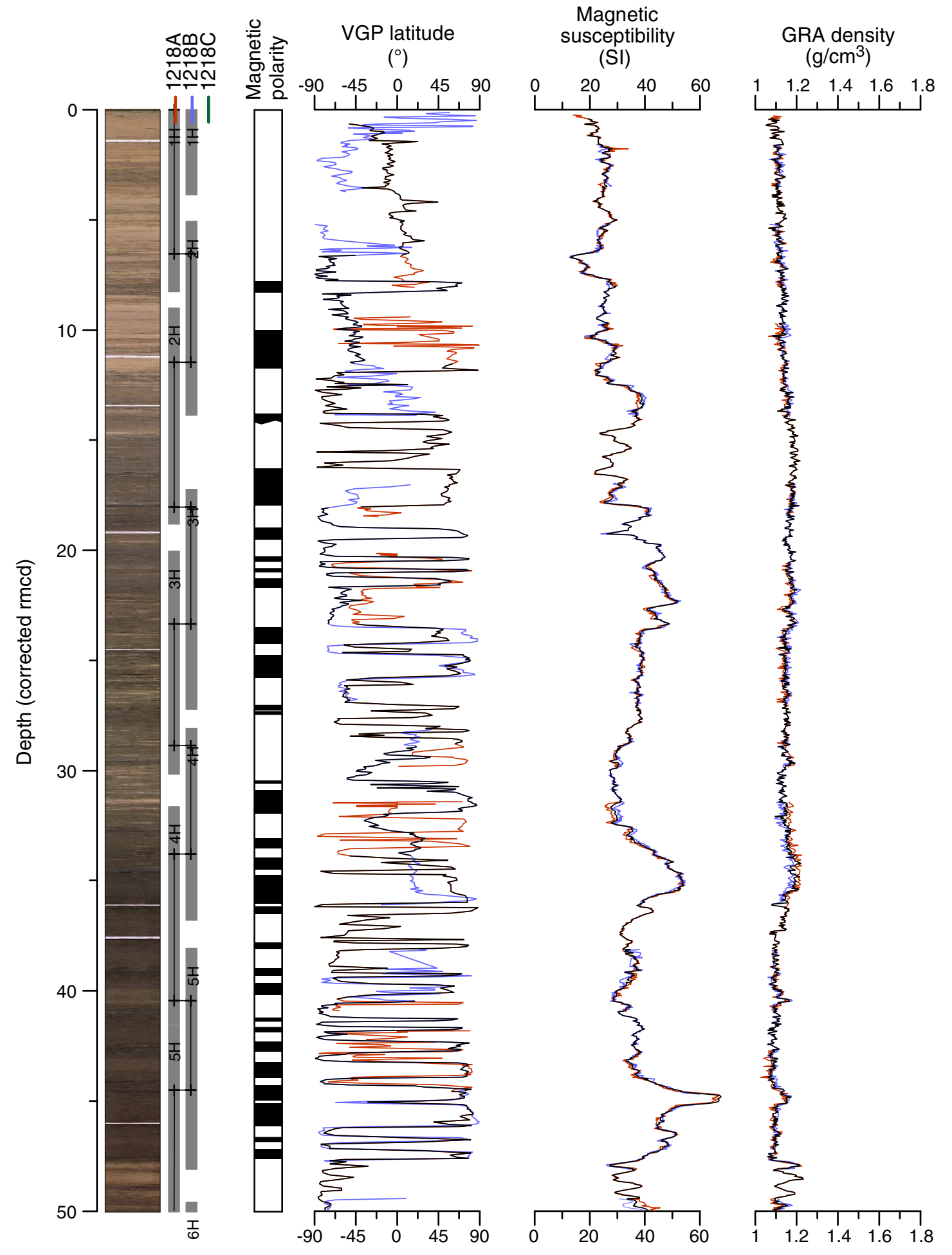


Figure F14 (continued). (Continued on next page.)

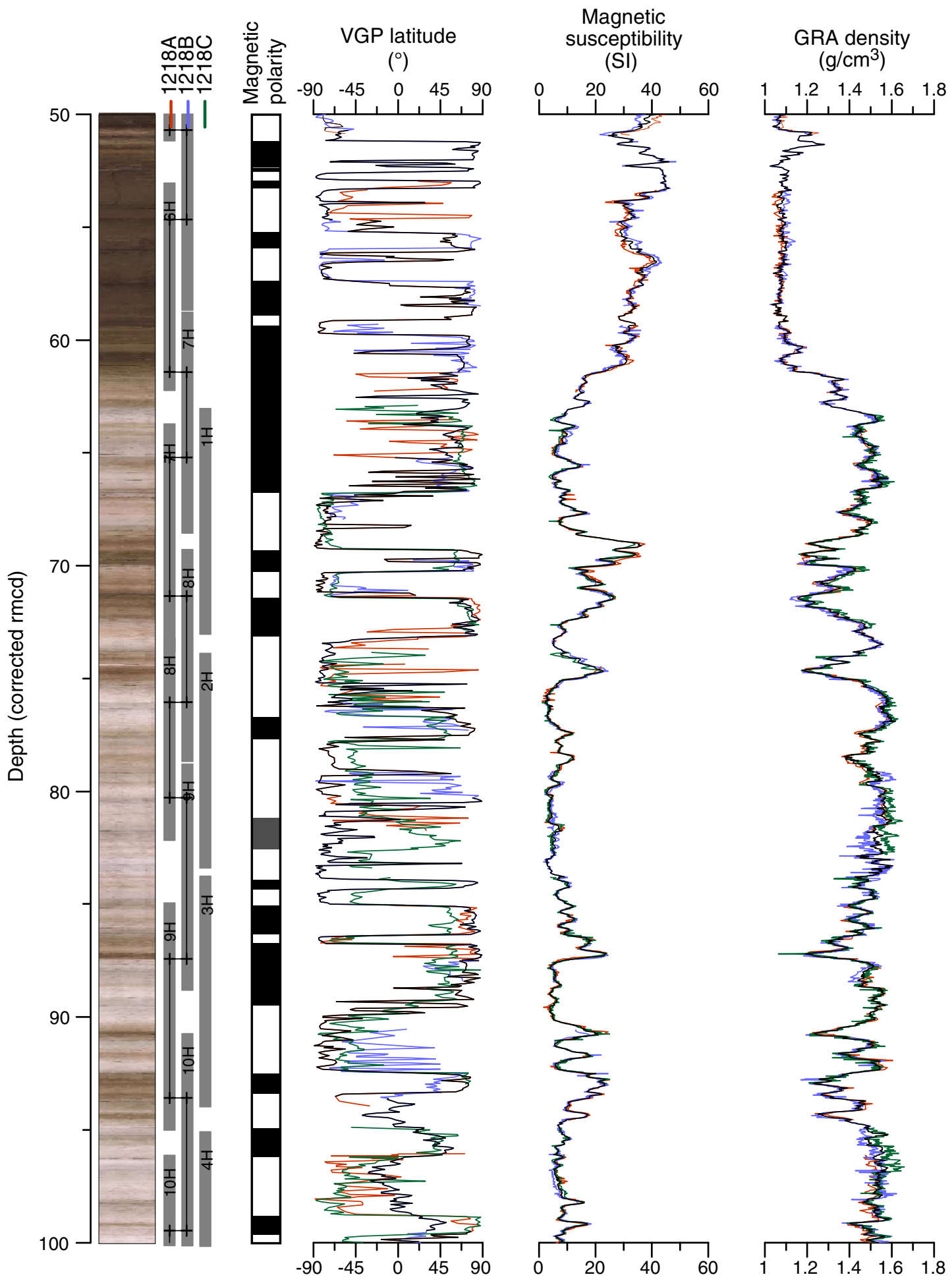


Figure F14 (continued). (Continued on next page.)

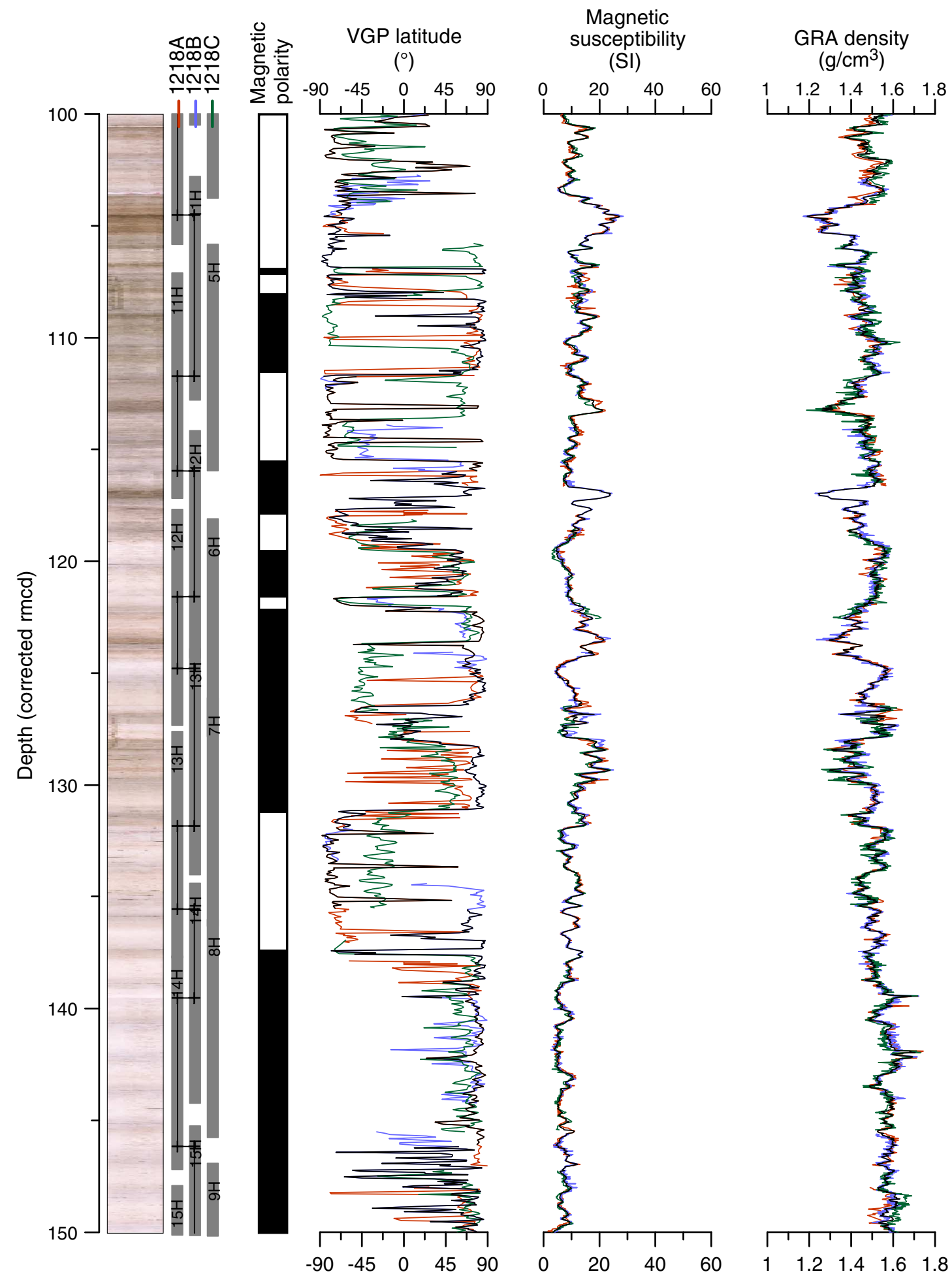


Figure F14 (continued). (Continued on next page.)

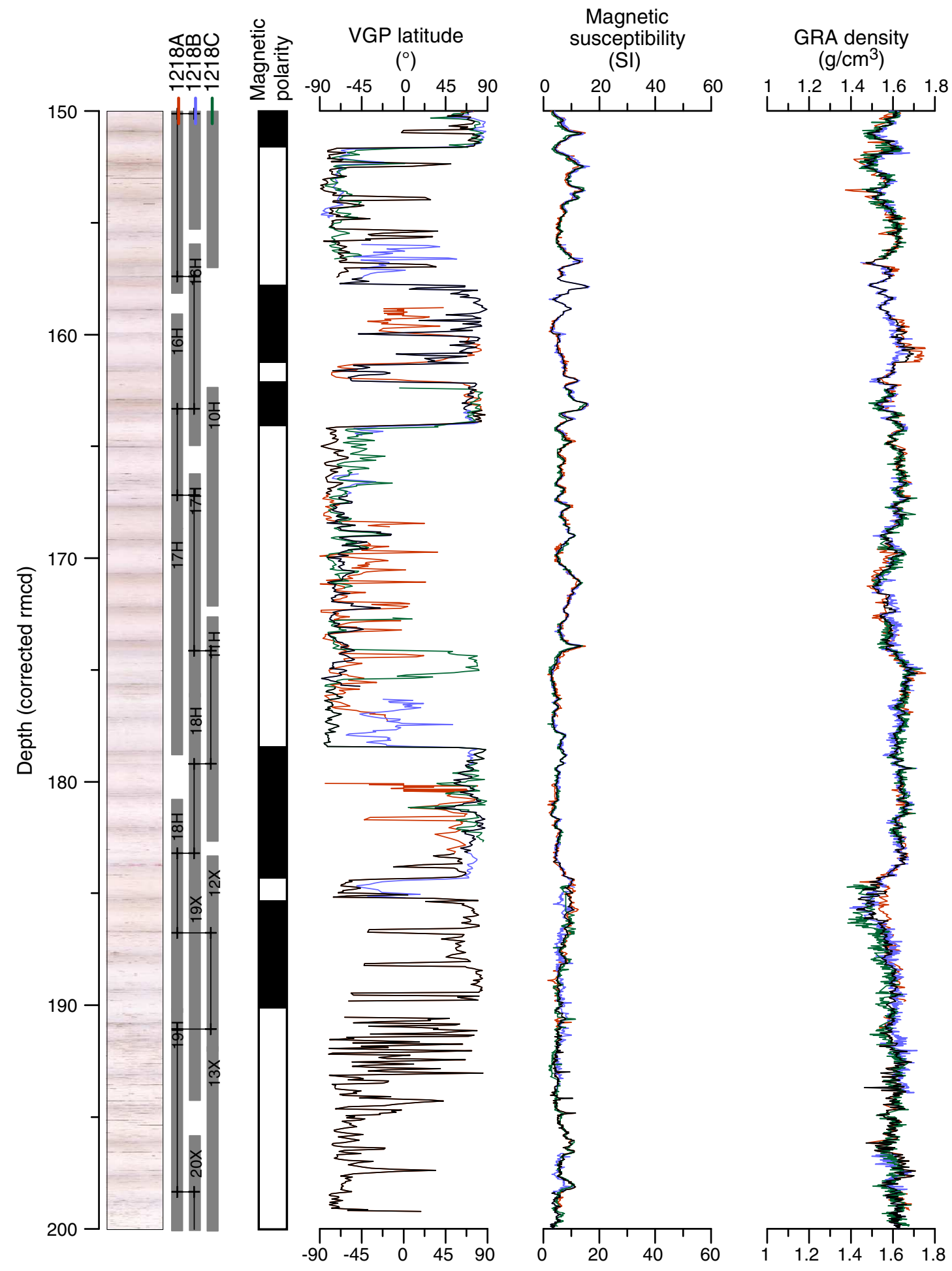


Figure F14 (continued). (Continued on next page.)

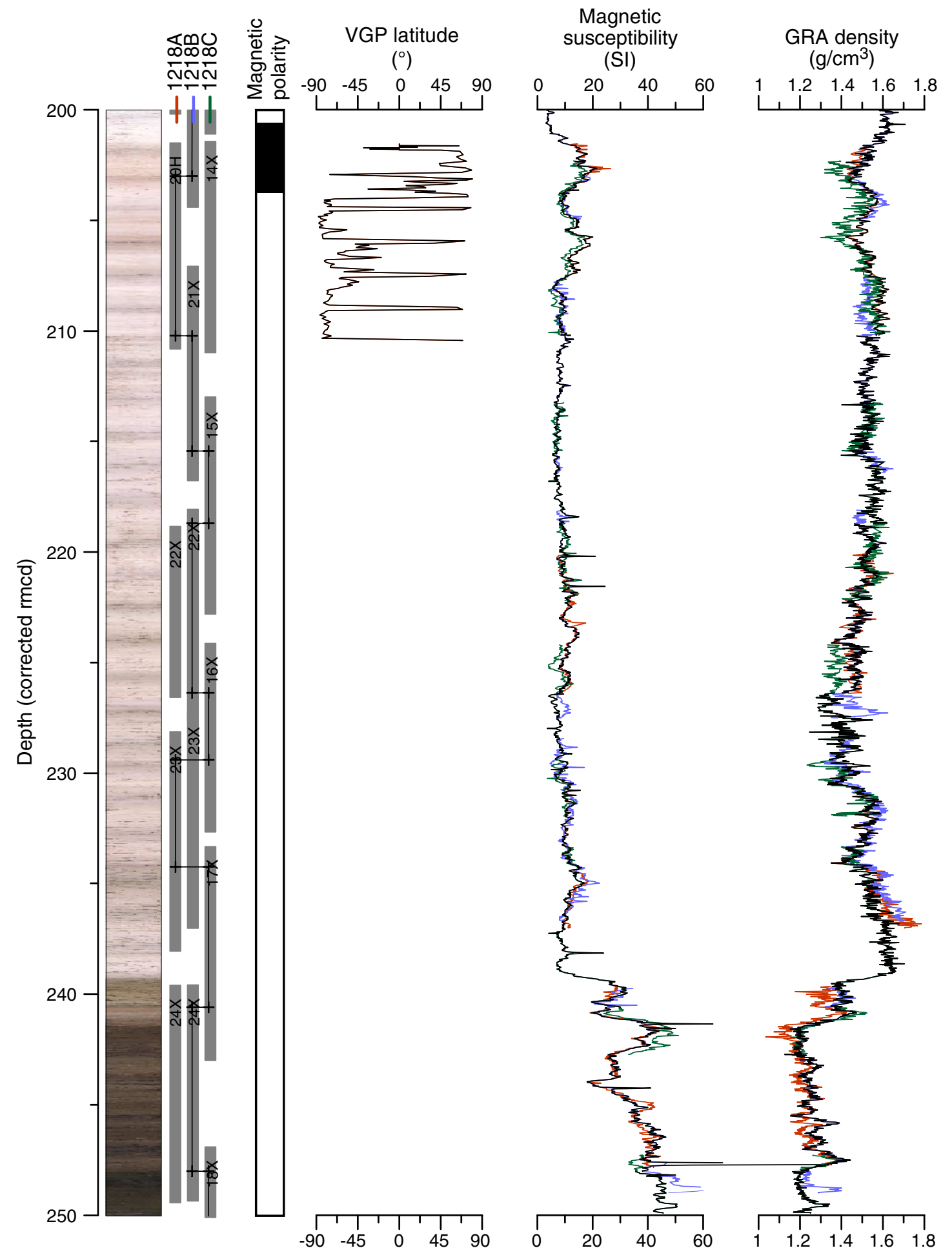


Figure F14 (continued).

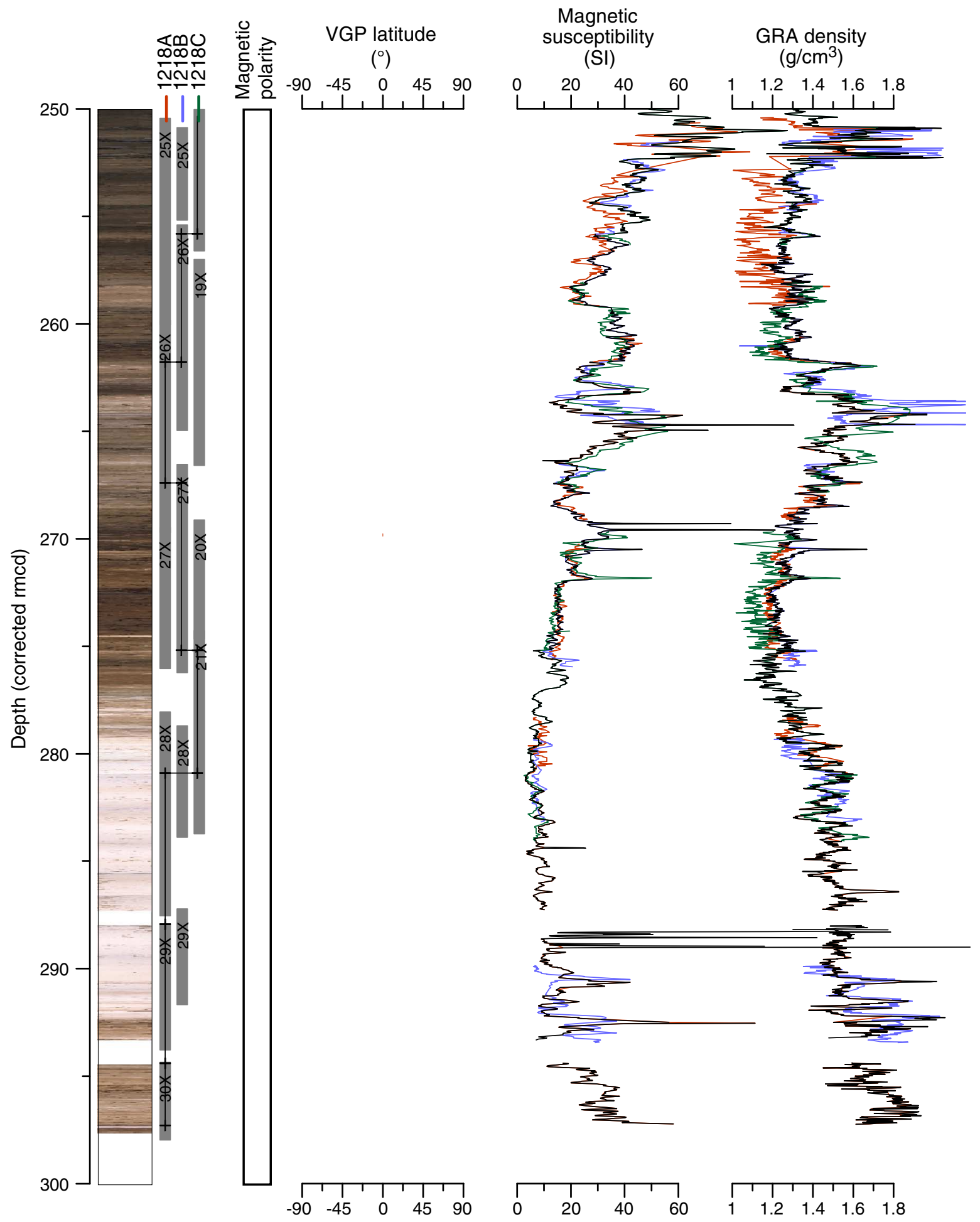


Figure F15. Digital line scan images, Site 1218. Red lines are the tops of splice sections and yellow lines are the bases of those sections (tie to next section). Images were depth registered by R. Wilkens using IGOR-Pro software.
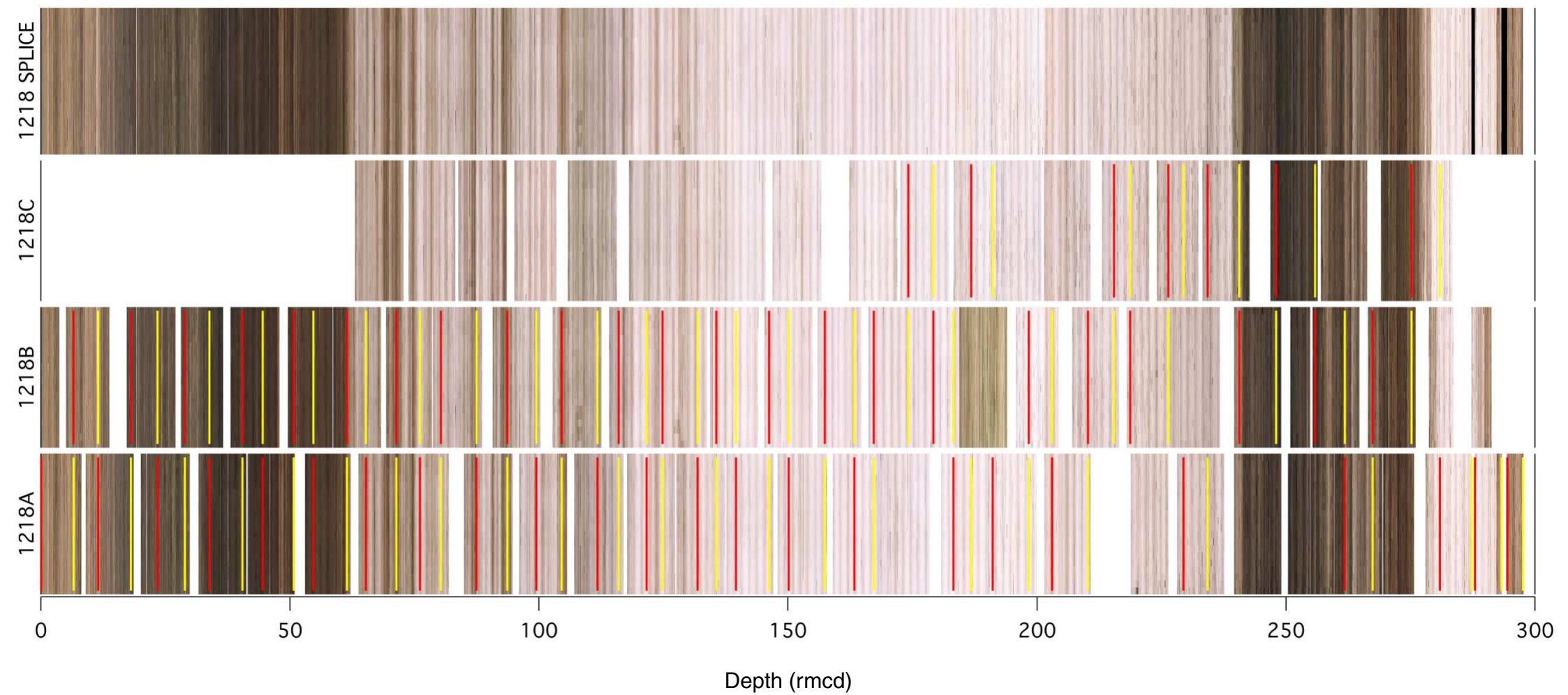
Figure F16. Growth factor calculated by plotting revised composite depth (rmcd) against drilled core depth (mbsf), Site 1218.

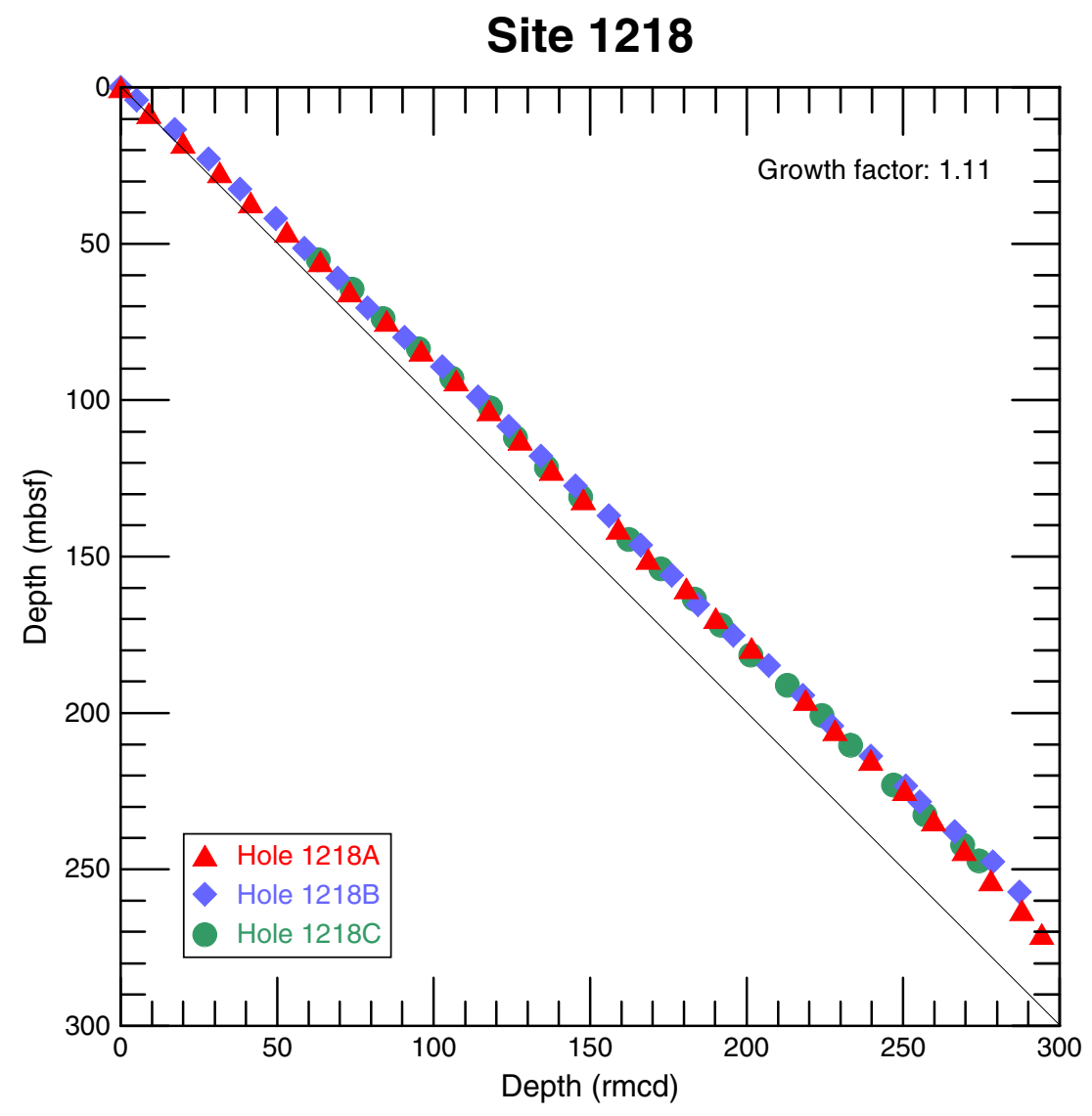


Figure F17. Site 1219 paleomagnetic and physical property data on rmcd scale. Splice map and spliced core image on the left side. VGP = virtual geomagnetic pole, GRA = gamma ray attenuation. Red = Hole 1219A, blue $=$ Hole 1219B, black $=$ composite record. Composite record line is discontinuous because of distortion and data gaps. (Continued on next four pages.)

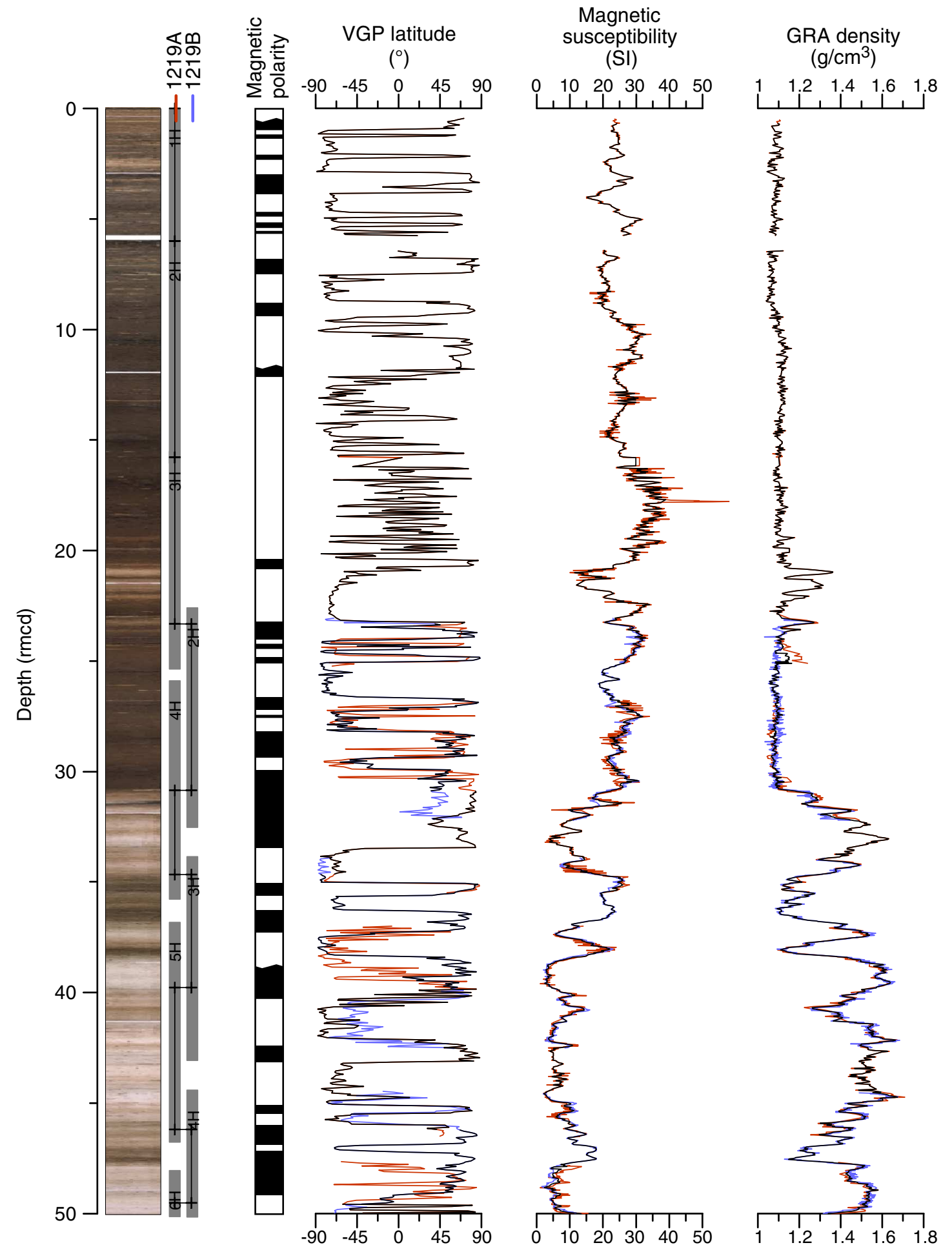


Figure F17 (continued). (Continued on next page.)

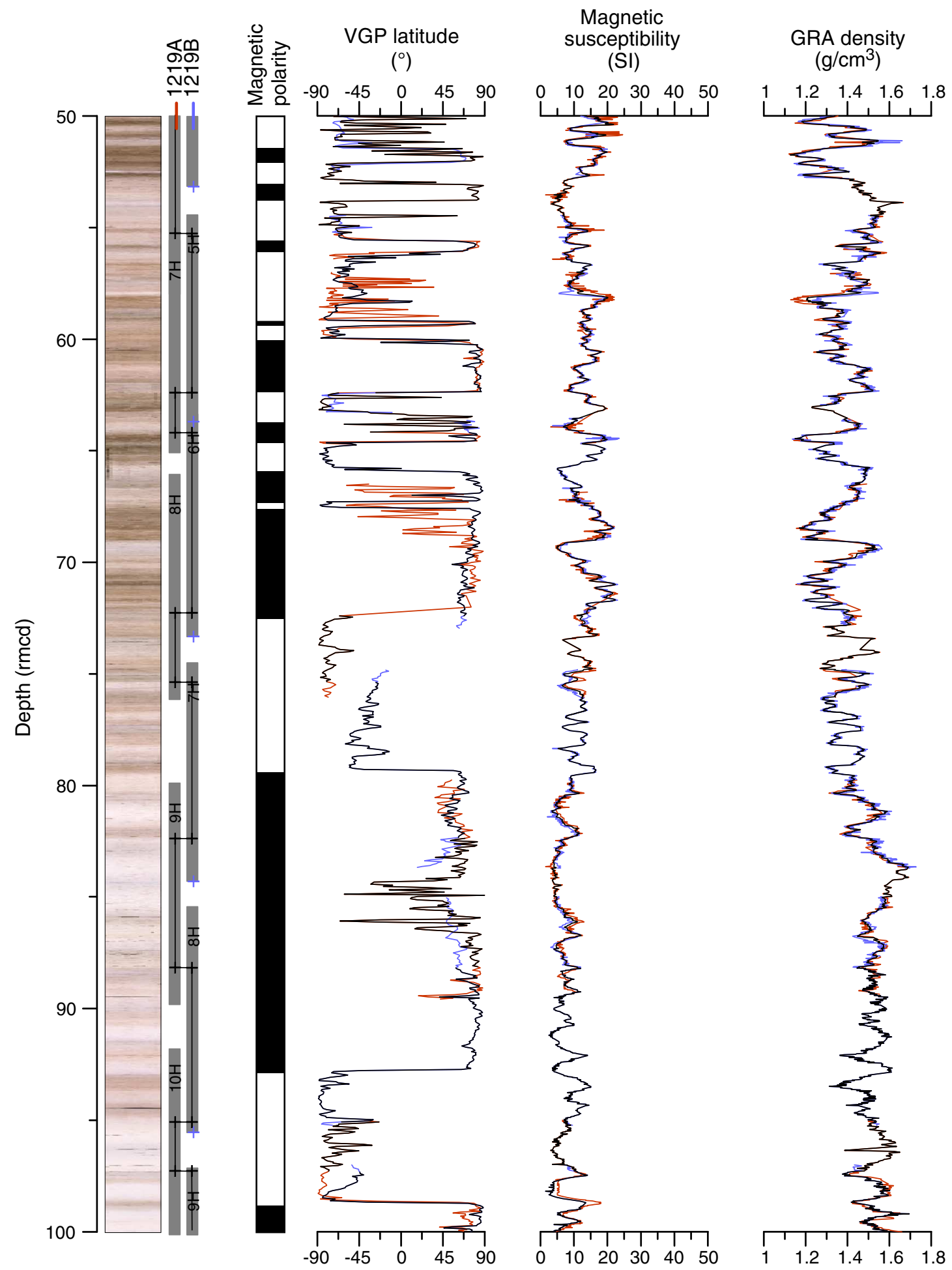


Figure F17 (continued). (Continued on next page.)

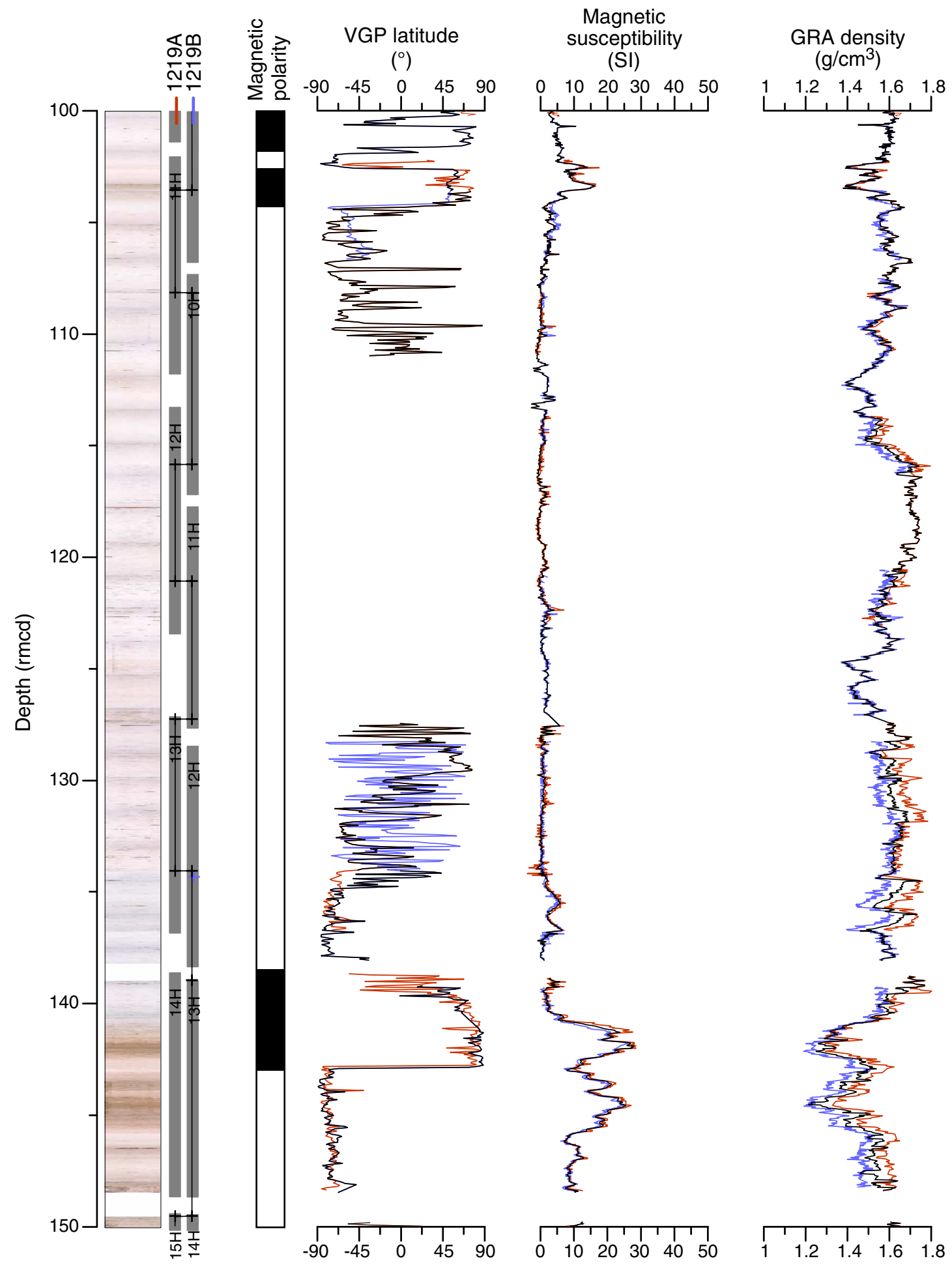


Figure F17 (continued). (Continued on next page.)

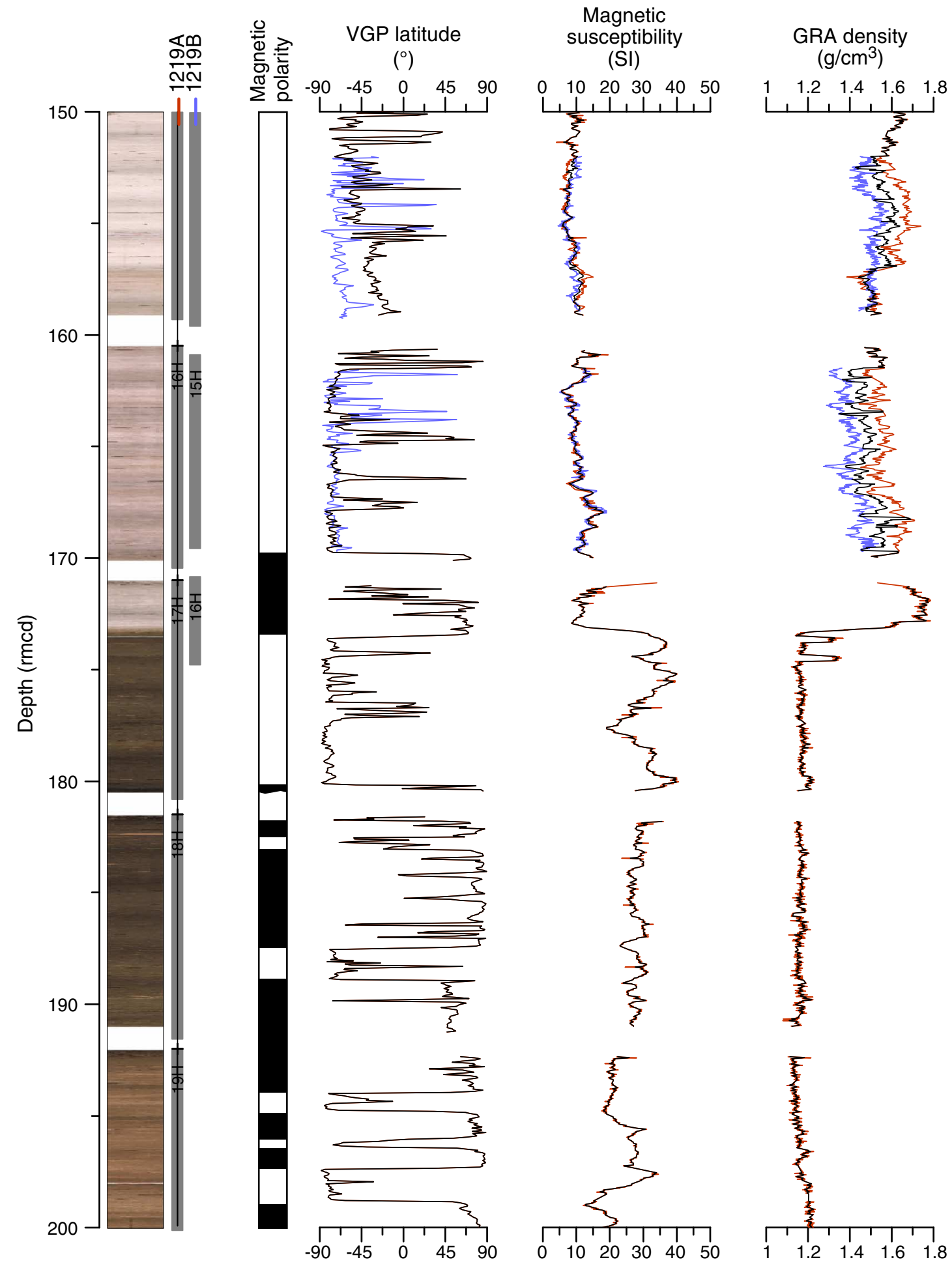


Figure F17 (continued).

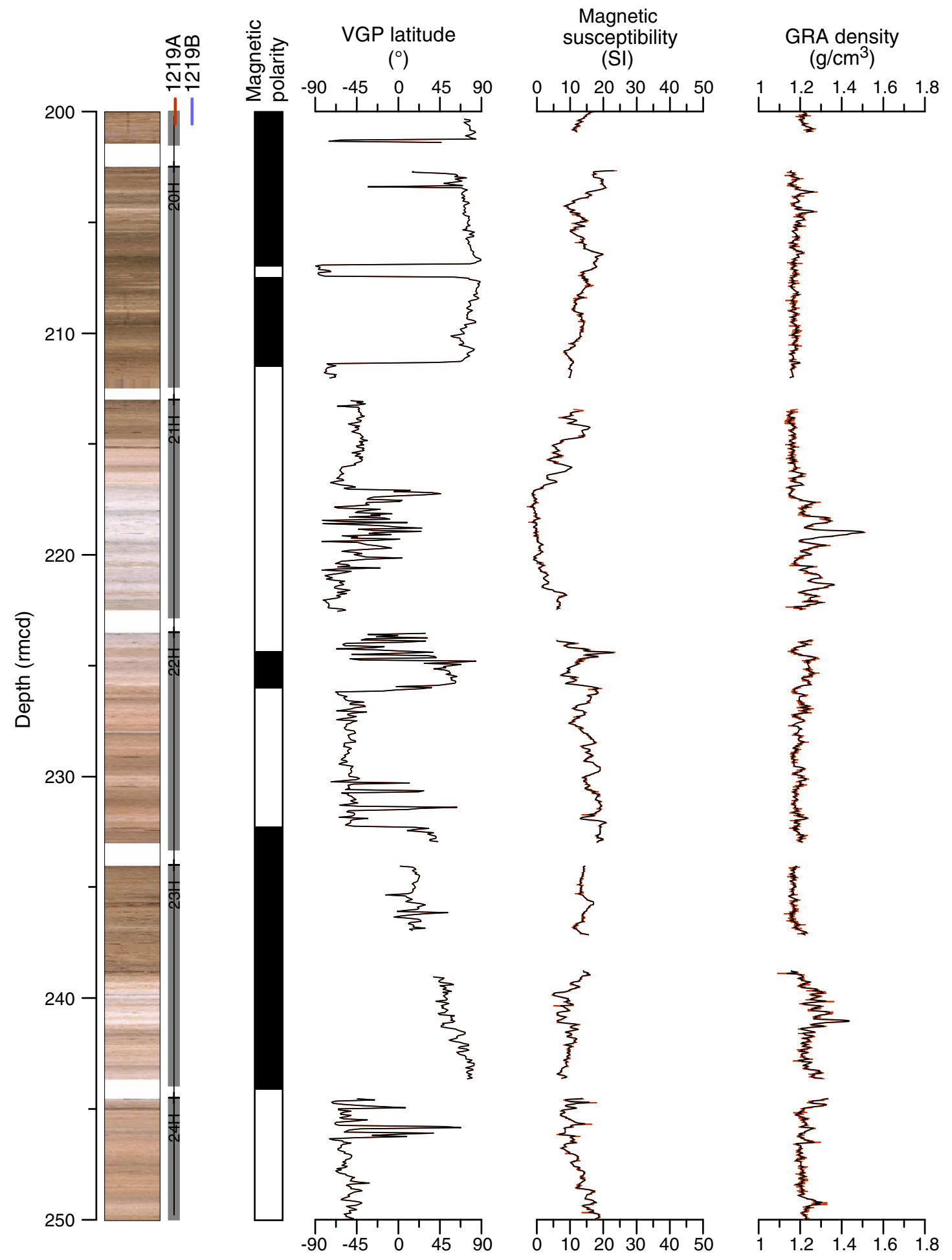


Figure F18. Digital line scan images, Site 1219. Red lines are the tops of splice sections and yellow lines are the bases of those sections (tie to next section). Images were depth registered by R. Wilkens using IGOR-Pro software.

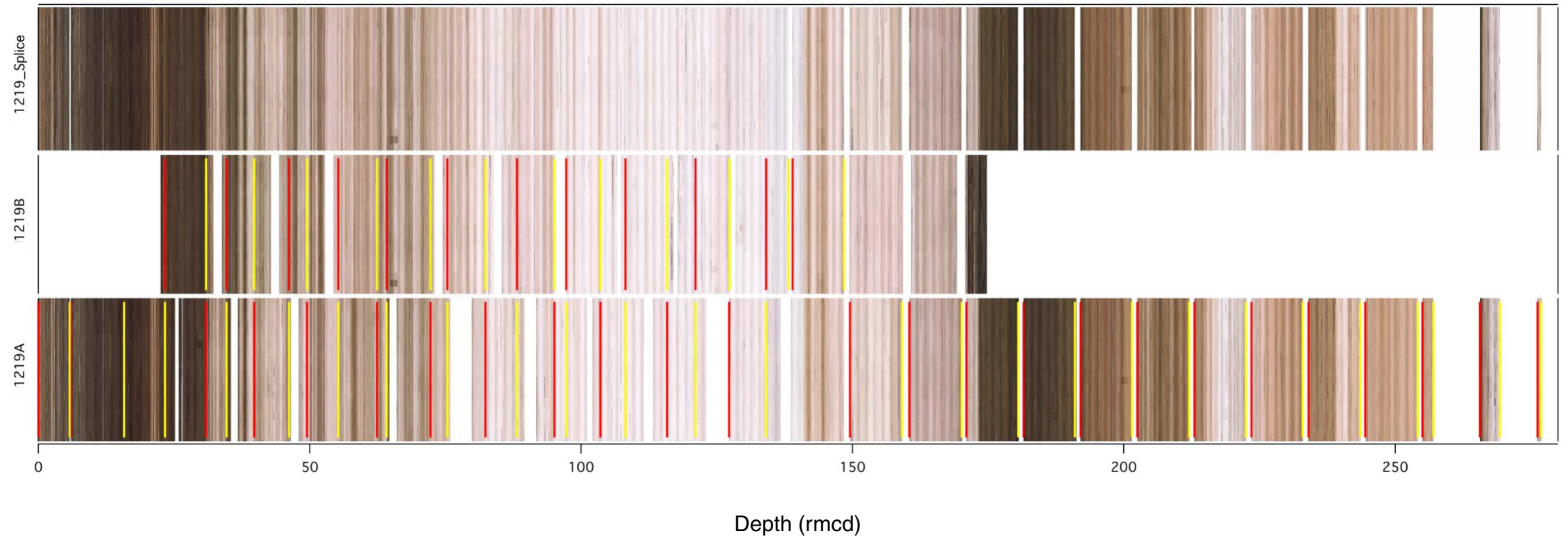

Depth $(\mathrm{rmcd})$ 
Figure F19. Site 1220 paleomagnetic and physical property data on rmcd scale. Splice map and spliced core image on the left side. VGP = virtual geomagnetic pole, GRA = gamma ray attenuation. Red = Hole 1220A, blue $=$ Hole $1220 \mathrm{~B}$, green $=$ Hole $1220 \mathrm{C}$, black $=$ composite record. Composite record line is discontinuous because of distortion and data gaps. (Continued on next three pages.)

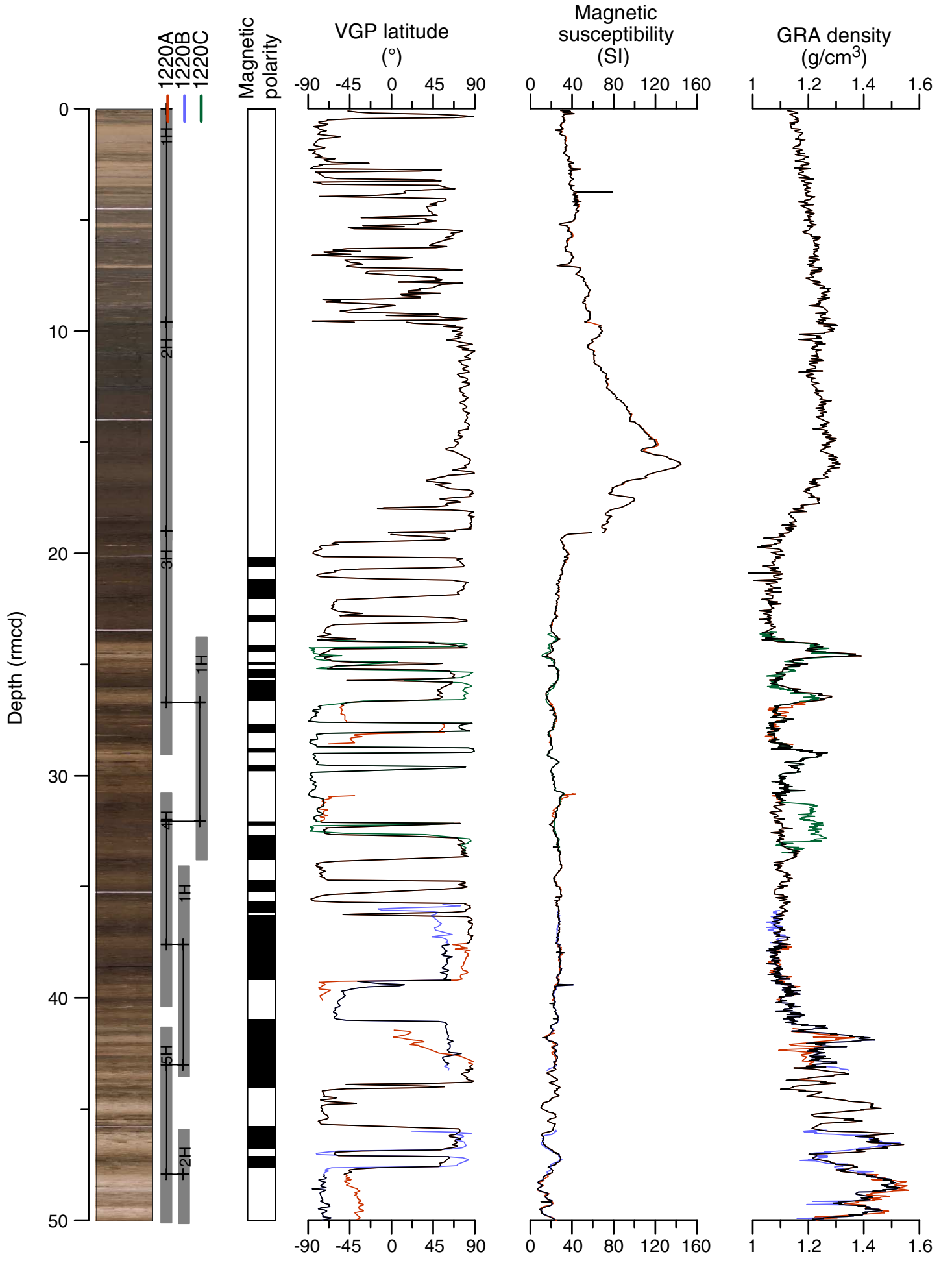


Figure F19 (continued). (Continued on next page.)
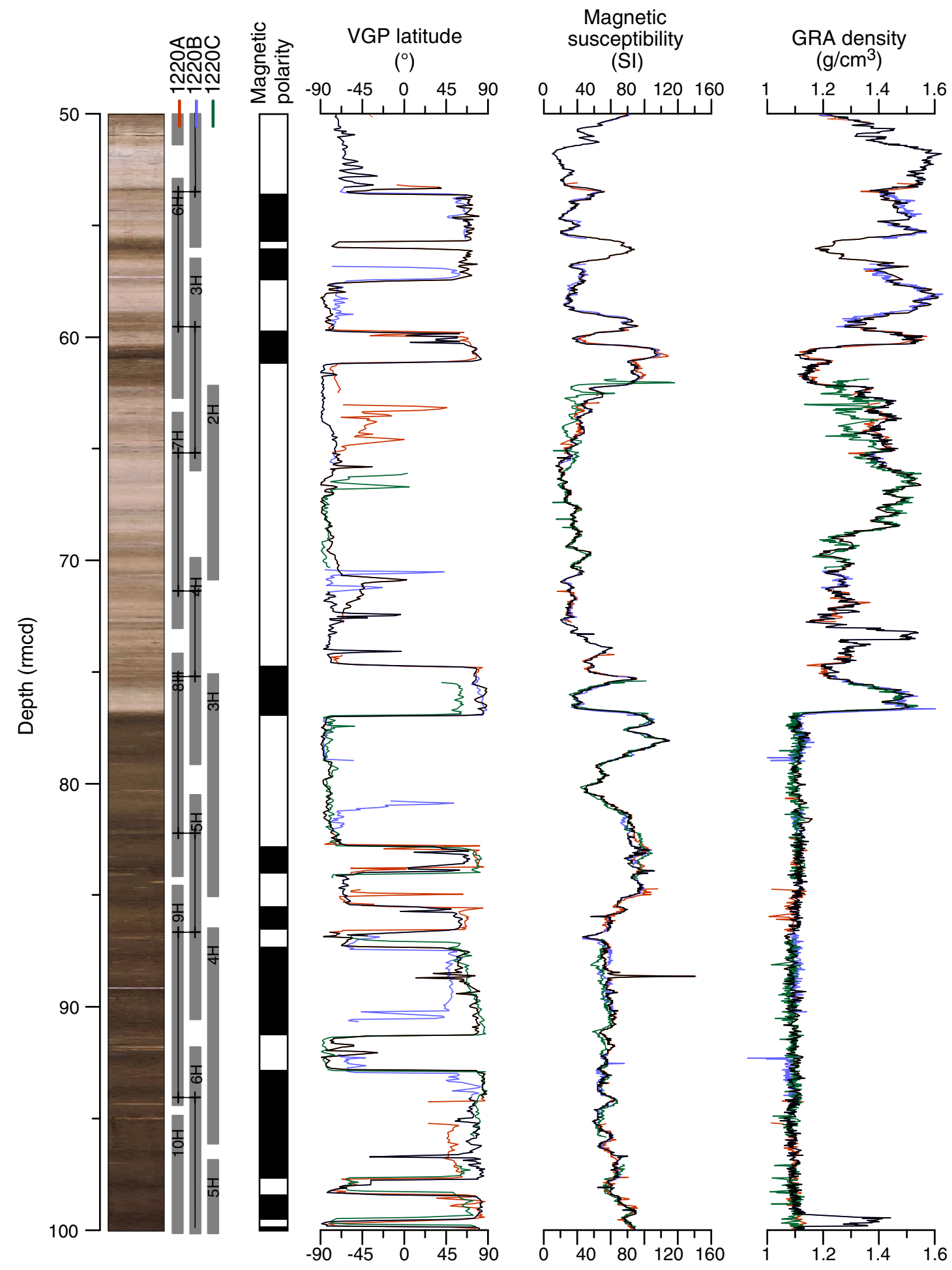
Figure F19 (continued). (Continued on next page.)

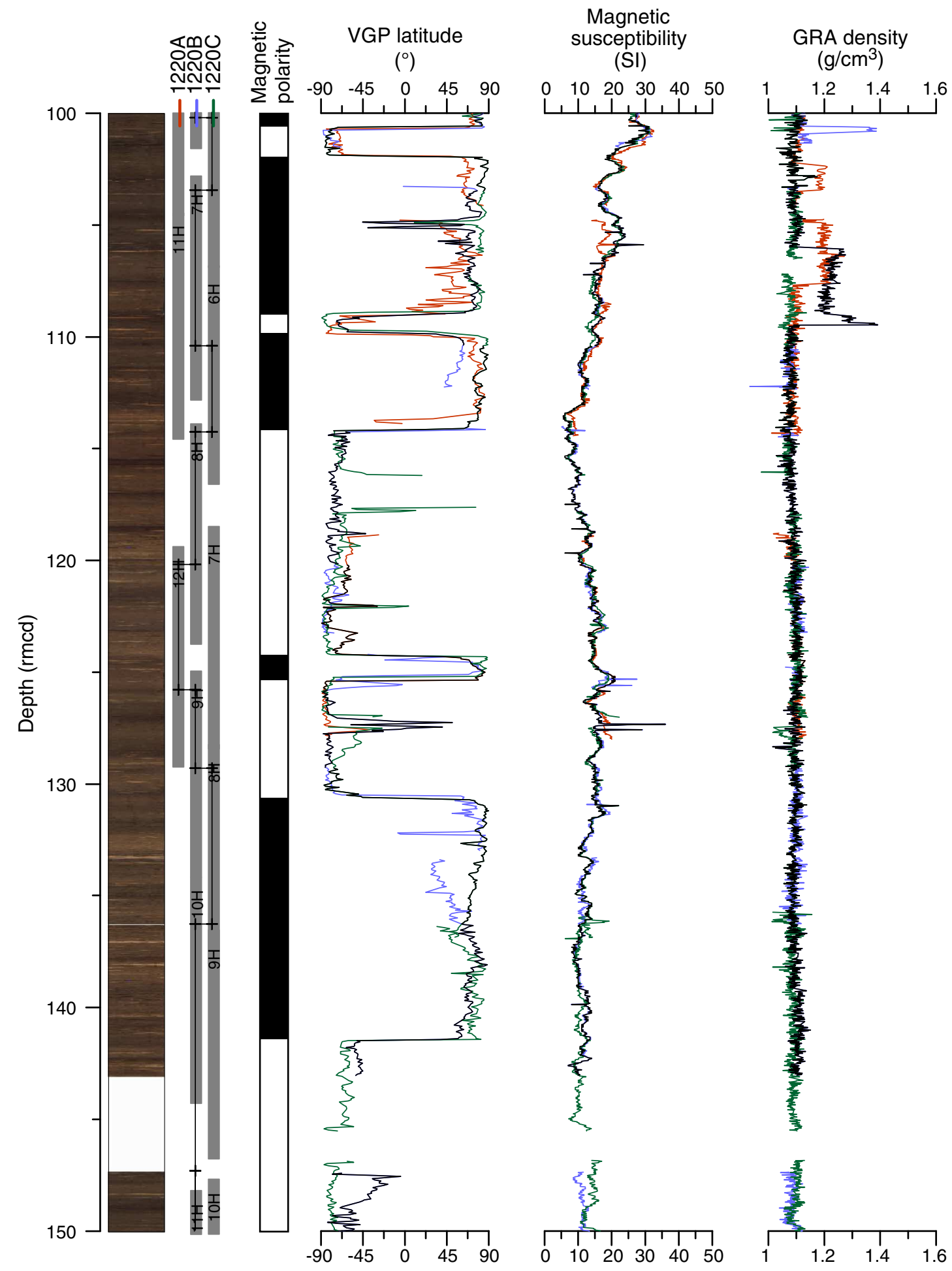


Figure F19 (continued).

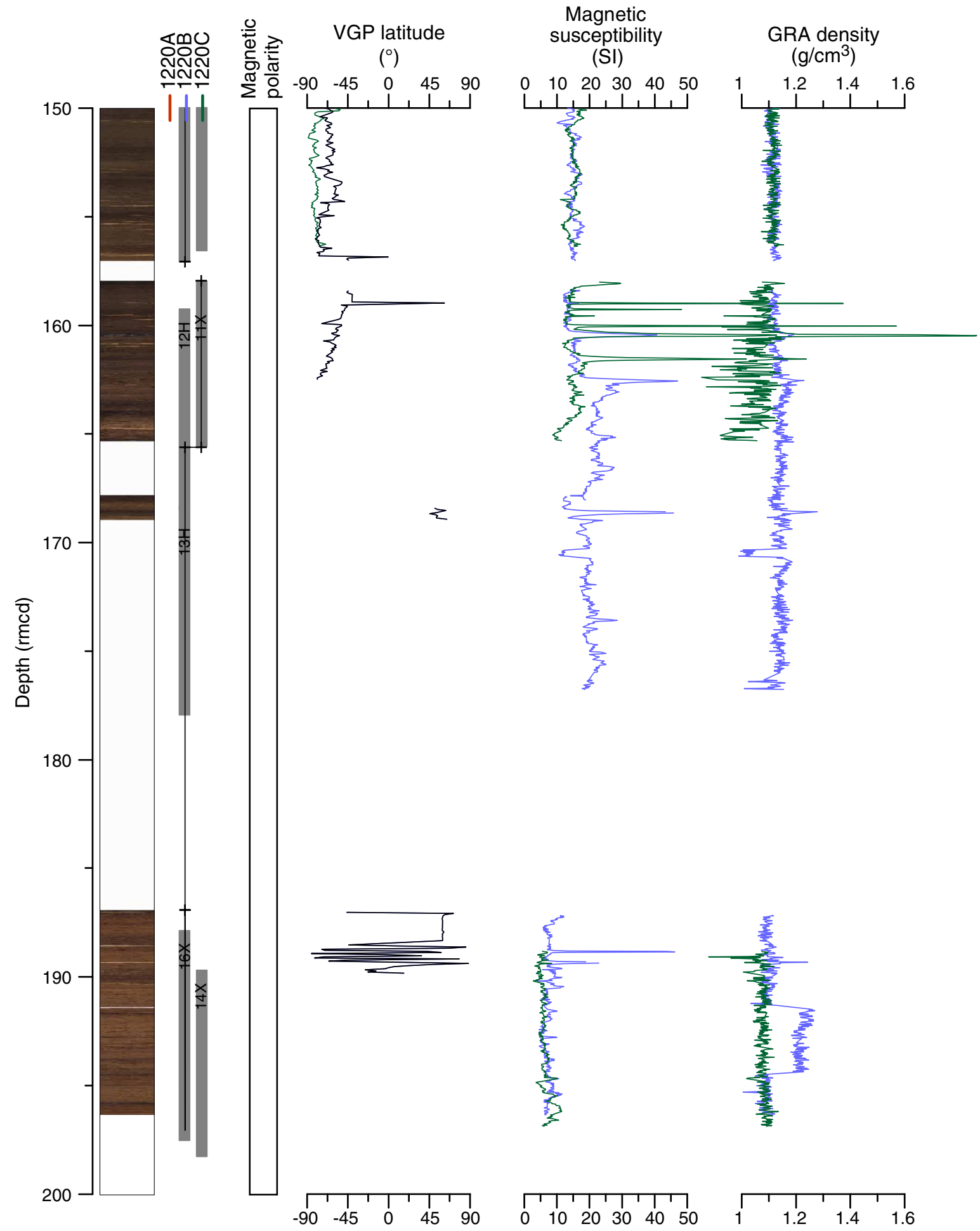


Figure F20. Digital line scan images, Site 1220. Red lines are the tops of splice sections and yellow lines are the bases of those sections (tie to next section). Images were depth registered by R. Wilkens using IGOR-Pro software.

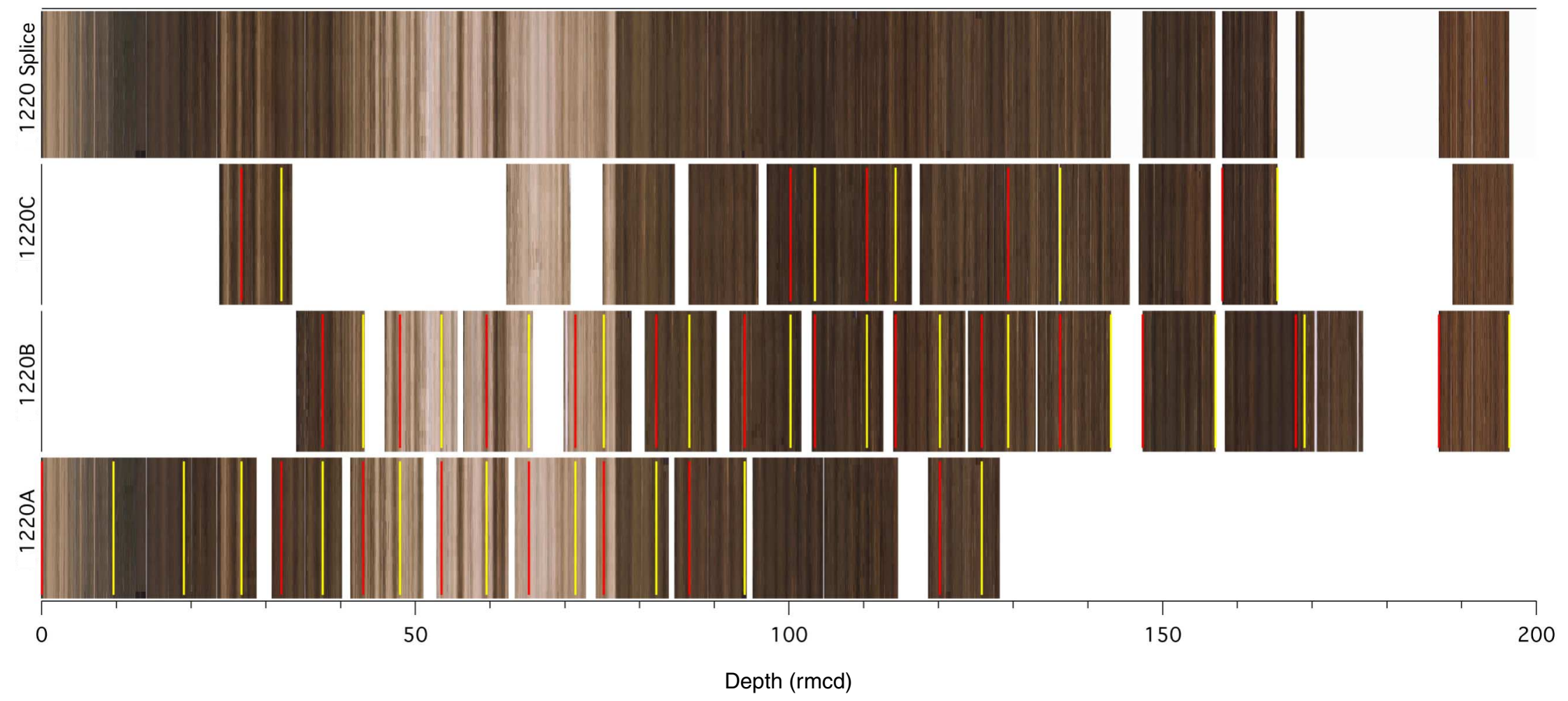


Figure F21. Growth factor calculated by plotting revised composite depth (rmcd) against drilled core depth (mbsf), Site 1220.

Site 1220

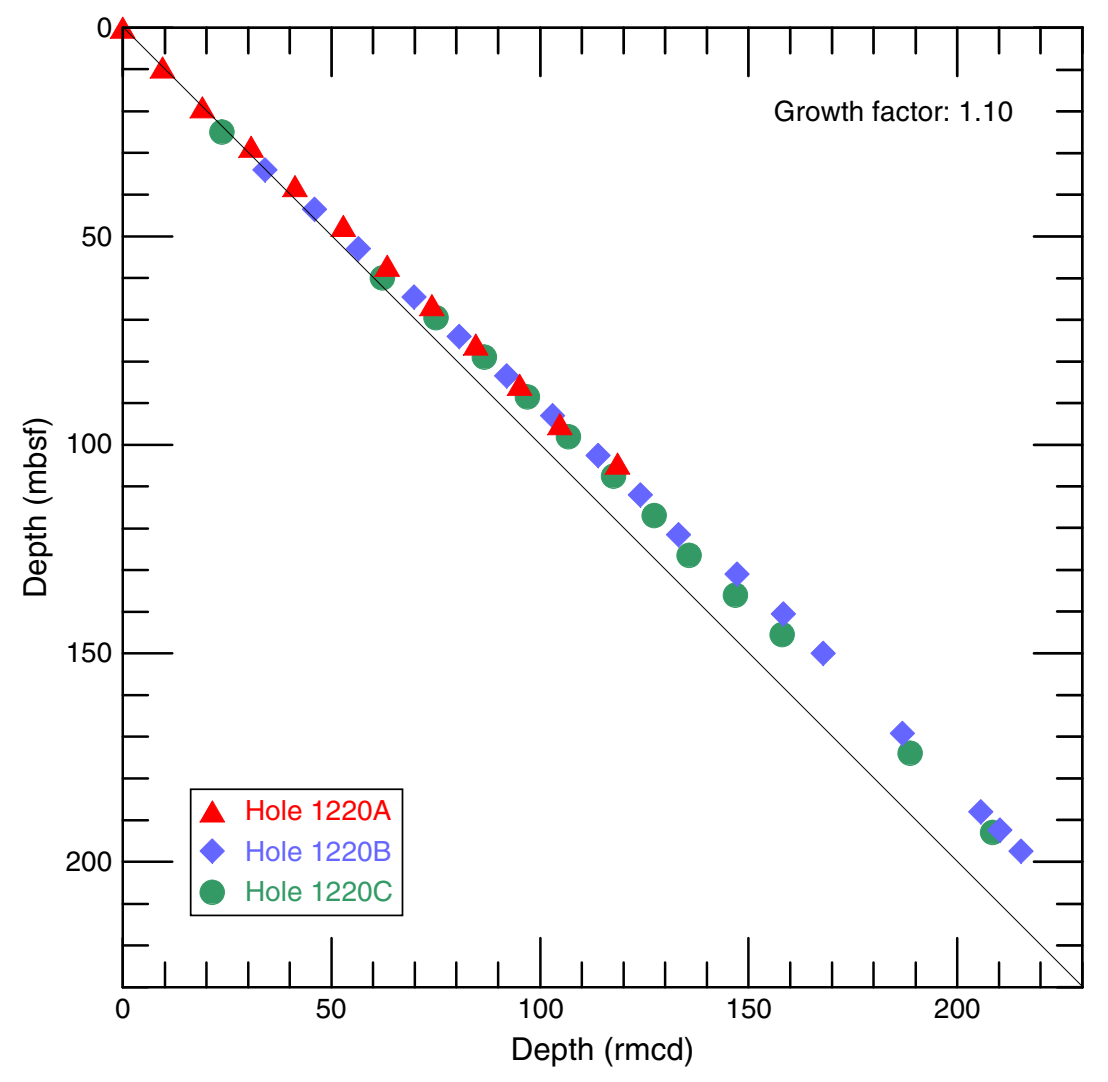


Figure F22. Site-to-site correlation of paleomagnetic and physical property data from Sites 1218, 1219, U1333, and U1334 on corrected rmcd scale of Site 1218. VGP = virtual geomagnetic pole, GRA = gamma ray attenuation . Black $=$ Site 1218, green $=$ Site 1219, blue $=$ Site U1333, red $=$ Site U1334. Composite record lines are discontinuous because of distortion and data gaps. (Continued on next five pages.)

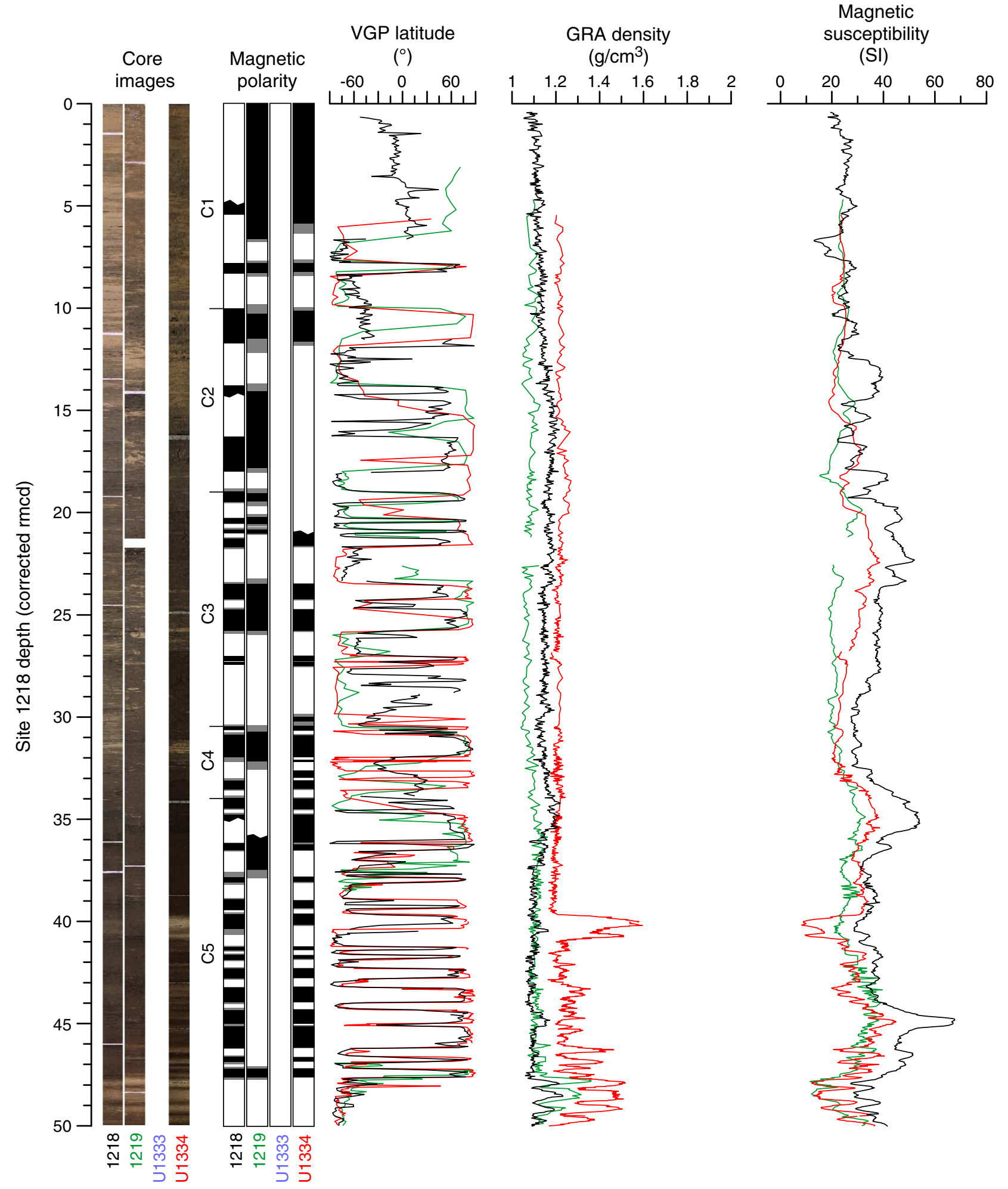


Figure F22 (continued). (Continued on next page.)

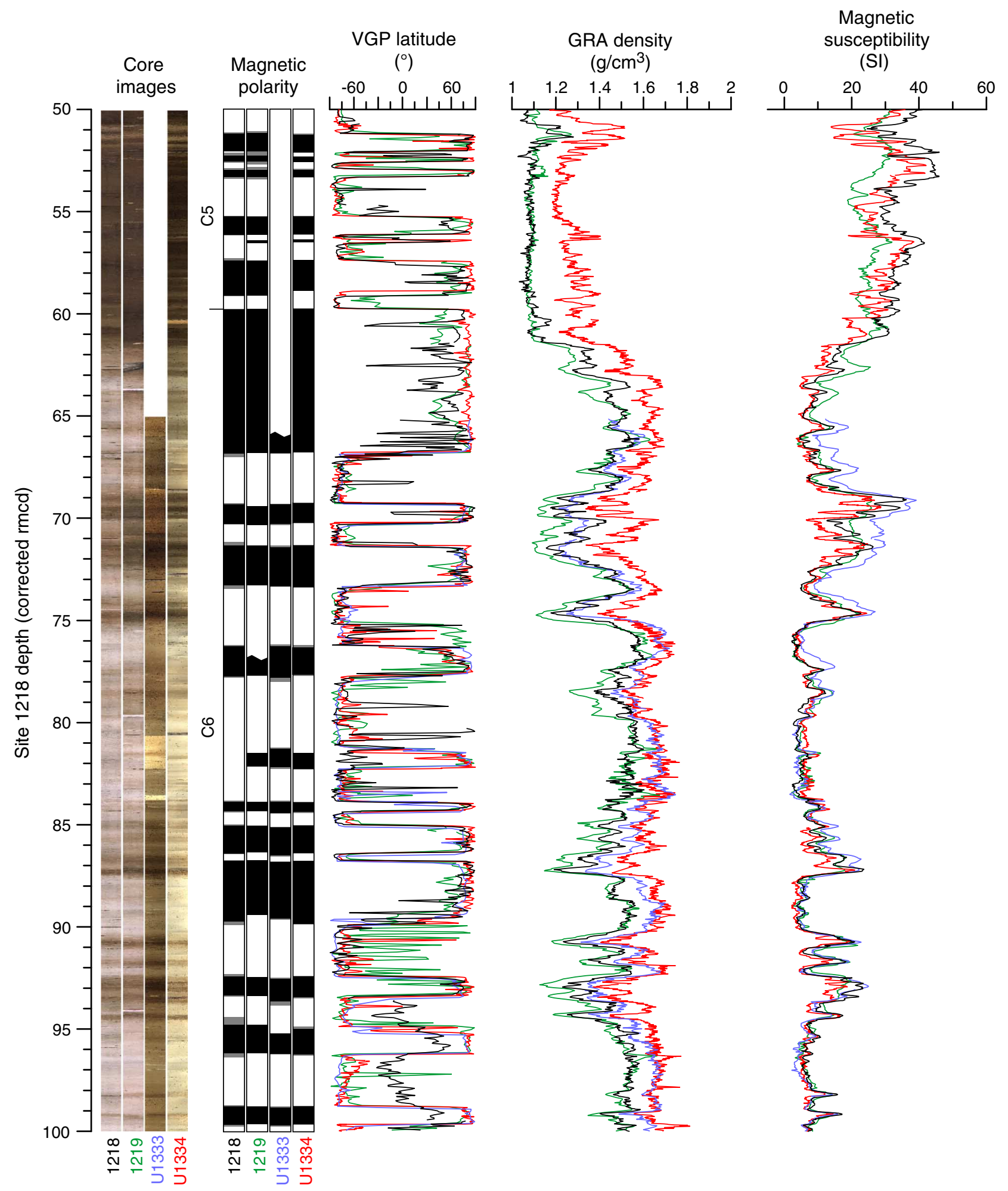


Figure F22 (continued). (Continued on next page.)

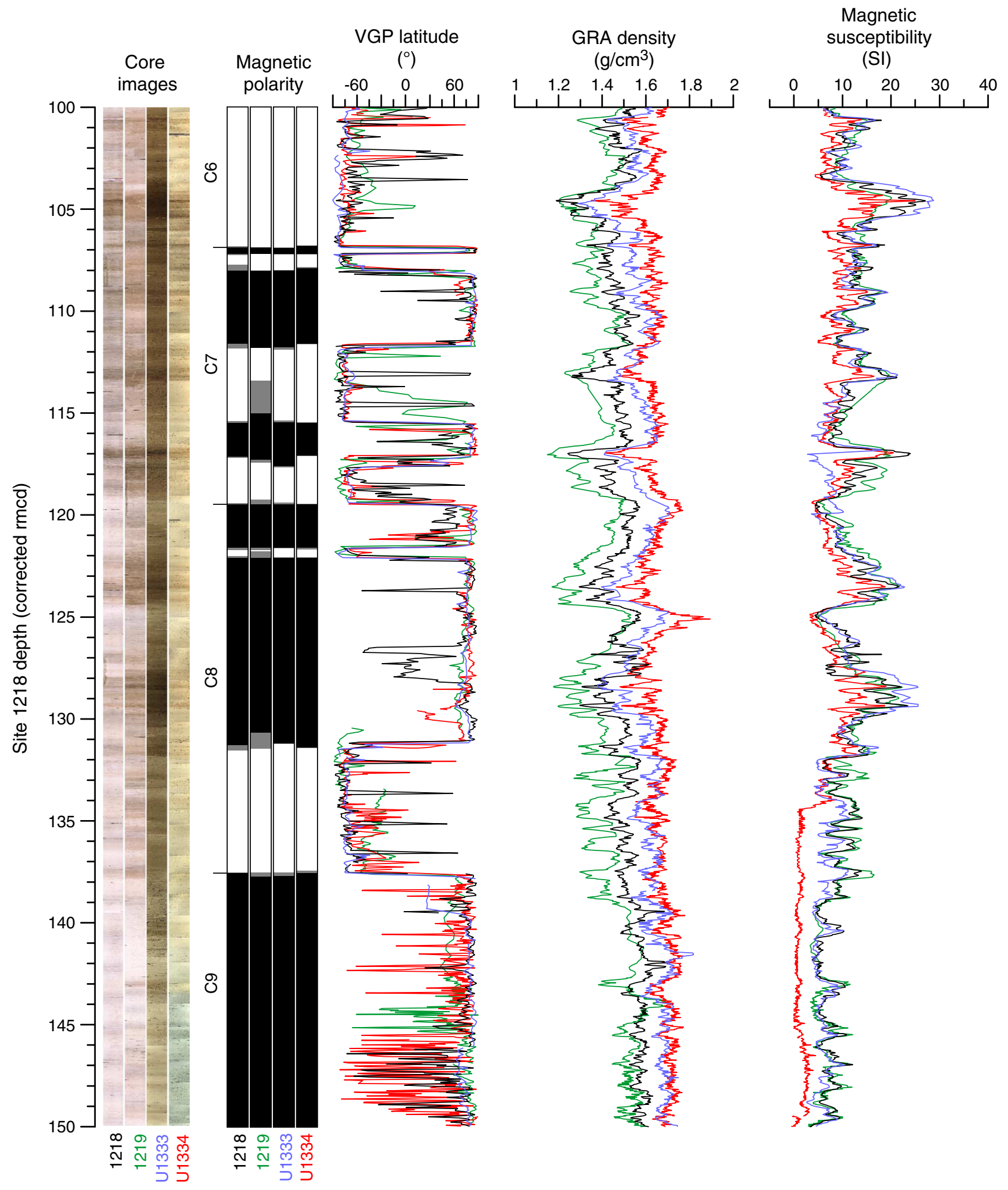


Figure F22 (continued). (Continued on next page.)

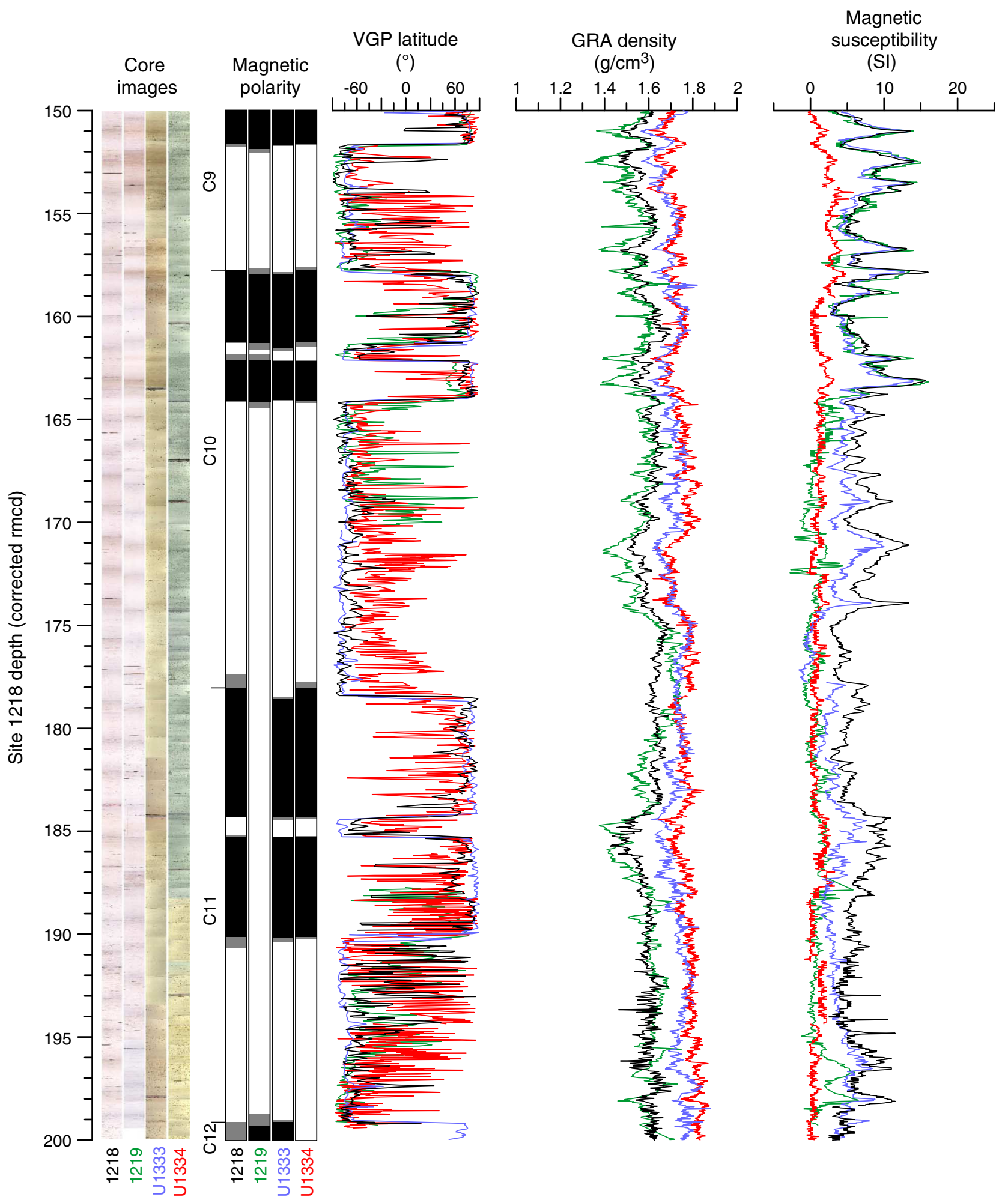


Figure F22 (continued). (Continued on next page.)




Figure F22 (continued).

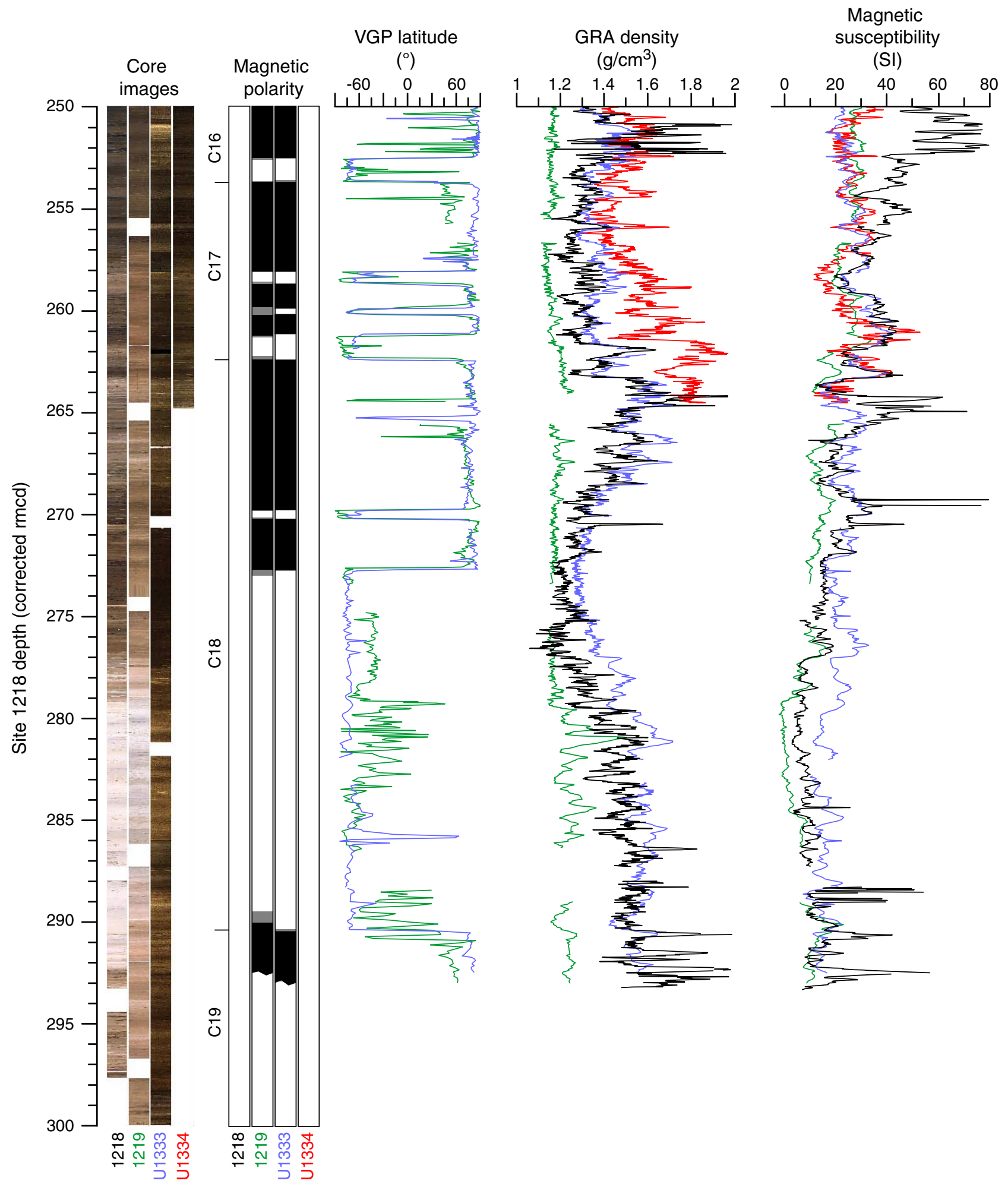


Figure F23. Site-to-site correlation of paleomagnetic and physical property data from Sites 1220, U1331, and U1332 on rmcd scale of Site 1220 . VGP = virtual geomagnetic pole, GRA = gamma ray attenuation. Black = Site 1220 , blue $=$ Site U1331, red $=$ Site U1332. Composite record lines are discontinuous because of distortion and data gaps. (Continued on next two pages.)

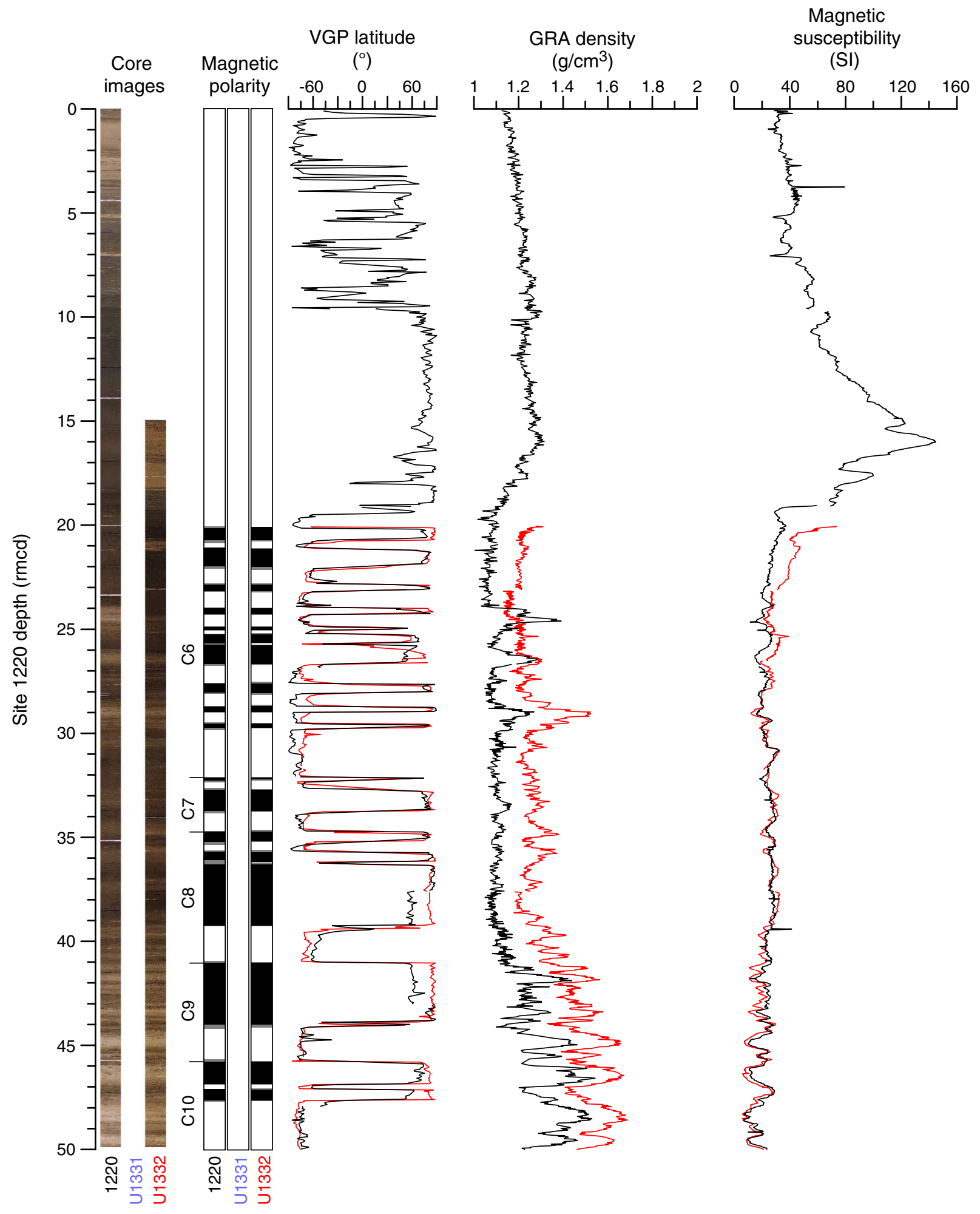


Figure F23 (continued). (Continued on next page.)

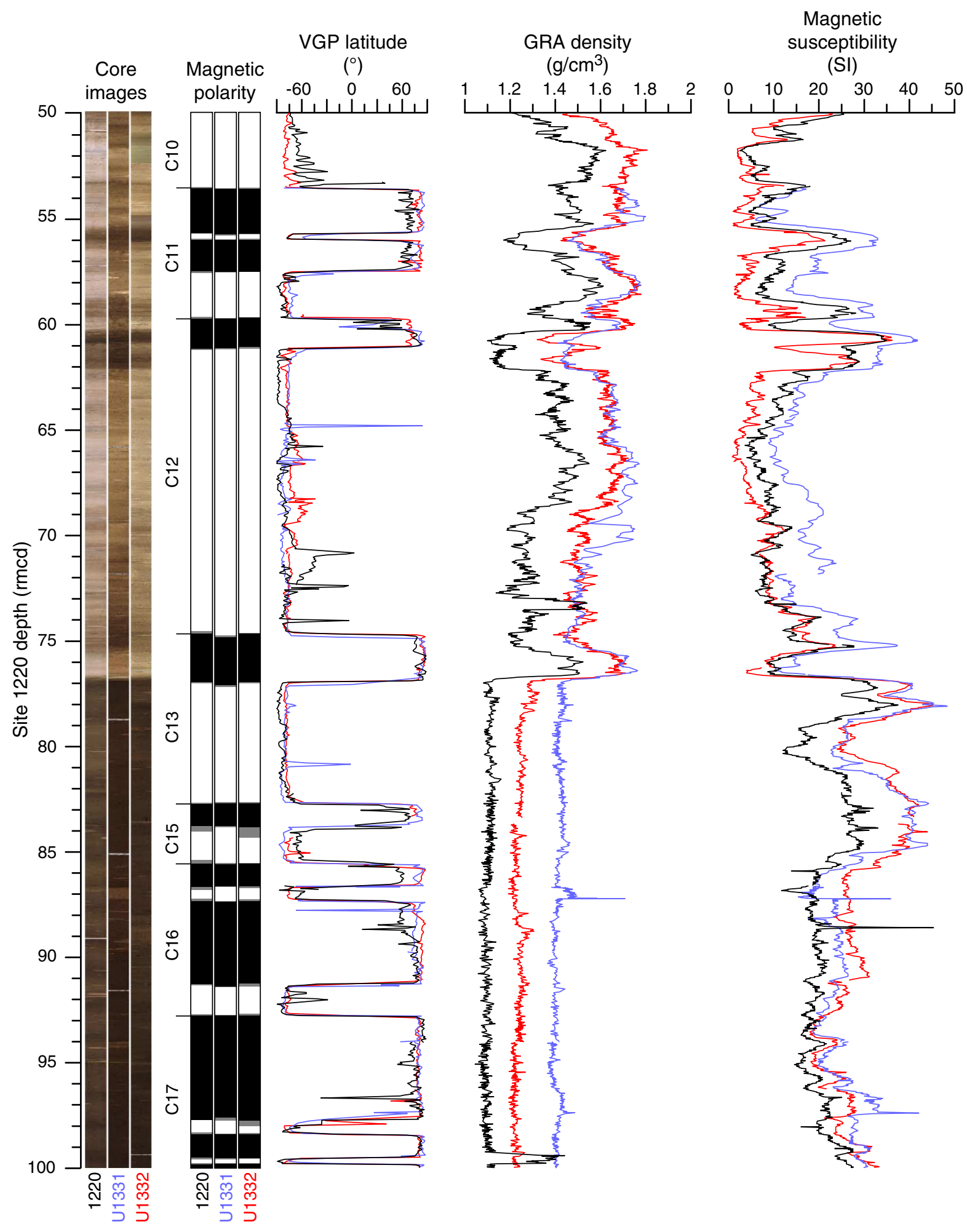


Figure F23 (continued).

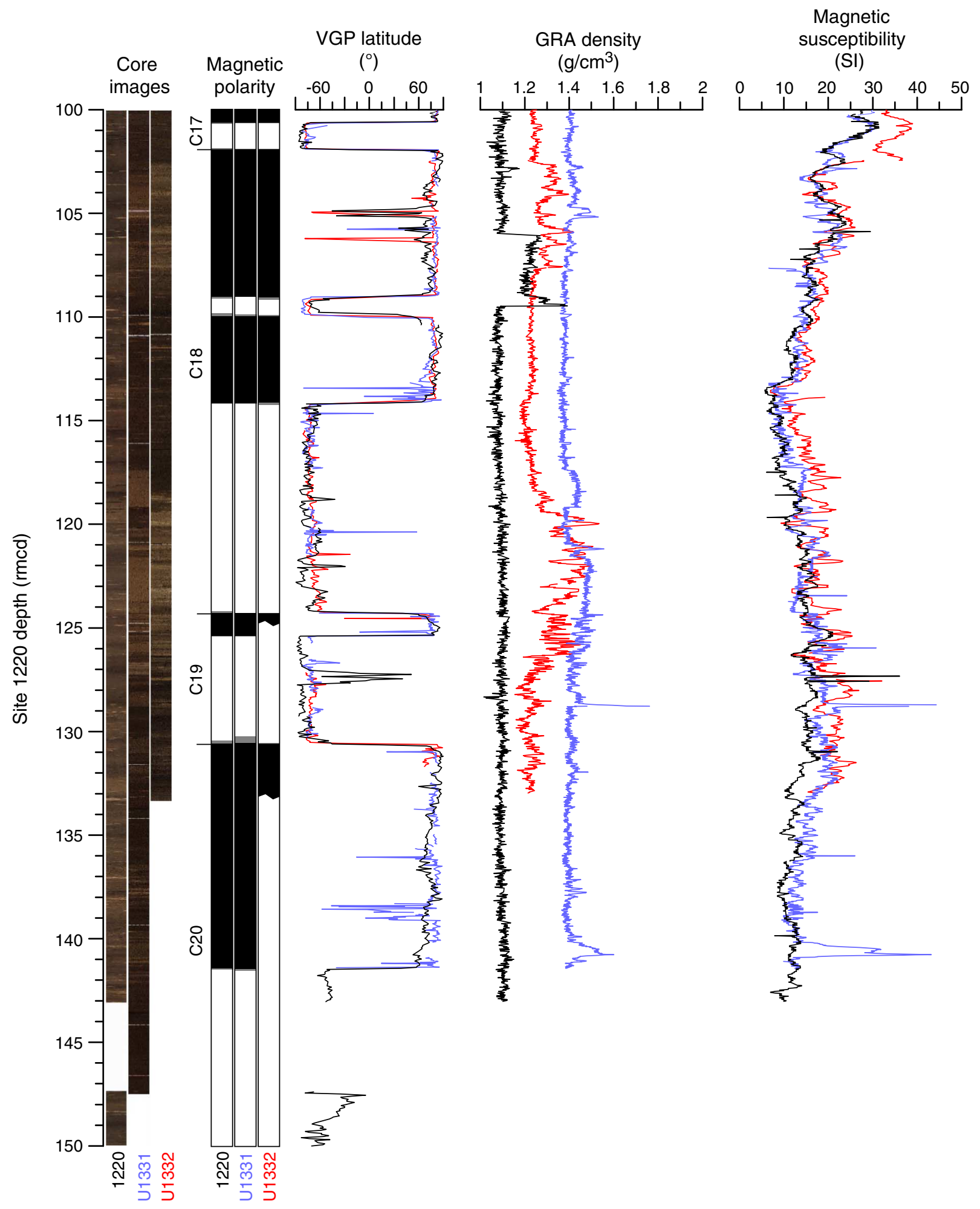


Figure F24. Estimate of linear sedimentation rates for Sites 1218, 1219, 1220, U1331, U1332, U1333, and U1334 by plotting paleomagnetic chronostratigraphic markers from Table T31.

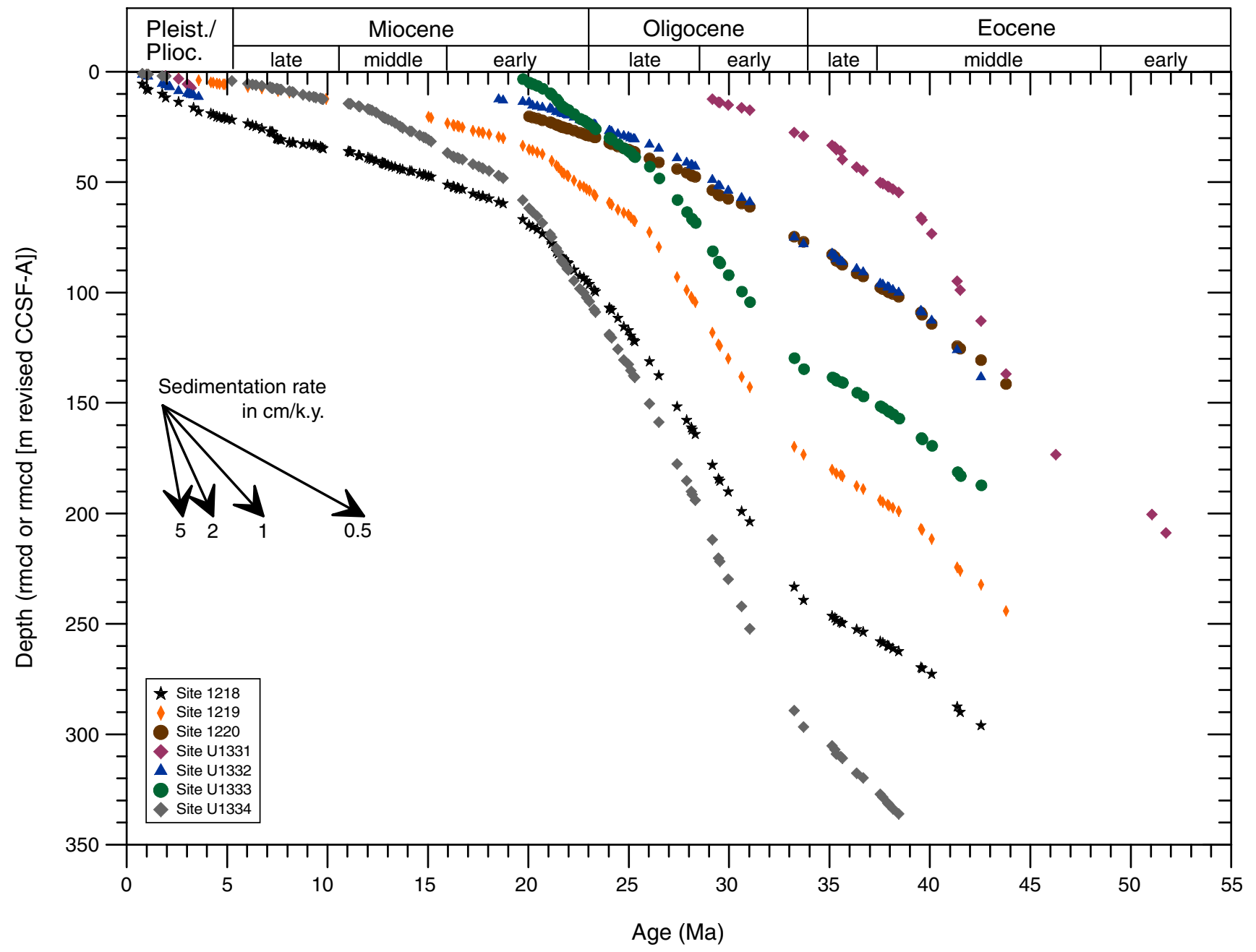


Table T1. Revised shipboard composite and corrected composite depths, Site U1331.

\begin{tabular}{|c|c|c|c|}
\hline Core & $\begin{array}{c}\text { Depth } \\
\text { (mbsf } \\
\text { [m CSF-A]) }\end{array}$ & $\begin{array}{l}\text { Offset } \\
(\mathrm{m})\end{array}$ & $\begin{array}{l}\text { Top depth } \\
\text { (rmcd } \\
\text { [m revised } \\
\text { CCSF-A]) }\end{array}$ \\
\hline \multicolumn{4}{|c|}{ 320-U1331A- } \\
\hline $1 \mathrm{H}$ & 0.00 & 0.00 & 0.00 \\
\hline $2 \mathrm{H}$ & 5.20 & 1.50 & 6.70 \\
\hline $3 \mathrm{H}$ & 14.70 & 2.91 & 17.61 \\
\hline $4 \mathrm{H}$ & 24.20 & 3.26 & 27.46 \\
\hline $5 \mathrm{H}$ & 33.70 & 5.25 & 38.95 \\
\hline $6 \mathrm{H}$ & 43.20 & 7.16 & 50.36 \\
\hline $7 \mathrm{H}$ & 52.70 & 9.81 & 62.51 \\
\hline $8 \mathrm{H}$ & 62.20 & 10.77 & 72.97 \\
\hline $9 \mathrm{H}$ & 71.70 & 11.36 & 83.06 \\
\hline $10 \mathrm{H}$ & 81.20 & 10.88 & 92.08 \\
\hline $11 \mathrm{H}$ & 90.70 & 13.22 & 103.92 \\
\hline $12 \mathrm{H}$ & 100.20 & 16.13 & 116.33 \\
\hline $13 \mathrm{H}$ & 109.70 & 17.97 & 127.67 \\
\hline $14 \mathrm{H}$ & 119.20 & 19.09 & 138.29 \\
\hline $15 \mathrm{H}$ & 128.70 & 20.96 & 149.66 \\
\hline $16 X$ & 138.20 & 19.81 & 158.01 \\
\hline $17 X$ & 147.60 & 20.40 & 168.00 \\
\hline $18 x$ & 157.30 & 20.40 & 177.70 \\
\hline $19 x$ & 160.50 & 20.40 & 180.90 \\
\hline $20 x$ & 167.00 & 20.40 & 187.40 \\
\hline $21 X$ & 176.70 & 20.40 & 197.10 \\
\hline $22 X$ & 186.40 & 20.40 & 206.80 \\
\hline \multicolumn{4}{|c|}{ 320-U1331B- } \\
\hline $1 \mathrm{H}$ & 0.00 & 0.85 & 0.85 \\
\hline $2 \mathrm{H}$ & 10.10 & 0.27 & 10.37 \\
\hline $3 \mathrm{H}$ & 19.60 & 2.98 & 22.58 \\
\hline $4 \mathrm{H}$ & 29.10 & 3.75 & 32.85 \\
\hline $5 \mathrm{H}$ & 38.60 & 5.51 & 44.11 \\
\hline $6 \mathrm{H}$ & 48.10 & 7.09 & 55.19 \\
\hline $7 \mathrm{H}$ & 57.60 & 8.31 & 65.91 \\
\hline $8 \mathrm{H}$ & 67.10 & 10.79 & 77.89 \\
\hline $9 \mathrm{H}$ & 76.60 & 12.45 & 89.05 \\
\hline $10 \mathrm{H}$ & 86.10 & 13.20 & 99.30 \\
\hline $11 \mathrm{H}$ & 95.60 & 15.03 & 110.63 \\
\hline $12 \mathrm{H}$ & 105.10 & 16.54 & 121.64 \\
\hline $13 \mathrm{H}$ & 114.60 & 17.73 & 132.33 \\
\hline $14 \mathrm{H}$ & 124.10 & 19.60 & 143.70 \\
\hline $15 \mathrm{H}$ & 133.60 & 20.30 & 153.90 \\
\hline $16 \mathrm{H}$ & 143.10 & 22.85 & 165.95 \\
\hline $17 \mathrm{H}$ & 152.60 & 24.93 & 177.53 \\
\hline $18 x$ & 156.60 & 24.93 & 181.53 \\
\hline \multicolumn{4}{|c|}{ 320-U1331C- } \\
\hline $1 \mathrm{H}$ & 0.00 & 0.54 & 0.54 \\
\hline $2 \mathrm{H}$ & 9.50 & 2.12 & 11.62 \\
\hline $3 \mathrm{H}$ & 19.00 & 2.40 & 21.40 \\
\hline $4 \mathrm{H}$ & 28.50 & 2.50 & 31.00 \\
\hline $6 \mathrm{H}$ & 59.00 & 11.46 & 70.46 \\
\hline $8 \mathrm{H}$ & 92.50 & 14.39 & 106.89 \\
\hline $10 \mathrm{H}$ & 102.50 & 15.09 & 117.59 \\
\hline $12 \mathrm{H}$ & 129.00 & 19.43 & 148.43 \\
\hline $13 \mathrm{H}$ & 138.50 & 20.50 & 159.00 \\
\hline $14 \mathrm{H}$ & 148.00 & 21.25 & 169.25 \\
\hline $16 \mathrm{H}$ & 177.00 & 21.25 & 198.25 \\
\hline $17 \mathrm{H}$ & 184.00 & 22.75 & 206.75 \\
\hline
\end{tabular}

Bold $=$ changes to ship CCSF, italics = ship splice, but CCSF shifted according to new offsets higher in the section. 
Table T2. Revised splice tie points, Site U1331.

\begin{tabular}{|c|c|c|c|c|c|c|}
\hline \multirow[b]{2}{*}{$\begin{array}{l}\text { Hole, core, section, } \\
\text { interval }(\mathrm{cm})\end{array}$} & \multicolumn{2}{|c|}{ Depth } & & \multirow[b]{2}{*}{$\begin{array}{l}\text { Hole, core, section, } \\
\text { interval }(\mathrm{cm})\end{array}$} & \multicolumn{2}{|c|}{ Depth } \\
\hline & $\begin{array}{c}\text { (mbsf } \\
{[\mathrm{m} \text { CSF-A] })}\end{array}$ & $\begin{array}{l}(\mathrm{rmcd} \\
{[\mathrm{m} \text { revised }} \\
\text { CCSF-A]) }\end{array}$ & & & $\begin{array}{c}\text { (mbsf } \\
{[\mathrm{m} \text { CSF-A] })}\end{array}$ & $\begin{array}{l}(\mathrm{rmcd} \\
\text { [m revised } \\
\text { CCSF-A]) }\end{array}$ \\
\hline $320-$ & & & & $320-$ & & \\
\hline U1331A-1H-3, 103 & 4.00 & 4.00 & Tie to & U1331C-1H-3, 46 & 3.46 & 4.00 \\
\hline U1331C-1H-5, 94 & 6.95 & 7.48 & Tie to & U1331A-2H-1, 79 & 5.98 & 7.48 \\
\hline U1331A-2H-5, 79 & 12.00 & 13.50 & Tie to & U1331C-2H-2, 38 & 11.38 & 13.50 \\
\hline U1331C-2H-6, 25 & 17.25 & 19.37 & Tie to & U1331A-3H-2, 26 & 16.46 & 19.37 \\
\hline U1331A-3H-6, 3 & 22.23 & 25.14 & Tie to & U1331B-3H-2, 106 & 22.16 & 25.14 \\
\hline U1331B-3H-5, 18 & 25.78 & 28.76 & Tie to & U1331A-4H-1, 130 & 25.50 & 28.76 \\
\hline U1331A-4H-6, 108 & 32.78 & 36.04 & Tie to & U1331B-4H-3, 19 & 32.29 & 36.04 \\
\hline U1331B-4H-6, 137 & 39.47 & 41.72 & Tie to & U1331A-5H-2, 127 & 36.47 & 41.72 \\
\hline U1331A-5H-5, 120 & 40.90 & 46.15 & Tie to & U1331B-5H-2, 54 & 40.64 & 46.15 \\
\hline U1331B-5H-7, 66 & 47.76 & 53.27 & Tie to & U1331A-6H-2, 141 & 46.11 & 53.27 \\
\hline U1331A-6H-6, 38 & 51.08 & 59.31 & Tie to & U1331B-6H-3, 5 & 51.15 & 58.24 \\
\hline U1331B-6H-6, 116 & 56.76 & 63.85 & Tie to & U1331A-7H-1, 134 & 54.04 & 63.85 \\
\hline U1331A-7H-7, 31 & 62.01 & 71.82 & Tie to & U1331C-6H-1, 136 & 60.36 & 71.82 \\
\hline U1331C-6H-4, 108 & 64.58 & 76.04 & Tie to & U1331A-8H-3, 7 & 65.27 & 76.04 \\
\hline U1331A-8H-5, 94 & 69.14 & 79.91 & Tie to & U1331B-8H-2, 52 & 69.12 & 79.91 \\
\hline U1331B-8H-6, 50 & 75.10 & 85.89 & Tie to & U1331A-9H-2, 133 & 74.53 & 85.89 \\
\hline U1331A-9H-6, 4 & 79.24 & 90.60 & Tie to & U1331B-9H-2, 4 & 78.15 & 90.60 \\
\hline U1331B-9H-3, 147 & 81.07 & 93.52 & Tie to & U1331A-10H-1, 144 & 82.64 & 93.52 \\
\hline U1331A-10H-5, 148 & 88.68 & 99.56 & Tie to & U1331B-10H-1, 26 & 86.36 & 99.56 \\
\hline U1331B-10H-6, 122 & 94.82 & 108.02 & Tie to & U1331A-11H-4, 42 & 94.80 & 108.02 \\
\hline U1331A-11H-6, 150 & 98.88 & 112.10 & Tie to & U1331B-11H-2, 147 & 97.07 & 112.10 \\
\hline U1331B-11H-7, 23 & 104.83 & 119.86 & Tie to & U1331A-12H-3, 53 & 103.73 & 119.86 \\
\hline U1331A-12H-5, 132 & 107.52 & 123.62 & Tie to & U1331B-12H-2, 48 & 107.08 & 123.62 \\
\hline U1331B-12H-6, 69 & 113.29 & 129.83 & Tie to & U1331A-13H-2, 66 & 111.86 & 129.83 \\
\hline U1331A-13H-6, 86 & 118.06 & 136.03 & Tie to & U1331B-13H-3, 70 & 118.30 & 136.03 \\
\hline U1331B-13H-6, 91 & 123.01 & 140.74 & Tie to & U1331A-14H-2, 98 & 121.65 & 140.74 \\
\hline U1331A-14H-5, 148 & 126.65 & 145.74 & Tie to & U1331B-14H-2, 54 & 126.14 & 145.74 \\
\hline U1331B-14H-5, 55 & 130.65 & 150.25 & Tie to & U1331C-12H-2, 32 & 130.82 & 150.25 \\
\hline U1331C-12H-7, 7 & 137.87 & 157.30 & Tie to & U1331A-15H-6, 14 & 136.34 & 157.30 \\
\hline U1331A-15H-7, 52 & 138.22 & 159.18 & Tie to & U1331B-15H-4, 78 & 138.88 & 159.18 \\
\hline U1331B-15H-5, 148 & 141.08 & 161.38 & Tie to & U1331C-13H-2, 88 & 140.88 & 161.38 \\
\hline U1331C-13H-6, 56 & 146.56 & 167.06 & Tie to & U1331B-16H-1, 111 & 144.21 & 167.06 \\
\hline U1331B-16H-3, 63 & 146.73 & 169.58 & Tie to & U1331A-17X-2, 8 & 149.18 & 169.58 \\
\hline
\end{tabular}

Bold $=$ new splice tie point, italics $=$ old splice tie points with new revised composite depth. 
Table T3. Mapping pairs for adjusting cores to the rmcd (m revised CCSF-A) splice, Site U1331. (Continued on next page.)

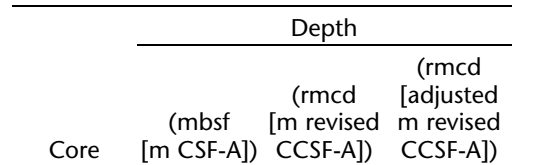

\begin{tabular}{llll}
\hline $320-U 1331 \mathrm{~A}-$ & & & \\
$1 \mathrm{H}$ & 0.01 & 0.01 & 0.01
\end{tabular}

$\begin{array}{rrrr}1 \mathrm{H} & 0.01 & 0.01 & 0.01 \\ 1 \mathrm{H} & 4.00 & 4.00 & 4.00 \\ 1 \mathrm{H} & 5.19 & 5.19 & 5.19 \\ 2 \mathrm{H} & 5.20 & 6.70 & 6.70 \\ 2 \mathrm{H} & 5.98 & 7.48 & 7.48 \\ 2 \mathrm{H} & 12.00 & 13.50 & 13.50 \\ 2 \mathrm{H} & 12.90 & 14.40 & 14.45\end{array}$

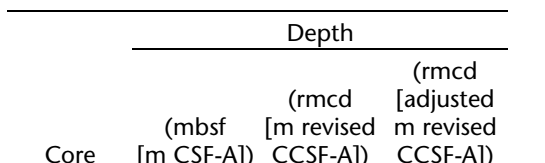

\begin{tabular}{lccc} 
& & & \\
\hline $12 \mathrm{H}$ & 100.20 & 116.33 & 116.20 \\
$12 \mathrm{H}$ & 101.50 & 117.63 & 117.50 \\
$12 \mathrm{H}$ & 103.73 & 119.86 & 119.86
\end{tabular}

$\begin{array}{llll}12 \mathrm{H} & 103.73 & 119.86 & 119.86 \\ 12 \mathrm{H} & 107.49 & 123.62 & 123.62 \\ 12 \mathrm{H} & 108.25 & 124.38 & 124.47\end{array}$

$\begin{array}{llll}12 \mathrm{H} & 108.25 & 124.38 & 124.47 \\ 12 \mathrm{H} & 109.70 & 125.83 & 126.17\end{array}$

$\begin{array}{llll}12 \mathrm{H} & 110.20 & 126.33 & 126.67\end{array}$

$\begin{array}{llll}13 \mathrm{H} & 109.70 & 127.67 & 127.39\end{array}$

$\begin{array}{llll}13 \mathrm{H} & 110.15 & 128.12 & 127.84\end{array}$

$\begin{array}{llll}13 \mathrm{H} & 111.86 & 129.83 & 129.83 \\ 13 \mathrm{H} & 118.06 & 136.03 & 136.03\end{array}$

$\begin{array}{llll}13 \mathrm{H} & 119.63 & 137.60 & 137.60\end{array}$

$\begin{array}{llll}14 \mathrm{H} & 119.20 & 138.29 & 138.29 \\ 14 \mathrm{H} & 121.65 & 140.74 & 140.74\end{array}$

$14 \mathrm{H} \quad 126.65 \quad 145.74 \quad 145.74$

$\begin{array}{llll}14 \mathrm{H} & 127.55 & 146.64 & 146.53\end{array}$

$14 \mathrm{H} \quad 128.17 \quad 147.26 \quad 147.19$

$\begin{array}{llll}14 \mathrm{H} & 129.28 & 148.37 & 148.37\end{array}$

$15 \mathrm{H} \quad 128.70 \quad 149.66 \quad 149.04$

$\begin{array}{llll}15 \mathrm{H} & 129.88 & 150.84 & 150.22 \\ 15 \mathrm{H} & 132.36 & 153.32 & 153.17\end{array}$

$\begin{array}{llll}15 \mathrm{H} & 132.36 & 153.32 & 153.17 \\ 15 \mathrm{H} & 136.34 & 157.30 & 157.30\end{array}$

$\begin{array}{llll}15 \mathrm{H} & 136.34 & 157.30 & 157.30 \\ 15 \mathrm{H} & 138.22 & 159.18 & 159.18\end{array}$

$15 \mathrm{H} \quad 138.60 \quad 159.56 \quad 159.56$

$\begin{array}{llll}16 \mathrm{X} & 138.20 & 158.01 & 158.01\end{array}$

$\begin{array}{llll}16 X & 139.30 & 159.11 & 159.11\end{array}$

$\begin{array}{llll}17 X & 147.60 & 168.00 & 168.00 \\ 17 X & 157.06 & 177.46 & 177.46\end{array}$

$\begin{array}{llll}17 X & 157.06 & 177.46 & 177.46 \\ 18 X & 157.30 & 177.70 & 177.70\end{array}$

$\begin{array}{llll}18 X & 157.30 & 177.70 & 177.70 \\ 18 X & 157.55 & 177.95 & 177.95\end{array}$

$\begin{array}{llll}19 \mathrm{X} & 160.50 & 180.90 & 180.90 \\ 19 \mathrm{X} & 162.41 & 182.81 & 182.81\end{array}$

320-U1331B-

$\begin{array}{lrrr}1 \mathrm{H} & 0.00 & 0.85 & 0.85 \\ 1 \mathrm{H} & 5.62 & 6.47 & 6.57 \\ 1 \mathrm{H} & 6.68 & 7.53 & 7.59 \\ 1 \mathrm{H} & 7.62 & 8.47 & 8.53 \\ 1 \mathrm{H} & 9.35 & 10.20 & 10.19 \\ 1 \mathrm{H} & 9.74 & 10.59 & 10.58 \\ 1 \mathrm{H} & 10.06 & 10.91 & 10.90 \\ 2 \mathrm{H} & 10.10 & 10.37 & 10.14 \\ 2 \mathrm{H} & 11.77 & 12.04 & 12.27 \\ 2 \mathrm{H} & 12.85 & 13.12 & 13.30 \\ 2 \mathrm{H} & 15.18 & 15.45 & 15.49 \\ 2 \mathrm{H} & 18.30 & 18.57 & 18.48 \\ 2 \mathrm{H} & 20.13 & 20.40 & 20.31 \\ 3 \mathrm{H} & 19.60 & 22.58 & 22.68 \\ 3 \mathrm{H} & 21.01 & 23.99 & 24.09 \\ 3 \mathrm{H} & 21.81 & 24.79 & 24.82 \\ 3 \mathrm{H} & 22.16 & 25.14 & 25.14 \\ 3 \mathrm{H} & 25.78 & 28.76 & 28.76 \\ 3 \mathrm{H} & 26.35 & 29.33 & 29.42 \\ 3 \mathrm{H} & 28.76 & 31.74 & 31.72 \\ 3 \mathrm{H} & 29.54 & 32.52 & 32.50 \\ 4 \mathrm{H} & 29.10 & 32.85 & 32.85 \\ 4 \mathrm{H} & 32.29 & 36.04 & 36.04 \\ 4 \mathrm{H} & 37.97 & 41.72 & 41.72 \\ 4 \mathrm{H} & 39.17 & 42.92 & 42.92 \\ 5 \mathrm{H} & 38.60 & 44.11 & 43.81 \\ 5 \mathrm{H} & 39.48 & 44.99 & 44.69 \\ 5 \mathrm{H} & 40.15 & 45.66 & 45.66 \\ 5 \mathrm{H} & 40.64 & 46.15 & 46.15 \\ 5 \mathrm{H} & 47.76 & 53.27 & 53.27 \\ 5 \mathrm{H} & 48.10 & 53.61 & 53.61 \\ 6 \mathrm{H} & 48.10 & 55.19 & 54.94\end{array}$

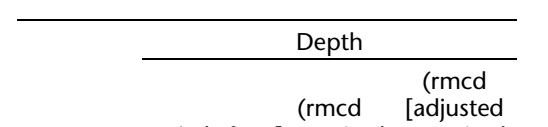

(mbsf [m revised $m$ revised

\begin{tabular}{cccc} 
Core & $[\mathrm{m}$ CSF-A $])$ & CCSF-A] $)$ & CCSF-A] $)$ \\
\hline $6 \mathrm{H}$ & 49.48 & 56.57 & 56.32 \\
$6 \mathrm{H}$ & 51.15 & 58.24 & 58.24
\end{tabular}

$\begin{array}{llll}6 \mathrm{H} & 56.76 & 63.85 & 63.85\end{array}$

$\begin{array}{llll}6 \mathrm{H} & 58.25 & 65.34 & 65.34\end{array}$

$\begin{array}{llll}7 \mathrm{H} & 57.60 & 65.91 & 66.33\end{array}$

$\begin{array}{llll}7 \mathrm{H} & 59.00 & 67.31 & 67.73\end{array}$

$\begin{array}{llll}7 \mathrm{H} & 59.68 & 67.99 & 68.02\end{array}$

$\begin{array}{llll}7 \mathrm{H} & 60.62 & 68.93 & 68.90\end{array}$

$\begin{array}{llll}7 \mathrm{H} & 60.62 & 68.93 & 68.90 \\ 7 \mathrm{H} & 63.46 & 71.77 & 71.77\end{array}$

$\begin{array}{llll}7 \mathrm{H} & 65.01 & 73.32 & 73.53\end{array}$

$\begin{array}{llll}7 \mathrm{H} & 66.11 & 74.42 & 74.45\end{array}$

$\begin{array}{llll}7 \mathrm{H} & 66.63 & 74.94 & 74.94\end{array}$

$\begin{array}{llll}7 \mathrm{H} & 67.21 & 75.52 & 75.52\end{array}$

$\begin{array}{llll}8 \mathrm{H} & 67.10 & 77.89 & 77.80\end{array}$

$\begin{array}{llll}8 \mathrm{H} & 68.10 & 78.89 & 78.80\end{array}$

$\begin{array}{llll}8 \mathrm{H} & 69.12 & 79.91 & 79.91\end{array}$

$\begin{array}{llll}8 \mathrm{H} & 75.10 & 85.89 & 85.89\end{array}$

$\begin{array}{llll}8 \mathrm{H} & 77.19 & 87.98 & 87.98\end{array}$

$\begin{array}{llll}9 \mathrm{H} & 76.60 & 89.05 & 89.05\end{array}$

$\begin{array}{llll}9 \mathrm{H} & 78.15 & 90.60 & 90.60\end{array}$

$\begin{array}{llll}9 \mathrm{H} & 81.07 & 93.52 & 93.52\end{array}$

$\begin{array}{llll}9 \mathrm{H} & 82.66 & 95.11 & 95.11\end{array}$

$\begin{array}{llll}9 \mathrm{H} & 83.05 & 95.50 & 95.48\end{array}$

$\begin{array}{llll}9 \mathrm{H} & 83.53 & 95.98 & 95.83\end{array}$

$\begin{array}{llll}9 \mathrm{H} & 84.98 & 97.43 & 97.19\end{array}$

$\begin{array}{llll}9 \mathrm{H} & 86.08 & 98.53 & 98.17\end{array}$

$\begin{array}{llll}9 \mathrm{H} & 86.65 & 99.10 & 98.74\end{array}$

$\begin{array}{llll}10 \mathrm{H} & 86.10 & 99.30 & 99.30\end{array}$

$\begin{array}{llll}10 \mathrm{H} & 86.36 & 99.56 & 99.56\end{array}$

$\begin{array}{llrr}10 \mathrm{H} & 94.82 & 108.02 & 108.02\end{array}$

$\begin{array}{llll}10 \mathrm{H} & 95.07 & 108.27 & 108.22\end{array}$

$\begin{array}{llll}10 \mathrm{H} & 96.14 & 109.34 & 109.29\end{array}$

$\begin{array}{llll}11 \mathrm{H} & 95.60 & 110.63 & 110.64\end{array}$

$\begin{array}{llll}11 \mathrm{H} & 96.13 & 111.16 & 111.17\end{array}$

$\begin{array}{llll}11 \mathrm{H} & 96.69 & 111.72 & 111.82\end{array}$

$\begin{array}{llll}11 \mathrm{H} & 97.07 & 112.10 & 112.10\end{array}$

$\begin{array}{llll}11 \mathrm{H} & 104.83 & 119.86 & 119.86\end{array}$

$\begin{array}{llll}11 \mathrm{H} & 105.47 & 120.50 & 120.38\end{array}$

$\begin{array}{llll}11 \mathrm{H} & 105.70 & 120.73 & 120.61\end{array}$

$\begin{array}{llll}12 \mathrm{H} & 105.10 & 121.64 & 121.64\end{array}$

$\begin{array}{llll}12 \mathrm{H} & 107.08 & 123.62 & 123.62\end{array}$

$\begin{array}{llll}12 \mathrm{H} & 113.29 & 129.83 & 129.83\end{array}$

$\begin{array}{llll}12 \mathrm{H} & 115.02 & 131.56 & 131.56\end{array}$

$\begin{array}{llll}13 \mathrm{H} & 114.60 & 132.33 & 132.81\end{array}$

$\begin{array}{llll}13 \mathrm{H} & 115.27 & 133.00 & 133.48\end{array}$

$\begin{array}{llll}13 \mathrm{H} & 115.69 & 135.29 & 135.36\end{array}$

$\begin{array}{llll}13 \mathrm{H} & 118.30 & 136.03 & 136.03\end{array}$

$\begin{array}{llll}13 \mathrm{H} & 123.01 & 140.74 & 140.74\end{array}$

$\begin{array}{llll}13 \mathrm{H} & 124.41 & 142.14 & 142.14\end{array}$

$\begin{array}{llll}14 \mathrm{H} & 124.10 & 143.70 & 143.51\end{array}$

$\begin{array}{llll}14 \mathrm{H} & 124.25 & 143.85 & 143.66\end{array}$

$\begin{array}{llll}14 \mathrm{H} & 124.68 & 144.28 & 144.07\end{array}$

$\begin{array}{llll}14 \mathrm{H} & 125.58 & 145.18 & 145.14\end{array}$

$\begin{array}{llll}14 \mathrm{H} & 126.14 & 145.74 & 145.74\end{array}$

$\begin{array}{llll}14 \mathrm{H} & 130.65 & 150.25 & 150.25\end{array}$

$\begin{array}{llll}14 \mathrm{H} & 132.41 & 152.01 & 152.01\end{array}$

$\begin{array}{llll}14 \mathrm{H} & 133.43 & 153.03 & 153.17\end{array}$

$\begin{array}{llll}14 \mathrm{H} & 134.03 & 153.63 & 153.63\end{array}$

$\begin{array}{llll}15 \mathrm{H} & 133.60 & 153.90 & 153.90\end{array}$

$\begin{array}{llll}15 \mathrm{H} & 138.88 & 159.18 & 159.18\end{array}$

$\begin{array}{llll}15 \mathrm{H} & 141.08 & 161.38 & 161.38\end{array}$

$\begin{array}{llll}15 \mathrm{H} & 143.62 & 163.92 & 163.92\end{array}$

$\begin{array}{llll}16 \mathrm{H} & 143.10 & 165.95 & 165.95\end{array}$

$\begin{array}{llll}16 \mathrm{H} & 144.21 & 167.06 & 167.06\end{array}$

$\begin{array}{llll}16 \mathrm{H} & 146.73 & 169.58 & 169.58\end{array}$ 
Table T3 (continued).

\begin{tabular}{|c|c|c|c|}
\hline \multirow[b]{2}{*}{ Core } & \multicolumn{3}{|c|}{ Depth } \\
\hline & $\begin{array}{c}\text { (mbsf } \\
{[\mathrm{m} \mathrm{CSF-A])}}\end{array}$ & $\begin{array}{c}(\mathrm{rmcd} \\
{[\mathrm{m} \text { revised }} \\
\text { CCSF-A]) }\end{array}$ & $\begin{array}{l}\text { (rmcd } \\
\text { [adjusted } \\
\text { m revised } \\
\text { CCSF-A]) }\end{array}$ \\
\hline $16 \mathrm{H}$ & 149.24 & 172.09 & 172.36 \\
\hline $16 \mathrm{H}$ & 149.21 & 174.14 & 174.21 \\
\hline $16 \mathrm{H}$ & 149.86 & 174.79 & 174.60 \\
\hline $16 \mathrm{H}$ & 152.50 & 175.35 & 175.16 \\
\hline $17 \mathrm{H}$ & 152.60 & 177.53 & 177.53 \\
\hline $17 \mathrm{H}$ & 156.88 & 181.81 & 181.81 \\
\hline \multicolumn{4}{|c|}{ 320-U1331C- } \\
\hline $1 \mathrm{H}$ & 0.00 & 0.54 & 0.54 \\
\hline $1 \mathrm{H}$ & 3.46 & 4.00 & 4.00 \\
\hline $1 \mathrm{H}$ & 6.94 & 7.48 & 7.48 \\
\hline $1 \mathrm{H}$ & 9.46 & 10.00 & 10.00 \\
\hline $2 \mathrm{H}$ & 9.50 & 11.62 & 11.54 \\
\hline $2 \mathrm{H}$ & 10.08 & 12.20 & 12.12 \\
\hline $2 \mathrm{H}$ & 10.80 & 12.92 & 12.90 \\
\hline $2 \mathrm{H}$ & 11.25 & 13.37 & 13.32 \\
\hline $2 \mathrm{H}$ & 11.38 & 13.50 & 13.50 \\
\hline $2 \mathrm{H}$ & 17.25 & 19.37 & 19.37 \\
\hline $2 \mathrm{H}$ & 17.83 & 19.95 & 20.00 \\
\hline $2 \mathrm{H}$ & 18.23 & 20.35 & 20.30 \\
\hline $2 \mathrm{H}$ & 18.46 & 20.58 & 20.64 \\
\hline $2 \mathrm{H}$ & 18.98 & 21.10 & 21.21 \\
\hline $2 \mathrm{H}$ & 19.52 & 21.64 & 21.75 \\
\hline $3 \mathrm{H}$ & 19.00 & 21.40 & 20.92 \\
\hline $3 \mathrm{H}$ & 20.34 & 22.74 & 22.26 \\
\hline $3 \mathrm{H}$ & 22.05 & 24.45 & 24.09 \\
\hline
\end{tabular}

\begin{tabular}{|c|c|c|c|}
\hline \multirow[b]{2}{*}{ Core } & \multicolumn{3}{|c|}{ Depth } \\
\hline & $\begin{array}{c}\text { (mbsf } \\
{[\mathrm{m} \mathrm{CSF-A]})}\end{array}$ & $\begin{array}{c}(\mathrm{rmcd} \\
{[\mathrm{m} \text { revised }} \\
\text { CCSF-A] })\end{array}$ & $\begin{array}{c}\text { (rmcd } \\
\text { [adjusted } \\
\text { m revised } \\
\text { CCSF-A]) }\end{array}$ \\
\hline $3 \mathrm{H}$ & 23.18 & 25.58 & 25.24 \\
\hline $3 \mathrm{H}$ & 24.84 & 27.24 & 27.00 \\
\hline $3 \mathrm{H}$ & 25.47 & 27.87 & 27.77 \\
\hline $3 \mathrm{H}$ & 25.94 & 28.34 & 28.15 \\
\hline $3 \mathrm{H}$ & 26.31 & 28.71 & 28.63 \\
\hline $3 \mathrm{H}$ & 27.02 & 29.42 & 29.42 \\
\hline $3 \mathrm{H}$ & 27.35 & 29.75 & 29.89 \\
\hline $3 \mathrm{H}$ & 28.25 & 30.65 & 30.70 \\
\hline $3 \mathrm{H}$ & 28.77 & 31.17 & 31.22 \\
\hline $4 \mathrm{H}$ & 28.50 & 31.00 & 31.00 \\
\hline $4 \mathrm{H}$ & 36.34 & 38.84 & 38.96 \\
\hline $4 \mathrm{H}$ & 38.49 & 40.99 & 40.99 \\
\hline $6 \mathrm{H}$ & 59.00 & 70.46 & 70.46 \\
\hline $6 \mathrm{H}$ & 60.36 & 71.82 & 71.82 \\
\hline $6 \mathrm{H}$ & 64.58 & 76.04 & 76.04 \\
\hline $6 \mathrm{H}$ & 65.63 & 77.09 & 76.94 \\
\hline $6 \mathrm{H}$ & 67.62 & 79.08 & 78.82 \\
\hline $6 \mathrm{H}$ & 68.25 & 79.71 & 79.29 \\
\hline $6 \mathrm{H}$ & 68.25 & 79.71 & 79.29 \\
\hline $6 \mathrm{H}$ & 68.54 & 80.00 & 79.58 \\
\hline $8 \mathrm{H}$ & 92.50 & 106.89 & 106.89 \\
\hline $8 \mathrm{H}$ & 95.48 & 109.87 & 109.87 \\
\hline $8 \mathrm{H}$ & 101.71 & 116.10 & 116.10 \\
\hline $10 \mathrm{H}$ & 102.50 & 117.59 & 117.59 \\
\hline $10 \mathrm{H}$ & 106.08 & 121.17 & 121.17 \\
\hline
\end{tabular}

\begin{tabular}{|c|c|c|c|}
\hline \multirow[b]{2}{*}{ Core } & \multicolumn{3}{|c|}{ Depth } \\
\hline & $\begin{array}{c}\text { (mbsf } \\
{[\mathrm{m} \text { CSF-A] })}\end{array}$ & $\begin{array}{c}\text { (rmcd } \\
\text { [m revised } \\
\text { CCSF-A]) }\end{array}$ & $\begin{array}{c}\text { (rmcd } \\
\text { [adjusted } \\
\text { m revised } \\
\text { CCSF-A]) }\end{array}$ \\
\hline $10 \mathrm{H}$ & 107.59 & 122.68 & 122.68 \\
\hline $10 \mathrm{H}$ & 108.19 & 123.28 & 123.28 \\
\hline $10 \mathrm{H}$ & 110.54 & 125.63 & 125.48 \\
\hline $10 \mathrm{H}$ & 110.98 & 126.07 & 125.92 \\
\hline $12 \mathrm{H}$ & 129.00 & 148.43 & 148.43 \\
\hline $12 \mathrm{H}$ & 129.10 & 148.53 & 148.35 \\
\hline $12 \mathrm{H}$ & 130.82 & 150.25 & 150.25 \\
\hline $12 \mathrm{H}$ & 137.87 & 157.30 & 157.30 \\
\hline $12 \mathrm{H}$ & 139.05 & 158.48 & 158.48 \\
\hline $13 \mathrm{H}$ & 138.50 & 159.00 & 159.00 \\
\hline $13 \mathrm{H}$ & 140.88 & 161.38 & 161.38 \\
\hline $13 \mathrm{H}$ & 146.56 & 167.06 & 167.06 \\
\hline $13 \mathrm{H}$ & 148.59 & 169.09 & 169.09 \\
\hline $14 \mathrm{H}$ & 148.00 & 169.25 & 168.92 \\
\hline $14 \mathrm{H}$ & 151.29 & 172.54 & 172.21 \\
\hline $14 \mathrm{H}$ & 149.60 & 170.85 & 170.20 \\
\hline $14 \mathrm{H}$ & 154.32 & 175.57 & 175.17 \\
\hline $14 \mathrm{H}$ & 155.52 & 176.77 & 176.20 \\
\hline $14 \mathrm{H}$ & 156.94 & 178.19 & 177.62 \\
\hline $16 \mathrm{H}$ & 177.00 & 198.25 & 198.25 \\
\hline $16 \mathrm{H}$ & 185.46 & 206.71 & 206.71 \\
\hline $17 \mathrm{H}$ & 184.00 & 206.75 & 206.75 \\
\hline $17 \mathrm{H}$ & 189.53 & 212.28 & 212.28 \\
\hline
\end{tabular}

Italics = splice tie points.

Table T4. Turbidites in Site U1331 revised splice.

\begin{tabular}{|c|c|c|c|c|c|c|c|}
\hline \multicolumn{3}{|c|}{ Top of turbidite } & & \multicolumn{3}{|c|}{ Base of turbidite } & \multirow{3}{*}{$\begin{array}{l}\text { Thickness } \\
\text { (rmcd } \\
\text { [m revised } \\
\text { CCSF-A]) }\end{array}$} \\
\hline \multirow[b]{2}{*}{$\begin{array}{l}\text { Hole, core, section, } \\
\text { interval }(\mathrm{cm})\end{array}$} & \multicolumn{2}{|c|}{ Depth } & & \multirow[b]{2}{*}{$\begin{array}{l}\text { Hole, core, section, } \\
\text { interval }(\mathrm{cm})\end{array}$} & \multicolumn{2}{|c|}{ Depth } & \\
\hline & $\begin{array}{c}(\mathrm{mbsf} \\
{[\mathrm{m} \mathrm{CSF-A]})}\end{array}$ & $\begin{array}{c}\text { (rmcd } \\
{[\mathrm{m} \text { revised }} \\
\text { CCSF-A] })\end{array}$ & & & $\begin{array}{c}\text { (mbsf } \\
{[\mathrm{m} \mathrm{CSF-A]})}\end{array}$ & $\begin{array}{c}\text { (rmcd } \\
{[\mathrm{m} \text { revised }} \\
\text { CCSF-A] }\end{array}$ & \\
\hline $320-$ & & & & $320-$ & & & \\
\hline U1331A-3H-4, 52 & 19.72 & 22.63 & Down to & U1331A-3H-4, 72.5 & 19.92 & 22.83 & 0.21 \\
\hline U1331A-4H-6, 106 & 32.76 & 36.02 & Down to & U1331B-4H-5, 8 & 36.68 & 38.94 & 2.92 \\
\hline U1331A-6H-4, 86 & 48.56 & 55.72 & Down to & U1331A-6H-4, 143 & 49.13 & 56.29 & 0.57 \\
\hline U1331A-6H-5, 148 & 50.68 & 57.84 & Down to & U1331B-6H-3, 88 & 51.98 & 59.07 & 1.23 \\
\hline U1331B-6H-3, 110 & 52.20 & 59.29 & Down to & U1331B-6H-4, 32 & 52.92 & 60.01 & 0.72 \\
\hline U1331B-8H-2, 66 & 69.26 & 80.05 & Down to & U1331B-8H-5, 6 & 73.16 & 83.95 & 3.90 \\
\hline U1331A-9H-4, 138 & 77.58 & 88.94 & Down to & U1331B-9H-4, 124 & 82.34 & 94.79 & 5.85 \\
\hline U1331A-10H-3, 38 & 84.58 & 95.46 & Down to & U1331A-10H-3, 44 & 84.64 & 95.52 & 0.06 \\
\hline U1331B-10H-1, 50 & 86.60 & 99.80 & Down to & U1331B-10H-2, 10 & 87.70 & 100.90 & 1.10 \\
\hline U1331B-10H-5, 85 & 92.95 & 106.15 & Down to & U1331A-11H-4, 43 & 94.84 & 108.03 & 1.88 \\
\hline U1331A-11H-6, 149 & 98.87 & 112.09 & Down to & U1331B-11H-3, 23 & 98.83 & 113.86 & 1.77 \\
\hline U1331B-11H-4, 10 & 100.20 & 115.23 & Down to & U1331B-11H-4, 79 & 100.89 & 115.92 & 0.69 \\
\hline U1331B-11H-4, 112 & 101.22 & 116.25 & Down to & U1331B-11H-5, 40 & 102.00 & 117.03 & 0.78 \\
\hline U1331A-12H-4, 86 & 105.56 & 121.69 & Down to & U1331A-12H-5, 22 & 106.42 & 122.55 & 0.86 \\
\hline U1331B-12H-2, 70 & 107.30 & 123.84 & Down to & U1331B-12H-2, 90 & 107.50 & 124.04 & 0.20 \\
\hline U1331A-13H-3, 22 & 112.92 & 130.89 & Down to & U1331A-13H-3, 35 & 113.05 & 131.05 & 0.16 \\
\hline U1331A-13H-5, 36 & 116.06 & 134.03 & Down to & U1331A-13H-6, 45 & 117.65 & 135.62 & 1.59 \\
\hline U1331B-14H-3, 40 & 127.50 & 147.10 & Down to & U1331B-14H-3, 55 & 127.65 & 147.25 & 0.15 \\
\hline U1331B-14H-4, 40 & 129.00 & 148.60 & Down to & U1331B-14H-4, 51 & 129.13 & 148.73 & 0.13 \\
\hline
\end{tabular}


Table T5. Revised shipboard composite and corrected composite depths, Site U1332.

\begin{tabular}{|c|c|c|c|}
\hline Core & $\begin{array}{c}\text { Depth } \\
\text { (mbsf } \\
\text { [m CSF-A]) }\end{array}$ & $\begin{array}{c}\text { Offset } \\
(\mathrm{m})\end{array}$ & $\begin{array}{l}\text { Top depth } \\
\text { (rmcd } \\
\text { [m revised } \\
\text { CCSF-A] })\end{array}$ \\
\hline \multicolumn{4}{|c|}{ 320-U1332A- } \\
\hline $1 \mathrm{H}$ & 0.00 & 0.00 & 0.00 \\
\hline $2 \mathrm{H}$ & 3.90 & 0.31 & 4.21 \\
\hline $3 \mathrm{H}$ & 13.40 & 0.65 & 14.05 \\
\hline $4 \mathrm{H}$ & 22.90 & 2.55 & 25.45 \\
\hline $5 \mathrm{H}$ & 32.40 & 5.05 & 37.45 \\
\hline $6 \mathrm{H}$ & 41.90 & 5.50 & 47.40 \\
\hline $7 \mathrm{H}$ & 51.40 & 7.75 & 59.15 \\
\hline $8 \mathrm{H}$ & 60.90 & 7.35 & 68.25 \\
\hline $9 \mathrm{H}$ & 70.40 & 2.63 & 73.03 \\
\hline $10 \mathrm{H}$ & 79.90 & 3.30 & 83.20 \\
\hline $11 \mathrm{H}$ & 89.40 & 3.38 & 92.78 \\
\hline $12 \mathrm{H}$ & 98.90 & 6.22 & 105.12 \\
\hline $13 \mathrm{H}$ & 108.40 & 8.62 & 117.02 \\
\hline $14 \mathrm{X}$ & 117.50 & 12.50 & 130.00 \\
\hline $15 x$ & 125.90 & 13.50 & 139.40 \\
\hline $16 \mathrm{X}$ & 135.50 & 13.50 & 149.00 \\
\hline $17 X$ & 144.50 & 13.50 & 158.00 \\
\hline $18 \mathrm{X}$ & 150.40 & 13.50 & 163.90 \\
\hline \multicolumn{4}{|c|}{ 320-U1332B- } \\
\hline $1 \mathrm{H}$ & 0.00 & 0.15 & 0.15 \\
\hline $2 \mathrm{H}$ & 2.10 & 0.65 & 2.75 \\
\hline $3 \mathrm{H}$ & 11.60 & 2.15 & 13.75 \\
\hline $4 \mathrm{H}$ & 19.60 & 4.15 & 23.75 \\
\hline $5 \mathrm{H}$ & 29.10 & 4.25 & 33.35 \\
\hline $6 \mathrm{H}$ & 38.60 & 7.05 & 45.65 \\
\hline $7 \mathrm{H}$ & 48.10 & 10.35 & 58.45 \\
\hline $8 \mathrm{H}$ & 57.60 & 10.05 & 67.65 \\
\hline $9 \mathrm{H}$ & 67.10 & 3.75 & 70.85 \\
\hline $10 \mathrm{H}$ & 76.60 & 5.53 & 82.13 \\
\hline $11 \mathrm{H}$ & 86.10 & 5.89 & 91.99 \\
\hline $12 \mathrm{H}$ & 91.10 & 9.27 & 100.37 \\
\hline $13 \mathrm{H}$ & 100.60 & 10.48 & 111.08 \\
\hline $14 X$ & 110.10 & 11.48 & 121.58 \\
\hline $15 X$ & 116.10 & 16.05 & 132.15 \\
\hline $16 \mathrm{X}$ & 124.60 & 18.08 & 142.68 \\
\hline $17 X$ & 134.30 & 18.08 & 152.38 \\
\hline $18 \mathrm{X}$ & 143.90 & 15.90 & 159.80 \\
\hline \multicolumn{4}{|c|}{ 320-U1332C- } \\
\hline $1 \mathrm{H}$ & 0.00 & 0.04 & 0.04 \\
\hline $2 \mathrm{H}$ & 7.50 & 0.49 & 7.99 \\
\hline $3 \mathrm{H}$ & 17.00 & 0.25 & 17.25 \\
\hline $4 \mathrm{H}$ & 26.50 & 2.35 & 28.85 \\
\hline $5 \mathrm{H}$ & 36.00 & 4.15 & 40.15 \\
\hline $6 \mathrm{H}$ & 45.50 & 3.35 & 48.85 \\
\hline $7 \mathrm{H}$ & 49.50 & 6.80 & 56.30 \\
\hline $8 \mathrm{H}$ & 59.00 & 7.51 & 66.51 \\
\hline $9 \mathrm{H}$ & 66.00 & 0.95 & 66.95 \\
\hline $10 \mathrm{H}$ & 75.50 & 0.55 & 76.05 \\
\hline $11 \mathrm{H}$ & 85.00 & 3.43 & 88.43 \\
\hline $12 \mathrm{H}$ & 94.50 & 4.84 & 99.34 \\
\hline $13 \mathrm{H}$ & 104.00 & 5.60 & 109.60 \\
\hline $14 X$ & 113.50 & 7.96 & 121.46 \\
\hline $15 X$ & 118.00 & 10.76 & 128.76 \\
\hline $16 \mathrm{X}$ & 127.60 & 11.82 & 139.42 \\
\hline $17 X$ & 137.20 & 11.82 & 149.02 \\
\hline $18 \mathrm{X}$ & 146.90 & 13.82 & 160.72 \\
\hline
\end{tabular}

Bold = changes to ship CCSF, italics = ship splice, but CCSF shifted according to new offsets higher up in the section. 
Table T6. Revised splice tie points, Site U1332.

\begin{tabular}{|c|c|c|c|c|c|c|}
\hline \multirow[b]{2}{*}{$\begin{array}{l}\text { Hole, core, section, } \\
\text { interval }(\mathrm{cm})\end{array}$} & \multicolumn{2}{|c|}{ Depth } & & \multirow[b]{2}{*}{$\begin{array}{l}\text { Hole, core, section, } \\
\text { interval }(\mathrm{cm})\end{array}$} & \multicolumn{2}{|c|}{ Depth } \\
\hline & $\begin{array}{c}\text { (mbsf } \\
{[\mathrm{m} \mathrm{CSF-A]})}\end{array}$ & $\begin{array}{l}(\text { rmcd } \\
{[\text { m revised }} \\
\text { CCSF-A]) }\end{array}$ & & & $\begin{array}{c}\text { (mbsf } \\
[\mathrm{m} \text { CSF-A }])\end{array}$ & $\begin{array}{l}\text { (rmcd } \\
\text { [m revised } \\
\text { CCSF-A]) }\end{array}$ \\
\hline $320-$ & & & & $320-$ & & \\
\hline U1332A-1H-2, 110 & 2.60 & 2.60 & Tie to & U1332C-1H-2, 104 & 2.55 & 2.60 \\
\hline U1332C-1H-5, 61 & 6.60 & 6.65 & Tie to & U1332A-2H-2, 94 & 6.34 & 6.65 \\
\hline U1332A-2H-5, 125 & 11.15 & 11.46 & Tie to & U1332C-2H-3, 47 & 10.97 & 11.46 \\
\hline U1332C-2H-6, 146 & 16.46 & 16.95 & Tie to & U1332A-3H-2, 138 & 16.28 & 16.95 \\
\hline U1332A-3H-5, 38 & 19.78 & 20.43 & Tie to & U1332C-3H-3, 18 & 20.18 & 20.43 \\
\hline U1332C-3H-7, 5 & 26.05 & 26.30 & Tie to & U1332A-4H-1, 85 & 23.75 & 26.30 \\
\hline U1332A-4H-5, 20 & 29.10 & 31.65 & Tie to & U1332C-4H-2, 130 & 29.30 & 31.65 \\
\hline U1332C-4H-5, 45 & 32.95 & 35.30 & Tie to & U1332B-5H-2, 45 & 31.05 & 35.30 \\
\hline U1332B-5H-5, 151 & 36.60 & 40.85 & Tie to & U1332C-5H-1, 70 & 36.70 & 40.85 \\
\hline U1332C-5H-6, 103 & 44.53 & 48.68 & Tie to & U1332A-6H-1, 128 & 43.18 & 48.68 \\
\hline U1332A-6H-7, 30 & 51.20 & 56.70 & Tie to & U1332C-7H-1, 40 & 49.90 & 56.70 \\
\hline U1332C-7H-7, 27 & 58.77 & 65.57 & Tie to & U1332B-7H-5, 112 & 55.22 & 65.57 \\
\hline U1332B-7H-7, 68 & 57.78 & 68.13 & Tie to & U1332C-8H-2, 12 & 60.62 & 68.13 \\
\hline$U 1332 \mathrm{C}-8 \mathrm{H}-4,34$ & 63.84 & 71.35 & Tie to & U1332B-9H-1, 50 & 67.60 & 71.35 \\
\hline U1332B-9H-5, 35 & 73.45 & 77.20 & Tie to & U1332A-9H-3, 117 & 74.57 & 77.20 \\
\hline U1332A-9H-CC, 16 & 83.38 & 83.01 & Tie to & U1332B-10H-1, 88 & 77.48 & 83.01 \\
\hline U1332B-10H-5, 92 & 83.52 & 89.05 & Tie to & U1332A-10H-4, 135 & 85.75 & 89.05 \\
\hline U1332A-10H-6, 108 & 88.48 & 91.78 & Tie to & U1332C-11H-3, 35 & 88.35 & 91.78 \\
\hline U1332C-11H-4, 140 & 90.90 & 94.33 & Tie to & U1332A-11H-2, 5 & 90.95 & 94.33 \\
\hline U1332A-11H-6, 28 & 97.18 & 100.56 & Tie to & U1332C-12H-1, 122 & 95.72 & 100.56 \\
\hline U1332C-12H-5, 131 & 101.81 & 106.65 & Tie to & U1332A-12H-2, 3 & 100.43 & 106.65 \\
\hline U1332A-12H-5, 122 & 106.12 & 112.34 & Tie to & U1332C-13H-2, 124 & 106.74 & 112.34 \\
\hline U1332C-13H-4, 26 & 108.76 & 114.36 & Tie to & U1332B-13H-3, 28 & 103.88 & 114.36 \\
\hline U1332B-13H-6, 28 & 108.38 & 118.86 & Tie to & U1332A-13H-2, 34 & 110.24 & 118.86 \\
\hline U1332A-13H-5, 118 & 115.58 & 124.20 & Tie to & U1332B-14X-2, 112 & 112.72 & 124.20 \\
\hline U1332B-14X-6, 138 & 118.98 & 130.46 & Tie to & U1332C-15X-2, 20 & 119.70 & 130.46 \\
\hline U1332C-15X-3, 115 & 122.15 & 132.91 & Tie to & U1332B-15X-1, 76 & 116.86 & 132.91 \\
\hline
\end{tabular}

Bold $=$ new splice tie point, italics = old splice tie points with new revised composite depth. 
Table T7. Mapping pairs for adjusting cores to the rmcd (m revised CCSF-A) splice, Site U1332. (Continued on next page.)

\begin{tabular}{|c|c|c|c|}
\hline \multirow[b]{2}{*}{ Core } & \multicolumn{3}{|c|}{ Depth } \\
\hline & $\begin{array}{c}(\mathrm{mbsf} \\
[\mathrm{m} \text { CSF-A }])\end{array}$ & $\begin{array}{l}(\mathrm{rmcd} \\
{[\mathrm{m} \text { revised }} \\
\text { CCSF-A]) }\end{array}$ & $\begin{array}{c}\text { (rmcd } \\
\text { [adjusted } \\
\text { m revised } \\
\text { CCSF-A]) }\end{array}$ \\
\hline \multicolumn{4}{|c|}{ 320-U1332A- } \\
\hline $1 \mathrm{H}$ & 0.01 & 0.01 & 0.01 \\
\hline $1 \mathrm{H}$ & 2.60 & 2.60 & 2.60 \\
\hline $1 \mathrm{H}$ & 3.91 & 3.91 & 3.91 \\
\hline $2 \mathrm{H}$ & 3.90 & 4.21 & 4.03 \\
\hline $2 \mathrm{H}$ & 4.10 & 4.41 & 4.23 \\
\hline $2 \mathrm{H}$ & 5.52 & 5.83 & 5.83 \\
\hline $2 \mathrm{H}$ & 6.34 & 6.65 & 6.65 \\
\hline $2 \mathrm{H}$ & 11.15 & 11.46 & 11.46 \\
\hline $2 \mathrm{H}$ & 11.49 & 11.80 & 12.16 \\
\hline $2 \mathrm{H}$ & 12.01 & 12.32 & 12.64 \\
\hline $2 \mathrm{H}$ & 12.83 & 13.14 & 13.60 \\
\hline $2 \mathrm{H}$ & 13.67 & 13.98 & 14.44 \\
\hline $3 \mathrm{H}$ & 13.40 & 14.05 & 14.05 \\
\hline $3 \mathrm{H}$ & 16.30 & 16.95 & 16.95 \\
\hline $3 \mathrm{H}$ & 19.78 & 20.43 & 20.43 \\
\hline $3 \mathrm{H}$ & 23.09 & 23.74 & 23.22 \\
\hline $3 \mathrm{H}$ & 23.56 & 24.21 & 23.69 \\
\hline $4 \mathrm{H}$ & 22.90 & 25.45 & 25.45 \\
\hline $4 \mathrm{H}$ & 23.75 & 26.30 & 26.30 \\
\hline $4 \mathrm{H}$ & 29.10 & 31.65 & 31.65 \\
\hline $4 \mathrm{H}$ & 30.72 & 33.27 & 33.41 \\
\hline $4 \mathrm{H}$ & 32.34 & 34.89 & 35.14 \\
\hline $4 \mathrm{H}$ & 33.00 & 35.55 & 35.90 \\
\hline $5 \mathrm{H}$ & 32.40 & 37.45 & 36.91 \\
\hline $5 \mathrm{H}$ & 32.83 & 37.88 & 37.34 \\
\hline $5 \mathrm{H}$ & 33.58 & 38.63 & 38.31 \\
\hline $5 \mathrm{H}$ & 34.22 & 39.27 & 39.01 \\
\hline $5 \mathrm{H}$ & 35.49 & 40.54 & 40.52 \\
\hline $5 \mathrm{H}$ & 35.82 & 40.87 & 40.92 \\
\hline $5 \mathrm{H}$ & 36.45 & 41.50 & 41.57 \\
\hline $5 \mathrm{H}$ & 37.44 & 42.49 & 42.47 \\
\hline $5 \mathrm{H}$ & 38.43 & 43.48 & 43.55 \\
\hline $5 \mathrm{H}$ & 39.38 & 44.43 & 44.47 \\
\hline $5 \mathrm{H}$ & 40.69 & 45.74 & 45.72 \\
\hline $5 \mathrm{H}$ & 41.49 & 46.54 & 46.45 \\
\hline $5 \mathrm{H}$ & 42.52 & 47.57 & 47.48 \\
\hline $6 \mathrm{H}$ & 41.90 & 47.40 & 47.20 \\
\hline $6 \mathrm{H}$ & 42.80 & 48.30 & 48.10 \\
\hline $6 \mathrm{H}$ & 43.18 & 48.68 & 48.68 \\
\hline $6 \mathrm{H}$ & 51.20 & 56.70 & 56.70 \\
\hline $6 \mathrm{H}$ & 51.52 & 57.02 & 57.00 \\
\hline $6 \mathrm{H}$ & 52.02 & 57.52 & 57.50 \\
\hline $7 \mathrm{H}$ & 51.40 & 59.15 & 59.03 \\
\hline $7 \mathrm{H}$ & 51.93 & 59.68 & 59.56 \\
\hline $7 \mathrm{H}$ & 52.99 & 60.74 & 60.87 \\
\hline $7 \mathrm{H}$ & 53.84 & 61.59 & 61.82 \\
\hline $7 \mathrm{H}$ & 54.90 & 62.65 & 62.69 \\
\hline $7 \mathrm{H}$ & 55.75 & 63.50 & 63.25 \\
\hline $7 \mathrm{H}$ & 56.41 & 64.16 & 64.45 \\
\hline $7 \mathrm{H}$ & 59.87 & 67.62 & 67.20 \\
\hline $7 \mathrm{H}$ & 61.54 & 69.29 & 68.68 \\
\hline $8 \mathrm{H}$ & 60.90 & 68.25 & 68.30 \\
\hline $8 \mathrm{H}$ & 63.92 & 71.27 & 71.32 \\
\hline $8 \mathrm{H}$ & 65.90 & 73.25 & 73.39 \\
\hline $8 \mathrm{H}$ & 66.15 & 73.50 & 73.57 \\
\hline $8 \mathrm{H}$ & 66.75 & 74.10 & 74.15 \\
\hline $8 \mathrm{H}$ & 67.71 & 75.06 & 74.97 \\
\hline $8 \mathrm{H}$ & 68.45 & 75.80 & 75.63 \\
\hline $8 \mathrm{H}$ & 68.87 & 76.22 & 76.04 \\
\hline $8 \mathrm{H}$ & 69.57 & 76.92 & 76.69 \\
\hline $8 \mathrm{H}$ & 70.55 & 77.90 & 77.57 \\
\hline $8 \mathrm{H}$ & 71.05 & 78.40 & 78.07 \\
\hline $9 \mathrm{H}$ & 70.40 & 73.03 & 72.84 \\
\hline $9 \mathrm{H}$ & 70.75 & 73.38 & 73.19 \\
\hline
\end{tabular}

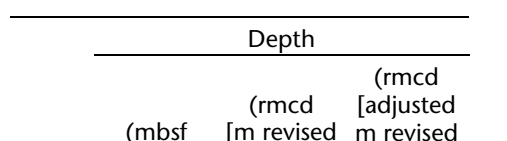
Core [m CSF-A]) CCSF-A]) CCSF-A])

\begin{tabular}{llll}
\hline $9 \mathrm{H}$ & 71.15 & 73.78 & 73.54 \\
$9 \mathrm{H}$ & 71.68 & 74.31 & 74.15 \\
$9 \mathrm{H}$ & 72.13 & 74.76 & 74.50
\end{tabular}

$\begin{array}{llll}9 \mathrm{H} & 72.56 & 75.19 & 75.00\end{array}$

$\begin{array}{llll}9 \mathrm{H} & 72.56 & 75.19 & 75.00 \\ 9 \mathrm{H} & 73.09 & 75.72 & 75.63\end{array}$

$\begin{array}{llll}9 \mathrm{H} & 73.50 & 76.13 & 76.03\end{array}$

$\begin{array}{llll}9 \mathrm{H} & 74.02 & 76.65 & 76.56\end{array}$

$\begin{array}{llll}9 \mathrm{H} & 74.57 & 77.20 & 77.20\end{array}$

$\begin{array}{llll}9 \mathrm{H} & 80.38 & 83.01 & 83.01\end{array}$

$\begin{array}{llll}9 \mathrm{H} & 80.38 & 83.01 & 83.01 \\ 9 \mathrm{H} & 80.52 & 83.15 & 83.15\end{array}$

$10 \mathrm{H} \quad 79.90 \quad 83.20 \quad 83.51$

$\begin{array}{llll}10 \mathrm{H} & 81.28 & 84.58 & 84.89\end{array}$

$\begin{array}{llll}10 \mathrm{H} & 82.91 & 86.21 & 86.73\end{array}$

$\begin{array}{llll}10 \mathrm{H} & 84.40 & 87.70 & 88.09\end{array}$

$\begin{array}{llll}10 \mathrm{H} & 85.75 & 89.05 & 89.05\end{array}$

$\begin{array}{llll}10 \mathrm{H} & 88.48 & 91.78 & 91.78\end{array}$

$\begin{array}{llll}10 \mathrm{H} & 89.94 & 93.24 & 93.24\end{array}$

$11 \mathrm{H} \quad 89.40 \quad 92.78 \quad 92.78$

$\begin{array}{llll}11 \mathrm{H} & 90.95 & 94.33 & 94.33\end{array}$

$\begin{array}{llll}11 \mathrm{H} & 97.18 & 100.56 & 100.56\end{array}$

$\begin{array}{llll}11 \mathrm{H} & 98.57 & 101.95 & 101.95\end{array}$

$\begin{array}{llll}12 \mathrm{H} & 98.90 & 105.12 & 105.12\end{array}$

$\begin{array}{llll}12 \mathrm{H} & 100.43 & 106.65 & 106.65\end{array}$

$\begin{array}{llll}12 \mathrm{H} & 106.12 & 112.34 & 112.34\end{array}$

$\begin{array}{llll}12 \mathrm{H} & 107.64 & 113.86 & 114.05\end{array}$

$\begin{array}{llll}12 \mathrm{H} & 108.15 & 114.37 & 114.85\end{array}$

$\begin{array}{llll}12 \mathrm{H} & 108.50 & 114.72 & 115.20\end{array}$

$\begin{array}{llll}13 \mathrm{H} & 108.40 & 117.02 & 117.17\end{array}$

$\begin{array}{llll}13 \mathrm{H} & 108.65 & 117.27 & 117.42\end{array}$

$\begin{array}{llll}13 \mathrm{H} & 110.24 & 118.86 & 118.86\end{array}$

$\begin{array}{llll}13 \mathrm{H} & 115.58 & 124.20 & 124.20\end{array}$

$\begin{array}{llll}13 \mathrm{H} & 116.87 & 125.49 & 125.64\end{array}$

$\begin{array}{llll}13 \mathrm{H} & 117.96 & 126.58 & 126.88\end{array}$

$\begin{array}{llll}13 \mathrm{H} & 118.43 & 127.05 & 127.35\end{array}$

$\begin{array}{llll}14 \mathrm{H} & 117.90 & 130.40 & 130.71\end{array}$

$\begin{array}{llll}14 \mathrm{H} & 120.29 & 132.79 & 133.10\end{array}$

$\begin{array}{llll}14 \mathrm{H} & 122.13 & 134.63 & 134.63\end{array}$

$\begin{array}{llll}14 \mathrm{H} & 125.24 & 137.74 & 138.92\end{array}$

$\begin{array}{llll}14 \mathrm{H} & 125.72 & 138.22 & 139.37\end{array}$

$\begin{array}{llll}14 \mathrm{H} & 126.33 & 138.83 & 140.01\end{array}$

$\begin{array}{llll}15 \mathrm{H} & 125.90 & 139.40 & 139.40\end{array}$

$\begin{array}{llll}15 \mathrm{H} & 132.98 & 146.48 & 146.48\end{array}$

$\begin{array}{llll}16 \mathrm{X} & 135.50 & 149.00 & 149.00\end{array}$

$\begin{array}{llll}16 X & 135.25 & 148.75 & 148.75\end{array}$

$\begin{array}{llll}17 X & 144.50 & 158.00 & 158.00\end{array}$

$\begin{array}{llll}17 X & 148.26 & 161.76 & 161.76\end{array}$

$\begin{array}{llll}18 X & 150.40 & 163.90 & 163.90\end{array}$

$\begin{array}{llll}18 \mathrm{X} & 150.56 & 164.06 & 164.06\end{array}$

320-U1332B-

$\begin{array}{rrrr}1 \mathrm{H} & 0.01 & 0.16 & 0.16 \\ 1 \mathrm{H} & 2.10 & 2.25 & 2.25 \\ 2 \mathrm{H} & 2.10 & 2.75 & 2.10 \\ 2 \mathrm{H} & 3.00 & 3.65 & 3.00 \\ 2 \mathrm{H} & 3.85 & 4.50 & 4.06 \\ 2 \mathrm{H} & 5.21 & 5.86 & 5.82 \\ 2 \mathrm{H} & 6.14 & 6.79 & 6.79 \\ 2 \mathrm{H} & 8.25 & 8.90 & 8.90 \\ 2 \mathrm{H} & 10.32 & 10.97 & 10.47 \\ 2 \mathrm{H} & 11.68 & 12.33 & 11.43 \\ 2 \mathrm{H} & 12.14 & 12.79 & 11.89 \\ 3 \mathrm{H} & 11.60 & 13.75 & 13.31 \\ 3 \mathrm{H} & 13.96 & 16.11 & 15.67 \\ 3 \mathrm{H} & 14.53 & 16.68 & 16.42 \\ 3 \mathrm{H} & 15.73 & 17.88 & 17.81 \\ 3 \mathrm{H} & 16.80 & 18.95 & 18.42\end{array}$

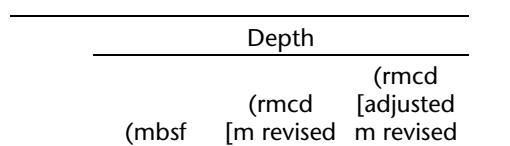
Core [m CSF-A]) CCSF-A]) CCSF-A])

\begin{tabular}{llll}
\hline $3 \mathrm{H}$ & 18.32 & 20.47 & 20.55 \\
$3 \mathrm{H}$ & 19.25 & 21.40 & 21.46 \\
$3 \mathrm{H}$ & 20.02 & 22.17 & 22.17 \\
$4 \mathrm{H}$ & 19.60 & 23.75 & 24.00 \\
$4 \mathrm{H}$ & 20.37 & 24.52 & 24.77 \\
$4 \mathrm{H}$ & 21.33 & 25.48 & 25.48
\end{tabular}

$\begin{array}{llll}4 \mathrm{H} & 23.29 & 27.44 & 27.44\end{array}$

$\begin{array}{llll}4 \mathrm{H} & 23.29 & 27.44 & 27.44 \\ 4 \mathrm{H} & 24.20 & 28.35 & 28.33\end{array}$

$\begin{array}{llll}4 \mathrm{H} & 24.81 & 28.96 & 29.04\end{array}$

$\begin{array}{llll}4 \mathrm{H} & 24.81 & 28.96 & 29.04 \\ 4 \mathrm{H} & 25.21 & 29.36 & 29.47\end{array}$

$\begin{array}{llll}4 \mathrm{H} & 28.71 & 32.86 & 33.40\end{array}$

$\begin{array}{llll}4 \mathrm{H} & 28.71 & 32.86 & 33.40 \\ 4 \mathrm{H} & 29.66 & 33.81 & 34.35\end{array}$

$\begin{array}{llll}5 \mathrm{H} & 29.10 & 33.35 & 33.35\end{array}$

$\begin{array}{llll}5 \mathrm{H} & 31.05 & 35.30 & 35.30\end{array}$

$\begin{array}{llll}5 \mathrm{H} & 36.60 & 40.85 & 40.85\end{array}$

$\begin{array}{llll}5 \mathrm{H} & 37.68 & 41.93 & 41.93\end{array}$

$\begin{array}{llll}5 \mathrm{H} & 37.68 & 41.93 & 41.93 \\ 6 \mathrm{H} & 38.60 & 45.65 & 45.23\end{array}$

$\begin{array}{llll}6 \mathrm{H} & 41.26 & 48.31 & 47.89\end{array}$

$\begin{array}{llll}6 \mathrm{H} & 41.84 & 48.89 & 48.64\end{array}$

$\begin{array}{llll}6 \mathrm{H} & 43.25 & 50.30 & 50.30\end{array}$

$\begin{array}{llll}6 \mathrm{H} & 44.52 & 51.57 & 51.72\end{array}$

$\begin{array}{llll}6 \mathrm{H} & 48.55 & 55.60 & 55.60\end{array}$

$\begin{array}{llll}7 \mathrm{H} & 48.10 & 58.45 & 58.45\end{array}$

$\begin{array}{llll}7 \mathrm{H} & 55.22 & 65.57 & 65.57\end{array}$

$\begin{array}{llll}7 \mathrm{H} & 57.78 & 68.13 & 68.13\end{array}$

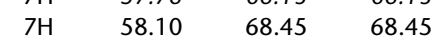

$\begin{array}{llll}8 \mathrm{H} & 57.60 & 67.65 & 68.18\end{array}$

$\begin{array}{llll}8 \mathrm{H} & 60.03 & 70.08 & 70.61\end{array}$

$\begin{array}{llll}8 \mathrm{H} & 63.10 & 73.15 & 73.21\end{array}$

$\begin{array}{llll}8 \mathrm{H} & 63.68 & 73.73 & 73.57\end{array}$

$\begin{array}{llll}8 \mathrm{H} & 65.02 & 75.07 & 74.57\end{array}$

$\begin{array}{llll}8 \mathrm{H} & 65.57 & 75.62 & 74.70\end{array}$

$\begin{array}{llll}8 \mathrm{H} & 67.30 & 77.35 & 76.43\end{array}$

$\begin{array}{llll}9 \mathrm{H} & 67.10 & 70.85 & 70.85\end{array}$

$\begin{array}{llll}9 \mathrm{H} & 67.60 & 71.35 & 71.35\end{array}$

$\begin{array}{llll}9 \mathrm{H} & 67.60 & 71.35 & 71.35 \\ 9 \mathrm{H} & 73.45 & 77.20 & 77.20\end{array}$

$\begin{array}{llll}9 \mathrm{H} & 74.16 & 77.91 & 77.87\end{array}$

$\begin{array}{llll}9 \mathrm{H} & 74.38 & 78.13 & 78.07\end{array}$

$\begin{array}{llll}9 \mathrm{H} & 74.38 & 78.13 & 78.07 \\ 9 \mathrm{H} & 74.64 & 78.39 & 78.32\end{array}$

$\begin{array}{llll}9 \mathrm{H} & 74.96 & 78.71 & 78.59\end{array}$

$\begin{array}{llll}9 \mathrm{H} & 75.11 & 78.86 & 78.77\end{array}$

$\begin{array}{llll}9 \mathrm{H} & 75.11 & 78.86 & 78.77 \\ 9 \mathrm{H} & 75.29 & 79.04 & 78.96\end{array}$

$\begin{array}{llll}9 \mathrm{H} & 76.12 & 79.87 & 79.78\end{array}$

$\begin{array}{llll}9 \mathrm{H} & 76.12 & 79.87 & 79.78 \\ 9 \mathrm{H} & 76.56 & 80.31 & 80.19\end{array}$

$\begin{array}{llll}9 \mathrm{H} & 77.12 & 80.87 & 80.74\end{array}$

$\begin{array}{llll}10 \mathrm{H} & 76.60 & 82.13 & 82.13\end{array}$

$\begin{array}{llll}10 \mathrm{H} & 77.48 & 83.01 & 83.01\end{array}$

$\begin{array}{llll}10 \mathrm{H} & 83.52 & 89.05 & 89.05\end{array}$

$\begin{array}{llll}10 \mathrm{H} & 86.69 & 92.22 & 92.22\end{array}$

$\begin{array}{llll}11 \mathrm{H} & 86.10 & 91.99 & 92.25\end{array}$

$\begin{array}{llll}11 \mathrm{H} & 86.10 & 91.99 & 92.25 \\ 11 \mathrm{H} & 88.15 & 94.04 & 94.30\end{array}$

$\begin{array}{llll}11 \mathrm{H} & 90.87 & 96.76 & 96.94\end{array}$

$\begin{array}{llll}11 \mathrm{H} & 92.63 & 98.52 & 98.81\end{array}$

$\begin{array}{lllr}11 \mathrm{H} & 93.87 & 99.76 & 100.05\end{array}$

$\begin{array}{llrr}12 \mathrm{H} & 91.10 & 100.37 & 99.64\end{array}$

$12 \mathrm{H} \quad 92.06 \quad 101.33 \quad 100.60$

$\begin{array}{llll}12 \mathrm{H} & 92.88 & 102.15 & 101.62\end{array}$

$\begin{array}{llll}12 \mathrm{H} & 93.69 & 102.96 & 102.58\end{array}$

$\begin{array}{llll}12 \mathrm{H} & 94.88 & 104.15 & 103.86\end{array}$

$\begin{array}{llll}12 \mathrm{H} & 95.66 & 104.93 & 104.88\end{array}$

$\begin{array}{llll}12 \mathrm{H} & 96.27 & 105.54 & 105.54\end{array}$

$\begin{array}{rrrr}12 \mathrm{H} & 100.19 & 109.46 & 109.82\end{array}$

$\begin{array}{llll}12 \mathrm{H} & 101.06 & 110.33 & 110.69\end{array}$

$\begin{array}{llll}13 \mathrm{H} & 100.60 & 111.08 & 111.08 \\ 13 \mathrm{H} & 103.88 & 114.36 & 114.36\end{array}$ 
Table T7 (continued).

\begin{tabular}{|c|c|c|c|}
\hline \multirow[b]{2}{*}{ Core } & \multicolumn{3}{|c|}{ Depth } \\
\hline & $\begin{array}{c}\text { (mbsf } \\
{[\mathrm{m} \mathrm{CSF-A])}}\end{array}$ & $\begin{array}{c}(\mathrm{rmcd} \\
{[\mathrm{m} \text { revised }} \\
\text { CCSF-A] })\end{array}$ & $\begin{array}{l}\text { (rmcd } \\
\text { [adjusted } \\
\text { m revised } \\
\text { CCSF-A]) }\end{array}$ \\
\hline $13 \mathrm{H}$ & 108.38 & 118.86 & 118.86 \\
\hline $13 \mathrm{H}$ & 109.65 & 120.13 & 120.28 \\
\hline $13 \mathrm{H}$ & 110.81 & 121.29 & 121.44 \\
\hline $14 X$ & 110.10 & 121.58 & 121.95 \\
\hline $14 X$ & 110.70 & 122.18 & 122.55 \\
\hline $14 X$ & 112.72 & 124.20 & 124.20 \\
\hline $14 X$ & 118.98 & 130.46 & 130.46 \\
\hline $14 X$ & 119.83 & 131.31 & 131.31 \\
\hline $15 X$ & 116.10 & 132.15 & 132.15 \\
\hline $15 X$ & 116.86 & 132.91 & 132.91 \\
\hline $15 X$ & 125.82 & 141.87 & 141.87 \\
\hline $16 X$ & 124.60 & 142.68 & 142.68 \\
\hline $16 X$ & 125.39 & 143.47 & 143.47 \\
\hline $17 X$ & 134.30 & 152.38 & 152.38 \\
\hline $17 X$ & 135.08 & 153.16 & 153.16 \\
\hline $18 x$ & 143.90 & 159.80 & 159.80 \\
\hline $18 X$ & 146.26 & 162.16 & 162.16 \\
\hline \multicolumn{4}{|c|}{ 320-U1332C- } \\
\hline $1 \mathrm{H}$ & 0.00 & 0.04 & 0.04 \\
\hline $1 \mathrm{H}$ & 2.56 & 2.60 & 2.60 \\
\hline $1 \mathrm{H}$ & 6.61 & 6.65 & 6.65 \\
\hline $1 \mathrm{H}$ & 7.49 & 7.53 & 7.53 \\
\hline $2 \mathrm{H}$ & 7.50 & 7.99 & 7.99 \\
\hline $2 \mathrm{H}$ & 7.50 & 7.99 & 7.85 \\
\hline $2 \mathrm{H}$ & 9.08 & 9.57 & 9.43 \\
\hline $2 \mathrm{H}$ & 9.99 & 10.48 & 10.48 \\
\hline $2 \mathrm{H}$ & 10.97 & 11.46 & 11.46 \\
\hline $2 \mathrm{H}$ & 16.46 & 16.95 & 16.95 \\
\hline $2 \mathrm{H}$ & 17.48 & 17.97 & 17.97 \\
\hline $3 \mathrm{H}$ & 17.00 & 17.25 & 17.25 \\
\hline $3 \mathrm{H}$ & 20.18 & 20.43 & 20.43 \\
\hline $3 \mathrm{H}$ & 26.05 & 26.30 & 26.30 \\
\hline $3 \mathrm{H}$ & 26.96 & 27.21 & 27.21 \\
\hline $4 \mathrm{H}$ & 26.50 & 28.85 & 29.00 \\
\hline $4 \mathrm{H}$ & 26.70 & 29.05 & 29.20 \\
\hline
\end{tabular}

\begin{tabular}{|c|c|c|c|}
\hline \multirow[b]{2}{*}{ Core } & \multicolumn{3}{|c|}{ Depth } \\
\hline & $\begin{array}{c}\text { (mbsf } \\
{[\mathrm{m} \mathrm{CSF-A]})}\end{array}$ & $\begin{array}{c}\text { (rmcd } \\
{[\mathrm{m} \text { revised }} \\
\text { CCSF-A] })\end{array}$ & $\begin{array}{c}\text { (rmcd } \\
\text { [adjusted } \\
\text { m revised } \\
\text { CCSF-A]) }\end{array}$ \\
\hline $4 \mathrm{H}$ & 28.17 & 30.52 & 30.64 \\
\hline $4 \mathrm{H}$ & 29.30 & 31.65 & 31.65 \\
\hline $4 \mathrm{H}$ & 32.95 & 35.30 & 35.30 \\
\hline $4 \mathrm{H}$ & 33.89 & 36.24 & 36.38 \\
\hline $4 \mathrm{H}$ & 34.78 & 37.13 & 37.30 \\
\hline $4 \mathrm{H}$ & 36.00 & 38.35 & 38.65 \\
\hline $4 \mathrm{H}$ & 36.55 & 38.90 & 39.20 \\
\hline $5 \mathrm{H}$ & 36.00 & 40.15 & 39.95 \\
\hline $5 \mathrm{H}$ & 36.10 & 40.25 & 40.05 \\
\hline $5 \mathrm{H}$ & 36.70 & 40.85 & 40.85 \\
\hline $5 \mathrm{H}$ & 44.53 & 48.68 & 48.68 \\
\hline $5 \mathrm{H}$ & 45.79 & 49.94 & 49.94 \\
\hline $6 \mathrm{H}$ & 45.50 & 48.85 & 48.91 \\
\hline $6 \mathrm{H}$ & 48.34 & 51.69 & 51.75 \\
\hline $6 \mathrm{H}$ & 49.37 & 52.72 & 52.72 \\
\hline $6 \mathrm{H}$ & 49.83 & 53.18 & 53.15 \\
\hline $6 \mathrm{H}$ & 52.15 & 55.50 & 55.45 \\
\hline $6 \mathrm{H}$ & 53.07 & 56.42 & 56.38 \\
\hline $7 \mathrm{H}$ & 49.50 & 56.30 & 56.30 \\
\hline $7 \mathrm{H}$ & 49.90 & 56.70 & 56.70 \\
\hline $7 \mathrm{H}$ & 58.77 & 65.57 & 65.57 \\
\hline $7 \mathrm{H}$ & 59.46 & 66.26 & 66.26 \\
\hline $8 \mathrm{H}$ & 59.00 & 66.51 & 66.51 \\
\hline $8 \mathrm{H}$ & 60.62 & 68.13 & 68.13 \\
\hline $8 \mathrm{H}$ & 63.84 & 71.35 & 71.35 \\
\hline $8 \mathrm{H}$ & 67.01 & 74.52 & 74.17 \\
\hline $8 \mathrm{H}$ & 68.22 & 75.73 & 75.20 \\
\hline $9 \mathrm{H}$ & 66.00 & 66.95 & 66.95 \\
\hline $9 \mathrm{H}$ & 68.59 & 69.54 & 69.54 \\
\hline $9 \mathrm{H}$ & 68.88 & 69.83 & 70.05 \\
\hline $9 \mathrm{H}$ & 69.72 & 70.67 & 70.90 \\
\hline $9 \mathrm{H}$ & 72.65 & 73.60 & 73.60 \\
\hline $9 \mathrm{H}$ & 74.74 & 75.69 & 75.69 \\
\hline $9 \mathrm{H}$ & 75.96 & 76.91 & 76.91 \\
\hline $10 \mathrm{H}$ & 75.50 & 76.05 & 76.05 \\
\hline
\end{tabular}

Italics = splice tie points. 
Table T8. Revised shipboard composite and corrected composite depths, Site U1333.

\begin{tabular}{|c|c|c|c|}
\hline Core & $\begin{array}{c}\text { Depth } \\
\text { (mbsf } \\
{[\mathrm{m} \mathrm{CSF-A])}}\end{array}$ & $\begin{array}{c}\text { Offset } \\
(\mathrm{m})\end{array}$ & $\begin{array}{l}\text { Top depth } \\
\text { (rmcd } \\
\text { [m revised } \\
\text { CCSF-A]) }\end{array}$ \\
\hline \multicolumn{4}{|c|}{ 320-U1333A- } \\
\hline $1 \mathrm{H}$ & 0.00 & 4.66 & 4.66 \\
\hline $2 \mathrm{H}$ & 9.50 & 3.68 & 13.18 \\
\hline $3 \mathrm{H}$ & 19.00 & 6.84 & 25.84 \\
\hline $4 \mathrm{H}$ & 28.50 & 6.57 & 35.07 \\
\hline $5 \mathrm{H}$ & 38.00 & 9.80 & 47.80 \\
\hline $6 \mathrm{H}$ & 47.50 & 11.75 & 59.25 \\
\hline $7 \mathrm{H}$ & 57.00 & 13.30 & 70.30 \\
\hline $8 \mathrm{H}$ & 66.50 & 15.38 & 81.88 \\
\hline $9 \mathrm{H}$ & 76.00 & 17.73 & 93.73 \\
\hline $10 \mathrm{H}$ & 85.50 & 19.36 & 104.86 \\
\hline $11 x$ & 95.00 & 20.72 & 115.72 \\
\hline $12 \mathrm{X}$ & 100.70 & 21.45 & 122.15 \\
\hline $13 x$ & 110.30 & 23.35 & 133.65 \\
\hline $14 X$ & 120.00 & 23.61 & 143.61 \\
\hline $15 X$ & 129.60 & 26.70 & 156.30 \\
\hline $16 X$ & 139.20 & 28.00 & 167.20 \\
\hline $17 X$ & 148.80 & 30.20 & 179.00 \\
\hline $18 \mathrm{X}$ & 158.40 & 26.90 & 185.30 \\
\hline $19 x$ & 168.00 & 25.75 & 193.75 \\
\hline $20 x$ & 177.60 & 25.75 & 203.35 \\
\hline $21 X$ & 181.60 & 25.75 & 207.35 \\
\hline $22 X$ & 182.60 & 25.75 & 208.35 \\
\hline \multicolumn{4}{|c|}{ 320-U1333B- } \\
\hline $1 \mathrm{H}$ & 0.00 & 0.00 & 0.00 \\
\hline $2 \mathrm{H}$ & 7.70 & 0.01 & 7.71 \\
\hline $3 \mathrm{H}$ & 17.20 & 2.07 & 19.27 \\
\hline $4 \mathrm{H}$ & 26.70 & 3.18 & 29.88 \\
\hline $5 \mathrm{H}$ & 36.20 & 3.98 & 40.18 \\
\hline $6 \mathrm{H}$ & 45.70 & 7.84 & 53.54 \\
\hline $7 \mathrm{H}$ & 55.20 & 10.03 & 65.23 \\
\hline $8 \mathrm{H}$ & 64.70 & 10.77 & 75.47 \\
\hline $9 \mathrm{H}$ & 74.20 & 12.03 & 86.23 \\
\hline $10 \mathrm{H}$ & 83.70 & 15.21 & 98.91 \\
\hline $11 \mathrm{H}$ & 93.20 & 17.33 & 110.53 \\
\hline $12 \mathrm{H}$ & 102.70 & 18.50 & 121.20 \\
\hline
\end{tabular}

\begin{tabular}{|c|c|c|c|}
\hline Core & $\begin{array}{c}\text { Depth } \\
\text { (mbsf } \\
\text { [m CSF-A]) }\end{array}$ & $\begin{array}{l}\text { Offset } \\
(\mathrm{m})\end{array}$ & $\begin{array}{l}\text { Top depth } \\
\text { (rmcd } \\
\text { [m revised } \\
\text { CCSF-A] })\end{array}$ \\
\hline $13 \mathrm{H}$ & 112.20 & 19.55 & 131.75 \\
\hline $14 \mathrm{H}$ & 121.70 & 21.45 & 143.15 \\
\hline $15 \mathrm{H}$ & 131.20 & 26.00 & 157.20 \\
\hline $16 \mathrm{H}$ & 140.70 & 26.00 & 166.70 \\
\hline $17 \mathrm{H}$ & 150.20 & 28.90 & 179.10 \\
\hline $18 \mathrm{H}$ & 159.70 & 28.90 & 188.60 \\
\hline $19 X$ & 162.70 & 25.65 & 188.35 \\
\hline $20 x$ & 172.30 & 26.00 & 198.30 \\
\hline \multicolumn{4}{|c|}{ 320-U1333C- } \\
\hline $1 \mathrm{H}$ & 0.00 & 0.00 & 0.00 \\
\hline $2 \mathrm{H}$ & 1.60 & 2.30 & 3.90 \\
\hline $3 \mathrm{H}$ & 11.10 & 2.66 & 13.76 \\
\hline $4 \mathrm{H}$ & 20.60 & 3.28 & 23.88 \\
\hline $5 \mathrm{H}$ & 30.10 & 6.55 & 36.65 \\
\hline $6 \mathrm{H}$ & 39.60 & 8.60 & 48.20 \\
\hline $7 \mathrm{H}$ & 49.10 & 11.13 & 60.23 \\
\hline $8 \mathrm{H}$ & 58.60 & 12.90 & 71.50 \\
\hline $9 \mathrm{H}$ & 68.10 & 15.34 & 83.44 \\
\hline $10 \mathrm{H}$ & 77.60 & 16.81 & 94.41 \\
\hline $11 \mathrm{H}$ & 87.10 & 16.60 & 103.70 \\
\hline $12 \mathrm{H}$ & 93.10 & 19.13 & 112.23 \\
\hline $13 \mathrm{H}$ & 98.10 & 19.00 & 117.10 \\
\hline $14 \mathrm{H}$ & 107.60 & 21.90 & 129.50 \\
\hline $15 \mathrm{H}$ & 117.10 & 22.20 & 139.30 \\
\hline $16 \mathrm{H}$ & 126.60 & 24.36 & 150.96 \\
\hline $17 \mathrm{H}$ & 131.10 & 25.70 & 156.80 \\
\hline $18 \mathrm{H}$ & 140.60 & 26.50 & 167.10 \\
\hline $19 \mathrm{H}$ & 150.10 & 26.50 & 176.60 \\
\hline $20 \mathrm{H}$ & 154.10 & 27.95 & 182.05 \\
\hline $21 \mathrm{H}$ & 163.20 & 28.10 & 191.30 \\
\hline $22 \mathrm{x}$ & 163.20 & 28.10 & 191.30 \\
\hline $23 x$ & 172.80 & 28.10 & 200.90 \\
\hline $24 X$ & 176.00 & 28.10 & 204.10 \\
\hline
\end{tabular}

Bold = changes to ship CCSF, italics = ship splice, but CCSF shifted according to new offsets higher up in the section. 
Table T9. Revised splice tie points, Site U1333.

\begin{tabular}{|c|c|c|c|c|c|c|}
\hline \multirow[b]{2}{*}{$\begin{array}{l}\text { Hole, core, section, } \\
\text { interval }(\mathrm{cm})\end{array}$} & \multicolumn{2}{|c|}{ Depth } & & \multirow[b]{2}{*}{$\begin{array}{l}\text { Hole, core, section, } \\
\text { interval }(\mathrm{cm})\end{array}$} & \multicolumn{2}{|c|}{ Depth } \\
\hline & $\begin{array}{c}\text { (mbsf } \\
{[\mathrm{m} \text { CSF-A] })}\end{array}$ & $\begin{array}{c}(\mathrm{rmcd} \\
{[\mathrm{m} \text { revised }} \\
\text { CCSF-A] })\end{array}$ & & & $\begin{array}{c}\text { (mbsf } \\
{[\mathrm{m} \text { CSF-A] })}\end{array}$ & $\begin{array}{c}(\mathrm{rmcd} \\
{[\mathrm{m} \text { revised }} \\
\text { CCSF-A] })\end{array}$ \\
\hline $320-$ & & & & $320-$ & & \\
\hline U1333B-1H-5, 22 & 6.22 & 6.22 & Tie to & U1333A-1H-2, 6 & 1.56 & 6.22 \\
\hline U1333A-1H-5, 76 & 6.76 & 11.42 & Tie to & U1333B-2H-3, 71 & 11.41 & 11.42 \\
\hline U1333B-2H-6, 31 & 15.51 & 15.51 & Tie to & U1333C-3H-2, 26 & 12.86 & 15.51 \\
\hline U1333C-3H-6, 63 & 19.23 & 21.88 & Tie to & U1333B-3H-2, 111 & 19.81 & 21.88 \\
\hline U1333B-3H-6, 93 & 25.63 & 27.70 & Tie to & U1333C-4H-3, 82 & 24.42 & 27.70 \\
\hline U1333C-4H-5, 83 & 27.43 & 30.70 & Tie to & U1333B-4H-1, 82 & 27.52 & 30.70 \\
\hline U1333B-4H-6, 35 & 34.55 & 37.73 & Tie to & U1333C-5H-1, 108 & 31.18 & 37.73 \\
\hline U1333C-5H-5, 98 & 37.08 & 43.63 & Tie to & U1333B-5H-3, 45 & 39.65 & 43.63 \\
\hline U1333B-5H-7, 30 & 45.00 & 48.98 & Tie to & U1333C-6H-1，77* & 40.37 & 48.98 \\
\hline U1333C-6H-4, 131 & 45.41 & 54.01 & Tie to & U1333B-6H-1, 46 & 46.16 & 54.01 \\
\hline U1333B-6H-6, 130 & 54.50 & 62.35 & Tie to & U1333A-6H-3, 9 & 50.59 & 62.35 \\
\hline U1333A-6H-5, 106 & 54.56 & 66.32 & Tie to & U1333B-7H-1, 109 & 56.29 & 66.32 \\
\hline U1333B-7H-6, 96 & 63.66 & 73.69 & Tie to & U1333C-8H-2, 69 & 60.79 & 73.69 \\
\hline U1333C-8H-7, 23 & 67.83 & 80.73 & Tie to & $3-8 H-4,76$ & 69.96 & 80.73 \\
\hline U1333B-8H-6, 81 & 73.01 & 83.78 & Tie to & U1333A-8H-2, 59 & 68.39 & 83.78 \\
\hline U1333A-8H-4, 46 & 71.26 & 86.65 & Tie to & U1333C-9H-3, 21 & 71.31 & 86.65 \\
\hline U1333C-9H-4, 21 & 72.81 & 88.15 & Tie to & U1333B-9H-2, 41 & 76.11 & 88.15 \\
\hline U1333B-9H-6, 95 & 82.65 & 94.68 & Tie to & U1333A-9H-1, 95 & 76.95 & 94.68 \\
\hline U13333-9H-6, 113 & 84.64 & 102.37 & Tie to & U1333B-10H-3, 46 & 87.16 & 102.37 \\
\hline U1333B-10H-6, 137 & 92.57 & 107.78 & Tie to & U1333A-10H-2, 142 & 88.42 & 107.78 \\
\hline U1333A-10H-6, 150 & 94.50 & 113.86 & Tie to & U1333B-11H-3, 33 & 96.53 & 113.86 \\
\hline U1333B-11H-5, 105 & 100.25 & 117.58 & Tie to & U1333A-11X-2, 36 & 96.86 & 117.58 \\
\hline U1333A-11X-4, 16 & 99.66 & 120.38 & Tie to & U1333C-13H-3, 28 & 101.38 & 120.38 \\
\hline U1333C-13H-7, 28 & 107.38 & 126.38 & Tie to & U1333B-12H-4, 68 & 107.88 & 126.38 \\
\hline U1333B-12H-6, 143 & 111.63 & 130.13 & Tie to & U1333C-14H-1, 63 & 108.23 & 130.13 \\
\hline U1333C-14H-3, 33 & 110.93 & 132.83 & Tie to & U1333B-13H-1, 108 & 113.28 & 132.83 \\
\hline U1333B-13H-5, 145 & 119.65 & 139.20 & Tie to & U1333A-13X-4, 105 & 115.85 & 139.20 \\
\hline U1333A-13X-6, 89 & 118.69 & 142.04 & Tie to & U1333C-15H-2, 124 & 119.84 & 142.04 \\
\hline U1333C- $15 H-5,125$ & 124.35 & 146.55 & Tie to & U1333B-14H-3, 40 & 125.10 & 146.55 \\
\hline U1333B-14H-6, 110 & 130.31 & 151.76 & Tie to & U1333C-16H-1, 80 & 127.40 & 151.76 \\
\hline U1333C-16H-4, 98 & 132.08 & 156.44 & Appenc & U1333C-17H-1, 0 & 131.10 & 156.80 \\
\hline U1333C-17H-7, 72 & 140.82 & 166.52 & Append to & U1333C-18H-1, 0 & 140.60 & 167.10 \\
\hline U1333C-18H-CC, 30 & 150.81 & 177.31 & Append to & U1333C-19H-1, 88 & 150.98 & 177.48 \\
\hline U1333C-19H-3, 70 & 153.80 & 180.30 & Tie to & U1333B-17H-1, 120 & 151.40 & 180.30 \\
\hline U1333B-17H-3, 140 & 154.60 & 183.50 & Tie to & U1333C-20H-1, 145 & 155.55 & 183.50 \\
\hline U1333C-20H-5, 95 & 161.50 & 189.00 & Tie to & U1333A-18X-3, 136* & 162.10 & 189.00 \\
\hline U1333A-18X-4, 74 & 162.98 & 189.88 & Tie to & U1333B-19X-2, 3 & 164.23 & 189.88 \\
\hline U1333B-19X-5, 17 & 168.87 & 194.52 & Tie to & U1333A-19X-1, 77 & 168.77 & 194.52 \\
\hline U1333A-19X-CC, 20 & 174.68 & 200.43 & & End of splice & & \\
\hline
\end{tabular}

* $=$ uncertain tie point. Bold = new splice tie point, italics = old splice tie points with new revised composite depth. 
Table T10. Mapping pairs for adjusting cores to the rmcd (m revised CCSF-A) splice, Site U1333. (Continued on next two pages.)

\begin{tabular}{|c|c|c|c|c|c|c|c|c|c|c|c|}
\hline \multirow[b]{2}{*}{ Core } & \multicolumn{3}{|c|}{ Depth } & \multirow[b]{2}{*}{ Core } & \multicolumn{3}{|c|}{ Depth } & \multicolumn{4}{|c|}{ Depth } \\
\hline & $\begin{array}{c}\text { (mbsf } \\
{[\mathrm{m} \text { CSF-A] })}\end{array}$ & $\begin{array}{c}(\mathrm{rmcd} \\
{[\mathrm{m} \text { revised }} \\
\text { CCSF-A] })\end{array}$ & $\begin{array}{c}\text { (rmcd } \\
\text { [adjusted } \\
\text { m revised } \\
\text { CCSF-A] })\end{array}$ & & $\begin{array}{c}(\mathrm{mbsf} \\
{[\mathrm{m} \text { CSF-A] })}\end{array}$ & $\begin{array}{l}(\text { rmcd } \\
\text { [m revised } \\
\text { CCSF-A]) }\end{array}$ & $\begin{array}{l}\text { (rmcd } \\
\text { [adjusted } \\
\text { m revised } \\
\text { CCSF-A]) }\end{array}$ & Core & $\begin{array}{c}\text { (mbsf } \\
{[\mathrm{m} \text { CSF-A] })}\end{array}$ & $\begin{array}{l}(\mathrm{rmcd} \\
{[\mathrm{m} \text { revised }} \\
\text { CCSF-A]) }\end{array}$ & $\begin{array}{l}\text { (rmcd } \\
\text { [adjusted } \\
\text { m revised } \\
\text { CCSF-A]) }\end{array}$ \\
\hline \multicolumn{4}{|c|}{ 320-U1333A- } & $8 \mathrm{H}$ & 68.41 & 83.79 & 83.79 & $14 X$ & 129.05 & 152.66 & 153.05 \\
\hline $1 \mathrm{H}$ & 0.01 & 4.67 & 3.90 & $8 \mathrm{H}$ & 71.27 & 86.65 & 86.65 & $14 X$ & 129.85 & 153.46 & 153.85 \\
\hline $1 \mathrm{H}$ & 0.50 & 5.16 & 4.97 & $8 \mathrm{H}$ & 71.82 & 87.20 & 87.20 & $15 X$ & 129.60 & 156.30 & 156.13 \\
\hline $1 \mathrm{H}$ & 1.01 & 5.67 & 5.67 & $8 \mathrm{H}$ & 73.33 & 88.71 & 88.65 & $15 X$ & 130.00 & 156.70 & 156.87 \\
\hline $1 \mathrm{H}$ & 1.56 & 6.22 & 6.22 & $8 \mathrm{H}$ & 73.74 & 89.12 & 89.10 & $15 X$ & 130.75 & 157.45 & 157.50 \\
\hline $1 \mathrm{H}$ & 6.76 & 11.42 & 11.42 & $8 \mathrm{H}$ & 75.11 & 90.49 & 90.38 & $15 X$ & 131.79 & 158.49 & 158.53 \\
\hline $1 \mathrm{H}$ & 7.59 & 12.25 & 12.25 & $8 \mathrm{H}$ & 75.54 & 90.92 & 90.76 & $15 x$ & 131.56 & 158.26 & 158.35 \\
\hline $1 \mathrm{H}$ & 8.18 & 12.84 & 12.88 & $8 \mathrm{H}$ & 76.19 & 91.57 & 91.41 & $15 X$ & 132.49 & 159.19 & 159.28 \\
\hline $1 \mathrm{H}$ & 10.05 & 14.71 & 14.75 & $9 \mathrm{H}$ & 76.00 & 93.73 & 93.73 & $15 x$ & 133.03 & 159.73 & 159.88 \\
\hline $2 \mathrm{H}$ & 9.50 & 13.18 & 13.13 & $9 \mathrm{H}$ & 76.96 & 94.69 & 94.69 & $15 x$ & 133.46 & 160.16 & 160.34 \\
\hline $2 \mathrm{H}$ & 9.83 & 13.51 & 13.46 & $9 \mathrm{H}$ & 84.64 & 102.37 & 102.37 & $15 X$ & 134.57 & 161.27 & 161.36 \\
\hline $2 \mathrm{H}$ & 10.08 & 13.76 & 13.66 & $9 \mathrm{H}$ & 85.92 & 103.65 & 103.65 & $15 X$ & 135.60 & 162.30 & 162.49 \\
\hline $2 \mathrm{H}$ & 10.70 & 14.38 & 14.36 & $10 \mathrm{H}$ & 85.50 & 104.86 & 104.91 & $15 X$ & 136.29 & 162.99 & 163.37 \\
\hline $2 \mathrm{H}$ & 11.11 & 14.79 & 14.74 & $10 \mathrm{H}$ & 86.02 & 105.38 & 105.43 & $15 x$ & 136.99 & 163.69 & 164.27 \\
\hline $2 \mathrm{H}$ & 11.38 & 15.06 & 15.06 & $10 \mathrm{H}$ & 86.22 & 105.58 & 105.57 & $15 X$ & 137.31 & 164.01 & 164.59 \\
\hline $2 \mathrm{H}$ & 12.04 & 15.72 & 15.69 & $10 \mathrm{H}$ & 86.42 & 105.78 & 105.77 & $15 x$ & 138.47 & 165.17 & 166.15 \\
\hline $2 \mathrm{H}$ & 12.29 & 15.97 & 16.00 & $10 \mathrm{H}$ & 86.82 & 106.18 & 106.15 & $15 X$ & 139.19 & 165.89 & 166.95 \\
\hline $2 \mathrm{H}$ & 12.86 & 16.54 & 16.50 & $10 \mathrm{H}$ & 87.38 & 106.74 & 106.79 & $16 \mathrm{X}$ & 139.20 & 167.20 & 166.57 \\
\hline $2 \mathrm{H}$ & 13.29 & 16.97 & 16.91 & $10 \mathrm{H}$ & 87.92 & 107.28 & 107.37 & $16 \mathrm{X}$ & 141.82 & 169.82 & 169.19 \\
\hline $2 \mathrm{H}$ & 14.28 & 17.96 & 17.86 & $10 \mathrm{H}$ & 88.42 & 107.78 & 107.78 & $16 \mathrm{X}$ & 142.23 & 170.23 & 170.00 \\
\hline $2 \mathrm{H}$ & 15.26 & 18.94 & 18.78 & $10 \mathrm{H}$ & 94.50 & 113.86 & 113.86 & $16 X$ & 144.37 & 172.37 & 171.96 \\
\hline $2 \mathrm{H}$ & 16.22 & 19.90 & 19.70 & $10 \mathrm{H}$ & 95.38 & 114.74 & 114.74 & $16 \mathrm{X}$ & 144.99 & 172.99 & 173.29 \\
\hline $2 \mathrm{H}$ & 19.62 & 23.30 & 23.10 & $11 X$ & 95.00 & 115.72 & 115.72 & $16 X$ & 145.78 & 173.78 & 173.92 \\
\hline $3 \mathrm{H}$ & 19.00 & 25.84 & 25.84 & $11 x$ & 96.86 & 117.58 & 117.58 & $16 \mathrm{X}$ & 146.90 & 174.90 & 174.90 \\
\hline $3 \mathrm{H}$ & 19.95 & 26.79 & 26.79 & $11 X$ & 99.66 & 120.38 & 120.38 & $16 X$ & 147.83 & 175.83 & 175.83 \\
\hline $3 \mathrm{H}$ & 20.10 & 26.94 & 26.88 & $11 x$ & 101.22 & 121.94 & 121.94 & $16 \mathrm{X}$ & 148.22 & 176.22 & 176.73 \\
\hline $3 \mathrm{H}$ & 21.56 & 28.40 & 28.25 & $12 X$ & 100.70 & 122.15 & 122.04 & $16 \mathrm{X}$ & 149.14 & 177.14 & 177.65 \\
\hline $3 \mathrm{H}$ & 23.04 & 29.88 & 29.64 & $12 X$ & 100.80 & 122.25 & 122.14 & $17 X$ & 148.80 & 179.00 & 178.95 \\
\hline $3 \mathrm{H}$ & 24.23 & 31.07 & 30.76 & $12 X$ & 101.70 & 123.15 & 123.15 & $17 X$ & 148.93 & 179.13 & 179.08 \\
\hline $3 \mathrm{H}$ & 25.01 & 31.85 & 31.52 & $12 X$ & 103.19 & 124.64 & 124.91 & $17 X$ & 149.38 & 179.58 & 179.58 \\
\hline $3 \mathrm{H}$ & 26.16 & 33.00 & 32.63 & $12 X$ & 103.66 & 125.11 & 125.34 & $17 X$ & 150.30 & 180.50 & 180.50 \\
\hline $3 \mathrm{H}$ & 27.28 & 34.12 & 33.69 & $12 x$ & 104.48 & 125.93 & 126.25 & $17 X$ & 151.22 & 181.42 & 181.29 \\
\hline $3 \mathrm{H}$ & 28.50 & 35.34 & 35.01 & $12 X$ & 105.39 & 126.84 & 127.26 & $17 X$ & 153.85 & 184.05 & 183.35 \\
\hline $3 \mathrm{H}$ & 28.92 & 35.76 & 35.43 & $12 X$ & 106.12 & 127.57 & 128.20 & $17 X$ & 154.22 & 184.42 & 183.72 \\
\hline $4 \mathrm{H}$ & 28.50 & 35.07 & 36.46 & $12 X$ & 106.70 & 128.15 & 129.03 & $17 X$ & 155.48 & 185.68 & 184.42 \\
\hline $4 \mathrm{H}$ & 31.74 & 38.31 & 39.70 & $12 X$ & 107.80 & 129.25 & 130.00 & $17 x$ & 155.91 & 186.11 & 184.80 \\
\hline $4 \mathrm{H}$ & 32.39 & 38.96 & 40.13 & $12 X$ & 107.99 & 129.44 & 130.19 & $17 X$ & 157.70 & 187.90 & 186.02 \\
\hline $4 \mathrm{H}$ & 33.13 & 39.70 & 40.63 & $13 x$ & 110.30 & 133.65 & 132.94 & $17 X$ & 158.72 & 188.92 & 187.04 \\
\hline $4 \mathrm{H}$ & 34.33 & 40.90 & 41.50 & $13 x$ & 111.82 & 135.17 & 134.46 & $18 \mathrm{X}$ & 158.40 & 185.30 & 185.30 \\
\hline $4 \mathrm{H}$ & 35.26 & 41.83 & 42.12 & $13 x$ & 112.30 & 135.65 & 135.26 & $18 \mathrm{X}$ & 163.91 & 190.81 & 190.81 \\
\hline $4 \mathrm{H}$ & 36.07 & 42.64 & 42.72 & $13 x$ & 112.80 & 136.15 & 135.83 & $19 \mathrm{X}$ & 168.00 & 193.75 & 193.75 \\
\hline $4 \mathrm{H}$ & 36.90 & 43.47 & 43.32 & $13 x$ & 114.25 & 137.60 & 137.32 & $19 x$ & 174.75 & 200.50 & 200.50 \\
\hline $4 \mathrm{H}$ & 37.76 & 44.33 & 44.33 & $13 x$ & 115.23 & 138.58 & 138.58 & $20 x$ & 177.60 & 203.35 & 203.35 \\
\hline $4 \mathrm{H}$ & 38.65 & 45.22 & 45.22 & $13 X$ & 115.85 & 139.20 & 139.20 & $20 x$ & 180.13 & 205.88 & 205.88 \\
\hline $5 \mathrm{H}$ & 38.00 & 47.80 & 47.83 & $13 x$ & 118.69 & 142.04 & 142.04 & $21 X$ & 181.60 & 207.35 & 207.35 \\
\hline $5 \mathrm{H}$ & 39.34 & 49.14 & 49.17 & $13 x$ & 119.10 & 142.45 & 142.61 & $21 x$ & 181.66 & 207.41 & 207.41 \\
\hline $5 \mathrm{H}$ & 40.58 & 50.38 & 50.28 & $13 X$ & 119.61 & 142.96 & 143.20 & $22 X$ & 182.60 & 208.35 & 208.35 \\
\hline $5 \mathrm{H}$ & 42.66 & 52.46 & 52.28 & $13 x$ & 119.98 & 143.33 & 143.57 & $22 X$ & 182.66 & 208.41 & 208.41 \\
\hline $5 \mathrm{H}$ & 45.05 & 54.85 & 54.59 & $14 X$ & 120.00 & 143.61 & 143.89 & $320-\mathrm{U} 1$ & |333B- & & \\
\hline $5 \mathrm{H}$ & 45.94 & 55.74 & 55.44 & $14 X$ & 120.05 & 143.66 & 143.94 & $1 \mathrm{H}$ & 0.01 & 0.01 & 0.01 \\
\hline $5 \mathrm{H}$ & 47.12 & 56.92 & 56.73 & $14 X$ & 121.00 & 144.61 & 144.72 & $1 \mathrm{H}$ & 1.58 & 6.22 & 6.22 \\
\hline $5 \mathrm{H}$ & 47.70 & 57.50 & 57.31 & $14 X$ & 121.32 & 144.93 & 145.02 & $1 \mathrm{H}$ & 7.73 & 7.73 & 7.73 \\
\hline $6 \mathrm{H}$ & 47.50 & 59.25 & 59.25 & $14 \mathrm{X}$ & 121.74 & 145.35 & 145.43 & $2 \mathrm{H}$ & 7.70 & 7.71 & 7.69 \\
\hline $6 \mathrm{H}$ & 50.60 & 62.35 & 62.35 & $14 \mathrm{X}$ & $\begin{array}{l}122.39 \\
12207\end{array}$ & 146.00 & $\begin{array}{l}145.96 \\
146.57\end{array}$ & $2 \mathrm{H}$ & 9.24 & 9.25 & 9.23 \\
\hline $6 \mathrm{H}$ & $\begin{array}{l}54.57 \\
5.32\end{array}$ & 66.32 & $\begin{array}{r}66.32 \\
69.07\end{array}$ & $\begin{array}{l}14 X \\
14 X\end{array}$ & $\begin{array}{l}122.97 \\
123.81\end{array}$ & $\begin{array}{l}146.58 \\
147.42\end{array}$ & $\begin{array}{l}146.57 \\
147.20\end{array}$ & $2 \mathrm{H}$ & 9.66 & 9.67 & 9.78 \\
\hline $6 \mathrm{H}$ & $\begin{array}{l}57.32 \\
57.00\end{array}$ & $\begin{array}{l}69.07 \\
70.30\end{array}$ & $\begin{array}{l}69.07 \\
69.93\end{array}$ & $\begin{array}{l}14 X \\
14 X\end{array}$ & $\begin{array}{l}123.81 \\
124.59\end{array}$ & $\begin{array}{l}147.42 \\
148.20\end{array}$ & $\begin{array}{l}147.20 \\
147.86\end{array}$ & $2 \mathrm{H}$ & 10.29 & 10.30 & 10.43 \\
\hline 7H & $\begin{array}{l}57.00 \\
58.65\end{array}$ & $\begin{array}{l}70.30 \\
71.95\end{array}$ & $\begin{array}{l}69.93 \\
71.58\end{array}$ & $\begin{array}{l}14 X \\
14 X\end{array}$ & $\begin{array}{l}124.59 \\
124.87\end{array}$ & 148.48 & $\begin{array}{l}14 / .86 \\
148.20\end{array}$ & $2 \mathrm{H}$ & 10.98 & 10.99 & 11.01 \\
\hline $\begin{array}{l}7 \mathrm{H} \\
7 \mathrm{H}\end{array}$ & $\begin{array}{l}58.65 \\
61.35\end{array}$ & 74.65 & 74.26 & $14 \mathrm{X}$ & 125.17 & 148.78 & 148.55 & $2 \mathrm{H}$ & 11.41 & 11.42 & 11.42 \\
\hline $\begin{array}{l}7 \mathrm{H} \\
7 \mathrm{H}\end{array}$ & $\begin{array}{l}61.35 \\
63.01\end{array}$ & 76.31 & 76.05 & $14 X$ & 126.01 & 149.62 & 149.40 & $2 \mathrm{H}$ & 15.50 & 15.51 & 15.51 \\
\hline 7H & $\begin{array}{l}63.01 \\
63.94\end{array}$ & 77.24 & 77.11 & $14 X$ & 126.28 & 149.89 & 149.67 & $2 \mathrm{H}$ & 17.65 & 17.66 & 17.66 \\
\hline $7 \mathrm{H}$ & $\begin{array}{l}63.94 \\
64.50\end{array}$ & 77.80 & 77.51 & $14 X$ & 126.78 & 150.39 & 150.22 & $3 \mathrm{H}$ & 17.20 & 19.27 & 19.15 \\
\hline $7 \mathrm{H}$ & $\begin{array}{l}64.50 \\
65.93\end{array}$ & 79.23 & 79.39 & $14 X$ & 127.53 & 151.14 & 151.14 & $3 \mathrm{H}$ & 18.30 & 20.37 & 20.25 \\
\hline $7 \mathrm{H}$ & $\begin{array}{l}65.93 \\
66.60\end{array}$ & 79.90 & 80.06 & $14 X$ & 128.21 & 151.82 & 151.91 & $3 \mathrm{H}$ & 18.88 & 20.95 & 20.85 \\
\hline 7H & $\begin{array}{l}66.60 \\
66.50\end{array}$ & 81.88 & 81.88 & $14 X$ & 128.96 & 152.57 & 152.91 & $3 \mathrm{H}$ & 19.81 & 21.88 & 21.88 \\
\hline $8 \mathrm{H}$ & 66.50 & & & & & & & $3 \mathrm{H}$ & 25.63 & 27.70 & 27.70 \\
\hline
\end{tabular}


Table T10 (continued). (Continued on next page.)

\begin{tabular}{|c|c|c|c|c|c|c|c|c|c|c|c|}
\hline \multirow[b]{2}{*}{ Core } & \multicolumn{3}{|c|}{ Depth } & \multicolumn{4}{|c|}{ Depth } & \multirow[b]{2}{*}{ Core } & \multicolumn{3}{|c|}{ Depth } \\
\hline & $\begin{array}{c}\text { (mbsf } \\
{[\mathrm{m} \mathrm{CSF-A]})}\end{array}$ & $\begin{array}{c}\text { (rmcd } \\
{[\mathrm{m} \text { revised }} \\
\text { CCSF-A] })\end{array}$ & $\begin{array}{l}\text { (rmcd } \\
\text { [adjusted } \\
\text { m revised } \\
\text { CCSF-A]) }\end{array}$ & Core & $\begin{array}{c}(\mathrm{mbsf} \\
{[\mathrm{m} \text { CSF-A]) }}\end{array}$ & $\begin{array}{c}(\mathrm{rmcd} \\
{[\mathrm{m} \text { revised }} \\
\text { CCSF-A] })\end{array}$ & $\begin{array}{l}\text { (rmcd } \\
\text { [adjusted } \\
\text { m revised } \\
\text { CCSF-A]) }\end{array}$ & & $\begin{array}{c}\text { (mbsf } \\
{[\mathrm{m} \mathrm{CSF-A])}}\end{array}$ & $\begin{array}{c}\text { (rmcd } \\
{[\mathrm{m} \text { revised }} \\
\text { CCSF-A]) }\end{array}$ & $\begin{array}{l}\text { (rmcd } \\
\text { [adjusted } \\
\text { m revised } \\
\text { CCSF-A]) }\end{array}$ \\
\hline $3 \mathrm{H}$ & 26.07 & 28.14 & 28.10 & $13 \mathrm{H}$ & 122.17 & 141.72 & 141.35 & $3 \mathrm{H}$ & 12.07 & 14.73 & 14.73 \\
\hline $3 \mathrm{H}$ & 27.23 & 29.30 & 29.25 & $14 \mathrm{H}$ & 121.70 & 143.15 & 143.05 & $3 \mathrm{H}$ & 12.85 & 15.51 & 15.51 \\
\hline $4 \mathrm{H}$ & 26.70 & 29.88 & 29.77 & $14 \mathrm{H}$ & 122.50 & 143.95 & 143.85 & $3 \mathrm{H}$ & 19.22 & 21.88 & 21.88 \\
\hline $4 \mathrm{H}$ & 26.98 & 30.16 & 30.05 & $14 \mathrm{H}$ & 123.05 & 144.50 & 144.50 & $3 \mathrm{H}$ & 19.97 & 22.63 & 22.71 \\
\hline $4 \mathrm{H}$ & 27.20 & 30.38 & 30.30 & $14 \mathrm{H}$ & 125.10 & 146.55 & 146.55 & $3 \mathrm{H}$ & 20.38 & 23.04 & 23.32 \\
\hline $4 \mathrm{H}$ & 27.38 & 30.56 & 30.58 & $14 \mathrm{H}$ & 130.31 & 151.76 & 151.76 & $3 \mathrm{H}$ & 21.10 & 23.76 & 24.04 \\
\hline $4 \mathrm{H}$ & 27.52 & 30.70 & 30.70 & $14 \mathrm{H}$ & 131.46 & 152.91 & 152.91 & $4 \mathrm{H}$ & 20.60 & 23.88 & 23.89 \\
\hline $4 \mathrm{H}$ & 34.55 & 37.73 & 37.73 & $15 \mathrm{H}$ & 131.20 & 157.20 & 156.53 & $4 \mathrm{H}$ & 22.51 & 25.79 & 25.89 \\
\hline $4 \mathrm{H}$ & 35.31 & 38.49 & 38.45 & $15 \mathrm{H}$ & 131.90 & 157.90 & 157.23 & $4 \mathrm{H}$ & 23.52 & 26.80 & 26.88 \\
\hline $4 \mathrm{H}$ & 35.74 & 38.92 & 38.82 & $15 \mathrm{H}$ & 132.40 & 158.40 & 157.91 & $4 \mathrm{H}$ & 24.21 & 27.49 & 27.51 \\
\hline $4 \mathrm{H}$ & 36.14 & 39.32 & 39.22 & $15 \mathrm{H}$ & 132.84 & 158.84 & 158.53 & $4 \mathrm{H}$ & 24.42 & 27.70 & 27.70 \\
\hline $4 \mathrm{H}$ & 36.66 & 39.84 & 39.74 & $15 \mathrm{H}$ & 134.00 & 160.00 & 159.88 & $4 \mathrm{H}$ & 27.42 & 30.70 & 30.70 \\
\hline $5 \mathrm{H}$ & 36.96 & 40.94 & 40.14 & $15 \mathrm{H}$ & 134.86 & 160.86 & 160.86 & $4 \mathrm{H}$ & 28.16 & 31.44 & 31.49 \\
\hline $5 \mathrm{H}$ & 37.28 & 41.26 & 40.63 & $15 \mathrm{H}$ & 136.13 & 162.13 & 162.49 & $4 \mathrm{H}$ & 28.84 & 32.12 & 32.23 \\
\hline $5 \mathrm{H}$ & 37.86 & 41.84 & 41.50 & $15 \mathrm{H}$ & 136.73 & 162.73 & 163.37 & $4 \mathrm{H}$ & 29.24 & 32.52 & 32.65 \\
\hline $5 \mathrm{H}$ & 38.19 & 42.17 & 41.98 & $15 \mathrm{H}$ & 137.59 & 163.59 & 164.44 & $4 \mathrm{H}$ & 30.72 & 34.00 & 34.13 \\
\hline $5 \mathrm{H}$ & 39.43 & 43.41 & 43.32 & $15 \mathrm{H}$ & 139.02 & 165.02 & 166.33 & $5 \mathrm{H}$ & 30.10 & 36.65 & 36.65 \\
\hline $5 \mathrm{H}$ & 39.65 & 43.63 & 43.63 & $15 \mathrm{H}$ & 139.84 & 165.84 & 167.15 & $5 \mathrm{H}$ & 31.18 & 37.73 & 37.73 \\
\hline $5 \mathrm{H}$ & 45.00 & 48.98 & 48.98 & $16 \mathrm{H}$ & 140.70 & 166.70 & 167.47 & $5 \mathrm{H}$ & 37.08 & 43.63 & 43.63 \\
\hline $5 \mathrm{H}$ & 45.90 & 49.88 & 49.88 & $16 \mathrm{H}$ & 141.73 & 167.73 & 168.50 & $5 \mathrm{H}$ & 37.65 & 44.20 & 44.26 \\
\hline $6 \mathrm{H}$ & 45.70 & 53.54 & 53.50 & $16 \mathrm{H}$ & 142.23 & 168.23 & 169.14 & $5 \mathrm{H}$ & 38.56 & 45.11 & 45.22 \\
\hline $6 \mathrm{H}$ & 45.90 & 53.74 & 53.60 & $16 \mathrm{H}$ & 143.79 & 169.79 & 170.83 & $5 \mathrm{H}$ & 39.03 & 45.58 & 45.77 \\
\hline $6 \mathrm{H}$ & 46.10 & 53.94 & 53.94 & $16 \mathrm{H}$ & 144.99 & 170.99 & 171.99 & $5 \mathrm{H}$ & 39.41 & 45.96 & 46.11 \\
\hline $6 \mathrm{H}$ & 54.51 & 62.35 & 62.35 & $16 \mathrm{H}$ & 145.69 & 171.69 & 173.07 & $5 \mathrm{H}$ & 39.89 & 46.44 & 46.59 \\
\hline $6 \mathrm{H}$ & 54.80 & 62.64 & 62.86 & $16 \mathrm{H}$ & 146.54 & 172.54 & 173.92 & $6 \mathrm{H}$ & 39.60 & 48.20 & 48.20 \\
\hline $6 \mathrm{H}$ & 55.60 & 63.44 & 63.66 & $16 \mathrm{H}$ & 147.28 & 173.28 & 174.57 & $6 \mathrm{H}$ & 40.38 & 48.98 & 48.98 \\
\hline $7 \mathrm{H}$ & 55.20 & 65.23 & 65.23 & $16 \mathrm{H}$ & 147.96 & 173.96 & 175.20 & $6 \mathrm{H}$ & 45.41 & 54.01 & 54.01 \\
\hline $7 \mathrm{H}$ & 56.29 & 66.32 & 66.32 & $16 \mathrm{H}$ & 148.73 & 174.73 & 175.86 & $6 \mathrm{H}$ & 45.97 & 54.57 & 54.60 \\
\hline $7 \mathrm{H}$ & 65.16 & 75.19 & 75.05 & $16 \mathrm{H}$ & 150.05 & 176.05 & 177.25 & $6 \mathrm{H}$ & 48.73 & 57.33 & 57.59 \\
\hline $8 \mathrm{H}$ & 64.70 & 75.47 & 75.32 & $17 \mathrm{H}$ & 150.20 & 179.10 & 179.10 & $6 \mathrm{H}$ & 49.41 & 58.01 & 58.46 \\
\hline $8 \mathrm{H}$ & 65.27 & 76.04 & 75.90 & $17 \mathrm{H}$ & 150.70 & 179.60 & 179.60 & $6 \mathrm{H}$ & 49.74 & 58.34 & 58.79 \\
\hline $8 \mathrm{H}$ & 66.38 & 77.15 & 77.11 & $17 \mathrm{H}$ & 151.13 & 180.03 & 179.90 & $7 \mathrm{H}$ & 49.10 & 60.23 & 60.23 \\
\hline $8 \mathrm{H}$ & 66.81 & 77.58 & 77.51 & $17 \mathrm{H}$ & 151.40 & 180.30 & 180.30 & $7 \mathrm{H}$ & 52.97 & 64.10 & 64.10 \\
\hline $8 \mathrm{H}$ & 69.45 & 80.22 & 80.22 & $17 \mathrm{H}$ & 154.60 & 183.50 & 183.50 & $7 \mathrm{H}$ & 53.57 & 64.70 & 64.93 \\
\hline $8 \mathrm{H}$ & 69.96 & 80.73 & 80.73 & $17 \mathrm{H}$ & 155.16 & 184.06 & 183.92 & $7 \mathrm{H}$ & 55.15 & 66.28 & 67.14 \\
\hline $8 \mathrm{H}$ & 73.01 & 83.78 & 83.78 & $17 \mathrm{H}$ & 155.70 & 184.60 & 184.42 & $7 \mathrm{H}$ & 55.60 & 66.73 & 67.87 \\
\hline $8 \mathrm{H}$ & 73.89 & 84.66 & 84.66 & $17 \mathrm{H}$ & 156.06 & 184.96 & 184.84 & $7 \mathrm{H}$ & 57.32 & 68.45 & 70.50 \\
\hline $8 \mathrm{H}$ & 74.23 & 85.00 & 85.15 & $17 \mathrm{H}$ & 156.33 & 185.23 & 185.12 & $8 \mathrm{H}$ & 58.60 & 71.50 & 71.50 \\
\hline $8 \mathrm{H}$ & 74.55 & 85.32 & 85.32 & $17 \mathrm{H}$ & 157.16 & 186.06 & 186.06 & $8 \mathrm{H}$ & 60.79 & 73.69 & 73.69 \\
\hline $9 \mathrm{H}$ & 74.20 & 86.13 & 86.02 & $17 \mathrm{H}$ & 158.00 & 186.90 & 186.80 & $8 \mathrm{H}$ & 67.83 & 80.73 & 80.73 \\
\hline $9 \mathrm{H}$ & 76.12 & 88.15 & 88.15 & $17 \mathrm{H}$ & 158.56 & 187.46 & 187.36 & $8 \mathrm{H}$ & 68.70 & 81.60 & 81.60 \\
\hline $9 \mathrm{H}$ & 82.65 & 94.68 & 94.68 & $18 \mathrm{H}$ & 159.70 & 188.60 & 188.60 & $9 \mathrm{H}$ & 68.10 & 83.34 & 83.02 \\
\hline $9 \mathrm{H}$ & 83.16 & 95.19 & 95.19 & $18 \mathrm{H}$ & 161.30 & 190.20 & 190.20 & $9 \mathrm{H}$ & 69.02 & 84.36 & 84.04 \\
\hline $10 \mathrm{H}$ & 83.70 & 98.91 & 98.53 & $18 \mathrm{H}$ & 162.35 & 191.25 & 191.05 & $9 \mathrm{H}$ & 69.80 & 85.14 & 84.90 \\
\hline $10 \mathrm{H}$ & 84.86 & 100.07 & 99.69 & $18 \mathrm{H}$ & 162.99 & 191.89 & 191.69 & $9 \mathrm{H}$ & 70.53 & 85.87 & 85.77 \\
\hline $10 \mathrm{H}$ & 87.16 & 102.37 & 102.37 & $19 x$ & 162.70 & 188.35 & 188.35 & $9 \mathrm{H}$ & 71.31 & 86.65 & 86.65 \\
\hline $10 \mathrm{H}$ & 92.57 & 107.78 & 107.78 & $19 x$ & 164.23 & 189.88 & 189.88 & $9 \mathrm{H}$ & 72.81 & 88.15 & 88.15 \\
\hline $10 \mathrm{H}$ & 93.46 & 108.67 & 108.67 & $19 X$ & 168.87 & 194.52 & 194.52 & $9 \mathrm{H}$ & 73.71 & 89.05 & 89.23 \\
\hline $11 \mathrm{H}$ & 93.20 & 110.53 & 110.53 & $19 X$ & 169.87 & 195.52 & 195.52 & $9 \mathrm{H}$ & 75.26 & 90.60 & 90.90 \\
\hline $11 \mathrm{H}$ & 96.53 & 113.86 & 113.86 & $20 x$ & 172.30 & 198.30 & 198.30 & $9 \mathrm{H}$ & 76.68 & 92.02 & 92.24 \\
\hline $11 \mathrm{H}$ & 100.25 & 117.58 & 117.58 & $20 x$ & 178.57 & 204.57 & 204.57 & $9 \mathrm{H}$ & 77.56 & 92.90 & 93.15 \\
\hline $11 \mathrm{H}$ & 101.82 & 119.15 & 119.15 & 320-U1 & $333 C-$ & & & $9 \mathrm{H}$ & 77.82 & 93.06 & 93.31 \\
\hline $11 \mathrm{H}$ & 103.31 & 120.64 & 120.20 & $1 \mathrm{H}$ & 0.01 & 0.01 & 0.01 & $10 \mathrm{H}$ & 77.60 & 94.41 & 94.04 \\
\hline $12 \mathrm{H}$ & 102.70 & 121.20 & 121.10 & $1 \mathrm{H}$ & 1.65 & 1.65 & 1.65 & $10 \mathrm{H}$ & 79.69 & 96.50 & 96.13 \\
\hline $12 \mathrm{H}$ & 103.63 & 122.13 & 122.13 & $2 \mathrm{H}$ & 1.60 & 3.90 & 3.40 & $10 \mathrm{H}$ & 80.72 & 97.53 & 97.10 \\
\hline $12 \mathrm{H}$ & 107.88 & 126.38 & 126.38 & $2 \mathrm{H}$ & 2.47 & 4.77 & 4.27 & $10 \mathrm{H}$ & 82.07 & 98.88 & 98.58 \\
\hline $12 \mathrm{H}$ & 111.63 & 130.13 & 130.13 & $2 \mathrm{H}$ & 5.64 & 7.94 & 7.94 & $10 \mathrm{H}$ & 82.89 & 99.70 & 99.46 \\
\hline $12 \mathrm{H}$ & 112.34 & 130.84 & 130.84 & $2 \mathrm{H}$ & 7.95 & 10.25 & 10.42 & $10 \mathrm{H}$ & 83.63 & 100.44 & 100.25 \\
\hline $13 \mathrm{H}$ & 112.20 & 131.75 & 131.75 & $2 \mathrm{H}$ & 8.53 & 10.83 & 11.05 & $10 \mathrm{H}$ & 85.56 & 102.37 & 102.37 \\
\hline $13 \mathrm{H}$ & 112.83 & 132.38 & 132.38 & $2 \mathrm{H}$ & 9.33 & 11.63 & 11.76 & $10 \mathrm{H}$ & 85.89 & 102.70 & 102.75 \\
\hline $13 \mathrm{H}$ & 119.65 & 139.20 & 139.20 & $2 \mathrm{H}$ & 10.40 & 12.70 & 12.88 & $10 \mathrm{H}$ & 86.17 & 102.98 & 103.00 \\
\hline
\end{tabular}


Table T10 (continued).

\begin{tabular}{|c|c|c|c|}
\hline \multirow[b]{2}{*}{ Core } & \multicolumn{3}{|c|}{ Depth } \\
\hline & $\begin{array}{c}\text { (mbsf } \\
{[\mathrm{m} \mathrm{CSF-A]})}\end{array}$ & $\begin{array}{c}(\mathrm{rmcd} \\
{[\mathrm{m} \text { revised }} \\
\text { CCSF-A] })\end{array}$ & $\begin{array}{c}\text { (rmcd } \\
\text { [adjusted } \\
\text { m revised } \\
\text { CCSF-A]) }\end{array}$ \\
\hline $11 \mathrm{H}$ & 87.10 & 103.70 & 103.51 \\
\hline $11 \mathrm{H}$ & 88.55 & 105.15 & 104.96 \\
\hline $11 \mathrm{H}$ & 89.51 & 106.11 & 105.80 \\
\hline $11 \mathrm{H}$ & 90.12 & 106.72 & 106.70 \\
\hline $11 \mathrm{H}$ & 90.82 & 107.42 & 107.42 \\
\hline $11 \mathrm{H}$ & 91.52 & 108.12 & 108.12 \\
\hline $11 \mathrm{H}$ & 93.64 & 110.24 & 109.88 \\
\hline $11 \mathrm{H}$ & 94.52 & 111.12 & 111.12 \\
\hline $11 \mathrm{H}$ & 96.25 & 112.85 & 112.85 \\
\hline $12 \mathrm{H}$ & 93.10 & 112.23 & 112.23 \\
\hline $12 \mathrm{H}$ & 97.16 & 116.29 & 116.28 \\
\hline $12 \mathrm{H}$ & 99.75 & 118.88 & 118.88 \\
\hline $13 \mathrm{H}$ & 98.10 & 117.10 & 117.95 \\
\hline $13 \mathrm{H}$ & 100.29 & 119.29 & 119.55 \\
\hline $13 \mathrm{H}$ & 101.38 & 120.38 & 120.38 \\
\hline $13 \mathrm{H}$ & 107.38 & 126.38 & 126.38 \\
\hline $13 \mathrm{H}$ & 108.06 & 127.06 & 126.80 \\
\hline $14 \mathrm{H}$ & 107.60 & 129.50 & 129.50 \\
\hline $14 \mathrm{H}$ & 108.23 & 130.13 & 130.13 \\
\hline
\end{tabular}

\begin{tabular}{|c|c|c|c|}
\hline \multirow[b]{2}{*}{ Core } & \multicolumn{3}{|c|}{ Depth } \\
\hline & $\begin{array}{c}\text { (mbsf } \\
{[\mathrm{m} \mathrm{CSF-A]})}\end{array}$ & $\begin{array}{c}(\mathrm{rmcd} \\
{[\mathrm{m} \text { revised }} \\
\text { CCSF-A]) }\end{array}$ & $\begin{array}{c}\text { (rmcd } \\
\text { [adjusted } \\
\text { m revised } \\
\text { CCSF-A]) }\end{array}$ \\
\hline $14 \mathrm{H}$ & 110.93 & 132.83 & 132.83 \\
\hline $14 \mathrm{H}$ & 115.00 & 136.90 & 136.90 \\
\hline $14 \mathrm{H}$ & 115.43 & 137.33 & 137.14 \\
\hline $14 \mathrm{H}$ & 116.96 & 138.86 & 138.58 \\
\hline $14 \mathrm{H}$ & 117.63 & 139.53 & 139.25 \\
\hline $15 \mathrm{H}$ & 117.10 & 139.30 & 139.30 \\
\hline $15 \mathrm{H}$ & 119.84 & 142.04 & 142.04 \\
\hline $15 \mathrm{H}$ & 124.35 & 146.55 & 146.55 \\
\hline $15 \mathrm{H}$ & 125.72 & 147.92 & 147.87 \\
\hline $15 \mathrm{H}$ & 126.06 & 148.26 & 148.17 \\
\hline $15 \mathrm{H}$ & 126.51 & 148.71 & 148.57 \\
\hline $15 \mathrm{H}$ & 126.84 & 149.04 & 149.12 \\
\hline $15 \mathrm{H}$ & 127.16 & 149.36 & 149.44 \\
\hline $16 \mathrm{H}$ & 126.60 & 150.96 & 150.96 \\
\hline $16 \mathrm{H}$ & 127.40 & 151.76 & 151.76 \\
\hline $16 \mathrm{H}$ & 132.13 & 156.49 & 156.49 \\
\hline $17 \mathrm{H}$ & 131.10 & 156.80 & 156.80 \\
\hline $17 \mathrm{H}$ & 141.11 & 166.81 & 166.81 \\
\hline $18 \mathrm{H}$ & 140.60 & 167.10 & 167.10 \\
\hline
\end{tabular}

Italics = splice tie points. 
Table T11. Revised shipboard composite and corrected composite depths, Site U1334.

\begin{tabular}{|c|c|c|c|}
\hline Core & $\begin{array}{c}\text { Depth } \\
\text { (mbsf } \\
{[\mathrm{m} \mathrm{CSF-A])}}\end{array}$ & $\begin{array}{l}\text { Offset } \\
(\mathrm{m})\end{array}$ & $\begin{array}{l}\text { Top depth } \\
\text { (rmcd } \\
\text { [m revised } \\
\text { CCSF-A]) }\end{array}$ \\
\hline \multicolumn{4}{|c|}{ 320-U1334A- } \\
\hline $1 \mathrm{H}$ & 0.00 & 0.00 & 0.00 \\
\hline $2 \mathrm{H}$ & 8.20 & 0.87 & 9.07 \\
\hline $3 \mathrm{H}$ & 17.70 & 1.97 & 19.67 \\
\hline $4 \mathrm{H}$ & 27.20 & 3.48 & 30.68 \\
\hline $5 \mathrm{H}$ & 36.70 & 4.77 & 41.47 \\
\hline $6 \mathrm{H}$ & 46.20 & 6.93 & 53.13 \\
\hline $7 \mathrm{H}$ & 55.70 & 7.42 & 63.12 \\
\hline $8 \mathrm{H}$ & 65.20 & 9.06 & 74.26 \\
\hline $9 \mathrm{H}$ & 74.70 & 11.22 & 85.92 \\
\hline $10 \mathrm{H}$ & 84.20 & 11.31 & 95.51 \\
\hline $11 \mathrm{H}$ & 93.70 & 12.92 & 106.62 \\
\hline $12 \mathrm{H}$ & 103.20 & 14.15 & 117.35 \\
\hline $13 \mathrm{H}$ & 112.70 & 15.44 & 128.14 \\
\hline $14 \mathrm{H}$ & 122.20 & 16.93 & 139.13 \\
\hline $15 \mathrm{H}$ & 131.70 & 18.86 & 150.56 \\
\hline $16 \mathrm{H}$ & 141.20 & 20.56 & 161.76 \\
\hline $17 \mathrm{H}$ & 150.70 & 23.22 & 173.92 \\
\hline $18 \mathrm{H}$ & 160.20 & 24.10 & 184.30 \\
\hline $19 \mathrm{H}$ & 169.70 & 26.42 & 196.12 \\
\hline $20 \mathrm{H}$ & 179.20 & 40.46 & 219.66 \\
\hline $21 \mathrm{H}$ & 188.70 & 41.16 & 229.86 \\
\hline $22 \mathrm{H}$ & 198.20 & 42.46 & 240.66 \\
\hline $23 x$ & 206.90 & 44.72 & 251.62 \\
\hline $24 X$ & 214.50 & 47.70 & 262.20 \\
\hline $25 x$ & 224.10 & 49.43 & 273.53 \\
\hline $26 x$ & 233.60 & 49.64 & 283.24 \\
\hline $27 X$ & 243.20 & 51.10 & 294.30 \\
\hline $28 \mathrm{X}$ & 252.80 & 53.30 & 306.10 \\
\hline $29 x$ & 262.40 & 54.07 & 316.47 \\
\hline $30 x$ & 272.10 & 55.27 & 327.37 \\
\hline $31 x$ & 281.80 & 55.78 & 337.58 \\
\hline $32 X$ & 285.00 & 55.78 & 340.78 \\
\hline \multicolumn{4}{|c|}{ 320-U1334B- } \\
\hline $1 \mathrm{H}$ & 3.70 & 4.00 & 7.70 \\
\hline $2 \mathrm{H}$ & 13.20 & 4.69 & 17.89 \\
\hline $3 \mathrm{H}$ & 22.70 & 4.64 & 27.34 \\
\hline $4 \mathrm{H}$ & 32.20 & 6.27 & 38.47 \\
\hline $5 \mathrm{H}$ & 41.70 & 5.99 & 47.69 \\
\hline $6 \mathrm{H}$ & 49.20 & 7.31 & 56.51 \\
\hline $7 \mathrm{H}$ & 58.70 & 9.29 & 67.99 \\
\hline $8 \mathrm{H}$ & 68.20 & 12.14 & 80.34 \\
\hline $9 \mathrm{H}$ & 77.70 & 14.35 & 92.05 \\
\hline $10 \mathrm{H}$ & 87.20 & 15.44 & 102.64 \\
\hline $11 \mathrm{H}$ & 96.70 & 17.09 & 113.79 \\
\hline $12 \mathrm{H}$ & 106.20 & 17.28 & 123.48 \\
\hline $13 \mathrm{H}$ & 115.70 & 18.99 & 134.69 \\
\hline $14 \mathrm{H}$ & 125.20 & 21.74 & 146.94 \\
\hline $15 \mathrm{H}$ & 134.70 & 22.49 & 157.19 \\
\hline $16 \mathrm{H}$ & 144.20 & 23.79 & 167.99 \\
\hline
\end{tabular}

\begin{tabular}{|c|c|c|c|}
\hline Core & $\begin{array}{c}\text { Depth } \\
\text { (mbsf } \\
{[\mathrm{m} \mathrm{CSF-A])}}\end{array}$ & $\begin{array}{l}\text { Offset } \\
(\mathrm{m})\end{array}$ & $\begin{array}{l}\text { Top depth } \\
\text { (rmcd } \\
\text { [m revised } \\
\text { CCSF-A] })\end{array}$ \\
\hline $17 \mathrm{H}$ & 153.70 & 25.14 & 178.84 \\
\hline $18 \mathrm{H}$ & 163.20 & 27.10 & 190.30 \\
\hline $19 \mathrm{H}$ & 172.70 & 28.25 & 200.95 \\
\hline $20 \mathrm{H}$ & 182.20 & 30.46 & 212.66 \\
\hline $21 \mathrm{H}$ & 191.70 & 32.51 & 224.21 \\
\hline $22 \mathrm{H}$ & 201.20 & 33.91 & 235.11 \\
\hline $23 x$ & 210.70 & 34.91 & 245.61 \\
\hline $24 X$ & 219.00 & 49.63 & 268.63 \\
\hline $25 x$ & 228.60 & 50.72 & 279.32 \\
\hline $26 x$ & 238.20 & 52.32 & 290.52 \\
\hline $27 x$ & 247.80 & 54.13 & 301.93 \\
\hline $28 \mathrm{X}$ & 257.40 & 55.21 & 312.61 \\
\hline $29 x$ & 267.00 & 57.43 & 324.43 \\
\hline $30 x$ & 276.60 & 58.04 & 334.64 \\
\hline $31 x$ & 283.90 & 58.04 & 341.94 \\
\hline \multicolumn{4}{|c|}{ 320-U1334C- } \\
\hline $1 \mathrm{H}$ & 0.00 & 3.65 & 3.65 \\
\hline $2 \mathrm{H}$ & 9.50 & 4.33 & 13.83 \\
\hline $3 \mathrm{H}$ & 19.00 & 6.65 & 25.65 \\
\hline $4 \mathrm{H}$ & 28.50 & 10.04 & 38.54 \\
\hline $5 \mathrm{H}$ & 38.00 & 10.11 & 48.11 \\
\hline $6 \mathrm{H}$ & 47.50 & 12.06 & 59.56 \\
\hline $7 \mathrm{H}$ & 57.00 & 12.06 & 69.06 \\
\hline $8 \mathrm{H}$ & 66.50 & 13.96 & 80.46 \\
\hline $9 \mathrm{H}$ & 76.00 & 16.30 & 92.30 \\
\hline $10 \mathrm{H}$ & 85.50 & 17.98 & 103.48 \\
\hline $11 \mathrm{H}$ & 95.00 & 19.69 & 114.69 \\
\hline $12 \mathrm{H}$ & 104.50 & 22.75 & 127.25 \\
\hline $13 \mathrm{H}$ & 114.00 & 25.13 & 139.13 \\
\hline $14 \mathrm{H}$ & 123.50 & 27.06 & 150.56 \\
\hline $15 \mathrm{H}$ & 133.00 & 28.23 & 161.23 \\
\hline $16 \mathrm{H}$ & 142.50 & 29.69 & 172.19 \\
\hline $17 \mathrm{H}$ & 152.00 & 30.35 & 182.35 \\
\hline $18 \mathrm{H}$ & 161.50 & 31.44 & 192.94 \\
\hline $19 \mathrm{H}$ & 171.00 & 32.52 & 203.52 \\
\hline $20 \mathrm{H}$ & 180.50 & 33.33 & 213.83 \\
\hline $21 \mathrm{H}$ & 190.00 & 34.06 & 224.06 \\
\hline $22 \mathrm{H}$ & 199.50 & 36.86 & 236.36 \\
\hline $23 x$ & 209.00 & 41.35 & 250.35 \\
\hline $24 X$ & 214.00 & 44.85 & 258.85 \\
\hline $25 x$ & 223.60 & 46.33 & 269.93 \\
\hline $26 X$ & 233.20 & 47.10 & 280.30 \\
\hline $27 x$ & 239.20 & 48.01 & 287.21 \\
\hline $28 \mathrm{X}$ & 248.80 & 49.53 & 298.33 \\
\hline $29 x$ & 258.40 & 58.28 & 316.68 \\
\hline $30 x$ & 268.00 & 58.81 & 326.81 \\
\hline $31 x$ & 277.70 & 61.02 & 338.72 \\
\hline
\end{tabular}

Bold = changes to ship CCSF, italics = ship splice, but CCSF shifted according to new offsets higher up in the section. 
Table T12. Revised splice tie points, Site U1334.

\begin{tabular}{|c|c|c|c|c|c|c|}
\hline \multirow[b]{2}{*}{$\begin{array}{l}\text { Hole, core, section, } \\
\text { interval }(\mathrm{cm})\end{array}$} & \multicolumn{2}{|c|}{ Depth } & & \multirow[b]{2}{*}{$\begin{array}{l}\text { Hole, core, section, } \\
\text { interval }(\mathrm{cm})\end{array}$} & \multicolumn{2}{|c|}{ Depth } \\
\hline & $\begin{array}{c}(\mathrm{mbsf} \\
{[\mathrm{m} \text { CSF-A] })}\end{array}$ & $\begin{array}{c}(\mathrm{rmcd} \\
{[\mathrm{m} \text { revised }} \\
\text { CCSF-A] })\end{array}$ & & & $\begin{array}{c}\text { (mbsf } \\
{[\mathrm{m} \text { CSF-A] })}\end{array}$ & $\begin{array}{c}(\mathrm{rmcd} \\
{[\mathrm{m} \text { revised }} \\
\text { CCSF-A] })\end{array}$ \\
\hline $320-$ & & & & $320-$ & & \\
\hline U1334A-1H-5, 80 & 6.80 & 6.80 & Tie to & U1334C-1H-3, 15 & 3.15 & 6.80 \\
\hline U1334C-1H-5, 69 & 6.69 & 10.35 & Tie to & U1334A-2H-1, 128 & 9.48 & 10.35 \\
\hline U1334A-2H-6, 132 & 17.02 & 17.89 & Tie to & U1334C-2H-4, 10 & 13.56 & 17.89 \\
\hline U1334C-2H-5, 108 & 16.04 & 20.37 & Tie to & U1334A-3H-1, 70 & 18.40 & 20.37 \\
\hline U1334A-3H-6, 145 & 26.65 & 28.62 & Tie to & U1334B-3H-1, 128 & 23.98 & 28.62 \\
\hline U1334B-3H-5, 114 & 29.84 & 34.48 & Tie to & U1334A-4H-3, 80 & 31.00 & 34.48 \\
\hline U1334A-4H-6, 139 & 36.09 & 39.57 & Tie to & U1334B-4H-1, 110 & 33.30 & 39.57 \\
\hline U1334B-4H-6, 145 & 41.15 & 47.42 & Tie to & U1334A-5H-4, 144 & 42.64 & 47.42 \\
\hline U1334A-5H-6, 30 & 44.50 & 49.27 & Tie to & U1334C-5H-1, 116 & 39.16 & 49.27 \\
\hline U1334C-5H-7, 5 & 47.05 & 57.16 & Tie to & U1334A-6H-3, 103 & 50.23 & 57.16 \\
\hline U1334A-6H-6, 23 & 53.93 & 60.86 & Tie to & U1334C-6H-1, 130 & 48.80 & 60.86 \\
\hline U1334C-6H-5, 149 & 54.99 & 67.05 & Tie to & U1334A-7H-3, 93 & 59.63 & 67.05 \\
\hline U1334A-7H-6, 68 & 63.88 & 71.30 & Tie to & U1334B-7H-3, 31 & 62.01 & 71.30 \\
\hline U1334B-7H-6, 102 & 67.22 & 76.51 & Tie to & U1334A-8H-2, 76 & 67.46 & 76.51 \\
\hline U1334A-8H-6, 128 & 73.98 & 83.04 & Tie to & U1334B-8H-2, 119 & 70.89 & 83.04 \\
\hline U1334B-8H-5, 75 & 74.95 & 87.10 & Tie to & U1334A-9H-1, 118 & 75.88 & 87.10 \\
\hline U1334A-9H-6, 20 & 82.40 & 93.61 & Tie to & U1334B-9H-2, 6 & 79.26 & 93.61 \\
\hline U1334B-9H-5, 74 & 84.44 & 98.80 & Tie to & U1334A-10H-3, 29 & 87.49 & 98.80 \\
\hline U1334A-10H-6, 142 & 93.12 & 104.43 & Tie to & U1334C-10H-1, 95 & 86.45 & 104.43 \\
\hline U1334C-10H-5, 37 & 91.87 & 109.84 & Tie to & U1334A-11H-3, 22 & 96.92 & 109.84 \\
\hline U1334A-11H-6, 132 & 102.52 & 115.44 & Tie to & U1334B-11H-2, 14 & 98.34 & 115.44 \\
\hline U1334B-11H-6, 100 & 105.20 & 122.30 & Tie to & U1334A-12H-4, 45 & 108.15 & 122.30 \\
\hline U1334A-12H-6, 43 & 111.13 & 125.27 & Tie to & U1334B-12H-2, 29 & 107.99 & 125.27 \\
\hline U1334B-12H-6, 39 & 114.09 & 131.37 & Tie to & U1334C-12H-3, 113 & 108.63 & 131.37 \\
\hline U1334C-12H-7, 36 & 113.86 & 136.61 & Tie to & U1334B-13H-2, 41 & 117.61 & 136.61 \\
\hline U1334B-13H-5, 5 & 121.75 & 140.75 & Tie to & U1334C-13H-2, 12 & 115.62 & 140.75 \\
\hline U1334C-13H-6, 86 & 122.36 & 147.49 & Tie to & U1334B-14H-1, 55 & 125.75 & 147.49 \\
\hline U1334B-14H-4, 101 & 130.71 & 152.45 & Tie to & U1334C-14H-2, 39 & 125.39 & 152.45 \\
\hline U1334C-14H-6, 44 & 131.44 & 158.49 & Tie to & U1334B-15H-1, 130 & 136.00 & 158.49 \\
\hline U1334B-15H-6, 129 & 143.49 & 165.99 & Tie to & U1334C-15H-4, 26 & 137.76 & 165.99 \\
\hline U1334C-15H-6, 74 & 141.24 & 169.47 & Tie to & U1334B-16H-1, 148 & 145.68 & 169.47 \\
\hline U1334B-16H-5, 75 & 150.95 & 174.74 & Tie to & U1334C-16H-2, 105 & 145.05 & 174.74 \\
\hline U1334C-16H-6, 53 & 150.53 & 180.22 & Tie to & U1334B-17H-1, 138 & 155.08 & 180.22 \\
\hline U1334B-17H-6, 67 & 161.87 & 187.01 & Tie to & U1334A-18H-2, 122 & 162.92 & 187.01 \\
\hline U1334A-18H-5, 53 & 166.73 & 190.83 & Tie to & U1334B-18H-1, 53 & 163.73 & 190.83 \\
\hline U1334B-18H-6, 126 & 171.96 & 199.06 & Tie to & U1334A-19H-3, 2 & 172.64 & 199.06 \\
\hline U1334A-19H-6, 19 & 177.31 & 203.72 & Tie to & U1334B-19H-2, 127 & 175.47 & 203.72 \\
\hline U1334B-19H-4, 12 & 177.32 & 205.57 & Tie to & U1334C-19H-2, 55 & 173.05 & 205.57 \\
\hline U1334C-19H-7, 66 & 180.66 & 213.18 & Tie to & U1334B-20H-1, 52 & 182.72 & 213.18 \\
\hline U1334B-20H-5, 110 & 189.30 & 219.76 & Tie to & U1334C-20H-4, 143 & 186.43 & 219.76 \\
\hline U1334C-20H-6, 142 & 189.42 & 222.75 & Tie to & U1334A-20H-3, 9 & 182.29 & 222.75 \\
\hline U1334A-20H-5, 99 & 186.19 & 226.65 & Tie to & U1334B-21H-2, 94 & 194.14 & 226.65 \\
\hline U1334B-21H-6, 11 & 199.31 & 231.82 & Tie to & U1334A-21H-2, 46 & 190.66 & 231.82 \\
\hline U1334A-21H-5, 65 & 195.35 & 236.51 & Tie to & U1334B-22H-1, 140 & 202.60 & 236.51 \\
\hline U1334B-22H-7, 13 & 210.33 & 244.24 & Tie to & U1334C-22H-6, 38 & 207.38 & 244.24 \\
\hline U1334C-22H-7, 44 & 208.94 & 245.80 & Tie to & U1334A-22H-4, 64 & 203.34 & 245.80 \\
\hline U1334A-22H-5, 70 & 204.90 & 247.36 & Tie to & U1334B-23X-2, 25 & 212.45 & 247.36 \\
\hline U1334B-23X-6, 139 & 219.59 & 254.50 & Tie to & U1334A-23X-2, 138 & 209.78 & 254.50 \\
\hline U1334A-23X-6, 27 & 214.67 & 259.39 & Tie to & U1334C-24X-1, 54 & 214.54 & 259.39 \\
\hline U1334C-24X-6, 143 & 222.93 & 267.78 & Tie to & U1334A-24X-4, 110 & 220.10 & 267.78 \\
\hline U1334A-24X-6, 134 & 223.34 & 271.02 & Tie to & U1334C-25X-1, 109 & 224.69 & 271.02 \\
\hline U1334C-25X-5, 21 & 229.81 & 276.14 & Tie to & U1334A-25X-2, 111 & 226.71 & 276.14 \\
\hline U1334A-25X-6, 97 & 232.57 & 282.00 & Tie to & U1334C-26X-2, 20 & 234.90 & 282.00 \\
\hline U1334C-26X-5, 23 & 239.43 & 286.53 & Tie to & U1334A-26X-3, 29 & 236.89 & 286.53 \\
\hline U1334A-26X-6, 93 & 242.03 & 291.67 & Tie to & U1334B-26X-1, 115 & 239.35 & 291.67 \\
\hline U1334B-26X-5, 86 & 245.06 & 297.38 & Tie to & U1334A-27X-3, 8 & 246.28 & 297.38 \\
\hline U1334A-27X-6, 27 & 249.97 & 301.07 & Tie to & U1334C-28X-2, 124 & 251.54 & 301.07 \\
\hline U1334C-28X-3, 145 & 253.25 & 302.78 & Tie to & U1334B-27X-1, 85 & 248.65 & 302.78 \\
\hline U1334B-27X-4, 14 & 252.44 & 306.57 & Tie to & U1334A-28X-1, 47 & 253.27 & 306.57 \\
\hline U1334A-28X-6, 110 & 261.40 & 314.70 & Tie to & U1334B-28X-2, 59 & 259.49 & 314.70 \\
\hline U1334B-28X-4, 92 & 262.82 & 318.03 & Tie to & U1334C-29X-1, 135 & 259.75 & 318.03 \\
\hline U1334C-29X-6, 121 & 267.11 & 325.39 & Tie to & U1334B-29X-1, 96 & 267.96 & 325.39 \\
\hline U1334B-29X-6, 90 & 275.40 & 332.83 & Tie to & U1334C-30X-5, 2 & 274.02 & 332.83 \\
\hline U1334C-30X-6, 144 & 276.94 & 335.75 & Tie to & U1334B-30X-1, 111 & 277.71 & 335.75 \\
\hline U1334B-30X-5, 78 & 283.38 & 341.42 & & End of splice & & \\
\hline
\end{tabular}

Bold $=$ new splice tie point, italics $=$ old splice tie points with new revised composite depth. 
Table T13. Mapping pairs for adjusting cores to the rmcd (m revised CCSF-A) splice, Site U1334. (Continued on next three pages.)

\begin{tabular}{|c|c|c|c|}
\hline & \multicolumn{3}{|c|}{ Depth } \\
\hline & & $\begin{array}{c}\text { (rmcd } \\
{[\mathrm{m} \text { revised }}\end{array}$ & $\begin{array}{l}\quad \text { rmcd } \\
\text { [adjusted } \\
\text { m revised }\end{array}$ \\
\hline
\end{tabular}

\begin{tabular}{cccc} 
& & & \\
\hline $320-U 1334 A-$ & & & \\
$1 \mathrm{H}$ & 0.01 & 0.01 & 0.01 \\
$1 \mathrm{H}$ & 6.60 & 6.60 & 6.60
\end{tabular}

$\begin{array}{rrrr}1 \mathrm{H} & 0.60 & 6.60 & 6.60 \\ 1 \mathrm{H} & 8.24 & 8.24 & 8.30 \\ 2 \mathrm{H} & 8.20 & 9.07 & 9.07 \\ 2 \mathrm{H} & 9.25 & 10.12 & 10.12 \\ 2 \mathrm{H} & 16.74 & 17.61 & 17.61\end{array}$

$\begin{array}{llll}2 \mathrm{H} & 16.74 & 17.61 & 17.61 \\ 2 \mathrm{H} & 18.16 & 19.03 & 19.03 \\ 3 \mathrm{H} & 17.70 & 19.67 & 19.67 \\ 3 \mathrm{H} & 18.20 & 20.17 & 20.17\end{array}$

$\begin{array}{llll}3 \mathrm{H} & 17.70 & 19.67 & 19.67 \\ 3 \mathrm{H} & 18.20 & 20.17 & 20.17 \\ 3 \mathrm{H} & 26.45 & 28.42 & 28.42 \\ 3 \mathrm{H} & 27.70 & 29.67 & 29.67\end{array}$

$\begin{array}{llll}3 \mathrm{H} & 27.70 & 29.67 & 29.67 \\ 4 \mathrm{H} & 27.20 & 30.68 & 30.60 \\ 4 \mathrm{H} & 27.32 & 30.80 & 31.00 \\ 4 \mathrm{H} & 27.68 & 31.16 & 31.34 \\ 4 \mathrm{H} & 27.84 & 31.32 & 31.52\end{array}$

$\begin{array}{llll}4 \mathrm{H} & 27.84 & 31.32 & 31.52 \\ 4 \mathrm{H} & 28.68 & 32.16 & 32.42 \\ 4 \mathrm{H} & 30.07 & 33.55 & 33.64\end{array}$

$\begin{array}{llll}4 \mathrm{H} & 30.07 & 33.55 & 33.64 \\ 4 \mathrm{H} & 30.49 & 33.97 & 34.00\end{array}$

$\begin{array}{llll}4 \mathrm{H} & 30.49 & 33.97 & 34.00 \\ 4 \mathrm{H} & 30.80 & 34.28 & 34.28\end{array}$

$\begin{array}{llll}4 \mathrm{H} & 30.80 & 34.28 & 34.28 \\ 4 \mathrm{H} & 35.89 & 39.37 & 39.37\end{array}$

$\begin{array}{llll}4 \mathrm{H} & 37.23 & 40.71 & 40.65 \\ 5 \mathrm{H} & 36.70 & 41.47 & 41.60\end{array}$

$\begin{array}{llll}5 \mathrm{H} & 36.70 & 41.47 & 41.60 \\ 5 \mathrm{H} & 37.25 & 42.02 & 42.15\end{array}$

$\begin{array}{llll}5 \mathrm{H} & 37.25 & 42.02 & 42.15 \\ 5 \mathrm{H} & 37.90 & 42.67 & 42.85 \\ 5 \mathrm{H} & 38.04 & 42.81 & 43.10\end{array}$

$\begin{array}{llll}5 \mathrm{H} & 38.91 & 43.68 & 43.87\end{array}$

$\begin{array}{llll}5 \mathrm{H} & 40.66 & 45.43 & 45.53 \\ 5 \mathrm{H} & 41.24 & 46.01 & 46.01\end{array}$

$\begin{array}{llll}5 \mathrm{H} & 41.24 & 46.01 & 46.01 \\ 5 \mathrm{H} & 42.47 & 47.24 & 47.24\end{array}$

$\begin{array}{llll}5 \mathrm{H} & 44.31 & 49.08 & 49.08\end{array}$

$\begin{array}{llll}5 \mathrm{H} & 44.88 & 49.65 & 49.63 \\ 5 \mathrm{H} & 45.70 & 50.47 & 50.52\end{array}$

$\begin{array}{llll}5 \mathrm{H} & 45.70 & 50.47 & 50.52 \\ 5 \mathrm{H} & 46.39 & 51.16 & 51.06\end{array}$

$\begin{array}{llll}5 \mathrm{H} & 46.39 & 51.16 & 51.06 \\ 5 \mathrm{H} & 46.83 & 51.60 & 51.50\end{array}$

$\begin{array}{llll}6 \mathrm{H} & 46.20 & 53.13 & 53.46\end{array}$

$\begin{array}{llll}6 \mathrm{H} & 47.10 & 54.03 & 54.36 \\ 6 \mathrm{H} & 49.22 & 56.15 & 56.15\end{array}$

$\begin{array}{llll}6 \mathrm{H} & 49.22 & 56.15 & 56.15 \\ 6 \mathrm{H} & 50.04 & 56.97 & 56.97\end{array}$

$\begin{array}{llll}6 \mathrm{H} & 53.76 & 60.69 & 60.69 \\ 6 \mathrm{H} & 54.68 & 61.61 & 61.55\end{array}$

$\begin{array}{llll}6 \mathrm{H} & 54.68 & 61.61 & 61.55 \\ 6 \mathrm{H} & 55.49 & 62.42 & 62.42\end{array}$

$\begin{array}{llll}6 \mathrm{H} & 55.49 & 62.42 & 62.42 \\ 6 \mathrm{H} & 55.86 & 62.79 & 62.82\end{array}$

$\begin{array}{llll}6 \mathrm{H} & 55.86 & 62.79 & 62.82 \\ 6 \mathrm{H} & 56.25 & 63.18 & 63.21\end{array}$

$\begin{array}{llll}7 \mathrm{H} & 55.70 & 63.12 & 63.70 \\ 7 \mathrm{H} & 57.18 & 64.60 & 64.83\end{array}$

$\begin{array}{llll}7 \mathrm{H} & 57.18 & 64.60 & 64.83 \\ 7 \mathrm{H} & 57.73 & 65.15 & 65.30\end{array}$

$\begin{array}{llll}7 \mathrm{H} & 57.73 & 65.15 & 65.30 \\ 7 \mathrm{H} & 58.55 & 65.97 & 66.11\end{array}$

$\begin{array}{llll}7 \mathrm{H} & 58.55 & 65.97 & 66.11 \\ 7 \mathrm{H} & 59.22 & 66.64 & 66.64\end{array}$

$\begin{array}{llll}7 \mathrm{H} & 59.43 & 66.85 & 66.85 \\ 7 \mathrm{H} & 63.84 & 71.26 & 71.26\end{array}$

$\begin{array}{llll}7 \mathrm{H} & 63.84 & 71.26 & 71.26 \\ 7 \mathrm{H} & 65.71 & 73.13 & 73.13\end{array}$

$\begin{array}{llll}7 \mathrm{H} & 65.71 & 73.13 & 73.13 \\ 8 \mathrm{H} & 65.20 & 74.26 & 74.60\end{array}$

$\begin{array}{llll}8 \mathrm{H} & 65.20 & 74.26 & 74.60 \\ 8 \mathrm{H} & 66.22 & 75.28 & 75.32 \\ 8 & 67.26 & 76.32 & 76.32\end{array}$

$\begin{array}{llll}8 \mathrm{H} & 66.22 & 75.28 & 75.32 \\ 8 \mathrm{H} & 67.26 & 76.32 & 76.32\end{array}$

$\begin{array}{llll}8 \mathrm{H} & 73.78 & 82.84 & 82.84 \\ 8 \mathrm{H} & 74.38 & 83.44 & 83.40\end{array}$

$\begin{array}{llll}8 \mathrm{H} & 74.38 & 83.44 & 83.40 \\ 8 \mathrm{H} & 75.11 & 84.17 & 84.17\end{array}$

$\begin{array}{llll}8 \mathrm{H} & 75.11 & 84.17 & 84.17 \\ 9 \mathrm{H} & 74.70 & 85.92 & 85.92\end{array}$

$\begin{array}{llll}9 \mathrm{H} & 74.70 & 85.92 & 85.92 \\ 9 \mathrm{H} & 75.70 & 86.92 & 86.92\end{array}$

$\begin{array}{llll}9 \mathrm{H} & 82.16 & 93.38 & 93.38\end{array}$

$\begin{array}{llll}9 \mathrm{H} & 84.79 & 96.01 & 95.65 \\ 10 \mathrm{H} & 84.20 & 95.51 & 95.82\end{array}$

$\begin{array}{llll}10 \mathrm{H} & 84.20 & 95.51 & 95.82 \\ 10 \mathrm{H} & 85.38 & 96.69 & 97.00\end{array}$

$\begin{array}{llll}10 \mathrm{H} & 87.38 & 96.69 & 97.00 \\ & & 98.51 & 98.51\end{array}$

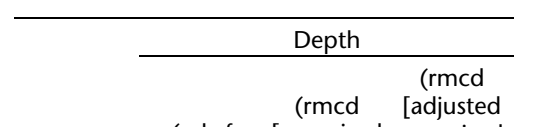

(mbsf [m revised m revised

\begin{tabular}{rrrr} 
& & & \\
\hline $10 \mathrm{H}$ & 87.29 & 98.60 & 98.60
\end{tabular}

\begin{tabular}{rrrr}
$10 \mathrm{H}$ & 92.97 & 104.28 & 104.28 \\
$10 \mathrm{H}$ & 94.00 & 105.31 & 105.00 \\
$11 \mathrm{H}$ & 93.70 & 106.62 & 106.53 \\
\hline $11 \mathrm{H}$ & 95.16 & 108.08 & 107.99
\end{tabular}

$\begin{array}{llll}11 \mathrm{H} & 95.16 & 108.08 & 107.99\end{array}$

$\begin{array}{rrrr}11 \mathrm{H} & 96.79 & 109.71 & 109.71 \\ 11 \mathrm{H} & 102.50 & 115.42 & 115.42\end{array}$

\begin{tabular}{llll}
$11 \mathrm{H}$ & 103.08 & 116.00 & 116.00 \\
\hline
\end{tabular}

$\begin{array}{llll}11 \mathrm{H} & 103.80 & 116.72 & 116.55\end{array}$

$\begin{array}{llll}12 \mathrm{H} & 103.20 & 117.35 & 117.43\end{array}$

$\begin{array}{llll}12 \mathrm{H} & 103.55 & 117.70 & 117.76 \\ 12 \mathrm{H} & 104.65 & 118.80 & 118.90\end{array}$

$\begin{array}{llll}12 \mathrm{H} & 105.17 & 119.32 & 119.33 \\ 12 \mathrm{H} & 105.52 & 119.67 & 119.82\end{array}$

$\begin{array}{llll}12 \mathrm{H} & 106.29 & 120.44 & 120.44\end{array}$

$\begin{array}{llll}12 \mathrm{H} & 106.13 & 122.44 & 120.44 \\ 12 \mathrm{H} & 108.28 & 122.28\end{array}$

$\begin{array}{llll}12 \mathrm{H} & 110.99 & 125.14 & 125.14\end{array}$

$\begin{array}{llll}12 \mathrm{H} & 112.90 & 127.05 & 127.05 \\ 13 \mathrm{H} & 112.70 & 128.14 & 128.18\end{array}$

$\begin{array}{llll}13 \mathrm{H} & 113.20 & 128.64 & 128.68\end{array}$

$\begin{array}{llll}13 \mathrm{H} & 113.88 & 129.32 & 129.36\end{array}$

$\begin{array}{llll}13 \mathrm{H} & 115.10 & 130.54 & 130.62 \\ 13 \mathrm{H} & 116.83 & 132.27 & 132.35\end{array}$

$\begin{array}{llll}13 \mathrm{H} & 117.50 & 132.94 & 133.10\end{array}$

$\begin{array}{llll}13 \mathrm{H} & 118.17 & 133.61 & 133.67\end{array}$

$\begin{array}{llll}13 \mathrm{H} & 119.06 & 134.50 & 134.62\end{array}$

$\begin{array}{llll}13 \mathrm{H} & 119.72 & 135.16 & 135.16 \\ 13 \mathrm{H} & 121.01 & 136.45 & 136.50\end{array}$

$\begin{array}{llll}13 \mathrm{H} & 121.80 & 137.24 & 137.24\end{array}$

$\begin{array}{llll}13 \mathrm{H} & 122.60 & 138.04 & 138.04\end{array}$

$\begin{array}{llll}14 \mathrm{H} & 122.20 & 139.13 & 139.05 \\ 14 \mathrm{H} & 122.68 & 139.61 & 139.45 \\ 14 \mathrm{H} & 123.15 & 140.08 & 140.08\end{array}$

$\begin{array}{llll}14 \mathrm{H} & 123.66 & 140.59 & 140.72 \\ 14 \mathrm{H} & 124.07 & 141.00 & 141.22\end{array}$

$\begin{array}{llll}14 \mathrm{H} & 124.07 & 141.00 & 141.22 \\ 14 \mathrm{H} & 124.74 & 141.67 & 142.00 \\ 14 \mathrm{H} & 126.83 & 143.76 & 144.45\end{array}$

$\begin{array}{llll}14 \mathrm{H} & 126.83 & 143.76 & 144.45 \\ 14 \mathrm{H} & 127.33 & 144.26 & 145.15 \\ 14 \mathrm{H} & 127.85 & 144.78 & 145.80\end{array}$

$\begin{array}{llll}14 \mathrm{H} & 127.85 & 144.78 & 145.80 \\ 14 \mathrm{H} & 128.68 & 145.61 & 146.89\end{array}$

$\begin{array}{llll}14 \mathrm{H} & 128.68 & 145.61 & 146.89 \\ 14 \mathrm{H} & 128.95 & 145.88 & 147.16\end{array}$

$\begin{array}{llll}14 \mathrm{H} & 128.95 & 145.88 & 147.16 \\ 14 \mathrm{H} & 129.28 & 146.21 & 147.54\end{array}$

$\begin{array}{llll}14 \mathrm{H} & 129.28 & 146.21 & 147.54 \\ 14 \mathrm{H} & 129.58 & 146.51 & 147.86 \\ 14 \mathrm{H} & 131.19 & 148.12 & 149.46\end{array}$

$\begin{array}{llll}14 \mathrm{H} & 131.19 & 148.12 & 149.46 \\ 14 \mathrm{H} & 132.01 & 148.94 & 150.28 \\ 15 \mathrm{H} & 131.70 & 150.56 & 150.65\end{array}$

$\begin{array}{llll}15 \mathrm{H} & 131.70 & 150.56 & 150.65 \\ 15 \mathrm{H} & 132.46 & 151.32 & 151.41\end{array}$

$\begin{array}{llll}15 \mathrm{H} & 132.46 & 151.32 & 151.41 \\ 15 \mathrm{H} & 133.35 & 152.21 & 152.21 \\ 134.67 & 153.53 & 153.58\end{array}$

$\begin{array}{llll}15 \mathrm{H} & 134.67 & 153.53 & 153.58 \\ 15 \mathrm{H} & 135.34 & 154.20 & 154.16\end{array}$

$\begin{array}{llll}15 \mathrm{H} & 135.34 & 154.20 & 154.16 \\ 15 \mathrm{H} & 136.67 & 155.53 & 155.62\end{array}$

$\begin{array}{llll}15 \mathrm{H} & 136.90 & 155.76 & 155.82 \\ 15 \mathrm{H} & 138.21 & 157.07 & 157.36\end{array}$

$\begin{array}{llll}15 \mathrm{H} & 138.21 & 157.07 & 157.36 \\ 15 \mathrm{H} & 139.52 & 158.38 & 158.71\end{array}$

$\begin{array}{llll}15 \mathrm{H} & 140.00 & 158.86 & 159.11 \\ 15 \mathrm{H} & 141.25 & 160.11 & 160.36\end{array}$

$\begin{array}{llll}15 \mathrm{H} & 141.25 & 160.11 & 160.36 \\ 16 \mathrm{H} & 141.20 & 161.76 & 161.95\end{array}$

$\begin{array}{llll}16 \mathrm{H} & 141.20 & 161.76 & 161.95 \\ 16 \mathrm{H} & 142.42 & 162.98 & 163.17\end{array}$

$\begin{array}{llll}16 \mathrm{H} & 145.44 & 166.00 & 166.01 \\ 16 \mathrm{H} & 145.99 & 166.55 & 166.56\end{array}$

$\begin{array}{llll}16 \mathrm{H} & 145.99 & 166.55 & 166.56 \\ 16 \mathrm{H} & 148.04 & 168.60 & 168.80\end{array}$

$\begin{array}{llll}16 \mathrm{H} & 148.49 & 169.05 & 169.31 \\ 16 \mathrm{H} & 149.62 & 170.18 & 170.44\end{array}$

$\begin{array}{llll}16 \mathrm{H} & 149.62 & 170.18 & 170.44 \\ 17 \mathrm{H} & 150.70 & 173.92 & 173.65\end{array}$

$\begin{array}{llll}17 \mathrm{H} & 150.70 & 173.92 & 173.65 \\ 17 \mathrm{H} & 152.57 & 175.79 & 175.40\end{array}$

\begin{tabular}{|c|c|c|c|}
\hline \multirow[b]{2}{*}{ Core } & \multicolumn{3}{|c|}{ Depth } \\
\hline & $\begin{array}{c}\text { (mbsf } \\
{[\mathrm{m} \mathrm{CSF-A]})}\end{array}$ & $\begin{array}{c}(\mathrm{rmcd} \\
{[\mathrm{m} \text { revised }} \\
\text { CCSF-A] })\end{array}$ & $\begin{array}{c}\text { (rmcd } \\
\text { [adjusted } \\
\text { m revised } \\
\text { CCSF-A]) }\end{array}$ \\
\hline $17 \mathrm{H}$ & 153.78 & 177.00 & 176.65 \\
\hline $17 \mathrm{H}$ & 155.62 & 178.84 & 178.55 \\
\hline $17 \mathrm{H}$ & 157.64 & 180.86 & 180.26 \\
\hline $17 \mathrm{H}$ & 158.80 & 182.02 & 181.35 \\
\hline $17 \mathrm{H}$ & 159.01 & 182.23 & 181.56 \\
\hline $18 \mathrm{H}$ & 160.20 & 184.30 & 184.14 \\
\hline $18 \mathrm{H}$ & 161.62 & 185.72 & 185.56 \\
\hline $18 \mathrm{H}$ & 162.33 & 186.43 & 186.34 \\
\hline $18 \mathrm{H}$ & 162.83 & 186.93 & 186.93 \\
\hline $18 \mathrm{H}$ & 166.65 & 190.75 & 190.75 \\
\hline $18 \mathrm{H}$ & 168.53 & 192.63 & 192.63 \\
\hline $18 \mathrm{H}$ & 170.22 & 194.32 & 194.32 \\
\hline $19 \mathrm{H}$ & 169.70 & 196.12 & 196.12 \\
\hline $19 \mathrm{H}$ & 172.55 & 198.97 & 198.97 \\
\hline $19 \mathrm{H}$ & 177.23 & 203.65 & 203.65 \\
\hline $19 \mathrm{H}$ & 178.54 & 204.96 & 204.96 \\
\hline $20 \mathrm{H}$ & 179.20 & 219.66 & 219.80 \\
\hline $20 \mathrm{H}$ & 180.58 & 221.04 & 221.18 \\
\hline $20 \mathrm{H}$ & 182.29 & 222.75 & 222.75 \\
\hline $20 \mathrm{H}$ & 186.19 & 226.65 & 226.65 \\
\hline $20 \mathrm{H}$ & 188.68 & 229.14 & 229.14 \\
\hline $21 \mathrm{H}$ & 188.70 & 229.86 & 229.86 \\
\hline $21 \mathrm{H}$ & 189.53 & 230.69 & 230.66 \\
\hline $21 \mathrm{H}$ & 189.92 & 231.08 & 231.11 \\
\hline $21 \mathrm{H}$ & 190.15 & 231.31 & 231.36 \\
\hline $21 \mathrm{H}$ & 190.66 & 231.82 & 231.82 \\
\hline $21 \mathrm{H}$ & 195.35 & 236.51 & 236.51 \\
\hline $21 \mathrm{H}$ & 196.27 & 237.43 & 237.44 \\
\hline $21 \mathrm{H}$ & 197.88 & 239.04 & 239.05 \\
\hline $21 \mathrm{H}$ & 198.10 & 239.26 & 239.34 \\
\hline $21 \mathrm{H}$ & 198.53 & 239.69 & 239.77 \\
\hline $22 \mathrm{H}$ & 198.20 & 240.66 & 240.66 \\
\hline $22 \mathrm{H}$ & 201.85 & 244.31 & 244.31 \\
\hline $22 \mathrm{H}$ & 203.34 & 245.80 & 245.80 \\
\hline $22 \mathrm{H}$ & 204.90 & 247.36 & 247.36 \\
\hline $22 \mathrm{H}$ & 205.24 & 247.70 & 247.96 \\
\hline $22 \mathrm{H}$ & 205.62 & 248.08 & 248.58 \\
\hline $22 \mathrm{H}$ & 206.91 & 249.37 & 249.87 \\
\hline $23 x$ & 206.90 & 251.62 & 251.47 \\
\hline $23 x$ & 207.25 & 251.97 & 251.81 \\
\hline $23 x$ & 208.08 & 252.80 & 252.87 \\
\hline $23 x$ & 208.95 & 253.67 & 253.92 \\
\hline $23 x$ & 209.23 & 253.95 & 254.17 \\
\hline $23 x$ & 209.78 & 254.50 & 254.50 \\
\hline $23 x$ & 214.67 & 259.39 & 259.39 \\
\hline $23 x$ & 215.12 & 259.84 & 259.95 \\
\hline $23 x$ & 215.43 & 260.15 & 260.32 \\
\hline $23 x$ & 215.65 & 260.37 & 260.60 \\
\hline $23 x$ & 215.77 & 260.49 & 260.88 \\
\hline $23 x$ & 216.22 & 260.94 & 260.33 \\
\hline $24 X$ & 214.50 & 262.18 & 262.18 \\
\hline $24 x$ & 220.10 & 267.78 & 267.78 \\
\hline $24 x$ & 223.34 & 271.02 & 271.02 \\
\hline $24 x$ & 224.27 & 271.95 & 271.95 \\
\hline $25 x$ & 224.10 & 273.53 & 273.42 \\
\hline $25 X$ & 224.60 & 274.03 & 273.92 \\
\hline $25 X$ & 224.80 & 274.23 & 274.10 \\
\hline $25 X$ & 225.00 & 274.43 & 274.28 \\
\hline $25 X$ & 225.35 & 274.78 & 274.64 \\
\hline $25 x$ & 225.94 & 275.37 & 275.44 \\
\hline $25 x$ & 226.29 & 275.72 & 275.72 \\
\hline $25 x$ & 226.71 & 276.14 & 276.14 \\
\hline $25 x$ & 232.57 & 282.00 & 282.00 \\
\hline $25 X$ & 233.84 & 283.27 & 282.75 \\
\hline $26 x$ & 233.60 & 283.24 & 283.79 \\
\hline
\end{tabular}


Table T13 (continued). (Continued on next page.)

\begin{tabular}{|c|c|c|c|c|c|c|c|c|c|c|c|}
\hline \multirow[b]{2}{*}{ Core } & \multicolumn{3}{|c|}{ Depth } & \multirow[b]{2}{*}{ Core } & \multicolumn{3}{|c|}{ Depth } & \multirow[b]{2}{*}{ Core } & \multicolumn{3}{|c|}{ Depth } \\
\hline & $\begin{array}{c}(\mathrm{mbsf} \\
{[\mathrm{m} \mathrm{CSF-A]})}\end{array}$ & $\begin{array}{c}(\mathrm{rmcd} \\
{[\mathrm{m} \text { revised }} \\
\text { CCSF-A] })\end{array}$ & $\begin{array}{c}\text { (rmcd } \\
\text { [adjusted } \\
\text { m revised } \\
\text { CCSF-A]) }\end{array}$ & & $\begin{array}{c}\text { (mbsf } \\
{[\mathrm{m} \mathrm{CSF-A])}}\end{array}$ & $\begin{array}{c}(\mathrm{rmcd} \\
{[\mathrm{m} \text { revised }} \\
\text { CCSF-A] })\end{array}$ & $\begin{array}{l}\text { (rmcd } \\
\text { [adjusted } \\
\text { m revised } \\
\text { CCSF-A]) }\end{array}$ & & $\begin{array}{c}\text { (mbsf } \\
{[\mathrm{m} \text { CSF-A] })}\end{array}$ & $\begin{array}{c}(\mathrm{rmcd} \\
{[\mathrm{m} \text { revised }} \\
\text { CCSF-A] })\end{array}$ & $\begin{array}{l}\text { (rmcd } \\
\text { [adjusted } \\
\text { m revised } \\
\text { CCSF-A] })\end{array}$ \\
\hline $26 X$ & 234.15 & 283.79 & 284.34 & $1 \mathrm{H}$ & 7.40 & 11.40 & 11.16 & $8 \mathrm{H}$ & 77.35 & 89.49 & 89.49 \\
\hline $26 X$ & 235.36 & 285.00 & 285.31 & $1 \mathrm{H}$ & 11.57 & 15.57 & 14.92 & $8 \mathrm{H}$ & 78.07 & 90.21 & 90.40 \\
\hline $26 X$ & 236.61 & 286.25 & 286.25 & $1 \mathrm{H}$ & 12.48 & 16.48 & 15.75 & $9 \mathrm{H}$ & 77.70 & 92.05 & 91.99 \\
\hline $26 x$ & 236.89 & 286.53 & 286.53 & $1 \mathrm{H}$ & 13.72 & 17.72 & 16.80 & $9 \mathrm{H}$ & 79.03 & 93.38 & 93.38 \\
\hline $26 X$ & 242.03 & 291.67 & 291.67 & $2 \mathrm{H}$ & 13.20 & 17.89 & 18.13 & $9 \mathrm{H}$ & 84.25 & 98.60 & 98.60 \\
\hline $26 X$ & 243.41 & 293.05 & 293.05 & $2 \mathrm{H}$ & 14.90 & 19.59 & 19.83 & $9 \mathrm{H}$ & 85.20 & 99.55 & 99.72 \\
\hline $27 X$ & 243.20 & 294.30 & 294.88 & $2 \mathrm{H}$ & 16.45 & 21.14 & 21.31 & $9 \mathrm{H}$ & 85.90 & 100.25 & 100.52 \\
\hline $27 X$ & 243.48 & 294.58 & 295.16 & $2 \mathrm{H}$ & 17.69 & 22.38 & 22.48 & $9 \mathrm{H}$ & 86.40 & 100.75 & 101.10 \\
\hline $27 X$ & 243.83 & 294.93 & 295.41 & $2 \mathrm{H}$ & 18.45 & 23.14 & 23.14 & $9 \mathrm{H}$ & 86.91 & 101.26 & 101.68 \\
\hline $27 X$ & 244.71 & 295.81 & 296.05 & $2 \mathrm{H}$ & 19.83 & 24.52 & 24.55 & $9 \mathrm{H}$ & 87.73 & 102.08 & 102.50 \\
\hline $27 X$ & 245.47 & 296.57 & 296.65 & $2 \mathrm{H}$ & 20.63 & 25.32 & 25.25 & $10 \mathrm{H}$ & 87.20 & 102.64 & 102.77 \\
\hline $27 X$ & 245.73 & 296.83 & 296.83 & $2 \mathrm{H}$ & 21.63 & 26.32 & 26.20 & $10 \mathrm{H}$ & 87.40 & 102.84 & 102.97 \\
\hline $27 X$ & 246.28 & 297.38 & 297.38 & $2 \mathrm{H}$ & 22.88 & 27.57 & 27.25 & $10 \mathrm{H}$ & 88.00 & 103.44 & 103.99 \\
\hline $27 X$ & 249.97 & 301.07 & 301.07 & $2 \mathrm{H}$ & 23.08 & 27.77 & 27.45 & $10 \mathrm{H}$ & 91.42 & 106.86 & 106.22 \\
\hline $27 X$ & 250.13 & 301.23 & 301.21 & $3 \mathrm{H}$ & 22.70 & 27.34 & 27.45 & $10 \mathrm{H}$ & 91.72 & 107.16 & 106.39 \\
\hline $27 X$ & 250.24 & 301.34 & 301.30 & $3 \mathrm{H}$ & 23.18 & 27.82 & 27.95 & $10 \mathrm{H}$ & 91.99 & 107.43 & 106.70 \\
\hline $27 X$ & 250.79 & 301.89 & 301.89 & $3 \mathrm{H}$ & 23.78 & 28.42 & 28.42 & $10 \mathrm{H}$ & 92.65 & 108.09 & 108.00 \\
\hline $28 \mathrm{X}$ & 252.80 & 306.10 & 306.20 & $3 \mathrm{H}$ & 29.64 & 34.28 & 34.28 & $10 \mathrm{H}$ & 93.12 & 108.56 & 108.56 \\
\hline $28 X$ & 252.92 & 306.22 & 306.32 & $3 \mathrm{H}$ & 31.08 & 35.72 & 36.18 & $10 \mathrm{H}$ & 93.80 & 109.24 & 109.14 \\
\hline $28 X$ & 253.09 & 306.39 & 306.47 & $3 \mathrm{H}$ & 32.05 & 36.69 & 37.32 & $10 \mathrm{H}$ & 94.72 & 110.16 & 109.75 \\
\hline $28 X$ & 253.27 & 306.57 & 306.57 & $3 \mathrm{H}$ & 32.30 & 36.94 & 37.57 & $10 \mathrm{H}$ & 94.89 & 110.33 & 110.01 \\
\hline $28 X$ & 261.40 & 314.70 & 314.70 & $3 \mathrm{H}$ & 32.87 & 37.51 & 38.14 & $10 \mathrm{H}$ & 95.36 & 110.80 & 110.38 \\
\hline $28 \mathrm{X}$ & 261.59 & 314.89 & 314.91 & $4 \mathrm{H}$ & 32.20 & 38.47 & 38.47 & $10 \mathrm{H}$ & 96.15 & 111.59 & 111.02 \\
\hline $28 X$ & 261.70 & 315.00 & 315.02 & $4 \mathrm{H}$ & 33.10 & 39.37 & 39.37 & $10 \mathrm{H}$ & 97.25 & 112.69 & 112.12 \\
\hline $28 X$ & 261.90 & 315.20 & 315.16 & $4 \mathrm{H}$ & 40.97 & 47.24 & 47.24 & $11 \mathrm{H}$ & 96.70 & 113.79 & 113.79 \\
\hline $28 X$ & 262.76 & 316.06 & 316.02 & $4 \mathrm{H}$ & 41.96 & 48.23 & 48.23 & $11 \mathrm{H}$ & 98.33 & 115.42 & 115.42 \\
\hline $29 x$ & 262.40 & 316.47 & 316.61 & $5 \mathrm{H}$ & 41.70 & 47.69 & 47.59 & $11 \mathrm{H}$ & 105.19 & 122.28 & 122.28 \\
\hline $29 x$ & 263.81 & 317.88 & 317.88 & $5 \mathrm{H}$ & 43.74 & 49.73 & 49.63 & $11 \mathrm{H}$ & 106.74 & 123.83 & 123.83 \\
\hline $29 x$ & 264.22 & 318.29 & 318.43 & $5 \mathrm{H}$ & 44.73 & 50.72 & 50.51 & $12 \mathrm{H}$ & 106.20 & 123.48 & 123.60 \\
\hline $29 x$ & 265.13 & 319.20 & 319.21 & $5 \mathrm{H}$ & 45.35 & 51.34 & 51.04 & $12 \mathrm{H}$ & 107.86 & 125.14 & 125.14 \\
\hline $29 x$ & 265.78 & 319.85 & 319.91 & $5 \mathrm{H}$ & 45.78 & 51.77 & 51.49 & $12 \mathrm{H}$ & 113.95 & 131.23 & 131.23 \\
\hline $29 x$ & 266.25 & 320.32 & 320.39 & $5 \mathrm{H}$ & 46.72 & 52.71 & 52.32 & $12 \mathrm{H}$ & 116.19 & 133.47 & 133.47 \\
\hline $29 x$ & 266.99 & 321.06 & 321.01 & $5 \mathrm{H}$ & 47.40 & 53.39 & 53.00 & $13 \mathrm{H}$ & 115.70 & 134.69 & 134.80 \\
\hline $29 x$ & 267.19 & 321.26 & 321.20 & $5 \mathrm{H}$ & 48.06 & 54.05 & 53.42 & $13 \mathrm{H}$ & 117.49 & 136.48 & 136.48 \\
\hline $29 x$ & 267.69 & 321.76 & 321.73 & $5 \mathrm{H}$ & 48.63 & 54.62 & 54.00 & $13 \mathrm{H}$ & 121.61 & 140.60 & 140.60 \\
\hline $29 x$ & 268.04 & 322.11 & 322.03 & $5 \mathrm{H}$ & 49.01 & 55.00 & 54.32 & $13 \mathrm{H}$ & 125.64 & 144.63 & 144.63 \\
\hline $29 x$ & 268.95 & 323.02 & 323.03 & $5 \mathrm{H}$ & 49.25 & 55.24 & 54.52 & $14 \mathrm{H}$ & 125.20 & 146.94 & 146.94 \\
\hline $29 x$ & 269.37 & 323.44 & 323.57 & $5 \mathrm{H}$ & 50.96 & 56.95 & 55.85 & $14 \mathrm{H}$ & 125.63 & 147.37 & 147.37 \\
\hline $29 x$ & 270.23 & 324.30 & 324.61 & $6 \mathrm{H}$ & 49.20 & 56.51 & 56.51 & $14 \mathrm{H}$ & 130.58 & 152.32 & 152.32 \\
\hline $29 x$ & 270.86 & 324.93 & 325.28 & $6 \mathrm{H}$ & 50.00 & 57.31 & 57.31 & $14 \mathrm{H}$ & 135.35 & 157.09 & 157.30 \\
\hline $29 X$ & 272.05 & 326.12 & 326.47 & $6 \mathrm{H}$ & 50.62 & 57.93 & 57.88 & $15 \mathrm{H}$ & 134.70 & 157.19 & 156.95 \\
\hline $30 x$ & 272.10 & 327.37 & 327.01 & $6 \mathrm{H}$ & 52.31 & 59.62 & 59.42 & $15 \mathrm{H}$ & 135.88 & 158.37 & 158.37 \\
\hline $30 x$ & 272.46 & 327.73 & 327.37 & $6 \mathrm{H}$ & 53.56 & 60.87 & 60.52 & $15 \mathrm{H}$ & 143.40 & 165.89 & 165.89 \\
\hline $30 x$ & 273.03 & 328.30 & 328.00 & $6 \mathrm{H}$ & 54.34 & 61.65 & 61.30 & $15 \mathrm{H}$ & 144.64 & 167.13 & 167.30 \\
\hline $30 x$ & 274.31 & 329.58 & 329.47 & $6 \mathrm{H}$ & 54.57 & 61.88 & 61.55 & $16 \mathrm{H}$ & 144.20 & 167.99 & 167.99 \\
\hline $30 x$ & 274.92 & 330.19 & 330.14 & $6 \mathrm{H}$ & 54.84 & 62.15 & 61.89 & $16 \mathrm{H}$ & 145.58 & 169.37 & 169.37 \\
\hline $30 x$ & 275.85 & 331.12 & 331.12 & $6 \mathrm{H}$ & 55.12 & 62.43 & 62.17 & $16 \mathrm{H}$ & 150.85 & 174.64 & 174.64 \\
\hline $30 x$ & 276.91 & 332.18 & 332.18 & $6 \mathrm{H}$ & 55.53 & 62.84 & 62.58 & $16 \mathrm{H}$ & 154.27 & 178.06 & 177.50 \\
\hline $30 x$ & 277.11 & 332.38 & 332.38 & $6 \mathrm{H}$ & 56.14 & 63.45 & 63.30 & $17 \mathrm{H}$ & 153.70 & 178.84 & 178.84 \\
\hline $30 x$ & 277.74 & 333.01 & 332.84 & $6 \mathrm{H}$ & 56.54 & 63.85 & 63.64 & $17 H$ & 154.98 & 180.12 & 180.12 \\
\hline $30 x$ & 278.01 & 333.28 & 333.15 & $6 \mathrm{H}$ & 56.72 & 64.03 & 64.08 & $17 H$ & 161.79 & 186.93 & 186.93 \\
\hline $30 x$ & 278.84 & 334.11 & 334.08 & $6 \mathrm{H}$ & 57.25 & 64.56 & 64.53 & $17 \mathrm{H}$ & 163.73 & 188.87 & 188.87 \\
\hline $30 x$ & 279.40 & 334.67 & 334.59 & $6 \mathrm{H}$ & 57.85 & 65.16 & 65.15 & $18 \mathrm{H}$ & 163.20 & 190.30 & 190.30 \\
\hline $30 x$ & 279.72 & 334.99 & 335.09 & $6 \mathrm{H}$ & 58.02 & 65.33 & 65.31 & $18 \mathrm{H}$ & 163.65 & 190.75 & 190.75 \\
\hline $30 x$ & 279.94 & 335.21 & 335.37 & $6 \mathrm{H}$ & 58.28 & 65.59 & 65.63 & $18 \mathrm{H}$ & 171.87 & 198.97 & 198.97 \\
\hline $30 x$ & 280.55 & 335.82 & 336.02 & $6 \mathrm{H}$ & 58.53 & 65.84 & 65.88 & $18 \mathrm{H}$ & 173.28 & 200.38 & 200.38 \\
\hline $30 x$ & 281.18 & 336.45 & 336.65 & $6 \mathrm{H}$ & 58.73 & 66.04 & 66.11 & $19 \mathrm{H}$ & 172.70 & 200.95 & 200.95 \\
\hline $30 x$ & 281.75 & 337.02 & 337.22 & $6 \mathrm{H}$ & 59.23 & 66.54 & 66.61 & $19 \mathrm{H}$ & 175.40 & 203.65 & 203.65 \\
\hline $31 x$ & 281.80 & 337.58 & 337.58 & $7 \mathrm{H}$ & 58.70 & 67.99 & 67.89 & $19 \mathrm{H}$ & 177.22 & 205.47 & 205.47 \\
\hline $31 x$ & 283.94 & 339.72 & 339.72 & $7 \mathrm{H}$ & 59.58 & 68.87 & 68.77 & $19 \mathrm{H}$ & 178.23 & 206.48 & 206.48 \\
\hline $32 x$ & 285.00 & 340.78 & 340.78 & $7 \mathrm{H}$ & 61.27 & 70.56 & 70.56 & $19 \mathrm{H}$ & 179.18 & 207.43 & 207.63 \\
\hline $32 x$ & 285.36 & 341.14 & 341.14 & $7 \mathrm{H}$ & 61.97 & 71.26 & 71.26 & $19 \mathrm{H}$ & 181.58 & 209.83 & 209.83 \\
\hline & & & & $7 \mathrm{H}$ & 67.03 & 76.32 & 76.32 & $19 \mathrm{H}$ & 182.69 & 210.94 & 210.94 \\
\hline 320-U13 & & & & $7 \mathrm{H}$ & 68.91 & 78.20 & 78.20 & $20 \mathrm{H}$ & 182.20 & 212.66 & 212.66 \\
\hline $1 \mathrm{H}$ & $\begin{array}{l}3.70 \\
3.95\end{array}$ & $\begin{array}{l}7.70 \\
7.95\end{array}$ & $\begin{array}{l}7.36 \\
7.78\end{array}$ & $8 \mathrm{H}$ & 68.20 & 80.34 & 80.34 & $20 \mathrm{H}$ & 182.72 & 213.18 & 213.18 \\
\hline $1 \mathrm{H}$ & $\begin{array}{l}3.95 \\
580\end{array}$ & $\begin{array}{l}7.95 \\
980\end{array}$ & $\begin{array}{l}7.78 \\
9.79\end{array}$ & $8 \mathrm{H}$ & 70.70 & 82.84 & 82.84 & $20 \mathrm{H}$ & 189.30 & 219.76 & 219.76 \\
\hline $1 \mathrm{H}$ & 5.80 & 9.80 & 9.79 & $8 \mathrm{H}$ & 74.78 & 86.92 & 86.92 & $20 \mathrm{H}$ & 189.76 & 220.22 & 220.32 \\
\hline
\end{tabular}


Table T13 (continued). (Continued on next page.)

\begin{tabular}{|c|c|c|c|c|c|c|c|c|c|c|c|}
\hline \multirow[b]{2}{*}{ Core } & \multicolumn{3}{|c|}{ Depth } & \multirow[b]{2}{*}{ Core } & \multicolumn{3}{|c|}{ Depth } & \multirow[b]{2}{*}{ Core } & \multicolumn{3}{|c|}{ Depth } \\
\hline & $\begin{array}{c}\text { (mbsf } \\
{[\mathrm{m} \mathrm{CSF-A])}}\end{array}$ & $\begin{array}{c}(\mathrm{rmcd} \\
{[\mathrm{m} \text { revised }} \\
\text { CCSF-A] })\end{array}$ & $\begin{array}{c}\text { (rmcd } \\
\text { [adjusted } \\
\text { m revised } \\
\text { CCSF-A]) }\end{array}$ & & $\begin{array}{c}\text { (mbsf } \\
{[\mathrm{m} \text { CSF-A]) }}\end{array}$ & $\begin{array}{c}\text { (rmcd } \\
{[\mathrm{m} \text { revised }} \\
\text { CCSF-A] })\end{array}$ & $\begin{array}{l}\text { (rmcd } \\
\text { [adjusted } \\
\text { m revised } \\
\text { CCSF-A]) }\end{array}$ & & $\begin{array}{c}\text { (mbsf } \\
{[\mathrm{m} \mathrm{CSF-A]})}\end{array}$ & $\begin{array}{c}(\mathrm{rmcd} \\
{[\mathrm{m} \text { revised }} \\
\text { CCSF-A] })\end{array}$ & $\begin{array}{l}\text { (rmcd } \\
\text { [adjusted } \\
\text { m revised } \\
\text { CCSF-A]) }\end{array}$ \\
\hline $20 \mathrm{H}$ & 190.39 & 220.85 & 220.77 & $27 X$ & 257.63 & 311.76 & 311.70 & $5 \mathrm{H}$ & 46.86 & 56.97 & 56.97 \\
\hline $20 \mathrm{H}$ & 190.75 & 221.21 & 221.14 & $28 X$ & 257.40 & 312.61 & 312.69 & $5 \mathrm{H}$ & 48.03 & 58.14 & 58.14 \\
\hline $20 \mathrm{H}$ & 191.36 & 221.82 & 221.72 & $28 X$ & 257.75 & 312.96 & 313.04 & $6 \mathrm{H}$ & 47.50 & 59.56 & 59.56 \\
\hline $20 \mathrm{H}$ & 191.58 & 222.04 & 221.94 & $28 X$ & 258.40 & 313.61 & 313.57 & $6 \mathrm{H}$ & 48.63 & 60.69 & 60.69 \\
\hline $21 \mathrm{H}$ & 191.70 & 224.21 & 223.91 & $28 X$ & 258.98 & 314.19 & 314.11 & $6 \mathrm{H}$ & 54.79 & 66.85 & 66.85 \\
\hline $21 \mathrm{H}$ & 192.38 & 224.89 & 224.59 & $28 X$ & 259.24 & 314.45 & 314.40 & $6 \mathrm{H}$ & 57.28 & 69.34 & 69.55 \\
\hline $21 \mathrm{H}$ & 192.80 & 225.31 & 225.06 & $28 X$ & 259.49 & 314.70 & 314.70 & 7H & 57.00 & 69.06 & 69.23 \\
\hline $21 \mathrm{H}$ & 193.30 & 225.81 & 225.68 & $28 X$ & 262.82 & 318.03 & 318.03 & $7 \mathrm{H}$ & 59.63 & 71.69 & 71.86 \\
\hline $21 \mathrm{H}$ & 194.14 & 226.65 & 226.65 & $28 X$ & 263.18 & 318.39 & 318.45 & $7 \mathrm{H}$ & 61.02 & 73.08 & 73.27 \\
\hline $21 \mathrm{H}$ & 199.31 & 231.82 & 231.82 & $28 X$ & 263.66 & 318.87 & 318.81 & 7H & 62.53 & 74.59 & 74.63 \\
\hline $21 \mathrm{H}$ & 199.64 & 232.15 & 232.14 & $28 X$ & 264.17 & 319.38 & 319.21 & 7H & 63.25 & 75.31 & 75.33 \\
\hline $21 \mathrm{H}$ & 200.48 & 232.99 & 232.94 & $28 X$ & 264.75 & 319.96 & 319.91 & $7 \mathrm{H}$ & 63.82 & 75.88 & 75.88 \\
\hline $21 \mathrm{H}$ & 201.31 & 233.82 & 233.77 & $28 X$ & 265.71 & 320.92 & 320.69 & $7 \mathrm{H}$ & 64.95 & 77.01 & 77.09 \\
\hline $22 \mathrm{H}$ & 201.20 & 235.11 & 235.11 & $28 X$ & 266.08 & 321.29 & 321.01 & $7 \mathrm{H}$ & 65.47 & 77.53 & 77.66 \\
\hline $22 \mathrm{H}$ & 202.60 & 236.51 & 236.51 & $28 X$ & 266.73 & 321.94 & 321.65 & $7 \mathrm{H}$ & 66.12 & 78.18 & 78.06 \\
\hline $22 \mathrm{H}$ & 210.33 & 244.24 & 244.24 & $28 X$ & 267.34 & 322.55 & 322.26 & $7 \mathrm{H}$ & 66.64 & 78.70 & 78.58 \\
\hline $22 \mathrm{H}$ & 211.13 & 245.04 & 245.04 & $29 x$ & 267.00 & 324.43 & 324.50 & $8 \mathrm{H}$ & 66.50 & 80.46 & 80.33 \\
\hline $23 x$ & 210.70 & 245.61 & 245.71 & $29 x$ & 267.96 & 325.39 & 325.39 & $8 \mathrm{H}$ & 67.70 & 81.66 & 81.79 \\
\hline $23 x$ & 211.33 & 246.24 & 246.34 & $29 x$ & 275.40 & 332.83 & 332.83 & $8 \mathrm{H}$ & 68.89 & 82.85 & 82.82 \\
\hline $23 x$ & 212.45 & 247.36 & 247.36 & $29 x$ & 277.03 & 334.46 & 334.40 & $8 \mathrm{H}$ & 69.74 & 83.70 & 83.59 \\
\hline $23 x$ & 219.59 & 254.50 & 254.50 & $30 x$ & 276.60 & 334.64 & 334.78 & $8 \mathrm{H}$ & 70.97 & 84.93 & 84.86 \\
\hline $23 x$ & 220.48 & 255.39 & 255.39 & $30 x$ & 276.67 & 334.71 & 334.85 & $8 \mathrm{H}$ & 71.36 & 85.32 & 85.18 \\
\hline $24 X$ & 219.00 & 268.63 & 268.63 & $30 x$ & 276.90 & 334.94 & 335.04 & $8 \mathrm{H}$ & 71.96 & 85.92 & 85.81 \\
\hline $24 X$ & 221.46 & 271.09 & 271.09 & $30 x$ & 277.11 & 335.15 & 335.23 & $8 \mathrm{H}$ & 73.11 & 87.07 & 87.00 \\
\hline $24 X$ & 222.02 & 271.65 & 271.61 & $30 x$ & 277.71 & 335.75 & 335.75 & $8 \mathrm{H}$ & 74.14 & 88.10 & 88.00 \\
\hline $24 X$ & 222.52 & 272.15 & 272.08 & $30 x$ & 283.83 & 341.87 & 341.87 & $8 \mathrm{H}$ & 75.59 & 89.55 & 89.55 \\
\hline $24 X$ & 222.92 & 272.55 & 272.41 & $31 x$ & 283.90 & 341.94 & 341.94 & $8 \mathrm{H}$ & 76.36 & 90.32 & 90.32 \\
\hline $24 X$ & 223.86 & 273.49 & 273.28 & $31 x$ & 284.02 & 342.06 & 342.06 & $9 \mathrm{H}$ & 76.00 & 92.30 & 92.15 \\
\hline $24 X$ & 224.61 & 274.24 & 274.10 & $320-U 13$ & & & & $9 \mathrm{H}$ & 76.80 & 93.10 & 92.95 \\
\hline $24 X$ & 225.09 & 274.72 & 274.64 & $\begin{array}{c}320-0 \\
1 \mathrm{H}\end{array}$ & 0.00 & 3.65 & & $9 \mathrm{H}$ & 77.57 & 93.87 & 93.87 \\
\hline $24 X$ & 226.06 & 275.69 & 275.75 & $1 \mathrm{H}$ & $\begin{array}{l}0.00 \\
1.67\end{array}$ & $\begin{array}{l}3.65 \\
5.32\end{array}$ & $\begin{array}{l}3.19 \\
5.46\end{array}$ & $9 \mathrm{H}$ & 79.29 & 95.59 & 95.32 \\
\hline $24 X$ & 226.97 & 276.60 & 276.74 & $1 \mathrm{H}$ & 2.84 & $\begin{array}{l}0.32 \\
6.49\end{array}$ & $\begin{array}{l}3.46 \\
6.49\end{array}$ & $9 \mathrm{H}$ & 79.74 & 96.04 & 95.70 \\
\hline $24 X$ & 228.21 & 277.84 & 278.03 & $1 \mathrm{H}$ & 6.47 & 10.12 & $\begin{array}{r}0.49 \\
10.12\end{array}$ & $9 \mathrm{H}$ & 81.31 & 97.61 & 97.16 \\
\hline $24 X$ & 228.73 & 278.36 & 278.55 & $1 \mathrm{H}$ & 9.83 & 13.48 & 13.48 & $9 \mathrm{H}$ & 82.28 & 98.58 & 98.04 \\
\hline $25 X$ & 228.60 & 279.32 & 279.72 & $2 \mathrm{H}$ & 9.50 & 13.83 & 13.83 & $9 \mathrm{H}$ & 82.93 & 99.23 & 98.64 \\
\hline $25 x$ & 229.15 & 279.87 & 280.27 & $2 \mathrm{H}$ & 13.28 & 17.61 & 17.61 & $9 \mathrm{H}$ & 83.25 & 99.55 & 98.97 \\
\hline $25 X$ & 229.75 & 280.47 & 280.76 & $2 \mathrm{H}$ & 15.84 & 20.17 & 20.17 & $9 \mathrm{H}$ & 84.70 & 101.00 & 100.65 \\
\hline $25 x$ & 230.93 & 281.65 & 281.95 & $2 \mathrm{H}$ & 18.72 & 23.05 & 23.05 & $9 \mathrm{H}$ & 85.82 & 102.12 & 101.80 \\
\hline $25 X$ & 232.77 & 283.49 & 283.61 & $3 \mathrm{H}$ & 19.00 & 25.65 & 25.65 & $10 \mathrm{H}$ & 85.50 & 103.48 & 103.48 \\
\hline $25 X$ & 233.50 & 284.22 & 284.35 & $3 \mathrm{H}$ & 20.95 & 27.60 & 27.60 & $10 \mathrm{H}$ & 86.30 & 104.28 & 104.28 \\
\hline $25 X$ & 234.13 & 284.85 & 284.98 & $3 \mathrm{H}$ & 21.84 & 28.49 & 28.32 & $10 \mathrm{H}$ & 91.73 & 109.71 & 109.71 \\
\hline $25 X$ & 234.50 & 285.22 & 285.28 & $3 \mathrm{H}$ & 22.92 & 29.57 & 29.36 & $10 \mathrm{H}$ & 92.43 & 110.41 & 110.41 \\
\hline $25 x$ & 235.29 & 286.01 & 286.01 & $3 \mathrm{H}$ & 23.75 & 30.40 & 30.32 & $10 \mathrm{H}$ & 93.97 & 111.95 & 111.79 \\
\hline $25 X$ & 235.81 & 286.53 & 286.53 & $3 \mathrm{H}$ & 24.25 & 30.90 & 30.92 & $10 \mathrm{H}$ & 94.90 & 112.88 & 112.72 \\
\hline $25 X$ & 237.43 & 288.15 & 288.38 & $3 \mathrm{H}$ & 24.78 & 31.43 & 31.50 & $10 \mathrm{H}$ & 95.17 & 113.15 & 112.99 \\
\hline $25 X$ & 238.42 & 289.14 & 289.37 & $3 \mathrm{H}$ & 25.58 & 32.23 & 32.42 & $11 \mathrm{H}$ & 95.00 & 114.69 & 114.79 \\
\hline $26 x$ & 238.20 & 290.52 & 290.52 & $3 \mathrm{H}$ & 26.92 & 33.57 & 33.70 & $11 \mathrm{H}$ & 95.98 & 115.67 & 115.77 \\
\hline $26 x$ & 239.35 & 291.67 & 291.67 & $3 \mathrm{H}$ & 27.78 & 34.43 & 34.43 & $11 \mathrm{H}$ & 96.43 & 116.12 & 116.22 \\
\hline $26 x$ & 245.06 & 297.38 & 297.38 & $3 \mathrm{H}$ & 28.12 & 34.77 & 34.77 & $11 \mathrm{H}$ & 98.08 & 117.77 & 117.77 \\
\hline $26 X$ & 245.28 & 297.60 & 297.59 & $4 \mathrm{H}$ & 28.50 & 38.54 & 38.45 & $11 \mathrm{H}$ & 99.23 & 118.92 & 118.92 \\
\hline $26 x$ & 245.40 & 297.72 & 297.79 & $4 \mathrm{H}$ & 28.80 & 38.84 & 38.75 & $11 \mathrm{H}$ & 99.66 & 119.35 & 119.41 \\
\hline $26 x$ & 245.81 & 298.13 & 298.29 & $4 \mathrm{H}$ & 28.98 & 39.02 & 39.05 & $11 \mathrm{H}$ & 99.92 & 119.61 & 119.51 \\
\hline $26 X$ & 246.26 & 298.58 & 298.72 & $4 \mathrm{H}$ & 29.80 & 39.84 & 39.62 & $11 \mathrm{H}$ & 100.22 & 119.91 & 119.91 \\
\hline $26 X$ & 246.71 & 299.03 & 299.20 & $4 \mathrm{H}$ & 30.58 & 40.62 & 40.58 & $11 \mathrm{H}$ & 100.77 & 120.46 & 120.46 \\
\hline $26 X$ & 246.95 & 299.27 & 299.46 & $4 \mathrm{H}$ & 31.24 & 41.28 & 41.28 & $11 \mathrm{H}$ & 101.84 & 121.53 & 121.55 \\
\hline $26 x$ & 247.30 & 299.62 & 299.84 & $4 \mathrm{H}$ & 32.63 & 42.67 & 42.85 & $11 \mathrm{H}$ & 102.16 & 121.85 & 121.85 \\
\hline $26 x$ & 247.46 & 299.78 & 299.99 & $4 \mathrm{H}$ & 33.14 & 43.18 & 43.38 & $11 \mathrm{H}$ & 102.41 & 122.10 & 122.10 \\
\hline $26 X$ & 247.90 & 300.22 & 300.43 & $4 \mathrm{H}$ & 33.64 & 43.68 & 43.83 & $11 \mathrm{H}$ & 104.01 & 123.70 & 123.70 \\
\hline $27 X$ & 247.80 & 301.93 & 302.05 & $\begin{array}{l}4 \mathrm{H} \\
4 \mathrm{H}\end{array}$ & 35.20 & 45.24 & 45.54 & $11 \mathrm{H}$ & 104.62 & 124.31 & 124.31 \\
\hline $27 x$ & 248.00 & 302.13 & 302.25 & $4 \mathrm{H}$ & 35.68 & 45.72 & 45.98 & $12 \mathrm{H}$ & 104.50 & 127.25 & 126.95 \\
\hline $27 x$ & 248.18 & 302.31 & 302.35 & $4 \mathrm{H}$ & 36.83 & 46.87 & 47.11 & $12 \mathrm{H}$ & 105.30 & 128.05 & 127.75 \\
\hline $27 X$ & 248.65 & 302.78 & 302.78 & $4 \mathrm{H}$ & 37.36 & 47.40 & 47.70 & $12 \mathrm{H}$ & 106.78 & 129.53 & 129.35 \\
\hline $27 X$ & 252.44 & 306.57 & 306.57 & $4 \mathrm{H}$ & 37.90 & 47.94 & 48.28 & $12 \mathrm{H}$ & 107.68 & 130.43 & 130.31 \\
\hline $27 x$ & 252.90 & 307.03 & 306.98 & $4 \mathrm{H}$ & 38.31 & 48.35 & 48.69 & $12 \mathrm{H}$ & 108.48 & 131.23 & 131.23 \\
\hline $27 X$ & 254.30 & 308.43 & 308.37 & $5 \mathrm{H}$ & 38.00 & 48.11 & 48.11 & $12 \mathrm{H}$ & 113.73 & 136.48 & 136.48 \\
\hline $27 X$ & 254.87 & 309.00 & 308.96 & $5 \mathrm{H}$ & 38.97 & 49.08 & 49.08 & $12 \mathrm{H}$ & 114.28 & 137.03 & 137.03 \\
\hline $27 X$ & 255.02 & 309.15 & 309.09 & $\mathrm{SH}$ & 38.91 & 49.08 & 49.08 & $13 \mathrm{H}$ & 114.00 & 139.13 & 139.00 \\
\hline
\end{tabular}


Table T13 (continued).

\begin{tabular}{|c|c|c|c|}
\hline \multirow[b]{2}{*}{ Core } & \multicolumn{3}{|c|}{ Depth } \\
\hline & $\begin{array}{c}\text { (mbsf } \\
{[\mathrm{m} \mathrm{CSF-A]})}\end{array}$ & $\begin{array}{c}(\mathrm{rmcd} \\
{[\mathrm{m} \text { revised }} \\
\text { CCSF-A] })\end{array}$ & $\begin{array}{l}\text { ( } \mathrm{rmcd} \\
\text { [adjusted } \\
\mathrm{m} \text { revised } \\
\text { CCSF-A]) }\end{array}$ \\
\hline $13 \mathrm{H}$ & 114.91 & 140.04 & 139.94 \\
\hline $13 \mathrm{H}$ & 115.32 & 140.45 & 140.35 \\
\hline $13 \mathrm{H}$ & 115.47 & 140.60 & 140.60 \\
\hline $13 \mathrm{H}$ & 122.24 & 147.37 & 147.37 \\
\hline $13 \mathrm{H}$ & 122.81 & 147.94 & 147.90 \\
\hline $13 \mathrm{H}$ & 123.85 & 148.98 & 148.80 \\
\hline $14 \mathrm{H}$ & 123.50 & 150.56 & 150.56 \\
\hline $14 \mathrm{H}$ & 125.26 & 152.32 & 152.32 \\
\hline $14 \mathrm{H}$ & 131.31 & 158.37 & 158.37 \\
\hline $14 \mathrm{H}$ & 133.03 & 160.09 & 160.09 \\
\hline $15 \mathrm{H}$ & 133.00 & 161.23 & 161.63 \\
\hline $15 \mathrm{H}$ & 137.66 & 165.89 & 165.89 \\
\hline $15 \mathrm{H}$ & 141.14 & 169.37 & 169.37 \\
\hline $15 \mathrm{H}$ & 142.50 & 170.73 & 170.73 \\
\hline $16 \mathrm{H}$ & 142.50 & 172.19 & 172.00 \\
\hline $16 \mathrm{H}$ & 143.88 & 173.57 & 173.57 \\
\hline $16 \mathrm{H}$ & 144.95 & 174.64 & 174.64 \\
\hline $16 \mathrm{H}$ & 150.43 & 180.12 & 180.12 \\
\hline $16 \mathrm{H}$ & 151.94 & 181.63 & 181.78 \\
\hline $17 \mathrm{H}$ & 152.00 & 182.35 & 182.23 \\
\hline $17 \mathrm{H}$ & 153.21 & 183.56 & 183.44 \\
\hline $17 \mathrm{H}$ & 153.72 & 184.07 & 183.94 \\
\hline $17 \mathrm{H}$ & 155.27 & 185.62 & 185.46 \\
\hline $17 \mathrm{H}$ & 156.68 & 187.03 & 187.07 \\
\hline $17 \mathrm{H}$ & 160.53 & 190.88 & 191.30 \\
\hline $17 \mathrm{H}$ & 161.52 & 191.87 & 191.29 \\
\hline $18 \mathrm{H}$ & 161.50 & 192.94 & 193.50 \\
\hline $18 \mathrm{H}$ & 162.31 & 193.75 & 194.31 \\
\hline $18 \mathrm{H}$ & 166.37 & 197.81 & 197.75 \\
\hline $18 \mathrm{H}$ & 167.45 & 198.89 & 198.90 \\
\hline $18 \mathrm{H}$ & 168.70 & 200.14 & 200.10 \\
\hline $18 \mathrm{H}$ & 170.56 & 202.00 & 201.94 \\
\hline $18 \mathrm{H}$ & 171.16 & 202.60 & 202.54 \\
\hline $19 \mathrm{H}$ & 171.00 & 203.52 & 203.48 \\
\hline $19 \mathrm{H}$ & 171.75 & 204.27 & 204.21 \\
\hline $19 \mathrm{H}$ & 172.88 & 205.40 & 205.44 \\
\hline $19 \mathrm{H}$ & 173.05 & 205.57 & 205.57 \\
\hline $19 \mathrm{H}$ & 180.66 & 213.18 & 213.18 \\
\hline $19 \mathrm{H}$ & 180.76 & 213.28 & 213.28 \\
\hline $20 \mathrm{H}$ & 180.50 & 213.83 & 213.40 \\
\hline $20 \mathrm{H}$ & 180.75 & 214.08 & 213.65 \\
\hline $20 \mathrm{H}$ & 181.79 & 215.12 & 214.63 \\
\hline $20 \mathrm{H}$ & 184.21 & 217.54 & 217.23 \\
\hline $20 \mathrm{H}$ & 184.67 & 218.00 & 217.76 \\
\hline $20 \mathrm{H}$ & 186.43 & 219.76 & 219.76 \\
\hline $20 \mathrm{H}$ & 189.42 & 222.75 & 222.75 \\
\hline $20 \mathrm{H}$ & 190.02 & 223.79 & 223.79 \\
\hline $21 \mathrm{H}$ & 190.00 & 224.06 & 224.06 \\
\hline $21 \mathrm{H}$ & 199.97 & 234.03 & 234.03 \\
\hline
\end{tabular}

\begin{tabular}{|c|c|c|c|}
\hline \multirow[b]{2}{*}{ Core } & \multicolumn{3}{|c|}{ Depth } \\
\hline & $\begin{array}{c}\text { (mbsf } \\
{[\mathrm{m} \text { CSF-A]) }}\end{array}$ & $\begin{array}{c}\text { (rmcd } \\
\text { [m revised } \\
\text { CCSF-A]) }\end{array}$ & $\begin{array}{c}\text { (rmcd } \\
\text { [adjusted } \\
\text { m revised } \\
\text { CCSF-A]) }\end{array}$ \\
\hline $22 \mathrm{H}$ & 199.50 & 236.36 & 236.31 \\
\hline $22 \mathrm{H}$ & 199.76 & 236.62 & 236.56 \\
\hline $22 \mathrm{H}$ & 200.68 & 237.54 & 237.36 \\
\hline $22 \mathrm{H}$ & 201.69 & 238.55 & 238.36 \\
\hline $22 \mathrm{H}$ & 202.59 & 239.45 & 239.28 \\
\hline $22 \mathrm{H}$ & 204.78 & 241.64 & 241.58 \\
\hline $22 \mathrm{H}$ & 205.77 & 242.63 & 242.72 \\
\hline $22 \mathrm{H}$ & 187.38 & 224.24 & 244.24 \\
\hline $22 \mathrm{H}$ & 208.94 & 245.80 & 245.80 \\
\hline $22 \mathrm{H}$ & 209.41 & 246.27 & 246.27 \\
\hline $23 x$ & 209.00 & 250.35 & 249.99 \\
\hline $23 x$ & 209.22 & 250.57 & 250.21 \\
\hline $23 x$ & 210.14 & 251.49 & 251.37 \\
\hline $23 X$ & 210.94 & 252.29 & 252.04 \\
\hline $23 x$ & 211.65 & 253.00 & 252.89 \\
\hline $23 X$ & 212.70 & 254.05 & 254.25 \\
\hline $23 x$ & 213.16 & 254.51 & 254.70 \\
\hline $23 x$ & 213.61 & 254.96 & 255.15 \\
\hline $24 X$ & 214.00 & 258.85 & 258.86 \\
\hline $24 X$ & 214.54 & 259.39 & 259.39 \\
\hline $24 X$ & 222.93 & 267.78 & 267.78 \\
\hline $24 X$ & 223.89 & 268.74 & 268.74 \\
\hline $25 X$ & 223.60 & 269.93 & 269.93 \\
\hline $25 X$ & 224.69 & 271.02 & 271.02 \\
\hline $25 X$ & 229.81 & 276.14 & 276.14 \\
\hline $25 X$ & 230.49 & 276.82 & 276.88 \\
\hline $25 X$ & 232.03 & 278.36 & 278.45 \\
\hline $25 x$ & 232.93 & 279.26 & 279.27 \\
\hline $25 X$ & 233.35 & 279.68 & 279.69 \\
\hline $26 x$ & 233.20 & 280.30 & 280.30 \\
\hline $26 x$ & 234.90 & 282.00 & 282.00 \\
\hline $26 x$ & 239.43 & 286.53 & 286.53 \\
\hline $26 x$ & 240.54 & 287.64 & 287.80 \\
\hline $27 X$ & 239.20 & 287.21 & 287.54 \\
\hline $27 X$ & 241.16 & 289.17 & 289.50 \\
\hline $27 X$ & 242.55 & 290.56 & 290.45 \\
\hline $27 X$ & 243.58 & 291.59 & 291.49 \\
\hline $27 X$ & 245.17 & 293.18 & 293.48 \\
\hline $27 X$ & 245.44 & 293.45 & 293.30 \\
\hline $27 X$ & 246.97 & 294.98 & 294.95 \\
\hline $27 X$ & 247.35 & 295.36 & 295.41 \\
\hline $27 X$ & 248.16 & 296.17 & 296.22 \\
\hline $28 X$ & 248.80 & 298.33 & 297.65 \\
\hline $28 X$ & 249.49 & 299.02 & 298.62 \\
\hline $28 X$ & 249.60 & 299.13 & 298.76 \\
\hline $28 X$ & 249.74 & 299.27 & 298.92 \\
\hline $28 X$ & 249.89 & 299.42 & 299.08 \\
\hline $28 X$ & 250.00 & 299.53 & 299.20 \\
\hline
\end{tabular}

\begin{tabular}{|c|c|c|c|}
\hline \multirow[b]{2}{*}{ Core } & \multicolumn{3}{|c|}{ Depth } \\
\hline & $\begin{array}{c}\text { (mbsf } \\
{[\mathrm{m} \mathrm{CSF-A]})}\end{array}$ & $\begin{array}{c}(\mathrm{rmcd} \\
{[\mathrm{m} \text { revised }} \\
\text { CCSF-A] })\end{array}$ & $\begin{array}{c}\text { (rmcd } \\
\text { [adjusted } \\
\text { m revised } \\
\text { CCSF-A]) }\end{array}$ \\
\hline $28 X$ & 250.22 & 299.75 & 299.46 \\
\hline $28 X$ & 250.29 & 299.82 & 299.58 \\
\hline $28 x$ & 250.38 & 299.91 & 299.68 \\
\hline $28 X$ & 250.44 & 299.97 & 299.74 \\
\hline $28 x$ & 250.54 & 300.07 & 299.87 \\
\hline $28 X$ & 250.80 & 300.33 & 300.19 \\
\hline $28 X$ & 250.95 & 300.48 & 300.38 \\
\hline $28 X$ & 251.25 & 300.78 & 300.76 \\
\hline $28 X$ & 251.54 & 301.07 & 301.07 \\
\hline $28 X$ & 253.25 & 302.78 & 302.78 \\
\hline $28 X$ & 253.48 & 303.01 & 303.05 \\
\hline $28 X$ & 253.68 & 303.21 & 303.31 \\
\hline $28 X$ & 254.01 & 303.54 & 303.83 \\
\hline $28 X$ & 254.26 & 303.79 & 304.09 \\
\hline $28 X$ & 254.67 & 304.20 & 304.64 \\
\hline $28 X$ & 254.96 & 304.49 & 305.09 \\
\hline $28 X$ & 255.06 & 304.59 & 305.24 \\
\hline $28 X$ & 255.26 & 304.79 & 305.61 \\
\hline $28 X$ & 255.42 & 304.95 & 305.86 \\
\hline $28 X$ & 255.82 & 305.35 & 306.45 \\
\hline $28 X$ & 256.34 & 305.87 & 307.26 \\
\hline $28 X$ & 256.94 & 306.47 & 308.46 \\
\hline $28 X$ & 257.21 & 306.74 & 308.76 \\
\hline $28 X$ & 257.52 & 307.05 & 309.15 \\
\hline $28 X$ & 258.62 & 308.15 & 310.15 \\
\hline $29 X$ & 258.40 & 316.68 & 316.68 \\
\hline $29 X$ & 258.56 & 316.84 & 316.95 \\
\hline $29 x$ & 258.76 & 317.04 & 317.09 \\
\hline $29 x$ & 259.10 & 317.38 & 317.35 \\
\hline $29 x$ & 259.75 & 318.03 & 318.03 \\
\hline $29 x$ & 267.11 & 325.39 & 325.39 \\
\hline $29 x$ & 268.29 & 326.57 & 326.57 \\
\hline $30 x$ & 268.00 & 326.81 & 326.78 \\
\hline $30 x$ & 268.69 & 327.50 & 327.37 \\
\hline $30 x$ & 269.78 & 328.59 & 328.45 \\
\hline $30 x$ & 271.40 & 330.21 & 330.14 \\
\hline $30 x$ & 271.78 & 330.59 & 330.57 \\
\hline $30 x$ & 271.91 & 330.72 & 330.62 \\
\hline $30 x$ & 272.88 & 331.69 & 331.55 \\
\hline $30 x$ & 273.08 & 331.89 & 331.84 \\
\hline $30 x$ & 273.63 & 332.44 & 332.28 \\
\hline $30 x$ & 274.02 & 332.83 & 332.83 \\
\hline $30 x$ & 276.94 & 335.75 & 335.75 \\
\hline $30 x$ & 277.96 & 336.77 & 336.77 \\
\hline $31 x$ & 277.70 & 338.72 & 338.72 \\
\hline $31 x$ & 280.11 & 341.13 & 341.13 \\
\hline $32 x$ & 279.70 & 279.70 & 279.70 \\
\hline $32 X$ & 280.17 & 280.17 & 280.17 \\
\hline
\end{tabular}

Italics = splice tie points. 
Table T14. Revised offset for composite depth section, Site 1218.

\begin{tabular}{crrr}
\hline & & & \\
Core & $\begin{array}{r}\text { Depth } \\
\text { (mbsf) }\end{array}$ & $\begin{array}{c}\text { Offset } \\
(\mathrm{m})\end{array}$ & $\begin{array}{r}\text { Depth } \\
\text { (corrected } \\
\text { rmcd) }\end{array}$ \\
\hline $199-1218 \mathrm{~A}-$ & & & \\
$1 \mathrm{H}$ & 0.00 & 0.00 & 0.00 \\
$2 \mathrm{H}$ & 8.20 & 0.78 & 8.98 \\
$3 \mathrm{H}$ & 17.70 & 2.34 & 20.04 \\
$4 \mathrm{H}$ & 27.20 & 4.42 & 31.62 \\
$5 \mathrm{H}$ & 36.70 & 4.80 & 41.50 \\
$6 \mathrm{H}$ & 46.20 & 6.84 & 53.04 \\
$7 \mathrm{H}$ & 55.70 & 8.06 & 63.76 \\
$8 \mathrm{H}$ & 65.20 & 7.96 & 73.16 \\
$9 \mathrm{H}$ & 74.70 & 10.26 & 84.96 \\
$10 \mathrm{H}$ & 84.20 & 11.90 & 96.10 \\
$11 \mathrm{H}$ & 93.70 & 13.40 & 107.10 \\
$12 \mathrm{H}$ & 103.20 & 14.47 & 117.67 \\
$13 \mathrm{H}$ & 112.70 & 14.91 & 127.61 \\
$14 \mathrm{H}$ & 122.20 & 15.42 & 137.62 \\
$15 \mathrm{H}$ & 131.70 & 16.22 & 147.92 \\
$16 \mathrm{H}$ & 141.20 & 17.84 & 159.04 \\
$17 \mathrm{H}$ & 150.70 & 17.90 & 168.60 \\
$18 \mathrm{H}$ & 160.20 & 20.56 & 180.76 \\
$19 \mathrm{H}$ & 169.70 & 20.38 & 190.08 \\
$20 \mathrm{H}$ & 179.20 & 22.28 & 201.48 \\
$22 \mathrm{X}$ & 195.90 & $\mathbf{2 2 . 9 3}$ & $\mathbf{2 1 8 . 8 3}$ \\
$23 \mathrm{X}$ & 205.50 & $\mathbf{2 2 . 6 4}$ & $\mathbf{2 2 8 . 1 4}$ \\
$24 \mathrm{X}$ & 215.10 & $\mathbf{2 4 . 4 8}$ & $\mathbf{2 3 9 . 5 8}$ \\
$25 \mathrm{X}$ & 224.70 & $\mathbf{2 5 . 7 3}$ & $\mathbf{2 5 0 . 4 3}$ \\
$26 \mathrm{X}$ & 234.30 & $\mathbf{2 5 . 5 3}$ & $\mathbf{2 5 9 . 8 3}$ \\
$27 \mathrm{X}$ & 243.90 & $\mathbf{2 5 . 5 9}$ & $\mathbf{2 6 9 . 4 9}$ \\
$28 \mathrm{X}$ & 253.50 & $\mathbf{2 4 . 5 3}$ & $\mathbf{2 7 8 . 0 3}$ \\
$29 \mathrm{X}$ & 263.10 & $\mathbf{2 4 . 8 3}$ & $\mathbf{2 8 7 . 9 3}$ \\
$30 \mathrm{X}$ & 270.70 & $\mathbf{2 3 . 6 3}$ & $\mathbf{2 9 4 . 3 3}$ \\
$199-1218 \mathrm{~B}-$ & & & \\
$1 \mathrm{H}$ & 0.00 & 0.00 & 0.00 \\
$2 \mathrm{H}$ & 3.90 & 1.16 & 5.06 \\
$3 \mathrm{H}$ & 13.40 & 3.82 & 17.22 \\
$4 \mathrm{H}$ & 22.90 & 5.18 & 28.08 \\
$5 \mathrm{H}$ & 32.40 & 5.66 & 38.06 \\
$6 \mathrm{H}$ & 41.90 & 7.66 & 49.56 \\
$7 \mathrm{H}$ & 51.40 & 7.29 & 58.69 \\
$8 \mathrm{H}$ & 60.90 & 8.39 & 69.29 \\
$9 \mathrm{H}$ & 70.40 & 8.34 & 78.74 \\
$10 \mathrm{H}$ & 79.90 & 10.80 & 90.70 \\
$11 \mathrm{H}$ & 89.40 & 13.38 & 102.78 \\
$12 \mathrm{H}$ & 98.90 & 15.24 & 114.14 \\
& & & \\
& & &
\end{tabular}

\begin{tabular}{crrr}
\hline Core & $\begin{array}{r}\text { Depth } \\
\text { (mbsf) }\end{array}$ & $\begin{array}{r}\text { Offset } \\
(\mathrm{m})\end{array}$ & $\begin{array}{c}\text { Depth } \\
\text { (corrected } \\
\text { rmcd) }\end{array}$ \\
\hline $13 \mathrm{H}$ & 108.40 & 15.49 & 123.89 \\
$14 \mathrm{H}$ & 117.90 & 16.46 & 134.36 \\
$15 \mathrm{H}$ & 127.40 & 17.82 & 145.22 \\
$16 \mathrm{H}$ & 136.90 & 19.02 & 155.92 \\
$17 \mathrm{H}$ & 146.40 & 19.78 & 166.18 \\
$18 \mathrm{H}$ & 155.90 & 20.12 & 176.02 \\
$19 \mathrm{X}$ & 165.40 & 19.04 & 184.44 \\
$20 \mathrm{X}$ & 175.10 & 20.70 & 195.80 \\
$21 \mathrm{X}$ & 184.80 & $\mathbf{2 2 . 2 7}$ & $\mathbf{2 0 7 . 0 7}$ \\
$22 \mathrm{X}$ & 194.40 & $\mathbf{2 3 . 6 5}$ & $\mathbf{2 1 8 . 0 5}$ \\
$23 \mathrm{X}$ & 204.10 & $\mathbf{2 3 . 1 8}$ & $\mathbf{2 2 7 . 2 8}$ \\
$24 \mathrm{X}$ & 213.70 & $\mathbf{2 5 . 8 6}$ & $\mathbf{2 3 9 . 5 6}$ \\
$25 \mathrm{X}$ & 223.30 & $\mathbf{2 7 . 5 9}$ & $\mathbf{2 5 0 . 8 9}$ \\
$26 \mathrm{X}$ & 228.30 & $\mathbf{2 7 . 0 7}$ & $\mathbf{2 5 5 . 3 7}$ \\
$27 \mathrm{X}$ & 237.90 & $\mathbf{2 8 . 5 9}$ & $\mathbf{2 6 6 . 4 9}$ \\
$28 \mathrm{X}$ & 247.50 & $\mathbf{3 1 . 2 0}$ & $\mathbf{2 7 8 . 7 0}$ \\
$29 \mathrm{X}$ & 257.20 & $\mathbf{3 0 . 0 0}$ & $\mathbf{2 8 7 . 2 0}$ \\
$199-1218 \mathrm{C}-$ & & & \\
$1 \mathrm{H}$ & 55.00 & 8.04 & 63.04 \\
$2 \mathrm{H}$ & 64.50 & 9.36 & 73.86 \\
$3 \mathrm{H}$ & 74.00 & 9.78 & 83.78 \\
$4 \mathrm{H}$ & 83.50 & 11.58 & 95.08 \\
$5 \mathrm{H}$ & 93.00 & 12.82 & 105.82 \\
$6 \mathrm{H}$ & 102.50 & 15.58 & 118.08 \\
$7 \mathrm{H}$ & 112.00 & 14.09 & 126.09 \\
$8 \mathrm{H}$ & 121.50 & 14.47 & 135.97 \\
$9 \mathrm{H}$ & 131.00 & 15.90 & 146.90 \\
$10 \mathrm{H}$ & 144.50 & 17.84 & 162.34 \\
$11 \mathrm{H}$ & 154.00 & 18.62 & 172.62 \\
$12 \mathrm{X}$ & 163.50 & 19.78 & 183.28 \\
$13 \mathrm{X}$ & 172.00 & 19.76 & 191.76 \\
$14 \mathrm{X}$ & 181.60 & 19.81 & 201.41 \\
$15 \mathrm{X}$ & 191.20 & $\mathbf{2 1 . 8 2}$ & $\mathbf{2 1 3 . 0 2}$ \\
$16 \mathrm{X}$ & 200.80 & $\mathbf{2 3 . 3 0}$ & $\mathbf{2 2 4 . 1 0}$ \\
$17 \mathrm{X}$ & 210.40 & $\mathbf{2 2 . 8 6}$ & $\mathbf{2 3 3 . 2 6}$ \\
$18 \mathrm{X}$ & 223.00 & $\mathbf{2 3 . 8 8}$ & $\mathbf{2 4 6 . 8 8}$ \\
$19 \mathrm{X}$ & 232.60 & $\mathbf{2 4 . 3 9}$ & $\mathbf{2 5 6 . 9 9}$ \\
$20 \mathrm{X}$ & 242.20 & $\mathbf{2 6 . 9 1}$ & $\mathbf{2 6 9 . 1 1}$ \\
$21 \mathrm{X}$ & 247.20 & $\mathbf{2 7 . 0 3}$ & $\mathbf{2 7 4 . 2 3}$ \\
\hline & & & \\
\hline & & &
\end{tabular}

Bold = changes to rmcd of Pälike et al. (2005). 
Table T15. Tie points used to create the corrected revised composite depth section (corrected rmcd), Site 1218.

\begin{tabular}{|c|c|c|c|c|c|c|}
\hline \multirow[b]{2}{*}{$\begin{array}{l}\text { Hole, core, section, } \\
\text { interval }(\mathrm{cm})\end{array}$} & \multicolumn{2}{|c|}{ Depth } & & \multirow[b]{2}{*}{$\begin{array}{l}\text { Hole, core, section, } \\
\text { interval }(\mathrm{cm})\end{array}$} & \multicolumn{2}{|c|}{ Depth } \\
\hline & (mbsf) & $\begin{array}{l}\text { (Corrected } \\
\text { rmcd) }\end{array}$ & & & (mbsf) & $\begin{array}{l}\text { (Corrected } \\
\text { rmcd) }\end{array}$ \\
\hline $199-$ & & & & $199-$ & & \\
\hline 1218A-1H-5, 54 & 6.54 & 6.54 & Tie to & $1218 \mathrm{~B}-2 \mathrm{H}-1,148$ & 5.38 & 6.54 \\
\hline $1218 \mathrm{~B}-2 \mathrm{H}-5,40$ & 10.30 & 11.46 & Tie to & $1218 \mathrm{~A}-2 \mathrm{H}-2,97$ & 10.68 & 11.46 \\
\hline $1218 \mathrm{~A}-2 \mathrm{H}-7,6$ & 17.26 & 18.04 & Tie to & $1218 \mathrm{~B}-3 \mathrm{H}-1,82$ & 14.22 & 18.04 \\
\hline 1218B-3H-5, 12 & 19.52 & 23.34 & Tie to & $1218 \mathrm{~A}-3 \mathrm{H}-3,30$ & 21.00 & 23.34 \\
\hline $1218 \mathrm{~A}-3 \mathrm{H}-6,132$ & 26.52 & 28.86 & Tie to & 1218B-4H-1, 78 & 23.68 & 28.86 \\
\hline $1218 \mathrm{~B}-4 \mathrm{H}-4,120$ & 28.60 & 33.78 & Tie to & $1218 \mathrm{~A}-4 \mathrm{H}-2,66$ & 29.36 & 33.78 \\
\hline $1218 \mathrm{~A}-4 \mathrm{H}-6,132$ & 36.02 & 40.44 & Tie to & $1218 \mathrm{~B}-5 \mathrm{H}-2,88$ & 34.78 & 40.44 \\
\hline $1218 \mathrm{~B}-5 \mathrm{H}-5,44$ & 38.84 & 44.50 & Tie to & $1218 \mathrm{~A}-5 \mathrm{H}-2,150$ & 39.70 & 44.50 \\
\hline $1218 \mathrm{~A}-5 \mathrm{H}-7,20$ & 45.90 & 50.70 & Tie to & $1218 \mathrm{~B}-6 \mathrm{H}-1,113$ & 43.04 & 50.70 \\
\hline $1218 \mathrm{~B}-6 \mathrm{H}-4,60$ & 47.00 & 54.66 & Tie to & $1218 \mathrm{~A}-6 \mathrm{H}-2,12$ & 47.82 & 54.66 \\
\hline $1218 \mathrm{~A}-6 \mathrm{H}-6,88$ & 54.58 & 61.42 & Tie to & 1218B-7H-2, 128 & 54.13 & 61.42 \\
\hline 1218B-7H-5, 56 & 57.91 & 65.20 & Tie to & $1218 \mathrm{~A}-7 \mathrm{H}-1,144$ & 57.14 & 65.20 \\
\hline 1218A-7H-6, 8 & 63.28 & 71.34 & Tie to & $1218 \mathrm{~B}-8 \mathrm{H}-2,54.5$ & 62.95 & 71.34 \\
\hline $1218 \mathrm{~B}-8 \mathrm{H}-5,76$ & 67.66 & 76.05 & Tie to & $1218 \mathrm{~A}-8 \mathrm{H}-2,138.5$ & 68.09 & 76.05 \\
\hline 1218A-8H-5, 112 & 72.32 & 80.28 & Tie to & $1218 \mathrm{~B}-9 \mathrm{H}-2,3$ & 71.94 & 80.28 \\
\hline 1218B-9H-6, 118 & 79.08 & 87.42 & Tie to & $1218 \mathrm{~A}-9 \mathrm{H}-2,96$ & 77.16 & 87.42 \\
\hline 1218A-9H-6, 112 & 83.32 & 93.58 & Tie to & 1218B-10H-2, 138 & 82.78 & 93.58 \\
\hline 1218B-10H-6, 126 & 88.66 & 99.46 & Tie to & $1218 \mathrm{~A}-10 \mathrm{H}-3,36$ & 87.56 & 99.46 \\
\hline 1218A-10H-6, 92 & 92.62 & 104.52 & Tie to & $1218 \mathrm{~B}-11 \mathrm{H}-2,24$ & 91.14 & 104.52 \\
\hline 1218B-11H-6, 144 & 98.34 & 111.72 & Tie to & $1218 \mathrm{~A}-11 \mathrm{H}-4,12$ & 98.32 & 111.72 \\
\hline 1218A-11H-6, 136 & 102.56 & 115.96 & Tie to & $1218 \mathrm{~B}-12 \mathrm{H}-2,32$ & 100.72 & 115.96 \\
\hline 1218B-12H-5, 144 & 106.34 & 121.58 & Tie to & $1218 \mathrm{~A}-12 \mathrm{H}-3,90.5$ & 107.11 & 121.58 \\
\hline $1218 \mathrm{~A}-12 \mathrm{H}-5,112$ & 110.32 & 124.79 & Tie to & $1218 \mathrm{~B}-13 \mathrm{H}-1,90$ & 109.30 & 124.79 \\
\hline 1218B-13H-6, 44 & 116.34 & 131.83 & Tie to & $1218 \mathrm{~A}-13 \mathrm{H}-3,122$ & 116.92 & 131.83 \\
\hline $1218 \mathrm{~A}-13 \mathrm{H}-6,44$ & 120.64 & 135.55 & Tie to & 1218B-14H-1, 118.5 & 119.09 & 135.55 \\
\hline $1218 \mathrm{~B}-14 \mathrm{H}-4,66$ & 123.06 & 139.52 & Tie to & $1218 \mathrm{~A}-14 \mathrm{H}-2,36$ & 124.10 & 139.52 \\
\hline 1218A-14H-6, 104 & 130.74 & 146.16 & Tie to & 1218B-15H-1, 94 & 128.34 & 146.16 \\
\hline $1218 \mathrm{~B}-15 \mathrm{H}-4,40$ & 132.30 & 150.12 & Tie to & $1218 \mathrm{~A}-15 \mathrm{H}-2,69$ & 133.90 & 150.12 \\
\hline $1218 \mathrm{~A}-15 \mathrm{H}-7,48$ & 141.18 & 157.40 & Tie to & 1218B-16H-1, 148 & 138.38 & 157.40 \\
\hline $1218 \mathrm{~B}-16 \mathrm{H}-6,40$ & 144.30 & 163.32 & Tie to & $1218 \mathrm{~A}-16 \mathrm{H}-3,128$ & 145.48 & 163.32 \\
\hline $1218 \mathrm{~A}-16 \mathrm{H}-6,64$ & 149.34 & 167.18 & Tie to & 1218B-17H-1, 99 & 147.40 & 167.18 \\
\hline 1218B-17H-6, 46 & 154.36 & 174.14 & Tie to & $1218 \mathrm{C}-11 \mathrm{H}-2,0$ & 155.52 & 174.14 \\
\hline $1218 \mathrm{C}-11 \mathrm{H}-5,58$ & 160.58 & 179.20 & Tie to & $1218 \mathrm{~B}-18 \mathrm{H}-3,18$ & 159.08 & 179.20 \\
\hline 1218B-18H-5, 118 & 163.08 & 183.20 & Tie to & $1218 \mathrm{~A}-18 \mathrm{H}-2,94$ & 162.64 & 183.20 \\
\hline $1218 \mathrm{~A}-18 \mathrm{H}-5,8$ & 166.20 & 186.76 & Tie to & $1218 C-12 X-3,48$ & 166.98 & 186.76 \\
\hline $1218 \mathrm{C}-12 \mathrm{X}-6,28$ & 171.28 & 191.06 & Tie to & $1218 \mathrm{~A}-19 \mathrm{H}-1,98$ & 170.68 & 191.06 \\
\hline $1218 \mathrm{~A}-19 \mathrm{H}-6,76$ & 177.96 & 198.34 & Tie to & 1218B-20X-2, 103 & 177.64 & 198.34 \\
\hline 1218B-20X-5, 120 & 182.30 & 203.00 & Tie to & $1218 \mathrm{~A}-20 \mathrm{H}-1,152$ & 180.72 & 203.00 \\
\hline 1218A-20H-6, 124 & 187.94 & 210.22 & Tie to & 1218B-21X-3, 15 & 187.95 & 210.22 \\
\hline 1218B-21X-6, 86 & 193.16 & 215.43 & Tie to & $1218 C-15 X-2,93$ & 193.63 & 215.43 \\
\hline 1218C-15X-4, 118 & 196.88 & 218.70 & Tie to & 1218B-22X-1, 65 & 195.05 & 218.70 \\
\hline 1218B-22X-6, 82 & 202.72 & 226.37 & Tie to & 1218C-16X-2, 77 & 203.08 & 226.37 \\
\hline $1218 \mathrm{C}-16 \mathrm{X}-4,80$ & 206.10 & 229.40 & Tie to & $1218 \mathrm{~A}-23 \mathrm{X}-1,126$ & 206.76 & 229.40 \\
\hline 1218A-23X-5, 10 & 211.60 & 234.24 & Tie to & 1218C-17X-1, 98 & 211.38 & 234.24 \\
\hline $1218 C-17 X-6,12$ & 217.72 & 240.58 & Tie to & $1218 B-24 X-1,102$ & 214.72 & 240.58 \\
\hline $1218 B-24 X-6,94$ & 222.14 & 248.00 & Tie to & $1218 C-18 X-1,112$ & 224.12 & 248.00 \\
\hline $1218 C-18 X-7,36$ & 231.93 & 255.81 & Tie to & 1218B-26X-1, 44 & 228.74 & 255.81 \\
\hline 1218B-26X-5, 90 & 234.70 & 261.77 & Tie to & $1218 \mathrm{~A}-26 \mathrm{X}-2,44$ & 236.24 & 261.77 \\
\hline 1218A-26X-6, 7 & 241.87 & 267.40 & Tie to & 1218B-27X-1, 91 & 238.81 & 267.40 \\
\hline 1218B-27X-6, 119 & 246.59 & 275.18 & Tie to & $1218 \mathrm{C}-21 \mathrm{X}-1,95$ & 248.15 & 275.18 \\
\hline $1218 C-21 X-5,66$ & 253.86 & 280.89 & Tie to & 1218A-28X-2, 136 & 256.36 & 280.89 \\
\hline $1218 A-28 X-7,74$ & 262.74 & 287.27 & Append to & $1218 A-29 X-1,0$ & 263.10 & 287.93 \\
\hline $1218 A-29 X-4,88$ & 268.48 & 292.17 & Append to & $1218 A-30 X-1,0$ & 270.70 & 294.39 \\
\hline $1218 A-30 X-2,140$ & 273.60 & 297.29 & & & & \\
\hline
\end{tabular}

Bold = changes to rmcd of Pälike et al. (2005), italics = shifts according to new offsets higher up in the section. 
Table T16. Revised mapping pairs for adjusting cores to the corrected rmcd splice, Site 1218.

\begin{tabular}{|c|c|c|c|c|c|c|c|}
\hline \multirow[b]{2}{*}{ Core } & \multicolumn{3}{|c|}{ Depth } & \multirow[b]{2}{*}{ Core } & \multicolumn{3}{|c|}{ Depth } \\
\hline & (mbsf) & $\begin{array}{c}\text { (Corrected } \\
\text { rmcd) }\end{array}$ & $\begin{array}{l}\text { Adjusted } \\
\text { (corrected } \\
\text { rmcd) }\end{array}$ & & (mbsf) & $\begin{array}{c}\text { (Corrected } \\
\text { rmcd) }\end{array}$ & $\begin{array}{c}\text { Adjusted } \\
\text { (corrected } \\
\text { rmcd) }\end{array}$ \\
\hline 199-1218A- & & & & $21 x$ & 185.04 & 207.31 & 207.62 \\
\hline $22 x$ & 195.90 & 218.83 & 218.83 & $21 x$ & 187.12 & 209.39 & 209.41 \\
\hline $22 x$ & 203.63 & 226.56 & 226.56 & $21 x$ & 187.95 & 210.22 & 210.22 \\
\hline $23 x$ & 205.50 & 228.14 & 228.14 & $21 x$ & 193.16 & 215.43 & 215.43 \\
\hline $23 x$ & 206.76 & 229.40 & 229.40 & $21 x$ & 194.50 & 216.77 & 216.77 \\
\hline $23 x$ & 211.60 & 234.24 & 234.24 & $22 x$ & 194.40 & 218.05 & 218.05 \\
\hline $23 x$ & 212.42 & 235.06 & 235.06 & $22 x$ & 195.05 & 218.70 & 218.70 \\
\hline $23 x$ & 212.88 & 235.52 & 235.52 & $22 x$ & 202.72 & 226.37 & 226.37 \\
\hline $23 x$ & 215.39 & 238.03 & 238.03 & $22 x$ & 203.99 & 227.64 & 227.64 \\
\hline $24 X$ & 215.10 & 239.58 & 239.58 & $23 x$ & 204.10 & 227.28 & 228.22 \\
\hline $24 X$ & 215.64 & 240.12 & 240.12 & $23 x$ & 205.48 & 228.66 & 229.58 \\
\hline $24 X$ & 216.02 & 240.50 & 240.56 & $23 x$ & 206.20 & 229.38 & 230.00 \\
\hline $24 X$ & 216.42 & 240.90 & 240.90 & $23 x$ & 206.99 & 230.17 & 230.71 \\
\hline $24 X$ & 216.70 & 241.18 & 241.20 & $23 x$ & 208.34 & 231.52 & 231.74 \\
\hline $24 X$ & 217.60 & 242.08 & 242.00 & $23 x$ & 209.65 & 232.83 & 233.04 \\
\hline $24 X$ & 218.19 & 242.67 & 242.40 & $23 x$ & 210.00 & 233.18 & 233.28 \\
\hline $24 X$ & 220.06 & 244.54 & 243.98 & $23 x$ & 211.88 & 235.06 & 235.09 \\
\hline $24 X$ & 221.46 & 245.94 & 245.24 & $23 x$ & 212.68 & 235.86 & 235.73 \\
\hline $24 X$ & 223.28 & 247.76 & 246.70 & $23 x$ & 213.02 & 236.20 & 236.26 \\
\hline $24 X$ & 223.86 & 248.34 & 247.24 & $23 x$ & 213.82 & 237.00 & 237.00 \\
\hline $24 X$ & 224.92 & 249.40 & 248.15 & $24 X$ & 213.70 & 239.56 & 239.61 \\
\hline $25 X$ & 224.70 & 250.43 & 250.33 & $24 X$ & 214.20 & 240.06 & 240.13 \\
\hline $25 X$ & 225.33 & 251.06 & 251.05 & $24 X$ & 214.72 & 240.58 & 240.58 \\
\hline $25 x$ & 225.85 & 251.58 & 251.57 & $24 X$ & 222.14 & 248.00 & 248.00 \\
\hline $25 X$ & 226.06 & 251.79 & 251.67 & $24 X$ & 223.46 & 249.32 & 249.32 \\
\hline $25 x$ & 226.24 & 251.97 & 251.96 & $25 x$ & 223.30 & 250.89 & 250.51 \\
\hline $25 X$ & 226.48 & 252.21 & 252.11 & $25 x$ & 223.78 & 251.37 & 251.24 \\
\hline $25 X$ & 227.14 & 252.87 & 252.45 & $25 x$ & 224.24 & 251.83 & 251.71 \\
\hline $25 X$ & 227.60 & 253.33 & 252.83 & $25 x$ & 224.61 & 252.20 & 252.03 \\
\hline $25 X$ & 228.28 & 254.01 & 253.83 & $25 x$ & 224.72 & 252.31 & 252.21 \\
\hline $25 X$ & 228.80 & 254.53 & 254.73 & $25 x$ & 224.72 & 253.05 & 252.83 \\
\hline $25 x$ & 229.51 & 255.24 & 255.17 & $25 X$ & 227.60 & 255.19 & 255.20 \\
\hline $25 X$ & 230.06 & 255.79 & 255.85 & $26 x$ & 228.30 & 255.37 & 255.37 \\
\hline $25 X$ & 232.54 & 258.27 & 258.14 & $26 x$ & 228.74 & 255.81 & 255.81 \\
\hline $25 X$ & 234.31 & 260.04 & 259.94 & $26 x$ & 234.70 & 261.77 & 261.77 \\
\hline $26 x$ & 234.30 & 259.83 & 260.41 & $26 x$ & 237.89 & 264.96 & 264.96 \\
\hline $26 x$ & 234.24 & 259.77 & 260.35 & $27 X$ & 237.90 & 266.49 & 266.35 \\
\hline $26 x$ & 236.24 & 261.77 & 261.77 & $27 X$ & 238.81 & 267.40 & 267.40 \\
\hline $26 x$ & 241.87 & 267.40 & 267.40 & $27 X$ & 246.59 & 275.18 & 275.18 \\
\hline $26 x$ & 244.17 & 269.70 & 269.50 & $27 X$ & 247.65 & 276.24 & 276.24 \\
\hline $27 X$ & 243.84 & 269.43 & 269.63 & $28 X$ & 247.50 & 278.70 & 278.70 \\
\hline $27 X$ & 246.24 & 271.83 & 271.83 & $28 X$ & 249.20 & 280.40 & 280.32 \\
\hline $27 X$ & 250.43 & 276.02 & 276.02 & $28 x$ & 249.80 & 281.00 & 280.99 \\
\hline $28 x$ & 253.50 & 278.03 & 278.03 & $28 X$ & 250.90 & 282.10 & 281.94 \\
\hline $28 x$ & 255.47 & 280.00 & 279.80 & $28 X$ & 252.67 & 283.87 & 284.07 \\
\hline $28 x$ & 256.36 & 280.89 & 280.89 & $29 x$ & 257.20 & 287.20 & 289.70 \\
\hline $28 x$ & 262.98 & 287.51 & 287.51 & $29 x$ & 258.52 & 288.52 & 290.64 \\
\hline 199-1218B- & & & & $29 X$ & 258.82 & 288.82 & 291.34 \\
\hline $21 \mathrm{X}$ & 184.80 & 207.07 & 207.38 & $29 x$ & 259.74 & 289.74 & 292.08 \\
\hline
\end{tabular}

\begin{tabular}{|c|c|c|c|}
\hline \multirow[b]{2}{*}{ Core } & \multicolumn{3}{|c|}{ Depth } \\
\hline & (mbsf) & $\begin{array}{c}\text { (Corrected } \\
\text { rmcd) }\end{array}$ & $\begin{array}{c}\text { Adjusted } \\
\text { (corrected } \\
\text { rmcd) }\end{array}$ \\
\hline $29 x$ & 261.64 & 291.64 & 293.94 \\
\hline \multicolumn{4}{|c|}{ 199-1218C- } \\
\hline $15 X$ & 191.20 & 213.02 & 213.20 \\
\hline $15 X$ & 192.90 & 214.72 & 214.72 \\
\hline $15 X$ & 193.07 & 214.89 & 214.89 \\
\hline $15 X$ & 193.61 & 215.43 & 215.43 \\
\hline $15 X$ & 196.88 & 218.70 & 218.70 \\
\hline $15 X$ & 199.64 & 221.46 & 221.27 \\
\hline $15 X$ & 200.36 & 222.18 & 221.95 \\
\hline $15 X$ & 200.99 & 222.91 & 222.68 \\
\hline $16 X$ & 200.80 & 224.10 & 223.80 \\
\hline $16 X$ & 202.18 & 226.08 & 225.90 \\
\hline $16 X$ & 203.07 & 226.37 & 226.37 \\
\hline $16 X$ & 206.10 & 229.40 & 229.40 \\
\hline $16 X$ & 207.86 & 231.76 & 231.88 \\
\hline $16 X$ & 209.34 & 233.24 & 233.24 \\
\hline $17 X$ & 210.40 & 233.26 & 233.26 \\
\hline $17 X$ & 211.38 & 234.24 & 234.24 \\
\hline $17 X$ & 217.72 & 240.58 & 240.58 \\
\hline $17 X$ & 218.12 & 240.80 & 240.80 \\
\hline $17 X$ & 218.50 & 241.22 & 241.30 \\
\hline $17 X$ & 218.47 & 241.33 & 241.46 \\
\hline $17 X$ & 219.40 & 241.85 & 241.95 \\
\hline $17 X$ & 220.12 & 243.58 & 243.68 \\
\hline $18 X$ & 223.00 & 246.88 & 246.98 \\
\hline $18 X$ & 224.12 & 248.00 & 248.00 \\
\hline $18 X$ & 231.93 & 255.81 & 255.81 \\
\hline $18 X$ & 232.74 & 257.16 & 257.16 \\
\hline $19 X$ & 232.60 & 256.99 & 257.94 \\
\hline $19 x$ & 234.22 & 259.15 & 259.69 \\
\hline $19 X$ & 235.36 & 260.29 & 260.78 \\
\hline $19 x$ & 236.15 & 261.08 & 261.18 \\
\hline $19 X$ & 237.58 & 262.51 & 262.51 \\
\hline $19 x$ & 239.06 & 263.45 & 263.65 \\
\hline $19 x$ & 239.62 & 264.01 & 264.30 \\
\hline $19 X$ & 240.80 & 265.19 & 266.47 \\
\hline $19 X$ & 242.20 & 267.13 & 269.00 \\
\hline $20 X$ & 242.20 & 269.11 & 268.91 \\
\hline $20 x$ & 244.98 & 272.43 & 272.48 \\
\hline $20 x$ & 247.73 & 275.18 & 275.18 \\
\hline $21 x$ & 247.20 & 274.23 & 274.23 \\
\hline $21 X$ & 248.15 & 275.18 & 275.18 \\
\hline $21 x$ & 253.86 & 280.89 & 280.89 \\
\hline $21 X$ & 255.38 & 282.41 & 283.19 \\
\hline $21 x$ & 256.29 & 283.32 & 284.39 \\
\hline $21 X$ & 256.68 & 284.25 & 285.32 \\
\hline
\end{tabular}

Italics = splice tie points. 
Table T17. Tie points used to create the revised composite depth section (rmcd from Pälike et al., 2005), Site 1219.

\begin{tabular}{|c|c|c|c|c|c|c|}
\hline \multirow{2}{*}{$\begin{array}{l}\text { Hole, core, section, } \\
\text { interval }(\mathrm{cm})\end{array}$} & \multicolumn{2}{|c|}{ Depth } & & \multirow{2}{*}{$\begin{array}{l}\text { Hole, core, section, } \\
\text { interval }(\mathrm{cm})\end{array}$} & \multicolumn{2}{|c|}{ Depth } \\
\hline & (mbsf) & (rmcd) & & & (mbsf) & (rmcd) \\
\hline $199-$ & & & & 199 & & \\
\hline 1219A-1H-4, 128 & 5.78 & 5.78 & Tie to & 1219A-2H-1, 0 & 6.00 & 6.00 \\
\hline $1219 \mathrm{~A}-2 \mathrm{H}-7,78$ & 15.78 & 15.78 & Tie to & $1219 \mathrm{~A}-3 \mathrm{H}-1,28$ & 15.78 & 15.78 \\
\hline 1219A-3H-6, 32 & 23.32 & 23.32 & Tie to & $1219 \mathrm{~B}-2 \mathrm{H}-1,76$ & 21.76 & 23.32 \\
\hline $1219 \mathrm{~B}-2 \mathrm{H}-6,88$ & 29.29 & 30.85 & Tie to & $1219 \mathrm{~A}-4 \mathrm{H}-4,46.5$ & 29.97 & 30.85 \\
\hline 1219A-4H-6, 128 & 33.78 & 34.66 & Tie to & 1219B-3Н-1, 80.5 & 31.31 & 34.66 \\
\hline 1219B-3H-4, 142 & 36.42 & 39.77 & Tie to & $1219 \mathrm{~A}-5 \mathrm{H}-2,148$ & 37.48 & 39.77 \\
\hline 1219A-5H-7, 40 & 43.90 & 46.19 & Tie to & 1219B-4H-2, 28 & 41.78 & 46.19 \\
\hline $1219 \mathrm{~B}-4 \mathrm{H}-4,60$ & 45.10 & 49.51 & Tie to & 1219A-6H-1, 149 & 45.49 & 49.51 \\
\hline 1219A-6H-5, 124 & 51.24 & 55.26 & Tie to & 1219B-5H-1, 86 & 50.36 & 55.26 \\
\hline 1219B-5H-6, 50 & 57.50 & 62.40 & Tie to & $1219 \mathrm{~A}-7 \mathrm{H}-5,98.5$ & 60.33 & 62.40 \\
\hline 1219A-7H-6, 128 & 62.12 & 64.19 & Tie to & 1219B-6H-1, 84.5 & 59.85 & 64.19 \\
\hline 1219B-6H-6, 142 & 67.92 & 72.26 & Tie to & $1219 \mathrm{~A}-8 \mathrm{H}-5,19.5$ & 69.21 & 72.26 \\
\hline 1219A-8H-7, 32 & 72.32 & 75.37 & Tie to & 1219B-7H-1, 88.5 & 69.39 & 75.37 \\
\hline 1219B-7H-6, 40 & 76.40 & 82.38 & Tie to & 1219A-9H-2, 100.5 & 75.01 & 82.38 \\
\hline 1219A-9H-6, 79 & 80.79 & 88.16 & Tie to & 1219B-8H-2, 124 & 80.74 & 88.16 \\
\hline 1219B-8H-7, 66 & 87.66 & 95.08 & Tie to & 1219A-10H-3, 31 & 85.31 & 95.08 \\
\hline 1219A-10H-4, 100 & 87.50 & 97.27 & Tie to & 1219B-9H-1, 31 & 87.81 & 97.27 \\
\hline 1219B-9H-5, 28 & 93.78 & 103.55 & Tie to & 1219A-11H-1, 148 & 92.98 & 103.55 \\
\hline 1219A-11H-5, 8 & 97.58 & 108.15 & Tie to & 1219B-10H-1, 78.5 & 97.79 & 108.15 \\
\hline 1219B-10H-6, 98 & 105.48 & 115.84 & Tie to & $1219 \mathrm{~A}-12 \mathrm{H}-2,103.5$ & 103.55 & 115.84 \\
\hline 1219A-12H-6, 28 & 108.78 & 121.07 & Tie to & 1219B-11H-3, 34 & 109.84 & 121.07 \\
\hline 1219B-11H-7, 64 & 116.14 & 127.37 & Tie to & 1219A-13H-1, 0 & 110.50 & 127.25 \\
\hline $1219 \mathrm{~A}-13 \mathrm{H}-5,124$ & 117.30 & 134.05 & Tie to & 1219B-12H-4, 109 & 121.60 & 134.05 \\
\hline 1219B-12H-7, 74 & 125.74 & 138.19 & Append to & 1219B-13H-1, 0 & 125.50 & 138.95 \\
\hline $1219 \mathrm{~B}-13 \mathrm{H}-7,48$ & 134.98 & 148.43 & Tie to & $1219 \mathrm{~A}-15 \mathrm{H}-1,0$ & 129.50 & 149.51 \\
\hline 1219A-15H-7, 62 & 139.12 & 159.13 & Append to & 1219A-16H-1, 0 & 139.00 & 160.49 \\
\hline $1219 \mathrm{~A}-16 \mathrm{H}-7,50$ & 148.61 & 170.10 & Append to & 1219A-17H-1, 0 & 148.50 & 170.99 \\
\hline 1219A-17H-8, 22 & 157.99 & 180.48 & Append to & 1219A-18H-1, 0 & 158.00 & 181.49 \\
\hline 1219A-18H-7, 48 & 167.48 & 190.97 & Append to & 1219A-19H-1, 0 & 167.50 & 191.99 \\
\hline $1219 \mathrm{~A}-19 \mathrm{H}-7,46$ & 176.96 & 201.45 & Append to & 1219A-20H-1, 0 & 177.00 & 202.49 \\
\hline 1219A-20H-7, 94 & 186.99 & 212.48 & Append to & 1219A-21H-1, 0 & 186.50 & 212.99 \\
\hline 1219A-21H-8, 48 & 195.98 & 222.47 & Append to & 1219A-22H-1, 0 & 196.00 & 223.49 \\
\hline 1219A-22H-7, 48 & 205.48 & 232.97 & Append to & 1219A-23H-1, 0 & 205.50 & 233.99 \\
\hline 1219A-23H-7, 64 & 215.14 & 243.63 & Append to & 1219A-24H-1, 0 & 215.00 & 244.49 \\
\hline 1219A-24H-7, 58 & 224.63 & 254.12 & Append to & 1219A-25X-1, 0 & 224.50 & 254.99 \\
\hline 1219A-25X-2, 52 & 226.52 & 257.01 & Append to & 1219A-26X-1, 0 & 234.10 & 265.59 \\
\hline $1219 \mathrm{~A}-26 \mathrm{X}-3,72$ & 237.82 & 269.31 & Append to & $1219 \mathrm{~A}-27 \mathrm{X}-1,0$ & 243.70 & 276.19 \\
\hline
\end{tabular}

Bold = changes to rmcd of Pälike et al. (2005). 
Table T18. Revised offset for composite depth section, Site 1219 (from Pälike et al., 2005).

\begin{tabular}{crrr}
\hline Core & $\begin{array}{r}\text { Depth } \\
(\mathrm{mbsf})\end{array}$ & $\begin{array}{c}\text { Offset } \\
(\mathrm{m})\end{array}$ & $\begin{array}{r}\text { Depth } \\
\text { (rmcd) }\end{array}$ \\
\hline $199-1219 \mathrm{~A}-$ & & & \\
$1 \mathrm{H}$ & 0.00 & 0.00 & 0.00 \\
$2 \mathrm{H}$ & 6.00 & 0.00 & 6.00 \\
$3 \mathrm{H}$ & 15.50 & 0.00 & 15.50 \\
$4 \mathrm{H}$ & 25.00 & 0.88 & 25.88 \\
$5 \mathrm{H}$ & 34.50 & 2.29 & 36.79 \\
$6 \mathrm{H}$ & 44.00 & 4.02 & 48.02 \\
$7 \mathrm{H}$ & 53.50 & 2.07 & 55.57 \\
$8 \mathrm{H}$ & 63.00 & 3.05 & 66.05 \\
$9 \mathrm{H}$ & 72.50 & 7.37 & 79.87 \\
$10 \mathrm{H}$ & 82.00 & $\mathbf{9 . 7 7}$ & $\mathbf{9 1 . 7 7}$ \\
$11 \mathrm{H}$ & 91.50 & $\mathbf{1 0 . 5 7}$ & $\mathbf{1 0 2 . 0 7}$ \\
$12 \mathrm{H}$ & 101.00 & $\mathbf{1 2 . 2 9}$ & $\mathbf{1 1 3 . 2 9}$ \\
$13 \mathrm{H}$ & 110.50 & $\mathbf{1 6 . 7 5}$ & $\mathbf{1 2 7 . 2 5}$ \\
$14 \mathrm{H}$ & 120.00 & $\mathbf{1 8 . 6 1}$ & $\mathbf{1 3 8 . 6 1}$ \\
$15 \mathrm{H}$ & 129.50 & $\mathbf{2 0 . 0 1}$ & $\mathbf{1 4 9 . 5 1}$ \\
$16 \mathrm{H}$ & 139.00 & $\mathbf{2 1 . 4 9}$ & $\mathbf{1 6 0 . 4 9}$ \\
$17 \mathrm{H}$ & 148.50 & $\mathbf{2 2 . 4 9}$ & $\mathbf{1 7 0 . 9 9}$ \\
$18 \mathrm{H}$ & 158.00 & $\mathbf{2 3 . 4 9}$ & $\mathbf{1 8 1 . 4 9}$ \\
$19 \mathrm{H}$ & 167.50 & $\mathbf{2 4 . 4 9}$ & $\mathbf{1 9 1 . 9 9}$ \\
$2 \mathrm{H}$ & 177.00 & $\mathbf{2 5 . 4 9}$ & $\mathbf{2 0 2 . 4 9}$ \\
$21 \mathrm{H}$ & 186.50 & $\mathbf{2 6 . 4 9}$ & $\mathbf{2 1 2 . 9 9}$ \\
$22 \mathrm{H}$ & 196.00 & $\mathbf{2 7 . 4 9}$ & $\mathbf{2 2 3 . 4 9}$ \\
$23 \mathrm{H}$ & 205.50 & $\mathbf{2 8 . 4 9}$ & $\mathbf{2 3 3 . 9 9}$ \\
$24 \mathrm{H}$ & 215.00 & $\mathbf{2 9 . 4 9}$ & $\mathbf{2 4 4 . 4 9}$ \\
$25 \mathrm{X}$ & 224.50 & $\mathbf{3 0 . 4 9}$ & $\mathbf{2 5 4 . 9 9}$ \\
$26 \mathrm{X}$ & 234.10 & $\mathbf{3 1 . 4 9}$ & $\mathbf{2 6 5 . 5 9}$ \\
$27 \mathrm{X}$ & 243.70 & $\mathbf{3 2 . 4 9}$ & $\mathbf{2 7 6 . 1 9}$ \\
$199-1219 \mathrm{~B}-$ & & & \\
$2 \mathrm{H}$ & 21.00 & 1.56 & 22.56 \\
$3 \mathrm{H}$ & 30.50 & 3.35 & 33.85 \\
$4 \mathrm{H}$ & 40.00 & 4.41 & 44.41 \\
$5 \mathrm{H}$ & 49.50 & 4.90 & 54.40 \\
$6 \mathrm{H}$ & 59.00 & 4.34 & 63.34 \\
$7 \mathrm{H}$ & 68.50 & 5.98 & 74.48 \\
$8 \mathrm{H}$ & 78.00 & $\mathbf{7 . 4 2}$ & $\mathbf{8 5 . 4 2}$ \\
$9 \mathrm{H}$ & 87.50 & $\mathbf{9 . 7 7}$ & $\mathbf{9 7 . 2 7}$ \\
$10 \mathrm{H}$ & 97.00 & $\mathbf{1 0 . 3 6}$ & $\mathbf{1 0 7 . 3 6}$ \\
$11 \mathrm{H}$ & 106.50 & $\mathbf{1 1 . 2 3}$ & $\mathbf{1 1 7 . 7 3}$ \\
$12 \mathrm{H}$ & 116.00 & $\mathbf{1 2 . 4 5}$ & $\mathbf{1 2 8 . 4 5}$ \\
$13 \mathrm{H}$ & 125.50 & $\mathbf{1 3 . 4 5}$ & $\mathbf{1 3 8 . 9 5}$ \\
$14 \mathrm{H}$ & 135.00 & $\mathbf{1 4 . 4 5}$ & $\mathbf{1 4 9 . 4 5}$ \\
$15 \mathrm{H}$ & 144.50 & $\mathbf{1 6 . 3 5}$ & $\mathbf{1 6 0 . 8 5}$ \\
$16 \mathrm{H}$ & 154.00 & $\mathbf{1 6 . 8 1}$ & $\mathbf{1 7 0 . 8 1}$ \\
\hline & & & \\
& & &
\end{tabular}

Bold = changes to ship mcd. 
Table T19. Revised offset for composite depth section, Site 1220.

\begin{tabular}{|c|c|c|c|}
\hline Core & $\begin{array}{l}\text { Depth } \\
\text { (mbsf) }\end{array}$ & $\begin{array}{l}\text { Offset } \\
(\mathrm{m})\end{array}$ & $\begin{array}{l}\text { Depth } \\
\text { (rmcd) }\end{array}$ \\
\hline \multicolumn{4}{|c|}{ 199-1220A- } \\
\hline $1 \mathrm{H}$ & 0.00 & 0.00 & 0.00 \\
\hline $2 \mathrm{H}$ & 9.50 & 0.00 & 9.50 \\
\hline $3 \mathrm{H}$ & 19.00 & 0.00 & 19.00 \\
\hline $4 \mathrm{H}$ & 28.50 & 2.25 & 30.75 \\
\hline $5 \mathrm{H}$ & 38.00 & 3.29 & 41.29 \\
\hline $6 \mathrm{H}$ & 47.50 & 5.34 & 52.84 \\
\hline $7 \mathrm{H}$ & 57.00 & 6.31 & 63.31 \\
\hline $8 \mathrm{H}$ & 66.50 & 7.64 & 74.14 \\
\hline $9 \mathrm{H}$ & 76.00 & 8.65 & 84.65 \\
\hline $10 \mathrm{H}$ & 85.50 & 9.61 & 95.11 \\
\hline $11 \mathrm{H}$ & 95.00 & 9.69 & 104.69 \\
\hline $12 \mathrm{H}$ & 104.50 & 14.10 & 118.60 \\
\hline \multicolumn{4}{|c|}{ 199-1220B- } \\
\hline $1 \mathrm{H}$ & 34.00 & 0.05 & 34.05 \\
\hline $2 \mathrm{H}$ & 43.50 & 2.39 & 45.89 \\
\hline $3 \mathrm{H}$ & 53.00 & 3.39 & 56.39 \\
\hline $4 \mathrm{H}$ & 64.50 & 5.32 & 69.82 \\
\hline $5 \mathrm{H}$ & 74.00 & 6.67 & 80.67 \\
\hline $6 \mathrm{H}$ & 83.50 & 8.53 & 92.03 \\
\hline $7 \mathrm{H}$ & 93.00 & 10.02 & 103.02 \\
\hline $8 \mathrm{H}$ & 102.50 & 11.45 & 113.95 \\
\hline $9 \mathrm{H}$ & 112.00 & 11.95 & 123.95 \\
\hline $10 \mathrm{H}$ & 121.50 & 11.74 & 133.24 \\
\hline $11 \mathrm{H}$ & 131.00 & 16.30 & 147.30 \\
\hline $12 \mathrm{H}$ & 140.50 & 17.82 & 158.32 \\
\hline $13 \mathrm{H}$ & 150.00 & 17.82 & 167.82 \\
\hline $16 X$ & 169.10 & 17.82 & 186.92 \\
\hline $18 X$ & 187.90 & 17.82 & 205.72 \\
\hline $19 x$ & 192.40 & 17.82 & 210.22 \\
\hline $20 X$ & 197.40 & 17.82 & 215.22 \\
\hline \multicolumn{4}{|c|}{ 199-1220C- } \\
\hline $1 \mathrm{H}$ & 25.00 & -1.25 & 23.75 \\
\hline $2 \mathrm{H}$ & 60.00 & 2.17 & 62.17 \\
\hline $3 \mathrm{H}$ & 69.50 & 5.54 & 75.04 \\
\hline $4 \mathrm{H}$ & 79.00 & 7.55 & 86.55 \\
\hline $5 \mathrm{H}$ & 88.50 & 8.50 & 97.00 \\
\hline $6 \mathrm{H}$ & 98.00 & 8.85 & 106.85 \\
\hline $7 \mathrm{H}$ & 107.50 & 10.02 & 117.52 \\
\hline $8 \mathrm{H}$ & 117.00 & 10.35 & 127.35 \\
\hline $9 \mathrm{H}$ & 126.50 & 9.17 & 135.67 \\
\hline $10 \mathrm{H}$ & 136.00 & 10.77 & 146.77 \\
\hline $11 X$ & 145.50 & 12.45 & 157.95 \\
\hline $14 X$ & 173.90 & 14.85 & 188.75 \\
\hline $16 X$ & 193.00 & 15.51 & 208.51 \\
\hline
\end{tabular}

Bold $=$ changes to ship mcd, italics = ship splice, but mcd shifted according to new offsets higher up in the section. 
Table T20. Tie points used to create the revised composite depth section (rmcd), Site 1220.

\begin{tabular}{|c|c|c|c|c|c|c|}
\hline \multirow{2}{*}{$\begin{array}{l}\text { Hole, core, section, } \\
\text { interval }(\mathrm{cm})\end{array}$} & \multicolumn{2}{|c|}{ Depth } & & \multirow{2}{*}{$\begin{array}{l}\text { Hole, core, section, } \\
\text { interval }(\mathrm{cm})\end{array}$} & \multicolumn{2}{|c|}{ Depth } \\
\hline & (mbsf) & $(\mathrm{rmcd})$ & & & (mbsf) & (rmcd) \\
\hline 199 & & & & 199 & & \\
\hline $1220 \mathrm{~A}-1 \mathrm{H}-7,60$ & 9.60 & 9.60 & Tie to & $1220 \mathrm{~A}-2 \mathrm{H}-1,24$ & 9.70 & 9.60 \\
\hline 1220A-2H-CC, 18 & 19.33 & 19.23 & Append to & $1220 \mathrm{~A}-3 \mathrm{H}-1,0$ & 19.00 & 19.00 \\
\hline $1220 \mathrm{~A}-3 \mathrm{H}-6,75$ & 26.70 & 26.70 & Tie to & $1220 \mathrm{C}-1 \mathrm{H}-2,145$ & 27.95 & 26.70 \\
\hline $1220 \mathrm{C}-1 \mathrm{H}-6,80$ & 33.30 & 32.05 & Tie to & $1220 \mathrm{~A}-4 \mathrm{H}-1,130$ & 29.80 & 32.05 \\
\hline $1220 \mathrm{~A}-4 \mathrm{H}-5,84$ & 35.34 & 37.59 & Tie to & $1220 \mathrm{~B}-1 \mathrm{H}-3,54$ & 37.54 & 37.59 \\
\hline 1220B-1H-7, 46 & 42.96 & 43.01 & Tie to & $1220 \mathrm{~A}-5 \mathrm{H}-2,22$ & 39.72 & 43.01 \\
\hline $1220 \mathrm{~A}-5 \mathrm{H}-5,64$ & 44.64 & 47.93 & Tie to & $1220 \mathrm{~B}-2 \mathrm{H}-2,54$ & 45.54 & 47.93 \\
\hline 1220B-2H-6, 10 & 51.10 & 53.49 & Tie to & $1220 \mathrm{~A}-6 \mathrm{H}-1,64.5$ & 48.15 & 53.49 \\
\hline $1220 \mathrm{~A}-6 \mathrm{H}-5,70$ & 54.20 & 59.54 & Tie to & 1220B-3H-3, 15 & 56.15 & 59.54 \\
\hline 1220B-3H-7, 30 & 61.80 & 65.19 & Tie to & $1220 \mathrm{~A}-7 \mathrm{H}-2,37$ & 58.88 & 65.19 \\
\hline $1220 \mathrm{~A}-7 \mathrm{H}-6,60$ & 65.06 & 71.37 & Tie to & 1220B-4H-2, 5 & 66.05 & 71.37 \\
\hline 1220B-4H-5, 28 & 69.88 & 75.20 & Tie to & 1220A-8H-1, 106 & 67.56 & 75.20 \\
\hline $1220 \mathrm{~A}-8 \mathrm{H}-6,58$ & 74.58 & 82.22 & Tie to & 1220B-5H-2, 5 & 75.55 & 82.22 \\
\hline 1220B-5H-4, 148 & 79.98 & 86.65 & Tie to & $1220 \mathrm{~A}-9 \mathrm{H}-2,50$ & 78.00 & 86.65 \\
\hline $1220 \mathrm{~A}-9 \mathrm{H}-7,40$ & 85.40 & 94.05 & Tie to & 1220B-6H-2, 59 & 85.52 & 94.05 \\
\hline 1220B-6H-6, 84 & 91.67 & 100.20 & Tie to & $1220 \mathrm{C}-5 \mathrm{H}-3,28$ & 100.00 & 100.20 \\
\hline $1220 \mathrm{C}-5 \mathrm{H}-5,52$ & 94.94 & 103.44 & Tie to & 1220B-7H-1, 42 & 93.42 & 103.44 \\
\hline 1220B-7H-5, 138 & 100.38 & 110.40 & Tie to & $1220 \mathrm{C}-6 \mathrm{H}-3,55$ & 101.55 & 110.40 \\
\hline 1220C-6H-5, 140 & 105.40 & 114.25 & Tie to & 1220B-8H-1, 30 & 102.80 & 114.25 \\
\hline 1220B-8H-5, 23 & 108.72 & 120.18 & Tie to & $1220 \mathrm{~A}-12 \mathrm{H}-2,8$ & 106.08 & 120.18 \\
\hline 1220A-12H-5, 118 & 111.68 & 125.78 & Tie to & 1220B-9H-2, 33 & 113.83 & 125.78 \\
\hline 1220B-9H-4, 84 & 117.34 & 129.29 & Tie to & $1220 \mathrm{C}-8 \mathrm{H}-2,44$ & 118.94 & 129.29 \\
\hline $1220 \mathrm{C}-8 \mathrm{H}-6,140$ & 125.90 & 136.25 & Tie to & $1220 \mathrm{~B}-10 \mathrm{OH}-3,8$ & 124.52 & 136.26 \\
\hline $1220 \mathrm{~B}-10 \mathrm{H}-7,88$ & 131.32 & 143.06 & Append to & $1220 \mathrm{~B}-11 \mathrm{H}-1,0$ & 131.00 & 147.30 \\
\hline $1220 \mathrm{~B}-11 \mathrm{H}-7,74$ & 140.74 & 157.04 & Append to & 1220C- $11 X-1,0$ & 145.50 & 157.95 \\
\hline $1220 C-11 X-5,140$ & 152.90 & 165.35 & Append to & $1220 B-13 H-1,0$ & 150.00 & 167.82 \\
\hline $1220 \mathrm{~B}-13 \mathrm{H}-1,114$ & 151.14 & 168.96 & Append to & $1220 B-16 X-1,0$ & 169.10 & 186.92 \\
\hline $1220 B-16 X-7,44$ & 178.54 & 196.36 & Append to & $1220 B-18 X-1,0$ & 187.90 & 205.72 \\
\hline $1220 B-18 X-3,144$ & 191.66 & 209.48 & Append to & 1220B-19X-1, 0 & 192.40 & 210.22 \\
\hline 1220B-19X-1, 110 & 193.50 & 211.32 & Append to & $1220 B-20 X-1,0$ & 197.40 & 215.22 \\
\hline 1220B-20X-2, 92 & 199.82 & 217.64 & & & & \\
\hline
\end{tabular}

Bold = changes to ship mcd, italics = ship splice, but mcd shifted according to new offsets higher up in the section. 
Table T21. Mapping pairs for adjusting cores to the corrected rmcd splice, Site 1220.

\begin{tabular}{|c|c|c|c|}
\hline \multirow[b]{2}{*}{ Core } & \multicolumn{3}{|c|}{ Depth } \\
\hline & (mbsf) & $\begin{array}{l}\text { (Corrected } \\
\text { rmcd) }\end{array}$ & $\begin{array}{c}\text { Adjusted } \\
\text { (corrected } \\
\text { rmcd) }\end{array}$ \\
\hline \multicolumn{4}{|c|}{ 199-1220A- } \\
\hline $1 \mathrm{H}$ & 0.01 & 0.01 & 0.01 \\
\hline $1 \mathrm{H}$ & 9.86 & 9.86 & 9.86 \\
\hline $2 \mathrm{H}$ & 9.50 & 9.50 & 9.50 \\
\hline $2 \mathrm{H}$ & 19.37 & 19.37 & 19.37 \\
\hline $3 \mathrm{H}$ & 19.00 & 19.00 & 19.00 \\
\hline $3 \mathrm{H}$ & 26.70 & 26.70 & 26.70 \\
\hline $3 \mathrm{H}$ & 28.99 & 28.99 & 28.85 \\
\hline $4 \mathrm{H}$ & 28.50 & 30.75 & 30.75 \\
\hline $4 \mathrm{H}$ & 29.80 & 32.05 & 32.05 \\
\hline $4 \mathrm{H}$ & 35.34 & 37.59 & 37.59 \\
\hline $4 \mathrm{H}$ & 38.14 & 40.39 & 40.39 \\
\hline $5 \mathrm{H}$ & 38.00 & 41.29 & 41.29 \\
\hline $5 \mathrm{H}$ & 38.47 & 41.76 & 41.79 \\
\hline $5 \mathrm{H}$ & 39.02 & 42.31 & 42.23 \\
\hline $5 \mathrm{H}$ & 39.31 & 42.60 & 42.49 \\
\hline $5 \mathrm{H}$ & 39.72 & 43.01 & 43.01 \\
\hline $5 \mathrm{H}$ & 44.64 & 47.93 & 47.93 \\
\hline $5 \mathrm{H}$ & 45.28 & 48.57 & 48.31 \\
\hline $5 \mathrm{H}$ & 45.64 & 48.93 & 48.63 \\
\hline $5 \mathrm{H}$ & 46.40 & 49.69 & 49.23 \\
\hline $5 \mathrm{H}$ & 47.34 & 50.63 & 50.05 \\
\hline $5 \mathrm{H}$ & 47.74 & 51.03 & 50.39 \\
\hline $5 \mathrm{H}$ & 48.05 & 51.34 & 50.70 \\
\hline $6 \mathrm{H}$ & 47.50 & 52.84 & 52.84 \\
\hline $6 \mathrm{H}$ & 48.15 & 53.49 & 53.49 \\
\hline $6 \mathrm{H}$ & 54.20 & 59.54 & 59.54 \\
\hline $6 \mathrm{H}$ & 55.66 & 61.00 & 61.00 \\
\hline $6 \mathrm{H}$ & 57.39 & 62.73 & 63.00 \\
\hline $7 \mathrm{H}$ & 57.00 & 63.31 & 62.85 \\
\hline $7 \mathrm{H}$ & 58.88 & 65.19 & 65.19 \\
\hline $7 \mathrm{H}$ & 65.06 & 71.37 & 71.37 \\
\hline $7 \mathrm{H}$ & 66.71 & 73.02 & 73.02 \\
\hline $8 \mathrm{H}$ & 66.50 & 74.14 & 74.14 \\
\hline $8 \mathrm{H}$ & 67.56 & 75.20 & 75.20 \\
\hline $8 \mathrm{H}$ & 74.58 & 82.22 & 82.22 \\
\hline $8 \mathrm{H}$ & 75.14 & 82.78 & 82.97 \\
\hline $8 \mathrm{H}$ & 76.51 & 84.15 & 84.34 \\
\hline $9 \mathrm{H}$ & 76.00 & 84.65 & 84.65 \\
\hline $9 \mathrm{H}$ & 76.88 & 85.53 & 85.53 \\
\hline $9 \mathrm{H}$ & 77.12 & 85.77 & 85.95 \\
\hline $9 \mathrm{H}$ & 78.00 & 86.65 & 86.65 \\
\hline $9 \mathrm{H}$ & 85.40 & 94.05 & 94.05 \\
\hline $9 \mathrm{H}$ & 85.89 & 94.54 & 94.54 \\
\hline $10 \mathrm{H}$ & 85.50 & 95.11 & 95.11 \\
\hline $10 \mathrm{H}$ & 89.92 & 99.53 & 99.53 \\
\hline $10 \mathrm{H}$ & 90.78 & 100.39 & 100.39 \\
\hline $10 \mathrm{H}$ & 92.76 & 102.37 & 102.06 \\
\hline $10 \mathrm{H}$ & 94.40 & 104.01 & 103.62 \\
\hline $10 \mathrm{H}$ & 95.23 & 104.84 & 104.45 \\
\hline $11 \mathrm{H}$ & 95.00 & 104.69 & 104.69 \\
\hline $11 \mathrm{H}$ & 96.30 & 105.99 & 105.88 \\
\hline $11 \mathrm{H}$ & 100.12 & 109.81 & 109.81 \\
\hline $11 \mathrm{H}$ & 102.38 & 112.07 & 111.61 \\
\hline $11 \mathrm{H}$ & 104.16 & 113.85 & 113.47 \\
\hline $11 \mathrm{H}$ & 105.12 & 114.81 & 114.81 \\
\hline $12 \mathrm{H}$ & 104.50 & 118.60 & 118.40 \\
\hline $12 \mathrm{H}$ & 106.08 & 120.18 & 120.18 \\
\hline
\end{tabular}

\begin{tabular}{|c|c|c|c|}
\hline \multirow[b]{2}{*}{ Core } & \multicolumn{3}{|c|}{ Depth } \\
\hline & (mbsf) & $\begin{array}{c}\text { (Corrected } \\
\text { rmcd) }\end{array}$ & $\begin{array}{c}\text { Adjusted } \\
\text { (corrected } \\
\text { rmcd) }\end{array}$ \\
\hline $12 \mathrm{H}$ & 111.68 & 125.78 & 125.78 \\
\hline $12 \mathrm{H}$ & 114.37 & 128.47 & 128.30 \\
\hline \multicolumn{4}{|c|}{ 199-1220B- } \\
\hline $1 \mathrm{H}$ & 34.00 & 34.05 & 34.05 \\
\hline $1 \mathrm{H}$ & 37.54 & 37.59 & 37.59 \\
\hline $1 \mathrm{H}$ & 42.96 & 43.01 & 43.01 \\
\hline $1 \mathrm{H}$ & 43.47 & 43.52 & 43.90 \\
\hline $1 \mathrm{H}$ & 43.47 & 43.52 & 43.90 \\
\hline $2 \mathrm{H}$ & 43.50 & 45.89 & 45.89 \\
\hline $2 \mathrm{H}$ & 45.54 & 47.93 & 47.93 \\
\hline $2 \mathrm{H}$ & 51.10 & 53.49 & 53.49 \\
\hline $2 \mathrm{H}$ & 53.59 & 55.98 & 55.98 \\
\hline $3 \mathrm{H}$ & 53.00 & 56.39 & 56.60 \\
\hline $3 \mathrm{H}$ & 56.15 & 59.54 & 59.54 \\
\hline $3 \mathrm{H}$ & 61.80 & 65.19 & 65.19 \\
\hline $3 \mathrm{H}$ & 62.59 & 65.98 & 65.98 \\
\hline $4 \mathrm{H}$ & 64.50 & 69.82 & 69.82 \\
\hline $4 \mathrm{H}$ & 66.05 & 71.37 & 71.37 \\
\hline $4 \mathrm{H}$ & 69.88 & 75.20 & 75.20 \\
\hline $4 \mathrm{H}$ & 70.68 & 76.00 & 76.08 \\
\hline $4 \mathrm{H}$ & 71.88 & 77.20 & 77.26 \\
\hline $4 \mathrm{H}$ & 72.64 & 77.96 & 78.10 \\
\hline $4 \mathrm{H}$ & 73.73 & 79.05 & 79.19 \\
\hline $5 \mathrm{H}$ & 74.00 & 80.67 & 80.67 \\
\hline $5 \mathrm{H}$ & 75.55 & 82.22 & 82.22 \\
\hline $5 \mathrm{H}$ & 79.98 & 86.65 & 86.65 \\
\hline $5 \mathrm{H}$ & 84.06 & 90.73 & 90.73 \\
\hline $6 \mathrm{H}$ & 83.50 & 92.03 & 92.03 \\
\hline $6 \mathrm{H}$ & 85.52 & 94.05 & 94.05 \\
\hline $6 \mathrm{H}$ & 91.67 & 100.20 & 100.20 \\
\hline $6 \mathrm{H}$ & 93.32 & 101.85 & 101.85 \\
\hline $7 \mathrm{H}$ & 93.00 & 103.02 & 103.02 \\
\hline $7 \mathrm{H}$ & 93.42 & 103.44 & 103.44 \\
\hline $7 \mathrm{H}$ & 100.38 & 110.40 & 110.40 \\
\hline $7 \mathrm{H}$ & 102.96 & 112.98 & 112.70 \\
\hline $8 \mathrm{H}$ & 102.50 & 113.95 & 113.95 \\
\hline $8 \mathrm{H}$ & 103.04 & 114.49 & 114.49 \\
\hline $8 \mathrm{H}$ & 108.73 & 120.18 & 120.18 \\
\hline $8 \mathrm{H}$ & 109.20 & 120.65 & 120.65 \\
\hline $8 \mathrm{H}$ & 112.39 & 123.84 & 123.50 \\
\hline $9 \mathrm{H}$ & 112.00 & 123.95 & 123.95 \\
\hline $9 \mathrm{H}$ & 113.83 & 125.78 & 125.78 \\
\hline $9 \mathrm{H}$ & 117.34 & 129.29 & 129.29 \\
\hline $9 \mathrm{H}$ & 121.23 & 133.18 & 133.30 \\
\hline $10 \mathrm{H}$ & 121.50 & 133.24 & 133.24 \\
\hline $10 \mathrm{H}$ & 124.52 & 136.26 & 136.26 \\
\hline $10 \mathrm{H}$ & 131.65 & 143.39 & 143.39 \\
\hline $11 \mathrm{H}$ & 131.00 & 147.30 & 147.30 \\
\hline $11 \mathrm{H}$ & 141.09 & 157.39 & 157.39 \\
\hline $12 \mathrm{H}$ & 140.50 & 158.32 & 158.32 \\
\hline $12 \mathrm{H}$ & 150.56 & 168.38 & 168.38 \\
\hline $13 x$ & 150.00 & 167.82 & 167.82 \\
\hline $13 x$ & 159.19 & 177.01 & 177.01 \\
\hline $16 X$ & 169.10 & 186.92 & 186.92 \\
\hline $16 X$ & 178.78 & 196.60 & 196.60 \\
\hline $18 X$ & 187.90 & 205.72 & 205.72 \\
\hline $18 X$ & 191.89 & 209.71 & 209.71 \\
\hline $19 X$ & 192.40 & 210.22 & 210.22 \\
\hline
\end{tabular}

\begin{tabular}{|c|c|c|c|}
\hline \multirow[b]{2}{*}{ Core } & \multicolumn{3}{|c|}{ Depth } \\
\hline & (mbsf) & $\begin{array}{c}\text { (Corrected } \\
\text { rmcd) }\end{array}$ & $\begin{array}{c}\text { Adjusted } \\
\text { (corrected } \\
\text { rmcd) }\end{array}$ \\
\hline $19 x$ & 193.73 & 211.55 & 211.55 \\
\hline $20 x$ & 197.40 & 215.22 & 215.22 \\
\hline $20 x$ & 200.03 & 217.85 & 217.85 \\
\hline \multicolumn{4}{|c|}{ 199-1220C- } \\
\hline $1 \mathrm{H}$ & 25.00 & 23.75 & 23.45 \\
\hline $1 \mathrm{H}$ & 26.16 & 24.91 & 24.61 \\
\hline $1 \mathrm{H}$ & 27.95 & 26.70 & 26.70 \\
\hline $1 \mathrm{H}$ & 33.30 & 32.05 & 32.05 \\
\hline $1 \mathrm{H}$ & 35.05 & 33.80 & 33.80 \\
\hline $2 \mathrm{H}$ & 60.00 & 62.17 & 61.80 \\
\hline $2 \mathrm{H}$ & 62.36 & 64.53 & 65.07 \\
\hline $2 \mathrm{H}$ & 63.85 & 66.02 & 66.63 \\
\hline $2 \mathrm{H}$ & 66.30 & 68.47 & 68.47 \\
\hline $2 \mathrm{H}$ & 68.66 & 70.83 & 70.83 \\
\hline $3 \mathrm{H}$ & 69.50 & 75.04 & 75.35 \\
\hline $3 \mathrm{H}$ & 71.20 & 76.74 & 76.74 \\
\hline $3 \mathrm{H}$ & 72.78 & 78.32 & 78.10 \\
\hline $3 \mathrm{H}$ & 75.08 & 80.62 & 80.22 \\
\hline $3 \mathrm{H}$ & 79.48 & 85.02 & 84.60 \\
\hline $4 \mathrm{H}$ & 79.00 & 86.55 & 86.55 \\
\hline $4 \mathrm{H}$ & 88.69 & 96.24 & 96.24 \\
\hline $5 \mathrm{H}$ & 88.50 & 97.00 & 97.00 \\
\hline $5 \mathrm{H}$ & 91.70 & 100.20 & 100.20 \\
\hline $5 \mathrm{H}$ & 94.94 & 103.44 & 103.44 \\
\hline $5 \mathrm{H}$ & 98.59 & 107.09 & 106.80 \\
\hline $6 \mathrm{H}$ & 98.00 & 106.85 & 106.85 \\
\hline $6 \mathrm{H}$ & 101.55 & 110.40 & 110.40 \\
\hline $6 \mathrm{H}$ & 105.64 & 114.49 & 114.49 \\
\hline $6 \mathrm{H}$ & 105.96 & 114.81 & 114.81 \\
\hline $6 \mathrm{H}$ & 107.78 & 116.63 & 116.40 \\
\hline $7 \mathrm{H}$ & 107.50 & 117.52 & 117.52 \\
\hline $7 \mathrm{H}$ & 108.08 & 118.10 & 118.10 \\
\hline $7 \mathrm{H}$ & 108.62 & 118.64 & 118.75 \\
\hline $7 \mathrm{H}$ & 109.16 & 119.18 & 119.43 \\
\hline $7 \mathrm{H}$ & 109.92 & 119.94 & 120.44 \\
\hline $7 \mathrm{H}$ & 111.10 & 121.12 & 121.24 \\
\hline $7 \mathrm{H}$ & 112.66 & 122.68 & 123.02 \\
\hline $7 \mathrm{H}$ & 115.16 & 125.18 & 125.24 \\
\hline $7 \mathrm{H}$ & 116.56 & 126.58 & 126.35 \\
\hline $7 \mathrm{H}$ & 117.46 & 127.48 & 127.25 \\
\hline $8 \mathrm{H}$ & 117.00 & 127.35 & 127.35 \\
\hline $8 \mathrm{H}$ & 118.94 & 129.29 & 129.29 \\
\hline $8 \mathrm{H}$ & 125.91 & 136.26 & 136.26 \\
\hline $8 \mathrm{H}$ & 126.76 & 137.11 & 137.11 \\
\hline $9 \mathrm{H}$ & 126.50 & 135.67 & 135.67 \\
\hline $9 \mathrm{H}$ & 136.66 & 145.83 & 145.83 \\
\hline $10 \mathrm{H}$ & 136.00 & 146.77 & 146.77 \\
\hline $10 \mathrm{H}$ & 145.86 & 156.63 & 156.63 \\
\hline $11 x$ & 145.50 & 157.95 & 157.95 \\
\hline $11 x$ & 153.09 & 165.54 & 165.54 \\
\hline $14 X$ & 173.90 & 188.75 & 188.75 \\
\hline $14 X$ & 182.48 & 197.33 & 197.33 \\
\hline $16 X$ & 193.00 & 208.51 & 208.51 \\
\hline $16 x$ & 193.97 & 209.48 & 209.48 \\
\hline
\end{tabular}

Italics = splice tie points. 
Table T22. Cleaned magnetic susceptibility and gamma ray attenuation composite records from Site U1331 on rmcd (m revised CCSF-A).

\begin{tabular}{|c|c|c|c|}
\hline \multicolumn{2}{|c|}{ Gamma ray attenuation } & \multicolumn{2}{|c|}{ Magnetic susceptibility } \\
\hline $\begin{array}{l}\text { Depth (rmcd } \\
\text { [m revised } \\
\text { CCSF-A]) }\end{array}$ & $\begin{array}{l}\text { Density } \\
\left(\mathrm{g} / \mathrm{cm}^{3}\right)\end{array}$ & $\begin{array}{l}\text { Depth (rmcd } \\
\text { [m revised } \\
\text { CCSF-A]) }\end{array}$ & $\left(10^{-5}\right)$ \\
\hline 1.57 & 1.384 & 0.07 & 22.4 \\
\hline 1.59 & 1.382 & 0.10 & 21.6 \\
\hline 1.62 & 1.376 & 0.12 & 21.6 \\
\hline 1.64 & 1.375 & 0.15 & 21.4 \\
\hline 1.67 & 1.386 & 0.17 & 21.6 \\
\hline 1.69 & 1.376 & 0.20 & 22.2 \\
\hline 1.72 & 1.380 & 0.22 & 23.0 \\
\hline 1.74 & 1.373 & 0.25 & 24.0 \\
\hline 1.77 & 1.374 & 0.27 & 24.8 \\
\hline 1.79 & 1.372 & 0.29 & 25.8 \\
\hline 1.82 & 1.378 & 0.32 & 26.4 \\
\hline 1.84 & 1.376 & 0.34 & 27.0 \\
\hline 1.87 & 1.381 & 0.37 & 27.0 \\
\hline 1.89 & 1.374 & 0.39 & 27.0 \\
\hline 1.92 & 1.377 & 0.42 & 27.4 \\
\hline 1.94 & 1.379 & 0.44 & 28.0 \\
\hline 1.97 & 1.372 & 0.47 & 28.0 \\
\hline 1.99 & 1.369 & 0.49 & 28.4 \\
\hline 2.02 & 1.377 & 0.51 & 28.4 \\
\hline 2.04 & 1.383 & 0.54 & 28.2 \\
\hline 2.07 & 1.376 & 0.56 & 27.8 \\
\hline 2.09 & 1.376 & 0.59 & 28.4 \\
\hline 2.12 & 1.388 & 0.61 & 28.8 \\
\hline 2.14 & 1.372 & 0.64 & 29.0 \\
\hline 2.17 & 1.380 & 0.66 & 29.8 \\
\hline 2.19 & 1.389 & 0.69 & 31.8 \\
\hline 2.22 & 1.361 & 0.71 & 34.0 \\
\hline 2.24 & 1.373 & 0.73 & 32.0 \\
\hline 2.27 & 1.392 & 0.76 & 36.8 \\
\hline 2.29 & 1.378 & 0.78 & 40.4 \\
\hline 2.32 & 1.384 & 0.81 & 39.0 \\
\hline 2.34 & 1.381 & 0.83 & 40.0 \\
\hline 2.37 & 1.383 & 0.86 & 39.0 \\
\hline 2.39 & 1.367 & 0.88 & 38.0 \\
\hline 2.42 & 1.374 & 0.91 & 38.0 \\
\hline 2.44 & 1.378 & 0.93 & 37.0 \\
\hline 2.47 & 1.393 & 0.96 & 36.4 \\
\hline 2.49 & 1.399 & 0.98 & 36.4 \\
\hline 2.52 & 1.399 & 1.00 & 35.6 \\
\hline 2.55 & 1.384 & 1.03 & 36.4 \\
\hline 2.57 & 1.395 & 1.05 & 36.0 \\
\hline 2.60 & 1.401 & 1.08 & 35.6 \\
\hline 2.62 & 1.415 & 1.10 & 35.6 \\
\hline 2.65 & 1.401 & 1.13 & 35.6 \\
\hline 2.67 & 1.393 & 1.15 & 36.0 \\
\hline 2.70 & 1.409 & 1.18 & 36.0 \\
\hline 2.72 & 1.400 & 1.20 & 35.6 \\
\hline 2.75 & 1.386 & 1.22 & 35.6 \\
\hline 2.77 & 1.406 & 1.25 & 35.6 \\
\hline 2.80 & 1.411 & 1.27 & 35.8 \\
\hline 2.82 & 1.396 & 1.30 & 35.0 \\
\hline 2.85 & 1.410 & 1.32 & 35.8 \\
\hline 2.87 & 1.394 & 1.35 & 35.2 \\
\hline 2.90 & 1.399 & 1.37 & 34.6 \\
\hline 2.92 & 1.395 & 1.40 & 34.0 \\
\hline 2.95 & 1.399 & 1.42 & 34.0 \\
\hline 2.97 & 1.425 & 1.44 & 31.0 \\
\hline 3.10 & 1.373 & 1.47 & 31.0 \\
\hline 3.13 & 1.388 & 1.54 & 30.0 \\
\hline 3.15 & 1.400 & 1.57 & 31.2 \\
\hline 3.18 & 1.403 & 1.59 & 32.0 \\
\hline 3.20 & 1.406 & 1.62 & 32.8 \\
\hline 3.23 & 1.425 & 1.64 & 32.8 \\
\hline 3.25 & 1.410 & 1.67 & 32.8 \\
\hline 3.28 & 1.398 & 1.69 & 32.4 \\
\hline 3.30 & 1.410 & 1.72 & 32.4 \\
\hline
\end{tabular}

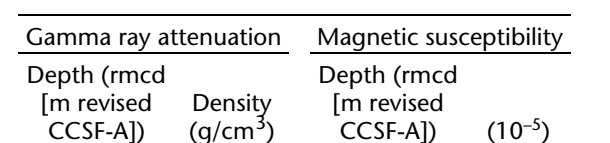

\begin{tabular}{cccc} 
CCSF-A $])$ & $\left(\mathrm{g} / \mathrm{cm}^{3}\right)$ & $\begin{array}{c}\text { CCSF-A }]) \\
\left(10^{-5}\right)\end{array}$ \\
\hline 3.33 & 1.410 & 1.74 & 32.6 \\
3.35 & 1.412 & 1.77 & 32.4 \\
3.38 & 1.414 & 1.79 & 31.8
\end{tabular}

3.38

3.40

3.43
3.45

3.48

3.50

3.53

3.55

3.58

3.60

3.63

3.65

3.68

3.70

3.73

3.75

3.78

3.80

3.83

3.85

3.88

3.90

3.93

3.95

3.98

4.00

4.03

4.05

4.08

4.10

4.13

4.15

4.18

4.20

4.23

4.25

4.28

4.30

4.33

4.35

4.38

4.40

4.43

4.45

4.48

4.50

4.53

4.55

4.58

4.60

4.63

4.65

4.68

4.70

4.73

4.75

4.78

4.80

4.83

4.85

4.88

4.90

4.93

$\begin{array}{lll}1.414 & 1.82 & 31.8\end{array}$

$\begin{array}{lll}1.406 & 1.85 & 31.4\end{array}$

$\begin{array}{lll}1.393 & 1.87 & 30.6\end{array}$

$\begin{array}{lll}1.391 & 1.90 & 30.8\end{array}$

$\begin{array}{lll}1.373 & 1.92 & 30.4\end{array}$

$\begin{array}{lll}1.420 & 1.95 & 30.2\end{array}$

$\begin{array}{lll}1.407 & 1.97 & 29.4\end{array}$

$\begin{array}{lll}1.400 & 2.00 & 29.4\end{array}$

1.398

1.419

1.400

1.397

1.393

1.397

1.412

1.397

1.387

1.405

1.403

1.395

1.410

1.418
1.410

1.399

1.403

1.416

1.418

1.415

1.413

1.414
1.401

1.413

1.406

1.404

1.415

1.413

1.412

1.406

1.411
1.413

1.413
1.416

1.433

1.414

1.406

1.411

1.424
1.419

1.407

1.408

1.415

1.407

1.404

1.409

1.408

1.412

1.398

1.407

1.401

1.407

1.401

1.402

1.403
2.02

$\begin{array}{ll}2.05 & 28.4\end{array}$

2.07

$2.10 \quad 28.2$

$2.12 \quad 28.0$

$2.15 \quad 27.4$

$2.17 \quad 27.6$

$2.20 \quad 27.0$

$2.22 \quad 27.0$

2.25

2.27

2.30

2.32

2.35

2.37

2.40

2.42

2.45

2.47

2.50

2.52

2.55

2.57

2.60

2.62

2.65

2.67

2.70

2.72

2.75

2.77

2.80

2.83

2.85

2.88

2.90

2.93

2.95

2.98

3.03

3.05

3.07

3.10

3.12

3.15

3.17

3.20

3.22

3.25

3.27

3.29

3.32

3.34

3.37

3.39

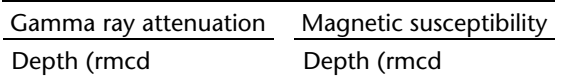

[ $\mathrm{m}$ revised Density [m revised

\begin{tabular}{cccc}
$\begin{array}{c}\text { CCSF-A] }) \\
\text { (g/cm }\end{array}$ & $\begin{array}{c}\text { CCSF-A }]) \\
\left(10^{-5}\right)\end{array}$ \\
\hline 4.95 & 1.416 & 3.42 & 27.0 \\
4.98 & 1.395 & 3.44 & 24.0
\end{tabular}

$3.44 \quad 24.0$

$\begin{array}{llll}5.00 & 1.405 & 3.46 & 25.0 \\ 5.03 & 1.417 & 3.49 & 25.4\end{array}$

$\begin{array}{llll}5.07 & 1.438 & 3.51 & 27.8\end{array}$

$\begin{array}{llll}5.07 & 1.438 & 3.51 & 27.8 \\ 5.10 & 1.430 & 3.54 & 27.2\end{array}$

$\begin{array}{llll}5.12 & 1.442 & 3.56 & 26.4\end{array}$

$\begin{array}{llll}5.12 & 1.442 & 3.56 & 26.4 \\ 5.15 & 1.430 & 3.59 & 26.4\end{array}$

$\begin{array}{llll}5.17 & 1.404 & 3.61 & 26.8\end{array}$

$\begin{array}{llll}5.20 & 1.414 & 3.64 & 27.0\end{array}$

$\begin{array}{llll}5.22 & 1.414 & 3.66 & 26.8\end{array}$

$\begin{array}{llll}5.25 & 1.430 & 3.68 & 26.6\end{array}$

$\begin{array}{llll}5.28 & 1.414 & 3.71 & 26.4\end{array}$

$\begin{array}{llll}5.30 & 1.422 & 3.73 & 26.8\end{array}$

$\begin{array}{llll}5.33 & 1.419 & 3.76 & 26.4\end{array}$

$\begin{array}{llll}5.35 & 1.420 & 3.78 & 27.0\end{array}$

$\begin{array}{llll}5.38 & 1.415 & 3.81 & 27.0\end{array}$

$\begin{array}{llll}5.40 & 1.410 & 3.83 & 27.2\end{array}$

$\begin{array}{llll}5.43 & 1.403 & 3.86 & 28.4\end{array}$

$\begin{array}{llll}5.45 & 1.419 & 3.88 & 28.8\end{array}$

$\begin{array}{llll}5.48 & 1.419 & 3.90 & 30.0\end{array}$

$\begin{array}{llll}5.50 & 1.413 & 3.93 & 30.8\end{array}$

$\begin{array}{llll}5.53 & 1.409 & 3.95 & 30.6\end{array}$

$\begin{array}{llll}5.55 & 1.424 & 3.98 & 30.0\end{array}$

$\begin{array}{llll}5.58 & 1.424 & 4.00 & 30.0\end{array}$

$\begin{array}{llll}5.60 & 1.408 & & \\ 5.63 & 1.422 & 4.03 & 30.8\end{array}$

$\begin{array}{llll}5.63 & 1.422 & 4.03 & 30.8 \\ 5.65 & 1.419 & 4.05 & 31.9\end{array}$

$\begin{array}{llll}5.68 & 1.421 & 4.08 & 32.2\end{array}$

$\begin{array}{llll}5.70 & 1.428 & 4.10 & 33.0\end{array}$

$\begin{array}{llll}5.73 & 1.428 & 4.13 & 33.0\end{array}$

$\begin{array}{llll}5.75 & 1.430 & 4.15 & 33.0\end{array}$

$\begin{array}{llll}5.78 & 1.414 & 4.18 & 33.3\end{array}$

$\begin{array}{llll}5.80 & 1.428 & 4.20 & 33.3\end{array}$

$\begin{array}{llll}5.83 & 1.402 & 4.23 & 33.7\end{array}$

$\begin{array}{llll}5.85 & 1.399 & 4.25 & 34.8\end{array}$

$\begin{array}{llll}5.88 & 1.412 & 4.28 & 34.8\end{array}$

$\begin{array}{llll}5.90 & 1.403 & 4.30 & 35.2\end{array}$

$\begin{array}{llll}5.93 & 1.412 & 4.33 & 35.2\end{array}$

$\begin{array}{llll}5.95 & 1.410 & 4.35 & 34.8\end{array}$

$\begin{array}{llll}5.98 & 1.400 & 4.38 & 35.2\end{array}$

$\begin{array}{llll}6.00 & 1.412 & 4.40 & 35.2\end{array}$

$\begin{array}{llll}6.03 & 1.433 & 4.43 & 35.2\end{array}$

$\begin{array}{llll}6.05 & 1.431 & 4.45 & 35.2\end{array}$

$\begin{array}{llll}6.08 & 1.406 & 4.48 & 34.1\end{array}$

$\begin{array}{llll}6.10 & 1.416 & 4.50 & 34.1\end{array}$

$\begin{array}{llll}6.13 & 1.417 & 4.53 & 34.1\end{array}$

$\begin{array}{llll}6.15 & 1.433 & 4.55 & 34.1\end{array}$

$\begin{array}{llll}6.18 & 1.430 & 4.58 & 34.4\end{array}$

$\begin{array}{llll}6.20 & 1.428 & 4.60 & 35.2\end{array}$

$\begin{array}{llll}6.23 & 1.430 & 4.63 & 34.8\end{array}$

$\begin{array}{llll}6.25 & 1.422 & 4.65 & 34.8\end{array}$

$\begin{array}{llll}6.28 & 1.413 & 4.68 & 34.8\end{array}$

$\begin{array}{llll}6.30 & 1.420 & 4.70 & 34.4\end{array}$

$\begin{array}{llll}6.33 & 1.420 & 4.73 & 34.4\end{array}$

$\begin{array}{llll}6.35 & 1.417 & 4.75 & 34.1\end{array}$

$\begin{array}{llll}6.38 & 1.401 & 4.78 & 34.4\end{array}$

$\begin{array}{llll}6.40 & 1.436 & 4.80 & 34.8\end{array}$

$\begin{array}{llll}6.43 & 1.415 & 4.83 & 34.4\end{array}$

$\begin{array}{llll}6.45 & 1.409 & 4.85 & 34.4\end{array}$

$\begin{array}{llll}6.48 & 1.405 & 4.88 & 34.4\end{array}$

$\begin{array}{llll}6.50 & 1.401 & 4.90 & 35.2\end{array}$

$\begin{array}{llll}6.53 & 1.414 & 4.93 & 35.5\end{array}$

Only a portion of this table appears here. The complete table is available in ASCII. 
Table T23. Cleaned magnetic susceptibility and gamma ray attenuation composite records from Site U1332 on rmcd (m revised CCSF-A).

\begin{tabular}{|c|c|c|c|}
\hline \multicolumn{2}{|c|}{ Gamma ray attenuation } & \multicolumn{2}{|c|}{ Magnetic susceptibility } \\
\hline $\begin{array}{l}\text { Depth (rmcd } \\
\text { [m revised } \\
\text { CCSF-A]) }\end{array}$ & $\begin{array}{l}\text { Density } \\
\left(\mathrm{g} / \mathrm{cm}^{3}\right)\end{array}$ & $\begin{array}{l}\text { Depth (rmcd } \\
\text { [m revised } \\
\text { CCSF-A]) }\end{array}$ & $\left(10^{-5}\right)$ \\
\hline 1.00 & 1.233 & 1.03 & 30.8 \\
\hline 1.00 & 1.257 & 1.05 & 30.4 \\
\hline 1.03 & 1.251 & 1.08 & 30.4 \\
\hline 1.05 & 1.229 & 1.10 & 30.4 \\
\hline 1.05 & 1.243 & 1.13 & 30.4 \\
\hline 1.08 & 1.237 & 1.15 & 30.4 \\
\hline 1.10 & 1.245 & 1.18 & 29.7 \\
\hline 1.10 & 1.260 & 1.20 & 29.3 \\
\hline 1.13 & 1.245 & 1.23 & 28.6 \\
\hline 1.15 & 1.240 & 1.25 & 28.9 \\
\hline 1.15 & 1.233 & 1.28 & 28.2 \\
\hline 1.18 & 1.253 & 1.30 & 27.5 \\
\hline 1.20 & 1.233 & 1.33 & 28.2 \\
\hline 1.20 & 1.243 & 1.35 & 28.6 \\
\hline 1.23 & 1.233 & 1.38 & 28.6 \\
\hline 1.25 & 1.213 & 1.40 & 28.9 \\
\hline 1.25 & 1.237 & 1.43 & 29.3 \\
\hline 1.28 & 1.220 & 1.45 & 28.6 \\
\hline 1.30 & 1.240 & 1.48 & 27.5 \\
\hline 1.30 & 1.230 & 1.52 & 23.1 \\
\hline 1.30 & 1.212 & 1.57 & 24.5 \\
\hline 1.33 & 1.232 & 1.59 & 26.0 \\
\hline 1.33 & 1.243 & 1.62 & 27.1 \\
\hline 1.35 & 1.231 & 1.64 & 28.2 \\
\hline 1.35 & 1.237 & 1.67 & 27.1 \\
\hline 1.35 & 1.234 & 1.69 & 25.6 \\
\hline 1.38 & 1.248 & 1.72 & 25.3 \\
\hline 1.38 & 1.247 & 1.74 & 24.5 \\
\hline 1.40 & 1.259 & 1.77 & 23.8 \\
\hline 1.40 & 1.244 & 1.79 & 25.3 \\
\hline 1.40 & 1.245 & 1.82 & 27.5 \\
\hline 1.43 & 1.252 & 1.84 & 28.9 \\
\hline 1.43 & 1.249 & 1.87 & 29.7 \\
\hline 1.45 & 1.231 & 1.89 & 30.0 \\
\hline 1.45 & 1.219 & 1.92 & 30.8 \\
\hline 1.45 & 1.248 & 1.94 & 31.9 \\
\hline 1.54 & 1.234 & 1.97 & 31.9 \\
\hline 1.56 & 1.222 & 1.99 & 32.6 \\
\hline 1.57 & 1.237 & 2.02 & 32.6 \\
\hline 1.57 & 1.212 & 2.04 & 32.2 \\
\hline 1.58 & 1.245 & 2.07 & 31.9 \\
\hline 1.59 & 1.217 & 2.09 & 31.9 \\
\hline 1.61 & 1.247 & 2.12 & 32.2 \\
\hline 1.62 & 1.239 & 2.14 & 32.6 \\
\hline 1.62 & 1.241 & 2.17 & 33.0 \\
\hline 1.63 & 1.242 & 2.19 & 32.6 \\
\hline 1.64 & 1.242 & 2.22 & 32.6 \\
\hline 1.66 & 1.241 & 2.24 & 31.9 \\
\hline 1.67 & 1.244 & 2.27 & 32.2 \\
\hline 1.67 & 1.229 & 2.29 & 30.8 \\
\hline 1.68 & 1.220 & 2.32 & 28.9 \\
\hline 1.69 & 1.226 & 2.34 & 25.3 \\
\hline 1.71 & 1.238 & 2.37 & 23.4 \\
\hline 1.72 & 1.230 & 2.39 & 22.0 \\
\hline 1.72 & 1.232 & 2.42 & 15.8 \\
\hline 1.73 & 1.236 & 2.44 & 18.0 \\
\hline 1.74 & 1.226 & 2.47 & 13.9 \\
\hline 1.76 & 1.260 & 2.49 & 16.1 \\
\hline 1.77 & 1.228 & 2.52 & 18.0 \\
\hline 1.77 & 1.237 & 2.54 & 19.8 \\
\hline 1.78 & 1.244 & 2.57 & 19.8 \\
\hline 1.79 & 1.252 & 2.59 & 22.0 \\
\hline 1.81 & 1.249 & & \\
\hline 1.82 & 1.236 & 2.62 & 22.0 \\
\hline 1.82 & 1.246 & 2.65 & 24.2 \\
\hline 1.83 & 1.272 & 2.67 & 25.0 \\
\hline
\end{tabular}

\begin{tabular}{|c|c|c|c|}
\hline \multicolumn{2}{|c|}{ Gamma ray attenuation } & \multicolumn{2}{|c|}{ Magnetic susceptibility } \\
\hline $\begin{array}{c}\text { Depth }(\mathrm{rmcd} \\
\text { [m revised } \\
\text { CCSF-A] })\end{array}$ & $\begin{array}{l}\text { Density } \\
\left(\mathrm{g} / \mathrm{cm}^{3}\right)\end{array}$ & $\begin{array}{l}\text { Depth }(\text { rmcd } \\
\text { [m revised } \\
\text { CCSF-A]) }\end{array}$ & $\left(10^{-5}\right)$ \\
\hline & 23 & 2.70 & 26.4 \\
\hline
\end{tabular}

$\begin{array}{llll}1.84 & 1.236 & 2.70 & 26.4 \\ 1.86 & 1.240 & 2.72 & 26.8\end{array}$

$\begin{array}{llll}1.87 & 1.238 & 2.75 & 26.4\end{array}$

$\begin{array}{llll}1.87 & 1.260 & 2.77 & 25.0\end{array}$

$1.88-1.247 \quad 2.80-24.2$

$\begin{array}{llll}1.88 & 1.247 & 2.80 & 24.2 \\ 1.89 & 1.257 & 2.82 & 24.0\end{array}$

$1.91-1.246$

$1.92 \quad 1.236$

$1.94 \quad 1.249$

$1.94 \quad 1.277$

$1.96 \quad 1.238$

$1.97 \quad 1.258$

$\begin{array}{ll}1.99 & 1.255\end{array}$

$\begin{array}{ll}1.99 & 1.258\end{array}$

2.01

2.02

2.04

2.04

2.06

2.07

2.09

2.09

2.11

2.12

2.12

2.14

2.14

2.16

2.17

2.17

2.19

2.19

2.21

2.22

2.22

2.24

2.24

2.26

2.27

2.27

2.29

2.29

2.31

2.32

2.32

2.34

2.34

2.36

2.37

2.37

2.39

2.39

2.41

2.42

2.42

2.44

2.44

2.46

2.47

2.49

2.49

2.51

2.52

2.54

2.54

2.56
1.261

1.264

1.257

1.241

1.272

1.259

1.246

1.259

1.268

1.262

1.250

1.247

1.266

1.237

1.253

1.256

1.258

1.241

1.262

1.259

1.248
1.252

1.254

1.261

1.270

1.264

1.242

1.259

1.256

1.261

1.254

1.236

1.233

1.236

1.238

1.236

1.254

1.238

1.249

1.230

1.253

1.250

1.250

1.245

1.252

1.244

1.245

1.249

1.236

1.250

1.235

$2.87 \quad 22.6$

$2.90 \quad 18.6$

$2.92 \quad 16.6$

$\begin{array}{ll}2.97 & 17.4\end{array}$

$3.00 \quad 17.0$

$3.08 \quad 21.2$

$\begin{array}{ll}3.10 & 23.6\end{array}$

$3.13 \quad 24.4$

$3.15 \quad 24.0$

$3.18 \quad 23.4$

$\begin{array}{ll}3.20 & 22.4\end{array}$

$3.23 \quad 22.4$

$\begin{array}{ll}3.25 & 24.6\end{array}$

$\begin{array}{ll}3.28 & 26.0\end{array}$

$\begin{array}{ll}3.30 & 26.4\end{array}$

$\begin{array}{ll}3.33 & 26.0\end{array}$

$3.35 \quad 25.0$

$3.40 \quad 24.0$

3.43

3.45

3.48

3.51

3.53

3.56

3.58

3.61

3.63
3.66

3.68

3.71

3.73

3.76

3.78

3.81

3.83
3.86

3.86

3.88

3.91

3.93

3.96

3.99

4.01

4.04

4.06

4.09

4.11

4.14

4.16

4.19

4.21

4.24

4.26

4.29

4.31

4.34

4.36

4.39

$2.85 \quad 24.0$

$2.95 \quad 17.0$

$3.38 \quad 24.4$

\begin{tabular}{|c|c|c|c|}
\hline \multicolumn{2}{|c|}{ Gamma ray attenuation } & \multicolumn{2}{|c|}{ Magnetic susceptibility } \\
\hline $\begin{array}{l}\text { Depth (rmcd } \\
\text { [m revised } \\
\text { CCSF-A]) }\end{array}$ & $\begin{array}{l}\text { Density } \\
\left(\mathrm{g} / \mathrm{cm}^{3}\right)\end{array}$ & $\begin{array}{l}\text { Depth (rmcd } \\
\text { [m revised } \\
\text { CCSF-A]) }\end{array}$ & $\left(10^{-5}\right)$ \\
\hline 2.57 & 1.230 & 4.41 & 22.8 \\
\hline
\end{tabular}

$\begin{array}{llll}2.57 & 1.230 & 4.41 & 22.8 \\ 2.59 & 1.231 & 4.44 & 22.6 \\ 2.59 & 1.231 & 4.47 & 22.0 \\ & & 4.49 & 22.4 \\ 2.62 & 1.230 & 4.52 & 21.4\end{array}$

$\begin{array}{llll}2.62 & 1.230 & 4.52 & 21.4 \\ 2.65 & 1.210 & 4.59 & 20.6\end{array}$

$2.67 \quad 1.222$

$\begin{array}{llll}2.70 & 1.240 & 4.64 & 20.4\end{array}$

$\begin{array}{llll}2.72 & 1.239 & 4.67 & 22.0\end{array}$

$\begin{array}{llll}2.75 & 1.222 & 4.69 & 23.0\end{array}$

$\begin{array}{llll}2.77 & 1.209 & 4.72 & 22.8\end{array}$

$\begin{array}{llll}2.80 & 1.217 & 4.74 & 21.6\end{array}$

$\begin{array}{llll}2.80 & 1.225 & 4.77 & 22.4\end{array}$

$\begin{array}{llll}2.85 & 1.225 & 4.79 & 22.8\end{array}$

$\begin{array}{llll}2.85 & 1.225 & 4.79 & 22.8 \\ 2.87 & 1.214 & 4.82 & 23.4\end{array}$

$\begin{array}{llll}2.90 & 1.226 & 4.84 & 23.4\end{array}$

$\begin{array}{llll}2.92 & 1.194 & 4.87 & 23.6\end{array}$

$\begin{array}{llll}2.95 & 1.212 & 4.89 & 23.6\end{array}$

$\begin{array}{llll}2.97 & 1.206 & 4.92 & 23.2\end{array}$

$\begin{array}{llll}3.07 & 1.204 & 4.94 & 24.0\end{array}$

$\begin{array}{llll}3.10 & 1.237 & 4.97 & 25.0\end{array}$

$\begin{array}{llll}3.13 & 1.228 & 4.99 & 25.8\end{array}$

$\begin{array}{llll}3.15 & 1.217 & 5.02 & 26.0\end{array}$

$\begin{array}{llll}3.18 & 1.210 & 5.04 & 26.0 \\ 3.20 & 1.219 & 5.07 & 28.6\end{array}$

$\begin{array}{llll}3.20 & 1.219 & 5.07 & 28.6\end{array}$

$\begin{array}{llll}3.23 & 1.217 & 5.09 & 28.4\end{array}$

$\begin{array}{llll}3.25 & 1.231 & 5.12 & 29.4\end{array}$

$\begin{array}{llll}3.25 & 1.231 & 5.12 & 29.4 \\ 3.28 & 1.243 & 5.14 & 29.6\end{array}$

$\begin{array}{llll}3.30 & 1.244 & 5.17 & 29.2\end{array}$

$\begin{array}{llll}3.33 & 1.225 & 5.19 & 30.2\end{array}$

$\begin{array}{llll}3.33 & 1.225 & 5.19 & 30.2 \\ 3.35 & 1.232 & 5.22 & 30.2\end{array}$

$\begin{array}{llll}3.35 & 1.232 & 5.22 & 30.2 \\ 3.38 & 1.224 & 5.24 & 30.0\end{array}$

$\begin{array}{llll}3.40 & 1.227 & 5.27 & 29.4\end{array}$

$\begin{array}{llll}3.43 & 1.220 & 5.29 & 29.0\end{array}$

$\begin{array}{llll}3.45 & 1.246 & 5.32 & 29.0\end{array}$

$\begin{array}{llll}3.48 & 1.249 & 5.34 & 29.2\end{array}$

$\begin{array}{llll}3.50 & 1.228 & 5.37 & 29.0 \\ 3.53 & 1.256 & 5.39 & 28.2\end{array}$

$\begin{array}{llll}3.53 & 1.256 & 5.39 & 28.2 \\ 3.56 & 1.239 & 5.42 & 27.0\end{array}$

$\begin{array}{llll}3.56 & 1.239 & 5.42 & 27.0\end{array}$

$\begin{array}{llll}3.58 & 1.252 & 5.42 & 27.0 \\ 3.61 & 1.266 & 5.47 & 25.0\end{array}$

$\begin{array}{llll}3.61 & 1.266 & 5.47 & 23.8\end{array}$

$\begin{array}{llll}3.63 & 1.255 & 5.49 & 20.8\end{array}$

$\begin{array}{llll}3.66 & 1.235 & 5.52 & 21.4 \\ 3.68 & 1.210 & 5.54\end{array}$

$\begin{array}{llll}3.68 & 1.210 & 5.54 & 23.8\end{array}$

$\begin{array}{llll}3.71 & 1.240 & 5.57 & 25.0\end{array}$

$\begin{array}{llll}3.73 & 1.224 & 5.60 & 25.0 \\ 3.76 & 1.213 & 5.62 & 23.6\end{array}$

$\begin{array}{llll}3.76 & 1.213 & 5.62 & 23.6 \\ 3.78 & 1.226 & 5.65 & 22.0 \\ 3.81 & 1.224 & 5.67 & 21.2\end{array}$

$\begin{array}{llll}3.78 & 1.226 & 5.65 & 22.0 \\ 3.81 & 1.224 & 5.67 & 21.2\end{array}$

$\begin{array}{llll}3.83 & 1.249 & 5.70 & 21.0\end{array}$

$\begin{array}{llll}3.86 & 1.237 & 5.72 & 18.4\end{array}$

$\begin{array}{llll}3.88 & 1.232 & 5.75 & 16.2\end{array}$

$\begin{array}{llll}3.88 & 1.232 & 5.75 & 16.2 \\ 3.91 & 1.222 & 5.77 & 16.0 \\ 3.93 & 1.231 & 5.80 & 15.6\end{array}$

$\begin{array}{llll}3.93 & 1.231 & 5.80 & 15.6\end{array}$

$\begin{array}{llll}3.96 & 1.223 & 5.82 & 16.6\end{array}$

$\begin{array}{llll}3.96 & 1.223 & 5.82 & 16.6 \\ 3.98 & 1.240 & 5.85 & 20.6\end{array}$

$\begin{array}{llll}4.01 & 1.223 & 5.87 & 24.0 \\ 4.04 & 1.205 & 5.90 & 26.2 \\ 4.06 & 1.213 & 5.92 & 27.0\end{array}$

$\begin{array}{llll}4.06 & 1.213 & 5.92 & 27.0\end{array}$

$\begin{array}{llll}4.09 & 1.218 & 5.95 & 27.0 \\ 4.11 & 1.223 & 5.97 & 27.6\end{array}$

$\begin{array}{llll}4.11 & 1.223 & 5.97 & 27.6\end{array}$

\begin{tabular}{llll}
4.14 & 1.230 & 6.00 & 27.8 \\
\hline 4.16 & 1.218 & 6.07 & 24.6
\end{tabular}

$\begin{array}{llll}4.16 & 1.218 & 6.07 & 24.6\end{array}$

Only a portion of this table appears here. The complete table is available in ASCII. 
Table T24. Cleaned magnetic susceptibility and gamma ray attenuation composite records from Site U1333 on rmcd (m revised CCSF-A).

\begin{tabular}{|c|c|c|c|}
\hline \multicolumn{2}{|c|}{ Gamma ray attenuation } & \multicolumn{2}{|c|}{ Magnetic susceptibility } \\
\hline $\begin{array}{l}\text { Depth (rmcd } \\
\text { [m revised } \\
\text { CCSF-A]) }\end{array}$ & $\begin{array}{l}\text { Density } \\
\left(\mathrm{g} / \mathrm{cm}^{3}\right)\end{array}$ & $\begin{array}{l}\text { Depth (rmcd } \\
\text { [m revised } \\
\text { CCSF-A]) }\end{array}$ & $\left(10^{-5}\right)$ \\
\hline 0.00 & 0.769 & 0.03 & 28.0 \\
\hline 0.03 & 1.080 & 0.05 & 33.0 \\
\hline 0.05 & 1.149 & 0.08 & 33.0 \\
\hline 0.08 & 1.061 & 0.10 & 36.2 \\
\hline 0.10 & 1.059 & 0.13 & 40.0 \\
\hline 0.13 & 1.149 & 0.15 & 40.8 \\
\hline 0.15 & 1.145 & 0.18 & 41.0 \\
\hline 0.18 & 1.141 & 0.20 & 41.0 \\
\hline 0.20 & 1.135 & 0.23 & 41.0 \\
\hline 0.23 & 1.149 & 0.25 & 41.4 \\
\hline 0.25 & 1.146 & 0.28 & 41.8 \\
\hline 0.28 & 1.152 & 0.30 & 42.6 \\
\hline 0.30 & 1.158 & 0.33 & 42.2 \\
\hline 0.33 & 1.125 & 0.35 & 42.4 \\
\hline 0.35 & 1.165 & 0.38 & 42.0 \\
\hline 0.38 & 1.157 & 0.40 & 41.0 \\
\hline 0.40 & 1.162 & 0.43 & 40.4 \\
\hline 0.43 & 1.147 & 0.45 & 39.4 \\
\hline 0.45 & 1.157 & 0.48 & 38.8 \\
\hline 0.48 & 1.156 & 0.50 & 38.4 \\
\hline 0.50 & 1.144 & 0.53 & 38.8 \\
\hline 0.53 & 1.162 & 0.55 & 39.6 \\
\hline 0.55 & 1.170 & 0.60 & 38.4 \\
\hline 0.58 & 1.169 & 0.63 & 38.0 \\
\hline 0.60 & 1.164 & 0.65 & 38.0 \\
\hline 0.63 & 1.150 & 0.68 & 38.0 \\
\hline 0.65 & 1.154 & 0.70 & 37.6 \\
\hline 0.68 & 1.172 & 0.73 & 37.6 \\
\hline 0.70 & 1.159 & 0.75 & 37.6 \\
\hline 0.73 & 1.172 & 0.78 & 37.8 \\
\hline 0.75 & 1.154 & 0.80 & 37.2 \\
\hline 0.78 & 1.150 & 0.83 & 38.0 \\
\hline 0.80 & 1.163 & 0.85 & 38.0 \\
\hline 0.83 & 1.163 & 0.88 & 37.2 \\
\hline 0.85 & 1.162 & 0.90 & 37.4 \\
\hline 0.88 & 1.162 & 0.93 & 37.4 \\
\hline 0.90 & 1.153 & 0.95 & 37.0 \\
\hline 0.93 & 1.158 & 0.98 & 36.8 \\
\hline 0.95 & 1.179 & 1.00 & 37.2 \\
\hline 0.98 & 1.156 & 1.03 & 37.2 \\
\hline 1.00 & 1.176 & 1.05 & 37.0 \\
\hline 1.03 & 1.165 & 1.08 & 36.8 \\
\hline 1.05 & 1.164 & 1.10 & 35.2 \\
\hline 1.08 & 1.158 & 1.13 & 34.4 \\
\hline 1.10 & 1.174 & 1.15 & 33.0 \\
\hline 1.13 & 1.169 & 1.18 & 32.2 \\
\hline 1.15 & 1.183 & 1.20 & 32.0 \\
\hline 1.18 & 1.153 & 1.23 & 31.0 \\
\hline 1.20 & 1.161 & 1.25 & 30.2 \\
\hline 1.23 & 1.157 & 1.28 & 30.2 \\
\hline 1.25 & 1.163 & 1.30 & 29.6 \\
\hline 1.28 & 1.179 & 1.33 & 29.4 \\
\hline 1.30 & 1.166 & 1.35 & 29.0 \\
\hline 1.33 & 1.155 & 1.38 & 30.0 \\
\hline 1.35 & 1.168 & 1.40 & 29.8 \\
\hline 1.38 & 1.159 & 1.43 & 29.0 \\
\hline 1.40 & 1.157 & 1.45 & 27.0 \\
\hline 1.43 & 1.165 & 1.53 & 26.0 \\
\hline 1.45 & 1.157 & 1.55 & 27.6 \\
\hline 1.48 & 1.185 & 1.58 & 28.4 \\
\hline 2.51 & 1.428 & 1.60 & 29.2 \\
\hline 2.53 & 1.441 & 1.63 & 29.8 \\
\hline 2.56 & 1.445 & 1.65 & 29.6 \\
\hline 2.58 & 1.457 & 1.68 & 29.2 \\
\hline 2.61 & 1.482 & 1.70 & 28.6 \\
\hline 2.63 & 1.454 & 1.73 & 27.8 \\
\hline
\end{tabular}

\begin{tabular}{ccccc}
\hline \multicolumn{2}{c}{ Gamma ray attenuation } & & \multicolumn{2}{c}{ Magnetic susceptibility } \\
\cline { 1 - 2 } $\begin{array}{c}\text { Depth (rmcd } \\
\text { [m revised } \\
\text { CCSF-A] })\end{array}$ & $\begin{array}{c}\text { Density } \\
\left(\mathrm{g} / \mathrm{cm}^{3}\right)\end{array}$ & $\begin{array}{c}\text { Depth (rmcd } \\
\text { [m revised } \\
\text { CCSF-A]) }\end{array}$ & $\left(10^{-5}\right)$ \\
\hline 2.66 & 1.471 & 1.76 & 27.6
\end{tabular}

$\begin{array}{llll}2.66 & 1.471 & 1.76 & 27.6 \\ 2.68 & 1.482 & 1.78 & 27.6\end{array}$

$\begin{array}{llll}2.71 & 1.482 & 1.81 & 27.0\end{array}$

2.73

2.76

2.78

2.81

2.83

2.86

2.88

2.91

2.93

2.96

2.98

3.03

3.05

3.08

3.10

3.13
3.15

3.15
3.18

3.18
3.20

3.23

3.25

3.28

3.30

3.33

3.35

3.38

3.40

3.43

3.45

3.48

3.50

3.53

3.55

3.58

3.60

3.63

3.65

3.68

3.70

3.73

3.75

3.78

3.80

3.83

3.85

3.88

3.90

3.93

3.95

3.98

4.00

4.03

4.05

4.08

4.10

4.13

4.15

4.18

4.20

4.23

4.25

4.28

4.30

$\begin{array}{lll}1.491 & 1.83 & 27.8\end{array}$

$\begin{array}{lll}1.472 & 1.86 & 28.2\end{array}$

$\begin{array}{lll}1.457 & 1.88 & 27.0\end{array}$

$\begin{array}{lll}1.480 & 1.91 & 25.6\end{array}$

$\begin{array}{lll}1.487 & 1.93 & 25.0\end{array}$

$\begin{array}{lll}1.513 & 1.96 & 24.2\end{array}$

$\begin{array}{lll}1.556 & 1.98 & 24.8\end{array}$

$\begin{array}{lll}1.541 & 2.01 & 23.6\end{array}$

1.544

1.554

1.601

1.607

1.596

1.568

1.550
1.579

1.560

1.558

1.584

1.585

1.576

1.551

1.561

1.543

1.532

1.501

1.470

1.486

1.463

1.459

1.469

1.466

1.491

1.504

1.492

1.502

1.516

1.503

1.498

1.520

1.504

1.496

1.449

1.475

1.439

1.454

1.424

1.446

1.427

1.419
1.422

1.432

1.452

1.462

1.473

1.523

1.477

1.544

1.543

1.531

1.531

1.514

1.519

$\begin{array}{ll}2.03 & 22.0 \\ 2.06 & 21.4\end{array}$

2.08

$\begin{array}{ll}2.11 & 21.8\end{array}$

$2.13 \quad 21.2$

$\begin{array}{ll}2.16 & 20.2\end{array}$

$2.18 \quad 20.6$

$\begin{array}{ll}2.21 & 20.2\end{array}$

$\begin{array}{ll}2.23 & 20.0\end{array}$

$\begin{array}{ll}2.26 & 19.8\end{array}$

$2.31 \quad 20.6$

$2.33 \quad 21.4$

$2.36 \quad 21.2$

$2.41 \quad 17.6$

$\begin{array}{ll}2.43 & 13.8\end{array}$

$2.46 \quad 12.0$

2.48

2.51

2.53

2.56

2.58

2.61
2.63

2.63
2.66

2.68

2.71

2.73

2.76

2.78

2.81

2.83
2.86

2.86

2.88
2.91

2.93

2.96

2.98

3.00

3.03

3.05

3.08

3.10

3.13

3.15

3.18

3.20

3.23

3.25

3.28

3.30

3.33

3.35

3.38 $\begin{array}{ll}2.38 & 20.4\end{array}$ $\begin{array}{ll}2.28 & 19.2\end{array}$

\begin{tabular}{|c|c|c|c|}
\hline \multicolumn{2}{|c|}{ Gamma ray attenuation } & \multicolumn{2}{|c|}{ Magnetic susceptibility } \\
\hline $\begin{array}{l}\text { Depth (rmcd } \\
\text { [m revised } \\
\text { CCSF-A]) }\end{array}$ & $\begin{array}{l}\text { Density } \\
\left(\mathrm{g} / \mathrm{cm}^{3}\right)\end{array}$ & $\begin{array}{c}\text { Depth (rmcd } \\
\text { [m revised } \\
\text { CCSF-A]) }\end{array}$ & $\left(10^{-5}\right)$ \\
\hline 4.33 & 1.495 & 3.40 & 16.0 \\
\hline & 1.496 & 3.43 & 160 \\
\hline
\end{tabular}

$\begin{array}{llll}4.38 & 1.467 & 3.45 & 16.0\end{array}$

$\begin{array}{llll}4.40 & 1.470 & 3.48 & 15.8\end{array}$

$\begin{array}{llll}4.43 & 1.460 & 3.50 & 15.2\end{array}$

$\begin{array}{llll}4.45 & 1.446 & 3.53 & 15.0\end{array}$

$\begin{array}{llll}4.48 & 1.453 & 3.55 & 15.0\end{array}$

$\begin{array}{llll}4.55 & 1.456 & 3.58 & 14.6\end{array}$

$\begin{array}{llll}4.57 & 1.483 & 3.60 & 14.8\end{array}$

$\begin{array}{llll}4.60 & 1.469 & 3.63 & 14.4\end{array}$

$\begin{array}{llll}4.62 & 1.451 & 3.65 & 13.8\end{array}$

$\begin{array}{llll}4.65 & 1.426 & 3.68 & 14.0\end{array}$

$\begin{array}{llll}4.67 & 1.424 & 3.70 & 14.0\end{array}$

$\begin{array}{llll}4.70 & 1.417 & 3.73 & 14.8\end{array}$

$\begin{array}{llll}4.72 & 1.396 & 3.75 & 15.6\end{array}$

$\begin{array}{llll}4.75 & 1.362 & 3.78 & 16.0\end{array}$

$\begin{array}{llll}4.82 & 1.309 & 3.80 & 17.4 \\ 4.85 & 1.328 & 3.83 & 19.0\end{array}$

$\begin{array}{llll}4.85 & 1.328 & 3.83 & 19.0\end{array}$

$\begin{array}{llll}4.87 & 1.271 & 3.85 & 19.0 \\ 4.90 & 1.284 & 3.88 & 19.8\end{array}$

$\begin{array}{llll}4.92 & 1.274 & 3.90 & 21.0\end{array}$

$\begin{array}{llll}4.95 & 1.262 & 3.90 & 21.0 \\ 4.97 & 1.279 & 3.95 & 21.6\end{array}$

$\begin{array}{llll}4.97 & 1.279 & 3.95 & 21.6\end{array}$

$\begin{array}{llll}5.00 & 1.270 & 3.98 & 21.2\end{array}$

$\begin{array}{llll}5.02 & 1.276 & 4.00 & 21.6\end{array}$

$\begin{array}{llll}5.05 & 1.288 & 4.03 & 21.0 \\ 5.07 & 1.301 & 4.05 & 20.4\end{array}$

$\begin{array}{llll}5.07 & 1.301 & 4.05 & 20.4 \\ 5.10 & 1.293 & 4.08 & 19.0\end{array}$

$\begin{array}{llll}5.12 & 1.279 & 4.10 & 17.2\end{array}$

$\begin{array}{llll}5.15 & 1.279 & 4.13 & 16.4\end{array}$

$\begin{array}{llll}5.17 & 1.267 & 4.13 & 16.4 \\ 5.20 & 1.253 & 4.18 & 15.0\end{array}$

$\begin{array}{llll}5.20 & 1.253 & 4.18 & 14.8\end{array}$

$\begin{array}{llll}5.22 & 1.249 & 4.20 & 13.6\end{array}$

$\begin{array}{llll}5.25 & 1.213 & 4.23 & 13.2\end{array}$

$\begin{array}{llll}5.27 & 1.231 & 4.25 & 13.8 \\ 5.30 & 1.257 & 4.28 & 14.0\end{array}$

$\begin{array}{llll}5.30 & 1.257 & 4.28 & 14.0\end{array}$

$\begin{array}{llll}5.32 & 1.251 & 4.30 & 14.8 \\ 5.35 & 1.246 & 4.33 & 15.4\end{array}$

$\begin{array}{llll}5.37 & 1.264 & 4.35 & 16.4\end{array}$

$\begin{array}{llll}5.40 & 1.260 & 4.38 & 17.4 \\ 5.42 & 1.261 & 4.40 & 17.4\end{array}$

$\begin{array}{llll}5.42 & 1.261 & 4.40 & 17.4\end{array}$

$\begin{array}{llll}5.45 & 1.263 & 4.43 & 18.0\end{array}$

$\begin{array}{llll}5.47 & 1.261 & 4.45 & 18.2\end{array}$

$\begin{array}{llll}5.50 & 1.242 & 4.48 & 18.8\end{array}$

$\begin{array}{llll}5.52 & 1.271 & 4.55 & 19.0\end{array}$

$\begin{array}{llll}5.55 & 1.264 & 4.57 & 19.6\end{array}$

$\begin{array}{llll}5.57 & 1.289 & 4.60 & 20.2\end{array}$

$\begin{array}{llll}5.60 & 1.311 & 4.62 & 20.8\end{array}$

$\begin{array}{llll}5.62 & 1.304 & 4.62 & 20.8 \\ 5.65 & 1.325 & 4.67 & 22.0\end{array}$

$\begin{array}{llll}5.65 & 1.325 & 4.67 & 22.8\end{array}$

$\begin{array}{llll}5.67 & 1.350 & 4.70 & 24.0 \\ 5.70 & 1.327 & 4.72\end{array}$

$\begin{array}{llll}5.70 & 1.327 & 4.72 & 27.0\end{array}$

$\begin{array}{llll}5.72 & 1.325 & 4.75 & 28.2\end{array}$

$\begin{array}{llll}5.75 & 1.324 & 4.77 & 29.0\end{array}$

$\begin{array}{llll}5.77 & 1.325 & 4.80 & 31.4\end{array}$

$\begin{array}{llll}5.77 & 1.325 & 4.80 & 31.4 \\ 5.80 & 1.328 & 4.82 & 32.0\end{array}$

$\begin{array}{llll}5.82 & 1.341 & 4.85 & 32.0\end{array}$

$\begin{array}{llll}5.82 & 1.341 & 4.85 & 32.0 \\ 5.85 & 1.328 & 4.87 & 34.0\end{array}$

$\begin{array}{llll}5.87 & 1.302 & 4.90 & 36.4\end{array}$

$\begin{array}{llll}5.90 & 1.300 & 4.92 & 37.0\end{array}$

$\begin{array}{llll}5.92 & 1.287 & 4.95 & 38.2\end{array}$

$\begin{array}{llll}5.95 & 1.288 & 4.97 & 39.2\end{array}$

$\begin{array}{llll}5.97 & 1.284 & 5.00 & 38.6\end{array}$

Only a portion of this table appears here. The complete table is available in ASCII. 
Table T25. Cleaned magnetic susceptibility and gamma ray attenuation composite records from Site U1334 on rmcd (m revised CCSF-A).

\begin{tabular}{|c|c|c|c|}
\hline \multicolumn{2}{|c|}{ Gamma ray attenuation } & \multicolumn{2}{|c|}{ Magnetic susceptibility } \\
\hline $\begin{array}{l}\text { Depth (rmcd } \\
\text { [m revised } \\
\text { CCSF-A]) }\end{array}$ & $\begin{array}{l}\text { Density } \\
\left(\mathrm{g} / \mathrm{cm}^{3}\right)\end{array}$ & $\begin{array}{l}\text { Depth (rmcd } \\
\text { [m revised } \\
\text { CCSF-A]) }\end{array}$ & $\left(10^{-5}\right)$ \\
\hline 0.13 & 1.202 & 0.03 & 15.8 \\
\hline 0.15 & 1.176 & 0.05 & 20.5 \\
\hline 0.18 & 1.178 & 0.08 & 19.8 \\
\hline 0.20 & 1.178 & 0.10 & 19.0 \\
\hline 0.23 & 1.200 & 0.13 & 20.5 \\
\hline 0.25 & 1.187 & 0.15 & 20.9 \\
\hline 0.28 & 1.190 & 0.18 & 22.3 \\
\hline 0.30 & 1.206 & 0.20 & 22.3 \\
\hline 0.33 & 1.204 & 0.23 & 22.7 \\
\hline 0.35 & 1.186 & 0.25 & 23.1 \\
\hline 0.38 & 1.228 & 0.28 & 24.2 \\
\hline 0.40 & 1.216 & 0.30 & 23.8 \\
\hline 0.43 & 1.203 & 0.33 & 24.5 \\
\hline 0.45 & 1.228 & 0.35 & 24.9 \\
\hline 0.48 & 1.208 & 0.38 & 24.9 \\
\hline 0.50 & 1.231 & 0.40 & 24.9 \\
\hline 0.53 & 1.204 & 0.43 & 25.3 \\
\hline 0.55 & 1.230 & 0.45 & 27.5 \\
\hline 0.58 & 1.235 & 0.48 & 26.4 \\
\hline 0.60 & 1.189 & 0.50 & 25.3 \\
\hline 0.63 & 1.222 & 0.53 & 26.0 \\
\hline 0.65 & 1.192 & 0.55 & 26.0 \\
\hline 0.68 & 1.216 & 0.58 & 25.3 \\
\hline 0.70 & 1.191 & 0.60 & 24.9 \\
\hline 0.73 & 1.197 & 0.63 & 24.5 \\
\hline 0.75 & 1.175 & 0.65 & 23.1 \\
\hline 0.78 & 1.224 & 0.68 & 22.3 \\
\hline 0.80 & 1.191 & 0.70 & 23.1 \\
\hline 0.83 & 1.202 & 0.73 & 23.4 \\
\hline 0.85 & 1.201 & 0.75 & 23.1 \\
\hline 0.88 & 1.196 & 0.78 & 23.1 \\
\hline 0.90 & 1.231 & 0.80 & 23.1 \\
\hline 0.93 & 1.231 & 0.83 & 23.4 \\
\hline 0.95 & 1.211 & 0.85 & 24.2 \\
\hline 0.98 & 1.204 & 0.88 & 23.8 \\
\hline 1.00 & 1.215 & 0.90 & 23.4 \\
\hline 1.03 & 1.223 & 0.93 & 23.1 \\
\hline 1.05 & 1.227 & 0.95 & 23.1 \\
\hline 1.08 & 1.231 & 0.98 & 23.1 \\
\hline 1.10 & 1.219 & 1.00 & 23.8 \\
\hline 1.13 & 1.237 & 1.03 & 24.2 \\
\hline 1.15 & 1.219 & 1.05 & 24.5 \\
\hline 1.18 & 1.224 & 1.08 & 24.9 \\
\hline 1.20 & 1.222 & 1.10 & 24.5 \\
\hline 1.23 & 1.198 & 1.15 & 24.9 \\
\hline 1.25 & 1.211 & 1.18 & 24.5 \\
\hline 1.28 & 1.198 & 1.20 & 24.2 \\
\hline 1.30 & 1.216 & 1.23 & 23.4 \\
\hline 1.33 & 1.219 & 1.25 & 23.1 \\
\hline 1.35 & 1.213 & 1.28 & 24.2 \\
\hline 1.38 & 1.193 & 1.30 & 23.1 \\
\hline 1.40 & 1.206 & 1.33 & 24.2 \\
\hline 1.56 & 1.216 & 1.35 & 23.4 \\
\hline 1.59 & 1.222 & 1.38 & 23.1 \\
\hline 1.61 & 1.218 & 1.40 & 22.0 \\
\hline 1.64 & 1.230 & 1.43 & 21.2 \\
\hline 1.66 & 1.228 & 1.45 & 20.1 \\
\hline 1.69 & 1.222 & 1.54 & 20.1 \\
\hline 1.71 & 1.223 & 1.56 & 21.2 \\
\hline 1.74 & 1.212 & 1.59 & 20.9 \\
\hline 1.76 & 1.214 & 1.61 & 19.8 \\
\hline 1.79 & 1.207 & 1.64 & 20.5 \\
\hline 1.81 & 1.222 & 1.66 & 20.1 \\
\hline 1.84 & 1.230 & 1.69 & 20.5 \\
\hline 1.86 & 1.206 & 1.71 & 20.5 \\
\hline 1.89 & 1.232 & 1.74 & 20.5 \\
\hline
\end{tabular}

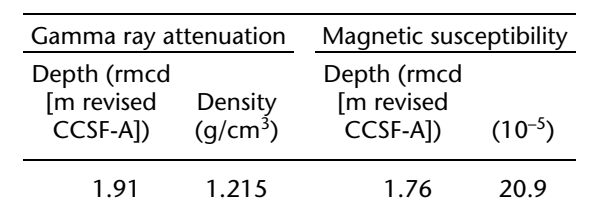

$\begin{array}{llll}1.91 & 1.215 & 1.76 & 20.9 \\ 1.94 & 1.212 & 1.79 & 22.3\end{array}$

$\begin{array}{llll}1.96 & 1.224 & 1.81 & 24.2\end{array}$

$\begin{array}{llll}1.99 & 1.236 & 1.84 & 24.9\end{array}$

$\begin{array}{llll}2.01 & 1.214 & 1.86 & 24.9\end{array}$

$\begin{array}{llll}2.04 & 1.221 & 1.89 & 25.3\end{array}$

$\begin{array}{llll}2.06 & 1.240 & 1.91 & 25.3\end{array}$

$\begin{array}{llll}2.09 & 1.227 & 1.94 & 25.3\end{array}$

$\begin{array}{llll}2.11 & 1.216 & 1.96 & 24.9\end{array}$

$\begin{array}{llll}2.14 & 1.226 & 1.99 & 24.9\end{array}$

$\begin{array}{llll}2.16 & 1.217 & 2.01 & 24.5\end{array}$

$\begin{array}{llll}2.19 & 1.209 & 2.04 & 24.2\end{array}$

$\begin{array}{llll}2.21 & 1.206 & 2.06 & 23.4\end{array}$

$\begin{array}{llll}2.24 & 1.220 & 2.09 & 22.7\end{array}$

$\begin{array}{llll}2.26 & 1.241 & 2.11 & 23.4\end{array}$

$\begin{array}{llll}2.29 & 1.231 & 2.14 & 23.1\end{array}$

$\begin{array}{llll}2.31 & 1.199 & 2.16 & 22.7\end{array}$

$\begin{array}{llll}2.34 & 1.202 & 2.19 & 22.3\end{array}$

$\begin{array}{llll}2.36 & 1.221 & 2.21 & 22.7\end{array}$

$\begin{array}{llll}2.39 & 1.203 & 2.24 & 22.0\end{array}$

$\begin{array}{llll}2.41 & 1.209 & 2.26 & 22.0\end{array}$

$\begin{array}{llll}2.44 & 1.212 & 2.29 & 20.9\end{array}$

$\begin{array}{llll}2.46 & 1.213 & 2.31 & 20.5\end{array}$

$\begin{array}{llll}2.49 & 1.216 & 2.34 & 20.5\end{array}$

$\begin{array}{llll}2.51 & 1.212 & 2.36 & 21.6\end{array}$

$\begin{array}{llll}2.54 & 1.222 & 2.39 & 20.9\end{array}$

$\begin{array}{llll}2.56 & 1.219 & 2.41 & 20.9\end{array}$

$\begin{array}{llll}2.59 & 1.227 & 2.44 & 21.2\end{array}$

$\begin{array}{llll}2.61 & 1.209 & 2.46 & 20.9\end{array}$

$\begin{array}{llll}2.64 & 1.203 & 2.49 & 20.1\end{array}$

$\begin{array}{llll}2.64 & 1.203 & 2.49 & 20.1 \\ 2.66 & 1.208 & 2.51 & 20.1\end{array}$

$\begin{array}{llll}2.69 & 1.216 & 2.54 & 20.9\end{array}$

$\begin{array}{llll}2.71 & 1.217 & 2.56 & 19.8\end{array}$

$\begin{array}{llll}2.74 & 1.211 & 2.59 & 19.0\end{array}$

$\begin{array}{llll}2.76 & 1.199 & 2.61 & 18.7\end{array}$

$\begin{array}{llll}2.79 & 1.224 & 2.64 & 19.0\end{array}$

$\begin{array}{llll}2.81 & 1.234 & 2.66 & 19.4\end{array}$

$\begin{array}{llll}2.84 & 1.224 & 2.69 & 19.8\end{array}$

$\begin{array}{llll}2.86 & 1.244 & 2.71 & 20.9\end{array}$

$\begin{array}{llll}2.89 & 1.239 & 2.74 & 22.3\end{array}$

$\begin{array}{llll}2.91 & 1.265 & 2.76 & 23.1\end{array}$

$\begin{array}{llll}3.08 & 1.207 & 2.79 & 23.8\end{array}$

$\begin{array}{llll}3.11 & 1.248 & 2.81 & 24.9\end{array}$

$\begin{array}{llll}3.13 & 1.205 & 2.84 & 27.5\end{array}$

$\begin{array}{llll}3.16 & 1.241 & 2.86 & 29.3\end{array}$

$\begin{array}{llll}3.18 & 1.238 & 2.89 & 29.3\end{array}$

$\begin{array}{llll}3.21 & 1.257 & 2.91 & 27.8\end{array}$

$\begin{array}{llll}3.23 & 1.238 & 3.03 & 28.6\end{array}$

$\begin{array}{llll}3.26 & 1.233 & 3.06 & 29.7\end{array}$

$\begin{array}{llll}3.28 & 1.240 & 3.08 & 31.1\end{array}$

$\begin{array}{llll}3.31 & 1.241 & 3.11 & 30.8\end{array}$

$\begin{array}{llll}3.33 & 1.241 & 3.13 & 30.8\end{array}$

$\begin{array}{llll}3.36 & 1.256 & 3.16 & 30.8\end{array}$

$\begin{array}{llll}3.38 & 1.239 & 3.18 & 30.0\end{array}$

$\begin{array}{llll}3.41 & 1.268 & 3.21 & 29.7\end{array}$

$\begin{array}{llll}3.43 & 1.267 & 3.23 & 30.4\end{array}$

$\begin{array}{llll}3.46 & 1.260 & 3.26 & 29.3\end{array}$

$\begin{array}{llll}3.48 & 1.258 & 3.28 & 27.5\end{array}$

$\begin{array}{llll}3.51 & 1.265 & 3.31 & 26.4\end{array}$

$\begin{array}{llll}3.53 & 1.248 & 3.33 & 25.6\end{array}$

$\begin{array}{llll}3.56 & 1.248 & 3.36 & 23.1\end{array}$

$\begin{array}{llll}3.58 & 1.233 & 3.38 & 23.1\end{array}$

$\begin{array}{llll}3.61 & 1.238 & 3.41 & 25.3\end{array}$

$\begin{array}{llll}3.63 & 1.259 & 3.43 & 26.7\end{array}$

$\begin{array}{llll}3.66 & 1.262 & 3.46 & 24.9\end{array}$

$\begin{array}{llll}3.68 & 1.255 & 3.48 & 24.2\end{array}$

\begin{tabular}{|c|c|c|c|}
\hline \multicolumn{2}{|c|}{ Gamma ray attenuation } & \multicolumn{2}{|c|}{ Magnetic susceptibilit) } \\
\hline $\begin{array}{c}\text { Depth (rmcd } \\
\text { [m revised } \\
\text { CCSF-A]) }\end{array}$ & $\begin{array}{l}\text { Density } \\
\left(\mathrm{g} / \mathrm{cm}^{3}\right)\end{array}$ & $\begin{array}{c}\text { Depth (rmcd } \\
\text { [m revised } \\
\text { CCSF-A]) }\end{array}$ & $\left(10^{-5}\right)$ \\
\hline 3.71 & 1.254 & 3.51 & 23.4 \\
\hline 3.73 & 1.260 & 3.53 & 23.1 \\
\hline 3.76 & 1.230 & 3.56 & 23.8 \\
\hline 3.78 & 1.223 & 3.58 & 24.2 \\
\hline 3.81 & 1.212 & 3.61 & 24.2 \\
\hline 3.83 & 1.219 & 3.63 & 24.2 \\
\hline 3.86 & 1.215 & 3.66 & 24.2 \\
\hline 3.88 & 1.212 & 3.68 & 25.3 \\
\hline
\end{tabular}

$\begin{array}{llll}3.91 & 1.214 & 3.71 & 28.2\end{array}$

$\begin{array}{llll}3.93 & 1.231 & 3.73 & 31.1\end{array}$

$\begin{array}{llll}3.96 & 1.210 & 3.76 & 33.0\end{array}$

$\begin{array}{llll}3.98 & 1.224 & 3.78 & 33.0\end{array}$

$\begin{array}{llll}4.01 & 1.215 & 3.81 & 33.0\end{array}$

$\begin{array}{llll}4.03 & 1.233 & 3.83 & 33.0\end{array}$

$\begin{array}{llll}4.06 & 1.227 & 3.86 & 33.3\end{array}$

$\begin{array}{llll}4.08 & 1.225 & 3.88 & 33.7\end{array}$

$\begin{array}{llll}4.11 & 1.210 & 3.91 & 34.1\end{array}$

$\begin{array}{llll}4.13 & 1.218 & 3.93 & 34.1\end{array}$

$\begin{array}{llll}4.16 & 1.229 & 3.96 & 34.1\end{array}$

$\begin{array}{llll}4.18 & 1.214 & 3.98 & 34.1\end{array}$

$\begin{array}{llll}4.21 & 1.210 & 4.01 & 34.8\end{array}$

$\begin{array}{llll}4.23 & 1.220 & 4.03 & 35.5\end{array}$

$\begin{array}{llll}4.26 & 1.221 & 4.06 & 35.5\end{array}$

$\begin{array}{llll}4.28 & 1.216 & 4.08 & 35.2\end{array}$

$\begin{array}{llll}4.31 & 1.208 & 4.11 & 34.8\end{array}$

$\begin{array}{llll}4.33 & 1.219 & 4.13 & 34.8\end{array}$

$\begin{array}{llll}4.36 & 1.230 & 4.16 & 34.8\end{array}$

$\begin{array}{llll}4.38 & 1.227 & 4.18 & 35.2\end{array}$

$\begin{array}{llll}4.41 & 1.219 & 4.21 & 35.2\end{array}$

$\begin{array}{llll}4.43 & 1.232 & 4.23 & 35.5\end{array}$

$\begin{array}{llll}4.46 & 1.216 & 4.26 & 36.3\end{array}$

$\begin{array}{llll}4.48 & 1.253 & 4.31 & 35.9\end{array}$

$\begin{array}{llll}4.54 & 1.231 & 4.33 & 36.3\end{array}$

$\begin{array}{llll}4.57 & 1.227 & 4.36 & 37.0\end{array}$

$\begin{array}{llll}4.59 & 1.240 & 4.38 & 36.6\end{array}$

$\begin{array}{llll}4.62 & 1.220 & 4.41 & 37.4\end{array}$

$\begin{array}{llll}4.64 & 1.207 & 4.43 & 38.1\end{array}$

$\begin{array}{llll}4.67 & 1.216 & 4.46 & 37.4\end{array}$

$\begin{array}{llll}4.69 & 1.222 & 4.54 & 37.4\end{array}$

$\begin{array}{llll}4.72 & 1.198 & 4.57 & 38.5\end{array}$

$\begin{array}{llll}4.74 & 1.201 & 4.59 & 38.1\end{array}$

$\begin{array}{llll}4.77 & 1.206 & 4.64 & 36.3\end{array}$

$\begin{array}{llll}4.79 & 1.203 & 4.67 & 35.5\end{array}$

$\begin{array}{llll}4.82 & 1.220 & 4.69 & 35.2\end{array}$

$\begin{array}{llll}4.84 & 1.210 & 4.72 & 34.8\end{array}$

$\begin{array}{llll}4.87 & 1.218 & 4.74 & 34.8\end{array}$

$\begin{array}{llll}4.89 & 1.221 & 4.77 & 34.1\end{array}$

$\begin{array}{llll}4.92 & 1.219 & 4.79 & 33.7\end{array}$

$\begin{array}{llll}4.94 & 1.222 & 4.82 & 33.7\end{array}$

$\begin{array}{llll}4.97 & 1.234 & 4.84 & 34.1\end{array}$

$\begin{array}{llll}4.99 & 1.230 & 4.87 & 34.4\end{array}$

$\begin{array}{llll}5.02 & 1.214 & 4.89 & 34.8\end{array}$

$\begin{array}{llll}5.04 & 1.224 & 4.92 & 34.1\end{array}$

$\begin{array}{llll}5.07 & 1.218 & 4.94 & 34.4\end{array}$

$\begin{array}{llll}5.09 & 1.224 & 4.97 & 35.2\end{array}$

$\begin{array}{llll}5.12 & 1.225 & 4.99 & 34.8\end{array}$

$\begin{array}{llll}5.14 & 1.221 & 5.02 & 34.4\end{array}$

$\begin{array}{llll}5.17 & 1.229 & 5.04 & 34.1\end{array}$

$\begin{array}{llll}5.19 & 1.226 & 5.07 & 34.4\end{array}$

$\begin{array}{llll}5.22 & 1.222 & 5.09 & 35.2\end{array}$

$\begin{array}{llll}5.24 & 1.219 & 5.12 & 35.9\end{array}$

$\begin{array}{llll}5.27 & 1.225 & 5.14 & 36.3\end{array}$

$\begin{array}{llll}5.29 & 1.222 & 5.17 & 35.9\end{array}$

Only a portion of this table appears here. The complete table is available in ASCII. 
Table T26. Cleaned virtual geomagnetic pole latitude, magnetic susceptibility, and gamma ray attenuation from Site 1218 on corrected rmcd.

\begin{tabular}{|c|c|c|c|c|c|}
\hline \multicolumn{2}{|c|}{$\begin{array}{c}\text { Virtual } \\
\text { geomagnetic pole } \\
\end{array}$} & \multicolumn{2}{|c|}{$\begin{array}{l}\text { Gamma ray } \\
\text { attenuation }\end{array}$} & \multicolumn{2}{|c|}{$\begin{array}{c}\text { Magnetic } \\
\text { susceptibility }\end{array}$} \\
\hline $\begin{array}{l}\text { Depth } \\
\text { (corrected } \\
\text { rmcd) }\end{array}$ & Latitude & $\begin{array}{l}\text { Depth } \\
\text { (corrected } \\
\text { rmcd) }\end{array}$ & $\begin{array}{l}\text { Density } \\
\left(\mathrm{g} / \mathrm{cm}^{3}\right)\end{array}$ & $\begin{array}{l}\text { Depth } \\
\text { (corrected } \\
\text { rmcd) }\end{array}$ & $\left(10^{-6}\right)$ \\
\hline 0.65 & -52.4 & 0.42 & 1.090 & 0.42 & 19.4 \\
\hline 0.70 & -42.5 & 0.44 & 1.067 & 0.44 & 20.0 \\
\hline 0.75 & -35.4 & 0.46 & 1.091 & 0.46 & 20.5 \\
\hline 0.80 & -27.4 & 0.48 & 1.114 & 0.48 & 20.7 \\
\hline 0.85 & -25.8 & 0.50 & 1.098 & 0.50 & 20.5 \\
\hline 0.90 & -28.3 & 0.52 & 1.081 & 0.52 & 20.4 \\
\hline 0.95 & -29.6 & 0.54 & 1.092 & 0.54 & 21.0 \\
\hline 1.00 & -15.2 & 0.56 & 1.104 & 0.56 & 22.0 \\
\hline 1.05 & -13.6 & 0.58 & 1.103 & 0.58 & 22.4 \\
\hline 1.10 & -19.3 & 0.60 & 1.100 & 0.60 & 22.2 \\
\hline 1.15 & -23.0 & 0.64 & 1.079 & 0.62 & 21.7 \\
\hline 1.20 & -28.2 & 0.68 & 1.058 & 0.64 & 18.4 \\
\hline 1.25 & -25.7 & 0.70 & 1.054 & 0.66 & 18.5 \\
\hline 1.30 & -21.4 & 0.72 & 1.053 & 0.68 & 19.8 \\
\hline 1.35 & -25.8 & 0.74 & 1.078 & 0.70 & 21.3 \\
\hline 1.40 & -30.4 & 0.76 & 1.104 & 0.72 & 22.1 \\
\hline 1.45 & 22.6 & 0.78 & 1.107 & 0.74 & 22.1 \\
\hline 1.55 & -16.4 & 0.80 & 1.107 & 0.76 & 22.3 \\
\hline 1.60 & -9.4 & 0.82 & 1.111 & 0.78 & 22.7 \\
\hline 1.65 & -6.7 & 0.84 & 1.116 & 0.80 & 23.0 \\
\hline 1.70 & -11.5 & 0.86 & 1.114 & 0.82 & 23.0 \\
\hline 1.75 & -9.1 & 0.88 & 1.111 & 0.84 & 23.1 \\
\hline 1.80 & -4.9 & 0.90 & 1.092 & 0.86 & 23.2 \\
\hline 1.85 & -8.7 & 0.92 & 1.074 & 0.88 & 23.0 \\
\hline 1.90 & -8.9 & 0.94 & 1.090 & 0.90 & 22.6 \\
\hline 1.95 & -13.2 & 0.96 & 1.108 & 0.92 & 22.4 \\
\hline 2.00 & -8.8 & 0.98 & 1.124 & 0.94 & 22.3 \\
\hline 2.05 & -4.7 & 1.00 & 1.138 & 0.96 & 22.5 \\
\hline 2.10 & -10.2 & 1.02 & 1.117 & 0.98 & 22.7 \\
\hline 2.15 & -8.3 & 1.04 & 1.093 & 1.00 & 22.6 \\
\hline 2.20 & -7.1 & 1.06 & 1.086 & 1.02 & 22.2 \\
\hline 2.25 & -9.2 & 1.08 & 1.081 & 1.04 & 21.4 \\
\hline 2.30 & -6.6 & 1.10 & 1.090 & 1.06 & 20.7 \\
\hline 2.35 & -2.2 & 1.12 & 1.101 & 1.08 & 20.7 \\
\hline 2.40 & -5.4 & 1.14 & 1.108 & 1.10 & 20.7 \\
\hline 2.45 & -10.7 & 1.16 & 1.114 & 1.12 & 21.0 \\
\hline 2.50 & -8.3 & 1.18 & 1.100 & 1.14 & 20.7 \\
\hline 2.55 & -4.3 & 1.20 & 1.087 & 1.16 & 19.7 \\
\hline 2.60 & -7.4 & 1.22 & 1.093 & 1.18 & 19.6 \\
\hline 2.65 & -15.7 & 1.24 & 1.099 & 1.20 & 21.0 \\
\hline 2.70 & -15.4 & 1.26 & 1.086 & 1.22 & 22.3 \\
\hline 2.75 & -10.5 & 1.28 & 1.072 & 1.24 & 23.0 \\
\hline 2.80 & -11.5 & 1.30 & 1.089 & 1.26 & 23.6 \\
\hline 2.85 & -10.8 & 1.32 & 1.108 & 1.28 & 23.8 \\
\hline 2.90 & -9.0 & 1.34 & 1.117 & 1.30 & 23.5 \\
\hline 2.95 & -3.5 & 1.36 & 1.125 & 1.32 & 23.1 \\
\hline 3.05 & -13.1 & 1.58 & 1.136 & 1.34 & 23.0 \\
\hline 3.10 & -12.2 & 1.60 & 1.113 & 1.56 & 23.2 \\
\hline 3.15 & -13.3 & 1.62 & 1.090 & 1.58 & 23.8 \\
\hline 3.20 & -7.1 & 1.64 & 1.091 & 1.60 & 24.2 \\
\hline 3.25 & -9.0 & 1.66 & 1.094 & 1.62 & 24.3 \\
\hline 3.30 & -14.6 & 1.68 & 1.103 & 1.64 & 24.7 \\
\hline 3.35 & -8.8 & 1.70 & 1.099 & 1.66 & 24.1 \\
\hline 3.40 & -9.5 & 1.72 & 1.104 & 1.68 & 24.6 \\
\hline 3.45 & -12.7 & 1.74 & 1.119 & 1.70 & 25.0 \\
\hline 3.50 & -12.7 & 1.76 & 1.122 & 1.72 & 25.5 \\
\hline 3.55 & -38.2 & 1.78 & 1.112 & 1.74 & 26.5 \\
\hline 3.60 & -10.4 & 1.80 & 1.106 & 1.76 & 27.6 \\
\hline 3.65 & -5.9 & 1.82 & 1.107 & 1.78 & 28.1 \\
\hline 3.70 & -3.7 & 1.84 & 1.110 & 1.80 & 27.2 \\
\hline 3.75 & -2.3 & 1.86 & 1.113 & 1.82 & 26.6 \\
\hline 3.80 & -1.6 & 1.88 & 1.108 & 1.84 & 26.8 \\
\hline 3.85 & 2.0 & 1.90 & 1.103 & 1.86 & 27.3 \\
\hline 3.90 & 3.1 & 1.92 & 1.102 & 1.88 & 27.2 \\
\hline 3.95 & 3.5 & 1.94 & 1.101 & 1.90 & 26.4 \\
\hline
\end{tabular}

\begin{tabular}{|c|c|c|c|c|c|}
\hline \multicolumn{2}{|c|}{$\begin{array}{c}\text { Virtual } \\
\text { geomagnetic pole }\end{array}$} & \multicolumn{2}{|c|}{$\begin{array}{l}\text { Gamma ray } \\
\text { attenuation }\end{array}$} & \multicolumn{2}{|c|}{$\begin{array}{c}\text { Magnetic } \\
\text { susceptibility }\end{array}$} \\
\hline $\begin{array}{l}\text { Depth } \\
\text { (corrected } \\
\text { rmcd) }\end{array}$ & Latitude & $\begin{array}{l}\text { Depth } \\
\text { (corrected } \\
\text { rmcd) }\end{array}$ & $\begin{array}{l}\text { Density } \\
\left(\mathrm{g} / \mathrm{cm}^{3}\right)\end{array}$ & $\begin{array}{l}\text { Depth } \\
\text { (corrected } \\
\text { rmcd) }\end{array}$ & $\left(10^{-6}\right)$ \\
\hline 4.00 & 3.7 & 1.96 & 1.099 & 1.92 & 25.4 \\
\hline 4.05 & 3.3 & 1.98 & 1.100 & 1.94 & 24.8 \\
\hline 4.10 & 5.6 & 2.00 & 1.102 & 1.96 & 24.6 \\
\hline 4.15 & 31.2 & 2.02 & 1.101 & 1.98 & 24.6 \\
\hline 4.20 & 44.4 & 2.04 & 1.108 & 2.00 & 25.1 \\
\hline 4.25 & 16.1 & 2.06 & 1.123 & 2.02 & 25.9 \\
\hline 4.30 & 8.0 & 2.08 & 1.131 & 2.04 & 26.2 \\
\hline 4.35 & 6.2 & 2.10 & 1.123 & 2.06 & 26.5 \\
\hline 4.40 & 6.9 & 2.12 & 1.119 & 2.08 & 26.9 \\
\hline 4.55 & 9.2 & 2.14 & 1.120 & 2.10 & 27.1 \\
\hline 4.60 & 20.5 & 2.16 & 1.121 & 2.12 & 27.4 \\
\hline 4.65 & -1.9 & 2.18 & 1.119 & 2.14 & 27.3 \\
\hline 4.70 & -8.2 & 2.20 & 1.119 & 2.16 & 27.1 \\
\hline 4.75 & -8.9 & 2.22 & 1.117 & 2.18 & 27.0 \\
\hline 4.80 & -6.4 & 2.24 & 1.099 & 2.20 & 26.3 \\
\hline 4.85 & -4.6 & 2.26 & 1.079 & 2.22 & 25.3 \\
\hline 4.90 & -8.1 & 2.28 & 1.088 & 2.24 & 24.3 \\
\hline 4.95 & -1.0 & 2.30 & 1.097 & 2.26 & 23.6 \\
\hline 5.00 & 1.8 & 2.32 & 1.101 & 2.28 & 23.9 \\
\hline 5.05 & 7.9 & 2.34 & 1.102 & 2.30 & 24.3 \\
\hline 5.10 & 3.5 & 2.36 & 1.097 & 2.32 & 24.6 \\
\hline 5.15 & 5.7 & 2.38 & 1.100 & 2.34 & 24.4 \\
\hline 5.20 & 6.4 & 2.40 & 1.111 & 2.36 & 24.1 \\
\hline 5.25 & 5.1 & 2.42 & 1.110 & 2.38 & 24.4 \\
\hline 5.30 & 4.0 & 2.44 & 1.106 & 2.40 & 24.9 \\
\hline 5.35 & 4.6 & 2.46 & 1.111 & 2.42 & 25.7 \\
\hline 5.40 & 3.6 & 2.48 & 1.118 & 2.44 & 26.3 \\
\hline 5.45 & 4.3 & 2.50 & 1.125 & 2.46 & 26.7 \\
\hline 5.50 & 5.7 & 2.52 & 1.123 & 2.48 & 27.2 \\
\hline 5.55 & 8.0 & 2.54 & 1.111 & 2.50 & 27.6 \\
\hline 5.60 & 9.9 & 2.56 & 1.113 & 2.52 & 27.6 \\
\hline 5.65 & 7.2 & 2.58 & 1.127 & 2.54 & 27.6 \\
\hline 5.70 & 8.9 & 2.60 & 1.124 & 2.56 & 27.7 \\
\hline 5.75 & 15.0 & 2.62 & 1.113 & 2.58 & 27.4 \\
\hline 5.80 & 15.5 & 2.64 & 1.110 & 2.60 & 27.2 \\
\hline 5.85 & 15.6 & 2.66 & 1.106 & 2.62 & 26.9 \\
\hline 5.90 & 18.2 & 2.68 & 1.111 & 2.64 & 26.8 \\
\hline 5.95 & 29.7 & 2.70 & 1.119 & 2.66 & 26.8 \\
\hline 6.05 & 5.9 & 2.72 & 1.116 & 2.68 & 26.6 \\
\hline 6.10 & 6.3 & 2.74 & 1.113 & 2.70 & 26.3 \\
\hline 6.15 & 6.3 & 2.76 & 1.114 & 2.72 & 25.9 \\
\hline 6.20 & 6.0 & 2.78 & 1.117 & 2.74 & 25.9 \\
\hline 6.25 & 2.0 & 2.80 & 1.118 & 2.76 & 26.1 \\
\hline 6.30 & 3.2 & 2.82 & 1.116 & 2.78 & 26.4 \\
\hline 6.35 & 6.0 & 2.84 & 1.108 & 2.80 & 26.6 \\
\hline 6.40 & 9.4 & 2.86 & 1.105 & 2.82 & 26.3 \\
\hline 6.45 & 12.2 & 2.88 & 1.094 & 2.84 & 25.7 \\
\hline 6.50 & 12.9 & 2.90 & 1.093 & 2.86 & 25.5 \\
\hline 6.55 & 8.7 & 3.04 & 1.144 & 2.88 & 25.2 \\
\hline \multirow[t]{2}{*}{6.61} & 5.2 & 3.06 & 1.144 & 2.90 & 24.7 \\
\hline & & 3.08 & 1.139 & 2.92 & 24.5 \\
\hline 6.61 & -45.7 & 3.10 & 1.133 & 3.02 & 26.0 \\
\hline 6.66 & -85.0 & 3.12 & 1.128 & 3.04 & 26.2 \\
\hline 6.71 & -75.9 & 3.14 & 1.108 & 3.06 & 26.2 \\
\hline 6.76 & -65.9 & 3.16 & 1.097 & 3.08 & 26.3 \\
\hline 6.81 & -74.7 & 3.18 & 1.099 & 3.10 & 26.5 \\
\hline 6.86 & -72.3 & 3.20 & 1.106 & 3.12 & 26.4 \\
\hline 6.91 & -73.0 & 3.22 & 1.106 & 3.14 & 26.1 \\
\hline 6.96 & -68.4 & 3.24 & 1.107 & 3.16 & 26.0 \\
\hline 7.01 & -70.2 & 3.26 & 1.114 & 3.18 & 25.6 \\
\hline 7.06 & -67.6 & 3.28 & 1.119 & 3.20 & 25.3 \\
\hline
\end{tabular}

Only a portion of this table appears here. The complete table is available in ASCII. 
Table T27. Cleaned virtual geomagnetic pole latitude, magnetic susceptibility, and gamma ray attenuation from Site 1219 on corrected rmcd.

\begin{tabular}{|c|c|c|c|c|c|}
\hline \multicolumn{2}{|c|}{$\begin{array}{c}\text { Virtual } \\
\text { geomagnetic pole }\end{array}$} & \multicolumn{2}{|c|}{$\begin{array}{l}\text { Gamma ray } \\
\text { attenuation }\end{array}$} & \multicolumn{2}{|c|}{$\begin{array}{c}\text { Magnetic } \\
\text { susceptibility }\end{array}$} \\
\hline $\begin{array}{l}\text { Depth } \\
\text { (rmcd) }\end{array}$ & Latitude & $\begin{array}{l}\text { Depth } \\
\text { (rmcd) }\end{array}$ & $\begin{array}{l}\text { Density } \\
\left(\mathrm{g} / \mathrm{cm}^{3}\right)\end{array}$ & $\begin{array}{l}\text { Depth } \\
\text { (rmcd) }\end{array}$ & $\left(10^{-6}\right)$ \\
\hline 0.45 & 70.9 & 0.68 & 1.096 & 0.68 & 24.2 \\
\hline 0.50 & 64.8 & 0.70 & 1.100 & 0.70 & 24.0 \\
\hline 0.55 & 60.6 & 0.72 & 1.104 & 0.72 & 23.9 \\
\hline 0.60 & 53.2 & 0.74 & 1.102 & 0.74 & 23.5 \\
\hline 0.65 & 54.2 & 0.76 & 1.100 & 0.76 & 23.1 \\
\hline 0.70 & 59.4 & 0.78 & 1.084 & 0.78 & 22.9 \\
\hline 0.75 & 66.1 & 0.80 & 1.067 & 0.80 & 22.8 \\
\hline 0.80 & 53.6 & 1.00 & 1.080 & 0.82 & 22.9 \\
\hline 0.85 & 49.1 & 1.02 & 1.081 & 0.98 & 23.3 \\
\hline 0.90 & 60.2 & 1.04 & 1.083 & 1.00 & 22.8 \\
\hline 1.00 & -76.4 & 1.06 & 1.098 & 1.02 & 22.5 \\
\hline 1.05 & -81.2 & 1.08 & 1.114 & 1.04 & 22.6 \\
\hline 1.10 & -84.2 & 1.10 & 1.113 & 1.06 & 23.1 \\
\hline 1.15 & -87.1 & 1.12 & 1.110 & 1.08 & 23.9 \\
\hline 1.20 & 62.2 & 1.14 & 1.100 & 1.10 & 24.2 \\
\hline 1.25 & 50.9 & 1.16 & 1.090 & 1.12 & 24.2 \\
\hline 1.30 & -81.7 & 1.18 & 1.084 & 1.14 & 24.4 \\
\hline 1.35 & -82.1 & 1.20 & 1.078 & 1.16 & 24.4 \\
\hline 1.40 & -80.3 & 1.22 & 1.085 & 1.18 & 24.3 \\
\hline 1.45 & -80.4 & 1.24 & 1.093 & 1.20 & 24.5 \\
\hline 1.55 & -69.5 & 1.26 & 1.102 & 1.22 & 24.6 \\
\hline 1.60 & -72.6 & 1.28 & 1.111 & 1.24 & 24.3 \\
\hline 1.65 & -78.4 & 1.30 & 1.108 & 1.26 & 24.2 \\
\hline 1.70 & -72.6 & 1.32 & 1.103 & 1.28 & 24.5 \\
\hline 1.75 & -70.9 & 1.34 & 1.090 & 1.30 & 24.7 \\
\hline 1.80 & -70.7 & 1.36 & 1.076 & 1.32 & 24.7 \\
\hline 1.85 & -66.1 & 1.38 & 1.076 & 1.34 & 24.8 \\
\hline 1.90 & -67.6 & 1.40 & 1.076 & 1.36 & 24.6 \\
\hline 1.95 & -73.4 & 1.58 & 1.084 & 1.38 & 24.6 \\
\hline 2.00 & -83.7 & 1.60 & 1.090 & 1.40 & 24.7 \\
\hline 2.05 & -79.2 & 1.62 & 1.096 & 1.42 & 24.6 \\
\hline 2.10 & 46.4 & 1.64 & 1.084 & 1.56 & 24.6 \\
\hline 2.15 & 77.6 & 1.66 & 1.072 & 1.58 & 24.9 \\
\hline 2.20 & 66.8 & 1.68 & 1.077 & 1.60 & 25.0 \\
\hline 2.25 & -48.8 & 1.70 & 1.084 & 1.62 & 24.7 \\
\hline 2.30 & -74.9 & 1.72 & 1.091 & 1.64 & 24.1 \\
\hline 2.35 & -77.5 & 1.74 & 1.099 & 1.66 & 23.5 \\
\hline 2.40 & -78.5 & 1.76 & 1.106 & 1.68 & 23.1 \\
\hline 2.45 & -77.9 & 1.78 & 1.114 & 1.70 & 23.0 \\
\hline 2.50 & -78.0 & 1.80 & 1.119 & 1.72 & 22.7 \\
\hline 2.55 & -75.6 & 1.82 & 1.123 & 1.74 & 22.3 \\
\hline 2.60 & -79.4 & 1.84 & 1.117 & 1.76 & 22.0 \\
\hline 2.65 & -82.2 & 1.86 & 1.110 & 1.78 & 22.1 \\
\hline 2.70 & -81.7 & 1.88 & 1.107 & 1.80 & 22.6 \\
\hline 2.75 & -79.9 & 1.90 & 1.104 & 1.82 & 23.4 \\
\hline 2.80 & -82.5 & 1.92 & 1.105 & 1.84 & 24.0 \\
\hline 2.85 & -88.8 & 1.94 & 1.106 & 1.86 & 24.4 \\
\hline 2.90 & 43.7 & 1.96 & 1.098 & 1.88 & 24.5 \\
\hline 2.95 & 77.6 & 1.98 & 1.088 & 1.90 & 24.4 \\
\hline 3.05 & 79.0 & 2.00 & 1.072 & 1.92 & 24.3 \\
\hline 3.10 & 79.3 & 2.02 & 1.058 & 1.94 & 24.5 \\
\hline 3.15 & 78.8 & 2.04 & 1.081 & 1.96 & 24.9 \\
\hline 3.20 & 77.1 & 2.06 & 1.106 & 1.98 & 25.0 \\
\hline 3.25 & 77.6 & 2.08 & 1.110 & 2.00 & 25.1 \\
\hline 3.30 & 80.9 & 2.10 & 1.112 & 2.02 & 25.3 \\
\hline 3.35 & 87.9 & 2.12 & 1.110 & 2.04 & 25.3 \\
\hline 3.40 & 51.9 & 2.14 & 1.107 & 2.06 & 25.5 \\
\hline 3.45 & 40.1 & 2.16 & 1.098 & 2.08 & 26.0 \\
\hline 3.50 & 28.8 & 2.18 & 1.090 & 2.10 & 26.4 \\
\hline 3.55 & -17.3 & 2.20 & 1.106 & 2.12 & 26.4 \\
\hline 3.60 & 10.1 & 2.22 & 1.122 & 2.14 & 26.1 \\
\hline 3.65 & 69.0 & 2.24 & 1.110 & 2.16 & 25.9 \\
\hline 3.70 & 74.3 & 2.26 & 1.096 & 2.18 & 25.6 \\
\hline 3.75 & 79.5 & 2.28 & 1.079 & 2.20 & 25.1 \\
\hline 3.80 & 76.1 & 2.30 & 1.064 & 2.22 & 24.5 \\
\hline
\end{tabular}

\begin{tabular}{|c|c|c|c|c|c|}
\hline \multicolumn{2}{|c|}{$\begin{array}{c}\text { Virtual } \\
\text { geomagnetic pole }\end{array}$} & \multicolumn{2}{|c|}{$\begin{array}{l}\text { Gamma ray } \\
\text { attenuation }\end{array}$} & \multicolumn{2}{|c|}{$\begin{array}{c}\text { Magnetic } \\
\text { susceptibility }\end{array}$} \\
\hline $\begin{array}{l}\text { Depth } \\
\text { (rmcd) }\end{array}$ & Latitude & $\begin{array}{l}\text { Depth } \\
\text { (rmcd) }\end{array}$ & $\begin{array}{l}\text { Density } \\
\left(\mathrm{g} / \mathrm{cm}^{3}\right)\end{array}$ & $\begin{array}{l}\text { Depth } \\
\text { (rmcd) }\end{array}$ & $\left(10^{-6}\right)$ \\
\hline 3.85 & -74.5 & 2.32 & 1.072 & 2.24 & 24.2 \\
\hline 3.90 & -73.4 & 2.34 & 1.080 & 2.26 & 23.6 \\
\hline 3.95 & -69.6 & 2.36 & 1.068 & 2.28 & 22.5 \\
\hline 4.00 & -71.3 & 2.38 & 1.055 & 2.30 & 21.9 \\
\hline 4.05 & -70.0 & 2.40 & 1.060 & 2.32 & 21.6 \\
\hline 4.10 & -66.9 & 2.42 & 1.065 & 2.34 & 21.4 \\
\hline 4.15 & -66.6 & 2.44 & 1.058 & 2.36 & 21.1 \\
\hline 4.20 & -69.0 & 2.46 & 1.050 & 2.38 & 21.1 \\
\hline 4.25 & -79.7 & 2.48 & 1.050 & 2.40 & 21.0 \\
\hline 4.30 & -73.1 & 2.50 & 1.051 & 2.42 & 20.5 \\
\hline 4.35 & -71.5 & 2.52 & 1.072 & 2.44 & 20.4 \\
\hline 4.40 & -73.6 & 2.54 & 1.093 & 2.46 & 20.6 \\
\hline 4.45 & -78.8 & 2.56 & 1.076 & 2.48 & 21.0 \\
\hline 4.55 & -72.0 & 2.58 & 1.057 & 2.50 & 21.1 \\
\hline 4.60 & -75.5 & 2.60 & 1.052 & 2.52 & 20.9 \\
\hline 4.65 & -66.6 & 2.62 & 1.049 & 2.54 & 21.0 \\
\hline 4.70 & -52.1 & 2.64 & 1.066 & 2.56 & 21.4 \\
\hline 4.75 & 56.5 & 2.66 & 1.083 & 2.58 & 21.7 \\
\hline 4.80 & 67.9 & 2.68 & 1.081 & 2.60 & 22.1 \\
\hline 4.85 & 69.1 & 2.70 & 1.077 & 2.62 & 22.2 \\
\hline 4.90 & 20.5 & 2.72 & 1.078 & 2.64 & 22.4 \\
\hline 4.95 & -80.5 & 2.74 & 1.080 & 2.66 & 22.5 \\
\hline 5.00 & -67.2 & 2.76 & 1.086 & 2.68 & 22.4 \\
\hline 5.05 & -73.0 & 2.78 & 1.091 & 2.70 & 22.2 \\
\hline 5.10 & -70.3 & 2.80 & 1.076 & 2.72 & 22.3 \\
\hline 5.15 & -63.2 & 2.82 & 1.060 & 2.74 & 22.1 \\
\hline 5.20 & 58.3 & 2.84 & 1.052 & 2.76 & 22.1 \\
\hline 5.25 & 69.1 & 2.86 & 1.044 & 2.78 & 22.4 \\
\hline 5.30 & 59.1 & 3.08 & 1.102 & 2.80 & 22.5 \\
\hline 5.35 & -64.0 & 3.10 & 1.100 & 2.82 & 22.4 \\
\hline 5.40 & -67.4 & 3.12 & 1.098 & 2.84 & 22.3 \\
\hline 5.45 & -72.8 & 3.14 & 1.113 & 2.86 & 22.5 \\
\hline 5.50 & -66.9 & 3.16 & 1.128 & 2.88 & 22.9 \\
\hline 5.55 & -20.6 & 3.18 & 1.126 & 3.06 & 26.4 \\
\hline 5.60 & 53.5 & 3.20 & 1.122 & 3.08 & 27.2 \\
\hline 5.65 & -59.0 & 3.22 & 1.120 & 3.10 & 28.1 \\
\hline 5.70 & -58.1 & 3.24 & 1.117 & 3.12 & 28.7 \\
\hline \multirow[t]{2}{*}{5.75} & 20.4 & 3.26 & 1.095 & 3.14 & 29.0 \\
\hline & & 3.28 & 1.074 & 3.16 & 28.7 \\
\hline 6.45 & -0.4 & 3.30 & 1.086 & 3.18 & 28.0 \\
\hline 6.50 & 8.8 & 3.32 & 1.099 & 3.20 & 27.2 \\
\hline 6.55 & 19.8 & 3.34 & 1.100 & 3.22 & 26.6 \\
\hline 6.60 & 15.3 & 3.36 & 1.099 & 3.24 & 25.6 \\
\hline 6.65 & 19.1 & 3.38 & 1.095 & 3.26 & 24.7 \\
\hline 6.70 & 11.0 & 3.40 & 1.092 & 3.28 & 24.3 \\
\hline 6.75 & -9.7 & 3.42 & 1.103 & 3.30 & 24.5 \\
\hline 6.80 & 86.5 & 3.44 & 1.114 & 3.32 & 25.2 \\
\hline 6.85 & 79.3 & 3.46 & 1.102 & 3.34 & 26.0 \\
\hline 6.90 & 82.5 & 3.48 & 1.089 & 3.36 & 26.4 \\
\hline 6.95 & 74.3 & 3.50 & 1.104 & 3.38 & 26.8 \\
\hline 7.00 & 72.2 & 3.52 & 1.119 & 3.40 & 27.0 \\
\hline 7.05 & 63.6 & 3.54 & 1.104 & 3.42 & 26.9 \\
\hline 7.10 & 87.8 & 3.56 & 1.088 & 3.44 & 26.5 \\
\hline 7.15 & 78.8 & 3.58 & 1.088 & 3.46 & 26.1 \\
\hline 7.20 & 80.4 & 3.60 & 1.090 & 3.48 & 25.7 \\
\hline 7.25 & 78.8 & 3.62 & 1.087 & 3.50 & 25.0 \\
\hline 7.30 & 79.2 & 3.64 & 1.084 & 3.52 & 24.0 \\
\hline 7.35 & 81.9 & 3.66 & 1.083 & 3.54 & 23.3 \\
\hline 7.40 & 80.8 & 3.68 & 1.083 & 3.56 & 23.0 \\
\hline 7.45 & 66.0 & 3.70 & 1.094 & 3.58 & 23.0 \\
\hline 7.55 & -86.1 & 3.72 & 1.105 & 3.60 & 22.9 \\
\hline
\end{tabular}

Only a portion of this table appears here. The complete table is available in ASCII. 
Table T28. Cleaned virtual geomagnetic pole latitude, magnetic susceptibility, and gamma ray attenuation from Site 1220 on corrected rmcd.

\begin{tabular}{|c|c|c|c|c|c|}
\hline \multicolumn{2}{|c|}{$\begin{array}{c}\text { Virtual } \\
\text { geomagnetic pole }\end{array}$} & \multicolumn{2}{|c|}{$\begin{array}{l}\text { Gamma ray } \\
\text { attenuation }\end{array}$} & \multicolumn{2}{|c|}{$\begin{array}{c}\text { Magnetic } \\
\text { susceptibility }\end{array}$} \\
\hline $\begin{array}{l}\text { Depth } \\
\text { (rmcd) }\end{array}$ & Latitude & $\begin{array}{l}\text { Depth } \\
\text { (rmcd) }\end{array}$ & $\begin{array}{l}\text { Density } \\
\left(\mathrm{g} / \mathrm{cm}^{3}\right)\end{array}$ & $\begin{array}{l}\text { Depth } \\
\text { (rmcd) }\end{array}$ & $\left(10^{-6}\right)$ \\
\hline 0.10 & -47.1 & 0.08 & 1.125 & 0.06 & 31.0 \\
\hline 0.15 & -37.0 & 0.12 & 1.142 & 0.08 & 29.0 \\
\hline 0.20 & -20.3 & 0.16 & 1.155 & 0.10 & 37.5 \\
\hline 0.25 & 47.8 & 0.20 & 1.162 & 0.12 & 38.5 \\
\hline 0.30 & 71.5 & 0.24 & 1.133 & 0.14 & 31.0 \\
\hline 0.35 & 88.6 & 0.28 & 1.143 & 0.16 & 31.0 \\
\hline 0.40 & 67.4 & 0.32 & 1.144 & 0.18 & 32.5 \\
\hline 0.45 & -39.9 & 0.36 & 1.153 & 0.20 & 39.5 \\
\hline 0.50 & -69.0 & 0.40 & 1.154 & 0.22 & 42.0 \\
\hline 0.55 & -69.8 & 0.44 & 1.163 & 0.24 & 35.0 \\
\hline 0.60 & -78.5 & 0.48 & 1.152 & 0.26 & 32.0 \\
\hline 0.65 & -77.6 & 0.52 & 1.164 & 0.28 & 31.5 \\
\hline 0.70 & -68.7 & 0.56 & 1.165 & 0.30 & 32.0 \\
\hline 0.75 & -71.3 & 0.60 & 1.161 & 0.32 & 32.0 \\
\hline 0.80 & -84.2 & 0.64 & 1.133 & 0.34 & 31.0 \\
\hline 0.85 & -78.9 & 0.68 & 1.151 & 0.36 & 30.5 \\
\hline 0.90 & -78.4 & 0.72 & 1.136 & 0.38 & 31.0 \\
\hline 0.95 & -75.1 & 0.76 & 1.135 & 0.40 & 32.0 \\
\hline 1.00 & -70.3 & 0.80 & 1.140 & 0.42 & 32.0 \\
\hline 1.05 & -77.2 & 0.84 & 1.138 & 0.44 & 32.0 \\
\hline 1.10 & -77.7 & 0.88 & 1.140 & 0.46 & 32.0 \\
\hline 1.15 & -79.7 & 0.92 & 1.173 & 0.48 & 32.0 \\
\hline 1.20 & -69.6 & 0.96 & 1.170 & 0.50 & 32.0 \\
\hline 1.25 & -55.2 & 1.00 & 1.152 & 0.52 & 33.0 \\
\hline 1.30 & -63.7 & 1.04 & 1.158 & 0.54 & 32.0 \\
\hline 1.35 & -77.2 & 1.08 & 1.179 & 0.56 & 32.0 \\
\hline 1.40 & -79.4 & 1.12 & 1.180 & 0.58 & 32.5 \\
\hline 1.60 & -83.5 & 1.16 & 1.175 & 0.60 & 32.0 \\
\hline 1.65 & -83.5 & 1.20 & 1.169 & 0.62 & 31.0 \\
\hline 1.70 & -78.9 & 1.24 & 1.165 & 0.64 & 30.0 \\
\hline 1.75 & -71.8 & 1.28 & 1.166 & 0.66 & 29.5 \\
\hline 1.80 & -75.3 & 1.32 & 1.167 & 0.68 & 30.0 \\
\hline 1.85 & -89.2 & 1.36 & 1.154 & 0.70 & 29.0 \\
\hline 1.90 & -82.2 & 1.40 & 1.189 & 0.72 & 29.0 \\
\hline 1.95 & -82.8 & 1.44 & 1.171 & 0.74 & 28.5 \\
\hline 2.00 & -85.0 & 1.58 & 1.173 & 0.76 & 29.0 \\
\hline 2.05 & -81.1 & 1.62 & 1.165 & 0.78 & 29.5 \\
\hline 2.10 & -77.6 & 1.66 & 1.169 & 0.80 & 29.0 \\
\hline 2.15 & -75.2 & 1.70 & 1.153 & 0.82 & 29.5 \\
\hline 2.20 & -82.5 & 1.74 & 1.147 & 0.84 & 29.5 \\
\hline 2.25 & -61.1 & 1.78 & 1.146 & 0.86 & 30.5 \\
\hline 2.30 & -72.8 & 1.82 & 1.174 & 0.88 & 29.5 \\
\hline 2.35 & -69.6 & 1.86 & 1.178 & 0.90 & 31.5 \\
\hline 2.40 & -64.6 & 1.90 & 1.155 & 0.92 & 30.5 \\
\hline 2.45 & -24.2 & 1.94 & 1.169 & 0.94 & 29.5 \\
\hline 2.50 & -78.1 & 1.98 & 1.160 & 0.96 & 24.0 \\
\hline 2.55 & -74.5 & 2.02 & 1.185 & 0.98 & 24.5 \\
\hline 2.60 & -84.0 & 2.06 & 1.153 & 1.00 & 28.0 \\
\hline 2.65 & -85.1 & 2.10 & 1.169 & 1.02 & 30.0 \\
\hline 2.70 & -87.4 & 2.14 & 1.191 & 1.04 & 30.0 \\
\hline 2.75 & 54.1 & 2.18 & 1.200 & 1.06 & 30.0 \\
\hline 2.80 & 31.0 & 2.22 & 1.185 & 1.08 & 29.0 \\
\hline 2.85 & -82.2 & 2.26 & 1.187 & 1.10 & 28.5 \\
\hline 2.90 & -78.5 & 2.30 & 1.174 & 1.12 & 28.5 \\
\hline 3.10 & -71.5 & 2.34 & 1.153 & 1.14 & 28.0 \\
\hline 3.15 & -58.4 & 2.38 & 1.182 & 1.16 & 29.0 \\
\hline 3.20 & 45.0 & 2.42 & 1.189 & 1.18 & 29.5 \\
\hline 3.25 & 53.7 & 2.46 & 1.202 & 1.20 & 31.0 \\
\hline 3.30 & -84.2 & 2.50 & 1.200 & 1.22 & 33.0 \\
\hline 3.35 & -77.1 & 2.54 & 1.174 & 1.24 & 33.5 \\
\hline 3.40 & -74.6 & 2.58 & 1.180 & 1.26 & 33.0 \\
\hline 3.45 & 50.6 & 2.62 & 1.181 & 1.28 & 33.5 \\
\hline 3.50 & 55.9 & 2.66 & 1.149 & 1.30 & 33.0 \\
\hline 3.55 & 59.6 & 2.70 & 1.207 & 1.32 & 34.0 \\
\hline 3.60 & 68.5 & 2.74 & 1.167 & 1.34 & 33.5 \\
\hline
\end{tabular}

\begin{tabular}{|c|c|c|c|c|c|}
\hline \multicolumn{2}{|c|}{$\begin{array}{c}\text { Virtual } \\
\text { geomagnetic pole }\end{array}$} & \multicolumn{2}{|c|}{$\begin{array}{l}\text { Gamma ray } \\
\text { attenuation }\end{array}$} & \multicolumn{2}{|c|}{$\begin{array}{c}\text { Magnetic } \\
\text { susceptibility }\end{array}$} \\
\hline $\begin{array}{l}\text { Depth } \\
\text { (rmcd) }\end{array}$ & Latitude & $\begin{array}{l}\text { Depth } \\
\text { (rmcd) }\end{array}$ & $\begin{array}{l}\text { Density } \\
\left(\mathrm{g} / \mathrm{cm}^{3}\right)\end{array}$ & $\begin{array}{l}\text { Depth } \\
\text { (rmcd) }\end{array}$ & $\left(10^{-6}\right)$ \\
\hline 3.65 & 36.2 & 2.78 & 1.171 & 1.36 & 33.5 \\
\hline 3.70 & 15.0 & 2.82 & 1.183 & 1.38 & 33.0 \\
\hline 3.75 & 13.5 & 2.86 & 1.181 & 1.40 & 32.5 \\
\hline 3.80 & 15.6 & 2.90 & 1.163 & 1.42 & 33.5 \\
\hline 3.85 & 3.9 & 2.94 & 1.165 & 1.44 & 33.5 \\
\hline 3.90 & -18.7 & 3.08 & 1.203 & 1.56 & 33.0 \\
\hline 3.95 & -77.7 & 3.12 & 1.166 & 1.58 & 33.0 \\
\hline 4.00 & 6.6 & 3.16 & 1.219 & 1.60 & 33.5 \\
\hline 4.05 & 58.5 & 3.20 & 1.194 & 1.62 & 34.0 \\
\hline 4.10 & 59.0 & 3.24 & 1.176 & 1.64 & 34.5 \\
\hline 4.15 & 56.0 & 3.28 & 1.187 & 1.66 & 35.0 \\
\hline 4.20 & 49.9 & 3.32 & 1.187 & 1.68 & 35.0 \\
\hline 4.25 & 48.1 & 3.36 & 1.211 & 1.70 & 34.0 \\
\hline 4.30 & 41.4 & 3.40 & 1.196 & 1.72 & 34.5 \\
\hline 4.35 & 48.7 & 3.44 & 1.183 & 1.74 & 33.5 \\
\hline 4.40 & 38.1 & 3.48 & 1.198 & 1.76 & 33.0 \\
\hline 4.60 & 40.4 & 3.52 & 1.194 & 1.78 & 34.0 \\
\hline 4.65 & 43.8 & 3.56 & 1.208 & 1.80 & 33.5 \\
\hline 4.70 & 43.6 & 3.60 & 1.190 & 1.82 & 33.0 \\
\hline 4.75 & 44.7 & 3.64 & 1.187 & 1.84 & 34.0 \\
\hline 4.80 & 49.6 & 3.68 & 1.189 & 1.86 & 34.0 \\
\hline 4.85 & 8.0 & 3.72 & 1.206 & 1.88 & 34.0 \\
\hline 4.90 & -32.7 & 3.76 & 1.198 & 1.90 & 34.0 \\
\hline 4.95 & 37.8 & 3.80 & 1.188 & 1.92 & 33.0 \\
\hline 5.00 & 36.7 & 3.84 & 1.195 & 1.94 & 33.0 \\
\hline 5.05 & 48.7 & 3.88 & 1.191 & 1.96 & 32.0 \\
\hline 5.10 & 38.5 & 3.92 & 1.193 & 1.98 & 32.0 \\
\hline 5.15 & 28.0 & 3.96 & 1.224 & 2.00 & 32.0 \\
\hline 5.20 & 29.1 & 4.00 & 1.211 & 2.02 & 32.0 \\
\hline 5.25 & -30.9 & 4.04 & 1.209 & 2.04 & 33.0 \\
\hline 5.30 & 13.9 & 4.08 & 1.207 & 2.06 & 33.5 \\
\hline 5.35 & -46.8 & 4.12 & 1.187 & 2.08 & 34.0 \\
\hline 5.40 & -40.5 & 4.16 & 1.189 & 2.10 & 35.0 \\
\hline 5.45 & 59.1 & 4.20 & 1.165 & 2.12 & 35.0 \\
\hline 5.50 & 76.4 & 4.24 & 1.196 & 2.14 & 36.0 \\
\hline 5.55 & 73.6 & 4.28 & 1.187 & 2.16 & 37.0 \\
\hline 5.60 & 64.4 & 4.32 & 1.218 & 2.18 & 37.5 \\
\hline 5.65 & 67.5 & 4.36 & 1.173 & 2.20 & 37.5 \\
\hline 5.70 & 66.0 & 4.58 & 1.214 & 2.22 & 37.0 \\
\hline 5.75 & 62.9 & 4.62 & 1.192 & 2.24 & 37.0 \\
\hline 5.80 & 57.9 & 4.66 & 1.189 & 2.26 & 37.0 \\
\hline 5.85 & 66.3 & 4.70 & 1.221 & 2.28 & 36.0 \\
\hline 5.90 & 58.9 & 4.74 & 1.219 & 2.30 & 35.0 \\
\hline 6.10 & 43.2 & 4.78 & 1.204 & 2.32 & 36.0 \\
\hline 6.15 & 41.1 & 4.82 & 1.197 & 2.34 & 36.0 \\
\hline 6.20 & 59.9 & 4.86 & 1.211 & 2.36 & 36.5 \\
\hline 6.25 & 47.8 & 4.90 & 1.200 & 2.38 & 38.5 \\
\hline 6.30 & -48.0 & 4.94 & 1.202 & 2.40 & 39.5 \\
\hline 6.35 & -63.1 & 4.98 & 1.209 & 2.42 & 41.5 \\
\hline 6.40 & -33.1 & 5.02 & 1.185 & 2.44 & 41.5 \\
\hline 6.45 & -72.9 & 5.06 & 1.219 & 2.46 & 37.5 \\
\hline 6.50 & -64.6 & 5.10 & 1.206 & 2.48 & 37.0 \\
\hline 6.55 & -43.5 & 5.14 & 1.207 & 2.50 & 36.5 \\
\hline 6.60 & -85.6 & 5.18 & 1.213 & 2.52 & 37.0 \\
\hline 6.65 & -43.2 & 5.22 & 1.228 & 2.54 & 38.5 \\
\hline 6.70 & 22.5 & 5.26 & 1.196 & 2.56 & 38.5 \\
\hline 6.75 & 8.0 & 5.30 & 1.217 & 2.58 & 39.0 \\
\hline 6.80 & -50.5 & 5.34 & 1.209 & 2.60 & 37.5 \\
\hline 6.85 & -47.7 & 5.38 & 1.205 & 2.62 & 37.0 \\
\hline 6.90 & -44.3 & 5.42 & 1.204 & 2.64 & 38.0 \\
\hline 6.95 & -34.3 & 5.46 & 1.202 & 2.66 & 38.0 \\
\hline
\end{tabular}

Only a portion of this table appears here. The complete table is available in ASCII. 
Table T29. Correlation of Sites 1219, U1333, and U1334 to corrected rmcd of Site 1218. (Continued on next page.)

\begin{tabular}{|c|c|c|c|c|c|}
\hline $\begin{array}{l}\text { Site } 1219 \\
\text { depth } \\
\text { (rmcd) }\end{array}$ & $\begin{array}{l}\text { Site } 1218 \\
\text { depth } \\
\text { (corrected } \\
\text { rmcd) }\end{array}$ & $\begin{array}{l}\text { Site U1333 } \\
\text { depth } \\
\text { (rmcd [m } \\
\text { revised } \\
\text { CCSF-A]) }\end{array}$ & $\begin{array}{l}\text { Site } 1218 \\
\text { depth } \\
\text { (corrected } \\
\text { rmcd) }\end{array}$ & $\begin{array}{l}\text { Site U1334 } \\
\text { depth } \\
\text { (rmcd [m } \\
\text { revised } \\
\text { CCSF-A]) }\end{array}$ & $\begin{array}{l}\text { Site } 1218 \\
\text { depth } \\
\text { (corrected } \\
\text { rmcd) }\end{array}$ \\
\hline 0.958 & 6.609 & 2.507 & 65.111 & 0.830 & 5.440 \\
\hline 1.178 & 7.798 & 3.530 & 66.830 & 1.130 & 7.790 \\
\hline 1.330 & 8.310 & 4.228 & 68.099 & 1.200 & 8.310 \\
\hline 2.100 & 10.020 & 5.097 & 69.299 & 1.880 & 10.020 \\
\hline 2.330 & 11.730 & 5.673 & 70.117 & 2.030 & 11.730 \\
\hline 2.895 & 13.817 & 6.183 & 70.984 & 4.080 & 21.680 \\
\hline 3.614 & 16.280 & 6.663 & 71.477 & 5.280 & 23.510 \\
\hline 3.850 & 17.980 & 7.566 & 72.896 & 5.730 & 24.240 \\
\hline 4.700 & 18.960 & 8.341 & 73.930 & 5.930 & 24.760 \\
\hline 4.880 & 19.490 & 8.734 & 74.641 & 6.330 & 25.790 \\
\hline 5.180 & 20.290 & 9.960 & 76.260 & 6.920 & 27.030 \\
\hline 5.400 & 20.520 & 10.654 & 77.814 & 7.220 & 27.270 \\
\hline 5.550 & 20.830 & 10.990 & 78.486 & 7.270 & 27.310 \\
\hline 5.650 & 20.990 & 11.597 & 79.698 & 7.390 & 27.440 \\
\hline 6.785 & 23.293 & 12.683 & 81.279 & 7.690 & 30.460 \\
\hline 7.491 & 25.740 & 15.405 & 83.685 & 7.950 & 30.610 \\
\hline 8.648 & 30.383 & 15.791 & 83.898 & 8.040 & 30.870 \\
\hline 9.177 & 31.522 & 16.175 & 84.596 & 8.720 & 31.960 \\
\hline 10.239 & 34.938 & 16.647 & 85.320 & 11.340 & 33.110 \\
\hline 11.241 & 36.427 & 17.048 & 85.951 & 11.620 & 33.520 \\
\hline 12.061 & 37.383 & 17.667 & 86.976 & 11.990 & 33.960 \\
\hline 14.760 & 40.409 & 18.881 & 89.008 & 12.170 & 34.490 \\
\hline 20.380 & 47.200 & 19.424 & 89.655 & 12.260 & 34.780 \\
\hline 20.830 & 47.620 & 20.248 & 90.746 & 14.420 & 36.190 \\
\hline 21.579 & 48.455 & 20.848 & 91.694 & 14.540 & 36.520 \\
\hline 22.137 & 49.203 & 21.781 & 92.900 & 15.540 & 37.820 \\
\hline 23.230 & 51.190 & 22.081 & 93.405 & 15.720 & 38.070 \\
\hline 24.040 & 52.040 & 22.716 & 94.336 & 16.790 & 38.900 \\
\hline 24.220 & 52.260 & 23.293 & 95.338 & 17.120 & 39.400 \\
\hline 24.450 & 52.550 & 23.940 & 96.200 & 17.340 & 39.630 \\
\hline 24.810 & 52.950 & 25.192 & 98.187 & 18.390 & 40.360 \\
\hline 25.120 & 53.290 & 25.884 & 99.147 & 20.170 & 41.220 \\
\hline 26.650 & 55.240 & 26.218 & 99.700 & 20.390 & 41.380 \\
\hline 27.220 & 56.120 & 26.779 & 100.420 & 20.790 & 41.610 \\
\hline 28.200 & 57.400 & 27.201 & 101.279 & 20.920 & 41.850 \\
\hline 29.370 & 59.120 & 27.651 & 102.220 & 21.640 & 42.300 \\
\hline 29.930 & 59.770 & 28.385 & 103.496 & 22.290 & 42.770 \\
\hline 31.219 & 62.108 & 28.744 & 104.590 & 23.070 & 43.230 \\
\hline 31.771 & 63.461 & 29.588 & 106.169 & 24.640 & 43.950 \\
\hline 32.699 & 65.583 & 30.048 & 106.765 & 25.390 & 44.320 \\
\hline 33.460 & 66.830 & 30.350 & 107.180 & 26.740 & 44.980 \\
\hline 33.942 & 67.664 & 30.960 & 108.020 & 26.970 & 45.100 \\
\hline 34.998 & 69.280 & 31.514 & 109.074 & 28.690 & 46.180 \\
\hline 35.522 & 70.167 & 32.217 & 110.194 & 29.570 & 46.600 \\
\hline 36.280 & 71.330 & 32.920 & 111.539 & 29.920 & 46.850 \\
\hline 37.280 & 73.270 & 33.641 & 113.071 & 30.720 & 47.200 \\
\hline 38.011 & 74.677 & 34.322 & 114.505 & 31.520 & 47.620 \\
\hline 39.257 & 76.355 & 34.632 & 115.076 & 32.394 & 48.131 \\
\hline 40.104 & 77.423 & 34.972 & 115.580 & 33.625 & 48.820 \\
\hline 40.742 & 78.549 & 35.916 & 116.973 & 35.024 & 49.957 \\
\hline 42.083 & 81.237 & 37.080 & 119.490 & 36.534 & 51.155 \\
\hline 45.090 & 83.880 & 38.520 & 121.590 & 38.176 & 52.032 \\
\hline 45.490 & 84.320 & 38.820 & 122.120 & 38.860 & 52.550 \\
\hline 45.990 & 85.040 & 39.498 & 123.517 & 39.280 & 52.950 \\
\hline 47.078 & 86.637 & 39.803 & 124.134 & 39.690 & 53.290 \\
\hline 47.499 & 87.197 & 40.142 & 124.932 & 41.740 & 55.240 \\
\hline 48.518 & 88.561 & 41.495 & 128.438 & 42.790 & 56.115 \\
\hline 49.117 & 89.341 & 41.962 & 129.354 & 43.777 & 56.434 \\
\hline 50.284 & 90.741 & 43.289 & 131.376 & 44.840 & 57.400 \\
\hline 50.850 & 91.714 & 44.281 & 132.786 & 47.236 & 59.009 \\
\hline 51.460 & 92.440 & 45.492 & 134.460 & 48.197 & 59.758 \\
\hline 52.120 & 93.370 & 46.117 & 135.409 & 53.042 & 62.814 \\
\hline 52.666 & 94.334 & 46.848 & 136.163 & 54.338 & 63.817 \\
\hline 53.040 & 94.790 & 48.388 & 137.538 & 56.376 & 65.537 \\
\hline 53.800 & 96.200 & 49.188 & 138.712 & 57.886 & 66.680 \\
\hline
\end{tabular}

\begin{tabular}{|c|c|c|c|c|c|}
\hline $\begin{array}{l}\text { Site } 1219 \\
\text { depth } \\
\text { (rmcd) }\end{array}$ & $\begin{array}{l}\text { Site } 1218 \\
\text { depth } \\
\text { (corrected } \\
\text { rmcd) }\end{array}$ & $\begin{array}{l}\text { Site U1333 } \\
\text { depth } \\
\text { (rmcd [m } \\
\text { revised } \\
\text { CCSF-A]) }\end{array}$ & $\begin{array}{l}\text { Site } 1218 \\
\text { depth } \\
\text { (corrected } \\
\text { rmcd) }\end{array}$ & $\begin{array}{l}\text { Site U1334 } \\
\text { depth } \\
\text { (rmcd [m } \\
\text { revised } \\
\text { CCSF-A]) }\end{array}$ & $\begin{array}{c}\text { Site } 1218 \\
\text { depth } \\
\text { (corrected } \\
\text { rmcd) }\end{array}$ \\
\hline 55.158 & 98.235 & 50.366 & 140.384 & 59.371 & 67.647 \\
\hline 55.580 & 98.780 & 52.244 & 143.350 & 60.507 & 68.494 \\
\hline 56.090 & 99.660 & 53.632 & 145.509 & 61.450 & 69.069 \\
\hline 56.669 & 100.678 & 54.561 & 146.945 & 62.241 & 69.522 \\
\hline 58.191 & 104.578 & 55.415 & 148.029 & 62.863 & 69.888 \\
\hline 59.180 & 106.870 & 56.581 & 149.398 & 63.550 & 70.237 \\
\hline 59.400 & 107.180 & 57.605 & 150.978 & 64.501 & 70.805 \\
\hline 60.050 & 108.020 & 58.240 & 151.630 & 65.420 & 71.330 \\
\hline 60.582 & 109.037 & 58.818 & 152.464 & 66.113 & 71.788 \\
\hline 62.291 & 111.617 & 59.799 & 153.536 & 67.889 & 72.890 \\
\hline 63.096 & 113.109 & 61.044 & 154.832 & 68.673 & 73.481 \\
\hline 63.730 & 115.490 & 62.865 & 156.729 & 69.789 & 74.127 \\
\hline 64.446 & 117.023 & 63.645 & 157.841 & 70.533 & 74.654 \\
\hline 64.680 & 117.329 & 64.494 & 158.854 & 73.589 & 76.263 \\
\hline 65.346 & 118.634 & 67.145 & 162.070 & 74.655 & 77.407 \\
\hline 65.920 & 119.490 & 67.876 & 163.185 & 76.141 & 78.527 \\
\hline 67.320 & 121.590 & 68.427 & 163.794 & 77.940 & 79.847 \\
\hline 67.620 & 122.120 & 69.188 & 164.780 & 79.983 & 81.591 \\
\hline 68.462 & 123.522 & 70.922 & 166.954 & 85.178 & 83.759 \\
\hline 68.857 & 124.103 & 71.526 & 167.718 & 85. & 84.164 \\
\hline 69.310 & 125.003 & 72.601 & 168.998 & 86.996 & 85.090 \\
\hline 69.609 & 125.917 & & & & 85.607 \\
\hline 69.983 & 127.251 & 75.806 & 172.615 & 89.170 & 86.370 \\
\hline 70.963 & 128.412 & 77.100 & 173.920 & 89.740 & 86.780 \\
\hline 71.670 & 129.354 & 80.889 & 177.936 & 90.580 & 87.211 \\
\hline 72.015 & 129.746 & 81.982 & 179.165 & 92.910 & 88.573 \\
\hline 72.883 & 131.338 & 83.989 & 181.671 & 94.625 & 89.872 \\
\hline 73.753 & 132.142 & 86.327 & 184.490 & 95.675 & 90.698 \\
\hline 75.224 & 133.274 & 86.910 & 185.310 & 97.134 & 91.695 \\
\hline 77.155 & 135.376 & 92.260 & 190.140 & 98.048 & 92.307 \\
\hline 77.922 & 136.148 & 97.073 & 196.498 & 99.015 & 92.941 \\
\hline 79.244 & 137.584 & 98.318 & 198.064 & 100.078 & 93.465 \\
\hline 80.784 & 139.231 & 102.640 & & & 94.360 \\
\hline 81.425 & 142.642 & 103.593 & 202.669 & 102.792 & 95.353 \\
\hline 86.720 & 145.492 & 104.510 & & & 96.221 \\
\hline 88.194 & 146.950 & 105.783 & 205.755 & 106.658 & 98.201 \\
\hline 89.237 & 148.064 & 106.831 & 207.262 & 107.570 & 98.780 \\
\hline 90.538 & 149.371 & 109.472 & 210.516 & 108.040 & 99.178 \\
\hline 92.093 & 150.994 & 112.025 & 213.602 & 108.783 & 99.719 \\
\hline 93.456 & 152.479 & 113.243 & 214.877 & 110.077 & 100.653 \\
\hline 94.539 & 153.743 & 116.247 & 218.396 & 110.626 & 101.037 \\
\hline 97.442 & 156.787 & 117.367 & 219.276 & 111.829 & 101.842 \\
\hline 98.715 & 157.837 & 118.913 & 221.243 & 113.161 & 102.929 \\
\hline 99.552 & 158.882 & 120.798 & 223.725 & 114.210 & 103.785 \\
\hline 101.870 & 161.260 & 123.116 & 226.463 & 114.722 & 104.129 \\
\hline 102.600 & 162.120 & 127.226 & 230.464 & & 104.582 \\
\hline 103.398 & 163.230 & 128.172 & 231.414 & 117.795 & 106.000 \\
\hline 104.361 & 164.245 & 129.980 & 233.390 & 118.935 & 106.766 \\
\hline 109.823 & 168.983 & 131.306 & 235.008 & 119.490 & 107.180 \\
\hline 112.145 & 171.332 & 131.879 & 235.949 & 120.432 & 107.887 \\
\hline 113.491 & 172.640 & 132.323 & 236.466 & 122.096 & 109.079 \\
\hline 114.670 & 174.053 & 133.083 & 237.446 & 123.632 & 110.230 \\
\hline 125.494 & 185.560 & 133.705 & 238.130 & 124.554 & 110.891 \\
\hline 136.744 & 198.107 & 134.700 & 239.128 & 125.680 & 111.590 \\
\hline 141.892 & 202.715 & 135.424 & 240.373 & 127.724 & 113.173 \\
\hline 143.173 & 204.344 & 135.841 & 240.792 & 128.983 & 114.247 \\
\hline 143.587 & 205.073 & 136.152 & 241.298 & 129.767 & 114.885 \\
\hline 144.388 & 205.994 & 136.562 & 242.640 & 130.487 & 115.494 \\
\hline 145.345 & 207.109 & 136.887 & 243.191 & 132.352 & 116.951 \\
\hline 146.213 & 208.052 & 137.381 & 244.118 & 133.177 & 117.705 \\
\hline 150.830 & 213.634 & 137.593 & 244.456 & 135.300 & 119.490 \\
\hline 151.739 & 214.370 & 138.016 & 245.254 & 137.690 & 121.590 \\
\hline 154.673 & 216.968 & 138.327 & 245.866 & 138.340 & 122.120 \\
\hline 156.025 & 218.425 & 138.652 & 246.618 & 138.942 & 122.556 \\
\hline 157.380 & 219.282 & 139.189 & 247.503 & 140.324 & 123.500 \\
\hline
\end{tabular}


Table T29 (continued).

\begin{tabular}{|c|c|c|c|c|c|}
\hline $\begin{array}{c}\text { Site } 1219 \\
\text { depth } \\
(\mathrm{rmcd})\end{array}$ & $\begin{array}{l}\text { Site } 1218 \\
\text { depth } \\
\text { (corrected } \\
\text { rmcd) }\end{array}$ & $\begin{array}{l}\text { Site U1333 } \\
\text { depth } \\
\text { (rmcd [m } \\
\text { revised } \\
\text { CCSF-A]) }\end{array}$ & $\begin{array}{l}\text { Site } 1218 \\
\text { depth } \\
\text { (corrected } \\
\text { rmcd) }\end{array}$ & $\begin{array}{l}\text { Site U1334 } \\
\text { depth } \\
\text { (rmcd [m } \\
\text { revised } \\
\text { CCSF-A]) }\end{array}$ & $\begin{array}{c}\text { Site } 1218 \\
\text { depth } \\
\text { (corrected } \\
\text { rmcd) }\end{array}$ \\
\hline 160.825 & 224.429 & 139.782 & 248.312 & 141.220 & 124.157 \\
\hline 161.651 & 225.609 & 142.321 & 250.911 & 142.040 & 124.906 \\
\hline 163.042 & 226.971 & 142.590 & 251.275 & 143.405 & 126.258 \\
\hline 164.515 & 228.152 & 143.593 & 251.627 & 145.447 & 128.420 \\
\hline 165.871 & 229.436 & 144.132 & 251.774 & 147.160 & 129.355 \\
\hline 166.274 & 229.806 & 145.345 & 252.454 & 147.877 & 129.741 \\
\hline 167.031 & 230.468 & 145.933 & 252.696 & 148.533 & 130.195 \\
\hline 167.937 & 231.362 & 146.915 & 253.321 & 149.892 & 131.113 \\
\hline 168.601 & 232.063 & 147.338 & 253.860 & 152.204 & 132.717 \\
\hline 169.972 & 233.378 & 147.856 & 254.374 & 153.575 & 133.668 \\
\hline 171.445 & 236.261 & 148.157 & 254.699 & 155.839 & 134.830 \\
\hline 172.461 & 238.150 & 148.440 & 255.017 & 157.155 & 135.395 \\
\hline 173.083 & 238.927 & 148.737 & 255.375 & 158.702 & 137.610 \\
\hline 173.427 & 239.426 & 148.962 & 255.640 & 160.400 & 138.729 \\
\hline 174.416 & 240.846 & 149.393 & 255.918 & 162.910 & 140.507 \\
\hline 177.591 & 243.969 & 149.852 & 256.280 & 166.656 & 143.504 \\
\hline 178.511 & 244.878 & 150.897 & 257.431 & 169.298 & 145.530 \\
\hline 180.057 & 246.446 & 151.575 & 258.003 & 173.489 & 148.528 \\
\hline 181.784 & 248.586 & 152.465 & 258.727 & 177.560 & 151.630 \\
\hline 182.520 & 249.211 & 152.663 & 258.873 & 180.377 & 153.873 \\
\hline 183.070 & 249.600 & 152.931 & 259.123 & 183.909 & 156.780 \\
\hline 187.470 & 252.525 & 153.184 & 259.415 & 185.170 & 157.770 \\
\hline 188.920 & 253.710 & 154.381 & 260.296 & 190.100 & 161.260 \\
\hline 193.940 & 258.080 & 154.782 & 260.628 & 191.358 & 162.069 \\
\hline 194.890 & 258.723 & 155.688 & 261.468 & 193.303 & 163.587 \\
\hline 195.452 & 259.174 & 157.197 & 262.379 & 194.050 & 164.090 \\
\hline 197.595 & 261.503 & 157.926 & 263.076 & 198.243 & 167.370 \\
\hline 198.970 & 262.430 & 158.510 & 263.656 & 201.693 & 169.824 \\
\hline 199.751 & 263.056 & 160.685 & 264.847 & 203.823 & 171.303 \\
\hline 203.627 & 266.398 & 161.392 & 265.427 & 205.282 & 172.317 \\
\hline 204.476 & 267.402 & 161.891 & 265.883 & 207.560 & 173.934 \\
\hline 206.920 & 269.794 & 162.458 & 266.440 & 212.750 & 178.050 \\
\hline 207.470 & 270.238 & 162.917 & 266.885 & 219.800 & 184.320 \\
\hline 211.500 & 272.721 & 163.355 & 267.415 & 221.330 & 185.310 \\
\hline 213.480 & 275.424 & 163.891 & 267.967 & 230.040 & 190.140 \\
\hline 214.349 & 276.645 & 164.428 & 268.492 & 237.380 & 194.921 \\
\hline 216.345 & 278.641 & 164.951 & 268.899 & 242.719 & 198.071 \\
\hline 217.641 & 279.590 & 165.389 & 269.301 & 249.948 & 202.691 \\
\hline 218.387 & 280.477 & 165.713 & 269.517 & 251.281 & 203.428 \\
\hline 218.978 & 280.952 & 166.448 & 270.071 & 254.529 & 205.729 \\
\hline 219.440 & 281.731 & 168.891 & 272.197 & 254.996 & 206.107 \\
\hline 219.841 & 282.340 & 172.210 & 274.760 & 255.734 & 206.523 \\
\hline 220.404 & 283.089 & 172.818 & 275.202 & 256.300 & 206.867 \\
\hline 221.733 & 284.948 & 173.256 & 275.660 & 256.817 & 207.255 \\
\hline 222.217 & 285.872 & 173.693 & 276.168 & 257.629 & 207.861 \\
\hline 224.071 & 289.456 & 174.810 & 277.880 & 258.466 & 208.486 \\
\hline 224.636 & 290.598 & 175.615 & 279.330 & 258.884 & 208.845 \\
\hline \multirow[t]{17}{*}{225.805} & 292.987 & 176.494 & 281.250 & 259.376 & 209.322 \\
\hline & & 178.575 & 285.334 & 260.336 & 210.228 \\
\hline & & 179.507 & 286.865 & 261.664 & 211.144 \\
\hline & & 180.466 & 288.557 & 262.870 & 211.913 \\
\hline & & 181.341 & 290.189 & 263.402 & 212.371 \\
\hline & & 182.089 & 291.400 & 264.568 & 213.225 \\
\hline & & 182.794 & 292.516 & 265.503 & 213.861 \\
\hline & & & & 266.610 & 214.875 \\
\hline & & & & 268.554 & 216.886 \\
\hline & & & & 270.153 & 218.494 \\
\hline & & & & 272.048 & 220.114 \\
\hline & & & & 273.179 & 221.176 \\
\hline & & & & 274.114 & 221.934 \\
\hline & & & & 275.418 & 222.996 \\
\hline & & & & 277.478 & 224.728 \\
\hline & & & & 278.411 & 225.465 \\
\hline & & & & 279.439 & 226.341 \\
\hline
\end{tabular}

\begin{tabular}{|c|c|c|c|c|c|}
\hline $\begin{array}{c}\text { Site } 1219 \\
\text { depth } \\
\text { (rmcd) }\end{array}$ & $\begin{array}{l}\text { Site } 1218 \\
\text { depth } \\
\text { (corrected } \\
\text { rmcd) }\end{array}$ & $\begin{array}{l}\text { Site U1333 } \\
\text { depth } \\
\text { (rmcd [m } \\
\text { revised } \\
\text { CCSF-A]) }\end{array}$ & $\begin{array}{l}\text { Site } 1218 \\
\text { depth } \\
\text { (corrected } \\
\text { rmcd) }\end{array}$ & $\begin{array}{l}\text { Site U1334 } \\
\text { depth } \\
\text { (rmcd [m } \\
\text { revised } \\
\text { CCSF-A]) }\end{array}$ & $\begin{array}{c}\text { Site } 1218 \\
\text { depth } \\
\text { (corrected } \\
\text { rmcd) }\end{array}$ \\
\hline & & & & 280.250 & 226.907 \\
\hline & & & & 283.318 & 229.156 \\
\hline & & & & 284.854 & 230.046 \\
\hline & & & & 286.495 & 231.405 \\
\hline & & & & 288.558 & 232.751 \\
\hline & & & & 289.537 & 233.436 \\
\hline & & & & 290.605 & 234.208 \\
\hline & & & & 291.499 & 235.035 \\
\hline & & & & 292.538 & 235.965 \\
\hline & & & & 293.181 & 236.532 \\
\hline & & & & 294.674 & 237.833 \\
\hline & & & & 295.047 & 238.130 \\
\hline & & & & 296.025 & 239.121 \\
\hline & & & & 296.482 & 239.500 \\
\hline & & & & 297.600 & 240.233 \\
\hline & & & & 298.562 & 240.800 \\
\hline & & & & 299.151 & 241.216 \\
\hline & & & & 299.476 & 241.450 \\
\hline & & & & 300.570 & 242.560 \\
\hline & & & & 300.762 & 242.799 \\
\hline & & & & 301.163 & 243.191 \\
\hline & & & & 302.109 & 243.937 \\
\hline & & & & 303.023 & 244.846 \\
\hline & & & & 303.600 & 245.238 \\
\hline & & & & 304.057 & 245.488 \\
\hline & & & & 304.546 & 245.866 \\
\hline & & & & 305.904 & 246.643 \\
\hline & & & & 306.457 & 246.999 \\
\hline & & & & 307.222 & 247.503 \\
\hline & & & & 308.476 & 248.312 \\
\hline & & & & 313.192 & 250.911 \\
\hline & & & & 313.617 & 251.275 \\
\hline & & & & 314.913 & 251.627 \\
\hline & & & & 315.580 & 251.774 \\
\hline & & & & 317.418 & 252.454 \\
\hline & & & & 318.080 & 252.696 \\
\hline & & & & 319.329 & 253.321 \\
\hline & & & & 319.918 & 253.860 \\
\hline & & & & 320.468 & 254.247 \\
\hline & & & & 321.264 & 254.696 \\
\hline & & & & 321.530 & 255.001 \\
\hline & & & & 322.016 & 255.375 \\
\hline & & & & 322.331 & 255.640 \\
\hline & & & & 323.037 & 255.918 \\
\hline & & & & 323.753 & 256.280 \\
\hline & & & & 324.525 & 256.989 \\
\hline & & & & 325.631 & 257.431 \\
\hline & & & & 326.792 & 257.992 \\
\hline & & & & 328.593 & 258.727 \\
\hline & & & & 328.959 & 258.873 \\
\hline & & & & 329.501 & 259.123 \\
\hline & & & & 329.962 & 259.415 \\
\hline & & & & 331.812 & 260.099 \\
\hline & & & & 332.866 & 260.621 \\
\hline & & & & 333.575 & 260.988 \\
\hline & & & & 334.609 & 261.452 \\
\hline & & & & 335.355 & 261.968 \\
\hline & & & & 336.004 & 262.375 \\
\hline & & & & 336.737 & 263.057 \\
\hline & & & & 337.722 & 263.656 \\
\hline & & & & 339.053 & 264.144 \\
\hline & & & & 340.377 & 264.538 \\
\hline
\end{tabular}


Table T30. Correlation of Sites U1331 and U1332 to rmcd of Site 1220.

\begin{tabular}{|c|c|c|c|}
\hline $\begin{array}{l}\text { Site U1331 } \\
\text { depth } \\
\text { (rmcd }[\mathrm{m} \\
\text { revised } \\
\text { CCSF-A]) }\end{array}$ & $\begin{array}{l}\text { Site } 1220 \\
\text { depth } \\
(\mathrm{rmcd})\end{array}$ & $\begin{array}{l}\text { Site U1332 } \\
\text { depth } \\
\text { (rmcd [m } \\
\text { revised } \\
\text { CCSF-A]) }\end{array}$ & $\begin{array}{c}\text { Site } 1220 \\
\text { depth } \\
(\mathrm{rmcd})\end{array}$ \\
\hline 13.660 & 20.150 & 12.350 & 53.560 \\
\hline 15.210 & 20.730 & 13.720 & 55.720 \\
\hline 15.660 & 21.130 & 13.900 & 56.040 \\
\hline 16.110 & 21.980 & 14.950 & 57.520 \\
\hline 16.560 & 22.850 & 16.300 & 59.770 \\
\hline 17.050 & 23.150 & 16.744 & 60.757 \\
\hline 17.980 & 23.980 & 17.087 & 60.932 \\
\hline 18.180 & 24.280 & 17.250 & 61.170 \\
\hline 18.700 & 24.900 & 17.573 & 61.806 \\
\hline 18.830 & 25.030 & 18.019 & 62.221 \\
\hline 19.050 & 25.250 & 18.579 & 63.251 \\
\hline 19.280 & 25.680 & 19.244 & 64.237 \\
\hline 19.750 & 25.750 & 19.670 & 64.802 \\
\hline 20.550 & 26.670 & 20.075 & 65.224 \\
\hline 21.780 & 27.630 & 20.526 & 65.810 \\
\hline 22.450 & 28.050 & 21.962 & 67.711 \\
\hline 22.850 & 28.730 & 22.912 & 69.682 \\
\hline 23.130 & 28.980 & 24.317 & 71.205 \\
\hline 23.680 & 29.550 & 25.426 & 72.166 \\
\hline 23.980 & 29.750 & 25.974 & 72.747 \\
\hline 25.459 & 30.835 & 26.680 & 73.406 \\
\hline 26.680 & 32.130 & 26.960 & 73.937 \\
\hline 26.730 & 32.250 & 27.422 & 74.771 \\
\hline 27.080 & 32.730 & 27.819 & 75.324 \\
\hline 28.130 & 33.750 & 28.818 & 76.706 \\
\hline 28.910 & 34.730 & 28.998 & 77.002 \\
\hline 29.380 & 35.200 & 29.383 & 77.601 \\
\hline 29.950 & 35.700 & 30.297 & 78.571 \\
\hline 30.330 & 36.180 & 30.814 & 79.289 \\
\hline 30.450 & 36.300 & 31.713 & 80.826 \\
\hline 31.606 & 37.542 & 33.310 & 82.750 \\
\hline 32.910 & 39.230 & 34.110 & 83.800 \\
\hline 34.730 & 41.030 & 35.230 & 85.600 \\
\hline 35.922 & 41.788 & 35.830 & 86.670 \\
\hline 37.313 & 42.673 & 39.600 & 87.380 \\
\hline 38.255 & 43.393 & 40.439 & 88.269 \\
\hline 39.050 & 43.990 & 42.897 & 91.112 \\
\hline 40.019 & 44.866 & 44.770 & 92.800 \\
\hline 41.030 & 45.790 & 45.631 & 93.578 \\
\hline 42.080 & 46.870 & 50.134 & 97.544 \\
\hline 42.330 & 47.120 & 50.590 & 98.440 \\
\hline 42.830 & 47.640 & 51.860 & 99.540 \\
\hline 43.664 & 48.440 & 52.140 & 99.840 \\
\hline 44.448 & 49.232 & 53.090 & 100.620 \\
\hline 45.097 & 50.067 & 54.690 & 101.920 \\
\hline 46.416 & 51.254 & 56.345 & 102.856 \\
\hline 48.651 & 53.471 & 57.578 & 103.553 \\
\hline 48.900 & 53.590 & 65.990 & 109.020 \\
\hline 50.494 & 55.002 & 67.040 & 109.980 \\
\hline 51.480 & 55.720 & 73.340 & 114.150 \\
\hline 51.680 & 56.040 & 94.800 & 124.300 \\
\hline 53.780 & 57.520 & 98.850 & 125.380 \\
\hline 56.063 & 59.239 & 112.930 & 130.600 \\
\hline 56.613 & 59.484 & 136.810 & 141.430 \\
\hline
\end{tabular}

\begin{tabular}{|c|c|c|c|}
\hline $\begin{array}{l}\text { Site U1331 } \\
\text { depth } \\
\text { (rmcd [m } \\
\text { revised } \\
\text { CCSF-A]) }\end{array}$ & $\begin{array}{l}\text { Site } 1220 \\
\text { depth } \\
\text { (rmcd) }\end{array}$ & $\begin{array}{l}\text { Site U1332 } \\
\text { depth } \\
\text { (rmcd [m } \\
\text { revised } \\
\text { CCSF-A]) }\end{array}$ & $\begin{array}{c}\text { Site } 1220 \\
\text { depth } \\
\text { (rmcd) }\end{array}$ \\
\hline 56.991 & 59.697 & & \\
\hline 58.251 & 60.360 & & \\
\hline 58.636 & 60.753 & & \\
\hline 59.691 & 61.810 & & \\
\hline 59.932 & 62.113 & & \\
\hline 61.470 & 63.373 & & \\
\hline 63.261 & 64.789 & & \\
\hline 67.161 & 67.730 & & \\
\hline 69.822 & 69.679 & & \\
\hline 70.990 & 70.556 & & \\
\hline 71.637 & 71.217 & & \\
\hline 73.582 & 73.481 & & \\
\hline 74.161 & 73.935 & & \\
\hline 75.000 & 74.700 & & \\
\hline 75.653 & 75.307 & & \\
\hline 77.605 & 76.574 & & \\
\hline 77.980 & 76.970 & & \\
\hline 78.540 & 77.752 & & \\
\hline 78.840 & 78.099 & & \\
\hline 80.139 & 79.624 & & \\
\hline 82.160 & 82.750 & & \\
\hline 83.260 & 83.800 & & \\
\hline 84.760 & 85.600 & & \\
\hline 85.580 & 86.670 & & \\
\hline 86.160 & 87.380 & & \\
\hline 89.200 & 91.300 & & \\
\hline 90.700 & 92.800 & & \\
\hline 91.876 & 94.134 & & \\
\hline 93.835 & 95.789 & & \\
\hline 95.860 & 97.740 & & \\
\hline 96.280 & 98.440 & & \\
\hline 97.460 & 99.540 & & \\
\hline 97.760 & 99.840 & & \\
\hline 98.660 & 100.620 & & \\
\hline 99.910 & 101.920 & & \\
\hline 102.110 & 103.729 & & \\
\hline 103.373 & 104.668 & & \\
\hline 105.897 & 106.764 & & \\
\hline 106.538 & 107.525 & & \\
\hline 108.120 & 109.020 & & \\
\hline 108.820 & 109.980 & & \\
\hline 112.600 & 114.150 & & \\
\hline 119.531 & 119.342 & & \\
\hline 122.558 & 121.810 & & \\
\hline 124.256 & 122.913 & & \\
\hline 125.850 & 124.300 & & \\
\hline 128.716 & 125.283 & & \\
\hline 130.606 & 126.307 & & \\
\hline 133.109 & 127.570 & & \\
\hline 135.818 & 128.959 & & \\
\hline 138.210 & 130.600 & & \\
\hline 141.272 & 132.974 & & \\
\hline
\end{tabular}


Table T31. Revised magnetochrons, Sites 1218, 1219, and 1220. (Continued on next three pages.)

\begin{tabular}{|c|c|c|c|c|c|c|c|c|c|c|c|c|c|}
\hline \multirow[b]{3}{*}{ Chron } & \multirow[b]{3}{*}{ CK95 } & \multirow[b]{3}{*}{ GPTS2004 } & & & \multicolumn{3}{|c|}{ Site 1218 depth } & \multicolumn{3}{|c|}{ Site 1219 depth } & \multicolumn{3}{|c|}{ Site 1220 depth } \\
\hline & & & \multicolumn{2}{|r|}{ Expedition 320} & \multirow{2}{*}{$\begin{array}{c}\text { Top } \\
\text { (corrected } \\
\text { rmcd) }\end{array}$} & \multirow{2}{*}{$\begin{array}{l}\text { Bottom } \\
\text { (corrected } \\
\text { rmcd) }\end{array}$} & \multirow{2}{*}{$\begin{array}{l}\text { Middle } \\
\text { (corrected } \\
\text { rmcd) }\end{array}$} & \multirow{2}{*}{$\begin{array}{c}\text { Top } \\
\text { (corrected } \\
\text { rmcd) }\end{array}$} & \multirow{2}{*}{$\begin{array}{l}\text { Bottom } \\
\text { (corrected } \\
\text { rmcd) }\end{array}$} & \multirow{2}{*}{$\begin{array}{l}\text { Middle } \\
\text { (corrected } \\
\text { rmcd) }\end{array}$} & \multirow{2}{*}{$\begin{array}{c}\text { Top } \\
\text { (corrected } \\
\text { rmcd) }\end{array}$} & \multirow{2}{*}{$\begin{array}{l}\text { Bottom } \\
\text { (corrected } \\
\text { rmcd) }\end{array}$} & \multirow{2}{*}{$\begin{array}{l}\text { Middle } \\
\text { (corrected } \\
\text { rmcd) }\end{array}$} \\
\hline & & & Age & Source & & & & & & & & & \\
\hline C1n (y) & 0.000 & 0.000 & 0.000 & Lourens et al., 2004 & & & & & & & & & \\
\hline C1n (o) & 0.780 & 0.781 & 0.781 & Lourens et al., 2004 & & & 5.44 & 0.90 & 1.10 & 1.00 & & & \\
\hline C1r.1n (y) & 0.990 & 0.988 & 0.988 & Lourens et al., 2004 & & & 7.79 & 1.15 & 1.20 & 1.18 & & & \\
\hline C1r.1n (o) & 1.070 & 1.072 & 1.072 & Lourens et al., 2004 & & & 8.31 & 1.25 & 1.40 & 1.33 & & & \\
\hline$C 2 n(y)$ & 1.770 & 1.778 & 1.778 & Lourens et al., 2004 & & & 10.02 & 2.00 & 2.20 & 2.10 & & & \\
\hline C2n (o) & 1.950 & 1.945 & 1.945 & Lourens et al., 2004 & & & 11.73 & 2.20 & 2.45 & 2.33 & & & \\
\hline C2r.1n (y) & 2.140 & 2.128 & 2.128 & Lourens et al., 2004 & & & & & & & & & \\
\hline C2r.1n (o) & 2.150 & 2.148 & 2.148 & Lourens et al., 2004 & & & & & & & & & \\
\hline C2An.1n (y) & 2.581 & 2.581 & 2.581 & Lourens et al., 2004 & & & 13.78 & 2.85 & 3.10 & 2.98 & & & \\
\hline C2An.1n (o) & 3.040 & 3.032 & 3.032 & Lourens et al., 2004 & & & & & & & & & \\
\hline C2An.2n (y) & 3.110 & 3.116 & 3.116 & Lourens et al., 2004 & & & & & & & & & \\
\hline C2An.2n (o) & 3.220 & 3.207 & 3.207 & Lourens et al., 2004 & & & & & & & & & \\
\hline C2An.3n (y) & 3.330 & 3.330 & 3.330 & Lourens et al., 2004 & & & 16.28 & & & & & & \\
\hline C2An.3n (o) & 3.580 & 3.596 & 3.596 & Lourens et al., 2004 & & & 17.98 & 3.80 & 3.90 & 3.85 & & & \\
\hline C3n.1n (y) & 4.180 & 4.187 & 4.187 & Lourens et al., 2004 & 18.81 & 19.10 & 18.96 & 4.60 & 4.80 & 4.70 & & & \\
\hline C $3 n .1 n(0)$ & 4.290 & 4.300 & 4.300 & Lourens et al., 2004 & 19.43 & 19.54 & 19.49 & 4.80 & 4.95 & 4.88 & & & \\
\hline C3n.2n (y) & 4.480 & 4.493 & 4.493 & Lourens et al., 2004 & 20.24 & 20.33 & 20.29 & 5.10 & 5.25 & 5.18 & & & \\
\hline C $3 n .2 n(0)$ & 4.620 & 4.631 & 4.631 & Lourens et al., 2004 & 20.48 & 20.56 & 20.52 & 5.30 & 5.50 & 5.40 & & & \\
\hline C3n.3n (y) & 4.800 & 4.799 & 4.799 & Lourens et al., 2004 & 20.77 & 20.88 & 20.83 & 5.50 & 5.60 & 5.55 & & & \\
\hline C $3 n .3 n(0)$ & 4.890 & 4.896 & 4.896 & Lourens et al., 2004 & 20.94 & 21.03 & 20.99 & 5.60 & 5.70 & 5.65 & & & \\
\hline C3n.4n (y) & 4.980 & 4.997 & 4.997 & Lourens et al., 2004 & 21.23 & 21.32 & 21.28 & & & & & & \\
\hline C $3 n .4 n(0)$ & 5.230 & 5.235 & 5.235 & Lourens et al., 2004 & 21.58 & 21.78 & 21.68 & & & & & & \\
\hline C3An.1n (y) & 5.894 & 6.033 & 6.033 & Lourens et al., 2004 & 23.40 & 23.62 & 23.51 & 6.75 & 6.90 & 6.83 & & & \\
\hline C3An.1n (o) & 6.137 & 6.252 & 6.252 & Lourens et al., 2004 & 24.18 & 24.29 & 24.24 & & & & & & \\
\hline C3An.2n (y) & 6.269 & 6.436 & 6.436 & Lourens et al., 2004 & 24.66 & 24.85 & 24.76 & & & & & & \\
\hline C3An.2n (o) & 6.567 & 6.733 & 6.733 & Lourens et al., 2004 & 25.65 & 25.92 & 25.79 & 7.45 & 7.55 & 7.50 & & & \\
\hline C3Bn (y) & 6.935 & 7.140 & 7.140 & Lourens et al., 2004 & & & 27.03 & & & & & & \\
\hline C3Bn (o) & 7.091 & 7.212 & 7.212 & Lourens et al., 2004 & & & 27.27 & & & & & & \\
\hline C3Br.1n (y) & 7.135 & 7.251 & 7.251 & Lourens et al., 2004 & & & 27.31 & & & & & & \\
\hline C3Br.1n (o) & 7.170 & 7.285 & 7.285 & Lourens et al., 2004 & & & 27.44 & & & & & & \\
\hline C3Br.2n (y) & 7.341 & 7.454 & 7.454 & Lourens et al., 2004 & & & & & & & & & \\
\hline C3Br.2n (o) & 7.375 & 7.489 & 7.489 & Lourens et al., 2004 & & & & & & & & & \\
\hline C4n.1n (y) & 7.432 & 7.528 & 7.528 & Lourens et al., 2004 & 30.37 & 30.54 & 30.46 & 8.65 & 8.95 & 8.80 & & & \\
\hline C $4 n .1 n(0)$ & 7.562 & 7.642 & 7.642 & Lourens et al., 2004 & 30.59 & 30.63 & 30.61 & & & & & & \\
\hline C4n.2n (y) & 7.650 & 7.695 & 7.695 & Lourens et al., 2004 & 30.76 & 30.98 & 30.87 & & & & & & \\
\hline C $4 n \cdot 2 n(0)$ & 8.072 & 8.108 & 8.108 & Lourens et al., 2004 & 31.72 & 32.20 & 31.96 & 9.25 & 9.50 & 9.38 & & & \\
\hline C4r.1n (y) & 8.225 & 8.254 & 8.254 & Lourens et al., 2004 & & & & & & & & & \\
\hline C4r.1n (o) & 8.257 & 8.300 & 8.300 & Lourens et al., 2004 & & & & & & & & & \\
\hline C4An (y) & 8.699 & 8.769 & 8.769 & Lourens et al., 2004 & & & & & & & & & \\
\hline C4An (o) & 9.025 & 9.098 & 9.098 & Lourens et al., 2004 & & & & & & & & & \\
\hline C4Ar.1n (y) & 9.230 & 9.312 & 9.312 & Lourens et al., 2004 & 32.98 & 33.23 & 33.11 & & & & & & \\
\hline C4Ar.1n (o) & 9.308 & 9.409 & 9.409 & Lourens et al., 2004 & 33.43 & 33.60 & 33.52 & & & & & & \\
\hline C4Ar.2n (y) & 9.580 & 9.656 & 9.656 & Lourens et al., 2004 & 33.85 & 34.06 & 33.96 & & & & & & \\
\hline C4Ar.2n (o) & 9.642 & 9.717 & 9.717 & Lourens et al., 2004 & 34.43 & 34.55 & 34.49 & & & & & & \\
\hline C5n.1n (y) & 9.740 & 9.779 & 9.779 & Lourens et al., 2004 & 34.68 & 34.88 & 34.78 & & & & & & \\
\hline C5n.1n (o) & 9.880 & 9.934 & 9.934 & Lourens et al., 2004 & & & & 11.80 & 12.50 & 12.15 & & & \\
\hline C5n.2n (y) & 9.920 & 9.987 & 9.987 & Lourens et al., 2004 & & & & & & & & & \\
\hline C5n.2n (o) & 10.949 & 11.040 & 11.040 & Lourens et al., 2004 & & & & & & & & & \\
\hline C5r.1n (y) & 11.052 & 11.118 & 11.118 & Lourens et al., 2004 & 36.17 & 36.21 & 36.19 & & & & & & \\
\hline
\end{tabular}


Table T31 (continued). (Continued on next page.)

\begin{tabular}{|c|c|c|c|c|c|c|c|c|c|c|c|c|c|}
\hline \multirow[b]{3}{*}{ Chron } & \multirow[b]{3}{*}{ CK95 } & \multirow[b]{3}{*}{ GPTS2004 } & & & \multicolumn{3}{|c|}{ Site 1218 depth } & \multicolumn{3}{|c|}{ Site 1219 depth } & \multicolumn{3}{|c|}{ Site 1220 depth } \\
\hline & & & \multicolumn{2}{|r|}{ Expedition 320} & \multirow{2}{*}{$\begin{array}{l}\text { Top } \\
\text { (corrected } \\
\text { rmcd) }\end{array}$} & \multirow{2}{*}{$\begin{array}{l}\text { Bottom } \\
\text { (corrected } \\
\text { rmcd) }\end{array}$} & \multirow{2}{*}{$\begin{array}{c}\text { Middle } \\
\text { (corrected } \\
\text { rmcd) }\end{array}$} & \multirow{2}{*}{$\begin{array}{c}\text { Top } \\
\text { (corrected } \\
\text { rmcd) }\end{array}$} & \multirow{2}{*}{$\begin{array}{l}\text { Bottom } \\
\text { (corrected } \\
\text { rmcd) }\end{array}$} & \multirow{2}{*}{$\begin{array}{l}\text { Middle } \\
\text { (corrected } \\
\text { rmcd) }\end{array}$} & \multirow{2}{*}{$\begin{array}{l}\text { Top } \\
\text { (corrected } \\
\text { rmcd) }\end{array}$} & \multirow{2}{*}{$\begin{array}{l}\text { Bottom } \\
\text { (corrected } \\
\text { rmcd) }\end{array}$} & \multirow{2}{*}{$\begin{array}{l}\text { Middle } \\
\text { (correctec } \\
\text { rmcd) }\end{array}$} \\
\hline & & & Age & Source & & & & & & & & & \\
\hline C5r.1n (o) & 11.099 & 11.154 & 11.154 & Lourens et al., 2004 & 36.46 & 36.58 & 36.52 & & & & & & \\
\hline C5r.2n (y) & 11.476 & 11.554 & 11.554 & Lourens et al., 2004 & 37.78 & 37.86 & 37.82 & & & & & & \\
\hline C5r.2n (o) & 11.531 & 11.614 & 11.614 & Lourens et al., 2004 & 38.02 & 38.14 & 38.07 & & & & & & \\
\hline C5An.1n (y) & 11.935 & 12.014 & 12.014 & Lourens et al., 2004 & 38.86 & 38.94 & 38.90 & & & & & & \\
\hline C5An.1n (o) & 12.078 & 12.116 & 12.116 & Lourens et al., 2004 & 39.35 & 39.44 & 39.40 & & & & & & \\
\hline C5An.2n (y) & 12.184 & 12.207 & 12.207 & Lourens et al., 2004 & 39.54 & 39.72 & 39.63 & & & & & & \\
\hline C5An.2n (o) & 12.401 & 12.415 & 12.415 & Lourens et al., 2004 & 40.07 & 40.64 & 40.36 & & & & & & \\
\hline C5Ar.1n (y) & 12.678 & 12.730 & 12.730 & Lourens et al., 2004 & 41.18 & 41.26 & 41.22 & & & & & & \\
\hline C5Ar.1n (o) & 12.708 & 12.765 & 12.765 & Lourens et al., 2004 & 41.30 & 41.46 & 41.38 & & & & & & \\
\hline C5Ar.2n (y) & 12.775 & 12.820 & 12.820 & Lourens et al., 2004 & 41.55 & 41.67 & 41.61 & & & & & & \\
\hline C5Ar.2n (o) & 12.819 & 12.878 & 12.878 & Lourens et al., 2004 & 41.80 & 41.92 & 41.85 & & & & & & \\
\hline C5AAn (y) & 12.991 & 13.015 & 13.015 & Lourens et al., 2004 & 42.21 & 42.38 & 42.30 & & & & & & \\
\hline C5AAn (o) & 13.139 & 13.183 & 13.183 & Lourens et al., 2004 & 42.71 & 42.83 & 42.77 & & & & & & \\
\hline C5ABn $(y)$ & 13.302 & 13.369 & 13.369 & Lourens et al., 2004 & 43.18 & 43.28 & 43.23 & & & & & & \\
\hline C5ABn (o) & 13.510 & 13.605 & 13.605 & Lourens et al., 2004 & 43.89 & 44.01 & 43.95 & & & & & & \\
\hline C5ACn $(y)$ & 13.703 & 13.734 & 13.734 & Lourens et al., 2004 & 44.21 & 44.42 & 44.32 & & & & & & \\
\hline C5ACn (o) & 14.076 & 14.095 & 14.095 & Lourens et al., 2004 & 44.90 & 45.05 & 44.98 & & & & & & \\
\hline C5ADn (y) & 14.178 & 14.194 & 14.194 & Lourens et al., 2004 & 45.05 & 45.15 & 45.10 & & & & & & \\
\hline C5ADn (o) & 14.612 & 14.581 & 14.581 & Lourens et al., 2004 & 46.15 & 46.20 & 46.18 & & & & & & \\
\hline C5Bn.1n (y) & 14.800 & 14.784 & 14.784 & Lourens et al., 2004 & 46.55 & 46.65 & 46.60 & & & & & & \\
\hline C5Bn.1n (o) & 14.888 & 14.877 & 14.877 & Lourens et al., 2004 & 46.76 & 46.97 & 46.85 & & & & & & \\
\hline C5Bn.2n (y) & 15.034 & 15.032 & 15.032 & Lourens et al., 2004 & 47.10 & 47.34 & 47.20 & 20.30 & 20.45 & 20.38 & & & \\
\hline C $5 B n .2 n(0)$ & 15.155 & 15.160 & 15.160 & Lourens et al., 2004 & 47.55 & 47.72 & 47.62 & 20.70 & 20.95 & 20.83 & & & \\
\hline C5Cn.1n (y) & 16.014 & 15.974 & 15.974 & Lourens et al., 2004 & 51.13 & 51.26 & 51.19 & 23.16 & 23.30 & 23.23 & & & \\
\hline C5Cn.1n (o) & 16.293 & 16.268 & 16.268 & Lourens et al., 2004 & 52.01 & 52.06 & 52.04 & 23.91 & 24.17 & 24.04 & & & \\
\hline C5Cn.2n (y) & 16.327 & 16.303 & 16.303 & Lourens et al., 2004 & 52.11 & 52.41 & 52.26 & 24.17 & 24.27 & 24.22 & & & \\
\hline C5Cn.2n (o) & 16.488 & 16.472 & 16.472 & Lourens et al., 2004 & 52.51 & 52.61 & 52.55 & 24.32 & 24.57 & 24.45 & & & \\
\hline C5Cn.3n (y) & 16.556 & 16.543 & 16.543 & Lourens et al., 2004 & 52.88 & 52.96 & 52.95 & 24.69 & 24.92 & 24.81 & & & \\
\hline C5Cn.3n (o) & 16.726 & 16.721 & 16.721 & Lourens et al., 2004 & 53.27 & 53.40 & 53.29 & 25.02 & 25.22 & 25.12 & & & \\
\hline C5Dn (y) & 17.277 & 17.235 & 17.235 & Lourens et al., 2004 & 55.19 & 55.29 & 55.24 & & & 26.65 & & & \\
\hline C5Dn (o) & & 17.533 & 17.533 & Lourens et al., 2004 & 56.09 & 56.14 & 56.12 & & & 27.22 & & & \\
\hline C5Dr.1n (y) & & 17.717 & 17.717 & Lourens et al., 2004 & & & & & & 27.43 & & & \\
\hline C5Dr.1n (o) & 17.615 & 17.740 & 17.740 & Lourens et al., 2004 & & & & & & 27.53 & & & \\
\hline C5En (y) & 18.281 & 18.056 & 18.056 & Lourens et al., 2004 & 57.28 & 57.58 & 57.40 & & & 28.20 & & & \\
\hline C5En (o) & 18.781 & 18.524 & 18.524 & Lourens et al., 2004 & 59.09 & 59.14 & 59.12 & & & 29.37 & & & \\
\hline C6n (y) & 19.048 & 18.748 & 18.748 & Lourens et al., 2004 & 59.74 & 59.79 & 59.77 & & & 29.93 & & & \\
\hline C6n (o) & 20.131 & 19.722 & 19.722 & Lourens et al., 2004 & 66.64 & 67.01 & 66.83 & & & 33.46 & & & \\
\hline C6An.1n (y) & 20.518 & 20.040 & 20.040 & Lourens et al., 2004 & 69.26 & 69.38 & 69.32 & & & 35.06 & 20.05 & 20.25 & 20.15 \\
\hline C6An.1n (o) & 20.725 & 20.213 & 20.213 & Lourens et al., 2004 & 70.21 & 70.31 & 70.25 & & & 35.63 & 20.60 & 20.85 & 20.73 \\
\hline C6An.2n (y) & 20.996 & 20.439 & 20.439 & Lourens et al., 2004 & 71.16 & 71.49 & 71.33 & & & 36.28 & 21.05 & 21.20 & 21.13 \\
\hline C6An.2n (o) & 21.320 & 20.709 & 20.709 & Lourens et al., 2004 & 73.09 & 73.44 & 73.27 & & & 37.28 & 21.85 & 22.10 & 21.98 \\
\hline C6AAn (y) & 21.768 & 21.083 & 21.083 & Lourens et al., 2004 & 76.21 & 76.31 & 76.26 & & & & 22.80 & 22.90 & 22.85 \\
\hline C6AAn (o) & 21.859 & 21.159 & 21.159 & Lourens et al., 2004 & 77.63 & 77.80 & 77.72 & & & 40.27 & 23.10 & 23.20 & 23.15 \\
\hline C6AAr.1n (y) & 22.151 & 21.403 & 21.403 & Lourens et al., 2004 & & & & & & 42.39 & 23.95 & 24.00 & 23.98 \\
\hline C6AAr.1n (o) & 22.248 & 21.483 & 21.483 & Lourens et al., 2004 & & & & & & 43.14 & 24.25 & 24.30 & 24.28 \\
\hline C6AAr.2n (y) & 22.459 & 21.659 & 21.659 & Lourens et al., 2004 & 83.81 & 83.94 & 83.88 & & & 45.09 & 24.85 & 24.95 & 24.90 \\
\hline C6AAr.2n (o) & 22.493 & 21.688 & 21.688 & Lourens et al., 2004 & 84.25 & 84.38 & 84.32 & & & 45.49 & 25.00 & 25.05 & 25.03 \\
\hline C6Bn.1n (y) & 22.588 & 21.767 & 21.767 & Lourens et al., 2004 & 84.99 & 85.08 & 85.04 & & & 45.99 & 25.20 & 25.30 & 25.25 \\
\hline C6Bn.1n (o) & 22.750 & 21.936 & 21.936 & Lourens et al., 2004 & 86.30 & 86.43 & 86.37 & & & 46.89 & 25.65 & 25.70 & 25.68 \\
\hline
\end{tabular}

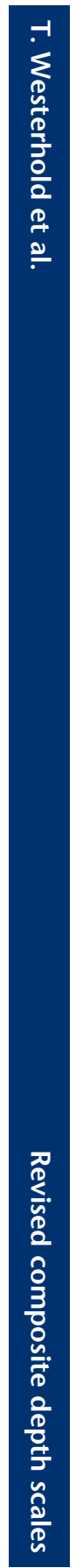


Table T31 (continued). (Continued on next page.)

\begin{tabular}{|c|c|c|c|c|c|c|c|c|c|c|c|c|c|}
\hline \multirow[b]{3}{*}{ Chron } & \multirow[b]{3}{*}{ CK95 } & \multirow[b]{3}{*}{ GPTS2004 } & & & \multicolumn{3}{|c|}{ Site 1218 depth } & \multicolumn{3}{|c|}{ Site 1219 depth } & \multicolumn{3}{|c|}{ Site 1220 depth } \\
\hline & & & \multicolumn{2}{|r|}{ Expedition 320} & \multirow{2}{*}{$\begin{array}{c}\text { Top } \\
\text { (corrected } \\
\text { rmcd) }\end{array}$} & \multirow{2}{*}{$\begin{array}{l}\text { Bottom } \\
\text { (corrected } \\
\text { rmcd) }\end{array}$} & \multirow{2}{*}{$\begin{array}{l}\text { Middle } \\
\text { (corrected } \\
\text { rmcd) }\end{array}$} & \multirow{2}{*}{$\begin{array}{c}\text { Top } \\
\text { (corrected } \\
\text { rmcd) }\end{array}$} & \multirow{2}{*}{$\begin{array}{l}\text { Bottom } \\
\text { (corrected } \\
\text { rmcd) }\end{array}$} & \multirow{2}{*}{$\begin{array}{l}\text { Middle } \\
\text { (corrected } \\
\text { rmcd) }\end{array}$} & \multirow{2}{*}{$\begin{array}{c}\text { Top } \\
\text { (corrected } \\
\text { rmcd) }\end{array}$} & \multirow{2}{*}{$\begin{array}{l}\text { Bottom } \\
\text { (corrected } \\
\text { rmcd) }\end{array}$} & \multirow{2}{*}{$\begin{array}{l}\text { Middle } \\
\text { (corrected } \\
\text { rmcd) }\end{array}$} \\
\hline & & & Age & Source & & & & & & & & & \\
\hline C6Bn.2n (y) & 22.804 & 21.992 & 21.992 & Lourens et al., 2004 & 86.73 & 86.82 & 86.78 & & & 47.16 & 25.70 & 25.80 & 25.75 \\
\hline C6Bn.2n (o) & 23.069 & 22.268 & 22.268 & Lourens et al., 2004 & 89.56 & 89.91 & 89.74 & & & 49.16 & 26.58 & 26.75 & 26.67 \\
\hline C6Cn.1n (y) & 23.353 & 22.564 & 22.564 & Lourens et al., 2004 & 92.31 & 92.56 & 92.44 & & & 51.46 & 27.60 & 27.65 & 27.63 \\
\hline C6Cn.1n (o) & 23.535 & 22.754 & 22.754 & Lourens et al., 2004 & 93.32 & 93.41 & 93.37 & & & 52.12 & 28.00 & 28.10 & 28.05 \\
\hline C6Cn.2n (y) & 23.677 & 22.902 & 22.902 & Lourens et al., 2004 & 94.42 & 95.15 & 94.79 & & & 53.04 & 28.70 & 28.75 & 28.73 \\
\hline C6Cn.2n (o) & 23.800 & 23.030 & 23.030 & Lourens et al., 2004 & 96.04 & 96.41 & 96.20 & & & 53.80 & 28.95 & 29.00 & 28.98 \\
\hline C6Cn.3n (y) & 23.999 & 23.249 & 23.278 & Pälike et al. 2006 & 98.73 & 98.83 & 98.78 & & & 55.58 & 29.50 & 29.60 & 29.55 \\
\hline C6Cn.3n (o) & 24.118 & 23.375 & 23.340 & Pälike et al. 2006 & 99.55 & 99.76 & 99.66 & & & 56.09 & 29.65 & 29.85 & 29.75 \\
\hline C7n.1n (y) & 24.730 & 24.044 & 24.022 & Pälike et al. 2006 & 106.81 & 106.92 & 106.87 & & & 59.18 & 32.10 & 32.15 & 32.13 \\
\hline C7n.1n (o) & 24.781 & 24.102 & 24.062 & Pälike et al. 2006 & 107.12 & 107.23 & 107.18 & & & 59.40 & 32.15 & 32.35 & 32.25 \\
\hline C7n.2n (y) & 24.835 & 24.163 & 24.147 & Pälike et al. 2006 & 107.72 & 108.32 & 108.02 & & & 60.05 & 32.65 & 32.80 & 32.73 \\
\hline C7n.2n (o) & 25.183 & 24.556 & 24.459 & Pälike et al. 2006 & 111.37 & 111.81 & 111.59 & & & 62.39 & 33.65 & 33.85 & 33.75 \\
\hline C7An (y) & 25.496 & 24.915 & 24.756 & Pälike et al. 2006 & 115.37 & 115.60 & 115.49 & 63.16 & 64.29 & 63.73 & 34.70 & 34.75 & 34.73 \\
\hline C7An (o) & 25.648 & 25.091 & 24.984 & Pälike et al. 2006 & 117.09 & 117.19 & 117.14 & 64.54 & 64.71 & 64.63 & 35.05 & 35.35 & 35.20 \\
\hline C8n.1n (y) & 25.823 & 25.295 & 25.110 & Pälike et al. 2006 & 119.41 & 119.56 & 119.49 & 65.74 & 66.09 & 65.92 & 35.65 & 35.75 & 35.70 \\
\hline C8n.1n (o) & 25.951 & 25.444 & 25.248 & Pälike et al. 2006 & 121.47 & 121.70 & 121.59 & 67.24 & 67.39 & 67.32 & 36.10 & 36.25 & 36.18 \\
\hline C8n.2n (y) & 25.992 & 25.492 & 25.306 & Pälike et al. 2006 & 122.00 & 122.23 & 122.12 & 67.44 & 67.79 & 67.62 & 36.25 & 36.35 & 36.30 \\
\hline C8n.2n (o) & 26.554 & 26.154 & 26.032 & Pälike et al. 2006 & 131.00 & 131.53 & 131.27 & 72.09 & 73.00 & 72.55 & 39.20 & 39.25 & 39.23 \\
\hline C9n (y) & 27.027 & 26.714 & 26.508 & Pälike et al. 2006 & 137.53 & 137.61 & 137.57 & 79.18 & 79.63 & 79.41 & 40.95 & 41.10 & 41.03 \\
\hline C9n (o) & 27.972 & 27.826 & 27.412 & Pälike et al. 2006 & 151.47 & 151.78 & 151.63 & 92.67 & 93.07 & 92.87 & 43.79 & 44.19 & 43.99 \\
\hline C10n.1n (y) & 28.283 & 28.186 & 27.886 & Pälike et al. 2006 & 157.76 & 157.78 & 157.77 & 98.47 & 99.12 & 98.80 & 45.69 & 45.89 & 45.79 \\
\hline C10n.1n (o) & 28.512 & 28.450 & 28.126 & Pälike et al. 2006 & 161.25 & 161.27 & 161.26 & 101.57 & 102.17 & 101.87 & 46.84 & 46.89 & 46.87 \\
\hline C10n.2n (y) & 28.578 & 28.525 & 28.164 & Pälike et al. 2006 & 161.85 & 162.38 & 162.12 & 102.37 & 102.82 & 102.60 & 47.09 & 47.14 & 47.12 \\
\hline C10n.2n (o) & 28.745 & 28.715 & 28.318 & Pälike et al. 2006 & 164.02 & 164.15 & 164.09 & 104.02 & 104.57 & 104.30 & 47.59 & 47.69 & 47.64 \\
\hline C11n.1n (y) & 29.401 & 29.451 & 29.166 & Pälike et al. 2006 & 177.41 & 178.69 & 178.05 & & & & 53.54 & 53.64 & 53.59 \\
\hline C11n.1n (o) & 29.662 & 29.740 & 29.467 & Pälike et al. 2006 & 184.31 & 184.33 & 184.32 & & & & 55.69 & 55.74 & 55.72 \\
\hline C11n.2n (y) & 29.765 & 29.853 & 29.536 & Pälike et al. 2006 & 185.19 & 185.43 & 185.31 & & & & 55.99 & 56.09 & 56.04 \\
\hline $\mathrm{C} 11 \mathrm{n} \cdot 2 \mathrm{n}(\mathrm{o})$ & 30.098 & 30.217 & 29.957 & Pälike et al. 2006 & 189.59 & 190.68 & 190.14 & & & & 57.44 & 57.59 & 57.52 \\
\hline $\mathrm{C} 12 \mathrm{n}(\mathrm{y})$ & 30.479 & 30.627 & 30.617 & Pälike et al. 2006 & & & & 137.45 & 138.77 & 138.11 & 59.69 & 59.84 & 59.77 \\
\hline $\mathrm{C} 12 \mathrm{n}(\mathrm{o})$ & 30.939 & 31.116 & 31.021 & Pälike et al. 2006 & & & & 142.77 & 142.87 & 142.82 & 61.09 & 61.24 & 61.17 \\
\hline C13n (y) & 33.058 & 33.266 & 33.232 & Pälike et al. 2006 & & & & 169.73 & 169.80 & 169.77 & 74.57 & 74.82 & 74.70 \\
\hline C13n (o) & 33.545 & 33.738 & 33.705 & Pälike et al. 2006 & & & & 173.42 & 173.43 & 173.43 & 76.89 & 77.04 & 76.97 \\
\hline C15n (y) & 34.655 & 34.782 & 35.126 & Pälike et al. 2006 & & & & 180.11 & 180.21 & 180.16 & 82.72 & 82.78 & 82.75 \\
\hline C15n (o) & 34.940 & 35.043 & 35.254 & Pälike et al. 2006 & & & & Core gap & & & 83.52 & 84.07 & 83.80 \\
\hline C16n.1n (y) & 35.343 & 35.404 & 35.328 & Pälike et al. 2006 & & & & 181.74 & 181.84 & 181.79 & 85.42 & 85.77 & 85.60 \\
\hline C16n.1n (o) & 35.526 & 35.567 & 35.554 & Pälike et al. 2006 & & & & 182.49 & 182.54 & 182.52 & 86.52 & 86.82 & 86.67 \\
\hline C16n.2n (y) & 35.685 & 35.707 & 35.643 & Pälike et al. 2006 & & & & 183.04 & 183.09 & 183.07 & 87.25 & 87.50 & 87.38 \\
\hline C16n.2n (o) & 36.341 & 36.276 & 36.355 & Pälike et al. 2006 & & & & 187.39 & 187.54 & 187.47 & 91.25 & 91.35 & 91.30 \\
\hline C17n.1n (y) & 36.618 & 36.512 & 36.668 & Pälike et al. 2006 & & & & 188.89 & 188.94 & 188.92 & 92.75 & 92.85 & 92.80 \\
\hline C17n.1n (o) & 37.473 & 37.235 & 37.520 & Pälike et al. 2006 & & & & 193.89 & 193.99 & 193.94 & 97.71 & 97.76 & 97.74 \\
\hline C17n.2n (y) & 37.604 & 37.345 & 37.656 & Pälike et al. 2006 & & & & 194.69 & 195.09 & 194.89 & 98.36 & 98.51 & 98.44 \\
\hline C17n.2n (o) & 37.848 & 37.549 & 37.907 & Pälike et al. 2006 & & & & 195.84 & 196.29 & 196.07 & 99.46 & 99.61 & 99.54 \\
\hline C17n.3n (y) & 37.920 & 37.610 & 37.956 & Pälike et al. 2006 & & & & 196.29 & 196.54 & 196.42 & 99.81 & 99.86 & 99.84 \\
\hline C17n.3n (o) & 38.113 & 37.771 & 38.159 & Pälike et al. 2006 & & & & 197.29 & 197.44 & 197.37 & 100.57 & 100.67 & 100.62 \\
\hline C18n.1n (y) & 38.426 & 38.032 & 38.449 & Pälike et al. 2006 & & & & 198.69 & 199.24 & 198.97 & 101.87 & 101.97 & 101.92 \\
\hline C18n.1n (o) & 39.552 & 38.975 & 39.554 & Pälike et al. 2006 & & & & 206.87 & 206.97 & 206.92 & 108.92 & 109.12 & 109.02 \\
\hline C18n.2n (y) & 39.631 & 39.041 & 39.602 & Pälike et al. 2006 & & & & 207.37 & 207.57 & 207.47 & 109.83 & 110.12 & 109.98 \\
\hline C18n.2n (o) & 40.130 & 39.464 & 40.084 & Pälike et al. 2006 & & & & 211.27 & 211.72 & 211.50 & 114.10 & 114.20 & 114.15 \\
\hline C19n (y) & 41.257 & 40.439 & 41.358 & Pälike et al. 2006 & & & & 224.09 & 224.64 & 224.37 & 124.20 & 124.40 & 124.30 \\
\hline
\end{tabular}


Table T31 (continued).

\begin{tabular}{|c|c|c|c|c|c|c|c|c|c|c|c|c|c|}
\hline \multirow[b]{3}{*}{ Chron } & \multirow[b]{3}{*}{ CK95 } & \multirow[b]{3}{*}{ GPTS2004 } & & & \multicolumn{3}{|c|}{ Site 1218 depth } & \multicolumn{3}{|c|}{ Site 1219 depth } & \multicolumn{3}{|c|}{ Site 1220 depth } \\
\hline & & & \multicolumn{2}{|r|}{ Expedition 320} & \multirow{2}{*}{$\begin{array}{l}\text { Top } \\
\text { (corrected } \\
\text { rmcd) }\end{array}$} & \multirow{2}{*}{$\begin{array}{l}\text { Bottom } \\
\text { (corrected } \\
\text { rmcd) }\end{array}$} & \multirow{2}{*}{$\begin{array}{l}\text { Middle } \\
\text { (corrected } \\
\text { rmcd) }\end{array}$} & \multirow{2}{*}{$\begin{array}{c}\text { Top } \\
\text { (corrected } \\
\text { rmcd) }\end{array}$} & \multirow{2}{*}{$\begin{array}{l}\text { Bottom } \\
\text { (corrected } \\
\text { rmcd) }\end{array}$} & \multirow{2}{*}{$\begin{array}{l}\text { Middle } \\
\text { (corrected } \\
\text { rmcd) }\end{array}$} & \multirow{2}{*}{$\begin{array}{c}\text { Top } \\
\text { (corrected } \\
\text { rmcd) }\end{array}$} & \multirow{2}{*}{$\begin{array}{l}\text { Bottom } \\
\text { (corrected } \\
\text { rmcd) }\end{array}$} & \multirow{2}{*}{$\begin{array}{l}\text { Middle } \\
\text { (corrected } \\
\text { rmcd) }\end{array}$} \\
\hline & & & Age & Source & & & & & & & & & \\
\hline C19n (o) & 41.521 & 40.671 & 41.510 & Pälike et al. 2006 & & & & 225.89 & 226.19 & 226.04 & 125.35 & 125.40 & 125.38 \\
\hline C20n (y) & 42.536 & 41.590 & 42.536 & Cande and Kent, 1995 & & & & 232.19 & 232.39 & 232.29 & 130.45 & 130.75 & 130.60 \\
\hline C20n (o) & 43.789 & 42.774 & 43.789 & Cande and Kent, 1995 & & & & 243.64 & 244.59 & 244.12 & 141.38 & 141.48 & 141.43 \\
\hline C21n (y) & 46.264 & 45.346 & 46.264 & Cande and Kent, 1995 & & & & & & & & & \\
\hline C21n (o) & 47.906 & 47.235 & 47.906 & Cande and Kent, 1995 & & & & & & & & & \\
\hline$C 22 n(y)$ & 49.037 & 48.599 & 49.037 & Cande and Kent, 1995 & & & & & & & & & \\
\hline$C 22 n(0)$ & 49.714 & 49.427 & 49.714 & Cande and Kent, 1995 & & & & & & & & & \\
\hline$C 23 n .1 n(y)$ & 50.778 & 50.730 & 50.778 & Cande and Kent, 1995 & & & & & & & & & \\
\hline C23n.1n (o) & 50.946 & 50.932 & 50.946 & Cande and Kent, 1995 & & & & & & & & & \\
\hline$C 23 n \cdot 2 n(y)$ & 51.047 & 51.057 & 51.047 & Cande and Kent, 1995 & & & & & & & & & \\
\hline$C 23 n \cdot 2 n(0)$ & 51.743 & 51.901 & 51.743 & Cande and Kent, 1995 & & & & & & & & & \\
\hline
\end{tabular}

Site 1219 data is from Pälike et al. (2005). 


\begin{tabular}{|c|c|c|c|c|c|c|c|c|c|c|c|c|c|c|c|}
\hline \multirow[b]{2}{*}{ Source } & \multirow[b]{2}{*}{ Event } & \multirow[b]{2}{*}{ Zone } & \multirow{2}{*}{$\begin{array}{l}\mathrm{Age}^{*} \\
(\mathrm{Ma})\end{array}$} & \multicolumn{2}{|c|}{ Core, section, interval $(\mathrm{cm})$} & \multicolumn{2}{|c|}{$\begin{array}{c}\text { Depth } \\
\text { (mbsf [m CSF-A]) }\end{array}$} & \multicolumn{3}{|c|}{$\begin{array}{l}\text { Depth (rmcd [adjusted } \\
\text { m revised CCSF-A]) }\end{array}$} & \multirow{2}{*}{$\begin{array}{l}\text { Error } \\
( \pm) \\
(\mathrm{m})\end{array}$} & \multicolumn{3}{|c|}{$\begin{array}{l}\text { Depth Site } 1220 \\
\text { (rmcd) }\end{array}$} & \multirow{2}{*}{$\begin{array}{c}\text { Error } \\
( \pm) \\
(\mathrm{m})\end{array}$} \\
\hline & & & & Top & Bottom & Top & Bottom & Top & Bottom & Mean & & Top & Bottom & Mean & \\
\hline Ship & B Theocyrtis annosa & RP21 & 28.330 & $\begin{array}{l}320-\mathrm{U} 1331 \mathrm{~A}- \\
2 \mathrm{H}-2,104-106\end{array}$ & $\begin{array}{l}320-\mathrm{U} 1331 \mathrm{~A}- \\
2 \mathrm{H}-4,104-106\end{array}$ & 7.75 & 10.75 & 9.25 & 12.25 & 10.75 & 1.50 & & & & \\
\hline Ship & T Tristylospyris triceros $>$ Dorcadospyris ateuchus & & 28.600 & $2 \mathrm{H}-4,104-106$ & $2 \mathrm{H}-\mathrm{CC}$ & 10.75 & 14.81 & 12.25 & 16.37 & 14.31 & 2.06 & & 59.93 & & \\
\hline Ship & B Dorcadospyris ateuchus & & 29.501 & $2 \mathrm{H}-4,104-106$ & $2 \mathrm{H}-\mathrm{CC}$ & 10.75 & 14.81 & 12.25 & 16.37 & 14.31 & 2.06 & & 59.93 & & \\
\hline Ship & B Dorcadospyris circulus & \multirow{17}{*}{ RP20 } & 29.960 & $2 \mathrm{H}-\mathrm{CC}$ & $3 \mathrm{H}-3,126-128$ & 14.81 & 18.97 & 16.37 & 21.88 & 19.13 & 2.76 & 59.93 & 67.60 & 63.76 & 3.84 \\
\hline Ship & T Lithocyclia crux & & 30.130 & $2 \mathrm{H}-\mathrm{CC}$ & $3 \mathrm{H}-3,126-128$ & 14.81 & 18.97 & 16.37 & 21.88 & 19.13 & 2.76 & 59.93 & 67.60 & 63.76 & 3.84 \\
\hline Ship & B Eucyrtidium plesiodiaphanes & & 30.567 & $2 \mathrm{H}-\mathrm{CC}$ & $3 \mathrm{H}-3,126-128$ & 14.81 & 18.97 & 16.37 & 21.88 & 19.13 & 2.76 & 59.93 & 67.60 & 63.76 & 3.84 \\
\hline Ship & B Lophocyrtis (C.) pegetrum & & 30.675 & $2 \mathrm{H}-\mathrm{CC}$ & $3 \mathrm{H}-3,126-128$ & 14.81 & 18.97 & 16.37 & 21.88 & 19.13 & 2.76 & 59.93 & 67.60 & 63.76 & 3.84 \\
\hline Ship & T Lophocyrtis (S.) oberhaensliae & & 30.740 & $3 \mathrm{H}-3,126-129$ & $3 \mathrm{H}-5,121-124$ & 18.97 & 21.92 & 21.88 & 24.83 & 23.36 & 1.48 & 67.60 & 71.65 & 69.63 & 2.02 \\
\hline Ship & B Dorcadospyris spinosa & & 30.842 & $3 \mathrm{H}-3,126-129$ & $3 \mathrm{H}-5,121-124$ & 18.97 & 21.92 & 21.88 & 24.83 & 23.36 & 1.48 & 67.60 & 71.65 & 69.63 & 2.02 \\
\hline Ship & T Dorcadospyris pseudopapilio & & 30.842 & $3 \mathrm{H}-3,126-129$ & $3 \mathrm{H}-5,121-124$ & 18.97 & 21.92 & 21.88 & 24.83 & 23.36 & 1.48 & 67.60 & 71.65 & 69.63 & 2.02 \\
\hline Ship & T Centrobotrys gravida & & 30.893 & $3 \mathrm{H}-5,121-124$ & $3 \mathrm{H}-\mathrm{CC}$ & 21.92 & 24.12 & 24.83 & 27.07 & 25.95 & 1.12 & 71.65 & 74.14 & 72.89 & 1.24 \\
\hline Ship & B Theocyrtis tuberosa & & 31.000 & $3 \mathrm{H}-\mathrm{CC}$ & $4 \mathrm{H}-2,104-106$ & 24.12 & 26.75 & 27.07 & 30.01 & 28.54 & 1.47 & 74.14 & 78.27 & 76.20 & 2.07 \\
\hline Ship & B Dorcadospyris pseudopapilio & & 31.003 & $3 \mathrm{H}-\mathrm{CC}$ & $4 \mathrm{H}-2,104-106$ & 24.12 & 26.75 & 27.07 & 30.01 & 28.54 & 1.47 & 74.14 & 78.27 & 76.20 & 2.07 \\
\hline Ship & B Centrobotrys gravida & & 31.008 & $3 \mathrm{H}-\mathrm{CC}$ & $4 \mathrm{H}-2,104-106$ & 24.12 & 26.75 & 27.07 & 30.01 & 28.54 & 1.47 & 74.14 & 78.27 & 76.20 & 2.07 \\
\hline Ship & B Lithocyclia crux & & 31.008 & $3 \mathrm{H}-\mathrm{CC}$ & $4 \mathrm{H}-2,104-106$ & 24.12 & 26.75 & 27.07 & 30.01 & 28.54 & 1.47 & 74.14 & 78.27 & 76.20 & 2.07 \\
\hline Ship & T Calocycletta anekathen & & 33.652 & $3 \mathrm{H}-\mathrm{CC}$ & $4 \mathrm{H}-2,104-106$ & 24.12 & 26.75 & 27.07 & 30.01 & 28.54 & 1.47 & 74.14 & 78.27 & 76.20 & 2.07 \\
\hline Ship & T Lophocyrtis (L.) jacchia & & 33.693 & $3 \mathrm{H}-\mathrm{CC}$ & $4 \mathrm{H}-2,104-106$ & 24.12 & 26.75 & 27.07 & 30.01 & 28.54 & 1.47 & 74.14 & 78.27 & 76.20 & 2.07 \\
\hline Ship & T Dictyoprora armadillo & & 33.693 & $3 \mathrm{H}-\mathrm{CC}$ & $4 \mathrm{H}-2,104-106$ & 24.12 & 26.75 & 27.07 & 30.01 & 28.54 & 1.47 & 74.14 & 78.27 & 76.20 & 2.07 \\
\hline Ship & T Lychnocanoma amphitrite & & 33.753 & $3 \mathrm{H}-\mathrm{CC}$ & $4 \mathrm{H}-2,104-106$ & 24.12 & 26.75 & 27.07 & 30.01 & 28.54 & 1.47 & 74.14 & 78.27 & 76.20 & 2.07 \\
\hline Ship & Lithocyclia aristotelis > Lithocyclia angusta & & 33.820 & $3 \mathrm{H}-\mathrm{CC}$ & $4 \mathrm{H}-2,104-106$ & 24.12 & 26.75 & 27.07 & 30.01 & 28.54 & 1.47 & 74.14 & 78.27 & 76.20 & 2.07 \\
\hline Ship & B Lithocyclia angusta & \multirow{3}{*}{ RP19 } & 34.125 & $4 \mathrm{H}-2,104-106$ & $4 \mathrm{H}-4,104-106$ & 26.75 & 29.75 & 30.01 & 33.01 & 31.51 & 1.50 & 78.27 & 82.39 & 80.33 & 2.06 \\
\hline Ship & T Calocyclas bandyca & & 34.616 & $4 \mathrm{H}-2,104-106$ & $4 \mathrm{H}-4,104-106$ & 26.75 & 29.75 & 30.01 & 33.01 & 31.51 & 1.50 & 78.27 & 82.39 & 80.33 & 2.06 \\
\hline Ship & T Thyrsocyrtis $(P$.$) tetracantha$ & & 35.300 & $4 \mathrm{H}-4,104-106$ & $4 \mathrm{H}-\mathrm{CC}$ & 29.75 & 33.95 & 33.01 & 37.21 & 35.11 & 2.10 & 82.39 & 86.93 & 84.66 & 2.27 \\
\hline Ship & B Lophocyrtis (C.) hadra & \multirow{4}{*}{ RP18 } & 35.338 & $4 \mathrm{H}-\mathrm{CC}$ & $5 \mathrm{H}-2,104-106$ & 33.95 & 36.25 & 37.21 & 41.50 & 39.36 & 2.15 & 86.93 & 89.50 & 88.21 & 1.28 \\
\hline Ship & T Lychnocanoma turgidum & & 35.772 & $4 \mathrm{H}-\mathrm{CC}$ & $5 \mathrm{H}-2,104-106$ & 33.95 & 36.25 & 37.21 & 41.50 & 39.36 & 2.15 & 86.93 & 89.50 & 88.21 & 1.28 \\
\hline Ship & T Lychnocanoma amphitrite & & 36.499 & $5 \mathrm{H}-4,104-106$ & $5 \mathrm{H}-\mathrm{CC}$ & 39.25 & 42.77 & 44.50 & 48.16 & 46.33 & 1.83 & 92.56 & 95.81 & 94.18 & 1.62 \\
\hline Ship & B Calocyclas bandyca & & 36.744 & $5 \mathrm{H}-4,104-106$ & $5 \mathrm{H}-\mathrm{CC}$ & 39.25 & 42.77 & 44.50 & 48.16 & 46.33 & 1.83 & 92.56 & 95.81 & 94.18 & 1.62 \\
\hline Ship & B Lophocyrtis (L.) jacchia & \multirow{3}{*}{ RP17 } & 37.065 & $5 \mathrm{H}-4,104-106$ & $5 \mathrm{H}-\mathrm{CC}$ & 39.25 & 42.77 & 44.50 & 48.16 & 46.33 & 1.83 & 92.56 & 95.81 & 94.18 & 1.62 \\
\hline Ship & B Thyrsocyrtis (P.) lochites & & 37.519 & $6 \mathrm{H}-2,105-107$ & $6 \mathrm{H}-4,105-107$ & 45.75 & 48.75 & 52.91 & 55.91 & 54.41 & 1.50 & 100.47 & 102.61 & 101.54 & 1.07 \\
\hline Ship & B Cryptocarpium azyx & & 37.519 & 6H-2, 105-107 & $6 \mathrm{H}-4,105-107$ & 45.75 & 48.75 & 52.91 & 55.91 & 54.41 & 1.50 & 100.47 & 102.61 & 101.54 & 1.07 \\
\hline Ship & T Anthocyrtoma spp. & \multirow{7}{*}{ RP16 } & 37.916 & $6 \mathrm{H}-4,105-107$ & $6 \mathrm{H}-\mathrm{CC}$ & 48.75 & 52.46 & 55.91 & 59.65 & 57.78 & 1.87 & 102.61 & 104.90 & 103.75 & 1.14 \\
\hline Ship & B Thyrsocyrtis (T.) bromia & & 38.068 & $6 \mathrm{H}-4,105-107$ & $6 \mathrm{H}-\mathrm{CC}$ & 48.75 & 52.46 & 55.91 & 59.65 & 57.78 & 1.87 & 102.61 & 104.90 & 103.75 & 1.14 \\
\hline Ship & B Thyrsocyrtis $(P$.$) tetracantha$ & & 38.118 & $6 \mathrm{H}-4,105-107$ & $6 \mathrm{H}-\mathrm{CC}$ & 48.75 & 52.46 & 55.91 & 59.65 & 57.78 & 1.87 & 102.61 & 104.90 & 103.75 & 1.14 \\
\hline Ship & T Dorcadospyris anastasis & & 38.447 & $6 \mathrm{H}-\mathrm{CC}$ & $7 \mathrm{H}-2,95-97$ & 52.46 & 55.16 & 59.65 & 64.97 & 62.31 & 2.66 & 104.90 & 108.36 & 106.63 & 1.73 \\
\hline Ship & B Calocyclas turris & & 38.667 & $6 \mathrm{H}-\mathrm{CC}$ & $7 \mathrm{H}-2,95-97$ & 52.46 & 55.16 & 59.65 & 64.97 & 62.31 & 2.66 & 104.90 & 108.36 & 106.63 & 1.73 \\
\hline Ship & B Lithocyclia aristotelis gr. & & 39.725 & 7H-2, 95-97 & 7H-4, 95-97 & 55.16 & 58.16 & 64.97 & 67.97 & 66.47 & 1.50 & 108.36 & 110.60 & 109.48 & 1.12 \\
\hline Ship & B Dorcadospyris anastasis & & 39.979 & 7H-4, 95-97 & $7 \mathrm{H}-\mathrm{CC}$ & 58.16 & 62.49 & 67.97 & 72.30 & 70.14 & 2.17 & 110.60 & 113.46 & 112.03 & 1.43 \\
\hline Ship & B Podocyrtis goetheana & & 40.156 & 7H-4, 95-97 & $7 \mathrm{H}-\mathrm{CC}$ & 58.16 & 62.49 & 67.97 & 72.30 & 70.14 & 2.17 & 110.60 & 113.46 & 112.03 & 1.43 \\
\hline Ship & T Lophocyrtis biaurita & RP15 & 40.362 & $8 \mathrm{H}-4,104-106$ & $8 \mathrm{H}-\mathrm{CC}$ & 67.75 & 72.00 & 78.52 & 82.77 & 80.65 & 2.13 & 116.60 & 118.61 & 117.61 & 1.01 \\
\hline Ship & Podocyrtis mitra > Podocyrtis chalara & & 40.700 & $9 \mathrm{H}-4,105-107$ & $9 \mathrm{H}-\mathrm{CC}$ & 77.26 & 81.47 & 88.62 & 92.83 & 90.73 & 2.11 & 121.38 & 123.37 & 122.37 & 1.00 \\
\hline Ship & T Podocyrtis trachodes & \multirow{9}{*}{ RP14 } & 41.227 & $10 \mathrm{H}-2,95-97$ & $10 \mathrm{H}-4,95-97$ & 83.66 & 86.66 & 94.54 & 97.54 & 96.04 & 1.50 & 124.18 & 125.03 & 124.60 & 0.43 \\
\hline Ship & B Podocyrtis chalara & & 41.542 & $10 \mathrm{H}-4,95-97$ & $10 \mathrm{H}-\mathrm{CC}$ & 86.66 & 90.29 & 97.54 & 100.92 & 99.23 & 1.69 & 125.03 & 126.15 & 125.59 & 0.56 \\
\hline Ship & B Cryptocarpium ornatum & & 42.098 & $10 \mathrm{H}-\mathrm{CC}$ & $11 \mathrm{H}-3,106-108$ & 90.29 & 93.95 & 100.92 & 106.57 & 103.75 & 2.83 & 126.15 & 128.24 & 127.19 & 1.05 \\
\hline Ship & B Sethochytris triconiscus & & 42.403 & $11 \mathrm{H}-3,106-108$ & $11 \mathrm{H}-5,106-108$ & 93.95 & 96.95 & 106.57 & 110.17 & 108.37 & 1.80 & 128.24 & 129.58 & 128.91 & 0.67 \\
\hline Ship & T Eusyringium lagena & & 42.687 & $11 \mathrm{H}-5,106-108$ & $11 \mathrm{H}-\mathrm{CC}$ & 96.95 & 99.32 & 110.17 & 112.54 & 111.36 & 1.19 & 129.58 & 130.46 & 130.02 & 0.44 \\
\hline Ship & B Theocyrtis perpumila & & 42.972 & $11 \mathrm{H}-\mathrm{CC}$ & $12 \mathrm{H}-2,106-108$ & 99.32 & 102.77 & 112.54 & 118.85 & 115.70 & 3.15 & 130.46 & 133.28 & 131.87 & 1.41 \\
\hline Ship & T Podocyrtis helenae & & 43.053 & $12 \mathrm{H}-2,106-108$ & $12 \mathrm{H}-4,106-108$ & 102.77 & 105.77 & 118.85 & 121.90 & 120.38 & 1.53 & 133.28 & 134.67 & 133.98 & 0.69 \\
\hline Ship & B Podocyrtis trachodes & & 43.219 & $12 \mathrm{H}-4,106-108$ & $12 \mathrm{H}-\mathrm{CC}$ & 105.77 & 109.99 & 121.90 & 126.46 & 124.18 & 2.28 & 134.67 & 136.74 & 135.70 & 1.03 \\
\hline Ship & B Zygocircus cimelium & & 43.351 & $12 \mathrm{H}-4,106-108$ & $12 \mathrm{H}-\mathrm{CC}$ & 105.77 & 109.99 & 121.90 & 126.46 & 124.18 & 2.28 & 134.67 & 136.74 & 135.70 & 1.03 \\
\hline
\end{tabular}




\begin{tabular}{|c|c|c|c|c|c|c|c|c|c|c|c|c|c|c|c|}
\hline \multirow[b]{2}{*}{ Source } & \multirow[b]{2}{*}{ Event } & \multirow[b]{2}{*}{ Zone } & \multirow{2}{*}{$\begin{array}{l}\mathrm{Age}^{*} \\
(\mathrm{Ma})\end{array}$} & \multicolumn{2}{|c|}{ Core, section, interval $(\mathrm{cm})$} & \multicolumn{2}{|c|}{$\begin{array}{c}\text { Depth } \\
\text { (mbsf [m CSF-A]) }\end{array}$} & \multicolumn{3}{|c|}{$\begin{array}{l}\text { Depth (rmcd [adjusted } \\
\text { m revised CCSF-A]) }\end{array}$} & \multirow{2}{*}{$\begin{array}{l}\text { Error } \\
( \pm) \\
(\mathrm{m})\end{array}$} & \multicolumn{3}{|c|}{$\begin{array}{c}\text { Depth Site } 1220 \\
\text { (rmcd) }\end{array}$} & \multirow{2}{*}{$\begin{array}{c}\text { Error } \\
( \pm) \\
(\mathrm{m})\end{array}$} \\
\hline & & & & Top & Bottom & Top & Bottom & Top & Bottom & Mean & & Top & Bottom & Mean & \\
\hline Ship & Podocyrtis sinuos $a>$ Podocyrtis mitra & & 43.840 & $13 \mathrm{H}-2,104-106$ & $13 \mathrm{H}-4,104-106$ & 112.25 & 115.25 & 130.22 & 133.22 & 131.72 & 1.50 & 138.44 & 139.80 & 139.12 & 0.68 \\
\hline Ship & B Podocyrtis helenae & & 44.145 & $13 \mathrm{H}-4,104-106$ & $13 \mathrm{H}-\mathrm{CC}$ & 115.25 & 119.38 & 133.22 & 137.35 & 135.29 & 2.07 & 139.80 & & & \\
\hline Ship & T Podocyrtis phyxis & RP13 & 44.444 & $13 \mathrm{H}-\mathrm{CC}$ & $14 \mathrm{H}-2,104-106$ & 119.38 & 121.72 & 137.35 & 140.81 & 139.08 & 1.73 & & & & \\
\hline Ship & Podocyrtis phyxis > Podocyrtis ampla & & 44.770 & $14 \mathrm{H}-2,104-106$ & $14 \mathrm{H}-4,104-106$ & 121.72 & 124.72 & 140.81 & 143.81 & 142.31 & 1.50 & & & & \\
\hline Ship & T Theocotyle venezuelensis & & 45.164 & $14 \mathrm{H}-4,104-106$ & $14 \mathrm{H}-\mathrm{CC}$ & 124.72 & 128.58 & 143.81 & 147.62 & 145.72 & 1.91 & & & & \\
\hline Ship & T Theocorys anaclasta & & 45.616 & $14 \mathrm{H}-\mathrm{CC}$ & $15 \mathrm{H}-2,94-96$ & 128.58 & 131.15 & 147.62 & 151.73 & 149.68 & 2.05 & & & & \\
\hline Ship & T Theocotyle cryptocephala & & 46.013 & $15 \mathrm{H}-2,94-96$ & $15 \mathrm{H}-4,94-96$ & 131.15 & 134.15 & 151.73 & 155.03 & 153.38 & 1.65 & & & & \\
\hline Ship & B Thyrsocyrtis orthotenes & & 46.160 & $15 \mathrm{H}-\mathrm{CC}$ & $16 \mathrm{X}-1,50-52$ & 138.47 & 138.71 & 159.43 & 158.52 & 158.98 & -0.45 & & & & \\
\hline Ship & T Thyrsocyrtis hirsuta & RP12 & 46.185 & $15 \mathrm{H}-\mathrm{CC}$ & $16 \mathrm{X}-1,50-52$ & 138.47 & 138.71 & 159.43 & 158.52 & 158.98 & -0.45 & & & & \\
\hline Ship & B Podocyrtis phyxis & & 46.435 & $16 X-1,50-52$ & $16 \mathrm{X}-\mathrm{CC}$ & 138.71 & 139.27 & 158.52 & 159.08 & 158.80 & 0.28 & & & & \\
\hline Ship & B Rhopalocanium ornatum & & 47.419 & $16 \mathrm{X}-\mathrm{CC}$ & $17 X-2,104-106$ & 139.27 & 150.15 & 159.08 & 170.55 & 164.82 & 5.74 & & & & \\
\hline Ship & B Thyrsocyrtis $(P$.$) triacantha$ & & 47.419 & $16 \mathrm{X}-\mathrm{CC}$ & $17 X-2,104-106$ & 139.27 & 150.15 & 159.08 & 170.55 & 164.82 & 5.74 & & & & \\
\hline Ship & B Eusyringium lagena & & 47.419 & $16 \mathrm{X}-\mathrm{CC}$ & $17 X-2,104-106$ & 139.27 & 150.15 & 159.08 & 170.55 & 164.82 & 5.74 & & & & \\
\hline Ship & T Phormocyrtis striata striata & & 48.141 & $17 X-4,104-106$ & 19X-CC & 153.15 & 162.10 & 173.55 & 182.50 & 178.03 & 4.47 & & & & \\
\hline Ship & B Dictyoprora mongolfieri & RP11 & 48.500 & $19 X-2,25-27$ & $19 \mathrm{X}-\mathrm{CC}$ & 161.75 & 162.10 & 182.15 & 182.50 & 182.33 & 0.17 & & & & \\
\hline Ship & Theocotyle nigriniae > Theocotyle cryptocephala & RP10 & 49.000 & $19 \mathrm{X}-\mathrm{CC}$ & $20 \mathrm{X}-\mathrm{CC}$ & 162.10 & 167.02 & 182.50 & 187.42 & 184.96 & 2.46 & & & & \\
\hline
\end{tabular}

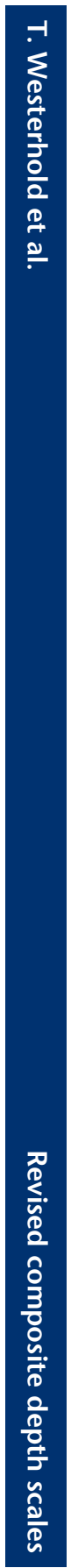




\begin{tabular}{|c|c|c|c|c|c|c|c|c|c|c|c|c|c|c|c|}
\hline \multirow[b]{2}{*}{ Source } & \multirow[b]{2}{*}{ Event } & \multirow[b]{2}{*}{ Zone } & \multirow{2}{*}{$\begin{array}{l}\mathrm{Age}^{*} \\
(\mathrm{Ma})\end{array}$} & \multicolumn{2}{|c|}{ Core, section, interval $(\mathrm{cm})$} & \multicolumn{2}{|c|}{$\begin{array}{c}\text { Depth } \\
\text { (mbsf [m CSF-A]) }\end{array}$} & \multicolumn{3}{|c|}{$\begin{array}{l}\text { Depth (rmcd [adjusted } \\
\text { m revised CCSF-A]) }\end{array}$} & \multirow{2}{*}{$\begin{array}{c}\text { Error } \\
( \pm) \\
(\mathrm{m})\end{array}$} & \multicolumn{3}{|c|}{$\begin{array}{l}\text { Depth Site } 1220 \\
\text { (rmcd) }\end{array}$} & \multirow{2}{*}{$\begin{array}{c}\text { Error } \\
( \pm) \\
(\mathrm{m})\end{array}$} \\
\hline & & & & Top & Bottom & Top & Bottom & Top & Bottom & Mean & & Top & Bottom & Mean & \\
\hline & & & & 320-U1332A- & 320-U1332A- & & & & & & & & & & \\
\hline Ship & B Cyrtocapsella cornuta & RN1 & 22.260 & $3 \mathrm{H}-2,93-95$ & $3 \mathrm{H}-4,93-95$ & 15.87 & 18.83 & 16.52 & 19.48 & 18.00 & 1.48 & 22.77 & 25.71 & 24.24 & 1.47 \\
\hline Ship & B Cyrtocapsella tetrapera & & 22.350 & $3 \mathrm{H}-4,93-95$ & $3 \mathrm{H}-\mathrm{CC}$ & 18.83 & 23.52 & 19.48 & 23.65 & 21.57 & 2.09 & 25.71 & 29.52 & 27.61 & 1.90 \\
\hline Ship & T Artophormis gracilis & \multirow{7}{*}{ RP22 } & 22.620 & $3 \mathrm{H}-4,93-95$ & $3 \mathrm{H}-\mathrm{CC}$ & 18.83 & 23.52 & 19.48 & 23.65 & 21.57 & 2.09 & 25.71 & 29.52 & 27.61 & 1.90 \\
\hline Ship & B Didymocyrtis bassanii & & 22.930 & $3 \mathrm{H}-4,93-95$ & $3 \mathrm{H}-\mathrm{CC}$ & 18.83 & 23.52 & 19.48 & 23.65 & 21.57 & 2.09 & 25.71 & 29.52 & 27.61 & 1.90 \\
\hline Ship & B Eucyrtidium diaphanes & & 22.950 & $3 \mathrm{H}-4,93-95$ & $3 \mathrm{H}-\mathrm{CC}$ & 18.83 & 23.52 & 19.48 & 23.65 & 21.57 & 2.09 & 25.71 & 29.52 & 27.61 & 1.90 \\
\hline Ship & T Dorcadospyris cyclacantha & & 22.980 & $3 \mathrm{H}-4,93-95$ & $3 \mathrm{H}-\mathrm{CC}$ & 18.83 & 23.52 & 19.48 & 23.65 & 21.57 & 2.09 & 25.71 & 29.52 & 27.61 & 1.90 \\
\hline Ship & T Dorcadospyris riedeli & & 23.010 & $3 \mathrm{H}-4,93-95$ & $3 \mathrm{H}-\mathrm{CC}$ & 18.83 & 23.52 & 19.48 & 23.65 & 21.57 & 2.09 & 25.71 & 29.52 & 27.61 & 1.90 \\
\hline Ship & B Dorcadospyris cyclacantha & & 23.290 & $3 \mathrm{H}-\mathrm{CC}$ & $4 \mathrm{H}-2,105-107$ & 23.52 & 25.50 & 23.65 & 28.05 & 25.85 & 2.20 & 29.52 & 33.67 & 31.60 & 2.08 \\
\hline Ship & T Liriospyris longicornuta & & 24.120 & $4 \mathrm{H}-2,105-107$ & $4 \mathrm{H}-4,105-107$ & 25.50 & 28.45 & 28.05 & 31.00 & 29.53 & 1.48 & 33.67 & 36.89 & 35.28 & 1.61 \\
\hline Ship & B Lychnocanoma elongata & & 25.050 & $4 \mathrm{H}-4,105-107$ & $4 \mathrm{H}-\mathrm{CC}$ & 28.45 & 32.96 & 31.00 & 35.85 & 33.43 & 2.43 & 36.89 & 41.74 & 39.32 & 2.43 \\
\hline Ship & B Dorcadospyris praeforcipata & \multirow{11}{*}{ RP21 } & 25.270 & $4 \mathrm{H}-4,105-107$ & $4 \mathrm{H}-\mathrm{CC}$ & 28.45 & 32.96 & 31.00 & 35.85 & 33.43 & 2.43 & 36.89 & 41.74 & 39.32 & 2.43 \\
\hline Ship & B Calocycletta (C.) robusta & & 25.270 & $4 \mathrm{H}-4,105-107$ & $4 \mathrm{H}-\mathrm{CC}$ & 28.45 & 32.96 & 31.00 & 35.85 & 33.43 & 2.43 & 36.89 & 41.74 & 39.32 & 2.43 \\
\hline Ship & B Didymocyrtis tubaria & & 25.270 & $4 \mathrm{H}-2,105-107$ & $4 \mathrm{H}-4,105-107$ & 25.50 & 28.45 & 28.05 & 31.00 & 29.53 & 1.48 & 33.67 & 36.89 & 35.28 & 1.61 \\
\hline Ship & B Liriospyris longicornuta & & 25.290 & $4 \mathrm{H}-4,105-107$ & $4 \mathrm{H}-\mathrm{CC}$ & 28.45 & 32.96 & 31.00 & 35.85 & 33.43 & 2.43 & 36.89 & 41.74 & 39.32 & 2.43 \\
\hline Ship & B Dorcadospyris scambos & & 25.330 & $4 \mathrm{H}-4,105-107$ & $4 \mathrm{H}-\mathrm{CC}$ & 28.45 & 32.96 & 31.00 & 35.85 & 33.43 & 2.43 & 36.89 & 41.74 & 39.32 & 2.43 \\
\hline Ship & T Dorcadospyris circulus (continuous) & & 26.170 & $4 \mathrm{H}-4,105-107$ & $4 \mathrm{H}-\mathrm{CC}$ & 28.45 & 32.96 & 31.00 & 35.85 & 33.43 & 2.43 & 36.89 & 41.74 & 39.32 & 2.43 \\
\hline Ship & T Eucyrtidium plesiodiaphanes & & 26.400 & $4 \mathrm{H}-\mathrm{CC}$ & $5 \mathrm{H}-2,110-112$ & 32.96 & 35.04 & 35.85 & 39.98 & 37.92 & 2.07 & 41.74 & 44.84 & 43.29 & 1.55 \\
\hline Ship & $\mathrm{T}$ Lithocyclia angusta & & 27.680 & $5 \mathrm{H}-2,110-112$ & $5 \mathrm{H}-4,105-107$ & 35.04 & 37.94 & 39.98 & 43.02 & 41.50 & 1.52 & 44.84 & 47.82 & 46.33 & 1.49 \\
\hline Ship & T Theocyrtis setanios & & 28.210 & $5 \mathrm{H}-4,105-107$ & $5 \mathrm{H}-\mathrm{CC}$ & 37.94 & 42.48 & 43.02 & 47.44 & 45.23 & 2.21 & 47.82 & 52.27 & 50.04 & 2.23 \\
\hline Ship & B Theocyrtis annosa & & 28.330 & $6 \mathrm{H}-2,105-107$ & $6 \mathrm{H}-4,105-107$ & 44.46 & 47.46 & 49.96 & 52.96 & 51.46 & 1.50 & 54.53 & 56.94 & 55.74 & 1.21 \\
\hline Ship & Tristylospyris triceros > Dorcadospyris ateuchus & & 28.600 & $5 \mathrm{H}-\mathrm{CC}$ & $6 \mathrm{H}-2,105-107$ & 42.48 & 44.46 & 47.44 & 49.96 & 48.70 & 1.26 & 52.27 & 54.53 & 53.40 & 1.13 \\
\hline Ship & B Dorcadospyris ateuchus & \multirow{20}{*}{ RP20 } & 29.503 & $6 \mathrm{H}-2,105-107$ & $6 \mathrm{H}-4,105-107$ & 44.46 & 47.46 & 49.96 & 52.96 & 51.46 & 1.50 & 54.53 & 56.94 & 55.74 & 1.21 \\
\hline Ship & B Eucyrtidium mitodes & & 29.410 & $6 \mathrm{H}-4,105-107$ & $6 \mathrm{H}-\mathrm{CC}$ & 47.46 & 51.98 & 52.96 & 57.46 & 55.21 & 2.25 & 56.94 & 59.94 & 58.44 & 1.50 \\
\hline Ship & B Dorcadospyris circulus & & 29.960 & $6 \mathrm{H}-4,105-107$ & $6 \mathrm{H}-\mathrm{CC}$ & 47.46 & 51.98 & 52.96 & 57.46 & 55.21 & 2.25 & 56.94 & 59.94 & 58.44 & 1.50 \\
\hline Ship & $\mathrm{T}$ Theocyrtis tuberosa & & 30.130 & $6 \mathrm{H}-\mathrm{CC}$ & 7H-3, 100-102 & 51.98 & 55.40 & 57.46 & 63.02 & 60.24 & 2.78 & 59.94 & 64.60 & 62.27 & 2.33 \\
\hline Ship & T Lithocyclia crux & & 30.130 & $6 \mathrm{H}-\mathrm{CC}$ & 7H-3, 100-102 & 51.98 & 55.40 & 57.46 & 63.02 & 60.24 & 2.78 & 59.94 & 64.60 & 62.27 & 2.33 \\
\hline Ship & B Eucyrtidium plesiodiaphanes & & 30.370 & $6 \mathrm{H}-\mathrm{CC}$ & 7H-3, 100-102 & 51.98 & 55.40 & 57.46 & 63.02 & 60.24 & 2.78 & 59.94 & 64.60 & 62.27 & 2.33 \\
\hline Ship & T Lophocyrtis (S.) oberhaensliae & & 30.740 & 7H-3, 100-102 & 7H-5, 100-102 & 55.40 & 58.41 & 63.02 & 66.04 & 64.53 & 1.51 & 64.60 & 66.88 & 65.74 & 1.14 \\
\hline Ship & B Dorcadospyris spinosa & & 30.840 & 7H-5, 100-102 & 7H-CC & 58.41 & 61.50 & 66.04 & 68.64 & 67.34 & 1.30 & 66.88 & 68.81 & 67.85 & 0.96 \\
\hline Ship & T Dorcadospyris pseudopapilio & & 30.840 & $7 \mathrm{H}-\mathrm{CC}$ & $8 \mathrm{H}-2,105-107$ & 61.50 & 63.45 & 68.64 & 70.85 & 69.75 & 1.11 & 68.81 & 70.45 & 69.63 & 0.82 \\
\hline Ship & T Centrobotrys gravida & & 30.890 & $7 \mathrm{H}-\mathrm{CC}$ & $8 \mathrm{H}-2,105-107$ & 61.50 & 63.45 & 68.64 & 70.85 & 69.75 & 1.11 & 68.81 & 70.45 & 69.63 & 0.82 \\
\hline Ship & B Lithocyclia crux & & 31.000 & $8 \mathrm{H}-4,105-107$ & $8 \mathrm{H}-\mathrm{CC}$ & 66.45 & 71.01 & 73.80 & 78.03 & 75.92 & 2.12 & 73.65 & 77.04 & 75.35 & 1.69 \\
\hline Ship & B Theocyrtis tuberosa & & 31.000 & $8 \mathrm{H}-4,105-107$ & $8 \mathrm{H}-\mathrm{CC}$ & 66.45 & 71.01 & 73.80 & 78.03 & 75.92 & 2.12 & 73.65 & 77.04 & 75.35 & 1.69 \\
\hline Ship & B Dorcadospyris pseudopapilio & & 31.000 & 9H-1, 92-98 & $9 \mathrm{H}-3,92-98$ & 71.35 & 74.38 & 73.77 & 76.98 & 75.38 & 1.61 & 73.63 & 76.17 & 74.90 & 1.27 \\
\hline Ship & B Centrobotrys gravida & & 31.010 & $8 \mathrm{H}-4,105-107$ & $8 \mathrm{H}-\mathrm{CC}$ & 66.45 & 71.01 & 73.80 & 78.03 & 75.92 & 2.12 & 73.65 & 77.04 & 75.35 & 1.69 \\
\hline Ship & T Thyrsocyrtis (P.) triacantha & & 33.340 & $\begin{array}{c}9 \mathrm{H}-3,92-98 \\
(8 \mathrm{H}-4,105)\end{array}$ & $\begin{array}{c}9 \mathrm{H}-5,92-98 \\
(8 \mathrm{H}-\mathrm{CC})\end{array}$ & 74.37 & 77.34 & 76.98 & 79.97 & 78.48 & 1.50 & 76.17 & 79.43 & 77.80 & 1.63 \\
\hline Ship & T Lithocy & & 33.510 & $9 \mathrm{H}-3,92-98$ & $9 \mathrm{H}-5,92-98$ & 74.37 & 77.34 & 76.98 & 79.97 & 78.48 & 1.50 & 76.17 & 79.43 & 77.80 & 1.63 \\
\hline Ship & T Calocyclas hispida & & 33.620 & $9 \mathrm{H}-3,92-98$ & $9 \mathrm{H}-5,92-98$ & 74.37 & 77.34 & 76.98 & 79.97 & 78.48 & 1.50 & 76.17 & 79.43 & 77.80 & 1.63 \\
\hline Ship & T Cryptocarpium ornatum & & 33.620 & $9 \mathrm{H}-3,92-98$ & $9 \mathrm{H}-5,92-98$ & 74.37 & 77.34 & 76.98 & 79.97 & 78.48 & 1.50 & 76.17 & 79.43 & 77.80 & 1.63 \\
\hline Ship & T Lychnocanoma babylonis & & 33.750 & $9 \mathrm{H}-3,92-98$ & $9 \mathrm{H}-5,92-98$ & 74.37 & 77.34 & 76.98 & 79.97 & 78.48 & 1.50 & 76.17 & 79.43 & 77.80 & 1.63 \\
\hline Ship & Lithocyclia aristotelis > Lithocyclia angusta & & 33.820 & 9H-5, 92-98 & $9 \mathrm{H}-\mathrm{CC}$ & 77.34 & 80.52 & 79.97 & 83.15 & 81.56 & 1.59 & 79.43 & 83.70 & 81.56 & 2.13 \\
\hline Ship & T Dorcadospyris copelata & \multirow{3}{*}{ RP19 } & 33.840 & $9 \mathrm{H}-5,92-98$ & 9H-CC & 77.34 & 80.52 & 79.97 & 83.15 & 81.56 & 1.59 & 79.43 & 83.70 & 81.56 & 2.13 \\
\hline Ship & B Lithocyclia angusta & & 34.130 & $9 \mathrm{H}-\mathrm{CC}$ & $10 \mathrm{H}-2,94-96$ & 80.52 & 82.34 & 83.15 & 86.09 & 84.62 & 1.47 & 83.70 & 87.29 & 85.49 & 1.80 \\
\hline Ship & T Calocyclas bandyca & & 34.620 & $9 \mathrm{H}-\mathrm{CC}$ & $10 \mathrm{H}-2,94-96$ & 80.52 & 82.34 & 83.15 & 86.09 & 84.62 & 1.47 & 83.70 & 87.29 & 85.49 & 1.80 \\
\hline Ship & $\mathrm{T}$ Thyrsocyrtis $(P$.$) tetracantha$ & & 35.300 & $9 \mathrm{H}-\mathrm{CC}$ & $10 \mathrm{H}-2,94-96$ & 80.52 & 82.34 & 83.15 & 86.09 & 84.62 & 1.47 & 83.70 & 87.29 & 85.49 & 1.80 \\
\hline Ship & B Lophocyrtis (C.) hadra & RP18 & 35.340 & $10 \mathrm{H}-2,94-96$ & $10 \mathrm{H}-4,94-96$ & 82.34 & 85.34 & 86.09 & 88.76 & 87.43 & 1.34 & 87.29 & 90.73 & 89.01 & 1.72 \\
\hline Ship & B Calocyclas bandyca & & 36.740 & $10 \mathrm{H}-4,94-96$ & $10 \mathrm{H}-\mathrm{CC}$ & 85.34 & 89.90 & 88.76 & 93.20 & 90.98 & 2.22 & 90.73 & 95.25 & 92.99 & 2.26 \\
\hline Ship & B Lophocyrtis (L.) jacchia & RP17 & 37.060 & $10 \mathrm{H}-\mathrm{CC}$ & $11 \mathrm{H}-2,105-107$ & 89.90 & 91.94 & 93.20 & 95.32 & 94.26 & 1.06 & 95.25 & 97.22 & 96.24 & 0.98 \\
\hline
\end{tabular}




\begin{tabular}{|c|c|c|c|c|c|c|c|c|c|c|c|c|c|c|c|}
\hline \multirow[b]{2}{*}{ Source } & \multirow[b]{2}{*}{ Event } & \multirow[b]{2}{*}{ Zone } & \multirow{2}{*}{$\begin{array}{l}\mathrm{Age}^{*} \\
(\mathrm{Ma})\end{array}$} & \multicolumn{2}{|c|}{ Core, section, interval $(\mathrm{cm})$} & \multicolumn{2}{|c|}{$\begin{array}{c}\text { Depth } \\
\text { (mbsf [m CSF-A]) }\end{array}$} & \multicolumn{3}{|c|}{$\begin{array}{l}\text { Depth (rmcd [adjusted } \\
\text { m revised CCSF-A]) }\end{array}$} & \multirow{2}{*}{$\begin{array}{l}\text { Error } \\
( \pm) \\
(\mathrm{m})\end{array}$} & \multicolumn{3}{|c|}{$\begin{array}{c}\text { Depth Site } 1220 \\
\text { (rmcd) }\end{array}$} & \multirow{2}{*}{$\begin{array}{c}\text { Error } \\
( \pm) \\
(\mathrm{m})\end{array}$} \\
\hline & & & & Top & Bottom & Top & Bottom & Top & Bottom & Mean & & Top & Bottom & Mean & \\
\hline Ship & B Cryptocarpium azyx & & 37.520 & $11 \mathrm{H}-2,105-107$ & $11 \mathrm{H}-4,105-107$ & 91.94 & 94.94 & 95.32 & 98.32 & 96.82 & 1.50 & 97.22 & 100.33 & 98.77 & 1.55 \\
\hline Ship & T Anthocyrtoma spp. & \multirow{7}{*}{ RP16 } & 37.920 & $11 \mathrm{H}-4,105-107$ & $11 \mathrm{H}-\mathrm{CC}$ & 94.94 & 98.53 & 98.32 & 101.91 & 100.12 & 1.80 & 100.33 & 103.56 & 101.94 & 1.62 \\
\hline Ship & B Thyrsocyrtis (T.) bromia & & 38.070 & $11 \mathrm{H}-4,105-107$ & $11 \mathrm{H}-\mathrm{CC}$ & 94.94 & 98.53 & 98.32 & 101.91 & 100.12 & 1.80 & 100.33 & 103.56 & 101.94 & 1.62 \\
\hline Ship & B Thyrsocyrtis $(P$.$) tetracantha$ & & 38.120 & $11 \mathrm{H}-4,105-107$ & $11 \mathrm{H}-\mathrm{CC}$ & 94.94 & 98.53 & 98.32 & 101.91 & 100.12 & 1.80 & 100.33 & 103.56 & 101.94 & 1.62 \\
\hline Ship & T Dorcadospyris anastasis & & 38.450 & $12 \mathrm{H}-2,105-107$ & $12 \mathrm{H}-4,105-107$ & 101.46 & 104.46 & 107.68 & 110.68 & 109.18 & 1.50 & 108.60 & 112.03 & 110.32 & 1.71 \\
\hline Ship & B Calocyclas turris & & 38.670 & $11 \mathrm{H}-\mathrm{CC}$ & $12 \mathrm{H}-2,105-107$ & 98.53 & 101.46 & 101.91 & 107.68 & 104.80 & 2.89 & 103.56 & 108.60 & 106.08 & 2.52 \\
\hline Ship & B Lithocyclia aristotelis gr. & & 39.730 & $12 \mathrm{H}-2,105-107$ & $12 \mathrm{H}-4,105-107$ & 101.46 & 104.46 & 107.68 & 110.68 & 109.18 & 1.50 & 108.60 & 112.03 & 110.32 & 1.71 \\
\hline Ship & B Dorcadospyris anastasis & & 39.980 & $12 \mathrm{H}-4,105-107$ & $12 \mathrm{H}-\mathrm{CC}$ & 104.46 & 108.46 & 110.68 & 115.16 & 112.92 & 2.24 & 112.03 & 116.07 & 114.05 & 2.02 \\
\hline Ship & B Podocyrtis goetheana & & 40.160 & $12 \mathrm{H}-4,105-107$ & $12 \mathrm{H}-\mathrm{CC}$ & 104.46 & 108.46 & 110.68 & 115.16 & 112.92 & 2.24 & 112.03 & 116.07 & 114.05 & 2.02 \\
\hline Ship & T Lophocyrtis biaurita & RP15 & 40.360 & $12 \mathrm{H}-4,105-107$ & $12 \mathrm{H}-\mathrm{CC}$ & 104.46 & 108.46 & 110.68 & 115.16 & 112.92 & 2.24 & 112.03 & 116.07 & 114.05 & 2.02 \\
\hline Ship & Podocyrtis mitra $>$ Podocyrtis chalara & & 40.700 & $13 \mathrm{H}-\mathrm{CC}$ & $14 \mathrm{H}-2,104-106$ & 118.39 & 120.44 & 127.31 & 133.22 & 130.27 & 2.96 & 124.80 & 127.63 & 126.21 & 1.41 \\
\hline Ship & T Podocyrtis trachodes & \multirow{10}{*}{ RP14 } & 41.230 & $13 \mathrm{H}-\mathrm{CC}$ & $14 \mathrm{H}-2,104-106$ & 118.39 & 120.44 & 127.31 & 133.22 & 130.27 & 2.96 & 124.80 & 127.63 & 126.21 & 1.41 \\
\hline Ship & B Podocyrtis chalara & & 41.540 & $14 \mathrm{H}-2,104-106$ & $14 \mathrm{H}-4,104-106$ & 120.44 & 123.45 & 133.22 & 136.45 & 134.84 & 1.61 & 127.63 & 129.39 & 128.51 & 0.88 \\
\hline Ship & B Cryptocarpium ornatum & & 42.100 & $14 \mathrm{H}-4,104-106$ & $14 \mathrm{H}-\mathrm{CC}$ & 123.45 & 126.29 & 136.45 & 139.97 & 138.21 & 1.76 & 129.39 & 131.96 & 130.68 & 1.29 \\
\hline Ship & B Sethochytris triconiscus & & 42.400 & $14 \mathrm{H}-\mathrm{CC}$ & $15 X-3,42-44$ & 126.29 & 129.36 & 139.97 & 142.86 & 141.42 & 1.45 & 131.96 & & & \\
\hline Ship & T Eusyringium lagena & & 42.690 & $14 \mathrm{H}-\mathrm{CC}$ & $15 X-3,42-44$ & 126.29 & 129.36 & 139.97 & 142.86 & 141.42 & 1.45 & 131.96 & & & \\
\hline Ship & B Theocyrtis perpumila & & 42.970 & $14 \mathrm{H}-\mathrm{CC}$ & $15 X-3,42-44$ & 126.29 & 129.36 & 139.97 & 142.86 & 141.42 & 1.45 & 131.96 & & & \\
\hline Ship & T Podocyrtis helenae & & 43.050 & $14 \mathrm{H}-\mathrm{CC}$ & $15 X-3,42-44$ & 126.29 & 129.36 & 139.97 & 142.86 & 141.42 & 1.45 & 131.96 & & & \\
\hline Ship & B Podocyrtis trachodes & & 43.220 & $16 \mathrm{X}-1,41-49$ & $16 \mathrm{X}-1,112-120$ & 135.94 & 136.66 & 149.44 & 150.16 & 149.80 & 0.36 & & & & \\
\hline Ship & B Zygocircus cimelium & & 43.350 & $15 X-5,40-42$ & $15 \mathrm{X}-\mathrm{CC}$ & 132.30 & 132.93 & 145.80 & 146.43 & 146.12 & 0.31 & & & & \\
\hline Ship & Podocyrtis sinuosa $>$ Podocyrtis mitra & & 43.840 & $16 X-1,112-120$ & $16 \mathrm{X}-2,37-44$ & 136.66 & 137.36 & 150.16 & 150.86 & 150.51 & 0.35 & & & & \\
\hline Ship & B Podocyrtis helenae & \multirow{6}{*}{ RP13 } & 44.140 & $\begin{array}{l}320-\mathrm{U} 1332 \mathrm{C}- \\
15 \mathrm{X}-\mathrm{CC}\end{array}$ & $\begin{array}{l}\text { 320-U1332C- } \\
16 \mathrm{X}-\mathrm{CC}\end{array}$ & 127.43 & 134.43 & 138.58 & 146.25 & 142.42 & 3.83 & & & & \\
\hline Ship & T Podocyrtis phyxis & & 44.440 & $15 \mathrm{X}-\mathrm{CC}$ & $16 \mathrm{X}-\mathrm{CC}$ & 127.43 & 134.43 & 138.58 & 146.25 & 142.42 & 3.83 & & & & \\
\hline Ship & T Podocyrtis diamesa & & 44.440 & $15 \mathrm{X}-\mathrm{CC}$ & $16 \mathrm{X}-\mathrm{CC}$ & 127.43 & 134.43 & 138.58 & 146.25 & 142.42 & 3.83 & & & & \\
\hline Ship & B Podocyrtis mitra & & 44.770 & $16 \mathrm{X}-\mathrm{CC}$ & $17 X-C C$ & 134.43 & 139.85 & 146.25 & 151.67 & 148.96 & 2.71 & & & & \\
\hline Ship & B Podocyrtis ampla & & 44.770 & $16 \mathrm{X}-\mathrm{CC}$ & $17 \mathrm{X}-\mathrm{CC}$ & 134.43 & 139.85 & 146.25 & 151.67 & 148.96 & 2.71 & & & & \\
\hline Ship & Podocyrtis phyxis > Podocyrtis ampla & & 44.770 & $16 \mathrm{X}-\mathrm{CC}$ & $17 X-C C$ & 134.43 & 139.85 & 146.25 & 151.67 & 148.96 & 2.71 & & & & \\
\hline
\end{tabular}

* = Nigrini et al. (2006). T = top, B = bottom. 


\begin{tabular}{|c|c|c|c|c|c|c|c|c|c|c|c|c|c|c|c|}
\hline \multirow[b]{2}{*}{ Source } & \multirow[b]{2}{*}{ Event } & \multirow[b]{2}{*}{ Zone } & \multirow{2}{*}{$\begin{array}{l}\mathrm{Age}^{*} \\
(\mathrm{Ma})\end{array}$} & \multicolumn{2}{|c|}{ Core, section, interval $(\mathrm{cm})$} & \multicolumn{2}{|c|}{$\begin{array}{c}\text { Depth } \\
\text { (mbsf [m CSF-A]) }\end{array}$} & \multicolumn{3}{|c|}{$\begin{array}{l}\text { Depth (rmcd [adjusted } \\
\text { m revised CCSF-A]) }\end{array}$} & \multirow{2}{*}{$\begin{array}{l}\text { Error } \\
( \pm) \\
(\mathrm{m})\end{array}$} & \multicolumn{3}{|c|}{$\begin{array}{l}\text { Depth Site } 1218 \\
\text { (corrected rmcd) }\end{array}$} & \multirow{2}{*}{$\begin{array}{c}\text { Error } \\
( \pm) \\
(\mathrm{m})\end{array}$} \\
\hline & & & & Top & Bottom & Top & Bottom & Top & Bottom & Mean & & Top & Bottom & Mean & \\
\hline Ship & B Stichocorys delmontensis & \multirow{3}{*}{ RN2 } & 20.680 & $\begin{array}{l}\text { 320-U1333A- } \\
1 \mathrm{H}-2,104-106\end{array}$ & $\begin{array}{l}320-U 1333 \mathrm{~A}- \\
1 \mathrm{H}-4,104-106\end{array}$ & 2.55 & 5.55 & 7.21 & 10.21 & 8.71 & 1.50 & 72.34 & 76.82 & 74.58 & 2.24 \\
\hline Ship & T Lophocyrtis (C.) pegetrum & & 20.890 & $1 \mathrm{H}-4,104-106$ & $1 \mathrm{H}-\mathrm{CC}$ & 5.55 & 9.92 & 10.21 & 14.62 & 12.42 & 2.21 & 76.82 & 85.40 & 81.11 & 4.29 \\
\hline Ship & T Theocyrtis annosa & & 21.380 & $1 \mathrm{H}-4,104-106$ & $1 \mathrm{H}-\mathrm{CC}$ & 5.55 & 9.92 & 10.21 & 14.62 & 12.42 & 2.21 & 76.82 & 85.40 & 81.11 & 4.29 \\
\hline Ship & B Calocycletta (C.) virginis & \multirow{5}{*}{ RN1 } & 21.390 & $1 \mathrm{H}-4,104-106$ & $1 \mathrm{H}-\mathrm{CC}$ & 5.55 & 9.92 & 10.21 & 14.62 & 12.42 & 2.21 & 76.82 & 85.40 & 81.11 & 4.29 \\
\hline Ship & B Lophocyrtis (C.) leptetrum & & 21.420 & $2 \mathrm{H}-4,104-106$ & $2 \mathrm{H}-\mathrm{CC}$ & 15.05 & 19.57 & 18.58 & 23.05 & 20.82 & 2.24 & 88.50 & 103.69 & 96.10 & 7.59 \\
\hline Ship & T Eucyrtidium mitodes & & 21.950 & $2 \mathrm{H}-2,104-106$ & $2 \mathrm{H}-4,104-106$ & 12.05 & 15.04 & 15.70 & 18.57 & 17.14 & 1.44 & 83.85 & 92.27 & 88.06 & 4.21 \\
\hline Ship & B Calocycletta (C.) serrata & & 22.040 & $1 \mathrm{H}-4,104-106$ & $1 \mathrm{H}-\mathrm{CC}$ & 5.55 & 9.92 & 10.21 & 14.62 & 12.42 & 2.21 & 76.82 & 85.40 & 81.11 & 4.29 \\
\hline Ship & B Cyrtocapsella cornuta & & 22.260 & $2 \mathrm{H}-4,104-106$ & $2 \mathrm{H}-\mathrm{CC}$ & 15.05 & 19.57 & 18.58 & 23.05 & 20.82 & 2.24 & 88.50 & 103.69 & 96.10 & 7.59 \\
\hline Ship & B Cyrtocapsella tetrapera & & 22.350 & $2 \mathrm{H}-4,104-106$ & $2 \mathrm{H}-\mathrm{CC}$ & 15.05 & 19.57 & 18.58 & 23.05 & 20.82 & 2.24 & 88.50 & 103.69 & 96.10 & 7.59 \\
\hline Ship & T Artophormis gracilis & \multirow{11}{*}{ RP22 } & 22.620 & $2 \mathrm{H}-4,104-106$ & $2 \mathrm{H}-\mathrm{CC}$ & 15.05 & 19.57 & 18.58 & 23.05 & 20.82 & 2.24 & 88.50 & 103.69 & 96.10 & 7.59 \\
\hline Ship & B Didymocyrtis bassanii & & 22.930 & $2 \mathrm{H}-4,104-106$ & $2 \mathrm{H}-\mathrm{CC}$ & 15.05 & 19.57 & 18.58 & 23.05 & 20.82 & 2.24 & 88.50 & 103.69 & 96.10 & 7.59 \\
\hline Ship & B Eucyrtidium diaphanes & & 22.950 & $3 \mathrm{H}-2,105-107$ & $3 \mathrm{H}-4,105-107$ & 21.56 & 24.56 & 28.25 & 31.08 & 29.67 & 1.42 & 103.26 & 114.54 & 108.90 & 5.64 \\
\hline Ship & T Dorcadospyris cyclacantha & & 22.980 & $2 \mathrm{H}-\mathrm{CC}$ & $3 \mathrm{H}-2,105-107$ & 19.57 & 21.56 & 26.41 & 28.25 & 27.33 & 0.92 & 99.95 & 105.75 & 102.85 & 2.90 \\
\hline Ship & T Dorcadospyris riedeli & & 23.010 & $4 \mathrm{H}-5,106-108$ & $4 \mathrm{H}-\mathrm{CC}$ & 35.57 & 38.60 & 42.35 & 45.17 & 43.76 & 1.41 & 129.95 & 137.22 & 133.58 & 3.64 \\
\hline Ship & B Dorcadospyris cyclacantha & & 23.290 & $3 \mathrm{H}-2,105-107$ & $3 \mathrm{H}-4,105-107$ & 21.56 & 24.56 & 28.25 & 31.08 & 29.67 & 1.42 & 103.26 & 114.54 & 108.90 & 5.64 \\
\hline Ship & T Dorcadospyris papilio & & 23.310 & $3 \mathrm{H}-2,105-107$ & $3 \mathrm{H}-4,105-107$ & 21.56 & 24.56 & 28.25 & 31.08 & 29.67 & 1.42 & 103.26 & 114.54 & 108.90 & 5.64 \\
\hline Ship & $\mathrm{T}$ Liriospyris longicornuta & & 24.120 & $3 \mathrm{H}-2,105-107$ & $3 \mathrm{H}-4,105-107$ & 21.56 & 24.56 & 28.25 & 31.08 & 29.67 & 1.42 & 103.26 & 114.54 & 108.90 & 5.64 \\
\hline Ship & T Acrocubus octopylus & & 24.380 & $3 \mathrm{H}-4,105-107$ & $3 \mathrm{H}-\mathrm{CC}$ & 24.56 & 28.87 & 31.08 & 35.38 & 33.23 & 2.15 & 108.25 & 122.10 & 115.18 & 6.93 \\
\hline Ship & T Lychnocanoma apodora & & 24.500 & $3 \mathrm{H}-4,105-107$ & $3 \mathrm{H}-\mathrm{CC}$ & 24.56 & 28.87 & 31.08 & 35.38 & 33.23 & 2.15 & 108.25 & 122.10 & 115.18 & 6.93 \\
\hline Ship & B Lychnocanoma elongata & & 25.050 & $4 \mathrm{H}-3,106-108$ & $4 \mathrm{H}-5,106-108$ & 32.57 & 35.57 & 40.25 & 42.35 & 41.30 & 1.05 & 125.21 & 132.72 & 128.97 & 3.75 \\
\hline Ship & B Acrocubus octopylus & \multirow{14}{*}{ RP21 } & 25.090 & $4 \mathrm{H}-5,106-108$ & $4 \mathrm{H}-\mathrm{CC}$ & 35.57 & 38.60 & 42.35 & 45.17 & 43.76 & 1.41 & 129.95 & 137.22 & 133.58 & 3.64 \\
\hline Ship & B Dorcadospyris praeforcipata & & 25.270 & $5 \mathrm{H}-4,105-107$ & $5 \mathrm{H}-\mathrm{CC}$ & 43.55 & 47.65 & 53.14 & 57.26 & 55.20 & 2.06 & 144.74 & 157.14 & 150.94 & 6.20 \\
\hline Ship & B Calocycletta (C.) robusta & & 25.270 & $4 \mathrm{H}-5,106-108$ & $4 \mathrm{H}-\mathrm{CC}$ & 35.57 & 38.60 & 42.35 & 45.17 & 43.76 & 1.41 & 129.95 & 137.22 & 133.58 & 3.64 \\
\hline Ship & B Didymocyrtis tubaria & & 25.270 & $4 \mathrm{H}-5,106-108$ & $4 \mathrm{H}-\mathrm{CC}$ & 35.57 & 38.60 & 42.35 & 45.17 & 43.76 & 1.41 & 129.95 & 137.22 & 133.58 & 3.64 \\
\hline Ship & B Liriospyris longicornuta & & 25.290 & $4 \mathrm{H}-3,106-108$ & $4 \mathrm{H}-5,106-108$ & 32.57 & 35.57 & 40.25 & 42.35 & 41.30 & 1.05 & 125.21 & 132.72 & 128.97 & 3.75 \\
\hline Ship & B Dorcadospyris scambos & & 25.330 & $4 \mathrm{H}-3,106-108$ & $4 \mathrm{H}-5,106-108$ & 32.57 & 35.57 & 40.25 & 42.35 & 41.30 & 1.05 & 125.21 & 132.72 & 128.97 & 3.75 \\
\hline Ship & B Lychnocanoma apodora & & 25.550 & $4 \mathrm{H}-5,106-108$ & $4 \mathrm{H}-\mathrm{CC}$ & 35.57 & 38.60 & 42.35 & 45.17 & 43.76 & 1.41 & 129.95 & 137.22 & 133.58 & 3.64 \\
\hline Ship & T Dorcadospyris circulus (continuous) & & 26.170 & $4 \mathrm{H}-5,106-108$ & $4 \mathrm{H}-\mathrm{CC}$ & 35.57 & 38.60 & 42.35 & 45.17 & 43.76 & 1.41 & 129.95 & 137.22 & 133.58 & 3.64 \\
\hline Ship & B Dorcadospyris riedeli & & 26.200 & $5 \mathrm{H}-4,105-107$ & $5 \mathrm{H}-\mathrm{CC}$ & 43.56 & 47.65 & 53.15 & 57.26 & 55.21 & 2.06 & 144.76 & 157.14 & 150.95 & 6.19 \\
\hline Ship & T Eucyrtidium plesiodiaphanes & & 26.400 & $5 \mathrm{H}-4,105-107$ & $5 \mathrm{H}-\mathrm{CC}$ & 43.56 & 47.65 & 53.15 & 57.26 & 55.21 & 2.06 & 144.76 & 157.14 & 150.95 & 6.19 \\
\hline Ship & T Lithocyclia angusta & & 27.680 & $6 \mathrm{H}-3,95-97$ & $6 \mathrm{H}-5,95-97$ & 51.46 & 54.46 & 63.21 & 66.21 & 64.71 & 1.50 & 157.22 & 162.91 & 160.07 & 2.85 \\
\hline Ship & T Theocyrtis setanios & & 28.210 & $6 \mathrm{H}-5,95-97$ & $6 \mathrm{H}-\mathrm{CC}$ & 54.46 & 57.27 & 66.21 & 69.02 & 67.62 & 1.41 & 160.94 & 169.65 & 165.29 & 4.36 \\
\hline Ship & B Theocyrtis annosa & & 28.330 & 7H-4, 95-97 & $7 \mathrm{H}-\mathrm{CC}$ & 62.46 & 66.55 & 75.45 & 80.01 & 77.73 & 2.28 & 172.26 & 180.01 & 176.14 & 3.87 \\
\hline Ship & B Dorcadospyris ateuchus & & 29.503 & 7H-4, 95-97 & $\mathrm{7H}-\mathrm{CC}$ & 62.46 & 66.55 & 75.45 & 80.01 & 77.73 & 2.28 & 172.26 & 180.01 & 176.14 & 3.87 \\
\hline Ship & Tristylospyris triceros $>$ Dorcadospyris ateuchus & & 28.600 & $7 \mathrm{H}-4,95-97$ & 7H-CC & 62.46 & 66.55 & 75.45 & 80.01 & 77.73 & 2.28 & 172.26 & 180.01 & 176.14 & 3.87 \\
\hline Ship & B Eucyrtidium mitodes & \multirow{14}{*}{ RP20 } & 29.410 & $8 \mathrm{H}-2,108-110$ & $8 \mathrm{H}-4,108-110$ & 68.89 & 71.89 & 84.27 & 87.17 & 85.72 & 1.45 & 182.01 & 188.18 & 185.10 & 3.09 \\
\hline Ship & B Theocyrtis setanios & & 29.510 & $9 \mathrm{H}-2,105-107$ & $9 \mathrm{H}-4,105-107$ & 78.55 & 81.56 & 96.28 & 99.29 & 97.79 & 1.51 & 195.45 & 203.30 & 199.38 & 3.93 \\
\hline Ship & B Dorcadospyris circulus & & 29.960 & $9 \mathrm{H}-2,105-107$ & $9 \mathrm{H}-4,105-107$ & 78.55 & 81.56 & 96.28 & 99.29 & 97.79 & 1.51 & 195.45 & 203.30 & 199.38 & 3.93 \\
\hline Ship & T Theocyrtis tuberosa & & 30.130 & $9 \mathrm{H}-\mathrm{CC}$ & $10 \mathrm{H}-2,105-107$ & 85.87 & 88.06 & 103.60 & 107.48 & 105.54 & 1.94 & 202.68 & 212.05 & 207.36 & 4.69 \\
\hline Ship & T Lithocyclia crux & & 30.130 & $9 \mathrm{H}-\mathrm{CC}$ & $10 \mathrm{H}-2,105-107$ & 85.87 & 88.06 & 103.60 & 107.48 & 105.54 & 1.94 & 202.68 & 212.05 & 207.36 & 4.69 \\
\hline Ship & B Eucyrtidium plesiodiaphanes & & 30.370 & $9 \mathrm{H}-\mathrm{CC}$ & $10 \mathrm{H}-2,105-107$ & 85.87 & 88.06 & 103.60 & 107.48 & 105.54 & 1.94 & 202.68 & 212.05 & 207.36 & 4.69 \\
\hline Ship & T Lophocyrtis (S.) oberhaensliae & & 30.740 & 9H-CC & $10 \mathrm{H}-2,105-107$ & 85.87 & 88.06 & 103.60 & 107.48 & 105.54 & 1.94 & 202.68 & 212.05 & 207.36 & 4.69 \\
\hline Ship & B Dorcadospyris spinosa & & 30.840 & $11 X-2,95-97$ & $11 X-4,95-97$ & 97.46 & 100.46 & 118.18 & 121.18 & 119.68 & 1.50 & 220.31 & 228.23 & 224.27 & 3.96 \\
\hline Ship & T Dorcadospyris pseudopapilio & & 30.840 & $11 X-2,95-97$ & $11 X-4,95-97$ & 97.46 & 100.46 & 118.18 & 121.18 & 119.68 & 1.50 & 220.31 & 228.23 & 224.27 & 3.96 \\
\hline Ship & T Centrobotrys gravida & & 30.890 & $11 X-4,95-97$ & $11 \mathrm{X}-\mathrm{CC}$ & 100.46 & 101.17 & 121.18 & 121.89 & 121.54 & 0.35 & 224.18 & 225.02 & 224.60 & 0.42 \\
\hline Ship & B Lychnocanoma plesioelongata & & & $11 \mathrm{X}-\mathrm{CC}$ & $12 X-2,105-107$ & 101.17 & 103.26 & 121.89 & 124.97 & 123.43 & 1.54 & 225.02 & 230.52 & 227.77 & 2.75 \\
\hline Ship & B Lithocyclia crux & & 31.000 & $12 X-2,105-107$ & $12 X-4,105-107$ & 103.26 & 106.26 & 124.97 & 128.40 & 126.69 & 1.72 & 228.27 & 237.19 & 232.73 & 4.46 \\
\hline Ship & B Theocyrtis tuberosa & & 31.000 & $12 X-4,105-107$ & $12 \mathrm{X}-\mathrm{CC}$ & 106.26 & 107.99 & 128.40 & 130.19 & 129.30 & 0.89 & 231.66 & 235.85 & 233.76 & 2.09 \\
\hline Ship & B Dorcadospyris pseudopapilio & & 31.000 & $12 X-2,105-107$ & $12 X-4,105-107$ & 103.26 & 106.26 & 124.97 & 128.40 & 126.69 & 1.72 & 228.27 & 237.19 & 232.73 & 4.46 \\
\hline
\end{tabular}




\begin{tabular}{|c|c|c|c|c|c|c|c|c|c|c|c|c|c|c|c|}
\hline \multirow[b]{2}{*}{ Source } & \multirow[b]{2}{*}{ Event } & \multirow[b]{2}{*}{ Zone } & \multirow{2}{*}{$\begin{array}{l}\mathrm{Age}^{*} \\
(\mathrm{Ma})\end{array}$} & \multicolumn{2}{|c|}{ Core, section, interval $(\mathrm{cm})$} & \multicolumn{2}{|c|}{$\begin{array}{c}\text { Depth } \\
\text { (mbsf [m CSF-A]) } \\
\end{array}$} & \multicolumn{3}{|c|}{$\begin{array}{l}\text { Depth (rmcd [adjusted } \\
\text { m revised CCSF-A]) }\end{array}$} & \multirow{2}{*}{$\begin{array}{c}\text { Error } \\
( \pm) \\
(\mathrm{m})\end{array}$} & \multicolumn{3}{|c|}{$\begin{array}{l}\text { Depth Site } 1218 \\
\text { (corrected rmcd) }\end{array}$} & \multirow{2}{*}{$\begin{array}{c}\text { Error } \\
( \pm) \\
(\mathrm{m})\end{array}$} \\
\hline & & & & Top & Bottom & Top & Bottom & Top & Bottom & Mean & & Top & Bottom & Mean & \\
\hline Ship & B Centrobotrys gravida & \multirow{9}{*}{ RP20 } & 31.010 & $12 X-2,105-107$ & $12 X-4,105-107$ & 103.26 & 106.26 & 124.97 & 128.40 & 126.69 & 1.72 & 228.27 & 237.19 & 232.73 & 4.46 \\
\hline Ship & $\mathrm{T}$ Thyrsocyrtis $(P$.$) triacantha$ & & 33.340 & $13 X-5,8-16$ & $13 X-5,96-104$ & 116.42 & 117.30 & 139.77 & 140.65 & 140.21 & 0.44 & 248.30 & 249.81 & 249.05 & 0.76 \\
\hline Ship & T Lithocyclia aristotelis gr. & & 33.510 & $13 \mathrm{X}-2,37-44$ & $13 X-2,88-95$ & 112.21 & 112.72 & 135.11 & 135.74 & 135.43 & 0.31 & 239.83 & 241.42 & 240.62 & 0.79 \\
\hline Ship & T Calocyclas hispida & & 33.620 & $13 \mathrm{X}-2,37-44$ & $13 \mathrm{X}-2,88-95$ & 112.21 & 112.72 & 135.11 & 135.74 & 135.43 & 0.31 & 239.83 & 241.42 & 240.62 & 0.79 \\
\hline Ship & T Cryptocarpium ornatum & & 33.620 & $13 X-1,127-134$ & $13 X-2,37-44$ & 111.61 & 112.21 & 134.25 & 135.11 & 134.68 & 0.43 & 238.68 & 241.55 & 240.11 & 1.43 \\
\hline Ship & T Lophocyrtis (C.) hadra & & 33.750 & $13 X-2,105-107$ & $13 X-3,0-8$ & 112.86 & 113.20 & 135.89 & 136.24 & 136.07 & 0.18 & 240.87 & 242.60 & 241.74 & 0.87 \\
\hline Ship & T Lychnocanoma amphitrite & & 33.750 & $13 X-2,37-44$ & $13 X-2,88-95$ & 112.21 & 112.72 & 135.11 & 135.74 & 135.43 & 0.31 & 239.83 & 241.42 & 240.62 & 0.79 \\
\hline Ship & T Lychnocanoma babylonis & & 33.750 & $13 X-2,105-107$ & $13 X-3,0-8$ & 112.86 & 113.34 & 135.89 & 136.38 & 136.14 & 0.25 & 240.87 & 243.06 & 241.97 & 1.10 \\
\hline Ship & Lithocyclia aristotelis > Lithocyclia angusta & & 33.820 & $13 X-2,88-95$ & $13 X-2,105-107$ & 112.72 & 112.86 & 135.74 & 135.89 & 135.82 & 0.07 & 240.69 & 241.55 & 241.12 & 0.43 \\
\hline Ship & T Dorcadospyris copelata & \multirow{8}{*}{ RP19 } & 33.840 & $12 \mathrm{X}-\mathrm{CC}$ & $13 X-1,127-134$ & 107.99 & 111.57 & 130.19 & 134.21 & 132.20 & 2.01 & 233.65 & 242.37 & 238.01 & 4.36 \\
\hline Ship & B Lithocyclia angusta & & 34.130 & $13 X-3,0-8$ & $13 X-3,122-129$ & 113.34 & 114.07 & 136.38 & 137.14 & 136.76 & 0.38 & 242.06 & 245.03 & 243.55 & 1.49 \\
\hline Ship & T Calocyclas bandyca & & 34.620 & $13 X-4,105-107$ & $13 X-4,120-128$ & 115.86 & 116.04 & 139.21 & 139.39 & 139.30 & 0.09 & 247.53 & 247.78 & 247.66 & 0.12 \\
\hline Ship & T Calocyclas turris & & 34.830 & $13 X-2,105-107$ & $13 X-3,0-8$ & 112.86 & 113.20 & 135.89 & 136.24 & 136.07 & 0.17 & 240.87 & 242.61 & 241.74 & 0.87 \\
\hline Ship & T Eusyringium fistuligerum & & 34.930 & $13 \mathrm{X}-\mathrm{CC}$ & $14 \mathrm{X}-2,95-97$ & 119.93 & 122.46 & 143.52 & 146.03 & 144.78 & 1.26 & 251.60 & 254.89 & 253.24 & 1.64 \\
\hline Ship & T Thyrsocyrtis (T.) bromia & & 33.936 & $13 X-4,120-128$ & $13 X-5,8-16$ & 116.04 & 116.42 & 139.39 & 139.77 & 139.58 & 0.19 & 247.78 & 248.30 & 248.04 & 0.26 \\
\hline Ship & T Thyrsocyrtis (P.) lochites & & 34.125 & $13 X-4,120-128$ & $13 X-5,8-16$ & 116.04 & 116.42 & 139.39 & 139.77 & 139.58 & 0.19 & 247.78 & 248.30 & 248.04 & 0.26 \\
\hline Ship & T Cryptocarpium azyx & & 35.070 & $13 X-4,105-107$ & $13 X-4,120-128$ & 114.07 & 116.04 & 137.14 & 139.39 & 138.26 & 1.13 & 243.66 & 250.92 & 247.29 & 3.63 \\
\hline Ship & T Thyrsocyrtis $(P$.$) tetracantha$ & & 35.300 & $13 X-4,120-128$ & $13 X-5,8-16$ & 116.04 & 116.42 & 139.39 & 139.77 & 139.58 & 0.19 & 247.78 & 248.30 & 248.04 & 0.26 \\
\hline Ship & B Lophocyrtis (C.) hadra & RP18 & 35.340 & $13 \mathrm{X}-4,72-80$ & $13 X-4,96-104$ & 115.52 & 115.76 & 138.87 & 139.11 & 138.99 & 0.12 & 246.98 & 247.37 & 247.18 & 0.20 \\
\hline Ship & B Calocyclas bandyca & & 36.740 & $13 \mathrm{X}-\mathrm{CC}$ & $14 X-2,95-97$ & 119.93 & 125.45 & 143.52 & 148.84 & 146.18 & 2.66 & 251.60 & 262.70 & 257.15 & 5.55 \\
\hline Ship & B Lophocyrtis (L.) jacchia & RP17 & 37.060 & $14 X-2,95-97$ & $14 X-4,95-97$ & 122.45 & 125.46 & 146.02 & 148.84 & 147.43 & 1.41 & 252.75 & 258.78 & 255.77 & 3.02 \\
\hline Ship & B Cryptocarpium azyx & & 37.520 & $14 X-4,95-97$ & $14 \mathrm{X}-\mathrm{CC}$ & 125.46 & 129.80 & 148.84 & 153.80 & 151.32 & 2.48 & 255.50 & 263.14 & 259.32 & 3.82 \\
\hline Ship & T Anthocyrtoma spp. & \multirow{9}{*}{ RP16 } & 37.920 & $14 \mathrm{X}-\mathrm{CC}$ & $15 X-2,96-98$ & 129.80 & 132.07 & 153.80 & 158.86 & 156.33 & 2.53 & 259.87 & 266.76 & 263.32 & 3.45 \\
\hline Ship & B Thyrsocyrtis (T.) bromia & & 38.070 & $14 \mathrm{X}-\mathrm{CC}$ & $15 X-2,96-98$ & 129.80 & 132.07 & 153.80 & 158.86 & 156.33 & 2.53 & 259.87 & 266.76 & 263.32 & 3.45 \\
\hline Ship & B Thyrsocyrtis $(P$.$) tetracantha$ & & 38.120 & $14 \mathrm{X}-\mathrm{CC}$ & $15 X-2,96-98$ & 129.80 & 132.07 & 153.80 & 158.86 & 156.33 & 2.53 & 259.87 & 266.76 & 263.32 & 3.45 \\
\hline Ship & T Dorcadospyris anastasis & & 38.450 & $14 \mathrm{X}-\mathrm{CC}$ & $15 X-2,96-98$ & 129.80 & 132.07 & 153.80 & 158.86 & 156.33 & 2.53 & 259.87 & 266.76 & 263.32 & 3.45 \\
\hline Ship & B Calocyclas turris & & 38.670 & $14 \mathrm{X}-\mathrm{CC}$ & $15 X-2,96-98$ & 129.80 & 132.07 & 153.80 & 158.86 & 156.33 & 2.53 & 259.87 & 266.76 & 263.32 & 3.45 \\
\hline Ship & B Lithocyclia aristotelis gr. & & 39.730 & $15 X-4,96-98$ & $15 \mathrm{X}-\mathrm{CC}$ & 135.07 & 139.14 & 161.91 & 166.89 & 164.40 & 2.49 & 265.90 & 274.42 & 270.16 & 4.26 \\
\hline Ship & T Podocyrtis mitra & & 39.850 & $15 \mathrm{X}-\mathrm{CC}$ & $16 \mathrm{X}-2,104-106$ & 139.14 & 141.75 & 166.89 & 169.12 & 168.01 & 1.12 & 270.46 & 274.26 & 272.36 & 1.90 \\
\hline Ship & B Dorcadospyris anastasis & & 39.980 & $15 X-C C$ & $16 \mathrm{X}-2,104-106$ & 139.14 & 141.75 & 166.89 & 169.12 & 168.01 & 1.12 & 270.46 & 274.26 & 272.36 & 1.90 \\
\hline Ship & B Podocyrtis goetheana & & 40.160 & $16 \mathrm{X}-2,104-106$ & $16 X-4,104-106$ & 141.75 & 144.75 & 169.12 & 172.78 & 170.95 & 1.83 & 272.37 & 277.59 & 274.98 & 2.61 \\
\hline Ship & T Lophocyrtis biaurita & RP15 & 40.360 & $16 \mathrm{X}-\mathrm{CC}$ & $17 X-2,105-107$ & 149.09 & 151.36 & 177.60 & 181.40 & 179.50 & 1.90 & 283.42 & 298.14 & 290.78 & 1.69 \\
\hline Ship & Podocyrtis mitra > Podocyrtis chalara & & 40.700 & $17 X-2,105-107$ & $17 X-4,105-107$ & 151.36 & 154.36 & 181.40 & 183.80 & 182.60 & 1.20 & 290.28 & & & \\
\hline Ship & T Podocyrtis trachodes & \multirow{9}{*}{ RP14 } & 41.230 & $17 X-2,105-107$ & $17 X-4,105-107$ & 151.36 & 154.36 & 181.40 & 183.80 & 182.60 & 1.20 & 290.28 & & & \\
\hline Ship & B Podocyrtis chalara & & 41.540 & $17 X-4,105-107$ & $17 \mathrm{X}-\mathrm{CC}$ & 154.36 & 158.46 & 183.80 & 186.78 & 185.29 & 1.49 & & & & \\
\hline Ship & B Cryptocarpium ornatum & & 42.100 & $17 X-4,105-107$ & $17 X-C C$ & 154.36 & 158.46 & 183.80 & 186.78 & 185.29 & 1.49 & & & & \\
\hline Ship & B Sethochytris triconiscus & & 42.400 & $18 X-1,95-97$ & $17 X-C C$ & 158.46 & 159.36 & 186.78 & 186.26 & 186.52 & 0.26 & & & & \\
\hline Ship & T Eusyringium lagena & & 42.690 & $18 X-1,95-97$ & $18 X-4,95-97$ & 159.36 & 163.20 & 186.26 & 190.10 & 188.18 & 1.92 & & & & \\
\hline Ship & B Theocyrtis perpumila & & 42.970 & $18 X-1,95-97$ & $18 X-4,95-97$ & 159.36 & 163.20 & 186.26 & 190.10 & 188.18 & 1.92 & & & & \\
\hline Ship & B Podocyrtis trachodes & & 43.220 & $18 X-1,95-97$ & $18 X-4,95-97$ & 159.36 & 163.20 & 186.26 & 190.10 & 188.18 & 1.92 & & & & \\
\hline Ship & B Zygocircus cimelium & & 43.350 & $18 X-1,95-97$ & $18 X-4,95-97$ & 159.36 & 163.20 & 186.26 & 190.10 & 188.18 & 1.92 & & & & \\
\hline Ship & Podocyrtis sinuosa > Podocyrtis mitra & & 43.840 & $18 \mathrm{X}-\mathrm{CC}$ & 19X-1, 104-106 & 163.86 & 169.05 & 190.76 & 194.80 & 192.78 & 2.02 & & & & \\
\hline Ship & T Podocyrtis phyxis & \multirow{3}{*}{ RP13 } & 44.440 & 19X-1, 104-106 & $19 X-3,104-106$ & 169.05 & 172.30 & 194.80 & 198.05 & 196.43 & 1.63 & & & & \\
\hline Ship & B Podocyrtis ampla & & 44.770 & 19X-1, 104-106 & $19 X-3,104-106$ & 169.05 & 172.30 & 194.80 & 198.05 & 196.43 & 1.63 & & & & \\
\hline Ship & Podocyrtis phyxis > Podocyrtis ampla & & 44.770 & 19X-1, 104-106 & $19 X-3,104-106$ & 169.05 & 172.30 & 194.80 & 198.05 & 196.43 & 1.63 & & & & \\
\hline
\end{tabular}

* = Nigrini et al. (2006). T = top, B = bottom 


\begin{tabular}{|c|c|c|c|c|c|c|c|c|c|c|c|c|c|c|c|}
\hline \multirow[b]{2}{*}{ Source } & \multirow[b]{2}{*}{ Event } & \multirow[b]{2}{*}{ Zone } & \multirow{2}{*}{$\begin{array}{l}\mathrm{Age}^{*} \\
(\mathrm{Ma})\end{array}$} & \multicolumn{2}{|c|}{ Hole, core, section, interval $(\mathrm{cm})$} & \multicolumn{2}{|c|}{$\begin{array}{c}\text { Depth } \\
\text { (mbsf [m CSF-A]) }\end{array}$} & \multicolumn{3}{|c|}{$\begin{array}{l}\text { Depth (rmcd [adjusted } \\
\text { m revised CCSF-A]) }\end{array}$} & \multirow{2}{*}{$\begin{array}{l}\text { Error } \\
( \pm) \\
(\mathrm{m})\end{array}$} & \multicolumn{3}{|c|}{$\begin{array}{l}\text { Depth Site } 1218 \\
\text { (corrected rmcd) }\end{array}$} & \multirow{2}{*}{$\begin{array}{c}\text { Error } \\
( \pm) \\
(\mathrm{m})\end{array}$} \\
\hline & & & & Top & Bottom & Top & Bottom & Top & Bottom & Mean & & Top & Bottom & Mean & \\
\hline & & & & $320-$ & $320-$ & & & & & & & & & & \\
\hline Ship & T Diartus petterssoni & RN7 & 8.629 & U1334A-1H-4, 105-107 & U1334A-1H-CC & 5.56 & 8.19 & 5.56 & 8.25 & 6.91 & 1.35 & 23.96 & 31.21 & 27.59 & 3.62 \\
\hline Ship & Diartus petterssoni > Diartus hughesi & & 8.760 & U1334A-1H-CC & U1334A-2H-2, 105-107 & 8.19 & 10.75 & 8.25 & 11.63 & 9.94 & 1.69 & 31.21 & 33.53 & 32.37 & 1.16 \\
\hline Ship & B Spongaster berminghami & & 8.760 & U1334A-1H-CC & U1334A-2H-2, 105-107 & 8.19 & 10.76 & 8.25 & 11.63 & 9.94 & 1.69 & 31.21 & 33.53 & 32.37 & 1.16 \\
\hline Ship & B Diartus hughesi & RN & 8.992 & U1334A-1H-CC & U1334A-2H-2, 105-107 & 8.19 & 10.76 & 8.25 & 11.63 & 9.94 & 1.69 & 31.21 & 33.53 & 32.37 & 1.16 \\
\hline Ship & B Diartus petterssoni & & 12.111 & U1334A-2H-4, 105-107 & U1334A-2H-CC & 13.76 & 18.11 & 14.63 & 18.98 & 16.81 & 2.18 & 36.64 & 40.65 & 38.64 & 2.00 \\
\hline Ship & B Lithopera neotera & & 12.950 & U1334A-2H-CC & U1334A-3H-2, 105-107 & 18.11 & 20.25 & 18.98 & 22.22 & 20.60 & 1.62 & 40.65 & 42.72 & 41.68 & 1.04 \\
\hline Ship & T Stichocorys armata & RN5 & 13.500 & U1334A-3H-2, 105-107 & U1334A-3H-4, 105-107 & 20.25 & 23.25 & 22.22 & 25.22 & 23.72 & 1.50 & 42.72 & 44.24 & 43.48 & 0.76 \\
\hline Ship & T Acrocubus octopyle & & 13.880 & U1334A-3H-2, 105-107 & U1334A-3H-4, 105-107 & 20.25 & 23.25 & 22.22 & 25.22 & 23.72 & 1.50 & 42.72 & 44.24 & 43.48 & 0.76 \\
\hline Ship & $\begin{array}{c}\text { Dorcadospyris dentata }> \\
\text { Dorcadospyris alata }\end{array}$ & & 14.780 & U1334A-3H-4, 105-107 & U1334A-3H-CC & 23.25 & 27.65 & 25.22 & 29.62 & 27.42 & 2.20 & 44.24 & 46.63 & 45.43 & 1.20 \\
\hline Ship & B Dorcadospyris alata & & 15.075 & U1334A-3H-CC & U1334A-4H-2, 105-107 & 27.65 & 29.76 & 29.62 & 33.37 & 31.49 & 1.88 & 46.63 & 48.68 & 47.66 & 1.02 \\
\hline Ship & B Liriospyris parkerae & & 15.034 & U1334A-4H-2, 105-107 & U1334A-4H-4, 105-107 & 29.76 & 32.76 & 33.37 & 36.24 & 34.81 & 1.44 & 48.68 & 50.92 & 49.80 & 1.12 \\
\hline Ship & T Carpocanopsis cingulata & RN4 & 15.129 & U1334A-4H-2, 105-107 & U1334A-4H-4, 105-107 & 29.76 & 32.76 & 33.37 & 36.24 & 34.81 & 1.44 & 48.68 & 50.92 & 49.80 & 1.12 \\
\hline Ship & T Lychnocanoma elongata & & 15.151 & U1334A-4H-4, 105-107 & U1334A-4H-CC & 32.76 & 37.18 & 36.24 & 40.60 & 38.42 & 2.18 & 50.92 & 54.16 & 52.54 & 1.62 \\
\hline Ship & B Lithopera renzae & & 16.770 & U1334A-4H-CC & U1334A-5H-2, 104-106 & 37.18 & 39.24 & 40.60 & 44.18 & 42.39 & 1.79 & 54.16 & 56.80 & 55.48 & 1.32 \\
\hline Ship & B Calocycletta costata & & 17.490 & U1334A-5H-2, 104-106 & U1334A-5H-4, 104-106 & 39.24 & 42.24 & 44.18 & 47.01 & 45.60 & 1.41 & 56.80 & 58.86 & 57.83 & 1.03 \\
\hline Ship & B Dorcadospyris dentata & & 17.720 & U1334A-5H-2, 104-106 & U1334A-5H-4, 104-106 & 39.24 & 42.24 & 44.18 & 47.01 & 45.60 & 1.41 & 56.80 & 58.86 & 57.83 & 1.03 \\
\hline Ship & B Liriospyris stauropora & RN3 & 17.724 & U1334A-5H-4, 104-106 & U1334A-5H-CC & 42.24 & 46.78 & 47.01 & 51.45 & 49.23 & 2.22 & 58.86 & 61.81 & 60.33 & 1.48 \\
\hline Ship & B Stichocorys wolffii & & 18.570 & U1334A-5H-4, 104-106 & U1334A-5H-CC & 42.24 & 46.78 & 47.01 & 51.45 & 49.23 & 2.22 & 58.86 & 61.81 & 60.33 & 1.48 \\
\hline Ship & B Dorcadospyris forcipata & & 18.610 & U1334A-5H-4, 104-106 & U1334A-5H-CC & 42.24 & 46.78 & 47.01 & 51.45 & 49.23 & 2.22 & 58.86 & 61.81 & 60.33 & 1.48 \\
\hline Ship & T Dorcadospyris simplex s.s. & & 18.687 & U1334A-5H-4, 104-106 & U1334A-5H-CC & 42.24 & 46.78 & 47.01 & 51.45 & 49.23 & 2.22 & 58.86 & 61.81 & 60.33 & 1.48 \\
\hline Ship & T Dorcadospyris praeforcipata & & 19.770 & U1334A-6H-4, 104-106 & U1334A-6H-CC & 51.74 & 56.20 & 58.67 & 63.16 & 60.92 & 2.25 & 67.19 & 70.04 & 68.61 & 1.42 \\
\hline Ship & B Dorcadospyris simplex & & 20.340 & U1334A-7H-2, 105-107 & U1334A-7H-4, 105-107 & 58.25 & 61.25 & 65.81 & 68.67 & 67.24 & 1.43 & 71.59 & 73.48 & 72.53 & 0.95 \\
\hline Ship & B Stichocorys delmontensis & RN2 & 20.680 & U1334A-7H-2, 105-107 & U1334A-7H-4, 105-107 & 58.25 & 61.25 & 65.81 & 68.67 & 67.24 & 1.43 & 71.59 & 73.48 & 72.53 & 0.95 \\
\hline Ship & T Lophocyrtis (C.) pegetrum & & 20.890 & U1334A-7H-4, 105-107 & U1334A-7H-CC & 61.25 & 65.66 & 68.67 & 73.08 & 70.88 & 2.21 & 73.48 & 76.00 & 74.74 & 1.26 \\
\hline Ship & B Calocycletta (C.) virginis & & 21.390 & U1334A-7H-CC & U1334A-8H-2, 105-107 & 65.66 & 67.76 & 73.08 & 76.82 & 74.95 & 1.87 & 76.00 & 79.03 & 77.51 & 1.52 \\
\hline Ship & B Lophocyrtis (C.) leptetrum & & 21.420 & U1334A-7H-CC & U1334A-8H-2, 105-107 & 65.66 & 67.76 & 73.08 & 76.82 & 74.95 & 1.87 & 76.00 & 79.03 & 77.51 & 1.52 \\
\hline Ship & T Theocyrtis annosa & & 21.380 & U1334A-8H-4, 105-107 & U1334A-8H-CC & 70.75 & 75.06 & 79.81 & 84.12 & 81.97 & 2.16 & 81.44 & 83.32 & 82.38 & 0.94 \\
\hline Ship & T Eucyrtidium mitodes & & 21.950 & U1334A-9H-2, 105-107 & U1334A-9H-4, 105-107 & 77.25 & 80.25 & 88.47 & 91.47 & 89.97 & 1.50 & 85.94 & 87.73 & 86.84 & 0.89 \\
\hline Ship & B Calocycletta (C.) serrata & RN1 & 22.040 & U1334A-9H-2, 105-107 & U1334A-9H-4, 105-107 & 77.25 & 80.25 & 88.47 & 91.47 & 89.97 & 1.50 & 85.94 & 87.73 & 86.84 & 0.89 \\
\hline Ship & B Cyrtocapsella cornuta & & 22.260 & U1334A-10H-2, 105-107 & U1334A-10H-4, 105-107 & 86.75 & 89.75 & 98.13 & 101.06 & 99.60 & 1.47 & 92.36 & 94.14 & 93.25 & 0.89 \\
\hline Ship & B Cyrtocapsella tetrapera & & 22.350 & U1334A-10H-2, 105-107 & U1334A-10H-4, 105-107 & 86.75 & 89.75 & 98.13 & 101.06 & 99.60 & 1.47 & 92.36 & 94.14 & 93.25 & 0.89 \\
\hline Ship & T Artophormis gracilis & & 22.620 & U1334A-10H-2, 105-107 & U1334A-10H-4, 105-107 & 86.75 & 89.75 & 98.13 & 101.06 & 99.60 & 1.47 & 92.36 & 94.14 & 93.25 & 0.89 \\
\hline Ship & B Didymocyrtis bassanii & & 22.930 & U1334A-10H-2, 105-107 & U1334A-10H-4, 105-107 & 86.75 & 89.75 & 98.13 & 101.06 & 99.60 & 1.47 & 92.36 & 94.14 & 93.25 & 0.89 \\
\hline Ship & B Eucyrtidium diaphanes & & 22.950 & U1334A-10H-4, 105-107 & U1334A-10H-CC & 89.75 & 93.95 & 101.06 & 105.26 & 103.16 & 2.10 & 94.14 & 97.17 & 95.65 & 1.52 \\
\hline Ship & T Dorcadospyris cyclacantha & & 22.980 & U1334A-10H-CC & U1334A-11H-2, 105-107 & 93.95 & 96.25 & 105.26 & 109.14 & 107.20 & 1.94 & 97.17 & 99.98 & 98.57 & 1.40 \\
\hline Ship & T Dorcadospyris riedeli (upper) & & 23.010 & U1 $334 \mathrm{~A}-10 \mathrm{H}-\mathrm{CC}$ & U1334A-11H-2, 105-107 & 93.95 & 96.25 & 105.26 & 109.14 & 107.20 & 1.94 & 97.17 & 99.98 & 98.57 & 1.40 \\
\hline Ship & T Dorcadospyris papilio & RP22 & 23.310 & U1 $334 \mathrm{~A}-10 \mathrm{H}-\mathrm{CC}$ & U1334A-11H-2, 105-107 & 93.95 & 96.25 & 105.26 & 109.14 & 107.20 & 1.94 & 97.17 & 99.98 & 98.57 & 1.40 \\
\hline Ship & B Dorcadospyris cyclacantha & & 23.290 & U1334A-11H-2, 105-107 & U1334A-11H-4, 105-107 & 96.25 & 99.25 & 109.14 & 112.17 & 110.66 & 1.52 & 99.98 & 102.12 & 101.05 & 1.07 \\
\hline Ship & T Liriospyris longicornuta & & 24.120 & U1334A-11H-4, 105-107 & U1334A-11H-CC & 99.25 & 103.75 & 112.17 & 116.51 & 114.34 & 2.17 & 102.12 & 105.19 & 103.66 & 1.54 \\
\hline Ship & T Acrocubus octopylus (lower) & & 24.380 & U1334A-13H-2, 105-107 & U1334A-13H-4, 105-107 & 114.25 & 117.25 & 129.74 & 132.82 & 131.28 & 1.54 & 114.86 & 117.38 & 116.12 & 1.26 \\
\hline Ship & T Lychnocanoma apodora & & 24.500 & U1334A-13H-2, 105-107 & U1334A-13H-4, 105-107 & 114.25 & 117.25 & 129.74 & 132.82 & 131.28 & 1.54 & 114.86 & 117.38 & 116.12 & 1.26 \\
\hline Ship & B Lychnocanoma elongata & & 25.050 & U1334A-13H-4, 105-107 & U1334A-13H-CC & 117.25 & 122.55 & 132.82 & 137.99 & 135.41 & 2.59 & 117.38 & 121.83 & 119.61 & 2.23 \\
\hline Ship & B Dorcadospyris praeforcipata & & 25.270 & U1334A-13H-CC & U1334A-14H-2, 105-107 & 122.55 & 124.75 & 137.99 & 142.01 & 140.00 & 2.01 & 121.83 & 124.88 & 123.36 & 1.52 \\
\hline Ship & B Didymocyrtis tubaria (lower) & & 25.270 & U1334A-13H-CC & U1334A-14H-2, 105-107 & 122.55 & 124.75 & 137.99 & 142.01 & 140.00 & 2.01 & 121.83 & 124.88 & 123.36 & 1.52 \\
\hline Ship & B Dorcadospyris scambos & & 25.330 & U1334C-13H-CC & U1334A-14H-CC & 123.72 & 131.96 & 148.69 & 150.23 & 149.46 & 0.77 & 130.30 & 131.34 & 130.82 & 0.52 \\
\hline Ship & B Calocycletta (C.) robusta & RP2 & 25.270 & U1334A-14H-4, 105-107 & U1334A-14H-CC & 127.75 & 131.96 & 145.67 & 150.23 & 147.95 & 2.28 & 128.54 & 131.34 & 129.94 & 1.40 \\
\hline Ship & B Liriospyris longicornuta & & 25.290 & U1334A-14H-4, 105-107 & U1334A-14H-CC & 127.25 & 131.96 & 145.67 & 150.23 & 147.95 & 2.28 & 128.54 & 131.34 & 129.94 & 1.40 \\
\hline Ship & B Lychnocanoma apodora & & 25.550 & U1334A-15H-4, 105-107 & U1334A-15H-CC & 137.25 & 141.20 & 156.24 & 160.31 & 158.28 & 2.04 & 135.00 & 138.67 & 136.84 & 1.83 \\
\hline
\end{tabular}


Table T35 (continued). (Continued on next page.)

\begin{tabular}{|c|c|c|c|c|c|c|c|c|c|c|c|c|c|c|c|}
\hline \multirow[b]{2}{*}{ Source } & \multirow[b]{2}{*}{ Event } & \multirow[b]{2}{*}{ Zone } & \multirow{2}{*}{$\begin{array}{l}\text { Age* }^{*} \\
(\mathrm{Ma})\end{array}$} & \multicolumn{2}{|c|}{ Hole, core, section, interval $(\mathrm{cm})$} & \multicolumn{2}{|c|}{$\begin{array}{c}\text { Depth } \\
(\mathrm{mbsf}[\mathrm{m} \text { CSF-A] }) \\
\end{array}$} & \multicolumn{3}{|c|}{$\begin{array}{l}\text { Depth (rmcd [adjusted } \\
\text { m revised CCSF-A]) }\end{array}$} & \multirow{2}{*}{$\begin{array}{l}\text { Error } \\
( \pm) \\
(\mathrm{m})\end{array}$} & \multicolumn{3}{|c|}{$\begin{array}{l}\text { Depth Site } 1218 \\
\text { (corrected rmcd) }\end{array}$} & \multirow{2}{*}{$\begin{array}{c}\text { Error } \\
( \pm) \\
(\mathrm{m})\end{array}$} \\
\hline & & & & Top & Bottom & Top & Bottom & Top & Bottom & Mean & & Top & Bottom & Mean & \\
\hline Ship & T Dorcadospyris circulus (continuous) & \multirow{7}{*}{ RP21 } & 26.170 & U1334A-16H-2, 104-106 & U1334A-16H-4, 104-106 & 143.74 & 146.74 & 164.41 & 167.38 & 165.90 & 1.49 & 141.71 & 144.06 & 142.88 & 1.18 \\
\hline Ship & T Dorcadospyris riedeli (upper) & & 26.200 & U1334A-16H-2, 104-106 & U1334A-16H-4, 104-106 & 143.74 & 146.74 & 164.41 & 167.38 & 165.90 & 1.49 & 141.71 & 144.06 & 142.88 & 1.18 \\
\hline Ship & B Eucyrtidium plesiodiaphanes & & 26.400 & U1334A-16H-2, 104-106 & U1334A-16H-4, 104-106 & 143.74 & 146.74 & 164.41 & 167.38 & 165.90 & 1.49 & 141.71 & 144.06 & 142.88 & 1.18 \\
\hline Ship & T Lithocyclia angusta (continuous) & & 27.680 & U1334A-17H-CC & U1334A-18H-2, 105-107 & 158.97 & 162.70 & 181.52 & 186.77 & 184.15 & 2.63 & 154.81 & 158.90 & 156.86 & 2.04 \\
\hline Ship & B Dorcadospyris ateuchus & & 29.503 & U1334B-20H-CC & U1334C-20H-CC & 191.55 & 189.97 & 221.91 & 223.30 & 222.61 & 0.69 & 185.63 & 186.40 & 186.02 & 0.39 \\
\hline Ship & B Theocyrtis annosa & & 28.330 & U1334A-20H-CC & U1334A-21H-2, 105-107 & 188.63 & 191.25 & 229.09 & 232.41 & 230.75 & 1.66 & 189.61 & 191.68 & 190.65 & 1.04 \\
\hline Ship & $\begin{array}{l}\text { Tristylospyris triceros > Dorcadospyris } \\
\text { ateuchus }\end{array}$ & & 28.600 & U1334A-20H-CC & U1334A-21H-2, 105-107 & 188.63 & 191.25 & 229.09 & 232.41 & 230.75 & 1.66 & 189.61 & 191.68 & 190.65 & 1.04 \\
\hline Ship & B Eucyrtidium mitodes & \multirow{29}{*}{ RP20 } & 29.410 & U1334A-21H-4, 105-107 & U1334A-21H-CC & 194.25 & 198.48 & 235.41 & 239.72 & 237.57 & 2.16 & 193.64 & 196.30 & 194.97 & 1.33 \\
\hline Ship & B Dorcadospyris circulus & & 29.960 & U1334A-21H-4, 105-107 & U1334A-21H-CC & 194.25 & 198.48 & 235.41 & 239.72 & 237.57 & 2.16 & 193.64 & 196.30 & 194.97 & 1.33 \\
\hline Revised & T Dorcadospyris spinosa & & 30.535 & U1334A-22H-3, 80-81 & U1334A-22H-3, 110-111 & 202.01 & 202.31 & 244.47 & 244.77 & 244.62 & 0.15 & 199.19 & 199.38 & 199.29 & 0.10 \\
\hline Revised & T Lithocyclia crux & & 30.130 & U1334A-22H-3, 110-111 & U1334B-22H-CC & 202.31 & 211.10 & 244.77 & 245.01 & 244.89 & 0.12 & 199.38 & 199.54 & 199.46 & 0.08 \\
\hline Ship & T Lophocyrtis (C.) milowi & & 29.534 & U1334C-19H-CC & U1334A-20H-CC & 180.68 & 188.63 & 213.20 & 229.09 & 221.15 & 7.95 & 178.45 & 189.61 & 184.03 & 5.58 \\
\hline Revised & T Theocyrtis tuberosa & & 30.130 & U1334A-22H-5, 140-141 & U1334A-22H-6, 20-21 & 205.61 & 205.91 & 248.56 & 248.87 & 248.72 & 0.15 & 201.81 & 202.00 & 201.90 & 0.10 \\
\hline Revised & T Dorcadospyris quadripes & & 30.535 & U1334B-23X-5, 90-91 & U1334A-23X-1, 100-101 & 217.61 & 207.91 & 252.52 & 252.65 & 252.59 & 0.07 & 204.31 & 204.40 & 204.35 & 0.05 \\
\hline Revised & B Eucyrtidium plesiodiaphanes & & 30.370 & U1334A-23X-4, 70-71 & U1334A-23X-4, 100-101 & 212.41 & 212.71 & 257.13 & 257.43 & 257.28 & 0.15 & 207.49 & 207.71 & 207.60 & 0.11 \\
\hline Ship & T Lophocyrtis (S.) oberhaensliae & & 30.740 & U1334A-23H-CC & U1334A-24H-2, 105-107 & 216.17 & 217.05 & 260.39 & 264.73 & 262.56 & 2.17 & 210.27 & 213.34 & 211.80 & 1.53 \\
\hline Revised & B Dorcadospyris spinosa & & 30.840 & U1334C-25X-2, 130-131 & U1334C-25X-3, 10-11 & 226.41 & 226.71 & 272.74 & 273.04 & 272.89 & 0.15 & 220.76 & 221.04 & 220.90 & 0.14 \\
\hline Revised & T Dorcadospyris pseudopapilio & & 30.840 & U1334C-25X-2, 130-131 & U1334C-25X-3, 10-11 & 226.41 & 226.71 & 272.74 & 273.04 & 272.89 & 0.15 & 220.76 & 221.04 & 220.90 & 0.14 \\
\hline Revised & $\mathrm{T}$ Centrobotrys gravida & & 30.890 & U1334C-25X-5, 100-101 & U1334C-25X-5, 130-131 & 230.61 & 230.91 & 277.00 & 277.31 & 277.16 & 0.15 & 224.33 & 224.59 & 224.46 & 0.13 \\
\hline Revised & B Centrobotrys petrushevskayae & & 30.909 & U1334A-25X-5, 10-11 & U1334A-25X-5, 40-41 & 230.21 & 230.51 & 279.64 & 279.94 & 279.79 & 0.15 & 226.48 & 226.69 & 226.59 & 0.10 \\
\hline Revised & T Theocyrtis careotuberosa & & 30.968 & U1334A-25X-6, 10-11 & U1334A-25X-6, 70-71 & 231.71 & 232.31 & 281.14 & 281.74 & 281.44 & 0.30 & 227.56 & 228.00 & 227.78 & 0.22 \\
\hline Revised & B Dorcadospyris quadripes & & 30.954 & U1334A-25X-7, 35-36 & U1334A-25X-CC & 233.46 & 233.79 & 282.53 & 282.72 & 282.62 & 0.10 & 228.57 & 228.72 & 228.65 & 0.07 \\
\hline Revised & B Centrobotrys gravida & & 31.010 & U1334A-26X-4, 50-51 & U1334A-26X-4, 80-81 & 238.61 & 238.91 & 288.25 & 288.55 & 288.40 & 0.15 & 232.55 & 232.75 & 232.65 & 0.10 \\
\hline Revised & B Lithocyclia crux & & 31.000 & U1334A-26X-4, 140-141 & U1334A-26X-5, 20-21 & 239.51 & 239.81 & 289.15 & 289.45 & 289.30 & 0.15 & 233.17 & 233.38 & 233.27 & 0.10 \\
\hline Revised & B Dorcadospyris pseudopapilio & & 31.000 & U1334A-26X-4, 140-141 & U1334A-26X-5, 20-21 & 239.51 & 239.81 & 289.15 & 289.45 & 289.30 & 0.15 & 233.17 & 233.38 & 233.27 & 0.10 \\
\hline Revised & T Artophormis barbadensis & & 33.974 & U1334B-26X-4, 40-41 & U1334B-26X-4, 70-71 & 243.11 & 243.41 & 295.43 & 295.73 & 295.58 & 0.15 & 238.52 & 238.82 & 238.67 & 0.15 \\
\hline Revised & T Artophormis dominasinensis & & & U1334B-26X-4, 40-41 & U1334B-26X-4, 70-71 & 243.11 & 243.41 & 295.43 & 295.73 & 295.58 & 0.15 & 238.52 & 238.82 & 238.67 & 0.15 \\
\hline Revised & B Lophocyrtis (S.) oberhaensliae & & 33.510 & U1334B-26X-4, 70-71 & U1334B-26X-4, 100-101 & 243.41 & 243.71 & 295.73 & 296.03 & 295.88 & 0.15 & 238.82 & 239.13 & 238.97 & 0.15 \\
\hline Revised & T Cryptocarpium ornatum & & 33.620 & U1334B-26X-5, 10-11 & U1334B-26X-5, 40-41 & 244.31 & 244.61 & 296.63 & 296.93 & 296.78 & 0.15 & 239.60 & 239.79 & 239.70 & 0.10 \\
\hline Revised & B Theocyrtis tuberosa & & 31.000 & U1334B-26X-5, 70-71 & U1334B-26X-5, 100-101 & 244.91 & 245.21 & 297.23 & 297.52 & 297.38 & 0.15 & 239.99 & 240.18 & 240.09 & 0.10 \\
\hline Revised & T Lychnocanoma amphitrite & & 33.750 & U1334B-26X-5, 130-131 & U1334C-28X-1, 40-41 & 245.51 & 249.21 & 297.92 & 298.23 & 298.08 & 0.15 & 240.42 & 240.61 & 240.52 & 0.09 \\
\hline Revised & T Dictyoprora mongolfieri & & 33.180 & U1334B-26X-5, 130-131 & U1334C-28X-1, 40-41 & 245.51 & 249.21 & 297.92 & 298.23 & 298.08 & 0.15 & 240.42 & 240.61 & 240.52 & 0.09 \\
\hline Revised & T Lophocyrtis (L.) jacchia & & 33.693 & U1334B-26X-5, 130-131 & U1334C-28X-1, 40-41 & 245.51 & 249.21 & 297.92 & 298.23 & 298.08 & 0.15 & 240.42 & 240.61 & 240.52 & 0.09 \\
\hline Revised & T Lithocyclia aristotelis gr. & & 33.510 & U1334C-28X-1, 40-41 & U1334B-26X-6, 40-41 & 249.21 & 246.11 & 298.23 & 298.58 & 298.40 & 0.17 & 240.61 & 240.81 & 240.71 & 0.10 \\
\hline Revised & T Lychnocanoma babylonis & & 33.750 & U1334C-28X-1, 40-41 & U1334B-26X-6, 40-41 & 249.21 & 246.11 & 298.23 & 298.58 & 298.40 & 0.17 & 240.61 & 240.81 & 240.71 & 0.10 \\
\hline Revised & $\begin{array}{l}\text { Lithocyclia aristotelis gr. > Lithocyclia } \\
\text { angusta }\end{array}$ & & 33.820 & U1334C-28X-1, 40-41 & U1334B-26X-6, 40-41 & 249.21 & 246.11 & 298.23 & 298.58 & 298.40 & 0.17 & 240.61 & 240.81 & 240.71 & 0.10 \\
\hline Revised & T Dictyoprora armadillo & \multirow{10}{*}{ RP19 } & 33.693 & U1334C-28X-1, 40-41 & U1334B-26X-6, 4 & 249.21 & 246.11 & 298.23 & 298.58 & 298.40 & 0.17 & 240.61 & 240.81 & 240.71 & 0.10 \\
\hline Revised & T Zealithapium mitra & & 33.974 & U1334C-28X-1, 40-41 & U1334B-26X-6, 40-41 & 249.21 & 246.11 & 298.23 & 298.58 & 298.40 & 0.17 & 240.61 & 240.81 & 240.71 & 0.10 \\
\hline Revised & B Lophocyrtis (C.) milowi & & 34.125 & U1334C-28X-1, 40-41 & U1334B-26X-6, 40-41 & 249.21 & 246.11 & 298.23 & 298.58 & 298.40 & 0.17 & 240.61 & 240.81 & 240.71 & 0.10 \\
\hline Revised & T Dorcadospyris copelata & & 33.840 & U1334C-28X-1, 70-71 & U1334B-26X-6, 70-71 & 249.51 & 246.41 & 298.65 & 298.88 & 298.77 & 0.11 & 240.86 & 241.02 & 240.94 & 0.08 \\
\hline Revised & T Calocycletta $(\mathrm{C}$.) anekathen & & 33.753 & U1334C-28X-1, 70-71 & U1334B-26X-6, 70-71 & 249.51 & 246.41 & 298.65 & 298.88 & 298.77 & 0.11 & 240.86 & 241.02 & 240.94 & 0.08 \\
\hline Revised & T Lophocyrtis (C.) hadra & & 33.750 & U1334C-28X-1, 100-101 & U1334C-28X-1, 130-131 & 249.81 & 250.11 & 299.00 & 299.34 & 299.17 & 0.17 & 241.11 & 241.35 & 241.23 & 0.12 \\
\hline Revised & B Lithocyclia angusta & & 34.130 & U1334B-26X-7, 10-11 & U1334B-26X-7, 35-36 & 247.31 & 247.56 & 299.85 & 300.09 & 299.97 & 0.12 & 241.83 & 242.07 & 241.95 & 0.12 \\
\hline Revised & $\begin{array}{l}\text { B Pteropilium sp. aff. Pterocanium } \\
\text { contiguum }\end{array}$ & & 34.251 & U1334B-26X-7, 10-11 & U1334B-26X-7, 35-36 & 247.31 & 247.56 & 299.85 & 300.09 & 299.97 & 0.12 & 241.83 & 242.07 & 241.95 & 0.12 \\
\hline Revised & T Dorcadospyris ombros (upper) & & 34.298 & U1334B-26H-CC & U1334B-27X-1, 10-11 & 247.87 & 246.24 & 300.40 & 302.16 & 301.28 & 0.88 & 242.39 & 243.98 & 243.19 & 0.80 \\
\hline Revised & $\begin{array}{l}\text { T Thyrsocyrtis }(P .) \text { lochites } \\
\text { (continuous) }\end{array}$ & & 34.125 & U1334A-27X-CC & U1334B-27X-2, 40-41 & 250.74 & 248.71 & 301.84 & 302.84 & 302.34 & 0.50 & 243.72 & 244.66 & 244.19 & 0.47 \\
\hline
\end{tabular}




\begin{tabular}{|c|c|c|c|c|c|c|c|c|c|c|c|c|c|c|c|}
\hline \multirow[b]{2}{*}{ Source } & \multirow[b]{2}{*}{ Event } & \multirow[b]{2}{*}{ Zone } & \multirow{2}{*}{$\begin{array}{l}\text { Age* }^{*} \\
(\mathrm{Ma})\end{array}$} & \multicolumn{2}{|c|}{ Hole, core, section, interval $(\mathrm{cm})$} & \multicolumn{2}{|c|}{$\begin{array}{c}\text { Depth } \\
\text { (mbsf [m CSF-A]) }\end{array}$} & \multicolumn{3}{|c|}{$\begin{array}{l}\text { Depth (rmcd [adjusted } \\
\text { m revised CCSF-A]) }\end{array}$} & \multirow{2}{*}{$\begin{array}{l}\text { Error } \\
( \pm) \\
(\mathrm{m})\end{array}$} & \multicolumn{3}{|c|}{$\begin{array}{l}\text { Depth Site } 1218 \\
\text { (corrected rmcd) }\end{array}$} & \multirow{2}{*}{$\begin{array}{l}\text { Error } \\
( \pm) \\
(\mathrm{m})\end{array}$} \\
\hline & & & & Top & Bottom & Top & Bottom & Top & Bottom & Mean & & Top & Bottom & Mean & \\
\hline Revised & T Cryptocarpium azyx & \multirow{5}{*}{ RP19 } & 35.070 & U1334B-27X-3, 10-11 & U1334B-27X-3, 70-71 & 250.91 & 251.51 & 305.64 & 305.64 & 305.64 & 0.00 & 246.49 & 246.49 & 246.49 & 0.00 \\
\hline Revised & T Calocyclas turris & & 34.830 & U1334B-27X-3, 130-131 & U1334B-27X-4, 40-41 & 252.11 & 252.71 & 306.81 & 306.81 & 306.81 & 0.00 & 247.23 & 247.23 & 247.23 & 0.00 \\
\hline Revised & B Lophocyrtis (C.) hadra & & 35.340 & U1334B-27X-4, 70-71 & U1334B-27X-4, 100-101 & 253.01 & 253.31 & 307.38 & 307.38 & 307.38 & 0.00 & 247.61 & 247.61 & 247.61 & 0.00 \\
\hline Revised & $\begin{array}{l}\text { T Thyrsocyrtis (T.) bromia } \\
\text { (continuous) }\end{array}$ & & 33.936 & U1334B-27X-4, 100-101 & U1334B-27X-4, 130-131 & 253.31 & 253.61 & 307.68 & 307.68 & 307.68 & 0.00 & 247.80 & 247.80 & 247.80 & 0.00 \\
\hline Revised & $\begin{array}{l}\text { T Thyrsocyrtis }(P .) \text { tetracantha } \\
\text { (continuous) }\end{array}$ & & 35.300 & U1334B-27X-4, 100-101 & U1334B-27X-4, 130-131 & 253.31 & 253.61 & 307.68 & 307.68 & 307.68 & 0.00 & 247.80 & 247.80 & 247.80 & 0.00 \\
\hline Revised & T Calocyclas bandyca & \multirow{10}{*}{ RP18 } & 34.620 & U1334B-27X-4, 100-101 & U1334B-27X-4, 130-131 & 253.31 & 253.61 & 307.68 & 307.68 & 307.68 & 0.00 & 247.80 & 247.80 & 247.80 & 0.00 \\
\hline Revised & T Thyrsocyrtis $(P$.$) krooni$ & & 35.440 & U1334B-27X-5, 100-101 & U1334B-27X-5, 130-131 & 254.81 & 255.11 & 309.18 & 309.18 & 309.18 & 0.00 & 248.70 & 248.70 & 248.70 & 0.00 \\
\hline Revised & T Thyrsocyrtis (T.) rhizodon & & 35.402 & U1334B-27X-6, 10-11 & U1334B-27X-6, 40-41 & 255.41 & 255.71 & 309.78 & 309.78 & 309.78 & 0.00 & 249.03 & 249.03 & 249.03 & 0.00 \\
\hline Revised & T Calocyclas hispida & & 33.620 & U1334A-28X-5, 10-11 & U1334A-28X-5, 40-41 & 258.71 & 258.91 & 312.01 & 312.21 & 312.11 & 0.10 & 250.26 & 250.37 & 250.31 & 0.06 \\
\hline Revised & $\begin{array}{l}\text { T Thyrsocyrtis (P.) triacantha } \\
\text { (continuous) }\end{array}$ & & 33.340 & U1334A-28X-5, 10-11 & U1334A-28X-5, 40-41 & 258.71 & 258.91 & 312.01 & 312.21 & 312.11 & 0.10 & 250.26 & 250.37 & 250.31 & 0.06 \\
\hline Revised & T Lithocyclia ocellus gr. & & 33.373 & U1334A-28X-5, 73-74 & U1334A-28X-5, 105-107 & 259.54 & 259.85 & 312.84 & 313.15 & 312.99 & 0.16 & 250.72 & 250.89 & 250.80 & 0.09 \\
\hline Revised & T Thyrsocyrtis (P.) orthotenes & & 36.893 & U1334A-28X-6, 10-11 & U1334A-28X-6, 40-41 & 260.40 & 260.71 & 313.70 & 314.01 & 313.85 & 0.15 & 251.30 & 251.38 & 251.34 & 0.04 \\
\hline Revised & B Artophormis gracilis & & 36.138 & U1334A-28X-6, 40-41 & U1334A-28X-7, 40-41 & 260.71 & 262.21 & 314.01 & 315.47 & 314.74 & 0.73 & 251.38 & 251.75 & 251.56 & 0.18 \\
\hline Revised & B Lychnocanoma amphitrite & & 36.490 & U1334A-29X-3, 30-31 & U1334A-29X-3, 60-61 & 264.22 & 264.52 & 318.43 & 318.69 & 318.56 & 0.13 & 252.87 & 253.00 & 252.94 & 0.06 \\
\hline Revised & T Theocyrtis perpumila & & 36.716 & U1334A-29X-3, 60-61 & U1334A-29X-3, 90-91 & 264.52 & 264.82 & 318.69 & 318.95 & 318.82 & 0.13 & 253.00 & 253.13 & 253.07 & 0.06 \\
\hline Revised & B Calocyclas bandyca & & 36.740 & U1334A-29X-4, 120-121 & U1334A-29X-4, 149-150 & 266.62 & 266.91 & 320.70 & 320.95 & 320.82 & 0.12 & 254.38 & 254.52 & 254.45 & 0.07 \\
\hline Revised & B Calocycletta (C.) anekathen & \multirow{5}{*}{ RP17 } & 36.413 & U1334A-29X-5, 30-31 & U1334A-29X-5, 60-61 & 267.22 & 267.52 & 321.24 & 321.55 & 321.39 & 0.16 & 254.68 & 255.02 & 254.85 & 0.17 \\
\hline Revised & B Lophocyrtis (L.) jacchia & & 37.065 & U1334B-28H-CC & U1334A-29X-5, 145-146 & 267.29 & 268.37 & 322.21 & 322.40 & 322.30 & 0.09 & 255.54 & 255.67 & 255.60 & 0.06 \\
\hline Ship & B Calocyclas turris & & 38.667 & U1334A-29X-CC & U1334C-29H-CC & 272.00 & 268.26 & 326.42 & 326.54 & 326.48 & 0.06 & 257.81 & 257.87 & 257.84 & 0.03 \\
\hline Ship & B Thyrsocyrtis (P.) lochites & & 37.519 & U1334C-29H-CC & U1334A-30X-2, 120-122 & 268.26 & 274.80 & 326.54 & 330.01 & 328.27 & 1.73 & 257.87 & 259.43 & 258.65 & 0.78 \\
\hline Ship & B Thyrsocyrtis $(P$.$) tetracantha$ & & 38.118 & U1334C-29H-CC & U1334A-30X-2, 120-122 & 268.26 & 274.80 & 326.54 & 330.01 & 328.27 & 1.73 & 257.87 & 259.43 & 258.65 & 0.78 \\
\hline
\end{tabular}

* = Nigrini et al. (2006). T = top, B = bottom. 


\begin{tabular}{|c|c|c|c|c|c|c|c|c|c|c|c|c|}
\hline \multirow[b]{2}{*}{ Source } & \multirow[b]{2}{*}{ Event } & \multirow[b]{2}{*}{ Zone } & \multirow{2}{*}{$\begin{array}{l}\mathrm{Age}^{*} \\
(\mathrm{Ma})\end{array}$} & \multicolumn{2}{|c|}{ Hole, core, section, interval $(\mathrm{cm})$} & \multicolumn{3}{|c|}{$\begin{array}{c}\text { Depth from } \\
\text { Pälike et al. (2005) (rmcd) } \\
\end{array}$} & \multicolumn{3}{|c|}{$\begin{array}{l}\text { Depth adjusted } \\
\text { (corrected rmcd) }\end{array}$} & \multirow{2}{*}{$\begin{array}{c}\text { Error } \\
( \pm) \\
(\mathrm{m})\end{array}$} \\
\hline & & & & Top & Bottom & Top & Bottom & Mean & Top & Bottom & Mean & \\
\hline & & \multirow{10}{*}{ RN2 } & & 199- & 199- & & & & & & & \\
\hline Nigrini et al., 2006 & T Dorcadospyris praeforcipata & & 20.070 & $1218 \mathrm{~A}-7 \mathrm{H}-4,46-48$ & $1218 \mathrm{~A}-7 \mathrm{H}-5,40-42$ & 68.97 & 70.32 & 69.65 & 68.97 & 70.32 & 69.65 & 0.67 \\
\hline Nigrini et al., 2006 & B Didymocyrtis violina & & 20.070 & $1218 \mathrm{~A}-7 \mathrm{H}-4,46-48$ & $1218 \mathrm{~A}-7 \mathrm{H}-5,40-42$ & 68.97 & 70.32 & 69.65 & 68.97 & 70.32 & 69.65 & 0.67 \\
\hline Nigrini et al., 2006 & B Didymocyrtis tubaria (upper) & & 20.070 & $1218 \mathrm{~A}-7 \mathrm{H}-4,46-48$ & $1218 \mathrm{~A}-7 \mathrm{H}-5,40-42$ & 68.97 & 70.32 & 69.65 & 68.97 & 70.32 & 69.65 & 0.67 \\
\hline Nigrini et al., 2006 & B Dorcadospyris simplex & & 20.565 & $1218 \mathrm{~A}-7 \mathrm{H}-6,46-48$ & $1218 \mathrm{~A}-7 \mathrm{H}-7,46-48$ & 71.65 & 73.37 & 72.51 & 71.65 & 73.37 & 72.51 & 0.86 \\
\hline Nigrini et al., 2006 & T Calocycletta (C.) serrata & & 20.565 & $1218 \mathrm{~A}-7 \mathrm{H}-6,46-48$ & $1218 \mathrm{~A}-7 \mathrm{H}-7,46-48$ & 71.65 & 73.37 & 72.51 & 71.65 & 73.37 & 72.51 & 0.86 \\
\hline Nigrini et al., 2006 & B Stichocorys delmontensis & & 21.175 & $1218 \mathrm{~A}-8 \mathrm{H}-3,46-48$ & $1218 \mathrm{~A}-8 \mathrm{H}-4,45-47$ & 76.64 & 78.15 & 77.40 & 76.64 & 78.15 & 77.40 & 0.76 \\
\hline Nigrini et al., 2006 & T Lophocyrtis (C.) pegetrum & & 21.175 & $1218 \mathrm{~A}-8 \mathrm{H}-3,46-48$ & $1218 \mathrm{~A}-8 \mathrm{H}-4,45-47$ & 76.64 & 78.15 & 77.40 & 76.64 & 78.15 & 77.40 & 0.76 \\
\hline Nigrini et al., 2006 & B Carpocanopsis bramlettei & & 21.550 & $1218 \mathrm{~A}-8 \mathrm{H}-6,46-48$ & $1218 \mathrm{~A}-8 \mathrm{H}-8-\mathrm{CC}$ & 81.21 & 82.19 & 81.70 & 81.21 & 82.19 & 81.70 & 0.49 \\
\hline Nigrini et al., 2006 & T Theocyrtis annosa & & 21.590 & $1218 \mathrm{~A}-8 \mathrm{H}-\mathrm{CC}$ & 1218B-9H-3, 45-47 & 82.19 & 82.19 & 82.19 & 82.19 & 82.19 & 82.19 & 0.00 \\
\hline Nigrini et al., 2006 & B Calocycletta (C.) virginis & \multirow{6}{*}{ RN1 } & 21.660 & $1218 \mathrm{~B}-9 \mathrm{H}-3,45-47$ & $1218 \mathrm{~B}-9 \mathrm{H}-4,45-47$ & 82.19 & 83.69 & 82.94 & 82.19 & 83.69 & 82.94 & 0.75 \\
\hline Nigrini et al., 2006 & B Lophocyrtis (C.) leptetrum & & 21.660 & $1218 \mathrm{~B}-9 \mathrm{H}-3,45-47$ & $1218 \mathrm{~B}-9 \mathrm{H}-4,45-47$ & 82.19 & 83.69 & 82.94 & 82.19 & 83.69 & 82.94 & 0.75 \\
\hline Nigrini et al., 2006 & T Didymocyrtis tubaria (lower) & & 21.850 & $1218 \mathrm{~B}-9 \mathrm{H}-5,45-47$ & $1218 \mathrm{~A}-9 \mathrm{H}-1,71-72$ & 84.19 & 85.67 & 84.93 & 84.19 & 85.67 & 84.93 & 0.74 \\
\hline Nigrini et al., 2006 & T Eucyrtidium mitodes & & 22.235 & $1218 \mathrm{~A}-9 \mathrm{H}-2,71-72$ & $1218 \mathrm{~A}-9 \mathrm{H}-4,74-75$ & 87.07 & 90.20 & 88.64 & 87.07 & 90.20 & 88.64 & 1.57 \\
\hline Nigrini et al., 2006 & B Calocycletta (C.) serrata & & 22.235 & $1218 \mathrm{~A}-9 \mathrm{H}-2,71-72$ & $1218 \mathrm{~A}-9 \mathrm{H}-4,74-75$ & 87.07 & 90.20 & 88.64 & 87.07 & 90.20 & 88.64 & 1.57 \\
\hline Nigrini et al., 2006 & B Cyrtocapsella cornuta & & 22.585 & $1218 \mathrm{~A}-9 \mathrm{H}-5,74-75$ & $1218 \mathrm{~B}-10 \mathrm{H}-2,70-72$ & 91.70 & 92.90 & 92.30 & 91.70 & 92.90 & 92.30 & 0.60 \\
\hline Nigrini et al., 2006 & B Cyrtocapsella tetrapera & & 22.585 & $1218 \mathrm{~A}-9 \mathrm{H}-5,74-75$ & $1218 \mathrm{~B}-10 \mathrm{H}-2,70-72$ & 91.70 & 92.90 & 92.30 & 91.70 & 92.90 & 92.30 & 0.60 \\
\hline Nigrini et al., 2006 & T Artophormis gracilis & \multirow{13}{*}{ RP22 } & 22.725 & $1218 \mathrm{~A}-9 \mathrm{H}-6,83-85$ & $1218 \mathrm{~B}-10 \mathrm{H}-3,46-48$ & 93.29 & 94.16 & 93.73 & 93.29 & 94.16 & 93.73 & 0.43 \\
\hline Nigrini et al., 2006 & B Eucyrtidium diaphanes & & 23.105 & $1218 \mathrm{~B}-10 \mathrm{H}-5,46-48$ & $1218 \mathrm{~A}-10 \mathrm{H}-1,74-75$ & 97.16 & 97.23 & 97.20 & 97.16 & 97.23 & 97.20 & 0.04 \\
\hline Nigrini et al., 2006 & B Didymocyrtis bassanii & & 22.910 & $1218 \mathrm{~A}-9 \mathrm{H}-\mathrm{CC}$ & $1218 \mathrm{~B}-10 \mathrm{H}-4,46-48$ & 95.04 & 95.66 & 95.35 & 95.04 & 95.66 & 95.35 & 0.31 \\
\hline Nigrini et al., 2006 & T Dorcadospyris cyclacantha & & 23.105 & $1218 \mathrm{~B}-10 \mathrm{H}-5,46-48$ & $1218 \mathrm{~A}-10 \mathrm{H}-1,74-75$ & 97.16 & 97.23 & 97.20 & 97.16 & 97.23 & 97.20 & 0.04 \\
\hline Nigrini et al., 2006 & T Dorcadospyris riedeli (upper) & & 23.165 & $1218 \mathrm{~A}-10 \mathrm{H}-1,74-75$ & $1218 \mathrm{~B}-10 \mathrm{H}-6,74-75$ & 97.23 & 104.34 & 100.79 & 97.23 & 104.34 & 100.79 & 3.56 \\
\hline Nigrini et al., 2006 & B Dorcadospyris cyclacantha & & 23.385 & $1218 \mathrm{~B}-10 \mathrm{H}-7,46-48$ & $1218 \mathrm{~A}-10 \mathrm{H}-4,74-75$ & 100.11 & 101.34 & 100.73 & 100.11 & 101.34 & 100.73 & 0.62 \\
\hline Nigrini et al., 2006 & T Dorcadospyris circulus (max) & & 23.575 & 1218A-10H-4, 74-76 & 1218A-10H-6, 74-75 & 101.34 & 104.34 & 102.84 & 101.34 & 104.34 & 102.84 & 1.50 \\
\hline Nigrini et al., 2006 & T Dorcadospyris papilio & & 23.760 & $1218 \mathrm{~A}-10 \mathrm{H}-6,74-75$ & $1218 \mathrm{~A}-10 \mathrm{H}-\mathrm{CC}$ & 104.34 & 105.49 & 104.92 & 104.34 & 105.49 & 104.92 & 0.57 \\
\hline Nigrini et al., 2006 & $\mathrm{~T}$ Liriospyris longicornuta & & 23.760 & $1218 \mathrm{~A}-10 \mathrm{H}-6,74-75$ & $1218 \mathrm{~A}-10 \mathrm{H}-\mathrm{CC}$ & 104.34 & 105.49 & 104.92 & 104.34 & 105.49 & 104.92 & 0.57 \\
\hline Nigrini et al., 2006 & B Carpocanopsis cingulata & & 24.435 & $1218 \mathrm{~A}-11 \mathrm{H}-3,44-46$ & $1218 \mathrm{~A}-11 \mathrm{H}-4,44-46$ & 110.46 & 112.04 & 111.25 & 110.46 & 112.04 & 111.25 & 0.79 \\
\hline Nigrini et al., 2006 & T Acrocubus octopylus (lower) & & 24.675 & $1218 \mathrm{~A}-11 \mathrm{H}-5,45-47$ & $1218 \mathrm{~A}-11 \mathrm{H}-6,45-47$ & 113.55 & 115.05 & 114.30 & 113.55 & 115.05 & 114.30 & 0.75 \\
\hline Nigrini et al., 2006 & T Lychnocanoma apodora & & 24.800 & $1218 \mathrm{~A}-11 \mathrm{H}-6,45-47$ & $1218 \mathrm{~A}-11 \mathrm{H}-7,45-47$ & 115.05 & 116.55 & 115.80 & 115.05 & 116.55 & 115.80 & 0.75 \\
\hline Nigrini et al., 2006 & B Lychnocanoma elongata & & 25.165 & $1218 \mathrm{~A}-12 \mathrm{H}-2,45-47$ & $1218 \mathrm{~A}-12 \mathrm{H}-3,45-47$ & 119.62 & 121.12 & 120.37 & 119.62 & 121.12 & 120.37 & 0.75 \\
\hline Nigrini et al., 2006 & B Acrocubus octopylus (lower) & \multirow{19}{*}{ RP21 } & 25.270 & $1218 \mathrm{~A}-12 \mathrm{H}-3,45-47$ & $1218 \mathrm{~A}-12 \mathrm{H}-4,45-47$ & 121.12 & 122.62 & 121.87 & 121.12 & 122.62 & 121.87 & 0.75 \\
\hline Nigrini et al., 2006 & B Didymocyrtis tubaria (lower) & & 25.505 & $1218 \mathrm{~A}-12 \mathrm{H}-5,45-47$ & $1218 \mathrm{~A}-12 \mathrm{H}-6,45-47$ & 124.12 & 125.75 & 124.94 & 124.12 & 125.75 & 124.94 & 0.81 \\
\hline Nigrini et al., 2006 & B Calocycletta (C.) robusta & & 25.505 & $1218 \mathrm{~A}-12 \mathrm{H}-5,45-47$ & $1218 \mathrm{~A}-12 \mathrm{H}-6,45-47$ & 124.12 & 125.75 & 124.94 & 124.12 & 125.75 & 124.94 & 0.81 \\
\hline Nigrini et al., 2006 & B Dorcadospyris praeforcipata & & 25.505 & $1218 \mathrm{~A}-12 \mathrm{H}-5,45-47$ & $1218 \mathrm{~A}-12 \mathrm{H}-6,45-47$ & 124.12 & 125.75 & 124.94 & 124.12 & 125.75 & 124.94 & 0.81 \\
\hline Nigrini et al., 2006 & B Liriospyris longicornuta & & 25.795 & $1218 \mathrm{~A}-13 \mathrm{H}-1,45-47$ & $1218 \mathrm{~A}-13 \mathrm{H}-2,45-47$ & 128.40 & 129.40 & 128.90 & 128.40 & 129.40 & 128.90 & 0.50 \\
\hline Nigrini et al., 2006 & B Dorcadospyris scambos & & 26.010 & $1218 \mathrm{~A}-13 \mathrm{H}-3,45-47$ & $1218 \mathrm{~A}-13 \mathrm{H}-4,45-47$ & 130.69 & 132.56 & 131.63 & 130.69 & 132.56 & 131.63 & 0.94 \\
\hline Nigrini et al., 2006 & T Theocorys puriri & & 26.125 & $1218 \mathrm{~A}-13 \mathrm{H}-4,45-47$ & $1218 \mathrm{~A}-13 \mathrm{H}-5,45-47$ & 132.56 & 134.06 & 133.31 & 132.56 & 134.06 & 133.31 & 0.75 \\
\hline Nigrini et al., 2006 & B Acrobotrys disolenia & & 26.125 & $1218 \mathrm{~A}-13 \mathrm{H}-4,45-47$ & $1218 \mathrm{~A}-13 \mathrm{H}-5,45-47$ & 132.56 & 134.06 & 133.31 & 132.56 & 134.06 & 133.31 & 0.75 \\
\hline Nigrini et al., 2006 & B Dorcadospyris papilio & & 26.125 & $1218 \mathrm{~A}-13 \mathrm{H}-4,45-47$ & $1218 \mathrm{~A}-13 \mathrm{H}-5,45-47$ & 132.56 & 134.06 & 133.31 & 132.56 & 134.06 & 133.31 & 0.75 \\
\hline Nigrini et al., 2006 & B Lychnocanoma apodora & & 26.125 & $1218 \mathrm{~A}-13 \mathrm{H}-4,45-47$ & $1218 \mathrm{~A}-13 \mathrm{H}-5,45-47$ & 132.56 & 134.06 & 133.31 & 132.56 & 134.06 & 133.31 & 0.75 \\
\hline Nigrini et al., 2006 & T Dorcadospyris circulus (continuous) & & 26.385 & $1218 \mathrm{~A}-13 \mathrm{H}-7,38-40$ & $1218 \mathrm{~A}-13 \mathrm{H}-\mathrm{CC}$ & 136.89 & 137.57 & 137.23 & 136.89 & 137.57 & 137.23 & 0.34 \\
\hline Nigrini et al., 2006 & B Dorcadospyris riedeli (upper) & & 26.505 & $1218 \mathrm{~A}-14 \mathrm{H}-1,45-47$ & $1218 \mathrm{~A}-14 \mathrm{H}-2,45-47$ & 138.18 & 139.57 & 138.88 & 138.18 & 139.57 & 138.88 & 0.69 \\
\hline Nigrini et al., 2006 & T Eucyrtidium plesiodiaphanes & & 27.355 & $1218 \mathrm{~A}-15 \mathrm{H}-2,45-47$ & $1218 \mathrm{~A}-15 \mathrm{H}-3,45-47$ & 149.87 & 151.37 & 150.62 & 149.87 & 151.37 & 150.62 & 0.75 \\
\hline Nigrini et al., 2006 & T Lithocyclia angusta (continuous) & & 28.015 & $1218 \mathrm{~A}-16 \mathrm{H}-1,45-47$ & $1218 \mathrm{~A}-16 \mathrm{H}-2,45-47$ & 159.21 & 160.76 & 159.99 & 159.21 & 160.76 & 159.99 & 0.77 \\
\hline Nigrini et al., 2006 & T Theocyrtis setanios & & 28.810 & $1218 \mathrm{~A}-17 \mathrm{H}-3,45-47$ & $1218 \mathrm{~A}-17 \mathrm{H}-4,45-47$ & 171.49 & 172.75 & 172.12 & 171.49 & 172.75 & 172.12 & 0.63 \\
\hline Nigrini et al., 2006 & $\mathrm{~T}$ Theocyrtis perysinos & & 29.075 & $1218 \mathrm{~A}-17 \mathrm{H}-6,45-47$ & $1218 \mathrm{~A}-17 \mathrm{H}-7,45-47$ & 175.51 & 177.01 & 176.26 & 175.51 & 177.01 & 176.26 & 0.75 \\
\hline Nigrini et al., 2006 & T Tristylospyris triceros & & 29.075 & $1218 \mathrm{~A}-17 \mathrm{H}-6,45-47$ & $1218 \mathrm{~A}-17 \mathrm{H}-7,45-47$ & 175.51 & 177.01 & 176.26 & 175.51 & 177.01 & 176.26 & 0.75 \\
\hline Nigrini et al., 2006 & B Theocyrtis annosa & & 29.130 & 1218A-17H-7, 45-47 & 1218A-17H-CC & 177.01 & 177.55 & 177.28 & 177.01 & 177.55 & 177.28 & 0.27 \\
\hline Nigrini et al., 2006 & Tristylospyris triceros > Dorcadospyris ateuchus & & 29.310 & $1218 \mathrm{~A}-18 \mathrm{H}-1,46-48$ & $1218 \mathrm{~A}-18 \mathrm{H}-2,46-48$ & 180.52 & 182.27 & 181.40 & 180.52 & 182.27 & 181.40 & 0.88 \\
\hline
\end{tabular}




\begin{tabular}{|c|c|c|c|c|c|c|c|c|c|c|c|c|}
\hline \multirow[b]{2}{*}{ Source } & \multirow[b]{2}{*}{ Event } & \multirow[b]{2}{*}{ Zone } & \multirow{2}{*}{$\begin{array}{l}\text { Age }^{*} \\
(\mathrm{Ma})\end{array}$} & \multicolumn{2}{|c|}{ Hole, core, section, interval $(\mathrm{cm})$} & \multicolumn{3}{|c|}{$\begin{array}{l}\text { Depth from } \\
\text { Pälike et al. (2005) (rmcd) } \\
\end{array}$} & \multicolumn{3}{|c|}{$\begin{array}{l}\text { Depth adjusted } \\
\text { (corrected rmcd) }\end{array}$} & \multirow{2}{*}{$\begin{array}{c}\text { Error } \\
( \pm) \\
(\mathrm{m})\end{array}$} \\
\hline & & & & Top & Bottom & Top & Bottom & Mean & Top & Bottom & Mean & \\
\hline Nigrini et al., 2006 & B Eucyrtidium mitodes & \multirow{33}{*}{ RP20 } & 29.515 & $1218 \mathrm{~A}-18 \mathrm{H}-3,46-48$ & $1218 \mathrm{~A}-18 \mathrm{H}-4,46-48$ & 184.22 & 185.72 & 184.97 & 184.22 & 185.72 & 184.97 & 0.75 \\
\hline Nigrini et al., 2006 & B Theocyrtis perysinos & & 29.735 & $1218 \mathrm{~A}-18 \mathrm{H}-5,46-48$ & $1218 \mathrm{~A}-18 \mathrm{H}-6,46-48$ & 187.14 & 188.58 & 187.86 & 187.14 & 188.58 & 187.86 & 0.72 \\
\hline Nigrini et al., 2006 & B Dorcadospyris ateuchus & & 30.175 & $1218 \mathrm{~A}-19 \mathrm{H}-3,46-48$ & $1218 \mathrm{~A}-19 \mathrm{H}-4,46-48$ & 193.54 & 195.04 & 194.29 & 193.54 & 195.04 & 194.29 & 0.75 \\
\hline Nigrini et al., 2006 & B Theocyrtis setanios & & 29.830 & $1218 \mathrm{~A}-18 \mathrm{H}-6,46-48$ & $1218 \mathrm{~A}-18 \mathrm{H}-\mathrm{CC}$ & 188.58 & 190.22 & 189.40 & 188.58 & 190.22 & 189.40 & 0.82 \\
\hline Nigrini et al., 2006 & T Lophocyrtis (C.) milowi & & 30.280 & $1218 \mathrm{~A}-19 \mathrm{H}-4,46-48$ & $1218 \mathrm{~A}-19 \mathrm{H}-5,46-48$ & 195.04 & 196.54 & 195.79 & 195.04 & 196.54 & 195.79 & 0.75 \\
\hline Nigrini et al., 2006 & B Didymocyrtis prismatica & & 30.510 & $1218 \mathrm{~A}-19 \mathrm{H}-6,46-48$ & $1218 \mathrm{~A}-19 \mathrm{H}-7,46-48$ & 198.04 & 199.11 & 198.58 & 198.04 & 199.11 & 198.58 & 0.54 \\
\hline Nigrini et al., 2006 & B Dorcadospyris circulus & & 30.710 & $1218 \mathrm{~A}-19 \mathrm{H}-\mathrm{CC}$ & $1218 \mathrm{~A}-20 \mathrm{H}-1,38-40$ & 199.72 & 201.86 & 200.79 & 199.72 & 201.86 & 200.79 & 1.07 \\
\hline Nigrini et al., 2006 & T Lophocyrtis (S.) oberhaensliae & & 30.885 & $1218 \mathrm{~A}-20 \mathrm{H}-1,38-40$ & $1218 \mathrm{~A}-20 \mathrm{H}-2,45-47$ & 201.86 & 203.43 & 202.65 & 201.86 & 203.43 & 202.65 & 0.78 \\
\hline Revised & $\mathrm{T}$ Theocyrtis tuberosa & & 30.710 & 1218B-20X-4, 98-99 & 1218B-20X-4, 123-124 & 201.28 & 201.74 & 201.51 & 201.28 & 201.74 & 201.51 & 0.23 \\
\hline Revised & T Lithocyclia crux & & 30.710 & 1218B-20X-3, 49-50 & 1218B-20X-3, 98-99 & 199.30 & 199.78 & 199.54 & 199.30 & 199.78 & 199.54 & 0.24 \\
\hline Revised & T Dorcadospyris spinosa & & 31.015 & $1218 \mathrm{~A}-20 \mathrm{H}-1,38-40$ & 1218B-20X-5, 48-49 & 201.86 & 202.28 & 202.07 & 201.86 & 202.28 & 202.07 & 0.21 \\
\hline Revised & T Dorcadospyris quadripes & & 31.015 & $1218 \mathrm{~A}-20 \mathrm{H}-2,45-47$ & $1218 \mathrm{~A}-20 \mathrm{H}-6,50-51$ & 203.43 & 203.80 & 203.62 & 203.43 & 204.93 & 204.18 & 0.75 \\
\hline Revised & B Eucyrtidium plesiodiaphanes & & 31.125 & $1218 \mathrm{~A}-20 \mathrm{H}-3,100-101$ & $1218 \mathrm{~A}-20 \mathrm{H}-3,144-145$ & 205.48 & 205.93 & 205.71 & 205.48 & 205.93 & 205.71 & 0.23 \\
\hline Nigrini et al., 2006 & B Lophocyrtis (C.) pegetrum & & 31.340 & $1218 \mathrm{~A}-20 \mathrm{H}-5,45-47$ & 1218B-21X-2, 45-47 & 207.93 & 209.27 & 208.60 & 207.93 & 209.09 & 208.51 & 0.58 \\
\hline Nigrini et al., 2006 & B Spirocyrtis subtilis & & 31.340 & $1218 \mathrm{~A}-20 \mathrm{H}-5,45-47$ & $1218 \mathrm{~B}-21 \mathrm{X}-2,45-47$ & 207.93 & 209.27 & 208.60 & 207.93 & 209.09 & 208.51 & 0.58 \\
\hline Revised & T Dorcadospyris pseudopapilo & & 32.165 & $1218 C-15 X-6,49-50$ & $1218 C-15 X-6,141-142$ & 222.02 & 223.02 & 222.52 & 220.86 & 221.72 & 221.29 & 0.43 \\
\hline Revised & B Dorcadospyris spinosa & & 32.165 & $1218 \mathrm{~A}-22 \mathrm{X}-2,45-47$ & $1218 C-15 X-6,49-50$ & 220.15 & 220.02 & 220.09 & 220.78 & 221.72 & 221.25 & 0.47 \\
\hline Revised & T Centrobotrys gravida & & 32.405 & 1218B-22X-4, 143-144 & $1218 \mathrm{~A}-22 \mathrm{X}-5,49-50$ & 223.36 & 223.92 & 223.64 & 223.99 & 224.55 & 224.27 & 0.28 \\
\hline Revised & B Centrobotrys petrushevskayae & & 32.645 & 1218A-22X-CC & 1218B-22X-7, 83-84 & 225.88 & 227.10 & 226.49 & 226.51 & 227.52 & 227.02 & 0.51 \\
\hline Revised & B Dorcadospyris quadripes & & 32.645 & $1218 C-16 X-3,143-144$ & $1218 \mathrm{~A}-23 \mathrm{X}-1,48-51$ & 229.14 & 230.22 & 229.68 & 228.54 & 228.62 & 228.58 & 0.04 \\
\hline Nigrini et al., 2006 & B Lychnodictyum audax & & 32.885 & $1218 \mathrm{~A}-23 \mathrm{X}-1,48-51$ & 1218A-23X-2, 105-107 & 230.22 & 231.82 & 231.02 & 228.62 & 230.69 & 229.66 & 1.04 \\
\hline Revised & $\mathrm{T}$ Theocyrtis careotuberosa & & 32.885 & $1218 C-16 X-3,49-50$ & $1218 C-16 X-3,143-144$ & 228.20 & 229.14 & 228.67 & 227.60 & 228.54 & 228.07 & 0.47 \\
\hline Revised & B Theocyrtis tuberosa & & 33.125 & $1218 C-17 X-3,73-74$ & $1218 C-17 X-3,99-100$ & 237.60 & 237.86 & 237.73 & 237.00 & 237.26 & 237.13 & 0.13 \\
\hline Revised & B Dorcadospyris pseudopapilio & & 33.025 & $1218 C-15 X-5,50-51$ & $1218 C-15 X-5,143-144$ & 219.87 & 221.01 & 220.44 & 219.47 & 220.34 & 219.90 & 0.44 \\
\hline Revised & B Lithocyclia crux & & 33.125 & 1218B-23X-4, 98-99 & $1218 \mathrm{~A}-23 \mathrm{X}-4,45-47$ & 233.65 & 233.89 & 233.77 & 232.97 & 233.09 & 233.03 & 0.06 \\
\hline Revised & B Centrobotrys gravida & & 33.125 & $1218 \mathrm{~A}-23 \mathrm{X}-3,115-117$ & 1218B-23X-4, 98-99 & 233.19 & 233.65 & 233.42 & 232.29 & 232.97 & 232.63 & 0.34 \\
\hline Revised & T Dictyoprora mongolfieri & & 33.235 & $1218 \mathrm{C}-17 \mathrm{X}-4,123-124$ & $1218 C-17 X-4,141-142$ & 239.60 & 239.78 & 239.69 & 239.00 & 239.18 & 239.09 & 0.09 \\
\hline Revised & T Lithocyclia ocellus gr. & & 33.355 & $1218 C-17 X-4,141-142$ & $1218 C-17 X-5,24-25$ & 239.78 & 240.10 & 239.94 & 239.18 & 239.50 & 239.34 & 0.16 \\
\hline Revised & T Lithocyclia aristotelis gr. & & 33.530 & $1218 C-17 X-4,123-124$ & 1218C-17X-4, 141-142 & 239.60 & 239.78 & 239.69 & 239.00 & 239.18 & 239.09 & 0.09 \\
\hline Revised & B Lophocyrtis (S.) oberhaensliae & & 33.125 & $1218 C-17 X-5,49-51$ & $1218 \mathrm{~A}-24 \mathrm{X}-1,45-47$ & 240.35 & 240.66 & 240.51 & 239.75 & 240.03 & 239.89 & 0.14 \\
\hline Revised & T Cryptocarpium ornatum & & 33.530 & $1218 C-17 X-4,141-142$ & $1218 C-17 X-5,24-25$ & 239.78 & 240.10 & 239.94 & 239.18 & 239.50 & 239.34 & 0.16 \\
\hline Revised & T Calocyclas hispida & & 33.530 & 1218B-24X-6, 50-51 & 1218B-24X-6, 78-79 & 248.16 & 248.44 & 248.30 & 247.56 & 247.84 & 247.70 & 0.14 \\
\hline Revised & Lithocyclia aristotelis gr. > Lithocyclia angusta & & 33.585 & $1218 C-17 X-5,117-118$ & $1218 C-17 X-6,23-24$ & 241.04 & 241.28 & 241.16 & 240.44 & 240.70 & 240.57 & 0.13 \\
\hline Revised & T Calocycletta $(C$.$) anekathen$ & \multirow{17}{*}{ RP19 } & 33.655 & $1218 C-17 X-4,24-25$ & 1218C-17X-5, 49-51 & 240.10 & 240.35 & 240.23 & 239.50 & 239.75 & 239.63 & 0.13 \\
\hline Revised & T Lophocyrtis (L.) jacchia & & 33.720 & $1218 C-17 X-6,52-54$ & $1218 \mathrm{~A}-24 \mathrm{X}-2,45-47$ & 241.50 & 241.76 & 241.63 & 241.01 & 241.24 & 241.13 & 0.12 \\
\hline Nigrini et al., 2006 & T Dictyoprora pirum & & 33.720 & $1218 C-17 X-5,49-51$ & $1218 \mathrm{~A}-24 \mathrm{X}-1,45-47$ & 240.35 & 240.61 & 240.48 & 239.75 & 240.03 & 239.89 & 0.14 \\
\hline Revised & T Dictyoprora armadillo & & 33.720 & $1218 C-17 X-5,117-118$ & $1218 C-17 X-6,23-24$ & 241.04 & 241.28 & 241.16 & 240.44 & 240.70 & 240.57 & 0.13 \\
\hline Revised & T Lychnocanoma babylonis & & 33.720 & $1218 C-17 X-5,117-118$ & $1218 C-17 X-6,23-24$ & 241.04 & 241.28 & 241.16 & 240.44 & 240.70 & 240.57 & 0.13 \\
\hline Revised & T Lophocyrtis (C.) hadra & & 33.720 & $1218 C-17 X-6,23-24$ & $1218 C-17 X-6,52-54$ & 241.28 & 241.50 & 241.39 & 240.70 & 241.01 & 240.86 & 0.16 \\
\hline Revised & T Lychnocanoma amphitrite & & 33.720 & 1218C-17X-5, 117-118 & $1218 C-17 X-6,23-24$ & 241.04 & 241.28 & 241.16 & 240.44 & 240.70 & 240.57 & 0.13 \\
\hline Revised & T Lophocyrtis (L.) exitelus & & 33.720 & $1218 C-17 X-6,23-24$ & $1218 C-17 X-6,52-54$ & 241.28 & 241.50 & 241.39 & 240.70 & 241.01 & 240.86 & 0.16 \\
\hline Revised & T Dorcadospyris copelata & & 33.720 & $1218 C-17 X-6,23-24$ & $1218 C-17 X-6,52-54$ & 241.28 & 241.50 & 241.39 & 240.70 & 241.01 & 240.86 & 0.16 \\
\hline Revised & T Zealithapium mitra & & 33.720 & $1218 C-17 X-6,23-24$ & $1218 C-17 X-6,52-54$ & 241.28 & 241.50 & 241.39 & 240.70 & 241.01 & 240.86 & 0.16 \\
\hline Revised & T Artophormis barbadensis & & 33.720 & $1218 C-17 X-4,123-124$ & $1218 C-17 X-4,141-142$ & 239.60 & 239.78 & 239.69 & 239.00 & 239.18 & 239.09 & 0.09 \\
\hline Revised & B Lithocyclia angusta & & 34.035 & $1218 C-17 X-6,123-124$ & 1218C-17X-6, 144-145 & 242.29 & 242.52 & 242.41 & 241.81 & 242.00 & 241.90 & 0.10 \\
\hline Revised & B Lophocyrtis (C.) milowi & & 34.035 & $1218 C-17 X-6,123-124$ & $1218 C-17 X-6,144-145$ & 242.29 & 242.52 & 242.41 & 241.81 & 242.00 & 241.90 & 0.10 \\
\hline Nigrini et al., 2006 & B Pteropilium sp. aff. Pterocanium contiguum & & 34.035 & 1218A-24X-2, 98-100 & $1218 \mathrm{~A}-24 \mathrm{X}-3,54-56$ & 242.21 & 243.04 & 242.63 & 241.61 & 242.44 & 242.03 & 0.42 \\
\hline Revised & T Dorcadospyris ombros (upper) & & 34.245 & $1218 C-17 X-7,23-24$ & $1218 \mathrm{~A}-24 \mathrm{X}-3,54-56$ & 242.85 & 243.04 & 242.95 & 242.30 & 242.44 & 242.37 & 0.07 \\
\hline Revised & B Lophocyrtis (L.) exitelus & & 34.245 & $1218 \mathrm{~B}-24 \mathrm{X}-3,48-49$ & 1218B-24X-3, 98-99 & 243.64 & 244.14 & 243.89 & 243.04 & 243.54 & 243.29 & 0.25 \\
\hline Revised & T Calocyclas bandyca & & 34.420 & $1218 B-24 X-6,22-23$ & $1218 C-18 X-1,50-52$ & 247.88 & 247.99 & 247.94 & 247.28 & 247.45 & 247.36 & 0.08 \\
\hline
\end{tabular}


Table T36 (continued). (Continued on next page.)

\begin{tabular}{|c|c|c|c|c|c|c|c|c|c|c|c|c|}
\hline \multirow[b]{2}{*}{ Source } & \multirow[b]{2}{*}{ Event } & \multirow[b]{2}{*}{ Zone } & \multirow{2}{*}{$\begin{array}{l}\text { Age* }^{*} \\
(\mathrm{Ma})\end{array}$} & \multicolumn{2}{|c|}{ Hole, core, section, interval $(\mathrm{cm})$} & \multicolumn{3}{|c|}{$\begin{array}{c}\text { Depth from } \\
\text { Pälike et al. (2005) (rmcd) }\end{array}$} & \multicolumn{3}{|c|}{$\begin{array}{l}\text { Depth adjusted } \\
\text { (corrected rmcd) }\end{array}$} & \multirow{2}{*}{$\begin{array}{c}\text { Error } \\
( \pm) \\
(\mathrm{m})\end{array}$} \\
\hline & & & & Top & Bottom & Top & Bottom & Mean & Top & Bottom & Mean & \\
\hline Revised & T Calocyclas turris & & 34.420 & $1218 \mathrm{~B}-24 \mathrm{X}-6,50-51$ & 1218B-24X-6, 78-79 & 248.16 & 248.44 & 248.30 & 247.56 & 247.84 & 247.70 & 0.14 \\
\hline Revised & T Cryptocarpium azyx & RP19 & 34.420 & $1218 \mathrm{~B}-24 \mathrm{X}-5,27-28$ & 1218B-24X-5, 48-49 & 246.42 & 246.64 & 246.53 & 245.82 & 246.04 & 245.93 & 0.11 \\
\hline Revised & T Thyrsocyrtis ( $T$. ) bromia (continuous) & & 35.405 & $1218 \mathrm{~B}-24 \mathrm{X}-6,22-23$ & $1218 \mathrm{C}-18 \mathrm{X}-1,50-52$ & 247.88 & 247.99 & 247.94 & 247.28 & 247.45 & 247.36 & 0.08 \\
\hline Revised & T Thyrsocyrtis (P.) tetracantha (continuous) & & 35.405 & 1218B-24X-5, 143-144 & 1218A-24X-7, 15-17 & 247.60 & 247.83 & 247.72 & 247.00 & 247.23 & 247.12 & 0.12 \\
\hline Revised & B Lophocyrtis (C.) hadra & & 35.405 & $1218 C-18 X-1,50-52$ & $1218 \mathrm{~B}-24 \mathrm{X}-6,50-51$ & 247.98 & 248.16 & 248.07 & 247.44 & 247.56 & 247.50 & 0.06 \\
\hline Revised & T Thyrsocyrtis $(P$.$) lochites (continuous)$ & & 35.405 & 1218B-24X-3, 123-124 & $1218 \mathrm{~B}-24 \mathrm{X}-3,143-144$ & 244.40 & 244.60 & 244.50 & 243.80 & 244.00 & 243.90 & 0.10 \\
\hline Revised & T Thyrsocyrtis (T.) rhizodon & & 35.405 & $1218 \mathrm{~B}-24 \mathrm{X}-6,50-51$ & $1218 \mathrm{~B}-24 \mathrm{X}-6,78-79$ & 248.16 & 248.44 & 248.30 & 247.56 & 247.84 & 247.70 & 0.14 \\
\hline Revised & T Thyrsocyrtis $(P$.$) krooni$ & & 35.520 & 1218B-24X-6, 104-106 & 1218B-24X-6, 78-79 & 248.91 & 248.44 & 248.68 & 244.16 & 247.84 & 246.00 & 1.84 \\
\hline Revised & T Thyrsocyrtis (P.) triacantha (continuous) & & 35.405 & $1218 \mathrm{~B}-25 \mathrm{X}-1,99-100$ & $1218 \mathrm{C}-18 \mathrm{X}-4,45-47$ & 252.05 & 252.45 & 252.25 & 251.51 & 251.91 & 251.71 & 0.20 \\
\hline Revised & T Artophormis dominasinensis & & 35.563 & $1218 C-18 X-3,20-22$ & $1218 \mathrm{~A}-25 \mathrm{X}-1,24-26$ & 250.69 & 251.14 & 250.92 & 250.15 & 250.60 & 250.38 & 0.23 \\
\hline Revised & T Eusyringium fistuligerum & RP18 & 33.910 & 1218B-25X-1, 144-145 & $1218 \mathrm{~A}-25 \mathrm{X}-2,82-84$ & 252.77 & 253.07 & 252.92 & 252.23 & 252.39 & 252.31 & 0.08 \\
\hline Revised & B Artophormis gracilis & & 36.100 & $1218 \mathrm{~B}-25 \mathrm{X}-1,20-21$ & $1218 \mathrm{~B}-25 \mathrm{X}-1,50-52$ & 251.35 & 251.92 & 251.64 & 250.81 & 251.26 & 251.04 & 0.22 \\
\hline Revised & T Lychnocanoma turgidum & & 35.195 & 1218B-24X-5, 143-144 & $1218 \mathrm{~A}-24 \mathrm{X}-7,15-17$ & 247.60 & 247.83 & 247.72 & 247.00 & 247.23 & 247.12 & 0.12 \\
\hline Revised & B Calocycletta (C.) anekathen & & 36.830 & 1218B-25X-3, 101-102 & $1218 \mathrm{~A}-25 \mathrm{X}-4,63-65$ & 255.25 & 255.96 & 255.61 & 254.78 & 255.32 & 255.05 & 0.27 \\
\hline Revised & T Theocyrtis perpumila & & 36.830 & $1218 \mathrm{~A}-25 \mathrm{X}-2,82-84$ & $1218 \mathrm{~B}-25 \mathrm{X}-2,50-51$ & 253.07 & 253.33 & 253.20 & 252.39 & 252.70 & 252.54 & 0.15 \\
\hline Revised & B Lychnocanoma amphitrite & & 36.100 & 1218B-25X-2, 100-101 & 1218B-25X-2, 137-138 & 253.83 & 254.21 & 254.02 & 253.21 & 253.63 & 253.42 & 0.21 \\
\hline Revised & B Calocyclas bandyca & & 36.830 & $1218 \mathrm{~B}-25 \mathrm{X}-3,50-51$ & $1218 \mathrm{~B}-25 \mathrm{X}-3,74-75$ & 254.73 & 254.97 & 254.85 & 254.20 & 254.47 & 254.34 & 0.13 \\
\hline Revised & B Lophocyrtis (L.) jacchia & & 36.830 & $1218 \mathrm{~A}-25 \mathrm{X}-4,63-65$ & $1218 \mathrm{~B}-26 \mathrm{X}-1,50-51$ & 255.96 & 256.41 & 256.19 & 255.32 & 255.87 & 255.59 & 0.28 \\
\hline Revised & T Thyrsocyrtis $(P$.$) orthotenes$ & RP17 & 36.315 & $1218 \mathrm{~B}-25 \mathrm{X}-1,99-100$ & $1218 C-18 X-4,45-47$ & 252.30 & 252.45 & 252.38 & 251.76 & 251.91 & 251.84 & 0.07 \\
\hline Revised & T Podocyrtis (P.) papalis (continuous) & & 36.830 & $1218 \mathrm{~B}-26 \mathrm{X}-3,73-74$ & 1218B-26X-3, 100-101 & 259.15 & 259.41 & 259.28 & 258.61 & 258.87 & 258.74 & 0.13 \\
\hline Revised & B Cryptocarpium azyx & & 37.475 & 1218B-26X-3, 73-74 & 1218B-26X-3, 100-101 & 259.15 & 259.41 & 259.28 & 258.61 & 258.87 & 258.74 & 0.13 \\
\hline Revised & B Thyrsocyrtis (P.) lochites & & 37.475 & 1218B-26X-2, 97-98 & $1218 \mathrm{~B}-26 \mathrm{X}-3,23-24$ & 258.39 & 258.65 & 258.52 & 257.85 & 258.11 & 257.98 & 0.13 \\
\hline Revised & T Anthocyrtoma spp. & & 37.925 & $1218 \mathrm{~B}-26 \mathrm{X}-3,22-23$ & $1218 \mathrm{~A}-26 \mathrm{X}-1,46-48$ & 260.15 & 261.28 & 260.72 & 259.61 & 260.72 & 260.16 & 0.55 \\
\hline Revised & B Dorcadospyris copelata & & 38.095 & $1218 \mathrm{~B}-26 \mathrm{X}-4,73-74$ & 1218B-26X-4, 100-101 & 260.05 & 260.91 & 260.48 & 259.51 & 260.37 & 259.94 & 0.43 \\
\hline Revised & T Calocycloma ampulla & & 38.095 & $1218 \mathrm{~B}-26 \mathrm{X}-4,73-74$ & 1218B-26X-4, 100-101 & 260.05 & 260.91 & 260.48 & 259.51 & 260.37 & 259.94 & 0.43 \\
\hline Revised & T Rhopalocanium ornatum & & 38.095 & 1218B-26X-4, 73-74 & $1218 \mathrm{~A}-25 \mathrm{X}-\mathrm{CC}$ & 260.05 & 261.06 & 260.56 & 259.51 & 259.89 & 259.70 & 0.19 \\
\hline Revised & B Botryocella sp. gr. & & 38.095 & 1218B-26X-4, 73-74 & 1218A-25X-CC & 260.05 & 261.06 & 260.56 & 259.51 & 259.89 & 259.70 & 0.19 \\
\hline Revised & T Spongatractus pachystylus & & 38.095 & 1218B-26X-4, 100-101 & 1218B-26X-4, 123-124 & 260.91 & 261.15 & 261.03 & 260.37 & 260.61 & 260.49 & 0.12 \\
\hline Revised & B Thyrsocyrtis (T.) bromia & & 38.095 & 1218B-26X-4, $123-124$ & $1218 \mathrm{~B}-26 \mathrm{X}-4,143-144$ & 261.15 & 261.35 & 261.25 & 260.61 & 260.81 & 260.71 & 0.10 \\
\hline Revised & B Thyrsocyrtis $(P$.$) tetracantha$ & & 38.245 & $1218 \mathrm{~B}-26 \mathrm{X}-5,73-74$ & $1218 \mathrm{~A}-26 \mathrm{X}-2,45-47$ & 262.15 & 262.34 & 262.25 & 261.61 & 261.78 & 261.70 & 0.08 \\
\hline Revised & B Theocyrtis careotuberosa & & 38.245 & $1218 \mathrm{~B}-26 \mathrm{X}-6,24-25$ & 1218B-26X-6, 50-51 & 263.30 & 263.61 & 263.46 & 262.61 & 262.87 & 262.74 & 0.13 \\
\hline Revised & T Zygocircus cimelium & & 38.675 & $1218 \mathrm{~A}-26 \mathrm{X}-3,123-124$ & $1218 \mathrm{~A}-26 \mathrm{X}-3,144-145$ & 264.61 & 264.81 & 264.71 & 264.07 & 264.27 & 264.17 & 0.10 \\
\hline Revised & B Dorcadospyris ombros (upper) & & 38.245 & $1218 B-26 X-5,23-24$ & 1218B-26X-5, 50-51 & 261.65 & 261.91 & 261.78 & 261.11 & 261.37 & 261.24 & 0.13 \\
\hline Revised & B Artophormis dominasinensis & & 38.334 & 1218B-26X-4, 123-124 & 1218B-26X-4, 143-144 & 261.15 & 261.35 & 261.25 & 260.61 & 260.81 & 260.71 & 0.10 \\
\hline Revised & B Tristylospyris triceros & & 38.245 & 1218B-26X-5, 143-144 & $1218 \mathrm{~B}-26 \mathrm{X}-6,24-25$ & 262.94 & 263.30 & 263.12 & 262.31 & 262.61 & 262.46 & 0.15 \\
\hline Revised & T Theocotylissa ficus & RP16 & 38.485 & 1218B-26X-5, 123-124 & $1218 \mathrm{~B}-26 \mathrm{X}-5,143-144$ & 262.70 & 262.94 & 262.82 & 262.11 & 262.31 & 262.21 & 0.10 \\
\hline Revised & T Dorcadospyris anastasis & & 38.485 & 1218B-26X-5, 123-124 & 1218B-26X-5, 143-144 & 262.70 & 262.94 & 262.82 & 262.11 & 262.31 & 262.21 & 0.10 \\
\hline Revised & B Calocyclas turris & & 38.485 & 1218A-26X-4, 73-74 & 1218A-26X-4, $100-101$ & 265.61 & 265.87 & 265.74 & 265.07 & 265.33 & 265.20 & 0.13 \\
\hline Revised & T Podocyrtis (L.) chalara (continuous) & & 38.485 & $1218 \mathrm{~A}-26 \mathrm{X}-5,23-24$ & $1218 \mathrm{~A}-26 \mathrm{X}-5,46-48$ & 266.61 & 266.83 & 266.72 & 266.07 & 266.29 & 266.18 & 0.11 \\
\hline Revised & T Podocyrtis (L.) goetheana (continuous) & & 38.675 & $1218 \mathrm{~A}-26 \mathrm{X}-3,123-124$ & $1218 \mathrm{~A}-26 \mathrm{X}-4,23-24$ & 264.61 & 264.81 & 264.71 & 264.07 & 264.27 & 264.17 & 0.10 \\
\hline Nigrini et al., 2006 & T Dictyophimus craticula & & 38.860 & 1218A-26X-4, 45-47 & $1218 \mathrm{~A}-26 \mathrm{X}-5,46-48$ & 265.32 & 266.83 & 266.08 & 264.78 & 266.29 & 265.54 & 0.76 \\
\hline Revised & B Thyrsocyrtis (P.) krooni & & 39.110 & $1218 \mathrm{~A}-26 \mathrm{X}-6,144-145$ & 1218A-26X-7, 9-11 & 269.24 & 269.41 & 269.33 & 268.65 & 268.79 & 268.72 & 0.07 \\
\hline Revised & T Podocyrtis (P.) apeza & & 39.355 & $1218 \mathrm{~B}-26 \mathrm{X}-5,73-74$ & $1218 \mathrm{~A}-26 \mathrm{X}-2,45-47$ & 262.15 & 262.34 & 262.25 & 261.61 & 261.78 & 261.70 & 0.08 \\
\hline Nigrini et al., 2006 & B Dictyoprora pirum & & 39.355 & 1218A-26X-6, 45-47 & 1218A-26X-7, 9-11 & 268.31 & 269.41 & 268.86 & 267.75 & 268.79 & 268.27 & 0.52 \\
\hline Revised & T Lithochytris vespertilio & & 39.355 & $1218 \mathrm{~A}-26 \mathrm{X}-6,144-145$ & 1218A-26X-7, 9-11 & 269.24 & 269.41 & 269.33 & 268.65 & 268.79 & 268.72 & 0.07 \\
\hline Nigrini et al., 2006 & T Sethochytris triconiscus & & 39.535 & $1218 \mathrm{~A}-26 \mathrm{X}-7,9-11$ & $1218 \mathrm{~A}-26 \mathrm{X}-\mathrm{CC}, 0$ & 269.41 & 270.38 & 269.90 & 268.79 & 269.45 & 269.12 & 0.33 \\
\hline Nigrini et al., 2006 & B Lithocyclia aristotelis gr. & & 39.625 & 1218A-26X-CC & $1218 \mathrm{~A}-27 \mathrm{X}-1,45-47$ & 270.38 & 270.64 & 270.51 & 269.45 & 270.10 & 269.78 & 0.32 \\
\hline Nigrini et al., 2006 & T Dorcadospyris ombros (lower) & & 39.730 & $1218 \mathrm{~A}-27 \mathrm{X}-1,45-47$ & $1218 \mathrm{~A}-27 \mathrm{X}-2,45-57$ & 270.64 & 272.02 & 271.33 & 270.10 & 271.47 & 270.79 & 0.69 \\
\hline Nigrini et al., 2006 & T Podocyrtis (L.) mitra & & 39.730 & $1218 \mathrm{~A}-27 \mathrm{X}-1,45-47$ & $1218 \mathrm{~A}-27 \mathrm{X}-2,45-57$ & 270.64 & 272.02 & 271.33 & 270.10 & 271.47 & 270.79 & 0.69 \\
\hline Nigrini et al., 2006 & B Dorcadospyris anastasis & & 39.930 & $1218 \mathrm{~A}-27 \mathrm{X}-2,45-57$ & $1218 \mathrm{~A}-27 \mathrm{X}-3,52-54$ & 272.02 & 273.55 & 272.79 & 271.47 & 273.01 & 272.24 & 0.77 \\
\hline
\end{tabular}


Table T36 (continued).

\begin{tabular}{|c|c|c|c|c|c|c|c|c|c|c|c|c|}
\hline \multirow[b]{2}{*}{ Source } & \multirow[b]{2}{*}{ Event } & \multirow[b]{2}{*}{ Zone } & \multirow{2}{*}{$\begin{array}{l}\text { Age }^{*} \\
(\mathrm{Ma})\end{array}$} & \multicolumn{2}{|c|}{ Hole, core, section, interval $(\mathrm{cm})$} & \multicolumn{3}{|c|}{$\begin{array}{c}\text { Depth from } \\
\text { Pälike et al. (2005) (rmcd) }\end{array}$} & \multicolumn{3}{|c|}{$\begin{array}{l}\text { Depth adjusted } \\
\text { (corrected } \mathrm{rmcd} \text { ) }\end{array}$} & \multirow{2}{*}{$\begin{array}{c}\text { Error } \\
( \pm) \\
(\mathrm{m})\end{array}$} \\
\hline & & & & Top & Bottom & Top & Bottom & Mean & Top & Bottom & Mean & \\
\hline \multirow{5}{*}{$\begin{array}{l}\text { Nigrini et al., } 2006 \\
\text { Nigrini et al., } 2006 \\
\text { Nigrini et al., } 2006 \\
\text { Nigrini et al., } 2006 \\
\text { Nigrini et al., } 2006\end{array}$} & \multirow{5}{*}{$\begin{array}{l}\text { B Dictyoprora armadillo } \\
\text { B Podocyrtis (L.) goetheana } \\
\text { T Lophocyrtis biaurita } \\
\text { Podocyrtis (L.) mitra > Podocyrtis (L.) chalara } \\
\text { B Podocyrtis (P.) apeza }\end{array}$} & RP16 & 38.860 & $1218 \mathrm{~A}-26 \mathrm{X}-4,45-47$ & $1218 \mathrm{~A}-26 \mathrm{X}-5$ & 265.32 & 266.83 & 26 & 264.78 & 266.29 & 54 & 0.76 \\
\hline & & & 39.930 & $1218 \mathrm{~A}-27 \mathrm{X}-2,45-57$ & $1218 \mathrm{~A}-27 \mathrm{X}-3,52-54$ & 272.02 & 273.55 & 272.79 & 271.47 & 273.01 & 272.24 & 0.77 \\
\hline & & RP15 & 40.345 & $1218 \mathrm{~A}-27 \mathrm{X}-4,40-42$ & $1218 \mathrm{~A}-27 \mathrm{X}-5,45-47$ & 274.93 & 275.98 & 275.46 & 274.39 & 275.44 & 274.92 & 0.53 \\
\hline & & & 41.335 & $1218 \mathrm{~A}-29 \mathrm{X}-1,51-53$ & $1218 \mathrm{~A}-29 \mathrm{X}-3,49-51$ & 288.98 & 291.96 & 290.47 & 288.44 & 291.42 & 289.93 & 1.49 \\
\hline & & RP14 & 41.335 & $1218 \mathrm{~A}-29 \mathrm{X}-1,51-53$ & $1218 \mathrm{~A}-29 \mathrm{X}-3,49-51$ & 288.98 & 291.96 & 290.47 & 288.44 & 291.42 & 289.93 & 1.49 \\
\hline
\end{tabular}

${ }^{*}=$ Nigrini et al. (2006). T = top, B = bottom. 


\begin{tabular}{|c|c|c|c|c|c|c|c|c|c|c|c|c|c|c|}
\hline \multirow[b]{2}{*}{ Source } & \multirow[b]{2}{*}{ Event } & \multirow[b]{2}{*}{ Zone } & \multirow{2}{*}{$\begin{array}{l}\text { Age }^{*} \\
(\mathrm{Ma})\end{array}$} & \multicolumn{2}{|c|}{ Hole, core, section, interval $(\mathrm{cm})$} & \multicolumn{2}{|c|}{$\begin{array}{l}\text { Depth } \\
(\mathrm{mcd})\end{array}$} & \multicolumn{3}{|c|}{$\begin{array}{c}\text { Depth from } \\
\text { Pälike et al. (2005) (rmcd) } \\
\end{array}$} & \multicolumn{3}{|c|}{$\begin{array}{l}\text { Depth Site } 1218 \\
\text { (corrected rmcd) }\end{array}$} & \multirow{2}{*}{$\begin{array}{c}\text { Erro } \\
( \pm) \\
(\mathrm{m})\end{array}$} \\
\hline & & & & Top & Bottom & Top & Bottom & Top & Bottom & Mean & Top & Bottom & Mean & \\
\hline & & \multirow{10}{*}{ RN2 } & & 199- & 199- & & & & & & & & & \\
\hline Nigrini et al., 2006 & T Dorcadospyris praeforcipata & & & $1219 \mathrm{~A}-4 \mathrm{H}-6,46-48$ & $1219 \mathrm{~A}-4 \mathrm{H}-7,46-48$ & 33.84 & 35.34 & 67.41 & 69.89 & 68.65 & 67.49 & 69.86 & 68.67 & 1.19 \\
\hline Nigrini et al., 2006 & B Didymocyrtis violina & & & $1219 \mathrm{~A}-4 \mathrm{H}-6,46-48$ & $1219 \mathrm{~A}-4 \mathrm{H}-7,46-48$ & 33.84 & 35.34 & 67.41 & 69.89 & 68.65 & 67.49 & 69.86 & 68.67 & 1.19 \\
\hline Nigrini et al., 2006 & B Didymocyrtis tubaria (upper) & & & $1219 \mathrm{~A}-4 \mathrm{H}-7,46-48$ & 1219A-4H-CC & 35.34 & 35.75 & 69.89 & 70.61 & 70.25 & 69.86 & 70.52 & 70.19 & 0.33 \\
\hline Nigrini et al., 2006 & B Dorcadospyris simplex & & & 1219A-4H-CC & $1219 \mathrm{~A}-5 \mathrm{H}-1,45-47$ & 35.75 & 37.24 & 70.61 & 73.18 & 71.90 & 70.52 & 73.19 & 71.85 & 1.34 \\
\hline Nigrini et al., 2006 & T Calocycletta (C.) serrata & & & $1219 \mathrm{~A}-5 \mathrm{H}-1,45-47$ & $1219 \mathrm{~A}-5 \mathrm{H}-2,55-57$ & 37.24 & 38.84 & 73.18 & 75.68 & 74.43 & 73.19 & 75.79 & 74.49 & 1.30 \\
\hline Nigrini et al., 2006 & B Stichocorys delmontensis & & & $1219 \mathrm{~A}-5 \mathrm{H}-2,55-57$ & $1219 \mathrm{~A}-5 \mathrm{H}-3,46-48$ & 38.84 & 38.84 & 75.68 & 75.68 & 75.68 & 75.79 & 75.79 & 75.79 & 0.00 \\
\hline Nigrini et al., 2006 & T Lophocyrtis (C.) pegetrum & & & $1219 \mathrm{~A}-5 \mathrm{H}-2,55-57$ & $1219 \mathrm{~A}-5 \mathrm{H}-3,45-47$ & 38.84 & 40.24 & 75.68 & 77.71 & 76.70 & 75.79 & 77.66 & 76.73 & 0.93 \\
\hline Nigrini et al., 2006 & B Carpocanopsis bramlettei & & & $1219 \mathrm{~A}-5 \mathrm{H}-4,45-47$ & 1219A-5H-5. 45-47 & 41.74 & 43.24 & 80.66 & 82.26 & 81.46 & 80.55 & 82.25 & 81.40 & 0.85 \\
\hline Nigrini et al., 2006 & $\mathrm{~T}$ Theocyrtis annosa & & & $1219 \mathrm{~A}-5 \mathrm{H}-4,45-47$ & $1219 \mathrm{~A}-5 \mathrm{H}-5,45-47$ & 41.74 & 43.24 & 80.66 & 82.26 & 81.46 & 80.55 & 82.25 & 81.40 & 0.85 \\
\hline Nigrini et al., 2006 & B Calocycletta (C.) virginis & \multirow{7}{*}{ RN1 } & & $1219 \mathrm{~A}-5 \mathrm{H}-4,45-47$ & $1219 \mathrm{~A}-5 \mathrm{H}-5.45-47$ & 41.74 & 43.24 & 80.66 & 82.26 & 81.46 & 80.55 & 82.25 & 81.40 & 0.85 \\
\hline Nigrini et al., 2006 & B Lophocyrtis (C.) leptetrum & & & $1219 \mathrm{~A}-5 \mathrm{H}-6,45-47$ & $1219 \mathrm{~A}-5 \mathrm{H}-7,45-47$ & 44.74 & 46.24 & 83.33 & 85.52 & 84.43 & 83.57 & 85.41 & 84.49 & 0.92 \\
\hline Nigrini et al., 2006 & T Didymocyrtis tubaria (lower) & & & 1219A-5H-CC, 14-18 & $1219 \mathrm{~A}-6 \mathrm{H}-1,45-47$ & 46.76 & 48.47 & 86.24 & 88.14 & 87.19 & 86.17 & 88.50 & 87.33 & 1.16 \\
\hline Nigrini et al., 2006 & T Eucyrtidium mitodes & & & 1219A-5H-CC, 14-18 & $1219 \mathrm{~A}-6 \mathrm{H}-1,45-47$ & 46.76 & 48.47 & 86.24 & 88.14 & 87.19 & 86.17 & 88.50 & 87.33 & 1.16 \\
\hline Nigrini et al., 2006 & B Calocycletta (C.) serrata & & & $1219 \mathrm{~A}-6 \mathrm{H}-2,45-47$ & $1219 \mathrm{~A}-6 \mathrm{H}-3,45-47$ & 49.97 & 51.47 & 90.32 & 92.54 & 91.43 & 90.36 & 92.45 & 91.41 & 1.04 \\
\hline Nigrini et al., 2006 & B Cyrtocapsella cornuta & & & $1219 \mathrm{~A}-6 \mathrm{H}-2,45-47$ & $1219 \mathrm{~A}-6 \mathrm{H}-3,45-47$ & 49.97 & 51.47 & 90.32 & 92.54 & 91.43 & 90.36 & 92.45 & 91.41 & 1.04 \\
\hline Nigrini et al., 2006 & B Cyrtocapsella tetrapera & & & $1219 \mathrm{~A}-6 \mathrm{H}-3,45-47$ & $1219 \mathrm{~A}-6 \mathrm{H}-4,45-47$ & 51.47 & 52.97 & 92.54 & 95.54 & 94.04 & 92.45 & 94.70 & 93.58 & 1.13 \\
\hline Nigrini et al., 2006 & T Artophormis gracilis & \multirow{13}{*}{ RP22 } & & $1219 \mathrm{~A}-6 \mathrm{H}-3,45-47$ & $1219 \mathrm{~A}-6 \mathrm{H}-4,45-47$ & 51.47 & 52.97 & 92.54 & 95.54 & 94.04 & 92.45 & 94.70 & 93.58 & 1.13 \\
\hline Nigrini et al., 2006 & B Eucyrtidium diaphanes & & & $1219 \mathrm{~A}-6 \mathrm{H}-4,45-47$ & $1219 \mathrm{~A}-6 \mathrm{H}-5,45-47$ & 52.97 & 54.97 & 95.54 & 97.31 & 96.43 & 94.70 & 97.95 & 96.33 & 1.62 \\
\hline Nigrini et al., 2006 & B Didymocyrtis bassanii & & & $1219 \mathrm{~A}-6 \mathrm{H}-5,45-47$ & $1219 \mathrm{~A}-6 \mathrm{H}-6,45-47$ & 54.97 & 55.97 & 97.31 & 99.42 & 98.37 & 97.95 & 99.45 & 98.70 & 0.75 \\
\hline Nigrini et al., 2006 & T Dorcadospyris cyclacantha & & & $1219 \mathrm{~A}-6 \mathrm{H}-5,45-47$ & $1219 \mathrm{~A}-6 \mathrm{H}-6,45-47$ & 54.97 & 55.97 & 97.31 & 99.42 & 98.37 & 97.95 & 99.45 & 98.70 & 0.75 \\
\hline Nigrini et al., 2006 & T Dorcadospyris riedeli (upper) & & & $1219 \mathrm{~A}-6 \mathrm{H}-5,45-47$ & $1219 \mathrm{~A}-6 \mathrm{H}-6,45-47$ & 54.97 & 55.97 & 97.31 & 99.42 & 98.37 & 97.95 & 99.45 & 98.70 & 0.75 \\
\hline Nigrini et al., 2006 & B Dorcadospyris cyclacantha & & & 1219A-6H-7, 18-20 & 1219A-6H-CC, 18-23 & 57.20 & 57.92 & 101.24 & 101.98 & 101.61 & 102.04 & 103.88 & 102.96 & 0.92 \\
\hline Nigrini et al., 2006 & T Dorcadospyris circulus (max) & & & 1219A-6H-7, 18-20 & $1219 \mathrm{~A}-6 \mathrm{H}-\mathrm{CC}, 18-23$ & 57.20 & 57.92 & 101.24 & 101.98 & 101.61 & 102.04 & 103.88 & 102.96 & 0.92 \\
\hline Nigrini et al., 2006 & T Dorcadospyris papilio & & & $1219 \mathrm{~A}-6 \mathrm{H}-7,18-20$ & $1219 \mathrm{~A}-6 \mathrm{H}-\mathrm{CC}, 18-23$ & 57.20 & 57.92 & 101.24 & 101.98 & 101.61 & 102.04 & 103.88 & 102.96 & 0.92 \\
\hline Nigrini et al., 2006 & T Liriospyris longicornuta & & & 1219A-7H-6, 45-47 & 1219A-7H-CC, 24-29 & 63.36 & 65.01 & 114.03 & 118.01 & 116.02 & 114.10 & 117.98 & 116.04 & 1.94 \\
\hline Nigrini et al., 2006 & B Carpocanopsis cingulata & & & 1219A-7H-6, 45-47 & 1219A-7H-CC, 24-29 & 63.36 & 65.01 & 114.03 & 118.01 & 116.02 & 114.10 & 117.98 & 116.04 & 1.94 \\
\hline Nigrini et al., 2006 & T Acrocubus octopylus (lower) & & & 1219A-7H-6, 45-47 & 1219A-7H-CC, 24-29 & 63.04 & 65.01 & 114.03 & 118.01 & 116.02 & 113.01 & 117.98 & 115.49 & 2.49 \\
\hline Nigrini et al., 2006 & T Lychnocanoma apodora & & & 1219A-7H-6, 45-47 & 1219A-7H-CC, 24-29 & 63.36 & 65.01 & 114.03 & 118.01 & 116.02 & 114.10 & 117.98 & 116.04 & 1.94 \\
\hline Nigrini et al., 2006 & B Lychnocanoma elongata & & & $1219 \mathrm{~A}-8 \mathrm{H}-1,43-45$ & 1219B-6H-3, 46-48 & 66.48 & 66.80 & 120.31 & 120.88 & 120.60 & 120.33 & 120.81 & 120.57 & 0.24 \\
\hline Nigrini et al., 2006 & B Acrocubus octopylus (lower) & \multirow{19}{*}{ RP21 } & & 1219A-8H-1, 43-45 & 1219B-6H-3, 46-48 & 66.48 & 66.80 & 120.31 & 120.88 & 120.60 & 120.33 & 120.81 & 120.57 & 0.24 \\
\hline Nigrini et al., 2006 & B Didymocyrtis tubaria (lower) & & & $1219 \mathrm{~A}-8 \mathrm{H}-2,45-47$ & $1219 \mathrm{~A}-8 \mathrm{H}-4,45-47$ & 68.00 & 71.00 & 123.01 & 128.45 & 125.73 & 122.75 & 128.46 & 125.61 & 2.85 \\
\hline Nigrini et al., 2006 & B Calocycletta (C.) robusta & & & $1219 \mathrm{~A}-8 \mathrm{H}-2,45-47$ & $1219 \mathrm{~A}-8 \mathrm{H}-4,45-47$ & 68.00 & 71.00 & 123.01 & 128.45 & 125.73 & 122.75 & 128.46 & 125.61 & 2.85 \\
\hline Nigrini et al., 2006 & B Dorcadospyris praeforcipata & & & $1219 \mathrm{~A}-8 \mathrm{H}-2,45-47$ & $1219 \mathrm{~A}-8 \mathrm{H}-4,45-47$ & 68.00 & 71.00 & 123.01 & 128.45 & 125.73 & 122.75 & 128.46 & 125.61 & 2.85 \\
\hline Nigrini et al., 2006 & B Liriospyris longicornuta & & & $1219 \mathrm{~A}-8 \mathrm{H}-2,45-47$ & $1219 \mathrm{~A}-8 \mathrm{H}-4,45-47$ & 68.00 & 71.00 & 123.01 & 128.45 & 125.73 & 122.75 & 128.46 & 125.61 & 2.85 \\
\hline Nigrini et al., 2006 & B Dorcadospyris scambos & & & $1219 \mathrm{~A}-8 \mathrm{H}-4,45-47$ & $1219 \mathrm{~A}-8 \mathrm{H}-6,45-47$ & 71.00 & 74.00 & 128.45 & 132.30 & 130.38 & 128.46 & 132.33 & 130.40 & 1.94 \\
\hline Nigrini et al., 2006 & T Theocorys puriri & & & $1219 \mathrm{~A}-8 \mathrm{H}-4,45-47$ & $1219 \mathrm{~A}-8 \mathrm{H}-6,45-47$ & 71.00 & 74.00 & 128.45 & 132.30 & 130.38 & 128.46 & 132.33 & 130.40 & 1.94 \\
\hline Nigrini et al., 2006 & B Acrobotrys disolenia & & & $1219 \mathrm{~A}-8 \mathrm{H}-4,45-47$ & $1219 \mathrm{~A}-8 \mathrm{H}-6,45-47$ & 71.00 & 74.00 & 128.45 & 132.30 & 130.38 & 128.46 & 132.33 & 130.40 & 1.94 \\
\hline Nigrini et al., 2006 & B Dorcadospyris papilio & & & $1219 \mathrm{~A}-8 \mathrm{H}-6,45-47$ & 1219A-8H-CC, 20-24 & 74.00 & 76.10 & 132.30 & 134.25 & 133.28 & 132.33 & 134.23 & 133.28 & 0.95 \\
\hline Nigrini et al., 2006 & B Lychnocanoma apodora & & & $1219 \mathrm{~A}-8 \mathrm{H}-6,45-47$ & 1219A-8H-CC, 20-24 & 74.00 & 76.10 & 132.30 & 134.25 & 133.28 & 132.33 & 134.23 & 133.28 & 0.95 \\
\hline Nigrini et al., 2006 & T Dorcadospyris circulus (continuous) & & & $1219 \mathrm{~A}-9 \mathrm{H}-5,46-48$ & 1219A-9H-CC, 10-14 & 86.33 & 89.72 & 143.32 & 148.56 & 145.94 & 145.28 & 148.55 & 146.92 & 1.63 \\
\hline Nigrini et al., 2006 & B Dorcadospyris riedeli (upper) & & & $1219 \mathrm{~A}-9 \mathrm{H}-5,46-48$ & 1219A-9H-CC, 10-14 & 86.33 & 89.72 & 143.32 & 148.56 & 145.94 & 145.28 & 148.55 & 146.92 & 1.63 \\
\hline Nigrini et al., 2006 & T Eucyrtidium plesiodiaphanes & & & $1219 \mathrm{~A}-9 \mathrm{H}-5,46-48$ & 1219A-9H-CC, 10-14 & 86.33 & 89.72 & 143.32 & 148.56 & 145.94 & 145.28 & 148.55 & 146.92 & 1.63 \\
\hline Nigrini et al., 2006 & T Lithocyclia angusta (continuous) & & & $1219 \mathrm{~A}-10 \mathrm{H}-4,45-47$ & $1219 \mathrm{~A}-10 \mathrm{H}-\mathrm{CC}, 27-32$ & 95.92 & 100.54 & 155.96 & 160.33 & 158.15 & 155.19 & 159.90 & 157.54 & 2.35 \\
\hline Nigrini et al., 2006 & T Theocyrtis setanios & & & $1219 \mathrm{~A}-11 \mathrm{H}-2,45-47$ & 1219A-11H-4, 45-47 & 106.86 & 109.86 & 163.88 & 166.63 & 165.26 & 166.41 & 169.02 & 167.72 & 1.30 \\
\hline Nigrini et al., 2006 & $\mathrm{~T}$ Theocyrtis perysinos & & & 1219A-11H-7, 45-47 & 1219A-11H-CC, 11-17 & 114.36 & 114.54 & 170.66 & 170.85 & 170.76 & 173.68 & 173.90 & 173.79 & 0.11 \\
\hline Nigrini et al., 2006 & T Tristylospyris triceros & & & $1219 \mathrm{~A}-12 \mathrm{H}-4,45-47$ & 1219A-12H-5, 45-47 & 121.08 & 122.58 & 177.46 & 178.98 & 178.22 & 180.87 & 182.46 & 181.66 & 0.80 \\
\hline Nigrini et al., 2006 & B Theocyrtis annosa & & & 1219A-12H-5, 45-47 & $1219 \mathrm{~A}-12 \mathrm{H}-6,45-47$ & 122.58 & 124.08 & 178.98 & 180.60 & 179.79 & 182.46 & 184.06 & 183.26 & 0.80 \\
\hline Nigrini et al., 2006 & $\begin{array}{l}\text { Tristylospyris triceros > Dorcadospyris } \\
\text { ateuchus }\end{array}$ & & & $1219 \mathrm{~A}-12 \mathrm{H}-6,45-47$ & $1219 \mathrm{~A}-12 \mathrm{H}-7,45-47$ & 124.08 & 125.58 & 180.60 & 182.26 & 181.43 & 184.06 & 185.66 & 184.86 & 0.80 \\
\hline
\end{tabular}


Table T37 (continued). (Continued on next page.)

\begin{tabular}{|c|c|c|c|c|c|c|c|c|c|c|c|c|c|c|}
\hline \multirow[b]{2}{*}{ Source } & \multirow[b]{2}{*}{ Event } & \multirow[b]{2}{*}{ Zone } & \multirow{2}{*}{$\begin{array}{l}\text { Age* }^{*} \\
(\mathrm{Ma})\end{array}$} & \multicolumn{2}{|c|}{ Hole, core, section, interval $(\mathrm{cm})$} & \multicolumn{2}{|c|}{$\begin{array}{l}\text { Depth } \\
\text { (mcd) }\end{array}$} & \multicolumn{3}{|c|}{$\begin{array}{c}\text { Depth from } \\
\text { Pälike et al. (2005) (rmcd) }\end{array}$} & \multicolumn{3}{|c|}{$\begin{array}{l}\text { Depth Site } 1218 \\
\text { (corrected rmcd) }\end{array}$} & \multirow{2}{*}{$\begin{array}{c}\text { Error } \\
( \pm) \\
(\mathrm{m})\end{array}$} \\
\hline & & & & Top & Bottom & Top & Bottom & Top & Bottom & Mean & Top & Bottom & Mean & \\
\hline Nigrini et al., 2006 & B Eucyrtidium mitodes & \multirow{37}{*}{ RP20 } & & $1219 \mathrm{~A}-12 \mathrm{H}-\mathrm{CC}, 24-30$ & 1219A-13H-5, 45-47 & 126.21 & 136.10 & 182.96 & 193.91 & 188.44 & 186.36 & 197.39 & 191.87 & 5.52 \\
\hline Nigrini et al., 2006 & B Theocyrtis perysinos & & & $1219 \mathrm{~A}-13 \mathrm{H}-5,45-47$ & $1219 \mathrm{~A}-13 \mathrm{H}-\mathrm{CC}, 7-12$ & 136.10 & 139.67 & 193.91 & 198.15 & 196.03 & 197.39 & 200.73 & 199.06 & 1.67 \\
\hline Nigrini et al., 2006 & B Dorcadospyris ateuchus & & & $1219 \mathrm{~A}-12 \mathrm{H}-7,45-47$ & $1219 \mathrm{~A}-12 \mathrm{H}-\mathrm{CC}, 24-30$ & 125.58 & 126.21 & 182.26 & 182.96 & 182.61 & 185.66 & 186.36 & 186.01 & 0.35 \\
\hline Nigrini et al., 2006 & B Theocyrtis setanios & & & $1219 \mathrm{~A}-12 \mathrm{H}-\mathrm{CC}, 24-30$ & $1219 \mathrm{~A}-13 \mathrm{H}-5,45-47$ & 126.21 & 136.10 & 182.96 & 193.91 & 188.44 & 186.36 & 197.39 & 191.87 & 5.52 \\
\hline Nigrini et al., 2006 & T Lophocyrtis (C.) milowi & & & $1219 \mathrm{~A}-12 \mathrm{H}-\mathrm{CC}, 24-30$ & $1219 \mathrm{~A}-13 \mathrm{H}-3,45-47$ & 126.21 & 133.10 & 182.96 & 190.59 & 186.78 & 186.36 & 194.04 & 190.20 & 3.84 \\
\hline Nigrini et al., 2006 & B Theocorys puriri & & & 1219A-13H-5, 45-47 & $1219 \mathrm{~A}-13 \mathrm{H}-\mathrm{CC}, 7-12$ & 136.10 & 139.67 & 193.91 & 198.15 & 196.03 & 197.39 & 200.73 & 199.06 & 1.67 \\
\hline Nigrini et al., 2006 & B Didymocyrtis prismatica & & & 1219A-13H-5, 45-47 & 1219A-13H-CC, 7-12 & 136.10 & 139.67 & 193.91 & 198.15 & 196.03 & 197.39 & 200.73 & 199.06 & 1.67 \\
\hline Nigrini et al., 2006 & B Dorcadospyris circulus & & & 1219A-13H-CC, 7-12 & $1219 \mathrm{~A}-14 \mathrm{H}-2,45-47$ & 139.67 & 143.40 & 198.15 & 201.17 & 199.66 & 200.73 & 204.74 & 202.73 & 2.01 \\
\hline Nigrini et al., 2006 & T Lophocyrtis (S.) oberhaensliae & & & 1219A-13H-CC, 7-12 & $1219 \mathrm{~A}-14 \mathrm{H}-2,45-47$ & 139.67 & 143.40 & 198.15 & 201.17 & 199.66 & 200.73 & 204.74 & 202.73 & 2.01 \\
\hline Nigrini et al., 2006 & $\mathrm{~T}$ Theocyrtis tuberosa & & & $1219 \mathrm{~A}-14 \mathrm{H}-2,45-47$ & $1219 \mathrm{~A}-14 \mathrm{H}-4,45-47$ & 143.40 & 146.40 & 201.17 & 204.84 & 203.01 & 204.74 & 208.28 & 206.51 & 1.77 \\
\hline Nigrini et al., 2006 & T Lithocyclia crux & & & $1219 \mathrm{~A}-14 \mathrm{H}-2,45-47$ & $1219 \mathrm{~A}-14 \mathrm{H}-4,45-47$ & 143.40 & 146.40 & 201.17 & 204.84 & 203.01 & 204.74 & 208.28 & 206.51 & 1.77 \\
\hline Nigrini et al., 2006 & T Dorcadospyris spinosa & & & $1219 \mathrm{~A}-14 \mathrm{H}-2,45-47$ & $1219 \mathrm{~A}-14 \mathrm{H}-4,45-47$ & 143.40 & 146.40 & 201.17 & 204.84 & 203.01 & 204.74 & 208.28 & 206.51 & 1.77 \\
\hline Nigrini et al., 2006 & T Dorcadospyris quadripes & & & $1219 \mathrm{~A}-14 \mathrm{H}-2,45-47$ & $1219 \mathrm{~A}-14 \mathrm{H}-4,45-47$ & 143.40 & 146.40 & 201.17 & 204.84 & 203.01 & 204.74 & 208.28 & 206.51 & 1.77 \\
\hline Nigrini et al., 2006 & B Eucyrtidium plesiodiaphanes & & & $1219 \mathrm{~A}-14 \mathrm{H}-2,45-47$ & $1219 \mathrm{~A}-14 \mathrm{H}-4,45-47$ & 143.40 & 146.40 & 201.17 & 204.84 & 203.01 & 204.74 & 208.28 & 206.51 & 1.77 \\
\hline Nigrini et al., 2006 & B Lophocyrtis (C.) pegetrum & & & $1219 \mathrm{~A}-14 \mathrm{H}-6,45-47$ & $1219 \mathrm{~A}-14 \mathrm{H}-\mathrm{CC}, 14-18$ & 149.40 & 151.42 & 208.41 & 210.95 & 209.68 & 211.91 & 214.11 & 213.01 & 1.10 \\
\hline Nigrini et al., 2006 & T Dorcadospyris riedeli (lower) & & & $1219 \mathrm{~A}-14 \mathrm{H}-\mathrm{CC}, 14-18$ & $1219 \mathrm{~A}-15 \mathrm{H}-2,46-48$ & 151.42 & 154.31 & 210.95 & 214.43 & 212.69 & 214.11 & 216.65 & 215.38 & 1.27 \\
\hline Nigrini et al., 2006 & B Spirocyrtis subtilis & & & $1219 \mathrm{~A}-14 \mathrm{H}-\mathrm{CC}, 14-18$ & $1219 \mathrm{~A}-15 \mathrm{H}-2,46-48$ & 151.42 & 154.31 & 210.95 & 214.43 & 212.69 & 214.11 & 216.65 & 215.38 & 1.27 \\
\hline Nigrini et al., 2006 & T Dorcadospyris pseudopapilc & & & $1219 \mathrm{~A}-15 \mathrm{H}-4,46-48$ & $1219 \mathrm{~A}-15 \mathrm{H}-6,46-48$ & 157.31 & 160.31 & 218.00 & 223.33 & 220.67 & 219.24 & 223.66 & 221.45 & 2.21 \\
\hline Nigrini et al., 2006 & B Dorcadospyris spinosa & & & $1219 \mathrm{~A}-15 \mathrm{H}-4,46-48$ & $1219 \mathrm{~A}-15 \mathrm{H}-6,46-48$ & 157.31 & 160.31 & 218.00 & 223.33 & 220.67 & 219.24 & 223.66 & 221.45 & 2.21 \\
\hline Nigrini et al., 2006 & B Dorcadospyris riedeli (lower) & & & $1219 \mathrm{~A}-15 \mathrm{H}-\mathrm{CC}$ & $1219 \mathrm{~A}-16 \mathrm{H}-2,45-47$ & 162.06 & 165.34 & 223.62 & 227.17 & 225.40 & 226.01 & 228.93 & 227.47 & 1.46 \\
\hline Nigrini et al., 2006 & T Centrobotrys gravida & & & 1219A-15H-CC & 1219A-16H-2, 45-47 & 162.06 & 165.34 & 223.62 & 227.17 & 225.40 & 226.01 & 228.93 & 227.47 & 1.46 \\
\hline Nigrini et al., 2006 & B Centrobotrys petrushevskayae & & & $1219 \mathrm{~A}-15 \mathrm{H}-6,46-48$ & $1219 \mathrm{~A}-15 \mathrm{H}-\mathrm{CC}$ & 160.31 & 162.06 & 223.33 & 223.62 & 223.48 & 223.66 & 226.01 & 224.83 & 1.17 \\
\hline Nigrini et al., 2006 & B Dorcadospyris quadripes & & & 1219A-16H-3, 45-47 & $1219 \mathrm{~A}-16 \mathrm{H}-4,45-47$ & 166.84 & 168.34 & 228.60 & 229.47 & 229.04 & 230.30 & 231.79 & 231.04 & 0.74 \\
\hline Nigrini et al., 2006 & B Lychnodictyum audax & & & $1219 \mathrm{~A}-16 \mathrm{H}-2,45-47$ & 1219A-16H-3, 45-47 & 165.34 & 166.84 & 227.17 & 228.60 & 227.89 & 228.93 & 230.30 & 229.62 & 0.68 \\
\hline Nigrini et al., 2006 & T Theocyrtis careotuberosa & & & $1219 \mathrm{~A}-16 \mathrm{H}-3,45-47$ & $1219 \mathrm{~A}-16 \mathrm{H}-4,45-47$ & 166.84 & 168.34 & 228.60 & 229.47 & 229.04 & 230.30 & 231.79 & 231.04 & 0.74 \\
\hline Nigrini et al., 2006 & B Theocyrtis tuberosa & & & 1219A-16H-5, 45-47 & $1219 \mathrm{~A}-16 \mathrm{H}-6,45-47$ & 169.89 & 171.39 & 230.47 & 231.91 & 231.19 & 233.30 & 236.15 & 234.73 & 1.43 \\
\hline Nigrini et al., 2006 & B Dorcadospyris pseudopapilio & & & $1219 \mathrm{~A}-16 \mathrm{H}-6,45-47$ & $1219 \mathrm{~A}-16 \mathrm{H}-7,45-47$ & 171.39 & 172.89 & 231.91 & 235.53 & 233.72 & 236.15 & 238.69 & 237.42 & 1.27 \\
\hline Nigrini et al., 2006 & B Lithocyclia crux & & & $1219 \mathrm{~A}-16 \mathrm{H}-6,45-47$ & $1219 \mathrm{~A}-16 \mathrm{H}-7,45-47$ & 171.39 & 172.89 & 231.91 & 235.53 & 233.72 & 236.15 & 238.69 & 237.42 & 1.27 \\
\hline Nigrini et al., 2006 & B Centrobotrys gravida & & & 1219A-16H-6, 45-47 & 1219A-16H-7, 45-47 & 171.39 & 172.89 & 231.91 & 235.53 & 233.72 & 236.15 & 238.69 & 237.42 & 1.27 \\
\hline Nigrini et al., 2006 & T Dictyoprora mongolfieri & & & $1219 \mathrm{~A}-16 \mathrm{H}-6,45-47$ & 1219A-16H-7, 45-47 & 171.39 & 172.89 & 231.91 & 235.53 & 233.72 & 236.15 & 238.69 & 237.42 & 1.27 \\
\hline Nigrini et al., 2006 & T Thyrsocyrtis $(P$.$) triacantha (max)$ & & & $1219 \mathrm{~A}-17 \mathrm{H}-2,20-22$ & $1219 \mathrm{~A}-17 \mathrm{H}-3,45-47$ & 175.53 & 176.55 & 239.04 & 240.60 & 239.82 & 241.94 & 242.95 & 242.44 & 0.50 \\
\hline Nigrini et al., 2006 & T Lithocyclia ocellus gr. & & & 1219A-17H-2, 20-22 & 1219A-17H-3, 45-47 & 175.53 & 176.55 & 239.04 & 240.60 & 239.82 & 241.94 & 242.95 & 242.44 & 0.50 \\
\hline Nigrini et al., 2006 & T Lithocyclia aristotelis gr. & & & 1219A-17H-2, 20-22 & 1219A-17H-3, 45-47 & 175.53 & 176.55 & 239.04 & 240.60 & 239.82 & 241.94 & 242.95 & 242.44 & 0.50 \\
\hline Nigrini et al., 2006 & B Lophocyrtis (S.) oberhaensliae & & & $1219 \mathrm{~A}-17 \mathrm{H}-2,20-22$ & $1219 \mathrm{~A}-17 \mathrm{H}-3,45-47$ & 175.53 & 176.55 & 239.04 & 240.60 & 239.82 & 241.94 & 242.95 & 242.44 & 0.50 \\
\hline Nigrini et al., 2006 & T Cryptocarpium ornatum & & & 1219A-17H-2, 20-22 & 1219A-17H-3, 45-47 & 175.53 & 176.55 & 239.04 & 240.60 & 239.82 & 241.94 & 242.95 & 242.44 & 0.50 \\
\hline Nigrini et al., 2006 & T Calocyclas hispida & & & $1219 \mathrm{~A}-17 \mathrm{H}-2,20-22$ & 1219A-17H-3, 45-47 & 175.53 & 176.55 & 239.04 & 240.60 & 239.82 & 241.94 & 242.95 & 242.44 & 0.50 \\
\hline Nigrini et al., 2006 & $\begin{array}{l}\text { Lithocyclia aristotelis gr. > Lithocyclia } \\
\text { angusta }\end{array}$ & & & $1219 \mathrm{~A}-17 \mathrm{H}-2,20-22$ & 1219A-17H-3, 45-47 & 175.53 & 176.55 & 239.04 & 240.60 & 239.82 & 241.94 & 242.95 & 242.44 & 0.50 \\
\hline Nigrini et al., 2006 & T Calocycletta $(\mathrm{C}$.$) anekathen$ & \multirow{12}{*}{ RP19 } & & 1219A-17H-2, 20-22 & $1219 \mathrm{~A}-17 \mathrm{H}-3,45-47$ & 175.53 & 176.55 & 239.04 & 240.60 & 239.82 & 241.94 & 242.95 & 242.44 & 0.50 \\
\hline Nigrini et al., 2006 & T Lophocyrtis (L.) jacchia & & & 1219A-17H-2, 20-22 & 1219A-17H-3, 45-47 & 175.53 & 176.55 & 239.04 & 240.60 & 239.82 & 241.94 & 242.95 & 242.44 & 0.50 \\
\hline Nigrini et al., 2006 & T Dictyoprora pirum & & & 1219A-17H-2, 20-22 & $1219 \mathrm{~A}-17 \mathrm{H}-3,45-47$ & 175.53 & 176.55 & 239.04 & 240.60 & 239.82 & 241.94 & 242.95 & 242.44 & 0.50 \\
\hline Nigrini et al., 2006 & T Dictyoprora armadillo & & & 1219A-17H-2, 20-22 & $1219 \mathrm{~A}-17 \mathrm{H}-3,45-47$ & 175.53 & 176.55 & 239.04 & 240.60 & 239.82 & 241.94 & 242.95 & 242.44 & 0.50 \\
\hline Nigrini et al., 2006 & T Lychnocanoma babylonis & & & 1219A-17H-3, 45-47 & $1219 \mathrm{~A}-17 \mathrm{H}-4,45-47$ & 176.55 & 177.34 & 240.60 & 241.06 & 240.83 & 242.95 & 243.72 & 243.33 & 0.39 \\
\hline Nigrini et al., 2006 & T Lophocyrtis (C.) hadra & & & $1219 \mathrm{~A}-17 \mathrm{H}-3,45-47$ & $1219 \mathrm{~A}-17 \mathrm{H}-4,45-47$ & 176.55 & 177.34 & 240.60 & 241.06 & 240.83 & 242.95 & 243.72 & 243.33 & 0.39 \\
\hline Nigrini et al., 2006 & T Lychnocanoma amphitrite & & & 1219A-17H-3, 45-47 & 1219A-17H-4, 45-47 & 176.55 & 177.34 & 240.60 & 241.06 & 240.83 & 242.95 & 243.72 & 243.33 & 0.39 \\
\hline Nigrini et al., 2006 & T Lophocyrtis (L.) exitelus & & & 1219A-17H-3, 45-47 & 1219A-17H-4, 45-47 & 176.55 & 177.34 & 240.60 & 241.06 & 240.83 & 242.95 & 243.72 & 243.33 & 0.39 \\
\hline Nigrini et al., 2006 & T Dorcadospyris copelata & & & $1219 \mathrm{~A}-17 \mathrm{H}-4,45-47$ & 1219A-17H-5, 32-34 & 177.34 & 179.01 & 241.06 & 242.92 & 241.99 & 243.72 & 245.38 & 244.55 & 0.83 \\
\hline Nigrini et al., 2006 & T Zealithapium mitra & & & 1219A-17H-4, 45-47 & 1219A-17H-5, 32-34 & 177.34 & 179.01 & 241.06 & 242.92 & 241.99 & 243.72 & 245.38 & 244.55 & 0.83 \\
\hline Nigrini et al., 2006 & T Artophormis barbadensis & & & 1219A-17H-4, 45-47 & 1219A-17H-5, 32-34 & 177.34 & 179.01 & 241.06 & 242.92 & 241.99 & 243.72 & 245.38 & 244.55 & 0.83 \\
\hline Nigrini et al., 2006 & B Lithocyclia angusta & & & $1219 \mathrm{~A}-17 \mathrm{H}-4,45-47$ & $1219 \mathrm{~A}-17 \mathrm{H}-5,32-34$ & 177.34 & 179.01 & 241.06 & 242.92 & 241.99 & 243.72 & 245.38 & 244.55 & 0.83 \\
\hline
\end{tabular}




\begin{tabular}{|c|c|c|c|c|c|c|c|c|c|c|c|c|c|c|}
\hline \multirow[b]{2}{*}{ Source } & \multirow[b]{2}{*}{ Event } & \multirow[b]{2}{*}{ Zone } & \multirow{2}{*}{$\begin{array}{l}\text { Age* }^{*} \\
(\mathrm{Ma})\end{array}$} & \multicolumn{2}{|c|}{ Hole, core, section, interval $(\mathrm{cm})$} & \multicolumn{2}{|c|}{$\begin{array}{l}\text { Depth } \\
\text { (mcd) }\end{array}$} & \multicolumn{3}{|c|}{$\begin{array}{l}\text { Depth from } \\
\text { Pälike et al. (2005) (rmcd) }\end{array}$} & \multicolumn{3}{|c|}{$\begin{array}{l}\text { Depth Site } 1218 \\
\text { (corrected rmcd) }\end{array}$} & \multirow{2}{*}{$\begin{array}{c}\text { Error } \\
( \pm) \\
(\mathrm{m})\end{array}$} \\
\hline & & & & Top & Bottom & Top & Bottom & Top & Bottom & Mean & Top & Bottom & Mean & \\
\hline Nigrini et al., 2006 & B Lophocyrtis (C.) milowi & \multirow{9}{*}{ RP19 } & & 1219A-17H-4, 45-47 & 1219A-17H-5, 32-34 & 177.34 & 179.01 & 241.06 & 242.92 & 241.99 & 243.72 & 245.38 & 244.55 & 0.83 \\
\hline Nigrini et al., 2006 & $\begin{array}{l}\text { B Pteropilium sp.aff. Pterocanium } \\
\text { contiguum }\end{array}$ & & & 1219A-17H-5, 32-34 & $1219 \mathrm{~A}-17 \mathrm{H}-6,45-47$ & 179.01 & 180.65 & 242.92 & 244.68 & 243.80 & 245.38 & 247.18 & 246.28 & 0.90 \\
\hline Nigrini et al., 2006 & T Dorcadospyris ombros (upper) & & & $1219 \mathrm{~A}-17 \mathrm{H}-6,45-47$ & 1219A-17H-7, 45-47 & 180.65 & 182.15 & 244.68 & 246.28 & 245.48 & 247.18 & 248.90 & 248.04 & 0.86 \\
\hline Nigrini et al., 2006 & B Lophocyrtis (L.) exitelus & & & 1219A-17H-6, 45-47 & 1219A-17H-7, 45-47 & 180.65 & 182.15 & 244.68 & 246.28 & 245.48 & 247.18 & 248.90 & 248.04 & 0.86 \\
\hline Nigrini et al., 2006 & T Calocyclas bandyca & & & $1219 \mathrm{~A}-17 \mathrm{H}-6,45-47$ & 1219A-17H-7, 45-47 & 180.65 & 182.15 & 244.68 & 246.28 & 245.48 & 247.18 & 248.90 & 248.04 & 0.86 \\
\hline Nigrini et al., 2006 & T Calocyclas turris & & & 1219A-17H-7, 45-47 & 1219A-17H-CC, $12-17$ & 182.15 & 183.22 & 246.28 & 247.38 & 246.83 & 248.90 & 249.70 & 249.30 & 0.40 \\
\hline Nigrini et al., 2006 & T Cryptocarpium azyx & & & 1219A-17H-CC, $12-17$ & $1219 \mathrm{~A}-18 \mathrm{H}-1,45-47$ & 183.22 & 184.78 & 247.38 & 248.62 & 248.00 & 249.70 & 250.74 & 250.22 & 0.52 \\
\hline Nigrini et al., 2006 & T Thyrsocyrtis (T.) bromia (continuous) & & & 1219A-17H-CC, $12-17$ & $1219 \mathrm{~A}-18 \mathrm{H}-1,45-47$ & 183.22 & 184.78 & 247.38 & 248.62 & 248.00 & 249.70 & 250.74 & 250.22 & 0.52 \\
\hline Nigrini et al., 2006 & $\begin{array}{l}\text { T Thyrsocyrtis }(P .) \text { tetracantha } \\
\text { (continuous) }\end{array}$ & & & $1219 \mathrm{~A}-17 \mathrm{H}-\mathrm{CC}, 12-17$ & $1219 \mathrm{~A}-18 \mathrm{H}-1,45-47$ & 183.22 & 184.78 & 247.38 & 248.62 & 248.00 & 249.70 & 250.74 & 250.22 & 0.52 \\
\hline Nigrini et al., 2006 & B Lophocyrtis (C.) hadra & \multirow{13}{*}{ RP18 } & & $1219 \mathrm{~A}-17 \mathrm{H}-\mathrm{CC}, 12-17$ & 1219A-18H-1, 45-47 & 183.22 & 184.78 & 247.38 & 248.62 & 248.00 & 249.70 & 250.74 & 250.22 & 0.52 \\
\hline Nigrini et al., 2006 & $\mathrm{~T}$ Thyrsocyrtis $(P$.$) lochites (continuous)$ & & & $1219 \mathrm{~A}-17 \mathrm{H}-\mathrm{CC}, 12-17$ & $1219 \mathrm{~A}-18 \mathrm{H}-1,45-47$ & 183.22 & 184.78 & 247.38 & 248.62 & 248.00 & 249.70 & 250.74 & 250.22 & 0.52 \\
\hline Nigrini et al., 2006 & T Thyrsocyrtis (T.) rhizodon & & & 1219A-17H-CC, $12-17$ & $1219 \mathrm{~A}-18 \mathrm{H}-1,45-47$ & 183.22 & 184.78 & 247.38 & 248.62 & 248.00 & 249.70 & 250.74 & 250.22 & 0.52 \\
\hline Nigrini et al., 2006 & T Thyrsocyrtis $(P$.$) krooni$ & & & $1219 \mathrm{~A}-17 \mathrm{H}-\mathrm{CC}, 12-17$ & $1219 \mathrm{~A}-18 \mathrm{H}-1,45-47$ & 183.22 & 184.78 & 247.38 & 248.62 & 248.00 & 249.70 & 250.74 & 250.22 & 0.52 \\
\hline Nigrini et al., 2006 & $\begin{array}{l}\text { T Thyrsocyrtis }(P .) \text { triacantha } \\
\text { (continuous) }\end{array}$ & & & $1219 \mathrm{~A}-17 \mathrm{H}-\mathrm{CC}, 12-17$ & 1219A-18H-1, 45-47 & 183.22 & 184.78 & 247.38 & 248.62 & 248.00 & 249.70 & 250.74 & 250.22 & 0.52 \\
\hline Nigrini et al., 2006 & T Artophormis dominasinensis & & & $1219 \mathrm{~A}-17 \mathrm{H}-\mathrm{CC}, 12-17$ & $1219 \mathrm{~A}-18 \mathrm{H}-1,45-47$ & 183.22 & 184.78 & 247.38 & 248.62 & 248.00 & 249.70 & 250.74 & 250.22 & 0.52 \\
\hline Nigrini et al., 2006 & T Eusyringium fistuligerum & & & 1219A-18H-1, 45-47 & $1219 \mathrm{~A}-18 \mathrm{H}-2,45-47$ & 184.78 & 186.28 & 248.62 & 249.19 & 248.91 & 250.74 & 251.73 & 251.24 & 0.50 \\
\hline Nigrini et al., 2006 & B Artophormis gracilis & & & $1219 \mathrm{~A}-18 \mathrm{H}-3,45-47$ & 1219A-18H-4, 45-47 & 187.78 & 189.28 & 250.69 & 252.23 & 251.46 & 252.78 & 254.02 & 253.40 & 0.62 \\
\hline Nigrini et al., 2006 & T Lychnocanoma turgidum & & & 1219A-18H-4, 45-47 & $1219 \mathrm{~A}-18 \mathrm{H}-5,45-47$ & 189.28 & 190.78 & 252.23 & 253.62 & 252.93 & 254.02 & 255.33 & 254.68 & 0.65 \\
\hline Nigrini et al., 2006 & B Calocycletta (C.) anekathen & & & 1219A-18H-5, 45-47 & $1219 \mathrm{~A}-18 \mathrm{H}-6,45-47$ & 190.78 & 192.28 & 253.62 & 255.10 & 254.36 & 255.33 & 256.63 & 255.98 & 0.65 \\
\hline Nigrini et al., 2006 & T Theocyrtis perpumila & & & 1219A-18H-CC, 17-22 & 1219A-19H-1, 45-47 & 194.30 & 195.25 & 256.40 & 257.03 & 256.72 & 258.32 & 259.01 & 258.67 & 0.34 \\
\hline Nigrini et al., 2006 & B Lychnocanoma amphitrite & & & $1219 \mathrm{~A}-18 \mathrm{H}-5,45-47$ & $1219 \mathrm{~A}-18 \mathrm{H}-6,45-47$ & 190.78 & 192.28 & 253.62 & 255.10 & 254.36 & 255.33 & 256.63 & 255.98 & 0.65 \\
\hline Nigrini et al., 2006 & B Calocyclas bandyca & & & 1219A-18H-5, 45-47 & $1219 \mathrm{~A}-18 \mathrm{H}-6,45-47$ & 190.78 & 192.28 & 253.62 & 255.10 & 254.36 & 255.33 & 256.63 & 255.98 & 0.65 \\
\hline Nigrini et al., 2006 & B Lophocyrtis (L.) jacchia & \multirow{3}{*}{ RP17 } & & 1219A-18H-CC, 17-22 & 1219A-19H-1, 45-47 & 194.30 & 195.25 & 256.40 & 257.03 & 256.72 & 258.32 & 259.01 & 258.67 & 0.34 \\
\hline Nigrini et al., 2006 & T Thyrsocyrtis $(P$.$) orthotenes$ & & & 1219A-18H-CC, 17-22 & 1219A-19H-1, 45-47 & 194.30 & 195.25 & 256.40 & 257.03 & 256.72 & 258.32 & 259.01 & 258.67 & 0.34 \\
\hline Nigrini et al., 2006 & T Podocyrtis (P.) papalis (continuous) & & & 1219A-19H-1, 45-47 & $1219 \mathrm{~A}-19 \mathrm{H}-2,45-47$ & 195.25 & 196.78 & 257.03 & 258.40 & 257.72 & 259.01 & 260.62 & 259.81 & 0.80 \\
\hline Nigrini et al., 2006 & B Cryptocarpium azyx & & & 1219A-19H-1, 45-47 & 1219A-19H-2, 45-47 & 195.25 & 196.78 & 257.03 & 258.40 & 257.72 & 259.01 & 260.62 & 259.81 & 0.80 \\
\hline Nigrini et al., 2006 & B Thyrsocyrtis $(P$.$) lochites$ & \multirow{20}{*}{ RP16 } & & 1219A-19H-1, 45-47 & 1219A-19H-2, 45-47 & 195.25 & 196.78 & 257.03 & 258.40 & 257.72 & 259.01 & 260.62 & 259.81 & 0.80 \\
\hline Nigrini et al., 2006 & T Anthocyrtoma spp. & & & $1219 \mathrm{~A}-19 \mathrm{H}-3,45-47$ & $1219 \mathrm{~A}-19 \mathrm{H}-4,45-47$ & 198.28 & 199.78 & 259.79 & 260.97 & 260.38 & 261.96 & 263.08 & 262.52 & 0.56 \\
\hline Nigrini et al., 2006 & B Dorcadospyris copelata & & & 1219A-19H-3, 45-47 & 1219A-19H-4, 45-47 & 198.28 & 199.78 & 259.79 & 260.97 & 260.38 & 261.96 & 263.08 & 262.52 & 0.56 \\
\hline Nigrini et al., 2006 & T Calocycloma ampulla & & & 1219A-19H-3, 45-47 & $1219 \mathrm{~A}-19 \mathrm{H}-4,45-47$ & 198.28 & 199.78 & 259.79 & 260.97 & 260.38 & 261.96 & 263.08 & 262.52 & 0.56 \\
\hline Nigrini et al., 2006 & T Rhopalocanium ornatum & & & 1219A-19H-3, 45-47 & $1219 \mathrm{~A}-19 \mathrm{H}-4,45-47$ & 198.28 & 199.78 & 259.79 & 260.97 & 260.38 & 261.96 & 263.08 & 262.52 & 0.56 \\
\hline Nigrini et al., 2006 & B Botryocella sp. gr. & & & $1219 \mathrm{~A}-20 \mathrm{H}-6,45-47$ & $1219 \mathrm{~A}-20 \mathrm{H}-7,45-47$ & 212.86 & 214.36 & 272.52 & 263.87 & 268.20 & 274.58 & 276.66 & 275.62 & 1.04 \\
\hline Nigrini et al., 2006 & T Spongatractus pachystylus & & & 1219A-19H-3, 45-47 & 1219A-19H-4, 45-47 & 198.28 & 199.78 & 259.79 & 260.97 & 260.38 & 261.96 & 263.08 & 262.52 & 0.56 \\
\hline Nigrini et al., 2006 & B Thyrsocyrtis (T.) bromia & & & $1219 \mathrm{~A}-19 \mathrm{H}-3,45-47$ & $1219 \mathrm{~A}-19 \mathrm{H}-4,45-47$ & 198.28 & 199.78 & 259.79 & 260.97 & 260.38 & 261.96 & 263.08 & 262.52 & 0.56 \\
\hline Nigrini et al., 2006 & B Thyrsocyrtis (Р.) tetracantha & & & $1219 \mathrm{~A}-19 \mathrm{H}-3,45-47$ & $1219 \mathrm{~A}-19 \mathrm{H}-4,45-47$ & 198.28 & 199.78 & 259.79 & 260.97 & 260.38 & 261.96 & 263.08 & 262.52 & 0.56 \\
\hline Nigrini et al., 2006 & B Theocyrtis careotuberosa & & & 1219A-19H-4, 45-47 & 1219A-19H-5, 45-47 & 199.78 & 201.28 & 260.97 & 262.23 & 261.60 & 263.08 & 264.37 & 263.73 & 0.65 \\
\hline Nigrini et al., 2006 & T Zygocircus cimelium & & & 1219A-19H-4, 45-47 & 1219A-19H-5, 45-47 & 199.78 & 201.28 & 260.97 & 262.23 & 261.60 & 263.08 & 264.37 & 263.73 & 0.65 \\
\hline Nigrini et al., 2006 & B Dorcadospyris ombros (upper) & & & $1219 \mathrm{~A}-19 \mathrm{H}-4,45-47$ & $1219 \mathrm{~A}-19 \mathrm{H}-5,45-47$ & 199.78 & 201.28 & 260.97 & 262.23 & 261.60 & 263.08 & 264.37 & 263.73 & 0.65 \\
\hline Nigrini et al., 2006 & B Artophormis dominasinensis & & & 1219A-19H-5, 45-47 & $1219 \mathrm{~A}-19 \mathrm{H}-6,45-47$ & 201.28 & 202.78 & 262.23 & 263.77 & 263.00 & 264.37 & 265.67 & 265.02 & 0.65 \\
\hline Nigrini et al., 2006 & B Tristylospyris triceros & & & 1219A-19H-5, 45-47 & $1219 \mathrm{~A}-19 \mathrm{H}-6,45-47$ & 201.28 & 202.78 & 262.23 & 263.77 & 263.00 & 264.37 & 265.67 & 265.02 & 0.65 \\
\hline Nigrini et al., 2006 & T Theocotylissa ficus & & & 1219A-19H-5, 45-47 & 1219A-19H-6, 45-47 & 201.28 & 202.78 & 262.23 & 263.77 & 263.00 & 264.37 & 265.67 & 265.02 & 0.65 \\
\hline Nigrini et al., 2006 & T Dorcadospyris anastasis & & & 1219A-19H-5, 45-47 & 1219A-19H-6, 45-47 & 201.28 & 202.78 & 262.23 & 263.77 & 263.00 & 264.37 & 265.67 & 265.02 & 0.65 \\
\hline Nigrini et al., 2006 & B Calocyclas turris & & & $1219 \mathrm{~A}-19 \mathrm{H}-5,45-47$ & 1219A-19H-6, 45-47 & 201.28 & 202.78 & 262.23 & 263.77 & 263.00 & 264.37 & 265.67 & 265.02 & 0.65 \\
\hline Nigrini et al., 2006 & $\mathrm{~T}$ Podocyrtis (L.) chalara (continuous) & & & $1219 \mathrm{~A}-19 \mathrm{H}-6,45-47$ & 1219A-19H-CC, 0-3 & 202.78 & 204.31 & 263.77 & 265.30 & 264.54 & 265.67 & 267.21 & 266.44 & 0.77 \\
\hline Nigrini et al., 2006 & $\begin{array}{l}\text { T Podocyrtis (L.) goetheana } \\
\text { (continuous) }\end{array}$ & & & 1219A-19H-6, 45-47 & 1219A-19H-CC, 0-3 & 202.78 & 204.31 & 263.77 & 265.30 & 264.54 & 265.67 & 267.21 & 266.44 & 0.77 \\
\hline Nigrini et al., 2006 & T Dictyophimus craticula & & & 1219A-19H-CC, 0-3 & $1219 \mathrm{~A}-20 \mathrm{H}-1,45-47$ & 204.31 & 205.78 & 265.30 & 266.76 & 266.03 & 267.21 & 268.68 & 267.94 & 0.74 \\
\hline
\end{tabular}




\begin{tabular}{|c|c|c|c|c|c|c|c|c|c|c|c|c|c|c|}
\hline \multirow[b]{2}{*}{ Source } & \multirow[b]{2}{*}{ Event } & \multirow[b]{2}{*}{ Zone } & \multirow{2}{*}{$\begin{array}{l}\text { Age* } \\
(\mathrm{Ma})\end{array}$} & \multicolumn{2}{|c|}{ Hole, core, section, interval $(\mathrm{cm})$} & \multicolumn{2}{|c|}{$\begin{array}{l}\text { Depth } \\
\text { (mcd) }\end{array}$} & \multicolumn{3}{|c|}{$\begin{array}{c}\text { Depth from } \\
\text { Pälike et al. (2005) (rmcd) }\end{array}$} & \multicolumn{3}{|c|}{$\begin{array}{l}\text { Depth Site } 1218 \\
\text { (corrected rmcd) }\end{array}$} & \multirow{2}{*}{$\begin{array}{c}\text { Error } \\
( \pm) \\
(\mathrm{m})\end{array}$} \\
\hline & & & & Top & Bottom & Top & Bottom & Top & Bottom & Mean & Top & Bottom & Mean & \\
\hline Nigrini et al., 2006 & B Thyrsocyrtis (P.) krooni & \multirow{10}{*}{ RP16 } & & 1219A-19H-CC, 0-3 & $1219 \mathrm{~A}-20 \mathrm{H}-1,45-47$ & 204.31 & 205.78 & 265.30 & 266.76 & 266.03 & 267.21 & 268.68 & 267.94 & 0.74 \\
\hline Nigrini et al., 2006 & T Podocyrtis (P.) apeza & & & $1219 \mathrm{~A}-20 \mathrm{H}-1,45-47$ & $1219 \mathrm{~A}-20 \mathrm{H}-2,45-47$ & 205.78 & 207.28 & 266.76 & 267.99 & 267.38 & 268.68 & 270.08 & 269.38 & 0.70 \\
\hline Nigrini et al., 2006 & B Dictyoprora pirum & & & $1219 \mathrm{~A}-20 \mathrm{H}-5,45-47$ & $1219 \mathrm{~A}-20 \mathrm{H}-6,45-47$ & 211.36 & 212.86 & 271.19 & 272.52 & 271.86 & 272.63 & 274.58 & 273.61 & 0.97 \\
\hline Nigrini et al., 2006 & T Lithochytris vespertilio & & & $1219 \mathrm{~A}-2 \mathrm{OH}-5,45-47$ & $1219 \mathrm{~A}-20 \mathrm{H}-6,45-47$ & 211.36 & 212.86 & 271.19 & 272.52 & 271.86 & 272.63 & 274.58 & 273.61 & 0.97 \\
\hline Nigrini et al., 2006 & T Sethochytris triconiscus & & & $1219 \mathrm{~A}-2 \mathrm{OH}-5,45-47$ & $1219 \mathrm{~A}-20 \mathrm{H}-6,45-47$ & 211.36 & 212.86 & 271.19 & 272.52 & 271.86 & 272.63 & 274.58 & 273.61 & 0.97 \\
\hline Nigrini et al., 2006 & B Lithocyclia aristotelis gr. & & & $1219 \mathrm{~A}-20 \mathrm{H}-5,45-47$ & 1219A-20H-6, 45-47 & 211.36 & 212.86 & 271.19 & 272.52 & 271.86 & 272.63 & 274.58 & 273.61 & 0.97 \\
\hline Nigrini et al., 2006 & T Dorcadospyris ombros (lower) & & & $1219 \mathrm{~A}-20 \mathrm{H}-4,45-47$ & $1219 \mathrm{~A}-20 \mathrm{H}-5,45-47$ & 209.81 & 211.36 & 269.95 & 271.19 & 270.57 & 271.68 & 272.63 & 272.16 & 0.48 \\
\hline Nigrini et al., 2006 & T Podocyrtis (L.) mitra & & & $1219 \mathrm{~A}-20 \mathrm{H}-5,45-47$ & 1219A-20H-6, 45-47 & 211.36 & 212.86 & 271.19 & 272.52 & 271.86 & 272.63 & 274.58 & 273.61 & 0.97 \\
\hline Nigrini et al., 2006 & B Dorcadospyris anastasis & & & $1219 \mathrm{~A}-20 \mathrm{H}-6,45-47$ & 1219A-20H-7, 45-47 & 212.86 & 214.36 & 272.52 & 263.87 & 268.20 & 274.58 & 276.66 & 275.62 & 1.04 \\
\hline Nigrini et al., 2006 & B Dictyoprora armadillo & & & $1219 \mathrm{~A}-20 \mathrm{H}-6,45-47$ & $1219 \mathrm{~A}-20 \mathrm{H}-7,45-47$ & 212.86 & 214.36 & 272.52 & 263.87 & 268.20 & 274.58 & 276.66 & 275.62 & 1.04 \\
\hline Nigrini et al., 2006 & B Podocyrtis (L.) goetheana & \multirow{4}{*}{ RP15 } & & $1219 \mathrm{~A}-20 \mathrm{H}-6,45-47$ & 1219A-20H-7, 45-47 & 212.86 & 214.36 & 272.52 & 263.87 & 268.20 & 274.58 & 276.66 & 275.62 & 1.04 \\
\hline Nigrini et al., 2006 & T Lophocyrtis biaurita & & & $1219 \mathrm{~A}-21 \mathrm{H}-1,45-47$ & 1219A-21H-2, 45-47 & 216.28 & 217.78 & 276.20 & 277.70 & 276.95 & & & & \\
\hline Nigrini et al., 2006 & T Podocyrtis (L.) trachodes & & & $1219 \mathrm{~A}-22 \mathrm{H}-1,46-48$ & $1219 \mathrm{~A}-22 \mathrm{H}-2,46-48$ & 226.79 & 228.29 & 290.43 & 292.62 & 291.53 & & & & \\
\hline Nigrini et al., 2006 & $\begin{array}{l}\text { Podocyrtis }(L .) \text { mitra }>\text { Podocyrtis }(L .) \\
\text { chalara }\end{array}$ & & & 1219A-22H-1, 46-48 & 1219A-22H-2, 46-48 & 226.79 & 228.29 & 290.43 & 292.62 & 291.53 & & & & \\
\hline Nigrini et al., 2006 & B Dorcadospyris ombros (lower) & \multirow{18}{*}{ RP14 } & & 1219A-22H-1, 46-48 & $1219 \mathrm{~A}-22 \mathrm{H}-2,46-48$ & 226.79 & 228.29 & 290.43 & 292.62 & 291.53 & & & & \\
\hline Nigrini et al., 2006 & B Artophormis barbadensis & & & $1219 \mathrm{~A}-22 \mathrm{H}-1,46-48$ & $1219 \mathrm{~A}-22 \mathrm{H}-2,46-48$ & 226.79 & 228.29 & 290.43 & 292.62 & 291.53 & & & & \\
\hline Nigrini et al., 2006 & B Podocyrtis (P.) apeza & & & $1219 \mathrm{~A}-22 \mathrm{H}-1,46-48$ & $1219 \mathrm{~A}-22 \mathrm{H}-2,46-48$ & 226.79 & 228.29 & 290.43 & 292.62 & 291.53 & & & & \\
\hline Nigrini et al., 2006 & B Podocyrtis (L.) chalara & & & $1219 \mathrm{~A}-22 \mathrm{H}-2,46-48$ & 1219A-22H-3, 46-48 & 228.29 & 299.79 & 292.62 & 294.86 & 293.74 & & & & \\
\hline Nigrini et al., 2006 & T Podocyrtis (P.) ampla & & & $1219 \mathrm{~A}-22 \mathrm{H}-3,46-48$ & $1219 \mathrm{~A}-22 \mathrm{H}-4,46-48$ & 299.79 & 231.29 & 294.86 & 296.78 & 295.82 & & & & \\
\hline Nigrini et al., 2006 & B Zealithapium mitra & & & $1219 \mathrm{~A}-22 \mathrm{H}-4,46-48$ & $1219 \mathrm{~A}-22 \mathrm{H}-5,46-48$ & 231.29 & 232.79 & 296.78 & 298.09 & 297.44 & & & & \\
\hline Nigrini et al., 2006 & T Zealithapium anoectum & & & $1219 \mathrm{~A}-22 \mathrm{H}-4,46-48$ & $1219 \mathrm{~A}-22 \mathrm{H}-5,46-48$ & 231.29 & 232.79 & 296.78 & 298.09 & 297.44 & & & & \\
\hline Nigrini et al., 2006 & B Cryptocarpium ornatum & & & $1219 \mathrm{~A}-22 \mathrm{H}-5,46-48$ & $1219 \mathrm{~A}-22 \mathrm{H}-6,46-48$ & 232.79 & 234.29 & 298.09 & & & & & & \\
\hline Nigrini et al., 2006 & B Sethochytris triconiscus & & & $1219 \mathrm{~A}-22 \mathrm{H}-5,46-48$ & $1219 \mathrm{~A}-22 \mathrm{H}-6,46-48$ & 232.79 & 234.29 & 298.09 & & & & & & \\
\hline Nigrini et al., 2006 & T Eusyringium lagena & & & $1219 \mathrm{~A}-23 \mathrm{H}-1,45-47$ & $1219 \mathrm{~A}-23 \mathrm{H}-2,45-47$ & 237.28 & 238.78 & & & & & & & \\
\hline Nigrini et al., 2006 & T Podocyrtis (L.) fasciolata & & & $1219 \mathrm{~A}-23 \mathrm{H}-1,45-47$ & $1219 \mathrm{~A}-23 \mathrm{H}-2,45-47$ & 237.28 & 238.78 & & & & & & & \\
\hline Nigrini et al., 2006 & T Zealithapium plegmacantha & & & $1219 \mathrm{~A}-23 \mathrm{H}-1,45-47$ & $1219 \mathrm{~A}-23 \mathrm{H}-2,45-47$ & 237.28 & 238.78 & & & & & & & \\
\hline Nigrini et al., 2006 & T Podocyrtis (L.) sinuosa & & & $1219 \mathrm{~A}-23 \mathrm{H}-1,45-47$ & $1219 \mathrm{~A}-23 \mathrm{H}-2,45-47$ & 237.28 & 238.78 & & & & & & & \\
\hline Nigrini et al., 2006 & $\begin{array}{l}\text { Zealithapium plegmacantha }> \\
\text { Zealithapium anoectum }\end{array}$ & & & 1219A-23H-1，45-47 & 1219A-23H-4, 49-50 & 237.28 & 241.82 & & & & & & & \\
\hline Nigrini et al., 2006 & B Theocyrtis perpumila & & & $1219 \mathrm{~A}-23 \mathrm{H}-2,45-47$ & $1219 \mathrm{~A}-23 \mathrm{H}-3,12-14$ & 238.78 & 239.95 & & & & & & & \\
\hline Nigrini et al., 2006 & T Podocyrtis (L.) helenae & & & $1219 \mathrm{~A}-23 \mathrm{H}-3,12-14$ & $1219 \mathrm{~A}-23 \mathrm{H}-4,49-50$ & 239.95 & 241.82 & & & & & & & \\
\hline Nigrini et al., 2006 & B Lychnocanoma turgidum & & & $1219 \mathrm{~A}-23 \mathrm{H}-3,12-14$ & $1219 \mathrm{~A}-23 \mathrm{H}-4,49-50$ & 239.95 & 241.82 & & & & & & & \\
\hline Nigrini et al., 2006 & $\begin{array}{l}\text { Eusyringium lagena }>\text { Eusyringium } \\
\text { fistuligerum }\end{array}$ & & & $1219 \mathrm{~A}-23 \mathrm{H}-2,45-47$ & $1219 \mathrm{~A}-23 \mathrm{H}-4,49-50$ & 238.78 & 241.82 & & & & & & & \\
\hline Nigrini et al., 2006 & $\begin{array}{l}\text { Podocyrtis (L.) sinuosa }>\text { Podocyrtis } \\
\text { (L.) mitra }\end{array}$ & & & 1219A-23H-4, 49-50 & 1219A-23H-5, 45-47 & 241.82 & 243.28 & & & & & & & \\
\hline Nigrini et al., 2006 & B Podocyrtis (L.) trachodes & \multirow{9}{*}{ RP13 } & & $1219 \mathrm{~A}-23 \mathrm{H}-5,45-47$ & $1219 \mathrm{~A}-23 \mathrm{H}-6,45-47$ & 243.28 & 244.78 & & & & & & & \\
\hline Nigrini et al., 2006 & B Zygocircus cimelium & & & $1219 \mathrm{~A}-23 \mathrm{H}-7,45-47$ & $1219 \mathrm{~A}-23 \mathrm{H}-\mathrm{CC}, 23-27$ & 246.28 & 246.76 & & & & & & & \\
\hline Nigrini et al., 2006 & B Podocyrtis (L.) fasciolata & & & $1219 \mathrm{~A}-24 \mathrm{H}-1,45-47$ & $1219 \mathrm{~A}-24 \mathrm{H}-2,45-47$ & 247.78 & 249.28 & & & & & & & \\
\hline Nigrini et al., 2006 & B Podocyrtis (L.) helenae & & & $1219 \mathrm{~A}-24 \mathrm{H}-1,45-47$ & 1219A-24H-2, 45-47 & 247.78 & 249.28 & & & & & & & \\
\hline Nigrini et al., 2006 & T Podocyrtis (P.) diamesa & & & $1219 \mathrm{~A}-24 \mathrm{H}-2,45-47$ & $1219 \mathrm{~A}-24 \mathrm{H}-3,45-47$ & 249.28 & 250.78 & & & & & & & \\
\hline Nigrini et al., 2006 & T Podocyrtis (P.) phyxis & & & $1219 \mathrm{~A}-24 \mathrm{H}-2,45-47$ & $1219 \mathrm{~A}-24 \mathrm{H}-3,45-47$ & 249.28 & 250.78 & & & & & & & \\
\hline Nigrini et al., 2006 & T Spongatractus balbis & & & $1219 \mathrm{~A}-24 \mathrm{H}-2,45-47$ & 1219A-24H-3, 45-47 & 249.28 & 250.78 & & & & & & & \\
\hline Nigrini et al., 2006 & B Podocyrtis (L.) mitra & & & $1219 \mathrm{~A}-24 \mathrm{H}-2,45-47$ & $1219 \mathrm{~A}-24 \mathrm{H}-3,45-47$ & 249.28 & 250.78 & & & & & & & \\
\hline Nigrini et al., 2006 & $\begin{array}{l}\text { Podocyrtis (P.) phyxis > Podocyrtis (P.) } \\
\text { ampla }\end{array}$ & & & 1219A-24H-2, 45-47 & $1219 \mathrm{~A}-24 \mathrm{H}-3,45-47$ & 249.28 & 250.78 & & & & & & & \\
\hline Nigrini et al., 2006 & B Podocyrtis (P.) ampla & \multirow{3}{*}{$\mathrm{RP} 12$} & & $1219 \mathrm{~A}-24 \mathrm{H}-2,45-47$ & $1219 \mathrm{~A}-24 \mathrm{H}-3,45-47$ & 249.28 & 250.78 & & & & & & & \\
\hline Nigrini et al., 2006 & B Eusyringium fistuligerum & & & $1219 \mathrm{~A}-24 \mathrm{H}-2,45-47$ & $1219 \mathrm{~A}-24 \mathrm{H}-3,45-47$ & 249.28 & 250.78 & & & & & & & \\
\hline Nigrini et al., 2006 & T Theocotyle venezuelensis & & & $1219 \mathrm{~A}-24 \mathrm{H}-3,45-47$ & $1219 \mathrm{~A}-24 \mathrm{H}-4,45-47$ & 250.78 & 252.28 & & & & & & & \\
\hline
\end{tabular}




\begin{tabular}{|c|c|c|c|c|c|c|c|c|c|c|c|c|c|c|}
\hline \multirow[b]{2}{*}{ Source } & \multirow[b]{2}{*}{ Event } & \multirow[b]{2}{*}{ Zone } & \multirow{2}{*}{$\begin{array}{l}\text { Age* }^{*} \\
(\mathrm{Ma})\end{array}$} & \multicolumn{2}{|c|}{ Hole, core, section, interval $(\mathrm{cm})$} & \multicolumn{2}{|c|}{$\begin{array}{l}\text { Depth } \\
\text { (mcd) }\end{array}$} & \multicolumn{3}{|c|}{$\begin{array}{c}\text { Depth from } \\
\text { Pälike et al. (2005) (rmcd) }\end{array}$} & \multicolumn{3}{|c|}{$\begin{array}{l}\text { Depth Site } 1218 \\
\text { (corrected rmcd) }\end{array}$} & \multirow{2}{*}{$\begin{array}{c}\text { Error } \\
( \pm) \\
(\mathrm{m})\end{array}$} \\
\hline & & & & Top & Bottom & Top & Bottom & Top & Bottom & Mean & Top & Bottom & Mean & \\
\hline Nigrini et al., 2006 & B Zealithapium anoectum & & & $1219 \mathrm{~A}-24 \mathrm{H}-3,45-47$ & $1219 \mathrm{~A}-24 \mathrm{H}-4,45-47$ & 250.78 & 252.28 & & & & & & & \\
\hline Nigrini et al., 2006 & T Theocotyle conica & & & 1219A-24H-3, 45-47 & 1219A-24H-4, 45-47 & 250.78 & 252.28 & & & & & & & \\
\hline Nigrini et al., 2006 & $\mathrm{~T}$ Theocotyle cryptocephala & & & $1219 \mathrm{~A}-24 \mathrm{H}-4,45-47$ & 1219A-24H-5, 45-47 & 252.28 & 253.83 & & & & & & & \\
\hline Nigrini et al., 2006 & B Thyrsocyrtis $(P$.$) orthotenes$ & & & $1219 \mathrm{~A}-24 \mathrm{H}-5,45-47$ & 1219A-24H-6, 45-47 & 253.83 & 255.33 & & & & & & & \\
\hline Nigrini et al., 2006 & T Thyrsocyrtis ( $T$. ) robusta & & & 1219A-24H-5, 45-47 & $1219 \mathrm{~A}-24 \mathrm{H}-6,45-47$ & 253.83 & 255.33 & & & & & & & \\
\hline Nigrini et al., 2006 & $\begin{array}{l}\text { Podocyrtis (P.) diamesa > Podocyrtis } \\
(P .) \text { phyxis }\end{array}$ & RP12 & & $1219 \mathrm{~A}-24 \mathrm{H}-5,45-47$ & $1219 \mathrm{~A}-24 \mathrm{H}-6,45-47$ & 253.83 & 255.33 & & & & & & & \\
\hline Nigrini et al., 2006 & B Podocyrtis (P.) phyxis & & & 1219A-24H-6, 45-47 & 1219A-24H-7, 45-47 & 255.33 & 256.83 & & & & & & & \\
\hline Nigrini et al., 2006 & T Periphaena tripyramis triangula & & & $1219 \mathrm{~A}-24 \mathrm{H}-6,45-47$ & 1219A-24H-7, 45-47 & 255.33 & 256.83 & & & & & & & \\
\hline Nigrini et al., 2006 & T Periphaena delta & & & 1219A-24H-7, 45-47 & $1219 \mathrm{~A}-24 \mathrm{H}-\mathrm{CC}, 21-24$ & 256.83 & 257.21 & & & & & & & \\
\hline Nigrini et al., 2006 & T Lamptonium fab. chaunothorax & & & 1219A-24H-7, 45-47 & $1219 \mathrm{~A}-24 \mathrm{H}-\mathrm{CC}, 21-24$ & 256.83 & 257.21 & & & & & & & \\
\hline Nigrini et al., 2006 & T Lamptonium fab. constrictum & & & $1219 \mathrm{~A}-25 \mathrm{X}-2,45-47$ & 1219A-25X-CC, 35-40 & 259.78 & 259.40 & & & & & & & \\
\hline
\end{tabular}

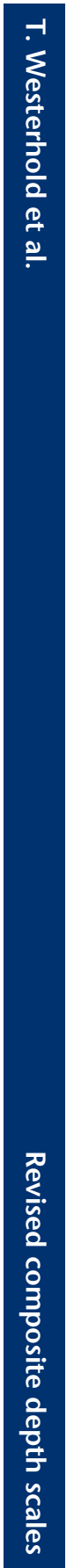




\begin{tabular}{|c|c|c|c|c|c|c|c|c|c|c|c|c|}
\hline \multirow[b]{2}{*}{ Source } & \multirow[b]{2}{*}{ Event } & \multirow[b]{2}{*}{ Zone } & \multirow{2}{*}{$\begin{array}{l}\text { Age* }^{*} \\
(\mathrm{Ma})\end{array}$} & \multicolumn{2}{|c|}{ Hole, core, section, interval $(\mathrm{cm})$} & \multicolumn{3}{|c|}{$\begin{array}{l}\text { Depth from } \\
\text { Pälike et al. (2005) (rmcd) }\end{array}$} & \multicolumn{3}{|c|}{$\begin{array}{c}\text { Depth } \\
\text { adjusted (rmcd) } \\
\end{array}$} & \multirow{2}{*}{$\begin{array}{l}\text { Error } \\
( \pm) \\
(\mathrm{m})\end{array}$} \\
\hline & & & & Top & Bottom & Top & Bottom & Mean & Top & Bottom & Mean & \\
\hline & & & & 199 & 199 & & & & & & & \\
\hline Nigrini et al., 2006 & T Theocyrtis annosa & & & $1220 \mathrm{~A}-3 \mathrm{H}-4,45-47$ & $1220 \mathrm{~A}-3 \mathrm{H}-5,45-47$ & 23.90 & 24.90 & 24.40 & 23.90 & 24.90 & 24.40 & 0.50 \\
\hline Nigrini et al., 2006 & T Eucyrtidium mitodes $\mathrm{n} . \mathrm{sp}$. & & & $1220 \mathrm{~A}-3 \mathrm{H}-6,45-47$ & $1220 \mathrm{~A}-3 \mathrm{H}-\mathrm{CC}$ & 26.40 & 28.94 & 27.67 & 26.40 & 28.80 & 27.60 & 1.20 \\
\hline Nigrini et al., 2006 & B Cyrtocapsella cornuta & $\mathrm{RNI}$ & & $1220 \mathrm{~A}-3 \mathrm{H}-6,45-47$ & $1220 \mathrm{~A}-3 \mathrm{H}-\mathrm{CC}$ & 26.40 & 28.94 & 27.67 & 26.40 & 28.80 & 27.60 & 1.20 \\
\hline Nigrini et al., 2006 & B Cyrtocapsella tetrapera & & & $1220 \mathrm{~A}-3 \mathrm{H}-6,45-47$ & $1220 \mathrm{~A}-3 \mathrm{H}-\mathrm{CC}$ & 26.40 & 28.94 & 27.67 & 26.40 & 28.80 & 27.60 & 1.20 \\
\hline Nigrini et al., 2006 & T Artophormis gracilis & & & $1220 \mathrm{~A}-3 \mathrm{H}-6,45-47$ & $1220 \mathrm{~A}-3 \mathrm{H}-\mathrm{CC}$ & 26.40 & 28.94 & 27.67 & 26.40 & 28.80 & 27.60 & 1.20 \\
\hline Nigrini et al., 2006 & B Eucyrtidium diaphanes & & & $1220 \mathrm{~A}-3 \mathrm{H}-6,45-47$ & $1220 \mathrm{~A}-3 \mathrm{H}-\mathrm{CC}$ & 26.40 & 28.94 & 27.67 & 26.40 & 28.80 & 27.60 & 1.20 \\
\hline Nigrini et al., 2006 & T Dorcadospyris circulus (max) & RP22 & & $1220 \mathrm{~A}-3 \mathrm{H}-\mathrm{CC}$ & $1220 \mathrm{~A}-4 \mathrm{H}-2,45-47$ & 28.94 & 32.70 & 30.82 & 28.80 & 32.70 & 30.75 & 1.95 \\
\hline Nigrini et al., 2006 & T Dorcadospyris papilio & KPZZZ & & $1220 \mathrm{~A}-3 \mathrm{H}-\mathrm{CC}$ & $1220 \mathrm{~A}-4 \mathrm{H}-2,45-47$ & 28.94 & 32.70 & 30.82 & 28.80 & 32.70 & 30.75 & 1.95 \\
\hline Nigrini et al., 2006 & T Liriospyris longicornuta & & & $1220 \mathrm{~A}-3 \mathrm{H}-\mathrm{CC}$ & $1220 \mathrm{~A}-4 \mathrm{H}-2,45-47$ & 28.94 & 32.70 & 30.82 & 28.80 & 32.70 & 30.75 & 1.95 \\
\hline Nigrini et al., 2006 & T Acrocubus octopylus (upper) & & & $1220 \mathrm{~A}-4 \mathrm{H}-2,45-47$ & $1220 \mathrm{~A}-4 \mathrm{H}-4,45-47$ & 32.70 & 35.70 & 34.20 & 32.70 & 35.70 & 34.20 & 1.50 \\
\hline Nigrini et al., 2006 & B Lychnocanoma elongata & & & $1220 \mathrm{~A}-4 \mathrm{H}-2,45-47$ & $1220 \mathrm{~A}-4 \mathrm{H}-3,45-47$ & 32.70 & 34.20 & 33.45 & 32.70 & 34.20 & 33.45 & 0.75 \\
\hline Nigrini et al., 2006 & B Acrocubus octopylus (lower) & & & $1220 \mathrm{~A}-4 \mathrm{H}-4,45-47$ & $1220 \mathrm{~A}-4 \mathrm{H}-6,45-47$ & 35.70 & 38.70 & 37.20 & 35.70 & 38.70 & 37.20 & 1.50 \\
\hline Nigrini et al., 2006 & B Didymocyrtis tubaria (lower) & & & $1220 \mathrm{~A}-3 \mathrm{H}-\mathrm{CC}$ & $1220 \mathrm{~A}-4 \mathrm{H}-2,45-47$ & 28.94 & 32.70 & 30.82 & 28.80 & 32.70 & 30.75 & 1.95 \\
\hline Nigrini et al., 2006 & B Calocycletta (C.) robusta & & & $1220 \mathrm{~A}-4 \mathrm{H}-4,45-47$ & $1220 \mathrm{~A}-4 \mathrm{H}-6,45-47$ & 35.70 & 38.70 & 37.20 & 35.70 & 38.70 & 37.20 & 1.50 \\
\hline Nigrini et al., 2006 & B Liriospyris longicornuta & & & $1220 \mathrm{~A}-4 \mathrm{H}-4,45-47$ & $1220 \mathrm{~A}-4 \mathrm{H}-6,45-47$ & 35.70 & 38.70 & 37.20 & 35.70 & 38.70 & 37.20 & 1.50 \\
\hline Nigrini et al., 2006 & B Dorcadospyris scambos n. sp. & & & $1220 \mathrm{~A}-4 \mathrm{H}-6,45-47$ & $1220 \mathrm{~A}-4 \mathrm{H}-\mathrm{CC}$ & 35.70 & 38.70 & 37.20 & 35.70 & 38.70 & 37.20 & 1.50 \\
\hline Nigrini et al., 2006 & T Theocorys puriri & & & $1220 \mathrm{~A}-4 \mathrm{H}-6,45-47$ & $1220 \mathrm{~A}-4 \mathrm{H}-\mathrm{CC}$ & 35.70 & 38.70 & 37.20 & 35.70 & 38.70 & 37.20 & 1.50 \\
\hline Nigrini et al., 2006 & B Dorcadospyris papilio & & & $1220 \mathrm{~A}-4 \mathrm{H}-6,45-47$ & $1220 \mathrm{~A}-4 \mathrm{H}-\mathrm{CC}$ & 38.70 & 40.39 & 39.55 & 38.70 & 40.34 & 39.52 & 0.82 \\
\hline Nigrini et al., 2006 & T Dorcadospyris circulus (continuous) & & & $1220 \mathrm{~A}-4 \mathrm{H}-\mathrm{CC}$ & $1220 \mathrm{~A}-5 \mathrm{H}-2,45-47$ & 38.70 & 43.24 & 40.97 & 38.70 & 43.24 & 40.97 & 2.27 \\
\hline Nigrini et al., 2006 & B Dorcadospyris riedeli (upper) & KPZI & & $1220 \mathrm{~A}-5 \mathrm{H}-2,45-47$ & 1220B-1H-CC & 43.24 & 43.47 & 43.36 & 43.24 & 43.81 & 43.53 & 0.29 \\
\hline Nigrini et al., 2006 & T Eucyrtidium plesiodiaphanes & & & 1220B-1H-CC & $1220 \mathrm{~A}-5 \mathrm{H}-4,45-47$ & 43.47 & 46.24 & 44.86 & 43.81 & 46.24 & 45.03 & 1.21 \\
\hline Nigrini et al., 2006 & T Lithocyclia angusta (continuous) & & & $1220 \mathrm{~A}-5 \mathrm{H}-4,45-47$ & $1220 \mathrm{~A}-5 \mathrm{H}-6,45-47$ & 46.24 & 49.24 & 47.74 & 46.24 & 48.87 & 47.56 & 1.32 \\
\hline Nigrini et al., 2006 & $\mathrm{~T}$ Theocyrtis setanios $\mathrm{n}$. sp. & & & $1220 \mathrm{~A}-5 \mathrm{H}-4,45-47$ & $1220 \mathrm{~A}-5 \mathrm{H}-6,45-47$ & 46.24 & 49.24 & 47.74 & 46.24 & 48.87 & 47.56 & 1.32 \\
\hline Nigrini et al., 2006 & T Theocyrtis perysinos $\mathrm{n} . \mathrm{sp}$. & & & $1220 \mathrm{~A}-5 \mathrm{H}-4,45-47$ & $1220 \mathrm{~A}-5 \mathrm{H}-6,45-47$ & 46.24 & 49.24 & 47.74 & 46.24 & 48.87 & 47.56 & 1.32 \\
\hline Nigrini et al., 2006 & T Tristylospyris triceros & & & $1220 \mathrm{~A}-5 \mathrm{H}-\mathrm{CC}$ & $1220 \mathrm{~A}-6 \mathrm{H}-1,45-47$ & 51.29 & 53.90 & 52.60 & 50.65 & 53.29 & 51.97 & 1.32 \\
\hline Nigrini et al., 2006 & B Theocyrtis annosa & & & $1220 \mathrm{~A}-5 \mathrm{H}-\mathrm{CC}$ & $1220 \mathrm{~A}-6 \mathrm{H}-1,45-47$ & 51.29 & 53.90 & 52.60 & 50.65 & 53.29 & 51.97 & 1.32 \\
\hline Nigrini et al., 2006 & Tristylospyris triceros > Dorcadospyris ateuchus & & & $1220 \mathrm{~A}-5 \mathrm{H}-\mathrm{CC}$ & $1220 \mathrm{~A}-6 \mathrm{H}-1,45-47$ & 51.29 & 53.90 & 52.60 & 50.65 & 53.29 & 51.97 & 1.32 \\
\hline Nigrini et al., 2006 & B Eucyrtidium mitodes $\mathrm{n} . \mathrm{sp}$. & & & $1220 \mathrm{~A}-6 \mathrm{H}-4,45-47$ & $1220 \mathrm{~A}-6 \mathrm{H}-6,45-47$ & 57.79 & 60.79 & 59.29 & 57.79 & 60.79 & 59.29 & 1.50 \\
\hline Nigrini et al., 2006 & B Theocyrtis perysinos $\mathrm{n}$. $\mathrm{sp}$. & & & $1220 \mathrm{~A}-6 \mathrm{H}-2,45-47$ & 1220B-2H-CC & 54.79 & 55.93 & 55.36 & 54.79 & 55.93 & 55.36 & 0.57 \\
\hline Nigrini et al., 2006 & B Dorcadospyris ateuchus & & & $1220 \mathrm{~B}-2 \mathrm{H}-\mathrm{CC}$ & $1220 \mathrm{~A}-6 \mathrm{H}-4,45-47$ & 55.93 & 57.79 & 56.86 & 55.93 & 57.79 & 56.86 & 0.93 \\
\hline Nigrini et al., 2006 & B Theocyrtis setanios $\mathrm{n} . \mathrm{sp}$. & & & $1220 \mathrm{~B}-2 \mathrm{H}-\mathrm{CC}$ & $1220 \mathrm{~A}-6 \mathrm{H}-4,45-47$ & 55.93 & 57.79 & 56.86 & 55.93 & 57.79 & 56.86 & 0.93 \\
\hline Nigrini et al., 2006 & T Lophocyrtis (C.) milowi & & & $1220 \mathrm{~A}-6 \mathrm{H}-4,45-47$ & $1220 \mathrm{~A}-6 \mathrm{H}-6,45-47$ & 57.79 & 60.79 & 59.29 & 57.79 & 60.79 & 59.29 & 1.50 \\
\hline Nigrini et al., 2006 & B Theocorys puriri & & & $1220 \mathrm{~A}-6 \mathrm{H}-2,45-47$ & $1220 \mathrm{~B}-2 \mathrm{H}-\mathrm{CC}$ & 54.79 & 55.93 & 55.36 & 54.79 & 55.93 & 55.36 & 0.57 \\
\hline Nigrini et al., 2006 & B Didymocyrtis prismatica & & & $1220 \mathrm{~A}-6 \mathrm{H}-4,45-47$ & $1220 \mathrm{~A}-6 \mathrm{H}-6,45-47$ & 57.79 & 60.79 & 59.29 & 57.79 & 60.79 & 59.29 & 1.50 \\
\hline Nigrini et al., 2006 & B Dorcadospyris circulus & & & $1220 \mathrm{~A}-6 \mathrm{H}-4,45-47$ & $1220 \mathrm{~A}-6 \mathrm{H}-6,45-47$ & 57.79 & 60.79 & 59.29 & 57.79 & 60.79 & 59.29 & 1.50 \\
\hline Nigrini et al., 2006 & T Lophocyrtis (S.) oberhaensliae & & & $1220 \mathrm{~A}-6 \mathrm{H}-4,45-47$ & $1220 \mathrm{~A}-6 \mathrm{H}-6,45-47$ & 57.79 & 60.79 & 59.29 & 57.79 & 60.79 & 59.29 & 1.50 \\
\hline Nigrini et al., 2006 & $\mathrm{~T}$ Theocyrtis tuberosa & & & $1220 \mathrm{~A}-6 \mathrm{H}-4,45-47$ & $1220 \mathrm{~A}-6 \mathrm{H}-6,45-47$ & 57.79 & 60.79 & 59.29 & 57.79 & 60.79 & 59.29 & 1.50 \\
\hline Nigrini et al., 2006 & T Lithocyclia crux & & & $1220 \mathrm{~A}-6 \mathrm{H}-6,45-47$ & $1220 \mathrm{~A}-6 \mathrm{H}-\mathrm{CC}$ & 60.79 & 62.68 & 61.74 & 60.79 & 62.94 & 61.87 & 1.08 \\
\hline Nigrini et al., 2006 & T Dorcadospyris spinosa & RP20 & & $1220 \mathrm{~A}-6 \mathrm{H}-4,45-47$ & $1220 \mathrm{~A}-6 \mathrm{H}-6,45-47$ & 57.79 & 60.79 & 59.29 & 57.79 & 60.79 & 59.29 & 1.50 \\
\hline Nigrini et al., 2006 & T Dorcadospyris quadripes & & & $1220 \mathrm{~A}-6 \mathrm{H}-6,45-47$ & $1220 \mathrm{~A}-6 \mathrm{H}-\mathrm{CC}$ & 60.79 & 62.68 & 61.74 & 60.79 & 62.94 & 61.87 & 1.08 \\
\hline Nigrini et al., 2006 & B Eucyrtidium plesiodiaphanes & & & $1220 \mathrm{~A}-6 \mathrm{H}-6,45-47$ & $1220 \mathrm{~A}-6 \mathrm{H}-\mathrm{CC}$ & 60.79 & 62.68 & 61.74 & 60.79 & 62.94 & 61.87 & 1.08 \\
\hline Nigrini et al., 2006 & B Lophocyrtis (C.) pegetrum & & & $1220 \mathrm{~A}-6 \mathrm{H}-\mathrm{CC}$ & $1220 \mathrm{~A}-7 \mathrm{H}-2,45-47$ & 62.68 & 65.26 & 63.97 & 62.94 & 65.26 & 64.10 & 1.16 \\
\hline Nigrini et al., 2006 & T Dorcadospyris riedeli (lower) & & & $1220 \mathrm{~A}-6 \mathrm{H}-\mathrm{CC}$ & $1220 \mathrm{~A}-7 \mathrm{H}-2,45-47$ & 62.68 & 65.26 & 63.97 & 62.94 & 65.26 & 64.10 & 1.16 \\
\hline Nigrini et al., 2006 & B Spirocyrtis subtilis & & & 1220B-3H-CC & $1220 \mathrm{~A}-7 \mathrm{H}-4,45-47$ & 65.94 & 68.22 & 67.08 & 65.94 & 68.22 & 67.08 & 1.14 \\
\hline Nigrini et al., 2006 & T Dorcadospyris pseudopapilo & & & $1220 \mathrm{~A}-7 \mathrm{H}-4,45-47$ & $1220 \mathrm{~A}-7 \mathrm{H}-6,45-47$ & 68.22 & 71.22 & 69.72 & 68.22 & 71.22 & 69.72 & 1.50 \\
\hline Nigrini et al., 2006 & B Dorcadospyris spinosa & & & $1220 \mathrm{~A}-7 \mathrm{H}-4,45-47$ & $1220 \mathrm{~A}-7 \mathrm{H}-6,45-47$ & 68.22 & 71.22 & 69.72 & 68.22 & 71.22 & 69.72 & 1.50 \\
\hline Nigrini et al., 2006 & B Dorcadospyris riedeli (lower) & & & 1220A-7H-4, 45-47 & 1220A-7H-6, 45-47 & 68.22 & 71.22 & 69.72 & 68.22 & 71.22 & 69.72 & 1.50 \\
\hline Nigrini et al., 2006 & T Centrobotrys gravida & & & $1220 \mathrm{~A}-7 \mathrm{H}-4,45-47$ & 1220A-7H-6, 45-47 & 68.22 & 71.22 & 69.72 & 68.22 & 71.22 & 69.72 & 1.50 \\
\hline Nigrini et al., 2006 & B Centrobotrys petrushevskayae & & & $1220 \mathrm{~A}-7 \mathrm{H}-4,45-47$ & 1220A-7H-6, 45-47 & 68.22 & 71.22 & 69.72 & 68.22 & 71.22 & 69.72 & 1.50 \\
\hline
\end{tabular}




\begin{tabular}{|c|c|c|c|c|c|c|c|c|c|c|c|c|}
\hline \multirow[b]{2}{*}{ Source } & \multirow[b]{2}{*}{ Event } & \multirow[b]{2}{*}{ Zone } & \multirow{2}{*}{$\begin{array}{l}\text { Age }^{*} \\
(\mathrm{Ma})\end{array}$} & \multicolumn{2}{|c|}{ Hole, core, section, interval $(\mathrm{cm})$} & \multicolumn{3}{|c|}{$\begin{array}{l}\text { Depth from } \\
\text { Pälike et al. (2005) (rmcd) }\end{array}$} & \multicolumn{3}{|c|}{$\begin{array}{c}\text { Depth } \\
\text { adjusted (rmcd) }\end{array}$} & \multirow{2}{*}{$\begin{array}{c}\text { Erro } \\
( \pm) \\
(\mathrm{m})\end{array}$} \\
\hline & & & & Top & Bottom & Top & Bottom & Mean & Top & Bottom & Mean & \\
\hline Nigrini et al., 2006 & B Dorcadospyris quadripes & \multirow{14}{*}{ RP20 } & & $1220 \mathrm{~A}-7 \mathrm{H}-4,45-47$ & $1220 \mathrm{~A}-7 \mathrm{H}-6,45-47$ & 68.22 & 71.22 & 69.72 & 68.22 & 71.22 & 69.72 & 1.50 \\
\hline Nigrini et al., 2006 & B Lychnodictyum audax & & & 1220A-7H-CC & $1220 \mathrm{~A}-8 \mathrm{H}-1,46-48$ & 72.99 & 74.60 & 73.80 & 72.99 & 74.60 & 73.80 & 0.81 \\
\hline Nigrini et al., 2006 & T Theocyrtis careotuberosa $\mathrm{n} . \mathrm{sp}$. & & & 1220A-7H-CC & $1220 \mathrm{~A}-8 \mathrm{H}-1,46-48$ & 72.99 & 74.60 & 73.80 & 72.99 & 74.60 & 73.80 & 0.81 \\
\hline Nigrini et al., 2006 & B Theocyrtis tuberosa & & & $1220 \mathrm{~A}-8 \mathrm{H}-1,46-48$ & $1220 \mathrm{~A}-8 \mathrm{H}-2,20-22$ & 74.60 & 75.84 & 75.22 & 74.60 & 75.84 & 75.22 & 0.62 \\
\hline Nigrini et al., 2006 & B Dorcadospyris pseudopapilio & & & 1220A-7H-CC & $1220 \mathrm{~A}-8 \mathrm{H}-1,46-48$ & 72.99 & 74.60 & 73.80 & 72.99 & 74.60 & 73.80 & 0.81 \\
\hline Nigrini et al., 2006 & B Lithocyclia crux & & & 1220A-7H-CC & $1220 \mathrm{~A}-8 \mathrm{H}-1,46-48$ & 72.99 & 74.60 & 73.80 & 72.99 & 74.60 & 73.80 & 0.81 \\
\hline Nigrini et al., 2006 & B Centrobotrys gravida & & & 1220A-7H-CC & $1220 \mathrm{~A}-8 \mathrm{H}-1,46-48$ & 72.99 & 74.60 & 73.80 & 72.99 & 74.60 & 73.80 & 0.81 \\
\hline Nigrini et al., 2006 & T Dictyoprora mongolfieri & & & 1220A-7H-CC & $1220 \mathrm{~A}-8 \mathrm{H}-1,46-48$ & 72.99 & 74.60 & 73.80 & 72.99 & 74.60 & 73.80 & 0.81 \\
\hline Nigrini et al., 2006 & $\mathrm{~T}$ Thyrsocyrtis $(P$.$) triacantha (\max )$ & & & 1220A-7H-CC & $1220 \mathrm{~A}-8 \mathrm{H}-1,46-48$ & 72.99 & 74.60 & 73.80 & 72.99 & 74.60 & 73.80 & 0.81 \\
\hline Nigrini et al., 2006 & T Lithocyclia ocellus gr. & & & 1220A-7H-CC & $1220 \mathrm{~A}-8 \mathrm{H}-1,46-48$ & 72.99 & 74.60 & 73.80 & 72.99 & 74.60 & 73.80 & 0.81 \\
\hline Nigrini et al., 2006 & T Lithocyclia aristotelis gr. & & & $1220 \mathrm{~A}-8 \mathrm{H}-1,46-48$ & $1220 \mathrm{~A}-8 \mathrm{H}-2,20-22$ & 74.60 & 75.84 & 75.22 & 74.60 & 75.84 & 75.22 & 0.62 \\
\hline Nigrini et al., 2006 & B Lophocyrtis (S.) oberhaensliae & & & $1220 \mathrm{~A}-8 \mathrm{H}-2,20-22$ & $1220 \mathrm{~A}-8 \mathrm{H}-3,46-48$ & 75.84 & 77.60 & 76.72 & 75.84 & 77.60 & 76.72 & 0.88 \\
\hline Nigrini et al., 2006 & T Cryptocarpium ornatum & & & $1220 \mathrm{~A}-8 \mathrm{H}-2,20-22$ & $1220 \mathrm{~A}-8 \mathrm{H}-3,46-48$ & 75.84 & 77.60 & 76.72 & 75.84 & 77.60 & 76.72 & 0.88 \\
\hline Nigrini et al., 2006 & T Calocyclas hispida & & & $1220 \mathrm{~A}-8 \mathrm{H}-2,20-22$ & $1220 \mathrm{~A}-8 \mathrm{H}-3,46-48$ & 75.84 & 77.60 & 76.72 & 75.84 & 77.60 & 76.72 & 0.88 \\
\hline Nigrini et al., 2006 & Lithocyclia aristotelis gr. > Lithocyclia angusta & \multirow{25}{*}{ RP19 } & & $1220 \mathrm{~A}-8 \mathrm{H}-2,20-22$ & $1220 \mathrm{~A}-8 \mathrm{H}-3,46-48$ & 75.84 & 77.60 & 76.72 & 75.84 & 77.60 & 76.72 & 0.88 \\
\hline Nigrini et al., 2006 & T Calocycletta (C.) anekathen n. sp. & & & $1220 \mathrm{~A}-8 \mathrm{H}-2,20-22$ & $1220 \mathrm{~A}-8 \mathrm{H}-3,46-48$ & 75.84 & 77.60 & 76.72 & 75.84 & 77.60 & 76.72 & 0.88 \\
\hline Nigrini et al., 2006 & T Lophocyrtis (L.) jacchia & & & $1220 \mathrm{~A}-8 \mathrm{H}-2,20-22$ & $1220 \mathrm{~A}-8 \mathrm{H}-3,46-48$ & 75.84 & 77.60 & 76.72 & 75.84 & 77.60 & 76.72 & 0.88 \\
\hline Nigrini et al., 2006 & T Dictyoprora pirum & & & $1220 \mathrm{~A}-8 \mathrm{H}-2,20-22$ & $1220 \mathrm{~A}-8 \mathrm{H}-3,46-48$ & 75.84 & 77.60 & 76.72 & 75.84 & 77.60 & 76.72 & 0.88 \\
\hline Nigrini et al., 2006 & T Dictyoprora armadillo & & & $1220 \mathrm{~A}-8 \mathrm{H}-2,20-22$ & $1220 \mathrm{~A}-8 \mathrm{H}-3,46-48$ & 75.84 & 77.60 & 76.72 & 75.84 & 77.60 & 76.72 & 0.88 \\
\hline Nigrini et al., 2006 & T Lychnocanoma babylonis & & & $1220 \mathrm{~A}-8 \mathrm{H}-2,20-22$ & $1220 \mathrm{~A}-8 \mathrm{H}-3,46-48$ & 75.84 & 77.60 & 76.72 & 75.84 & 77.60 & 76.72 & 0.88 \\
\hline Nigrini et al., 2006 & T Lophocyrtis (C.) hadra & & & $1220 \mathrm{~A}-8 \mathrm{H}-2,20-22$ & $1220 \mathrm{~A}-8 \mathrm{H}-3,46-48$ & 75.84 & 77.60 & 76.72 & 75.84 & 77.60 & 76.72 & 0.88 \\
\hline Nigrini et al., 2006 & T Lychnocanoma amphitrite & & & $1220 \mathrm{~A}-8 \mathrm{H}-2,20-22$ & $1220 \mathrm{~A}-8 \mathrm{H}-3,46-48$ & 75.84 & 77.60 & 76.72 & 75.84 & 77.60 & 76.72 & 0.88 \\
\hline Nigrini et al., 2006 & T Lophocyrtis (L.) exitelus & & & $1220 \mathrm{~A}-8 \mathrm{H}-2,20-22$ & $1220 \mathrm{~A}-8 \mathrm{H}-3,46-48$ & 75.84 & 77.60 & 76.72 & 75.84 & 77.60 & 76.72 & 0.88 \\
\hline Nigrini et al., 2006 & T Dorcadospyris copelata n. sp. & & & $1220 \mathrm{~A}-8 \mathrm{H}-2,20-22$ & $1220 \mathrm{~A}-8 \mathrm{H}-3,46-48$ & 75.84 & 77.60 & 76.72 & 75.84 & 77.60 & 76.72 & 0.88 \\
\hline Nigrini et al., 2006 & T Zealithapium mitra & & & $1220 \mathrm{~A}-8 \mathrm{H}-3,46-48$ & 1220B-4H-CC & 77.60 & 79.08 & 78.34 & 77.60 & 79.15 & 78.38 & 0.78 \\
\hline Nigrini et al., 2006 & T Artophormis barbadensis & & & $1220 \mathrm{~A}-8 \mathrm{H}-3,46-48$ & 1220B-4H-CC & 77.60 & 79.08 & 78.34 & 77.60 & 79.15 & 78.38 & 0.78 \\
\hline Nigrini et al., 2006 & $\mathrm{~T}$ Thyrsocyrtis (T.) bromia (max) & & & & & & & & & & & \\
\hline Nigrini et al., 2006 & T Thyrsocyrtis $(P$.$) lochites (max)$ & & & $1220 \mathrm{~A}-8 \mathrm{H}-2,20-22$ & $1220 \mathrm{~A}-8 \mathrm{H}-3,46-48$ & 75.84 & 77.60 & 76.72 & 75.84 & 77.60 & 76.72 & 0.88 \\
\hline Nigrini et al., 2006 & B Lithocyclia angusta & & & $1220 \mathrm{~A}-8 \mathrm{H}-3,46-48$ & 1220B-4H-CC & 77.60 & 79.08 & 78.34 & 77.60 & 79.15 & 78.38 & 0.78 \\
\hline Nigrini et al., 2006 & B Lophocyrtis (C.) milowi & & & $1220 \mathrm{~A}-8 \mathrm{H}-3,46-48$ & 1220B-4H-CC & 77.60 & 79.08 & 78.34 & 77.60 & 79.15 & 78.38 & 0.78 \\
\hline Nigrini et al., 2006 & T Thyrsocyrtis (P.) tetracantha (max) & & & $1220 \mathrm{~A}-8 \mathrm{H}-3,46-48$ & 1220B-4H-CC & 77.60 & 79.08 & 78.34 & 77.60 & 79.15 & 78.38 & 0.78 \\
\hline Nigrini et al., 2006 & B Pteropilium sp.aff. Pterocanium contiguum & & & $1220 \mathrm{~A}-8 \mathrm{H}-4,46-48$ & $1220 \mathrm{~A}-8 \mathrm{H}-5,46-48$ & 79.10 & 80.60 & 79.85 & 79.10 & 80.60 & 79.85 & 0.75 \\
\hline Nigrini et al., 2006 & T Dorcadospyris ombros n. sp. & & & $1220 \mathrm{~A}-8 \mathrm{H}-2,20-22$ & $1220 \mathrm{~A}-8 \mathrm{H}-3,46-48$ & 75.84 & 77.60 & 76.72 & 75.84 & 77.60 & 76.72 & 0.88 \\
\hline Nigrini et al., 2006 & B Lophocyrtis (L.) exitelus & & & $1220 \mathrm{~A}-8 \mathrm{H}-5,46-48$ & $1220 \mathrm{~A}-8 \mathrm{H}-6,46-48$ & 80.60 & 82.10 & 81.35 & 80.60 & 82.10 & 81.35 & 0.75 \\
\hline Nigrini et al., 2006 & T Calocyclas bandyca & & & $1220 \mathrm{~A}-8 \mathrm{H}-4,46-48$ & $1220 \mathrm{~A}-8 \mathrm{H}-5,46-48$ & 79.10 & 80.60 & 79.85 & 79.10 & 80.60 & 79.85 & 0.75 \\
\hline Nigrini et al., 2006 & T Calocyclas turris & & & $1220 \mathrm{~A}-8 \mathrm{H}-5,46-48$ & $1220 \mathrm{~A}-8 \mathrm{H}-6,46-48$ & 80.60 & 82.10 & 81.35 & 80.60 & 82.10 & 81.35 & 0.75 \\
\hline Nigrini et al., 2006 & T Cryptocarpium azyx & & & $1220 \mathrm{~A}-8 \mathrm{H}-7,46-48$ & $1220 \mathrm{~A}-8 \mathrm{H}-\mathrm{CC}$ & 83.60 & 84.12 & 83.86 & 83.79 & 84.31 & 84.05 & 0.26 \\
\hline Nigrini et al., 2006 & T Thyrsocyrtis (T.) bromia (continuous) & & & $1220 \mathrm{~A}-8 \mathrm{H}-5,46-48$ & $1220 \mathrm{~A}-8 \mathrm{H}-6,46-48$ & 80.60 & 82.10 & 81.35 & 80.60 & 82.10 & 81.35 & 0.75 \\
\hline Nigrini et al., 2006 & T Thyrsocyrtis $(P$.$) tetracantha (continuous)$ & & & $1220 \mathrm{~A}-8 \mathrm{H}-6,46-48$ & $1220 \mathrm{~A}-8 \mathrm{H}-7,46-48$ & 82.10 & 83.60 & 82.85 & 82.10 & 83.79 & 82.95 & 0.85 \\
\hline Nigrini et al., 2006 & B Lophocyrtis (C.) hadra & \multirow{11}{*}{ RP18 } & & $1220 \mathrm{~A}-8 \mathrm{H}-7,46-48$ & $1220 \mathrm{~A}-8 \mathrm{H}-\mathrm{CC}$ & 83.60 & 84.12 & 83.86 & 83.79 & 84.31 & 84.05 & 0.26 \\
\hline Nigrini et al., 2006 & T Thyrsocyrtis (P.) lochites (continuous) & & & $1220 \mathrm{~A}-8 \mathrm{H}-7,46-48$ & $1220 \mathrm{~A}-8 \mathrm{H}-\mathrm{CC}$ & 83.60 & 84.12 & 83.86 & 83.79 & 84.31 & 84.05 & 0.26 \\
\hline Nigrini et al., 2006 & T Thyrsocyrtis (T.) rhizodon & & & $1220 \mathrm{~A}-8 \mathrm{H}-\mathrm{CC}$ & $1220 \mathrm{~A}-9 \mathrm{H}-2,45-47$ & 84.12 & 86.44 & 85.28 & 84.31 & 86.61 & 85.46 & 1.15 \\
\hline Nigrini et al., 2006 & T Thyrsocyrtis $(P$.$) krooni$ & & & $1220 \mathrm{~A}-8 \mathrm{H}-\mathrm{CC}$ & $1220 \mathrm{~A}-9 \mathrm{H}-2,45-47$ & 84.12 & 86.44 & 85.28 & 84.31 & 86.61 & 85.46 & 1.15 \\
\hline Nigrini et al., 2006 & T Thyrsocyrtis (P.) triacantha (continuous) & & & $1220 \mathrm{~A}-9 \mathrm{H}-2,45-47$ & $1220 \mathrm{~A}-9 \mathrm{H}-4,45-47$ & 86.44 & 89.44 & 87.94 & 86.61 & 89.60 & 88.11 & 1.49 \\
\hline Nigrini et al., 2006 & T Artophormis dominasinensis & & & $1220 \mathrm{~A}-9 \mathrm{H}-2,45-47$ & $1220 \mathrm{~A}-9 \mathrm{H}-4,45-47$ & 86.44 & 89.44 & 87.94 & 86.61 & 89.60 & 88.11 & 1.49 \\
\hline Nigrini et al., 2006 & T Eusyringium fistuligerum & & & $1220 \mathrm{~A}-8 \mathrm{H}-6,46-48$ & $1220 \mathrm{~A}-8 \mathrm{H}-7,46-48$ & 82.10 & 83.60 & 82.85 & 82.10 & 83.79 & 82.95 & 0.85 \\
\hline Nigrini et al., 2006 & B Artophormis gracilis & & & $1220 \mathrm{~A}-9 \mathrm{H}-6,45-47$ & $1220 \mathrm{~A}-9 \mathrm{H}-7,45-47$ & 92.44 & 93.94 & 93.19 & 92.60 & 94.10 & 93.35 & 0.75 \\
\hline Nigrini et al., 2006 & T Lychnocanoma turgidum & & & $1220 \mathrm{~A}-9 \mathrm{H}-2,45-47$ & $1220 \mathrm{~A}-9 \mathrm{H}-4,45-47$ & 86.44 & 89.44 & 87.94 & 86.61 & 89.60 & 88.11 & 1.49 \\
\hline Nigrini et al., 2006 & B Calocycletta (C.) anekathen n. sp. & & & $1220 \mathrm{~A}-9 \mathrm{H}-2,45-47$ & $1220 \mathrm{~A}-9 \mathrm{H}-4,45-47$ & 86.44 & 89.44 & 87.94 & 86.61 & 89.60 & 88.11 & 1.49 \\
\hline Nigrini et al., 2006 & T Theocyrtis perpumila n. sp. & & & $1220 \mathrm{~A}-9 \mathrm{H}-4,45-47$ & 1220B-5H-CC & 89.44 & 90.46 & 89.95 & 89.60 & 90.69 & 90.15 & 0.55 \\
\hline
\end{tabular}




\begin{tabular}{|c|c|c|c|c|c|c|c|c|c|c|c|c|}
\hline \multirow[b]{2}{*}{ Source } & \multirow[b]{2}{*}{ Event } & \multirow[b]{2}{*}{ Zone } & \multirow{2}{*}{$\begin{array}{l}\mathrm{Age}^{*} \\
(\mathrm{Ma})\end{array}$} & \multicolumn{2}{|c|}{ Hole, core, section, interval $(\mathrm{cm})$} & \multicolumn{3}{|c|}{$\begin{array}{c}\text { Depth from } \\
\text { Pälike et al. (2005) (rmcd) }\end{array}$} & \multicolumn{3}{|c|}{$\begin{array}{c}\text { Depth } \\
\text { adjusted (rmcd) }\end{array}$} & \multirow{2}{*}{$\begin{array}{c}\text { Error } \\
( \pm) \\
(\mathrm{m})\end{array}$} \\
\hline & & & & Top & Bottom & Top & Bottom & Mean & Top & Bottom & Mean & \\
\hline Nigrini et al., 2006 & B Lychnocanoma amphitrite & RP18 & & $1220 \mathrm{~A}-9 \mathrm{H}-6,45-47$ & $1220 \mathrm{~A}-9 \mathrm{H}-7,45-47$ & 92.44 & 93.94 & 93.19 & 92.60 & 94.10 & 93.35 & 0.75 \\
\hline Nigrini et al., 2006 & B Calocyclas bandyca & & & $1220 \mathrm{~A}-9 \mathrm{H}-6,45-47$ & $1220 \mathrm{~A}-9 \mathrm{H}-7,45-47$ & 92.44 & 93.94 & 93.19 & 92.60 & 94.10 & 93.35 & 0.75 \\
\hline Nigrini et al., 2006 & B Lophocyrtis (L.) jacchia & & & $1220 \mathrm{~A}-9 \mathrm{H}-\mathrm{CC}$ & $1220 \mathrm{~A}-10 \mathrm{H}-2,45-47$ & 94.35 & 96.77 & 95.56 & 94.51 & 97.06 & 95.79 & 1.28 \\
\hline Nigrini et al., 2006 & T Thyrsocyrtis $(P$.$) orthotenes \mathrm{n} . \mathrm{sp}$. & RP17 & & $1220 \mathrm{~A}-9 \mathrm{H}-\mathrm{CC}$ & $1220 \mathrm{~A}-10 \mathrm{H}-2,45-47$ & 94.35 & 96.77 & 95.56 & 94.51 & 97.06 & 95.79 & 1.28 \\
\hline Nigrini et al., 2006 & T Podocyrtis (P.) papalis (continuous) & & & $1220 \mathrm{~A}-10 \mathrm{H}-2,45-47$ & $1220 \mathrm{~A}-10 \mathrm{H}-4,45-47$ & 96.77 & 99.77 & 98.27 & 97.06 & 100.06 & 98.56 & 1.50 \\
\hline Nigrini et al., 2006 & B Cryptocarpium azyx & & & $1220 \mathrm{~A}-10 \mathrm{H}-2,45-47$ & $1220 \mathrm{~A}-10 \mathrm{H}-4,45-47$ & 96.77 & 99.77 & 98.27 & 97.06 & 100.06 & 98.56 & 1.50 \\
\hline Nigrini et al., 2006 & B Thyrsocyrtis (P.) lochites & \multirow{31}{*}{ RP16 } & & $1220 \mathrm{~A}-10 \mathrm{H}-2,45-47$ & $1220 \mathrm{~A}-10 \mathrm{H}-4,45-47$ & 96.77 & 99.77 & 98.27 & 97.06 & 100.06 & 98.56 & 1.50 \\
\hline Nigrini et al., 2006 & T Anthocyrtoma spp. & & & $1220 \mathrm{~A}-10 \mathrm{H}-4,45-47$ & $1220 \mathrm{~A}-10 \mathrm{H}-5,45-47$ & 99.77 & 101.27 & 100.52 & 100.06 & 101.38 & 100.72 & 0.66 \\
\hline Nigrini et al., 2006 & B Dorcadospyris copelata n. sp. & & & $1220 \mathrm{~A}-10 \mathrm{H}-2,45-47$ & $1220 \mathrm{~A}-10 \mathrm{H}-4,45-47$ & 96.77 & 99.77 & 98.27 & 97.06 & 100.06 & 98.56 & 1.50 \\
\hline Nigrini et al., 2006 & T Calocycloma ampulla & & & $1220 \mathrm{~A}-10 \mathrm{H}-4,45-47$ & $1220 \mathrm{~A}-10 \mathrm{H}-5,45-47$ & 99.77 & 101.27 & 100.52 & 100.06 & 101.38 & 100.72 & 0.66 \\
\hline Nigrini et al., 2006 & T Rhopalocanium ornatum & & & $1220 \mathrm{~A}-10 \mathrm{H}-6,45-47$ & $1220 \mathrm{~A}-10 \mathrm{H}-\mathrm{CC}$ & 102.77 & 104.51 & 103.64 & 102.72 & 104.41 & 103.56 & 0.85 \\
\hline Nigrini et al., 2006 & B Botryocella sp. gr. & & & $1220 \mathrm{~A}-11 \mathrm{H}-4,45-47$ & $1220 \mathrm{~A}-11 \mathrm{H}-6,45-47$ & 109.37 & 112.37 & 110.87 & 109.55 & 112.21 & 110.88 & 1.33 \\
\hline Nigrini et al., 2006 & T Spongatractus pachystylus & & & $1220 \mathrm{~A}-10 \mathrm{H}-2,45-47$ & $1220 \mathrm{~A}-10 \mathrm{H}-4,45-47$ & 96.77 & 99.77 & 98.27 & 97.06 & 100.06 & 98.56 & 1.50 \\
\hline Nigrini et al., 2006 & B Thyrsocyrtis (T.) bromia & & & $1220 \mathrm{~A}-10 \mathrm{H}-4,45-47$ & $1220 \mathrm{~A}-10 \mathrm{H}-5,45-47$ & 99.77 & 101.27 & 100.52 & 100.06 & 101.38 & 100.72 & 0.66 \\
\hline Nigrini et al., 2006 & B Thyrsocyrtis $(P$.$) tetracantha$ & & & $1220 \mathrm{~A}-10 \mathrm{H}-4,45-47$ & $1220 \mathrm{~A}-10 \mathrm{H}-5,45-47$ & 99.77 & 101.27 & 100.52 & 100.06 & 101.38 & 100.72 & 0.66 \\
\hline Nigrini et al., 2006 & B Theocyrtis careotuberosa $\mathrm{n}$. sp. & & & $1220 \mathrm{~A}-10 \mathrm{H}-5,45-47$ & 1220B-6H-CC & 101.27 & 101.52 & 101.40 & 101.38 & 101.81 & 101.59 & 0.22 \\
\hline Nigrini et al., 2006 & T Zygocircus cimelium & & & $1220 \mathrm{~A}-10 \mathrm{H}-2,45-47$ & $1220 \mathrm{~A}-10 \mathrm{H}-4,45-47$ & 96.77 & 99.77 & 98.27 & 97.06 & 100.06 & 98.56 & 1.50 \\
\hline Nigrini et al., 2006 & B Dorcadospyris ombros $\mathrm{n}$. sp. & & & $1220 \mathrm{~A}-10 \mathrm{H}-5,45-47$ & $1220 \mathrm{~B}-6 \mathrm{H}-\mathrm{CC}$ & 101.27 & 101.52 & 101.40 & 101.38 & 101.81 & 101.59 & 0.22 \\
\hline Nigrini et al., 2006 & B Artophormis dominasinensis & & & $1220 \mathrm{~A}-10 \mathrm{H}-4,45-47$ & $1220 \mathrm{~A}-10 \mathrm{H}-5,45-47$ & 99.77 & 101.27 & 100.52 & 100.06 & 101.38 & 100.72 & 0.66 \\
\hline Nigrini et al., 2006 & B Tristylospyris triceros & & & $1220 \mathrm{~A}-10 \mathrm{H}-4,45-47$ & $1220 \mathrm{~A}-10 \mathrm{H}-5,45-47$ & 99.77 & 101.27 & 100.52 & 100.06 & 101.38 & 100.72 & 0.66 \\
\hline Nigrini et al., 2006 & T Theocotylissa ficus & & & $1220 \mathrm{~A}-10 \mathrm{H}-5,45-47$ & $1220 \mathrm{~B}-6 \mathrm{H}-\mathrm{CC}$ & 101.27 & 101.52 & 101.40 & 101.38 & 101.81 & 101.59 & 0.22 \\
\hline Nigrini et al., 2006 & T Dorcadospyris anastasis n. sp. & & & $1220 \mathrm{~A}-10 \mathrm{H}-5,45-47$ & 1220B-6H-CC & 101.27 & 101.52 & 101.40 & 101.38 & 101.81 & 101.59 & 0.22 \\
\hline Nigrini et al., 2006 & B Calocyclas turris & & & 1220A-10H-CC & $1220 \mathrm{~A}-11 \mathrm{H}-2,45-47$ & 104.51 & 106.37 & 105.44 & 104.41 & 106.55 & 105.48 & 1.07 \\
\hline Nigrini et al., 2006 & T Podocyrtis (L.) chalara (continuous) & & & 1220A-10H-CC & $1220 \mathrm{~A}-11 \mathrm{H}-2,45-47$ & 104.51 & 106.37 & 105.44 & 104.41 & 106.55 & 105.48 & 1.07 \\
\hline Nigrini et al., 2006 & T Podocyrtis (L.) goetheana continuous) & & & $1220 \mathrm{~A}-10 \mathrm{H}-6,45-47$ & $1220 \mathrm{~A}-10 \mathrm{H}-\mathrm{CC}$ & 102.77 & 104.51 & 103.64 & 102.72 & 104.41 & 103.56 & 0.85 \\
\hline Nigrini et al., 2006 & T Dictyophimus craticula & & & $1220 \mathrm{~A}-10 \mathrm{H}-2,45-47$ & $1220 \mathrm{~A}-10 \mathrm{H}-4,45-47$ & 96.77 & 99.77 & 98.27 & 97.06 & 100.06 & 98.56 & 1.50 \\
\hline Nigrini et al., 2006 & B Thyrsocyrtis (P.) krooni & & & $1220 \mathrm{~A}-11 \mathrm{H}-6,45-47$ & 1220B-7H-CC & 112.37 & 112.73 & 112.55 & 112.21 & 112.66 & 112.43 & 0.22 \\
\hline Nigrini et al., 2006 & T Podocyrtis (P.) apeza & & & $1220 \mathrm{~A}-10 \mathrm{H}-5,45-47$ & 1220B-6H-CC & 101.27 & 101.52 & 101.40 & 101.38 & 101.81 & 101.59 & 0.22 \\
\hline Nigrini et al., 2006 & B Dictyoprora pirum & & & $1220 \mathrm{~A}-11 \mathrm{H}-4,45-47$ & $1220 \mathrm{~A}-11 \mathrm{H}-6,45-47$ & 109.37 & 112.37 & 110.87 & 109.64 & 112.21 & 110.92 & 1.29 \\
\hline Nigrini et al., 2006 & T Lithochytris vespertilio & & & $1220 \mathrm{~A}-11 \mathrm{H}-4,45-47$ & $1220 \mathrm{~A}-11 \mathrm{H}-6,45-47$ & 109.37 & 112.37 & 110.87 & 109.64 & 112.21 & 110.92 & 1.29 \\
\hline Nigrini et al., 2006 & T Sethochytris triconiscus & & & $1220 \mathrm{~A}-11 \mathrm{H}-4,45-47$ & $1220 \mathrm{~A}-11 \mathrm{H}-6,45-47$ & 109.37 & 112.37 & 110.87 & 109.64 & 112.21 & 110.92 & 1.29 \\
\hline Nigrini et al., 2006 & B Lithocyclia aristotelis gr. & & & $1220 \mathrm{~A}-11 \mathrm{H}-4,45-47$ & $1220 \mathrm{~A}-11 \mathrm{H}-6,45-47$ & 109.37 & 112.37 & 110.87 & 109.64 & 112.21 & 110.92 & 1.29 \\
\hline Nigrini et al., 2006 & T Dorcadospyris ombros n. sp. & & & $1220 \mathrm{~A}-11 \mathrm{H}-4,45-47$ & $1220 \mathrm{~A}-11 \mathrm{H}-6,45-47$ & 109.37 & 112.37 & 110.87 & 109.64 & 112.21 & 110.92 & 1.29 \\
\hline Nigrini et al., 2006 & T Podocyrtis (L.) mitra & & & 1220B-7H-CC & $1220 \mathrm{~A}-11 \mathrm{H}-7,45-47$ & 112.73 & 113.87 & 113.30 & 112.66 & 113.87 & 113.27 & 0.61 \\
\hline Nigrini et al., 2006 & B Dorcadospyris anastasis $\mathrm{n} . \mathrm{sp}$. & & & 1220B-7H-CC & $1220 \mathrm{~A}-11 \mathrm{H}-7,45-47$ & 112.73 & 113.87 & 113.30 & 112.66 & 113.87 & 113.27 & 0.61 \\
\hline Nigrini et al., 2006 & B Dictyoprora armadillo & & & 1220B-7H-CC & $1220 \mathrm{~A}-11 \mathrm{H}-7,45-47$ & 112.73 & 113.87 & 113.30 & 112.66 & 113.87 & 113.27 & 0.61 \\
\hline Nigrini et al., 2006 & B Podocyrtis (L.) goetheana & & & 1220A-11H-CC & $1220 \mathrm{~A}-12 \mathrm{H}-2,45-47$ & 114.51 & 121.32 & 117.92 & 114.77 & 120.55 & 117.66 & 2.89 \\
\hline Nigrini et al., 2006 & T Lophocyrtis biaurita & \multirow{3}{*}{ RP15 } & & $1220 \mathrm{~A}-11 \mathrm{H}-\mathrm{CC}$ & $1220 \mathrm{~A}-12 \mathrm{H}-2,45-47$ & 114.51 & 121.32 & 117.92 & 114.77 & 120.55 & 117.66 & 2.89 \\
\hline Nigrini et al., 2006 & T Podocyrtis (L.) trachodes & & & $1220 \mathrm{~A}-12 \mathrm{H}-2,45-47$ & 1220B-8H-CC & 121.32 & 123.71 & 122.52 & 120.55 & 123.46 & 122.00 & 1.45 \\
\hline Nigrini et al., 2006 & Podocyrtis (L.) mitra > Podocyrtis (L.) chalara & & & $1220 \mathrm{~B}-9 \mathrm{H}-1,45-47$ & $1220 \mathrm{~A}-12 \mathrm{H}-5,45-47$ & 125.38 & 125.82 & 125.60 & 124.40 & 125.05 & 124.73 & 0.32 \\
\hline Nigrini et al., 2006 & B Dorcadospyris ombros n. sp. (lower) & \multirow{10}{*}{ RP14 } & & $1220 \mathrm{~B}-9 \mathrm{H}-2,45-47$ & $1220 \mathrm{~A}-12 \mathrm{H}-6,45-47$ & 126.88 & 127.33 & 127.11 & 125.90 & 126.50 & 126.20 & 0.30 \\
\hline Nigrini et al., 2006 & B Artophormis barbadensis & & & $1220 \mathrm{~A}-12 \mathrm{H}-6,45-47$ & $1220 \mathrm{~A}-12 \mathrm{H}-\mathrm{CC}$ & 127.33 & 129.20 & 128.27 & 126.50 & 128.26 & 127.38 & 0.88 \\
\hline Nigrini et al., 2006 & B Podocyrtis (P.) apeza & & & $1220 \mathrm{~A}-12 \mathrm{H}-\mathrm{CC}$ & $1220 \mathrm{~B}-9 \mathrm{H}-4,45-47$ & 129.20 & 129.88 & 129.54 & 128.26 & 128.90 & 128.58 & 0.32 \\
\hline Nigrini et al., 2006 & B Podocyrtis (L.) chalara & & & $1220 \mathrm{~A}-12 \mathrm{H}-5,45-47$ & $1220 \mathrm{~B}-9 \mathrm{H}-2,45-47$ & 125.82 & 126.88 & 126.35 & 125.05 & 125.90 & 125.48 & 0.43 \\
\hline Nigrini et al., 2006 & T Podocyrtis (P.) ampla & & & $1220 \mathrm{~A}-12 \mathrm{H}-5,45-47$ & $1220 \mathrm{~B}-9 \mathrm{H}-2,45-47$ & 125.82 & 126.88 & 126.35 & 125.05 & 125.90 & 125.48 & 0.43 \\
\hline Nigrini et al., 2006 & B Zealithapium mitra & & & $1220 \mathrm{~A}-12 \mathrm{H}-6,45-47$ & $1220 \mathrm{~A}-12 \mathrm{H}-\mathrm{CC}$ & 127.33 & 129.20 & 128.27 & 126.50 & 128.26 & 127.38 & 0.88 \\
\hline Nigrini et al., 2006 & T Zealithapium anoectum & & & $1220 \mathrm{~A}-12 \mathrm{H}-6,45-47$ & $1220 \mathrm{~A}-12 \mathrm{H}-\mathrm{CC}$ & 127.33 & 129.20 & 128.27 & 126.50 & 128.26 & 127.38 & 0.88 \\
\hline Nigrini et al., 2006 & B Cryptocarpium ornatum & & & $1220 \mathrm{~A}-12 \mathrm{H}-6,45-47$ & $1220 \mathrm{~A}-12 \mathrm{H}-\mathrm{CC}$ & 127.33 & 129.20 & 128.27 & 126.50 & 128.26 & 127.38 & 0.88 \\
\hline Nigrini et al., 2006 & B Sethochytris triconiscus & & & 1220B-9H-4, 45-47 & 1220B-9H-6, 45-47 & 129.88 & 132.88 & 131.38 & 128.90 & 131.98 & 130.44 & 1.54 \\
\hline Nigrini et al., 2006 & T Eusyringium lagena & & & $1220 \mathrm{~B}-9 \mathrm{H}-4,45-47$ & 1220B-9H-6, 45-47 & 129.88 & 132.88 & 131.38 & 128.90 & 131.98 & 130.44 & 1.54 \\
\hline
\end{tabular}




\begin{tabular}{|c|c|c|c|c|c|c|c|c|c|c|c|c|}
\hline \multirow[b]{2}{*}{ Source } & \multirow[b]{2}{*}{ Event } & \multirow[b]{2}{*}{ Zone } & \multirow{2}{*}{$\begin{array}{l}\text { Age* } \\
(\mathrm{Ma})\end{array}$} & \multicolumn{2}{|c|}{ Hole, core, section, interval $(\mathrm{cm})$} & \multicolumn{3}{|c|}{$\begin{array}{c}\text { Depth from } \\
\text { Pälike et al. (2005) (rmcd) }\end{array}$} & \multicolumn{3}{|c|}{$\begin{array}{c}\text { Depth } \\
\text { adjusted (rmcd) }\end{array}$} & \multirow{2}{*}{$\begin{array}{c}\text { Error } \\
( \pm) \\
(\mathrm{m})\end{array}$} \\
\hline & & & & Top & Bottom & Top & Bottom & Mean & Top & Bottom & Mean & \\
\hline Nigrini et al., 2006 & T Podocyrtis (L.) fasciolata & \multirow{9}{*}{ RP14 } & & 1220B-9H-6, 45-47 & 1220B-9H-CC & 132.88 & 134.12 & 133.50 & 131.98 & 133.26 & 132.62 & 0.64 \\
\hline Nigrini et al., 2006 & T Zealithapium plegmacantha & & & 1220B-9H-6, 45-47 & 1220B-9H-CC & 132.88 & 134.12 & 133.50 & 131.98 & 133.26 & 132.62 & 0.64 \\
\hline Nigrini et al., 2006 & T Podocyrtis (L.) sinuosa & & & $1220 \mathrm{~B}-9 \mathrm{H}-\mathrm{CC}$ & $1220 \mathrm{~B}-10 \mathrm{H}-2,45-47$ & 134.12 & 136.08 & 135.10 & 133.26 & 135.19 & 134.22 & 0.97 \\
\hline Nigrini et al., 2006 & Zealithapium plegmacantha $>$ Zealithapium anoectum & & & 1220B-9H-CC & $1220 \mathrm{~B}-10 \mathrm{H}-4,45-47$ & 134.12 & 139.02 & 136.57 & 133.26 & 138.13 & 135.69 & 2.44 \\
\hline Nigrini et al., 2006 & B Theocyrtis perpumila $\mathrm{n} . \mathrm{sp}$. & & & $1220 \mathrm{~B}-9 \mathrm{H}-\mathrm{CC}$ & $1220 \mathrm{~B}-10 \mathrm{H}-2,45-47$ & 134.12 & 136.08 & 135.10 & 133.26 & 135.19 & 134.22 & 0.97 \\
\hline Nigrini et al., 2006 & T Podocyrtis (L.) helenae & & & 1220B-9H-CC & $1220 \mathrm{~B}-10 \mathrm{H}-2,45-47$ & 134.12 & 136.08 & 135.10 & 133.26 & 135.19 & 134.22 & 0.97 \\
\hline Nigrini et al., 2006 & B Lychnocanoma turgidum & & & $1220 \mathrm{~B}-9 \mathrm{H}-\mathrm{CC}$ & $1220 \mathrm{~B}-10 \mathrm{H}-2,45-47$ & 134.12 & 136.08 & 135.10 & 133.26 & 135.19 & 134.22 & 0.97 \\
\hline Nigrini et al., 2006 & Eusyringium lagena $>$ Eusyringium fistuligerum & & & $1220 \mathrm{~B}-10 \mathrm{H}-3,45-47$ & $1220 \mathrm{~B}-10 \mathrm{H}-4,45-47$ & 137.52 & 139.02 & 138.27 & 136.63 & 138.13 & 137.38 & 0.75 \\
\hline Nigrini et al., 2006 & Podocyrtis (L.) sinuosa ?Podocyrtis (L.) mitra & & & 1220B-10H-3, 45-47 & 1220B-10H-4, 45-47 & 137.52 & 139.02 & 138.27 & 136.63 & 138.13 & 137.38 & 0.75 \\
\hline Nigrini et al., 2006 & B Podocyrtis (L.) trachodes & \multirow{8}{*}{ RP13 } & & 1220B-10H-4, 45-47 & $1220 \mathrm{~B}-10 \mathrm{H}-6,45-47$ & 139.02 & 142.02 & 140.52 & 138.13 & 141.13 & 139.63 & 1.50 \\
\hline Nigrini et al., 2006 & B Zygocircus cimelium & & & $1220 \mathrm{~B}-9 \mathrm{H}-\mathrm{CC}$ & $1220 \mathrm{~B}-10 \mathrm{H}-2,45-47$ & 134.12 & 136.08 & 135.10 & 133.26 & 135.19 & 134.22 & 0.97 \\
\hline Nigrini et al., 2006 & B Podocyrtis (L.) fasciolata & & & 1220B-10H-3, 45-47 & $1220 \mathrm{~B}-10 \mathrm{H}-4,45-47$ & 137.52 & 139.02 & 138.27 & 136.63 & 138.13 & 137.38 & 0.75 \\
\hline Nigrini et al., 2006 & B Podocyrtis (L.) helenae & & & $1220 \mathrm{~B}-10 \mathrm{H}-6,45-47$ & 1220B-10H-CC & 142.02 & 144.25 & 143.14 & 141.13 & 143.36 & 142.25 & 1.12 \\
\hline Nigrini et al., 2006 & T Podocyrtis (P.) diamesa & & & 1220B-10H-6, 45-47 & 1220B-10H-CC & 142.02 & 144.25 & 143.14 & 141.13 & 143.36 & 142.25 & 1.12 \\
\hline Nigrini et al., 2006 & T Podocyrtis (P.) phyxis & & & 1220B-10H-6, 45-47 & 1220B-10H-CC & 142.02 & 144.25 & 143.14 & 141.13 & 143.36 & 142.25 & 1.12 \\
\hline Nigrini et al., 2006 & T Spongatractus balbis & & & 1220B-11H-1, 45-47 & $1220 \mathrm{~B}-11 \mathrm{H}-2,45-47$ & 148.64 & 150.14 & 149.39 & 147.75 & 149.25 & 148.50 & 0.75 \\
\hline Nigrini et al., 2006 & B Podocyrtis (L.) mitra & & & $1220 \mathrm{~B}-11 \mathrm{H}-1,45-47$ & $1220 \mathrm{~B}-11 \mathrm{H}-2,45-47$ & 148.64 & 150.14 & 149.39 & 147.75 & 149.25 & 148.50 & 0.75 \\
\hline Nigrini et al., 2006 & Podocyrtis (P.) phyxis > Podocyrtis (P.) ampla & \multirow{23}{*}{ RP12 } & & 1220B-11H-1, 45-47 & 1220B-11H-2, 45-47 & 148.64 & 150.14 & 149.39 & 147.75 & 149.25 & 148.50 & 0.75 \\
\hline Nigrini et al., 2006 & B Podocyrtis $(P$.$) ampla$ & & & 1220B-11H-1, 45-47 & $1220 \mathrm{~B}-11 \mathrm{H}-2,45-47$ & 148.64 & 150.14 & 149.39 & 147.75 & 149.25 & 148.50 & 0.75 \\
\hline Nigrini et al., 2006 & B Eusyringium fistuligerum & & & 1220B-11H-2, 45-47 & 1220B-11H-4, 45-47 & 150.14 & 153.14 & 151.64 & 149.25 & 152.25 & 150.75 & 1.50 \\
\hline Nigrini et al., 2006 & T Theocotyle venezuelensis & & & 1220B-11H-2, 45-47 & $1220 \mathrm{~B}-11 \mathrm{H}-4,45-47$ & 150.14 & 153.14 & 151.64 & 149.25 & 152.25 & 150.75 & 1.50 \\
\hline Nigrini et al., 2006 & B Zealithapium anoectum & & & 1220B-11H-4, 45-47 & $1220 \mathrm{~B}-11 \mathrm{H}-6,45-47$ & 153.14 & 156.14 & 154.64 & 152.25 & 155.25 & 153.75 & 1.50 \\
\hline Nigrini et al., 2006 & T Theocotyle conica & & & 1220B-11H-4, 45-47 & $1220 \mathrm{~B}-11 \mathrm{H}-6,45-47$ & 153.14 & 156.14 & 154.64 & 152.25 & 155.25 & 153.75 & 1.50 \\
\hline Nigrini et al., 2006 & T Thyrsocyrtis $(P$.$) tensa$ & & & $1220 \mathrm{~B}-11 \mathrm{H}-6,45-47$ & $1220 \mathrm{~B}-11 \mathrm{H}-\mathrm{CC}$ & 156.14 & 158.25 & 157.20 & 155.25 & 157.36 & 156.31 & 1.06 \\
\hline Nigrini et al., 2006 & T Theocotyle cryptocephala & & & $1220 \mathrm{C}-11 \mathrm{H}-3,10-12$ & $1220 \mathrm{C}-11 \mathrm{H}-4,10-12$ & 161.95 & 163.45 & 162.70 & 161.05 & 162.55 & 161.80 & 0.75 \\
\hline Nigrini et al., 2006 & B Thyrsocyrtis $(P$.$) orthotenes \mathrm{n} . \mathrm{sp}$. & & & 1220B-11H-CC & $1220 \mathrm{C}-11 \mathrm{H}-3,10-12$ & 158.25 & 161.95 & 160.10 & 157.36 & 161.05 & 159.21 & 1.85 \\
\hline Nigrini et al., 2006 & T Thyrsocyrtis (T.) robusta & & & 1220B-11H-CC & $1220 \mathrm{C}-11 \mathrm{H}-3,10-12$ & 158.25 & 161.95 & 160.10 & 157.36 & 161.05 & 159.21 & 1.85 \\
\hline Nigrini et al., 2006 & Podocyrtis $(P$.$) diamesa >$ Podocyrtis $(P$.$) phyxis$ & & & 1220B-11H-CC & $1220 \mathrm{C}-11 \mathrm{H}-3,10-12$ & 158.25 & 161.95 & 160.10 & 157.36 & 161.05 & 159.21 & 1.85 \\
\hline Nigrini et al., 2006 & B Podocyrtis (P.) phyxis & & & 1220B-11H-CC & $1220 \mathrm{C}-11 \mathrm{H}-3,10-12$ & 158.25 & 161.95 & 160.10 & 157.36 & 161.05 & 159.21 & 1.85 \\
\hline Nigrini et al., 2006 & T Periphaena tripyramis triangula & & & $1220 \mathrm{~B}-11 \mathrm{H}-4,45-47$ & $1220 \mathrm{~B}-11 \mathrm{H}-6,45-47$ & 153.14 & 156.13 & 154.64 & 152.25 & 155.25 & 153.75 & 1.50 \\
\hline Nigrini et al., 2006 & T Periphaena delta & & & $1220 \mathrm{C}-11 \mathrm{H}-3,10-12$ & $1220 \mathrm{C}-11 \mathrm{H}-4,10-12$ & 161.95 & 163.45 & 162.70 & 161.05 & 162.55 & 161.80 & 0.75 \\
\hline Nigrini et al., 2006 & T Lamptonium fab. chaunothorax & & & 1220B-11H-CC & $1220 \mathrm{C}-11 \mathrm{H}-3,10-12$ & 158.25 & 161.95 & 160.10 & 157.36 & 161.05 & 159.21 & 1.85 \\
\hline Nigrini et al., 2006 & T Theocorys anaclasta & & & 1220B-11H-CC & $1220 \mathrm{C}-11 \mathrm{H}-3,10-12$ & 158.25 & 161.95 & 160.10 & 157.36 & 161.05 & 159.21 & 1.85 \\
\hline Nigrini et al., 2006 & T Lamptonium fab. constrictum & & & 1220B-11H-CC & $1220 \mathrm{C}-11 \mathrm{H}-3,10-12$ & 158.25 & 161.95 & 160.10 & 157.36 & 161.05 & 159.21 & 1.85 \\
\hline Nigrini et al., 2006 & T Lamptonium fab. fabaeforme & & & $1220 \mathrm{C}-11 \mathrm{H}-3,10-12$ & $1220 \mathrm{C}-11 \mathrm{H}-4,10-12$ & 161.95 & 163.45 & 162.70 & 161.05 & 162.55 & 161.80 & 0.75 \\
\hline Nigrini et al., 2006 & T Thyrsocyrtis (T.) hirsuta & & & $1220 \mathrm{C}-11 \mathrm{H}-4,10-12$ & $1220 \mathrm{C}-11 \mathrm{H}-5,57-59$ & 163.45 & 167.82 & 165.64 & 162.55 & 164.52 & 163.54 & 0.98 \\
\hline Nigrini et al., 2006 & T Calocycloma castum & & & $1220 \mathrm{~B}-12 \mathrm{H}-\mathrm{CC}$ & $1220 \mathrm{~B}-13 \mathrm{H}-1,45-47$ & 168.99 & 169.16 & 169.08 & 168.10 & 168.27 & 168.19 & 0.09 \\
\hline Nigrini et al., 2006 & B Thyrsocyrtis (P.) triacantha & & & $1220 \mathrm{~B}-12 \mathrm{H}-\mathrm{CC}$ & $1220 \mathrm{~B}-13 \mathrm{H}-1,45-47$ & 168.99 & 169.16 & 169.08 & 168.10 & 168.27 & 168.19 & 0.09 \\
\hline Nigrini et al., 2006 & B Rhopalocanium ornatum & & & $1220 \mathrm{~B}-12 \mathrm{H}-\mathrm{CC}$ & 1220B-13H-1, 45-47 & 168.99 & 169.16 & 169.08 & 168.10 & 168.27 & 168.19 & 0.09 \\
\hline Nigrini et al., 2006 & B Eusyringium lagena & & & 1220B-12H-CC & $1220 \mathrm{~B}-13 \mathrm{H}-1,45-47$ & 168.99 & 169.16 & 169.08 & 168.10 & 168.27 & 168.19 & 0.09 \\
\hline Nigrini et al., 2006 & T Buryella clinata & \multirow{3}{*}{ RP11 } & & 1220B-13H-1, 45-47 & 1220B-13H-CC & 169.16 & 177.87 & 173.52 & 168.27 & 176.98 & 172.63 & 4.35 \\
\hline Nigrini et al., 2006 & T Phormocyrtis striata striata & & & 1220B-13H-1, 45-47 & 1220B-13H-CC & 169.16 & 177.87 & 173.52 & 168.27 & 176.98 & 172.63 & 4.35 \\
\hline Nigrini et al., 2006 & T Amphicraspedum murrayanum & & & 1220B-13H-CC & 1220B-16X-1, 100-102 & 177.87 & 188.81 & 183.34 & 176.98 & 187.92 & 182.45 & 5.47 \\
\hline Nigrini et al., 2006 & T Dendrospyris fragoides & \multirow{7}{*}{ RP11-RP8 } & & 1220B-13H-CC & 1220B-16X-1, 100-102 & 177.87 & 188.81 & 183.34 & 176.98 & 187.92 & 182.45 & 5.47 \\
\hline Nigrini et al., 2006 & B Dictyophimus craticula & & & 1220B-13H-CC & 1220B-16X-1, 100-102 & 177.87 & 188.81 & 183.34 & 176.98 & 187.92 & 182.45 & 5.47 \\
\hline Nigrini et al., 2006 & B Dictyoprora mongolfieri & & & 1220B-13H-CC & 1220B-16X-1, 100-102 & 177.87 & 188.81 & 183.34 & 176.98 & 187.92 & 182.45 & 5.47 \\
\hline Nigrini et al., 2006 & T Diploplegma somphum & & & 1220B-13H-CC & 1220B-16X-1, 100-102 & 177.87 & 188.81 & 183.34 & 176.98 & 187.92 & 182.45 & 5.47 \\
\hline Nigrini et al., 2006 & T Giraffospyris lata & & & $1220 \mathrm{~B}-13 \mathrm{H}-\mathrm{CC}$ & 1220B-16X-1, 100-102 & 177.87 & 188.81 & 183.34 & 176.98 & 187.92 & 182.45 & 5.47 \\
\hline Nigrini et al., 2006 & B Lamptonium fab. constrictum & & & 1220B-13H-CC & 1220B-16X-1, 100-102 & 177.87 & 188.81 & 183.34 & 176.98 & 187.92 & 182.45 & 5.47 \\
\hline Nigrini et al., 2006 & T Lamptonium pennatum & & & 1220B-13H-CC & 1220B-16X-1, 100-102 & 177.87 & 188.81 & 183.34 & 176.98 & 187.92 & 182.45 & 5.47 \\
\hline
\end{tabular}




\begin{tabular}{|c|c|c|c|c|c|c|c|c|c|c|c|c|}
\hline \multirow[b]{2}{*}{ Source } & \multirow[b]{2}{*}{ Event } & \multirow[b]{2}{*}{ Zone } & \multirow{2}{*}{$\begin{array}{l}\mathrm{Age}^{*} \\
(\mathrm{Ma})\end{array}$} & \multicolumn{2}{|c|}{ Hole, core, section, interval $(\mathrm{cm})$} & \multicolumn{3}{|c|}{$\begin{array}{c}\text { Depth from } \\
\text { Pälike et al. (2005) (rmcd) }\end{array}$} & \multicolumn{3}{|c|}{$\begin{array}{c}\text { Depth } \\
\text { adjusted (rmcd) }\end{array}$} & \multirow{2}{*}{$\begin{array}{l}\text { Error } \\
( \pm) \\
(\mathrm{m})\end{array}$} \\
\hline & & & & Top & Bottom & Top & Bottom & Mean & Top & Bottom & Mean & \\
\hline Nigrini et al., 2006 & T Lamptonium sanfilippoae & \multirow{25}{*}{ RP11-RP8 } & & 1220B-13H-CC & 1220B-16X-1, 100-102 & 177.87 & 188.81 & 183.34 & 176.98 & 187.92 & 182.45 & 5.47 \\
\hline Nigrini et al., 2006 & B Zealithapium plegmacantha & & & 1220B-13H-CC & 1220B-16X-1, 100-102 & 177.87 & 188.81 & 183.34 & 176.98 & 187.92 & 182.45 & 5.47 \\
\hline Nigrini et al., 2006 & $\mathrm{~T}$ Lithochytris archaea & & & 1220B-13H-CC & 1220B-16X-1, 100-102 & 177.87 & 188.81 & 183.34 & 176.98 & 187.92 & 182.45 & 5.47 \\
\hline Nigrini et al., 2006 & B Lithochytris vespertilio & & & 1220B-13H-CC & 1220B-16X-1, 100-102 & 177.87 & 188.81 & 183.34 & 176.98 & 187.92 & 182.45 & 5.47 \\
\hline Nigrini et al., 2006 & B Lithocyclia ocellus gr. & & & 1220B-13H-CC & 1220B-16X-1, 100-102 & 177.87 & 188.81 & 183.34 & 176.98 & 187.92 & 182.45 & 5.47 \\
\hline Nigrini et al., 2006 & B Lophocyrtis biaurita & & & 1220B-13H-CC & 1220B-16X-1, 100-102 & 177.87 & 188.81 & 183.34 & 176.98 & 187.92 & 182.45 & 5.47 \\
\hline Nigrini et al., 2006 & T Lychnocanoma auxilla & & & 1220B-13H-CC & 1220B-16X-1, 100-102 & 177.87 & 188.81 & 183.34 & 176.98 & 187.92 & 182.45 & 5.47 \\
\hline Nigrini et al., 2006 & B Periphaena tripyramis triangula & & & 1220B-13H-CC & 1220B-16X-1, 100-102 & 177.87 & 188.81 & 183.34 & 176.98 & 187.92 & 182.45 & 5.47 \\
\hline Nigrini et al., 2006 & T Phormocyrtis cubensis & & & 1220B-13H-CC & 1220B-16X-1, 100-102 & 177.87 & 188.81 & 183.34 & 176.98 & 187.92 & 182.45 & 5.47 \\
\hline Nigrini et al., 2006 & T Phormocyrtis striata exquisita & & & 1220B-13H-CC & 1220B-16X-1, 100-102 & 177.87 & 188.81 & 183.34 & 176.98 & 187.92 & 182.45 & 5.47 \\
\hline Nigrini et al., 2006 & B Podocyrtis (L.) sinuosa & & & 1220B-13H-CC & 1220B-16X-1, 100-102 & 177.87 & 188.81 & 183.34 & 176.98 & 187.92 & 182.45 & 5.47 \\
\hline Nigrini et al., 2006 & B Podocyrtis (P.) diamesa & & & 1220B-13H-CC & 1220B-16X-1, 100-102 & 177.87 & 188.81 & 183.34 & 176.98 & 187.92 & 182.45 & 5.47 \\
\hline Nigrini et al., 2006 & T Pterocodon tenellus & & & 1220B-13H-CC & 1220B-16X-1, 100-102 & 177.87 & 188.81 & 183.34 & 176.98 & 187.92 & 182.45 & 5.47 \\
\hline Nigrini et al., 2006 & B Spongatractus pachystylus & & & 1220B-13H-CC & 1220B-16X-1, 100-102 & 177.87 & 188.81 & 183.34 & 176.98 & 187.92 & 182.45 & 5.47 \\
\hline Nigrini et al., 2006 & B Theocorys anaclasta & & & 1220B-13H-CC & 1220B-16X-1, 100-102 & 177.87 & 188.81 & 183.34 & 176.98 & 187.92 & 182.45 & 5.47 \\
\hline Nigrini et al., 2006 & B Theocotyle conica & & & 1220B-13H-CC & 1220B-16X-1, 100-102 & 177.87 & 188.81 & 183.34 & 176.98 & 187.92 & 182.45 & 5.47 \\
\hline Nigrini et al., 2006 & B Theocotyle cryptocephala & & & 1220B-13H-CC & 1220B-16X-1, 100-102 & 177.87 & 188.81 & 183.34 & 176.98 & 187.92 & 182.45 & 5.47 \\
\hline Nigrini et al., 2006 & Theocotyle nigriniae > Theocotyle cryptocephala & & & 1220B-13H-CC & 1220B-16X-1, 100-102 & 177.87 & 188.81 & 183.34 & 176.98 & 187.92 & 182.45 & 5.47 \\
\hline Nigrini et al., 2006 & $\mathrm{~T}$ Theocotyle nigriniae & & & 1220B-13H-CC & 1220B-16X-1, 100-102 & 177.87 & 188.81 & 183.34 & 176.98 & 187.92 & 182.45 & 5.47 \\
\hline Nigrini et al., 2006 & B Theocotyle venezuelensis & & & 1220B-13H-CC & 1220B-16X-1, 100-102 & 177.87 & 188.81 & 183.34 & 176.98 & 187.92 & 182.45 & 5.47 \\
\hline Nigrini et al., 2006 & T Theocotylissa alpha & & & 1220B-13H-CC & 1220B-16X-1, 100-102 & 177.87 & 188.81 & 183.34 & 176.98 & 187.92 & 182.45 & 5.47 \\
\hline Nigrini et al., 2006 & T Theocotylissa fimbria & & & 1220B-13H-CC & 1220B-16X-1, 100-102 & 177.87 & 188.81 & 183.34 & 176.98 & 187.92 & 182.45 & 5.47 \\
\hline Nigrini et al., 2006 & B Thyrsocyrtis (T.) rhizodon & & & 1220B-13H-CC & 1220B-16X-1, 100-102 & 177.87 & 188.81 & 183.34 & 176.98 & 187.92 & 182.45 & 5.47 \\
\hline Nigrini et al., 2006 & B Thyrsocyrtis (T.) robusta & & & 1220B-13H-CC & 1220B-16X-1, 100-102 & 177.87 & 188.81 & 183.34 & 176.98 & 187.92 & 182.45 & 5.47 \\
\hline Nigrini et al., 2006 & T Buryella tetradica & & & 1220B-16X-1, 100-102 & 1220B-16X-2, 12-14 & 188.81 & 189.43 & 189.12 & 187.92 & 188.54 & 188.23 & 0.31 \\
\hline Nigrini et al., 2006 & T Lychnocanoma pileus & \multirow{15}{*}{ RP8 } & & 1220B-16X-1, 100-102 & 1220B-16X-2, 12-14 & 188.81 & 189.43 & 189.12 & 187.92 & 188.54 & 188.23 & 0.31 \\
\hline Nigrini et al., 2006 & B Periphaena delta & & & 1220B-16X-2, 12-14 & 1220B-16X-3, 45-47 & 189.43 & 191.26 & 190.35 & 188.54 & 190.37 & 189.46 & 0.92 \\
\hline Nigrini et al., 2006 & Theocotylissa alpha $>$ Theocotylissa ficus & & & 1220B-16X-3, 45-47 & 1220B-16X-4, 46-48 & 191.26 & 192.77 & 192.02 & 190.37 & 191.88 & 191.13 & 0.75 \\
\hline Nigrini et al., 2006 & B Lamptonium sanfilippoae & & & 1220B-16X-4, 46-48 & $1220 \mathrm{C}-14 \mathrm{X}-4,60-62$ & 192.77 & 194.75 & 193.76 & 191.88 & 196.82 & 194.35 & 2.47 \\
\hline Nigrini et al., 2006 & B Spongatractus balbis & & & 1220B-16X-4, 46-48 & $1220 \mathrm{C}-14 \mathrm{X}-4,60-62$ & 192.77 & 194.75 & 193.76 & 191.88 & 196.82 & 194.35 & 2.47 \\
\hline Nigrini et al., 2006 & B Thyrsocyrtis $(P$.$) tensa$ & & & 1220B-16X-6, 46-48 & 1220B-16X-7, 15-17 & 195.77 & 196.96 & 196.37 & 194.88 & 196.07 & 195.48 & 0.59 \\
\hline Nigrini et al., 2006 & T Pterocodon anteclinata & & & 1220B-16X-7, 15-17 & 1220B-16X-CC & 196.96 & 197.45 & 197.21 & 196.07 & 196.56 & 196.32 & 0.25 \\
\hline Nigrini et al., 2006 & B Lychnocanoma babylonis & & & 1220B-16X-CC & $1220 \mathrm{C}-14 \mathrm{X}-6,60-62$ & 197.45 & 197.75 & 197.60 & 196.56 & 196.85 & 196.71 & 0.14 \\
\hline Nigrini et al., 2006 & B Theocotylissa ficus & & & $1220 \mathrm{C}-14 \mathrm{X}-6,60-62$ & 1220B-18X-2, 10-12 & 197.75 & 208.21 & 202.98 & 196.85 & 207.32 & 202.09 & 5.24 \\
\hline Nigrini et al., 2006 & B Anthocyrtoma spp. & & & $1220 \mathrm{C}-14 \mathrm{X}-6,60-62$ & 1220B-18X-2, 10-12 & 197.75 & 208.21 & 202.98 & 196.85 & 207.32 & 202.09 & 5.24 \\
\hline Nigrini et al., 2006 & T Phormocyrtis turgida & & & $1220 \mathrm{C}-14 \mathrm{X}-6,60-62$ & 1220B-18X-2, 10-12 & 197.75 & 208.21 & 202.98 & 196.85 & 207.32 & 202.09 & 5.24 \\
\hline Nigrini et al., 2006 & T Pterocodon ampla & & & $1220 \mathrm{C}-14 \mathrm{X}-6,60-62$ & 1220B-18X-2, 10-12 & 197.75 & 208.21 & 202.98 & 196.85 & 207.32 & 202.09 & 5.24 \\
\hline Nigrini et al., 2006 & B Phormocyrtis striata striata & & & $1220 \mathrm{C}-14 \mathrm{X}-6,60-62$ & 1220B-18X-2, 10-12 & 197.75 & 208.21 & 202.98 & 196.85 & 207.32 & 202.09 & 5.24 \\
\hline Nigrini et al., 2006 & B Theocotylissa fimbria & & & $1220 \mathrm{C}-14 \mathrm{X}-6,60-62$ & 1220B-18X-2, 10-12 & 197.75 & 208.21 & 202.98 & 196.85 & 207.32 & 202.09 & 5.24 \\
\hline Nigrini et al., 2006 & Pterocodon anteclinata $>$ Buryella clinata & & & $1220 \mathrm{C}-14 \mathrm{X}-6,60-62$ & 1220B-18X-2, 10-12 & 197.75 & 208.21 & 202.98 & 196.85 & 207.32 & 202.09 & 5.24 \\
\hline Nigrini et al., 2006 & B Buryella clinata & RP7 & & $1220 \mathrm{C}-14 \mathrm{X}-6,60-62$ & 1220B-18X-2, 10-12 & 197.75 & 208.21 & 202.98 & 196.85 & 207.32 & 202.09 & 5.24 \\
\hline
\end{tabular}


Table T39. Site U1331 calcareous nannofossil datums.

\begin{tabular}{|c|c|c|c|c|c|c|c|c|c|c|c|c|c|c|}
\hline \multirow[b]{2}{*}{ Source } & \multirow[b]{2}{*}{ Event } & \multirow{2}{*}{$\begin{array}{l}\text { Age* }^{*} \\
(\mathrm{Ma})\end{array}$} & \multicolumn{2}{|c|}{ Core, section, interval $(\mathrm{cm})$} & \multicolumn{2}{|c|}{$\begin{array}{c}\text { Depth } \\
\text { (mbsf [m CSF-A]) }\end{array}$} & \multicolumn{3}{|c|}{$\begin{array}{l}\text { Depth (rmcd [adjusted } \\
\text { m revised CCSF-A]) }\end{array}$} & \multirow{2}{*}{$\begin{array}{l}\text { Error } \\
( \pm) \\
(\mathrm{m})\end{array}$} & \multicolumn{3}{|c|}{$\begin{array}{l}\text { Depth Site } 1220 \\
\text { (rmcd) }\end{array}$} & \multirow{2}{*}{$\begin{array}{c}\text { Error } \\
( \pm) \\
(\mathrm{m})\end{array}$} \\
\hline & & & Top & Bottom & Top & Bottom & Top & Bottom & Mean & & Top & Bottom & Mean & \\
\hline Ship & B Sphenolithus distentus & 30.00 & $\begin{array}{c}320-U 1331 \mathrm{~A}- \\
2 \mathrm{H}-4,70\end{array}$ & $\begin{array}{c}320-\mathrm{U} 1331 \mathrm{~A}- \\
2 \mathrm{H}-5,70\end{array}$ & 10.40 & 11.90 & 90 & 0 & 12 & 0.75 & & 55.22 & & \\
\hline Ship & T Reticulofenestra umbilicus & 32.00 & $3 \mathrm{H}-5,110$ & $3 \mathrm{H}-6,110$ & 21.80 & 23.30 & 24.71 & 26.22 & 25.47 & 0.75 & 71.55 & 72.98 & 72.26 & 0.71 \\
\hline Ship & T Coccolithus formosus & 32.90 & $3 \mathrm{H}-6,110$ & $3 \mathrm{H}-\mathrm{CC}$ & 23.30 & 24.34 & 26.22 & 27.29 & 26.76 & 0.53 & 72.98 & 74.53 & 73.75 & 0.78 \\
\hline Ship & T Discoaster saipanensis & 34.40 & $4 \mathrm{H}-1,110$ & $4 \mathrm{H}-7,10$ & 25.30 & 33.30 & 28.56 & 36.56 & 32.56 & 4.00 & 76.35 & 86.81 & 81.58 & 5.23 \\
\hline Ship & T Chiasmolithus grandis & 37.10 & $6 \mathrm{H}-5,70$ & $6 \mathrm{H}-7,9$ & 49.90 & 51.79 & 57.06 & 59.08 & 58.07 & 1.01 & 103.26 & 104.53 & 103.89 & 0.63 \\
\hline Ship & B Dictyococcites bisectus & 38.00 & $6 \mathrm{H}-\mathrm{CC}$ & $7 \mathrm{H}-1,30$ & 52.60 & 53.00 & 59.87 & 62.81 & 61.34 & 1.47 & 105.04 & 106.95 & 106.00 & 0.96 \\
\hline Ship & T Chiasmolithus solitus & 40.40 & $7 \mathrm{H}-2,30$ & $8 \mathrm{H}-6,50$ & 54.50 & 70.20 & 64.31 & 80.97 & 72.64 & 8.33 & 107.93 & 117.76 & 112.84 & 4.92 \\
\hline Ship & B Reticulofenestra umbilicus $>14 \mu \mathrm{m}$ & 42.50 & $11 \mathrm{H}-4,30$ & $11 \mathrm{H}-5,30$ & 94.68 & 96.18 & 107.90 & 109.40 & 108.65 & 0.75 & 128.74 & 129.29 & 129.01 & 0.28 \\
\hline Ship & T Nannotetrina fulgens & 43.40 & $13 \mathrm{H}-2,5$ & $13 \mathrm{H}-5,70$ & 111.25 & 116.40 & 129.12 & 134.37 & 131.75 & 2.63 & 137.94 & 140.32 & 139.13 & 1.19 \\
\hline Ship & T Tribrachiatus orthostylus & 50.70 & $\begin{array}{c}320-U 1331 \mathrm{C}- \\
17 \mathrm{H}-3,100\end{array}$ & $\begin{array}{c}320-\mathrm{U} 1331 \mathrm{C}- \\
17 \mathrm{H}-3,109\end{array}$ & 188.00 & 188.09 & 210.75 & 210.84 & 210.80 & 0.05 & & & & \\
\hline
\end{tabular}

* = Expedition 320/321 Scientists (2010a). $\mathrm{T}=$ top, B = bottom.

Table T40. Site U1332 calcareous nannofossil datums.

\begin{tabular}{|c|c|c|c|c|c|c|c|c|c|c|c|c|c|c|}
\hline \multirow[b]{2}{*}{ Source } & \multirow[b]{2}{*}{ Event } & \multirow{2}{*}{$\begin{array}{l}\text { Age* }^{*} \\
(\mathrm{Ma})\end{array}$} & \multicolumn{2}{|c|}{ Core, section, interval $(\mathrm{cm})$} & \multicolumn{2}{|c|}{$\begin{array}{c}\text { Depth } \\
\text { (mbsf [m CSF-A]) } \\
\end{array}$} & \multicolumn{3}{|c|}{$\begin{array}{l}\text { Depth (rmcd [adjusted } \\
\text { m revised CCSF-A]) }\end{array}$} & \multirow{2}{*}{$\begin{array}{l}\text { Error } \\
( \pm) \\
(\mathrm{m})\end{array}$} & \multicolumn{3}{|c|}{$\begin{array}{c}\text { Depth Site } 1220 \\
(\mathrm{rmcd})\end{array}$} & \multirow{2}{*}{$\begin{array}{c}\text { Error } \\
( \pm) \\
(\mathrm{m})\end{array}$} \\
\hline & & & Top & Bottom & Top & Bottom & Top & Bottom & Mean & & Top & Bottom & Mean & \\
\hline & & & 320-U1332A- & 320-U1332A- & & & & & & & & & & \\
\hline Ship & T Triquetrorhabdulus carinatus & 18.28 & $3 \mathrm{H}-4,100$ & $3 \mathrm{H}-5,100$ & 18.90 & 20.40 & 19.55 & 20.95 & 20.25 & 0.70 & 25.72 & 26.98 & 26.35 & 0.63 \\
\hline Ship & T Sphenolithus delphix & 23.11 & $3 \mathrm{H}-7,60$ & $3 \mathrm{H}-\mathrm{CC}$ & 23.00 & 23.51 & 23.14 & 23.52 & 23.33 & 0.19 & 28.99 & 29.38 & 29.19 & 0.20 \\
\hline Ship & B Sphenolithus delphix & 23.21 & $3 \mathrm{H}-\mathrm{CC}$ & $4 \mathrm{H}-2,60$ & 23.51 & 23.50 & 23.52 & 26.05 & 24.79 & 1.27 & 29.38 & 31.46 & 30.42 & 1.04 \\
\hline Ship & $\begin{array}{l}\text { X Triquetrorhabdulus longus/ } \\
\text { Triquetrorhabdulus carinatus }\end{array}$ & 24.70 & $4 \mathrm{H}-2,60$ & $4 \mathrm{H}-3,60$ & 25.00 & 26.50 & 27.55 & 29.05 & 28.30 & 0.75 & 33.19 & 34.87 & 34.03 & 0.84 \\
\hline Ship & T ac Coccolithus abisectus & 25.50 & $4 \mathrm{H}-2,60$ & $4 \mathrm{H}-3,60$ & 25.00 & 26.50 & 27.55 & 29.05 & 28.30 & 0.75 & 33.19 & 34.87 & 34.03 & 0.84 \\
\hline Ship & T Sphenolithus predistentus & 26.90 & $4 \mathrm{H}-\mathrm{CC}$ & $5 \mathrm{H}-1,80$ & 32.95 & 33.20 & 35.72 & 37.80 & 36.76 & 1.04 & 41.66 & 43.04 & 42.35 & 0.69 \\
\hline Ship & T Sphenolithus pseudoradians & 29.10 & $5 \mathrm{H}-3,80$ & $5 \mathrm{H}-4,80$ & 36.20 & 37.70 & 41.30 & 42.80 & 42.05 & 0.75 & 46.07 & 47.61 & 46.84 & 0.77 \\
\hline Ship & B Sphenolithus distentus & 30.00 & $6 \mathrm{H}-5,70$ & $6 \mathrm{H}-6,70$ & 48.60 & 50.10 & 54.10 & 55.60 & 54.85 & 0.75 & 57.76 & 58.89 & 58.33 & 0.56 \\
\hline Ship & T Reticulofenestra umbilicus & 32.00 & $7 \mathrm{H}-6,80$ & $7 \mathrm{H}-7,80$ & 59.69 & 61.18 & 67.06 & 68.36 & 67.71 & 0.65 & 67.65 & 68.61 & 68.13 & 0.48 \\
\hline Ship & T Coccolithus formosus & 32.90 & $8 \mathrm{H}-4,50$ & $8 \mathrm{H}-5,50$ & 65.90 & 67.40 & 73.39 & 74.71 & 74.05 & 0.66 & 73.26 & 74.44 & 73.85 & 0.59 \\
\hline Ship & T Discoaster saipanensis & 34.40 & $9 \mathrm{H}-4,110$ & $10 \mathrm{H}-3,80$ & 76.00 & 83.70 & 78.63 & 87.45 & 83.04 & 4.41 & 77.86 & 89.04 & 83.45 & 5.59 \\
\hline Ship & T Chiasmolithus grandis & 37.10 & $11 \mathrm{H}-5,70$ & $11 \mathrm{H}-6,70$ & 96.10 & 97.60 & 99.48 & 100.98 & 100.23 & 0.75 & 101.47 & 102.80 & 102.14 & 0.66 \\
\hline Ship & B Dictyococcites bisectus & 38.00 & $11 \mathrm{H}-\mathrm{CC}$ & $12 \mathrm{H}-2,100$ & 98.52 & 101.40 & 101.90 & 107.62 & 104.76 & 2.86 & 103.56 & 108.55 & 106.05 & 2.50 \\
\hline Ship & T Chiasmolithus solitus & 40.40 & $12 \mathrm{H}-2,100$ & $13 \mathrm{H}-1,140$ & 101.40 & 109.80 & 107.62 & 118.46 & 113.04 & 5.42 & 108.55 & 118.54 & 113.54 & 5.00 \\
\hline Ship & T Nannotetrina spp. & 42.30 & $14 \mathrm{H}-5,40$ & $14 \mathrm{H}-6,35$ & 124.30 & 125.74 & 137.63 & 139.39 & 138.51 & 0.88 & 130.20 & 131.51 & 130.86 & 0.66 \\
\hline Ship & B Reticulofenestra umbilicus $>14 \mu \mathrm{m}$ & 42.50 & $14 \mathrm{H}-\mathrm{CC}$ & $15 X-1,113$ & 126.28 & 127.03 & 139.96 & 140.53 & 140.25 & 0.28 & 131.96 & 132.40 & 132.18 & 0.22 \\
\hline Ship & T Nannotetrina fulgens & 43.40 & $15 X-2,137$ & $15 X-3,66$ & 128.77 & 129.56 & 142.27 & 143.06 & 142.67 & 0.39 & & & & \\
\hline Ship & B Nannotetrina fulgens & 46.80 & $16 \mathrm{X}-2,39$ & $16 \mathrm{X}-\mathrm{CC}$ & 137.39 & 138.20 & 150.89 & 151.70 & 151.30 & 0.41 & & & & \\
\hline Ship & T Discoaster lodoensis & 48.40 & $\begin{array}{l}320-\mathrm{U} 1332 \mathrm{~B}- \\
17 \mathrm{X}-\mathrm{CC}\end{array}$ & $\begin{array}{c}320-U 1332 \mathrm{~B}- \\
18 \mathrm{X}-2,48\end{array}$ & 135.06 & 145.88 & 153.14 & 161.78 & 157.46 & 4.32 & & & & \\
\hline
\end{tabular}

* = Expedition 320/321 Scientists (2010a). $\mathrm{T}=$ top, $\mathrm{B}=$ bottom, $\mathrm{X}=$ crossover, $\mathrm{ac}=\mathrm{acme}$. 
Table T41. Site U1333 calcareous nannofossil datums.

\begin{tabular}{|c|c|c|c|c|c|c|c|c|c|c|c|c|c|c|}
\hline \multirow[b]{2}{*}{ Source } & \multirow[b]{2}{*}{ Event } & \multirow{2}{*}{$\begin{array}{l}\text { Age }^{*} \\
(\mathrm{Ma})\end{array}$} & \multicolumn{2}{|c|}{ Core, section, interval $(\mathrm{cm})$} & \multicolumn{2}{|c|}{$\begin{array}{c}\text { Depth } \\
\text { (mbsf [m CSF-A]) }\end{array}$} & \multicolumn{3}{|c|}{$\begin{array}{l}\text { Depth (rmcd [adjusted } \\
\text { m revised CCSF-A]) }\end{array}$} & \multirow{2}{*}{$\begin{array}{l}\text { Error } \\
( \pm) \\
(\mathrm{m})\end{array}$} & \multicolumn{3}{|c|}{$\begin{array}{l}\text { Depth Site } 1218 \\
\text { (corrected rmcd) }\end{array}$} & \multirow{2}{*}{$\begin{array}{c}\text { Error } \\
( \pm) \\
(\mathrm{m})\end{array}$} \\
\hline & & & Top & Bottom & Top & Bottom & Top & Bottom & Mean & & Top & Bottom & Mean & \\
\hline & & & 320-U1333A- & 320-U1333A- & & & & & & & & & & \\
\hline Ship & B Sphenolithus disbelemnos & 22.76 & $2 \mathrm{H}-5,70$ & $2 \mathrm{H}-6,70$ & 16.20 & 17.70 & 19.68 & 21.18 & 20.43 & 0.75 & 89.99 & 93.96 & 91.98 & 1.98 \\
\hline Ship & T Sphenolithus delphix & 23.11 & $2 \mathrm{H}-7,70$ & $2 \mathrm{H}-\mathrm{CC}$ & 19.20 & 19.57 & 22.68 & 23.05 & 22.86 & 0.19 & 94.28 & 96.02 & 95.15 & 0.87 \\
\hline Ship & B Sphenolithus delphix & 23.21 & $2 \mathrm{H}-\mathrm{CC}$ & $3 \mathrm{H}-1,70$ & 19.57 & 19.70 & 23.05 & 26.54 & 24.79 & 1.75 & 94.92 & 104.61 & 99.76 & 4.85 \\
\hline Ship & T Sphenolithus ciperoensis & 24.40 & $4 \mathrm{H}-5,70$ & $4 \mathrm{H}-6,70$ & 35.20 & 36.70 & 42.08 & 43.18 & 42.63 & 0.55 & 129.53 & 131.21 & 130.37 & 0.84 \\
\hline Ship & $\begin{array}{l}\text { X Triquetrorhabdulus longus/ } \\
\text { Triquetrorhabdulus carinatus }\end{array}$ & 24.70 & $3 \mathrm{H}-\mathrm{CC}$ & $4 \mathrm{H}-1,70$ & 28.87 & 29.20 & 35.38 & 37.16 & 36.27 & 0.89 & 116.18 & 122.68 & 119.43 & 3.25 \\
\hline Ship & T ac Coccolithus abisectus & 25.50 & $3 \mathrm{H}-5,70$ & $3 \mathrm{H}-6,70$ & 25.70 & 27.20 & 32.19 & 33.62 & 32.91 & 0.72 & 110.15 & 116.01 & 113.08 & 2.93 \\
\hline Ship & T Sphenolithus distentus & 26.80 & $5 \mathrm{H}-2,70$ & $5 \mathrm{H}-3,70$ & 40.20 & 41.70 & 49.94 & 51.36 & 50.65 & 0.71 & 139.78 & 143.81 & 141.80 & 2.02 \\
\hline Ship & T Sphenolithus predistentus & 26.90 & $5 \mathrm{H}-3,70$ & $5 \mathrm{H}-4,70$ & 41.70 & 43.20 & 51.36 & 52.80 & 52.08 & 0.72 & 141.95 & 147.14 & 144.55 & 2.59 \\
\hline Ship & B Sphenolithus ciperoensis & 27.10 & $5 \mathrm{H}-4,70$ & $5 \mathrm{H}-6,70$ & 43.20 & 46.20 & 52.80 & 55.72 & 54.26 & 1.46 & 144.22 & 152.12 & 148.17 & 3.95 \\
\hline Ship & T Sphenolithus pseudoradians & 29.10 & $6 \mathrm{H}-4,70$ & $6 \mathrm{H}-6,70$ & 52.70 & 55.70 & 64.45 & 67.45 & 65.95 & 1.50 & 158.80 & 167.87 & 163.34 & 4.54 \\
\hline Ship & B Sphenolithus distentus & 30.00 & $9 \mathrm{H}-3,50$ & $9 \mathrm{H}-4,50$ & 79.50 & 81.00 & 97.23 & 98.73 & 97.98 & 0.75 & 196.70 & 199.30 & 198.00 & 1.30 \\
\hline Ship & T Reticulofenestra umbilicus & 32.00 & $11 X-4,70$ & $11 \mathrm{X}-\mathrm{CC}$ & 100.20 & 101.17 & 120.92 & 121.89 & 121.41 & 0.48 & 223.87 & 225.02 & 224.44 & 0.57 \\
\hline Ship & T Coccolithus formosus & 32.90 & $12 X-3,70$ & $12 X-4,70$ & 104.40 & 105.90 & 126.16 & 127.92 & 127.04 & 0.88 & 229.43 & 235.29 & 232.36 & 2.93 \\
\hline Ship & T Discoaster saipanensis & 34.40 & $13 X-3,70$ & $13 X-3,140$ & 114.00 & 114.70 & 137.06 & 137.90 & 137.48 & 0.42 & 243.52 & 246.36 & 244.94 & 1.42 \\
\hline Ship & $\mathrm{T}$ Chiasmolithus grandis & 37.10 & $14 X-6,70$ & $14 \mathrm{X}-\mathrm{CC}$ & 128.20 & 129.80 & 151.89 & 153.80 & 152.85 & 0.96 & 258.26 & 261.05 & 259.66 & 1.40 \\
\hline Ship & B Dictyococcites bisectus & 38.00 & $15 X-5,70$ & $15 X-6,70$ & 136.30 & 137.80 & 163.38 & 165.25 & 164.32 & 0.94 & 267.44 & 270.64 & 269.04 & 1.60 \\
\hline Ship & T Chiasmolithus solitus & 40.40 & $16 X-4,40$ & $16 X-5,40$ & 144.10 & 145.60 & 171.71 & 173.78 & 172.75 & 1.04 & 274.37 & 281.28 & 277.82 & 3.45 \\
\hline Ship & T Nannotetrina spp. & 42.30 & $17 X-6,110$ & $17 X-C C$ & 157.40 & 158.46 & 185.82 & 186.78 & 186.30 & 0.48 & & & & \\
\hline Ship & B Reticulofenestra umbilicus $>14 \mu \mathrm{m}$ & 42.50 & $18 X-1,60$ & $18 \mathrm{X}-2,34$ & 159.00 & 160.24 & 185.90 & 187.14 & 186.52 & 0.62 & & & & \\
\hline Ship & T Nannotetrina fulgens & 43.40 & $18 X-4,88$ & $18 \mathrm{X}-\mathrm{CC}$ & 163.12 & 163.86 & 190.02 & 190.76 & 190.39 & 0.37 & & & & \\
\hline Ship & B Nannotetrina fulgens & 46.80 & $20 X-1,73$ & $20 X-2,50$ & 178.33 & 179.60 & 204.08 & 205.35 & 204.72 & 0.63 & & & & \\
\hline Ship & B Sphenolithus furcatolithoides & 45.80 & $20 X-2,50$ & $20 \mathrm{X}-\mathrm{CC}$ & 179.60 & 180.12 & 205.35 & 205.87 & 205.61 & 0.26 & & & & \\
\hline Ship & B Nannotetrina spp. & 48.00 & $20 x-2,50$ & $20 \mathrm{X}-\mathrm{CC}$ & 179.60 & 180.12 & 205.35 & 205.87 & 205.61 & 0.26 & & & & \\
\hline Ship & catolithoides & 45.80 & $\begin{array}{l}320-\mathrm{U} 1333 \mathrm{~B}- \\
20 \mathrm{X}-1,46\end{array}$ & $\begin{array}{l}320-\mathrm{U} 1333 \mathrm{~B}- \\
20 \mathrm{X}-2,86\end{array}$ & 172.76 & 1 & 76 & 56 & 11 & 0.95 & & & & \\
\hline Ship & B Nannotetrina spp. & 48.00 & $20 X-3,102$ & $20 X-4,37$ & 176.32 & 177.17 & 202.32 & 203.17 & 202.75 & 0.42 & & & & \\
\hline
\end{tabular}

* = Expedition 320/321 Scientists (2010a). $\mathrm{T}=$ top, $\mathrm{B}=$ bottom, $\mathrm{X}=$ crossover, $\mathrm{ac}=\mathrm{acme}$. 
Table T42. Site U1334 calcareous nannofossil datums.

\begin{tabular}{|c|c|c|c|c|c|c|c|c|c|c|c|c|c|c|}
\hline \multirow[b]{2}{*}{ Source } & \multirow[b]{2}{*}{ Event } & \multirow{2}{*}{$\begin{array}{l}\text { Age }^{*} \\
(\mathrm{Ma})\end{array}$} & \multicolumn{2}{|c|}{ Core, section, interval $(\mathrm{cm})$} & \multicolumn{2}{|c|}{$\begin{array}{c}\text { Depth } \\
\text { (mbsf [m CSF-A] }) \\
\end{array}$} & \multicolumn{3}{|c|}{$\begin{array}{l}\text { Depth (rmcd [adjusted } \\
\text { m revised CCSF-A]) }\end{array}$} & \multirow{2}{*}{$\begin{array}{l}\text { Error } \\
( \pm) \\
(\mathrm{m})\end{array}$} & \multicolumn{3}{|c|}{$\begin{array}{l}\text { Depth Site } 1218 \\
\text { (corrected rmcd) }\end{array}$} & \multirow{2}{*}{$\begin{array}{c}\text { Error } \\
( \pm) \\
(\mathrm{m})\end{array}$} \\
\hline & & & Top & Bottom & Top & Bottom & Top & Bottom & Mean & & Top & Bottom & Mean & \\
\hline & & & 320-U1334A- & 320-U1334A- & & & & & & & & & & \\
\hline Ship & Coronocyclus nitescens present & $>12.12$ & & $2 \mathrm{H}-6,120$ & & $16.90^{*}$ & & 17.77 & 17.77 & & & 39.93 & & \\
\hline Ship & Calcidiscus premacintyrei present & $>12.45$ & & $2 \mathrm{H}-6,120$ & & $16.90^{*}$ & & 17.77 & 17.77 & & & 39.93 & & \\
\hline Ship & T ac Cyclicargolithus floridanus & 13.33 & $3 \mathrm{H}-2,15$ & $3 \mathrm{H}-3,50$ & 19.35 & 21.20 & 21.32 & 23.17 & 22.25 & 0.93 & 42.10 & 43.28 & 42.69 & 0.59 \\
\hline Ship & T Sphenolithus heteromorphus & 13.53 & $3 \mathrm{H}-4,50$ & $3 \mathrm{H}-5,50$ & 22.70 & 24.20 & 24.67 & 26.17 & 25.42 & 0.75 & 43.96 & 44.70 & 44.33 & 0.37 \\
\hline Ship & B Discoaster petaliformis & 15.70 & $3 \mathrm{H}-\mathrm{CC}$ & $4 \mathrm{H}-2,70$ & 27.65 & 29.40 & 29.62 & 33.05 & 31.34 & 1.72 & 46.64 & 48.50 & 47.57 & 0.93 \\
\hline Ship & T Discoaster deflandrei & 15.80 & $3 \mathrm{H}-\mathrm{CC}$ & $4 \mathrm{H}-2,70$ & 27.65 & 29.40 & 29.62 & 33.05 & 31.34 & 1.72 & 46.64 & 48.50 & 47.57 & 0.93 \\
\hline Ship & B Sphenolithus heteromorphus & 17.71 & $4 \mathrm{H}-6,20$ & $4 \mathrm{H}-7,15$ & 34.90 & 36.35 & 38.38 & 39.81 & 39.09 & 0.71 & 52.19 & 53.40 & 52.80 & 0.61 \\
\hline Ship & T Triquetrorhabdulus carinatus & 18.28 & $5 \mathrm{H}-3,100$ & $5 \mathrm{H}-4,120$ & 40.70 & 42.40 & 45.56 & 47.17 & 46.37 & 0.80 & 57.89 & 58.97 & 58.43 & 0.54 \\
\hline Ship & $\mathrm{T}$ ac Triquetrorhabdulus carinatus & 22.09 & $9 \mathrm{H}-3,20$ & $9 \mathrm{H}-4,100$ & 77.90 & 80.20 & 89.12 & 91.42 & 90.27 & 1.15 & 86.34 & 87.70 & 87.02 & 0.68 \\
\hline Ship & B Sphenolithus disbelemnos & 22.80 & $9 \mathrm{H}-7,30$ & $9 \mathrm{H}-\mathrm{CC}$ & 84.00 & 84.74 & 94.97 & 95.61 & 95.29 & 0.32 & 90.14 & 90.64 & 90.39 & 0.25 \\
\hline Ship & T Sphenolithus delphix & 23.11 & $10 \mathrm{H}-7,30$ & $10 \mathrm{H}-\mathrm{CC}$ & 93.50 & 93.95 & 104.65 & 104.97 & 104.81 & 0.16 & 96.72 & 96.95 & 96.84 & 0.12 \\
\hline Ship & B Sphenolithus delphix & 23.21 & $11 \mathrm{H}-1,20$ & $11 \mathrm{H}-2,20$ & 94.70 & 96.20 & 107.53 & 109.09 & 108.31 & 0.78 & 98.75 & 99.94 & 99.35 & 0.59 \\
\hline Ship & T Sphenolithus ciperoensis & 24.40 & $12 \mathrm{H}-7,30$ & $12 \mathrm{H}-\mathrm{CC}$ & 112.27 & 112.58 & 126.42 & 126.73 & 126.58 & 0.16 & 112.16 & 112.40 & 112.28 & 0.12 \\
\hline Revised & $\begin{array}{l}\text { X Triquetrorhabdulus longus/ } \\
\text { Triquetrorhabdulus carinatus }\end{array}$ & 24.70 & $12 \mathrm{H}-7,30$ & $12 \mathrm{H}-\mathrm{CC}$ & 112.27 & 112.58 & 126.42 & 126.73 & 126.58 & 0.16 & 112.16 & 112.40 & 112.28 & 0.12 \\
\hline Ship & T ac Cyclicargolithus abisectus & 25.50 & $12 \mathrm{H}-\mathrm{CC}$ & $13 \mathrm{H}-1,45$ & 112.58 & 113.15 & 126.73 & 128.63 & 127.68 & 0.95 & 112.40 & 113.95 & 113.17 & 0.77 \\
\hline Ship & T Sphenolithus distentus & 26.80 & $16 \mathrm{H}-6,40$ & $16 \mathrm{H}-\mathrm{CC}$ & 149.10 & 149.57 & 169.92 & 170.39 & 170.16 & 0.23 & 145.97 & 146.31 & 146.14 & 0.17 \\
\hline Ship & T Sphenolithus predistentus & 26.90 & $16 \mathrm{H}-6,40$ & $16 \mathrm{H}-\mathrm{CC}$ & 149.10 & 149.57 & 169.92 & 170.39 & 170.16 & 0.23 & 145.97 & 146.31 & 146.14 & 0.17 \\
\hline Ship & B Sphenolithus ciperoensis & 27.10 & $18 \mathrm{H}-\mathrm{CC}$ & $19 \mathrm{H}-\mathrm{CC}$ & 170.17 & 178.49 & 194.27 & 204.91 & 199.59 & 5.32 & 164.26 & 172.06 & 168.16 & 3.90 \\
\hline Ship & T Sphenolithus pseudoradians & 29.10 & $19 \mathrm{H}-\mathrm{CC}$ & $20 \mathrm{H}-\mathrm{CC}$ & 178.49 & 188.63 & 204.91 & 229.09 & 217.00 & 12.09 & 172.06 & 189.61 & 180.84 & 8.78 \\
\hline Ship & B Sphenolithus distentus & 30.00 & $21 \mathrm{H}-\mathrm{CC}$ & $22 \mathrm{H}-2,70$ & 198.48 & 200.40 & 239.72 & 242.86 & 241.29 & 1.57 & 196.30 & 198.16 & 197.23 & 0.93 \\
\hline Ship & T Reticulofenestra umbilicus & 32.00 & $24 \mathrm{X}-\mathrm{CC}$ & $25 X-1,80$ & 224.22 & 224.90 & 271.90 & 274.19 & 273.05 & 1.15 & 219.99 & 222.00 & 220.99 & 1.00 \\
\hline Revised & T Ismolithus recurvus & 32.50 & $26 \mathrm{X}-6,100$ & $26 \mathrm{X}-\mathrm{CC}$ & 242.10 & 243.36 & 291.74 & 293.00 & 292.37 & 0.63 & 235.25 & 236.37 & 235.81 & 0.56 \\
\hline Ship & T Coccolithus formosus & 32.90 & $26 \mathrm{X}-2,100$ & $26 X-3,100$ & 236.10 & 237.60 & 285.87 & 287.24 & 286.55 & 0.69 & 230.88 & 231.89 & 231.39 & 0.50 \\
\hline Ship & T Discoaster saipanensis & 34.40 & $27 X-5,150$ & $27 X-C C$ & 249.70 & 250.76 & 300.80 & 301.86 & 301.33 & 0.53 & 242.84 & 243.74 & 243.29 & 0.45 \\
\hline Revised & T Reticulofenestra reticulata & 35.20 & $27 \mathrm{X}-\mathrm{CC}$ & $28 \mathrm{X}-\mathrm{CC}$ & 250.76 & 262.62 & 301.86 & 315.88 & 308.87 & 7.01 & 243.74 & 251.88 & 247.81 & 4.07 \\
\hline Ship & B Ismolithus recurvus & 36.60 & $29 \mathrm{X}-\mathrm{CC}$ & $30 X-1,66$ & 272.00 & 272.76 & 326.42 & 327.70 & 327.06 & 0.64 & 257.81 & 258.36 & 258.09 & 0.28 \\
\hline Revised & T Chiasmolithus oamaruensis & 37.00 & $29 X-8,36$ & 29X-CC & 271.44 & 272.00 & 325.86 & 326.42 & 326.14 & 0.28 & 257.54 & 257.81 & 257.68 & 0.14 \\
\hline Ship & T Chiasmolithus grandis & 37.10 & $30 X-1,66$ & $30 X-2,74$ & 272.76 & 274.34 & 327.70 & 329.50 & 328.60 & 0.90 & 258.36 & 259.12 & 258.74 & 0.38 \\
\hline Ship & Dictyococcites bisectus present & $<38.0$ & & $32 \mathrm{X}-\mathrm{CC}$ & & $285.21^{*}$ & & 340.99 & 340.99 & & & & & \\
\hline
\end{tabular}

* = Expedition 320/321 Scientists (2010a). $\mathrm{T}=$ top, $\mathrm{B}=$ bottom, $\mathrm{X}=$ crossover, ac $=$ acme. 
Table T43. Site 1218 calcareous nannofossil datums.

\begin{tabular}{|c|c|c|c|c|c|c|c|c|c|c|}
\hline \multirow[b]{2}{*}{ Source } & \multirow[b]{2}{*}{ Event } & \multirow{2}{*}{$\begin{array}{l}\text { Age }^{*} \\
(\mathrm{Ma})\end{array}$} & \multicolumn{2}{|c|}{ Core, section, interval $(\mathrm{cm})$} & \multicolumn{2}{|c|}{ Depth (mbsf) } & \multicolumn{3}{|c|}{$\begin{array}{l}\text { Depth adjusted } \\
\text { (corrected rmcd) }\end{array}$} & \multirow{2}{*}{$\begin{array}{c}\text { Error } \\
( \pm) \\
(\mathrm{m})\end{array}$} \\
\hline & & & Top & Bottom & Top & Bottom & Top & Bottom & Mean & \\
\hline Pälike et al., 2005 & B Catinaster coalitus & 10.89 & $\begin{array}{c}199-1218 \mathrm{~A}- \\
4 \mathrm{H}-7,50\end{array}$ & $\begin{array}{c}199-1218 \mathrm{~A}- \\
4 \mathrm{H}-\mathrm{CC}\end{array}$ & 36.70 & 37.08 & 40.90 & 41.18 & 41.04 & 0.14 \\
\hline Pälike et al., 2005 & T Sphenolithus heteromorphus & 13.53 & $5 \mathrm{H}-7,15$ & $5 \mathrm{H}-\mathrm{CC}$ & 45.85 & 46.40 & 50.65 & 51.20 & 50.93 & 0.28 \\
\hline Pälike et al., 2005 & T ac Discoaster deflandrei & 15.80 & $5 \mathrm{H}-7,15$ & $5 \mathrm{H}-\mathrm{CC}$ & 45.85 & 46.40 & 50.65 & 51.20 & 50.93 & 0.28 \\
\hline Pälike et al., 2005 & T Triquetrorhabdulus carinatus & 18.28 & $6 \mathrm{HCC}$ & $7 \mathrm{H}-1,120$ & 55.32 & 56.90 & 62.35 & 64.91 & 63.63 & 1.28 \\
\hline Pälike et al., 2005 & T ac Triquetrorhabdulus carinatus & 22.09 & $9 \mathrm{H}-2,80$ & $9 \mathrm{H}-2,130$ & 77.00 & 77.50 & 87.26 & 87.76 & 87.51 & 0.25 \\
\hline Pälike et al., 2005 & B Sphenolithus disbelemnos & 22.80 & $9 \mathrm{H}-4,80$ & $9 \mathrm{H}-4,130$ & 80.00 & 80.50 & 90.26 & 90.76 & 90.51 & 0.25 \\
\hline Pälike et al., 2005 & T Sphenolithus delphix & 23.10 & $10 \mathrm{H}-1,140$ & $10 \mathrm{H}-2,10$ & 85.60 & 85.80 & 97.44 & 97.65 & 97.55 & 0.11 \\
\hline Pälike et al., 2005 & B Sphenolithus delphix & 23.20 & $10 \mathrm{H}-3,120$ & $10 \mathrm{H}-3,140$ & 88.40 & 88.60 & 100.30 & 100.50 & 100.40 & 0.10 \\
\hline Pälike et al., 2005 & T Sphenolithus ciperoensis & 24.40 & $11 \mathrm{H}-2,130$ & $11 \mathrm{H}-3,130$ & 96.50 & 98.00 & 109.85 & 111.27 & 110.56 & 0.71 \\
\hline Pälike et al., 2005 & T ac Cyclicargolithus abisectus & 25.50 & $11 \mathrm{H}-4,130$ & $11 \mathrm{H}-5,38$ & 99.50 & 100.08 & 112.90 & 113.48 & 113.19 & 0.29 \\
\hline Pälike et al., 2005 & T Sphenolithus distentus & 26.80 & $13 \mathrm{H}-3,75$ & $13 \mathrm{H}-4,45$ & 116.45 & 117.65 & 131.42 & 132.56 & 131.99 & 0.57 \\
\hline Pälike et al., 2005 & B Sphenolithus ciperoensis & 27.10 & $14 \mathrm{H}-5,80$ & $14 \mathrm{H}-6,80$ & 129.00 & 130.50 & 144.42 & 145.92 & 145.17 & 0.75 \\
\hline Pälike et al., 2005 & B Sphenolithus distentus & 30.00 & $18 \mathrm{H}-\mathrm{CC}$ & $19 \mathrm{H}-1,120$ & 169.54 & 170.90 & 190.10 & 191.28 & 190.69 & 0.59 \\
\hline Pälike et al., 2005 & T Reticulofenestra umbilicus > $14 \mu \mathrm{m}$ & 32.00 & $22 X-2,70$ & $22 X-2,147$ & 198.10 & 198.87 & 221.03 & 221.80 & 221.42 & 0.39 \\
\hline Pälike et al., 2005 & T Coccolithus formosus & 32.90 & $23 X-2,148$ & $23 X-3,35$ & 208.48 & 208.85 & 231.12 & 231.49 & 231.31 & 0.19 \\
\hline Pälike et al., 2005 & T Discoaster saipanensis & 34.40 & $24 X-4,78$ & $24 X-4,90$ & 219.98 & 220.10 & 244.46 & 244.58 & 244.52 & 0.06 \\
\hline Pälike et al., 2005 & T Discoaster barbadiensis & 34.80 & $24 X-5,56$ & $24 X-5,85$ & 221.26 & 221.55 & 245.74 & 246.03 & 245.89 & 0.15 \\
\hline Pälike et al., 2005 & T Chiasmolithus grandis & 37.10 & $25 X-7,70$ & $25 \mathrm{X}-\mathrm{CC}$ & 233.90 & 234.31 & 259.63 & 260.04 & 259.84 & 0.21 \\
\hline Pälike et al., 2005 & B Dictyococcites bisectus & 38.00 & $26 X-5,60$ & $26 \mathrm{X}-6,125$ & 240.90 & 243.05 & 266.43 & 268.58 & 267.51 & 1.07 \\
\hline Pälike et al., 2005 & T Chiasmolithus solitus & 40.40 & $27 X-C C$ & $28 X-1,50$ & 250.11 & 254.00 & 275.70 & 278.53 & 277.12 & 1.41 \\
\hline \multirow[t]{2}{*}{ Pälike et al., 2005} & T Nannotetrina spp. & 42.30 & $30 X-2,90$ & $30 X-3,2$ & 273.10 & 273.72 & 296.73 & 297.35 & 297.04 & 0.31 \\
\hline & & & $\begin{array}{c}199-1218 \mathrm{~B}- \\
5 \mathrm{H}-7 \quad 45\end{array}$ & $\begin{array}{c}199-1218 \mathrm{~B}- \\
5 \mathrm{H}-\mathrm{CC}\end{array}$ & & & & & & \\
\hline Pälike et al., 2005 & T Cyclicargolithus floridanus & 13.33 & $5 \mathrm{H}-7,45$ & $5 \mathrm{H}-\mathrm{CC}$ & 41.85 & 42.42 & 47.75 & 48.38 & 48.07 & $\begin{array}{l}0.32 \\
0.40\end{array}$ \\
\hline Pälike et al., 2005 & T Sphenolithus delphix & 23.10 & $10 \mathrm{H}-4,140$ & $10 \mathrm{H}-5,70$ & 85.80 & 86.60 & 96.60 & 97.40 & 97.00 & $\begin{array}{l}0.40 \\
0.35\end{array}$ \\
\hline Pälike et al., 2005 & B Sphenolithus delphix & 23.20 & $10 \mathrm{H}-6,70$ & $10 \mathrm{H} 6,140$ & 88.10 & 88.80 & 98.90 & 99.60 & 99.25 & 0.35 \\
\hline Pälike et al., 2005 & T Sphenolithus ciperoensis & 24.40 & $11 \mathrm{H}-5,80$ & $11 \mathrm{H}-6,80$ & 96.20 & 97.70 & 109.58 & 111.08 & 110.33 & 0.75 \\
\hline Pälike et al., 2005 & T Sphenolithus distentus & 26.80 & $13 \mathrm{H}-6,100$ & $13 \mathrm{H}-6,130$ & 116.90 & 117.20 & 132.39 & 132.69 & 132.54 & 0.15 \\
\hline Pälike et al., 2005 & B Sphenolithus ciperoensis & 27.10 & $15 \mathrm{H}-1,100$ & $15 \mathrm{H}-1,120$ & 128.40 & 128.60 & 146.22 & 146.42 & 146.32 & 0.10 \\
\hline
\end{tabular}

* = Expedition 320/321 Scientists (2010a). $\mathrm{T}=$ top, $\mathrm{B}=$ bottom, $\mathrm{ac}=\mathrm{acme}$. 
Table T44. Site 1219 calcareous nannofossil datums.

\begin{tabular}{|c|c|c|c|c|c|c|c|c|c|c|c|c|c|c|}
\hline \multirow[b]{2}{*}{ Source } & \multirow[b]{2}{*}{ Event } & \multirow{2}{*}{$\begin{array}{l}\mathrm{Age}^{*} \\
(\mathrm{Ma})\end{array}$} & \multicolumn{2}{|c|}{ Core, section, interval $(\mathrm{cm})$} & \multicolumn{2}{|c|}{$\begin{array}{l}\text { Depth } \\
\text { (mbsf) }\end{array}$} & \multicolumn{3}{|c|}{$\begin{array}{c}\text { Depth from Pälike et al. (2005) } \\
\text { (rmcd) }\end{array}$} & \multirow{2}{*}{$\begin{array}{c}\text { Error } \\
( \pm) \\
(\mathrm{m})\end{array}$} & \multicolumn{3}{|c|}{$\begin{array}{l}\text { Depth Site } 1218 \\
\text { (corrected rmcd) }\end{array}$} & \multirow{2}{*}{$\begin{array}{c}\text { Error } \\
( \pm) \\
(\mathrm{m})\end{array}$} \\
\hline & & & Top & Bottom & Top & Bottom & Top & Bottom & Mean & & Top & Bottom & Mean & \\
\hline & & & 199-1219A- & 199-1219A- & & & & & & & & & & \\
\hline Pälike et al., 2005 & T Cyclicargolithus floridanus & 13.33 & $3 \mathrm{H}-3,92$ & $3 \mathrm{H}-3,138$ & 19.42 & 19.88 & 19.48 & 19.93 & 19.71 & 0.23 & 46.11 & 46.66 & 46.38 & 0.27 \\
\hline Pälike et al., 2005 & T Sphenolithus heteromorphus & 13.53 & $3 \mathrm{H}-3,138$ & $3 \mathrm{H}-4,145$ & 19.88 & 21.45 & 19.93 & 21.48 & 20.71 & 0.78 & 46.66 & 48.34 & 47.50 & 0.84 \\
\hline Pälike et al., 2005 & T ac Discoaster deflandrei & 15.80 & $3 \mathrm{H}-5,25$ & $3 \mathrm{H}-6,26$ & 21.75 & 23.62 & 21.77 & 23.64 & 22.71 & 0.94 & 48.71 & 51.62 & 50.17 & 1.45 \\
\hline Pälike et al., 2005 & T Triquetrorhabdulus carinatus & 18.28 & $4 \mathrm{H}-4,92$ & $4 \mathrm{H}-4,140$ & 30.42 & 30.90 & 31.30 & 31.78 & 31.54 & 0.24 & 62.31 & 63.48 & 62.89 & 0.59 \\
\hline Pälike et al., 2005 & T ac Triquetrorhabdulus carinatus & 22.09 & $5 \mathrm{H}-\mathrm{CC}$ & $6 \mathrm{H}-1,90$ & 44.47 & 44.90 & 46.76 & 48.91 & 47.84 & 1.08 & 86.17 & 89.07 & 87.62 & 1.45 \\
\hline Pälike et al., 2005 & B Sphenolithus disbelemnos & 22.76 & $6 \mathrm{H}-2,40$ & $6 \mathrm{H}-2,105$ & 45.90 & 46.55 & 50.08 & 50.71 & 50.40 & 0.32 & 90.50 & 91.47 & 90.98 & 0.49 \\
\hline Pälike et al., 2005 & T Sphenolithus delphix & 23.11 & $6 \mathrm{H}-5,30$ & $6 \mathrm{H}-5,90$ & 50.30 & 50.90 & 54.35 & 54.93 & 54.64 & 0.29 & 97.02 & 97.89 & 97.46 & 0.43 \\
\hline Pälike et al., 2005 & B Sphenolithus delphix & 23.21 & $6 \mathrm{H}-6,120$ & $6 \mathrm{H}-7,20$ & 52.70 & 53.20 & 56.60 & 56.99 & 56.80 & 0.20 & 100.56 & 101.50 & 101.03 & 0.47 \\
\hline Pälike et al., 2005 & T Sphenolithus ciperoensis & 24.40 & $7 \mathrm{H}-5,10$ & $7 \mathrm{H}-5,80$ & 59.44 & 60.14 & 61.45 & 62.20 & 61.83 & 0.38 & 110.35 & 111.48 & 110.91 & 0.57 \\
\hline Pälike et al., 2005 & T ac Cyclicargolithus abisectus & 25.50 & $7 \mathrm{H}-5,80$ & $7 \mathrm{H}-6,90$ & 60.14 & 61.74 & 62.20 & 64.00 & 63.10 & 0.90 & 111.48 & 116.07 & 113.77 & 2.29 \\
\hline Pälike et al., 2005 & T Sphenolithus distentus & 26.80 & $8 \mathrm{H}-3,100$ & $8 \mathrm{H}-4,100$ & 67.00 & 68.50 & 70.05 & 71.55 & 70.80 & 0.75 & 127.33 & 129.19 & 128.26 & 0.93 \\
\hline Pälike et al., 2005 & B Sphenolithus ciperoensis & 27.10 & $9 \mathrm{H}-2,140$ & $9 \mathrm{H}-3,140$ & 75.40 & 76.90 & 82.77 & 84.27 & 83.52 & 0.75 & 143.37 & 144.17 & 143.77 & 0.40 \\
\hline Pälike et al., 2005 & T Sphenolithus pseudoradians & 29.10 & $12 \mathrm{H}-5,80$ & $12 \mathrm{H}-6,80$ & 107.80 & 109.30 & 120.31 & 121.77 & 121.04 & 0.73 & 180.05 & 181.60 & 180.82 & 0.78 \\
\hline Pälike et al., 2005 & B Sphenolithus distentus & 30.00 & $13 \mathrm{H}-3,80$ & $13 \mathrm{H}-4,80$ & 113.86 & 115.36 & 133.45 & 134.95 & 134.20 & 0.75 & 194.43 & 196.11 & 195.27 & 0.84 \\
\hline Pälike et al., 2005 & T Reticulofenestra umbilicus $>14 \mu \mathrm{m}$ & 32.00 & $15 \mathrm{H}-5,140$ & $15 \mathrm{H}-6,10$ & 136.90 & 137.10 & 159.75 & 159.95 & 159.85 & 0.10 & 222.82 & 223.12 & 222.97 & 0.15 \\
\hline Pälike et al., 2005 & T Coccolithus formosus & 32.90 & $16 \mathrm{H}-4,140$ & $16 \mathrm{H}-5,140$ & 144.96 & 146.51 & 169.29 & 170.84 & 170.07 & 0.78 & 232.72 & 235.08 & 233.90 & 1.18 \\
\hline Pälike et al., 2005 & T Discoaster saipanensis & 34.40 & $17 \mathrm{H}-2,90$ & $19 \mathrm{H}-7,5$ & 150.90 & 176.55 & 176.23 & 203.88 & 190.06 & 13.83 & 242.63 & 266.70 & 254.66 & 12.03 \\
\hline Pälike et al., 2005 & T Discoaster barbadiensis & 34.80 & $17 \mathrm{H}-2,90$ & $19 \mathrm{H}-7,5$ & 150.90 & 176.55 & 176.23 & 203.88 & 190.06 & 13.83 & 242.63 & 266.70 & 254.66 & 12.03 \\
\hline Pälike et al., 2005 & T Chiasmolithus grandis & 37.10 & $17 \mathrm{H}-2,90$ & $20 \mathrm{H}-1,114$ & 150.90 & 178.14 & 176.23 & 206.47 & 191.35 & 15.12 & 242.63 & 269.35 & 255.99 & 13.36 \\
\hline Pälike et al., 2005 & B Dictyococcites bisectus & 38.00 & $20 \mathrm{H}-2,52$ & $21 \mathrm{H}-3,80$ & 179.02 & 190.30 & 207.35 & 219.63 & 213.49 & 6.14 & 270.14 & 282.02 & 276.08 & 5.94 \\
\hline Pälike et al., 2005 & B Reticulofenestra umbilicus $>14 \mu \mathrm{m}$ & 42.50 & $22 \mathrm{H}-3,49$ & $22 \mathrm{H}-3,125$ & 199.49 & 200.25 & 229.82 & 230.58 & 230.20 & 0.38 & & & & \\
\hline Pälike et al., 2005 & Nannotetrina spp. present & $>42.3$ & $22 \mathrm{H}-\mathrm{CC}$ & $24 \mathrm{H}-1,36$ & 205.84 & 215.36 & 236.17 & 247.69 & 241.93 & 5.76 & & & & \\
\hline \multirow[t]{2}{*}{ Pälike et al., 2005} & T Fasciculithus tympaniformis & 54.70 & $26 X-3,52$ & $27 X-C C$ & 237.62 & 244.92 & 271.95 & 280.25 & 276.10 & 4.15 & & & & \\
\hline & T Sphenolithus delphix & 23.11 & $\begin{array}{c}199-1219 \mathrm{~B}- \\
4 \mathrm{H}-\mathrm{CC}\end{array}$ & $\begin{array}{c}199-1219 \mathrm{~B}- \\
5 \mathrm{H}-1,30\end{array}$ & 48.75 & 49.80 & 52.86 & 54.70 & 53.78 & 0.92 & 94.57 & 97.55 & 96.06 & 1.49 \\
\hline Pälike et al., 2005 & B Sphenolithus delphix & 23.21 & $5 \mathrm{H}-2,80$ & $5 \mathrm{H}-2,90$ & 51.80 & 51.90 & 56.70 & 56.80 & 56.75 & 0.05 & 100.76 & 101.01 & 100.89 & 0.13 \\
\hline Pälike et al., 2005 & T Sphenolithus ciperoensis & 24.40 & $5 \mathrm{H}-\mathrm{CC}$ & $6 \mathrm{H}-1,60$ & 58.79 & 59.60 & 63.81 & 64.14 & 63.98 & 0.16 & 115.66 & 116.37 & 116.01 & 0.35 \\
\hline Pälike et al., 2005 & T Sphenolithus pseudoradians & 29.10 & $11 \mathrm{H}-3,85$ & $11 \mathrm{H}-3,120$ & 110.35 & 110.70 & 121.78 & 122.11 & 121.95 & 0.16 & 181.61 & 181.96 & 181.79 & 0.18 \\
\hline Pälike et al., 2005 & T Reticulofenestra umbilicus $>14 \mu \mathrm{m}$ & 32.00 & $14 \mathrm{H}-7,40$ & $14 \mathrm{H}-7,70$ & 144.40 & 144.70 & 161.69 & 161.99 & 161.84 & 0.15 & 225.65 & 225.94 & 225.79 & 0.15 \\
\hline Pälike et al., 2005 & T Coccolithus formosus & 32.90 & $15 \mathrm{H}-5,76$ & $15 \mathrm{H}-5,104$ & 150.82 & 151.10 & 170.17 & 170.45 & 170.31 & 0.14 & 233.77 & 234.31 & 234.04 & 0.27 \\
\hline
\end{tabular}

* = Expedition 320/321 Scientists (2010a). $\mathrm{T}=$ top, $\mathrm{B}=$ bottom, ac $=$ acme 


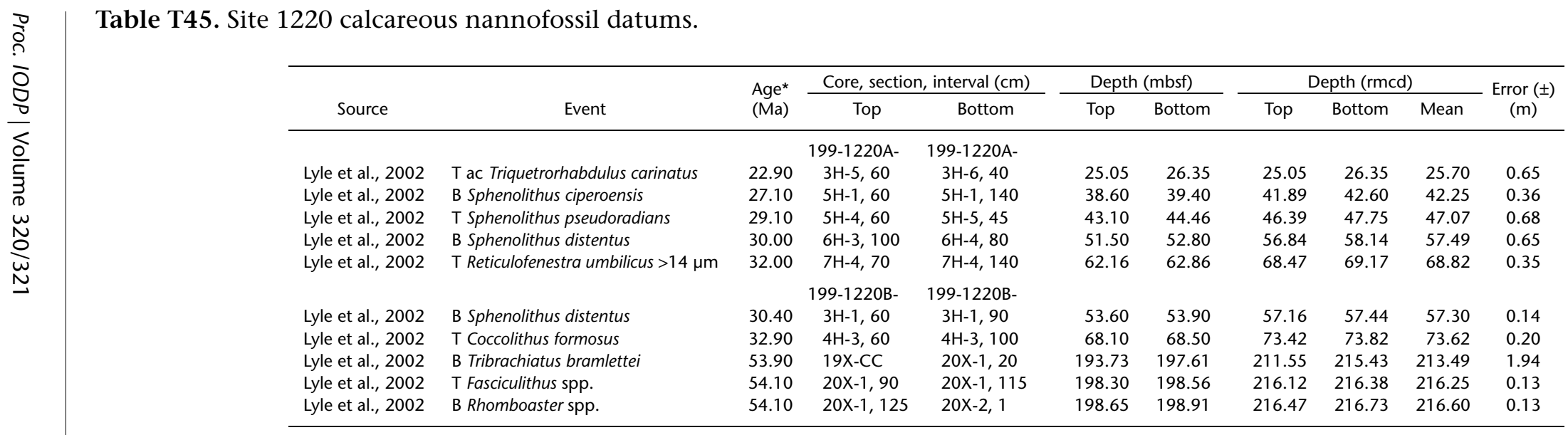

* = Expedition 320/321 Scientists (2010a). T = top, B = bottom, ac = acme. 